\title{
tst 2 (2)

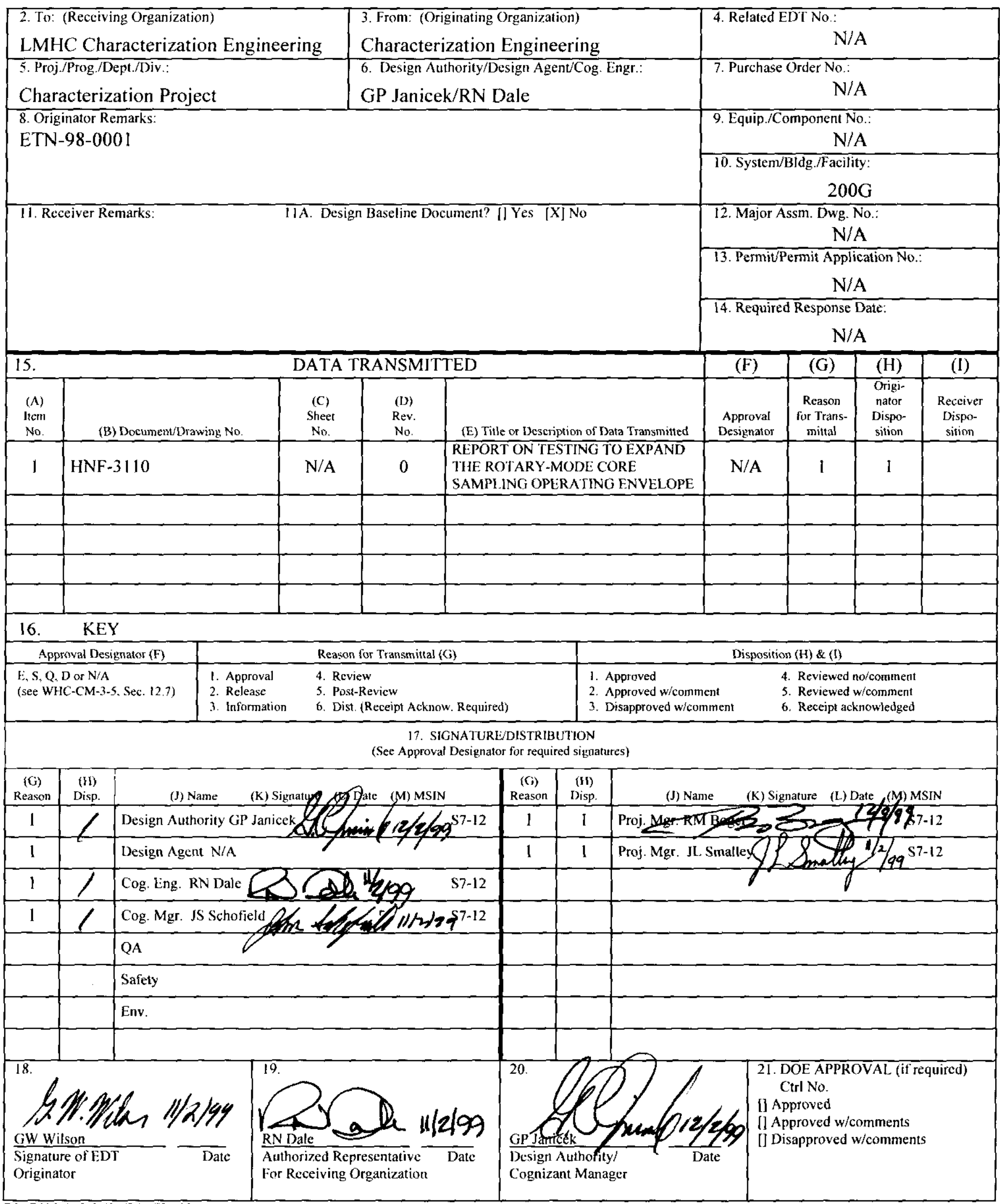




\title{
REPORT ON TESTING TO EXPAND THE ROTARY MODE CORE SAMPLING OPERATING ENVELOPE
}

\author{
R. M. Boger \\ Lockheed Martin Hanford Corporation \\ Richland, WA 99352 \\ U.S. Department of Energy Contract DE-AC06-96RL13200 \\ EDT/ECN: 627874 \\ UC: 2070 \\ Org Code: 74900 \\ Charge Code: 102250/BO00 \\ B\&R Code: EW3120074 Total Pages: $28+202$
}

Key Words: Sampling, Operating Envelope, RMCST

Abstract: This report documents the results of testing to expand the rotary mode core sampling operating envelope.

TRADEMARK DISCI.AIMER. Reference herein to any specific commercial product, process, or service by trade name, trademark, manufacturer, or otherwise, does not necessarily constitute or imply its endorsement, recommendation, or favoring by the United States Government or any agency thereof or its contractors or subcontractors.

ichland WA 99352, Phone (509) 372-2420; Fax (509) 376-4989.

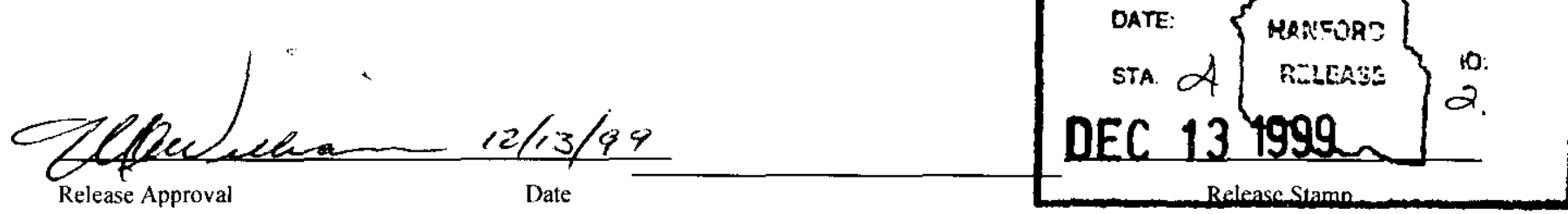

\section{Approved for Public Release}


HNF-3110

Rev. 0

REPORT ON TESTING TO EXPAND THE ROTARY-MODE CORE SAMPLING OPERATING ENVELOPE

December 1999

Keith S. Witwer, Mechanical Engineer

Engineering Testing Laboratory

Numatec Hanford Corporation

Richland, Washington 
HNF-3110

Rev. 0

\section{TABLE OF CONTENTS}

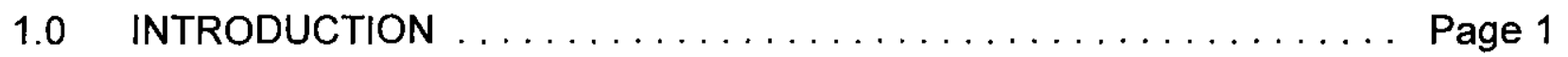

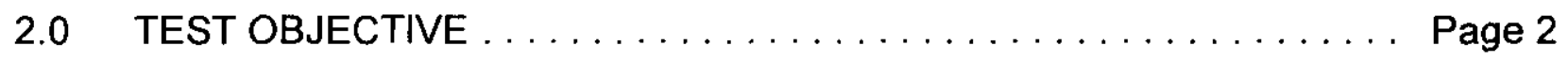

3.0 TEST EQUIPMENT $\ldots \ldots \ldots \ldots \ldots \ldots \ldots \ldots \ldots \ldots \ldots \ldots \ldots \ldots \ldots \ldots$

3.1 CORE DRILLING MACHINE $\ldots \ldots \ldots \ldots \ldots \ldots \ldots \ldots \ldots$ Page 3

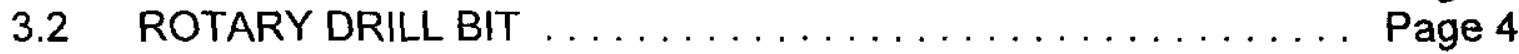

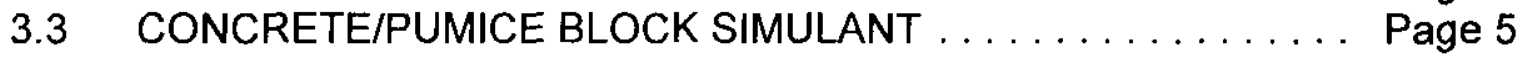

3.4 STEEL PLATE $\ldots \ldots \ldots \ldots \ldots \ldots \ldots \ldots \ldots \ldots \ldots \ldots \ldots \ldots \ldots \ldots \ldots$

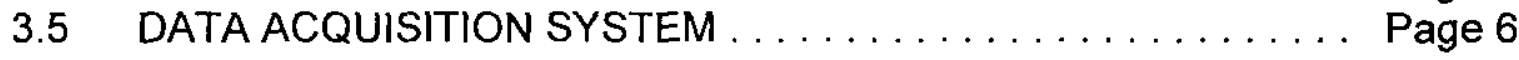

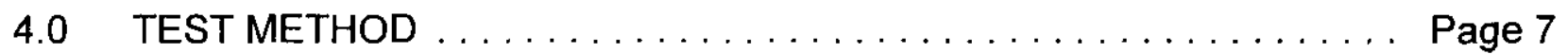

4.1 PUMICE BLOCK $\ldots \ldots \ldots \ldots \ldots \ldots \ldots \ldots \ldots \ldots \ldots \ldots \ldots \ldots \ldots \ldots \ldots$

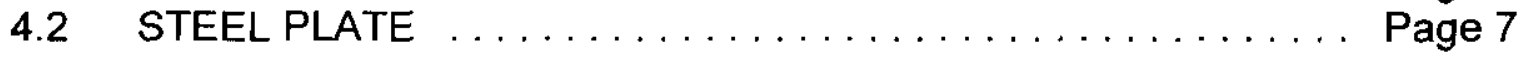

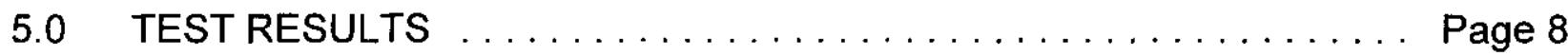

5.1 PRELIMINARY TESTS $\ldots \ldots \ldots \ldots \ldots \ldots \ldots \ldots \ldots \ldots \ldots$ Page 8

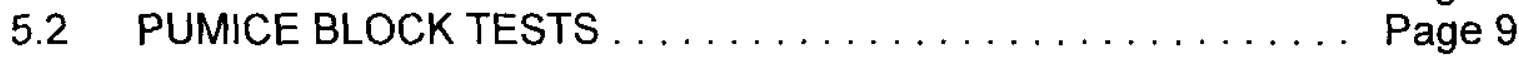

6.0 CONCLUSIONS $\ldots \ldots \ldots \ldots \ldots \ldots \ldots \ldots \ldots \ldots \ldots \ldots$ Page 13

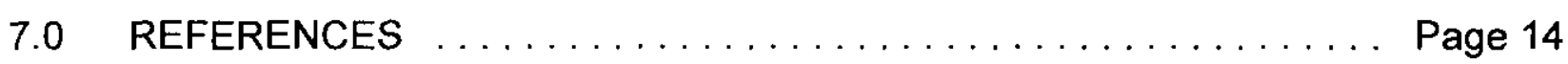

APPENDIX A - 900 lbf TEST PLOTS: DOWN FORCE VS TEMPERATURE . Page A-1 APPENDIX B - CONTROLLED LOGBOOK NOTES ............. Page B-1 APPENDIX C - SIMULANT THERMAL CONDUCTIVITY RESULTS ....... Page C-1 APPENDIX D - EQUIPMENT CALIBRATION DATA ............ Page D-1 APPENDIX E - TEST DATA FILES $\ldots \ldots \ldots \ldots \ldots \ldots \ldots \ldots \ldots$ Page E-1 
HNF-3110

Rev. 0

\section{LIST OF FIGURES}

Figure 1. RMCS Test Drilling Machine $\ldots \ldots \ldots \ldots \ldots \ldots \ldots \ldots \ldots \ldots$ Page 3

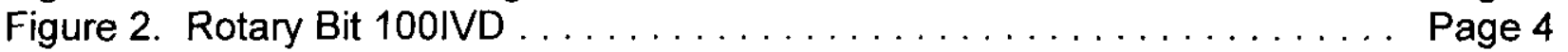

Figure 3. Concrete/Pumice Block Simulant ................ Page 5

Figure 4. 100IVD Bit in ASTM A36 Steel Plate ................ Page 6

\section{LIST OF TABLES}

Table 1. Preliminary Tests in Pumice Simulant ............... Page 8

Table 2. Test in Steel Plate . . . . . . . . . . . . . . . . . . Page 9

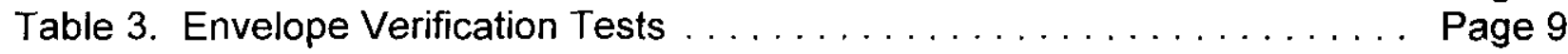

Table 4. Envelope Test Results . . . . . . . . . . . . . . . . . . . . Page 11 
HNF-3110

Rev. 0

\section{REPORT ON TESTING TO EXPAND THE ROTARY-MODE CORE SAMPLING OPERATING ENVELOPE}

\subsection{INTRODUCTION}

The Tank Waste Remediation System (TWRS) Characterization Equipment Group requested that the Numatec Hanford Corporation - Engineering Testing Laboratory (ETL) perform Rotary Mode Core Sampling (RMCS) Operating Envelope (OE) testing. This testing was based upon Witwer 1998a and was performed at different time periods between May and September 1998.

The RMCS OE defines limiting drilling parameters such as the maximum downward force, maximum rotational speed, minimum penetration rate and minimum purge gas volumetric flow rate allowed during drilling operations of the Hanford Core Sampling Trucks. The current OE was developed in 1996 at the ETL by observing the temperature rise on the rotary drill bit face while drilling into a selected waste simulant. Previous OE testing (Ralston and Witwer 1996), which helped define the current OE, identified a concrete/pumice block material as a "bounding" waste simulant. It was considered "bounding" because of key material properties such as its lower thermal conductivity and higher compressive strength compared to the known properties of hard tank waste.

The maximum waste temperature in single-shell tanks (SST) is considered to be approximately $90^{\circ} \mathrm{C}$, and the critical drill bit temperature, which is the temperature at which an exothermic reaction could be initiated in the tank waste, was previously determined to be $150^{\circ} \mathrm{C}$ (Waste Tank Safety Analysis Team 1996). Thus, the drill bit temperature increase was limited to $60^{\circ} \mathrm{C}$ for envelope testing. Any temperature increase seen in testing using the "bounding" concrete/pumice simulant under a specific OE would, therefore, be higher than the increase seen during drilling into actual waste material under the same $\mathrm{OE}$. The previous $\mathrm{OE}$ testing identified a value of $750 \mathrm{lb}$ down force at $55 \mathrm{rpm}$ at $30 \mathrm{scfm}$ purge gas to be the maximum down force, maximum bit rotational speed, and minimum purge gas flow rate at which the drill bit could be operated and still remain within the $60^{\circ} \mathrm{C}$ temperature rise.

It was surmised that higher down forces could increase the ability of the sampling system to penetrate harder waste forms and improve sample recovery. This report describes $O E$ testing where higher down forces were applied while attempting to stay below the maximum $60^{\circ} \mathrm{C}$ temperature rise--with the intent to expand the $\mathrm{OE}$.

This testing immediately followed other testing, RMCSS Bit Improvement, which was done in an attempt to improve sample recovery by experimenting with other rotary drill bit designs. The results of that testing helped define the testing to be done here, and the results are documented in Witwer 1998b. Some results from that testing are discussed in this document. 
HNF-3110

Rev. 0

\subsection{TEST OBJECTIVE}

The purpose of this testing was to raise the maximum down force limit for rotary mode core sampling as outlined in the current $O E$. If testing could show that a higher down force could be used while drilling into a concrete/pumice block simulant while still remaining below the $60^{\circ} \mathrm{C}$ limitation, then the current $O E$ could be revised to include the new, higher, down force limit. Although the Test Plan discussed varying the purge flow rate and rotation rate to find "optimal" drilling conditions, the number of drill bits that could be destructively tested was limited. Testing was subsequently limited in scope such that only the down force would be varied while the purge flow rate and rotation rate were kept constant at $30 \mathrm{scfm}$ and $55 \mathrm{rpm}$ respectively.

A second objective, which was not part of the original test plan, was added prior to testing. The Bit Improvement testing, mentioned previously, revealed that the drill bits tested in the OE testing were made of a slightly different metal matrix than the ones currently used. The older bits, a Longyear ${ }^{1}$ part number $100 \mathrm{IVD} / 5$ (/5 bit), had tungsten carbide mixed into the metal matrix that forms the cutting teeth. The currently used bits, Longyear part number 100IVD/8 (/8 bit), instead have tungsten metal in the matrix and no tungsten carbide. Rockwell $\mathrm{C}$ hardness testing showed that the $/ 5$ bit was significantly harder than the $/ 8 \mathrm{bit}$, with values of $18 \mathrm{vs} .8$, respectively. The change from the $/ 5$ bit to the $/ 8$ bit was made immediately after the previous OE testing in 1996 because of sparking concerns with the tungsten carbide in the $/ 5$ bit. This difference in hardness between the two bit materials was discovered in the Bit Improvement Testing and was expected to affect this $O E$ testing. The second objective, therefore, was to quantify what affect this change in material had and define the $O E$, based on the current $/ 8$ bit design rather than the old $/ 5$ bit design. 
HNF-3110

Rev. 0

\subsection{TEST EQUIPMENT}

\subsection{CORE DRILLING MACHINE}

The drilling machine used was a Longyear Model $34^{1}$. This testing drill, in its basic form, is the same type of drill installed on the core sampling trucks for field operations.

The drill rig is shown in Figure 1.

Figure 1. RMCS Test Drilling Machine

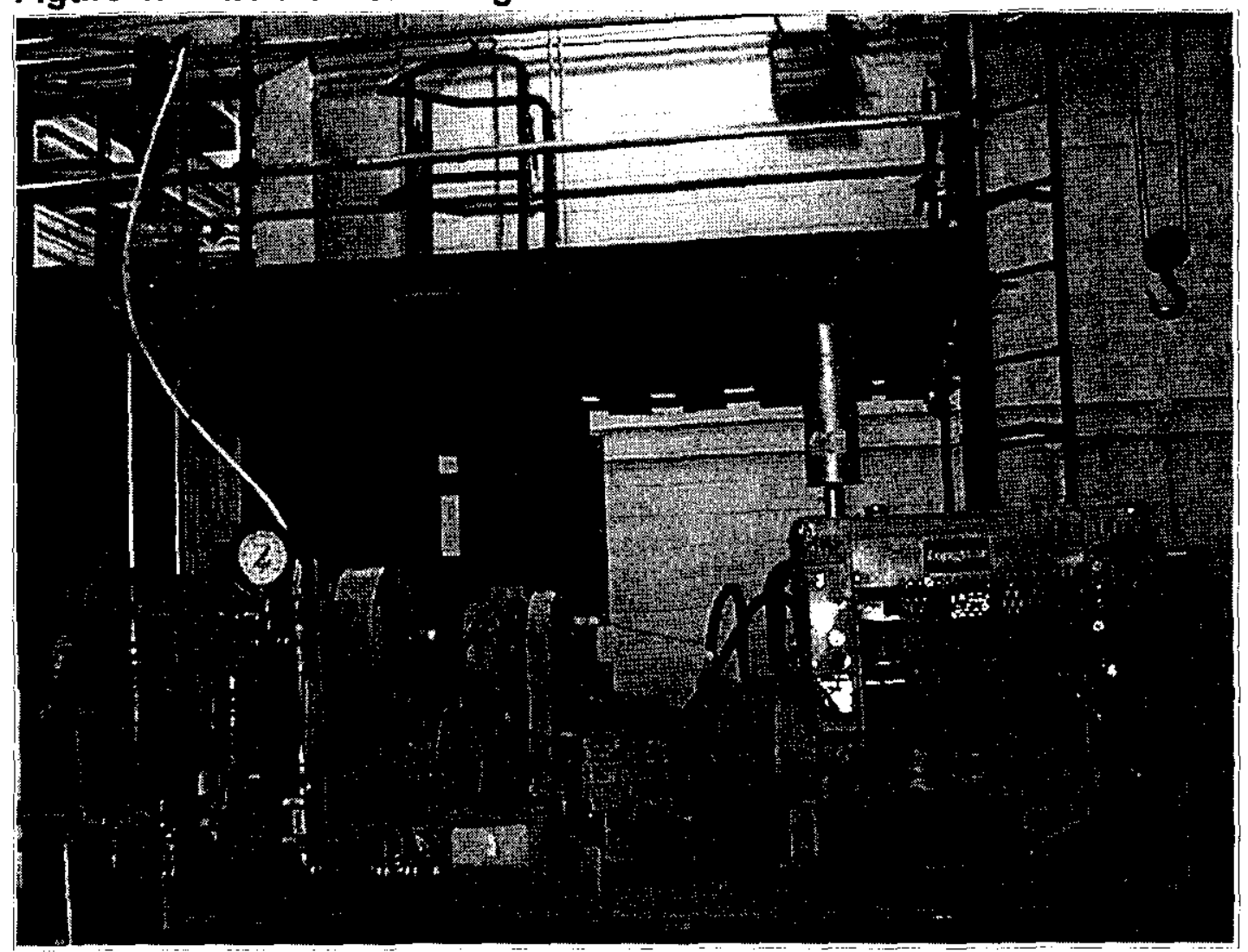

${ }^{1}$ Longyear is a trademark of Boart Longyear Incorporated 


\subsection{ROTARY DRILL BIT}

The drill bit used for the majority of this testing was Longyear part number 100IVD/8, shown in Figure 2. The bit is composed of a sintered bronze and tungsten metal matrix. Each bit tested had a unique serial number and received a QC inspection when received. Each bit had a 0.040 " diameter, grounded junction, stainless steel thermocouple silver soldered in the top section of one of the leading cutting teeth. The thermocouple was characterized, after silver soldering, in both a freezing and a boiling water bath to verify its performance - prior to testing. The record of calibration for these thermocouples is included in the log book entries found in Appendix B.

Four of the six purge holes were intentionally sealed with silver solder for all of the tests except one - BT1TST10. BT1TST10 was run with all the purge holes left open. Four purge holes could conceivably be plugged during field sampling operations and still allow $30 \mathrm{scfm}$ flow through the remaining two holes. Having the four holes plugged was previously determined to be a bounding condition in terms of maximum temperature rise, and a requirement was made that all remaining bits to be tested were to have four purge holes sealed. The thermocouple

Figure 2. Rotary Bit 100IVD/8

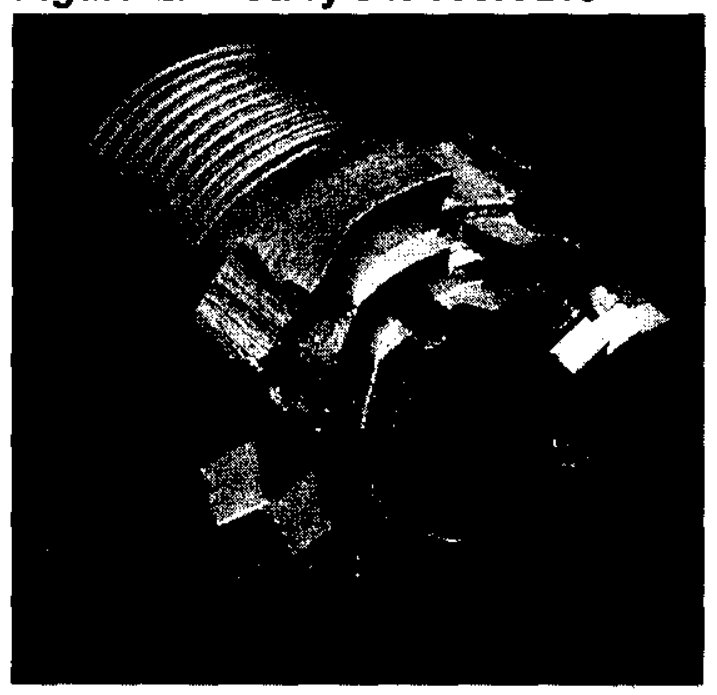
attached to the face of the drill bit was to be in the area of the bit where the least amount of purge gas was present, in the center of the silver-soldered section. This was the hottest part of the bit during drilling and, therefore, the location of interest for envelope testing.

As mentioned earlier, previous envelope testing used a Longyear 100IVD/5 (/5) drill bit, which is identical in geometry to the $/ 8$ bit but differs in the metal matrix used to form the cutting teeth. 


\title{
3.3 CONCRETE/PUMICE BLOCK SIMULANT
}

\begin{abstract}
A commercially available concrete and pumice block, shown in Figure 3, was used as the test simulant. Previous envelope testing (Ralston and Witwer1996) used this same type of material; but thermal conductivity tests (Appendix C) of representative samples from the current and previous simulant differed by about $23 \%$. The previously used simulant had a thermal conductivity of $0.969 \pm 0.094$

Figure 3. Concrete/Pumice Block Simulant

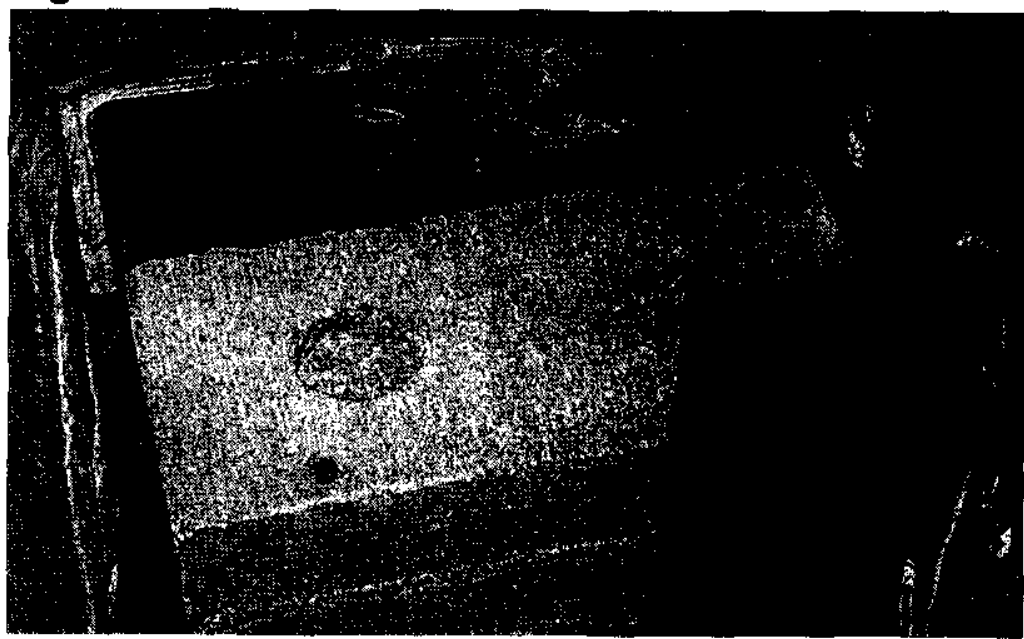

$\mathrm{W} / \mathrm{m} / \mathrm{K}$, while the simulant used in this testing had a lower thermal conductivity of $0.748 \pm 0.055 \mathrm{~W} / \mathrm{m} / \mathrm{K}$. This lower conductivity value caused the simulant used in this testing to lose applied heat more slowly than the previous concrete/pumice simulant. This effectively caused the drill bit to reach higher temperatures and to reach them sooner with the current concrete/pumice simulant, under otherwise identical conditions.
\end{abstract}




\subsection{STEEL PLATE}

One penetration test of the / 8 drill bit against ASTM A36 carbon steel plate was also performed. This test was run because of the uncertainty associated with the difference in bit material in the $/ 5 \mathrm{vs}$. $/ 8$ bit. Previous OE testing with the $/ 5$ bit included drilling into a steel plate to confirm that the bit would not penetrate more than $1 / 16$ " when drilling at the maximum envelope values of $55 \mathrm{rpm}, 750 \mathrm{lbf}$, and $30 \mathrm{scfm}$. The softer matrix of the $/ 8$ bit was expected to penetrate even less than the $/ 5$ bit, but one test with a /8 bit was run to confirm this. A photo of this test is shown in Figure 4.

Figure 4. 100IVD Bit in ASTM A36 Steel Plate

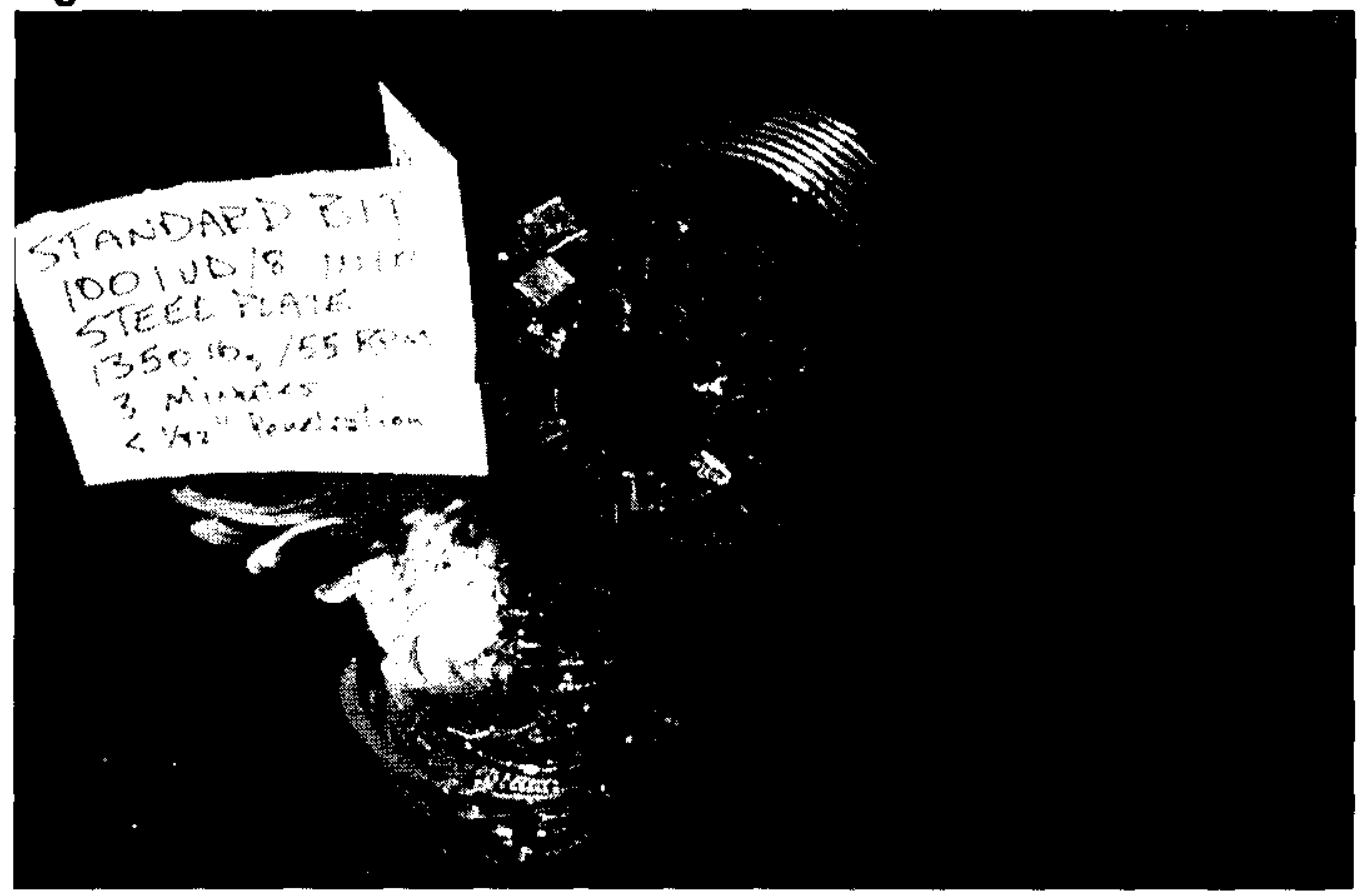

\subsection{DATA ACQUISITION SYSTEM}

A micro-computer-based data acquisition system acquired data from various sensors mounted on the drill rig. The data from these sensors included the following:

- Bit rpm

- Downward Force

- Bit Depth

- Torque

- $\quad$ Bit Tooth Temperature

- $\quad$ Purge Gas Temperature, Pressure, and Flow Rate 
HNF-3110

Rev. 0

Appendix $\mathrm{D}$ contains pertinent calibration information on these sensors.

Information was recorded to a computer file for each test at one second

intervals. These raw data files are included in Appendix E.

\subsection{TEST METHOD}

Following is a brief description of the method used for testing. A more detailed description can be found in the associated test plan (Witwer 1998a.)

\subsection{PUMICE BLOCK}

With a stack of pumice blocks placed below the drill string, the instrumented drill bit was lowered to the surface of the top pumice block. The down force control, purge flow rate, and bit rotation rate were all set to the desired values. A unique test name was input to the data acquisition system, and recording was started. Drilling was then started, and the test was allowed to run until the bit stopped penetrating (due to the bit teeth wearing down)--plus an additional two more minutes of drilling to monitor bit temperature rise. Purge flow, rotation rate, and down force were monitored and controlled throughout each test. The parameter of interest for this testing was the maximum drill bit temperature reached.

\subsection{STEEL. PLATE}

For the test involving drilling against A36 carbon steel plate, the procedure was identical to drilling into pumice block except that a guide-pipe fixture was used to keep the drill bit centered. The geometry of the drill bit face, combined with the high down force and rotation rate, caused the drill bit to tend to "walk" around the steel plate rather than stay centered. The inner diameter of the guide-pipe fixture was just slightly bigger than the outer diameter of the drill bit. With the guide-pipe firmly attached to the drill machine frame, the drill bit was kept centered during drilling. It was observed that this method of keeping the drill bit centered was more conservative, in terms of measuring damage done to the steel plate, than if the bit were allowed to cut over a larger area. This was because the drill bit would work against the same location on the steel plate, subsequently causing deeper cuts in one location rather than shallow cuts in many locations.

The purpose of this testing was to verify that the drill bit could not penetrate more than $1 / 16$ " into the steel plate (which simulated the steel liner of a radioactive waste tank). 
HNF-3110

Rev. 0

\subsection{TEST RESULTS}

\subsection{PRELIMINARY TESTS}

Table 1 shows pertinent information about preliminary tests in the pumice block simulant. A test (BT1TST10) with the /8 bit was run without any purge holes sealed to provide baseline data. This was the only test run with all the purge holes open and correspondingly showed the lowest temperature rise of any test run.

One test (BT1TST11) with the old /5 bit was run at $750 \mathrm{lbf}, 55 \mathrm{rpm}$, and $30 \mathrm{scfm}$ in the new concrete/pumice simulant. This test provided baseline information to compare against previous envelope testing which also used the $/ 5$ bit - but in the old concrete/pumice block simulant. This test showed that the 15 bit tested with the new simulant produced a higher maximum bit temperature than it did in the old concrete/pumice simulant. A maximum temperature rise of $75^{\circ} \mathrm{C}$ was seen with the new pumice block compared to a maximum temperature rise of $40^{\circ} \mathrm{C}$ seen in earlier envelope testing with the old pumice block. These results support the prediction mentioned in Section 3.3 that higher temperatures would occur in the new pumice block because of its lower thermal conductivity.

Table 1. Preliminary Tests in Pumice Simulant

\begin{tabular}{|l|l|l|l|l|l|l|}
\hline TEST NAME & BIT STYLE & $\begin{array}{l}\text { MATERIAL } \\
\text { DRILLED }\end{array}$ & $\begin{array}{c}\text { DOWN } \\
\text { FORCE } \\
(\text { LBF })\end{array}$ & RPM & $\begin{array}{c}\text { PURGE } \\
\text { FLOW } \\
(\text { SCFM) })\end{array}$ & $\begin{array}{c}\text { MAX } \\
\text { TEMP } \\
\text { Rise }\left({ }^{\circ} \mathrm{C}\right)\end{array}$ \\
\hline BT1TST10 & $\begin{array}{l}18, \text { All } 6 \\
\text { purge holes } \\
\text { open }\end{array}$ & $\begin{array}{l}\text { Pumice } \\
\text { Block }\end{array}$ & 750 & 55 & 30 & 23 \\
\hline BT1TST11 & $\begin{array}{l}154 \text { purge } \\
\text { holes } \\
\text { plugged }\end{array}$ & $\begin{array}{l}\text { Pumice } \\
\text { Block }\end{array}$ & 750 & 55 & 30 & 75 \\
\hline
\end{tabular}

Table 2 shows the result of a test of the $/ 8$ bit run at $1350 \mathrm{lbf}$ and $55 \mathrm{rpm}$ against the A36 steel plate for four minutes. A value of $1350 \mathrm{lbf}$ was chosen because (1) This is the maximum allowable down force for drilling (per Bureau of Mines Ignitability Testing), and it was desired that a single test provide bounding data and not need to be repeated, and (2) there was high confidence that the drill bit would not penetrate the steel plate even at this high down force because of the bit material properties.

Depth measurements showed the deepest penetration at 0.015 " below the surface. This was well below the $0.0625^{\prime \prime}$ maximum depth allowed in TWRS safety documentation. 
HNF-3110

Rev. 0

Table 2. Test in Steel Plate

\begin{tabular}{|l|l|l|l|l|l|l|}
\hline TEST NAME & BIT STYLE & $\begin{array}{l}\text { MATERIAL } \\
\text { DRILLED }\end{array}$ & $\begin{array}{c}\text { DOWN } \\
\text { FORCE } \\
\text { (LBF) }\end{array}$ & RPM & $\begin{array}{c}\text { PURGE } \\
\text { FLOW } \\
\text { (SCFM) }\end{array}$ & $\begin{array}{c}\text { MAX } \\
\text { DEPTH } \\
\text { IN STEEL } \\
\text { PLATE } \\
\text { (INCHES) }\end{array}$ \\
\hline BT1TST13 & $\begin{array}{l}18,4 \text { purge } \\
\text { holes } \\
\text { plugged }\end{array}$ & $\begin{array}{l}\text { A36 Steel } \\
\text { Plate }\end{array}$ & 1350 & 55 & 0 & .015 \\
\hline
\end{tabular}

\subsection{PUMICE BLOCK TESTS}

Three tests (BT1TST12, 14, 15) were run at $750 \mathrm{lbf}, 55 \mathrm{rpm}$, and $30 \mathrm{scfm}$ with the $/ 8$ bit having four of the six purge holes plugged. These three were run primarily to verify that the values used in the current $O E$ with the $/ 8$ bit were appropriate. The maximum temperature rises during the tests were $33^{\circ} \mathrm{C}, 44^{\circ} \mathrm{C}$, and $45^{\circ} \mathrm{C}$ respectively well below the maximum allowed $60^{\circ} \mathrm{C}$ rise. These results are shown in Table 3 .

Table 3. Envelope Verification Tests

\begin{tabular}{|l|l|l|l|l|l|l|}
\hline TEST NAME & BIT STYLE & $\begin{array}{l}\text { MATERIAL } \\
\text { DRILLED }\end{array}$ & $\begin{array}{c}\text { DOWN } \\
\text { FORCE } \\
\text { (LBF) }\end{array}$ & RPM & $\begin{array}{c}\text { PURGE } \\
\text { FLOW } \\
\text { (SCFM) }\end{array}$ & $\begin{array}{c}\text { MAX } \\
\text { TEMP. } \\
\left.\text { Rise ( }{ }^{\circ} \mathrm{C}\right)\end{array}$ \\
\hline BT1TST12 & $\begin{array}{l}18,4 \text { purge } \\
\text { holes } \\
\text { plugged }\end{array}$ & $\begin{array}{l}\text { Pumice } \\
\text { Block }\end{array}$ & 750 & 55 & 30 & 33 \\
\hline BT1TST14 & $\begin{array}{l}18,4 \text { purge } \\
\text { holes } \\
\text { plugged }\end{array}$ & $\begin{array}{l}\text { Pumice } \\
\text { Block }\end{array}$ & 750 & 55 & 30 & 44 \\
\hline BT1TST15 & $\begin{array}{l}18,4 \text { purge } \\
\text { holes } \\
\text { plugged }\end{array}$ & $\begin{array}{l}\text { Pumice } \\
\text { Block }\end{array}$ & 750 & 55 & 30 & 45 \\
\hline
\end{tabular}

A test was then run at $1000 \mathrm{lbf}, 55 \mathrm{rpm}$, and $30 \mathrm{scfm}$ for four minutes with the down force increased to $1350 \mathrm{lbf}$ for six additional minutes. The maximum temperature recorded was $38^{\circ} \mathrm{C}$ at $1000 \mathrm{lbf}$ and $46^{\circ} \mathrm{C}$ at $1350 \mathrm{lbf}$. Three more tests were run to verify the results with maximum temperatures recorded at $44^{\circ} \mathrm{C}, 44^{\circ} \mathrm{C}$, and $53^{\circ} \mathrm{C}$. Seven days passed until an additional confirmatory test was run. This next test (SN10776) was invalidated because the thermocouple wires were improperly 
connected to the drill bit. An additional confirmatory test (SN10759) was run at the same drilling parameters, but a maximum temperature rise of $70^{\circ} \mathrm{C}$ was recorded. This was $17^{\circ} \mathrm{C}$ higher than any test yet run at $1350 \mathrm{lbf}, 55 \mathrm{rpm}$, and $30 \mathrm{scfm}$. Attempts were made to identify a cause for the dramatic increase but the only change between tests that could be found was a crack running across the bottom diameter of the hole in the pumice block, introduced during the last test. The crack could have created an edge against which the drill bit could drag, creating friction and, ultimately, more heat. Further analysis of torque values recorded during each test revealed that the increased temperature corresponded with an increased torque value. This is graphically represented in Figure 5. The corresponding change in torque value confirms that a physical property change in either the simulant or the drill bit was the cause of the temperature change. Boart Longyear, the manufacturer of the drill bit, claims to control material properties such that the drill bits were probably not the cause of the change. Either way, the test showed that too high a temperature could be reached at the $1350 \mathrm{lbf}$ down force.

Figure 5. Drilling Torque Comparison

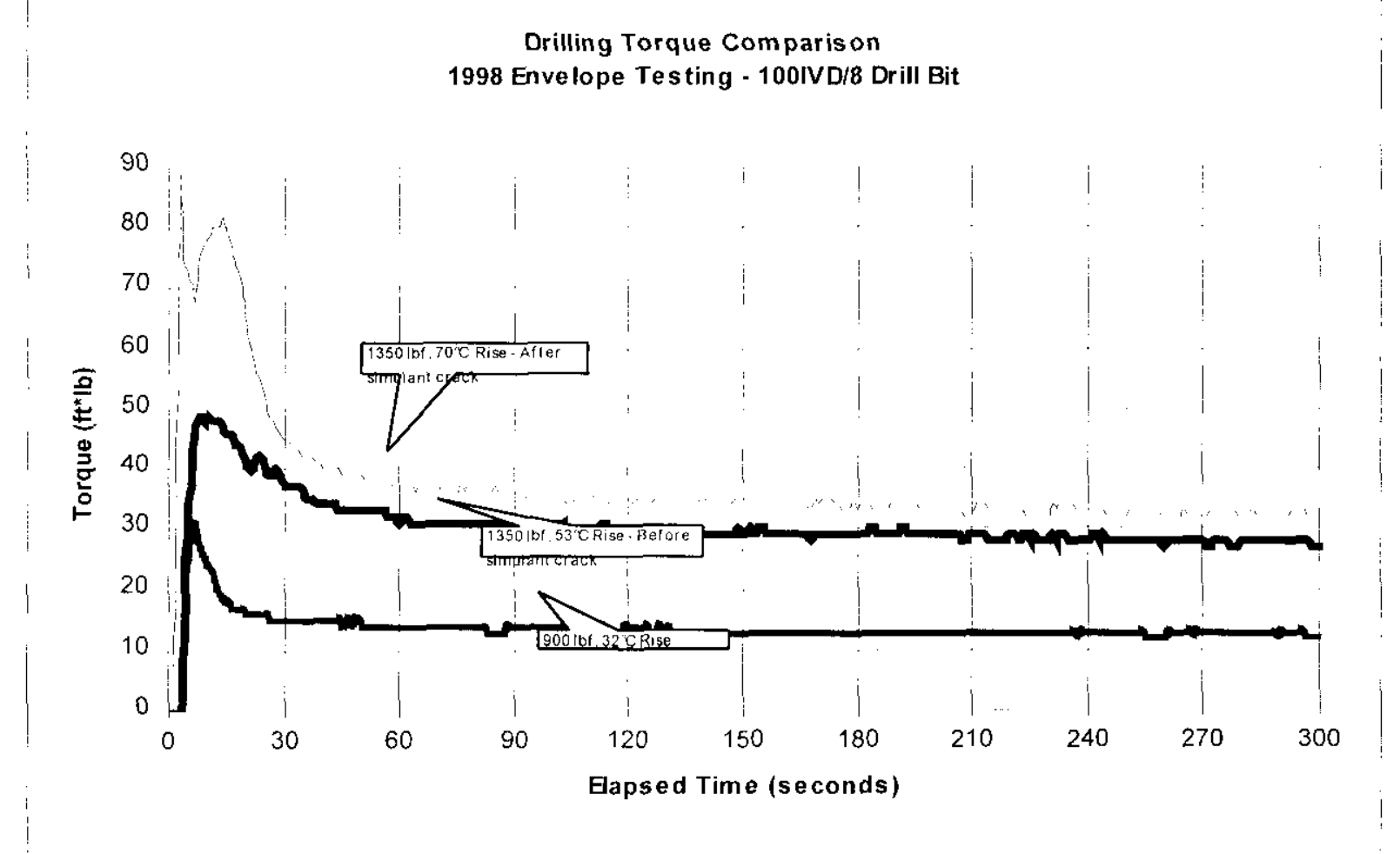


HNF-3110

Rev. 0

Several more tests were run with this cracked pumice block at incrementally decreasing down forces to determine the highest possible down force which could be used and still remain below the $60^{\circ}$ limit. Testing at $1350 \mathrm{lbf}, 1170 \mathrm{lbf}$, and $1000 \mathrm{lbf}$ were successfully run, but five repeat runs at any of these values were not completed without exceeding the $60^{\circ} \mathrm{C}$ limit. With the down force reduced to $900 \mathrm{lbs}$, five runs were successfully completed without exceeding the $60^{\circ} \mathrm{C}$ limit. Table 4 summarizes these test results.

Table 4. Envelope Test Results

\begin{tabular}{|c|c|c|c|c|c|c|}
\hline TEST NAME & BIT STYLE & $\begin{array}{l}\text { MATERIAL } \\
\text { DRILLED }\end{array}$ & $\begin{array}{c}\text { DOWN } \\
\text { FORCE } \\
\text { (LBF) }\end{array}$ & RPM & $\begin{array}{l}\text { PURGE } \\
\text { FLOW } \\
\text { (SCFM) }\end{array}$ & $\begin{array}{l}\text { MAX } \\
\text { TEMP. } \\
\text { Rise }\left({ }^{\circ} \mathrm{C}\right)\end{array}$ \\
\hline SN10764 & $\begin{array}{l}18,4 \text { purge } \\
\text { holes } \\
\text { plugged }\end{array}$ & $\begin{array}{l}\text { Pumice } \\
\text { Block }\end{array}$ & $\begin{array}{l}1000 \\
1350\end{array}$ & 55 & 30 & $\begin{array}{l}38 \\
46\end{array}$ \\
\hline SN76360 & $\begin{array}{l}18,4 \text { purge } \\
\text { holes ugged }\end{array}$ & $\begin{array}{l}\text { Pumice } \\
\text { Block }\end{array}$ & 1350 & 55 & 30 & 44 \\
\hline SN10781 & $\begin{array}{l}18,4 \text { purge } \\
\text { holes } \\
\text { plugged }\end{array}$ & $\begin{array}{l}\text { Pumice } \\
\text { Block }\end{array}$ & 1350 & 55 & 30 & 44 \\
\hline SN76358 & $\begin{array}{l}18,4 \text { purge } \\
\text { holes } \\
\text { plugged }\end{array}$ & $\begin{array}{l}\text { Pumice } \\
\text { Block }\end{array}$ & 1350 & 55 & 30 & 53 \\
\hline SN10776 & $\begin{array}{l}18,4 \text { purge } \\
\text { holes } \\
\text { plugged }\end{array}$ & $\begin{array}{l}\text { Pumice } \\
\text { Block }\end{array}$ & 1350 & 55 & 30 & $\begin{array}{l}\text { BAD } \\
\text { TEST* }\end{array}$ \\
\hline SN10759 & $\begin{array}{l}18,4 \text { purge } \\
\text { holes } \\
\text { plugged }\end{array}$ & $\begin{array}{l}\text { Pumice } \\
\text { Block }\end{array}$ & 1350 & 55 & 30 & 70 \\
\hline SN10777 & $\begin{array}{l}/ 8,4 \text { purge } \\
\text { holes } \\
\text { plugged }\end{array}$ & $\begin{array}{l}\text { Pumice } \\
\text { Block }\end{array}$ & 1170 & 55 & 30 & 59 \\
\hline SN10756 & $\begin{array}{l}18,4 \text { purge } \\
\text { holes } \\
\text { plugged }\end{array}$ & $\begin{array}{l}\text { Pumice } \\
\text { Block }\end{array}$ & 1000 & 55 & 30 & 63 \\
\hline
\end{tabular}


HNF-3110

Rev. 0

\begin{tabular}{|l|l|l|l|l|l|l|}
\hline TEST NAME & BIT STYLE & $\begin{array}{l}\text { MATERIAL } \\
\text { DRILLED }\end{array}$ & $\begin{array}{c}\text { DOWN } \\
\text { FORCE } \\
\text { (LBF) }\end{array}$ & RPM & $\begin{array}{c}\text { PURGE } \\
\text { FLOW } \\
\text { (SCFM) }\end{array}$ & $\begin{array}{c}\text { MAX } \\
\text { TEMP. } \\
\left.\text { Rise }{ }^{\circ} \mathrm{C}\right)\end{array}$ \\
\hline SN10773 & $\begin{array}{l}/ 8,4 \text { purge } \\
\text { holes } \\
\text { plugged }\end{array}$ & $\begin{array}{l}\text { Pumice } \\
\text { Block }\end{array}$ & 900 & 55 & 30 & 32 \\
\hline SN10359 & $\begin{array}{l}/ 8,4 \text { purge } \\
\text { holes } \\
\text { plugged }\end{array}$ & $\begin{array}{l}\text { Pumice } \\
\text { Block }\end{array}$ & 900 & 55 & 30 & 39 \\
\hline SN10782 & $\begin{array}{l}/ 8,4 \text { purge } \\
\text { holes } \\
\text { plugged }\end{array}$ & $\begin{array}{l}\text { Pumice } \\
\text { Block }\end{array}$ & 900 & 55 & 30 & 37 \\
\hline $\begin{array}{l}\text { SN76361 } \\
\text { holes } \\
\text { plugged }\end{array}$ & $\begin{array}{l}\text { Pumice } \\
\text { Block }\end{array}$ & 900 & 55 & 30 & 33 \\
\hline $\begin{array}{l}18,4 \text { purge } \\
\text { holes } \\
\text { plugged }\end{array}$ & $\begin{array}{l}\text { Pumice } \\
\text { Block }\end{array}$ & 900 & 55 & 30 & 34 \\
\hline
\end{tabular}


HNF-3110

Rev. 0

Figure 6 shows a Temperature vs. Downward force plot of Test SN10359, one of the five successful runs at $900 \mathrm{lbf}$. The temperature performance of this piot is typical of each of the five tests at $900 \mathrm{lbs}, 55 \mathrm{rpm}$ and $30 \mathrm{scfm}$. Plots of each of the other four test runs are included in Appendix A.

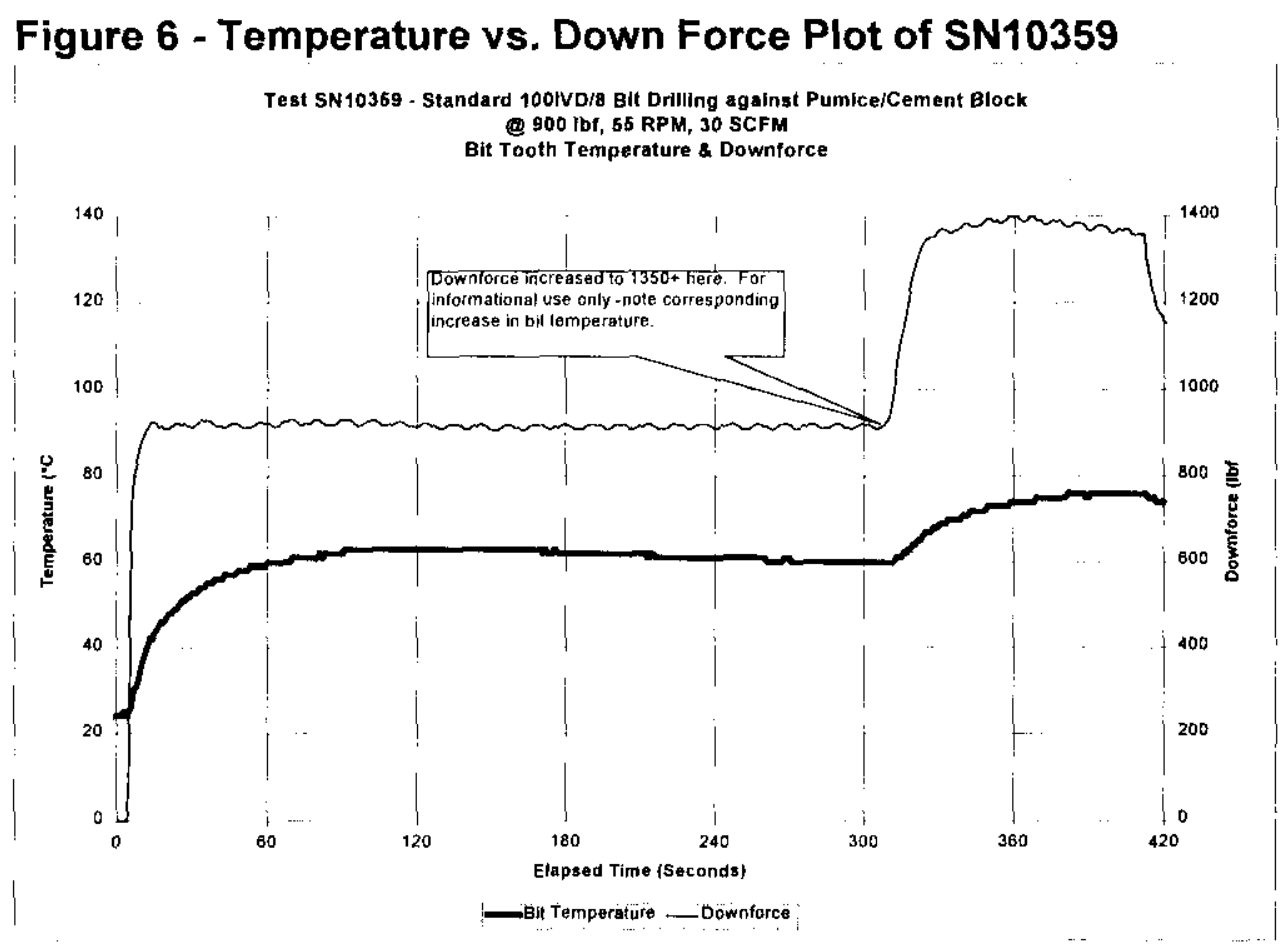

\subsection{CONCLUSIONS}

1. Rotary drilling into the bounding concrete/pumice block material with the current rotary bit (PN 100IVD/8) was possible at $900 \mathrm{lbs}, 55 \mathrm{rpm}$, and $30 \mathrm{scfm}$ purge flow for five repeat test runs while staying below the maximum $60^{\circ} \mathrm{C}$ temperature increase. These results at $900 \mathrm{lbf}$ are a $150 \mathrm{lbf}$ increase over the $750 \mathrm{lbf}$ achieved during previous envelope testing. Temperature results during repeated testing at down forces higher than $900 \mathrm{lbf}$ showed a higher-than- $60^{\circ} \mathrm{C}$ temperature rise.

2. Because of insufficient data and some inconsistencies in test results, changes to the existing $\mathrm{OE}$ are not recommended at this time. Testing with the 100IVD/8 bit did confirm its ability to stay below the maximum allowed $60^{\circ} \mathrm{C}$ temperature rise under the current $\mathrm{OE}$. 
HNF-3110

Rev. 0

3. Because of the many variables encountered, a thermal model should be developed to "correct" this and any future core drilling test data to represent actual waste conditions and properties. This model could be used to predict numerous drilling parameter combinations, followed by limited validation testing to verify the model.

\subsection{REFERENCES}

Ralston, G. L. and K. S. Witwer, 1996, "Standard Rotary Bit Temperature Testing Supporting the Self Assessment for Rotary Mode Core Sampling in Flammable Gas Tanks," WHC-SD-WM-TRP-252, Rev. 0, Westinghouse Hanford Company, Richland, Washington.

Waste Tank Safety Analysis Team, 1996, "A Safety Assessment of Push-Mode and Rotary Mode in Flammable Gas Single Shell Tanks," WHC-SD-WM-SAD-035, Rev. 0, Los Alamos National Laboratory, Los Alamos, New Mexico.

Witwer, K. S., 1998a, "Testing to Expand the Rotary Mode Core Sampling System Operating Envelope," HNF-SD-WM-TP-536, Rev. 0, Numatec Hanford Corporation, Richland, Washington.

Witwer, K. S., 1998b, "Results of No-Flow Rotary Drill Bit Comparison Testing," HNF- 3557, Rev. 0, Numatec Hanford Corporation, Richland, Washington. 
HNF-3110

Rev. 0

APPENDIX A - 900 Ibf TEST PLOTS: DOWN FORCE VS TEMPERATURE 
HNF-3110

Rev. 0

Test SN10773 - Standard 100IVD/8 Bit Drilling against Pumlce/Cement Block (i) 900 Ibf, 55 RPM, 30 SCFM

Bit Tooth Temperature \& Downforce
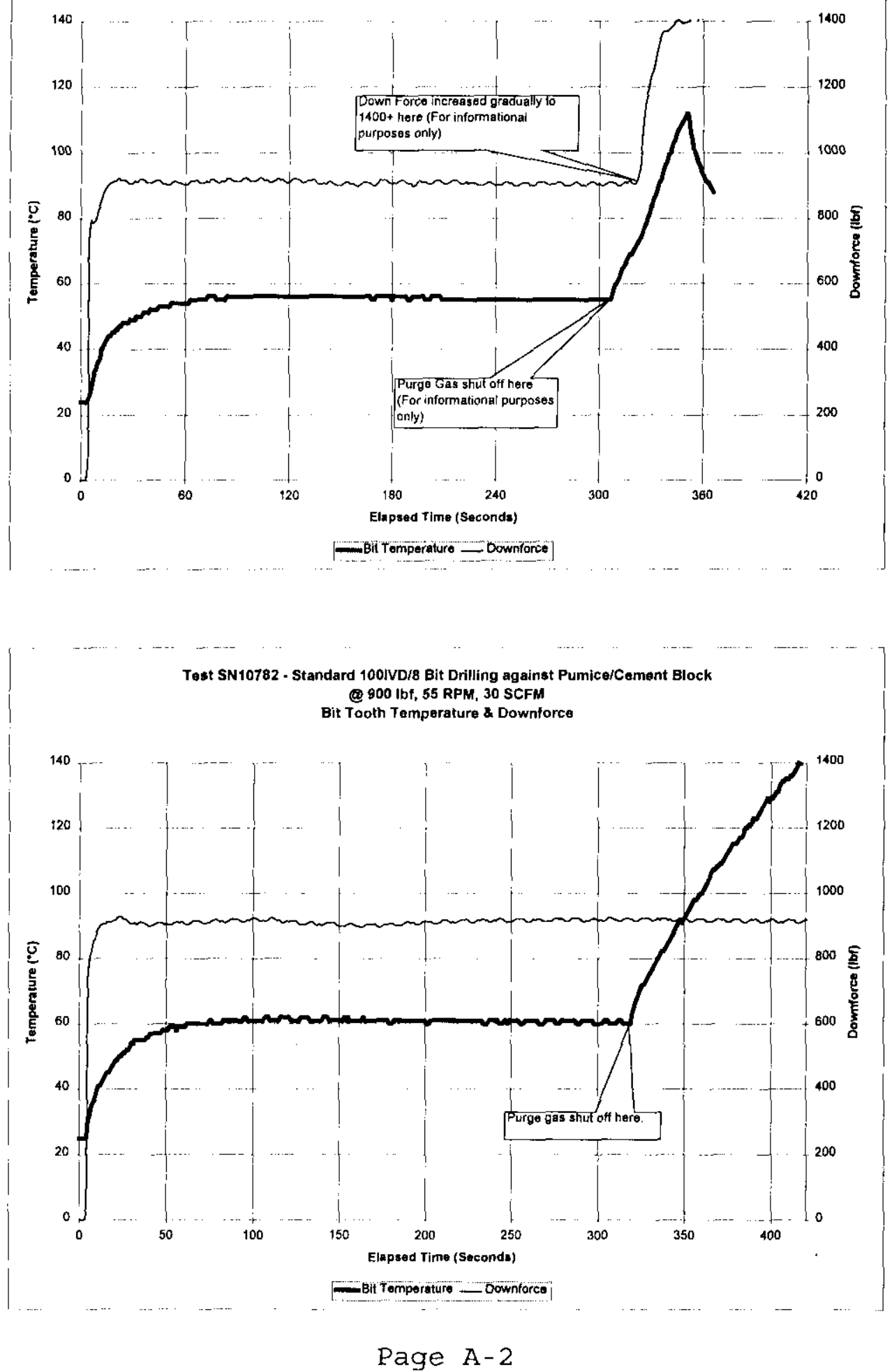
HNF-3110

Rev. 0

Test SN76361 - Standard 100IVD/B Bit Drilling against Pumice/Cement Block

(9) $900 \mathrm{Jbf}, 55$ RPM, 30 SCFM

Bit Jooth Temperature \& Downforce

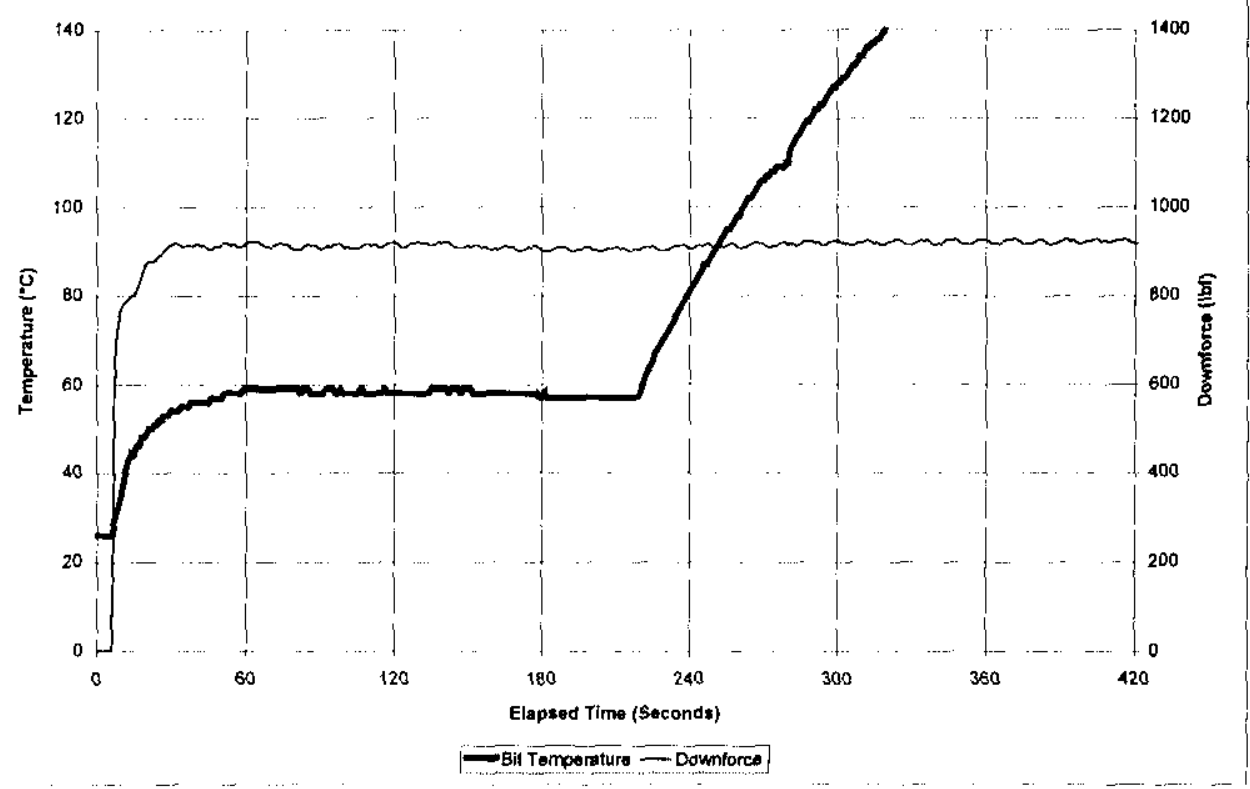

Test SN10763 - Standard 100IVD/B Bit Drilling against Pumice/Cement Block

900 lbf, 55 RPM, 30 SCFM

Bit Tooth Temperature \& Downiorce

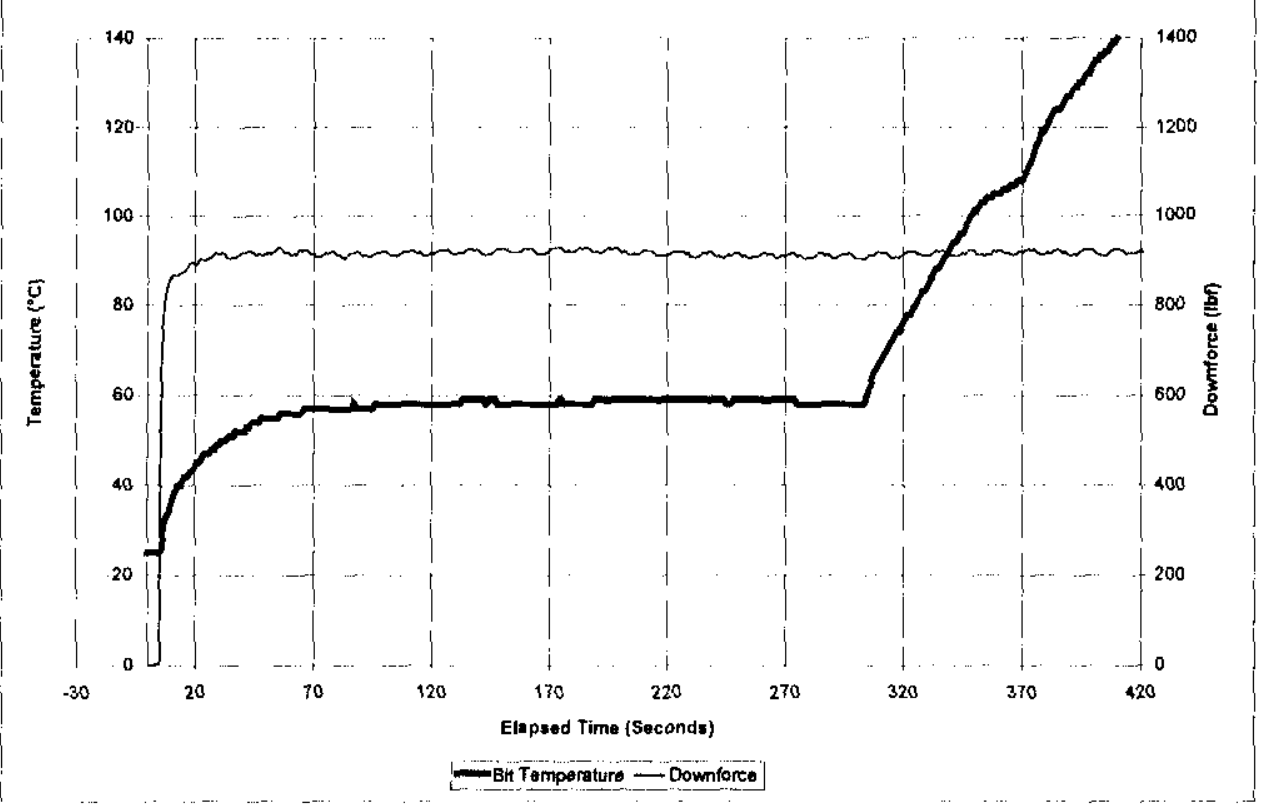

Page $\mathrm{A}-3$ 
HNF-3110

Rev. 0

APPENDIX B - CONTROLLED LOGBOOK NOTES

Page $B-1$ 
HNF-3110

54

Rev. 0

Test Brits io

- Drilling into Pumice block. This simulant is simitar to pumice used For Rm cs Envelope testing (witter \&Ralston) previous done which Found a linting envelope with the following values

-55 RPM Rotational speed (max)

Result

-750 (b down Force (max)

- 30 SCFM Purge gas Flow (min)

Envelope Testing is being feinitiated to try and expand these values to allow greater operator Flexbibilut and therefore improve lit penetration this test will set a baseline value by which all succeeding texts will be based on, ie:-@

750 of downtorce 55 RP M

-BO SCFM

using a 100 wo $/ 8$ Rotary bit with 2 Tope $t 5$ Thermocouple embedded in it the Temperature rise should not be greater than $60^{\circ} \mathrm{C}$.

- Two main reasons for This Test.

1) Pumice blocks are of a slight power thermal conductive Than the ones previously. used $(248 \mathrm{w} / \mathrm{m} / \mathrm{K}$ us $969 \mathrm{w} / \mathrm{m} / \mathrm{K})$

2) Recent hardness checks show the Rot un frill bits now being supplied to be of Rockwell $C$ Hardness $=5-8$ whereas the bits previously used $(1997002)$ showed acts ow (100IUD/5) have a Rockwell C Had ness of 15-18.

These Factors could impact, adversely, the envelope values previously obtained

- test parameters:

- Bit Serial I176356

- TE serial 03 (see dits sheet on following page)

- Downterce $=750$ 16F

$-R M=55$

- Par ge $R_{2 t e}=30$ Sc

page $B-2$ 
- Using Torque sensor Wireless Datz Corportion Madel 124 . to obtin torque volues during d 0 sw test

s Pesult:- Bit tempenature rose From $24^{\circ} \mathrm{C}\left(75^{\circ} \mathrm{F}\right)$ to $47^{\circ} \mathrm{C}\left(117^{\circ} \mathrm{F}\right)$ or a $23^{\circ} \mathrm{C}$ rise. This is well below the $60^{\circ} \mathrm{C}$ maxima temperature rise allowed in RmCs Saft $\rightarrow$ Documentstion.

- Bit lenetrated approximately $1 / a l l$

- Torave measured durina test showed a maxmum value of about $27 \mathrm{ft}$. lbs this occured during the first 15 seconds of drilling while the bit was actually penetrating/cuttiu Atter about 25 seconds the bit stopped penetrating due to the teuth bing worn down and the toraue also dropped down to about I I ft-ibs for the semainder of the test.

- These temperature and torane values are lower than similar tosts done in January 1996. Durring a similar test (ToRQTSTZ1 (@7501b-55PPM, 30 SCFM) the torque values averaged

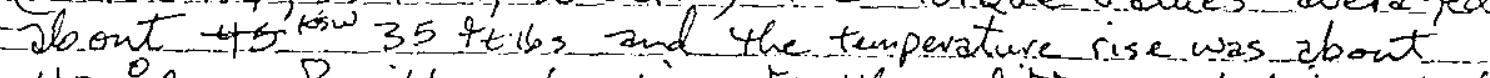
$40{ }^{\circ} \mathrm{C}$ Possible exptanstions for these ditterences between test: are 1) The pumice block composition (now use senced) wears the bit

2) The punice block (used now) has more concrete filler (less punince) and is havder which prevents the bit from cutting into it as much and developing the higher torequer temperatuve values.

3). The dr ll bit metal matrix appears to he sotter now thet ks whan the matevial previcoush used (Rockiel C of 5-8 us $15-8$ - (older 6its). This would cause the meewer (soffer 6 its to wear out to ter transter he t taster, not grab the simulant 25 much 25 . the ordor.

4) These test This test was rim aqainst a flat face of the punice block. Previous tests were rum into a prevousily dvilled hole in the panice block This ettectively trapped more of the heat infaround the drull bit. 2 -continued $\Rightarrow$ 
, 56

HNF-3110

Bav. 0

$4 / 28 / 18$

5) Previous pumice tests used a drill bit with 4 of the six purge holes plugged with silver solder. The tharmocouple was placed in a tooth betind one of the plugged holes. This causes the Mermocouple to read abont 5 to $10^{\circ} \mathrm{C}$ higher than a thermocouple placed wehind an open purge hole. The test run today (Bti tstoo) had all the purge holes. open.

- ETITSTII

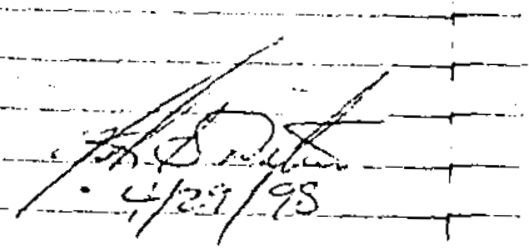

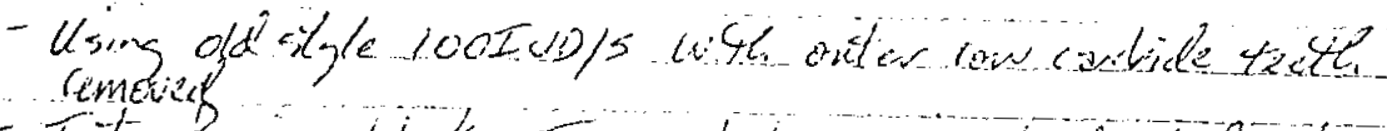

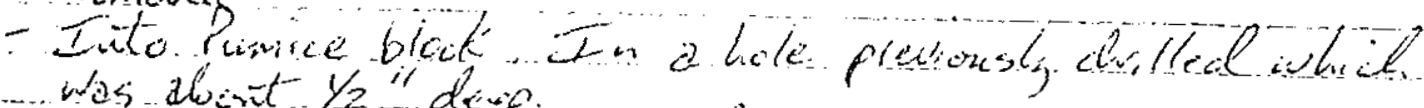

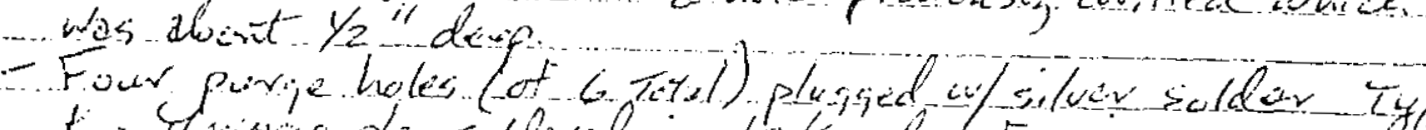

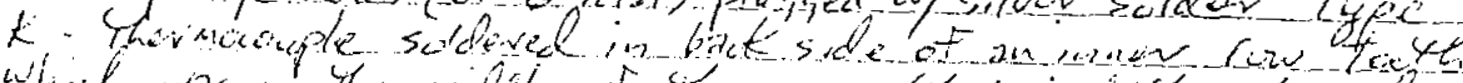
which was in the mide of the av2 htat had the plagsed

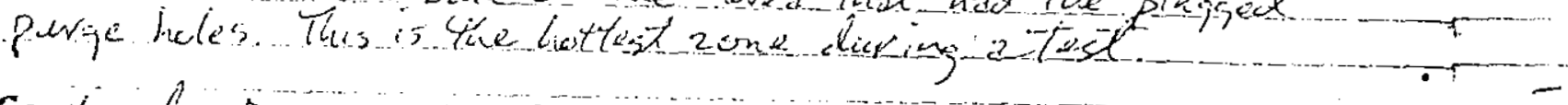

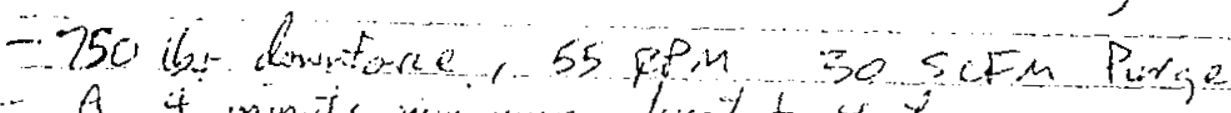

- A 4 ininude minimum dor de do yest

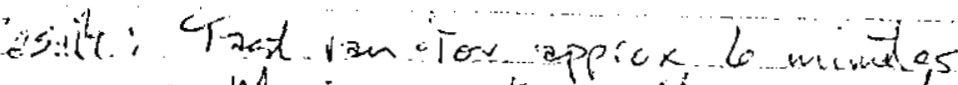

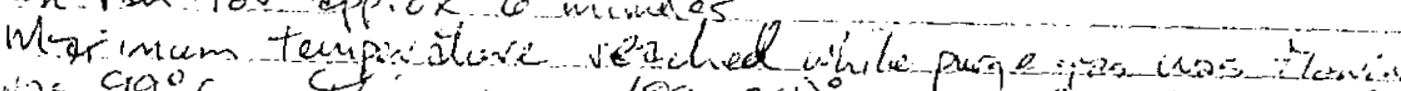

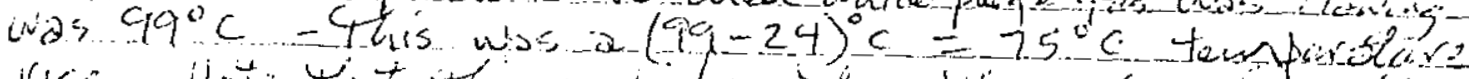

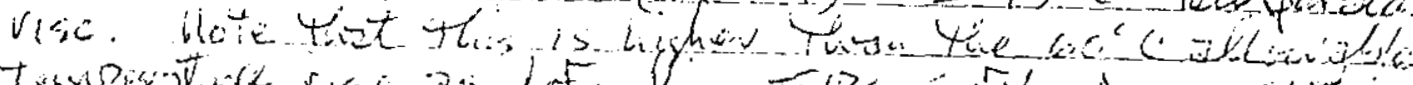

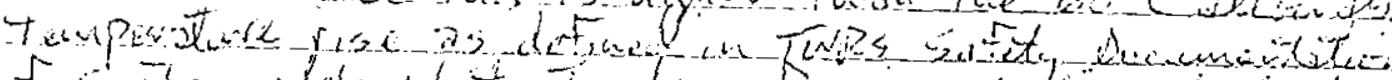

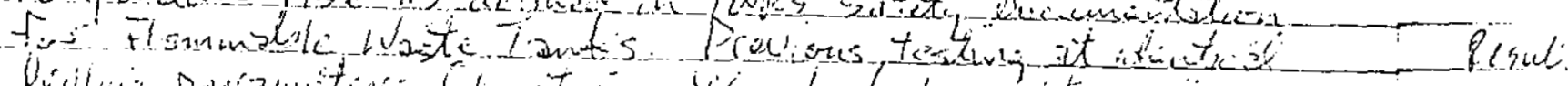

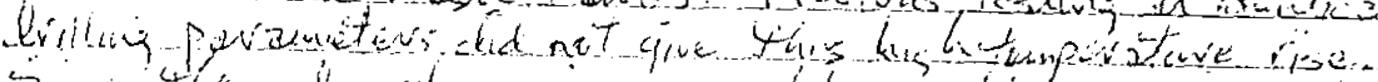

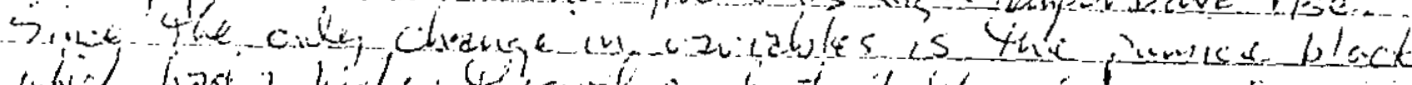

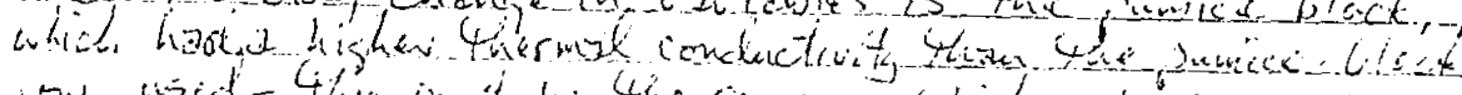

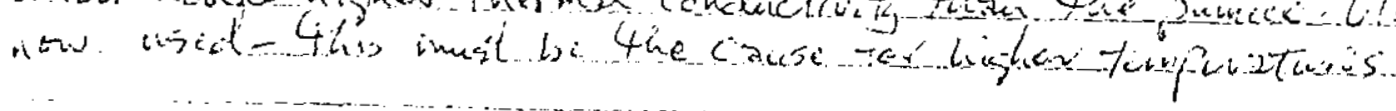




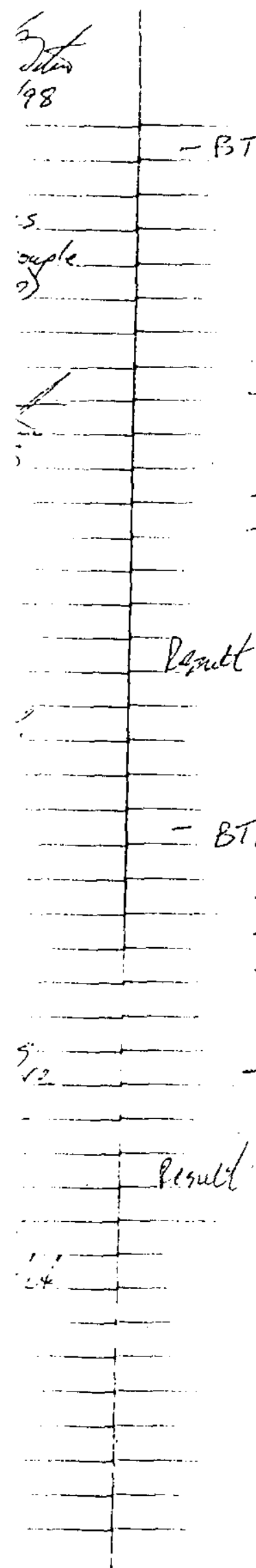

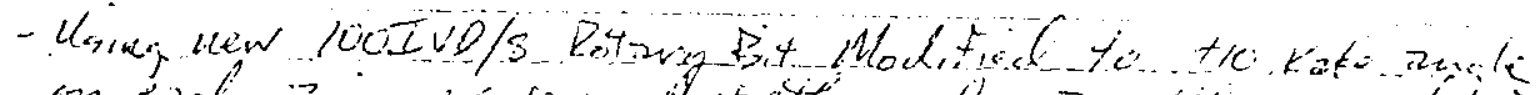

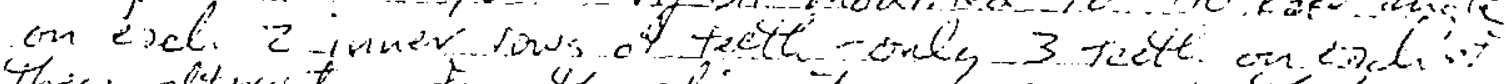

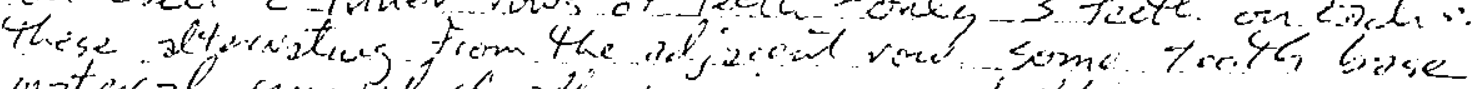

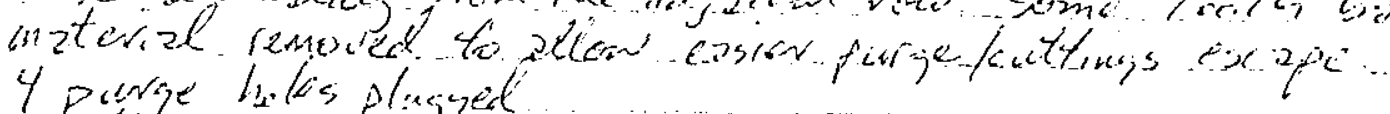

- Ypunge lis las plugiged.

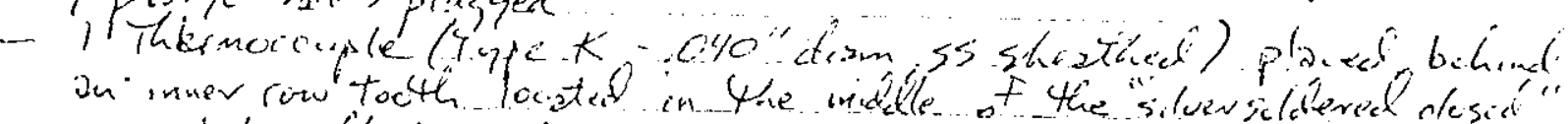
zurge boles (hot zrez)

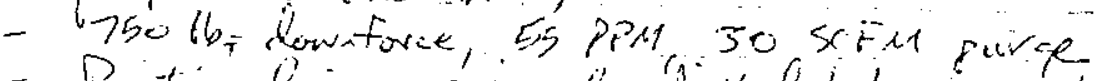

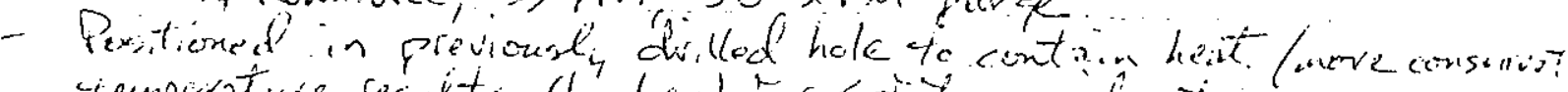

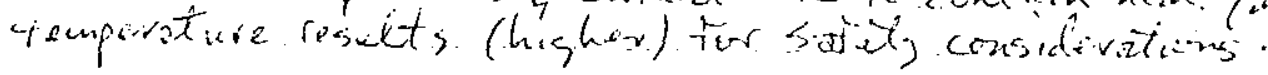

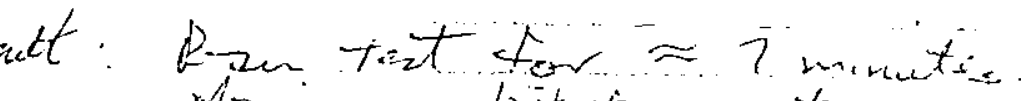

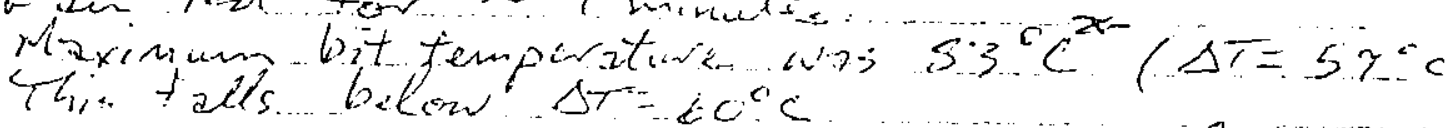

BTiकात?

$$
\text { Ex-while puripe gas fisured }
$$

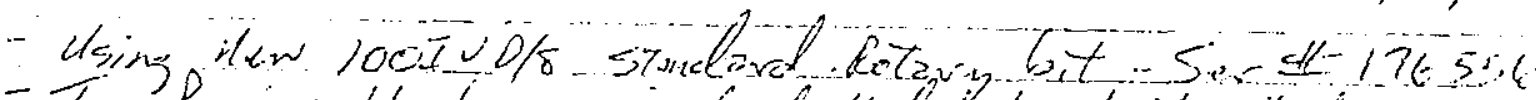

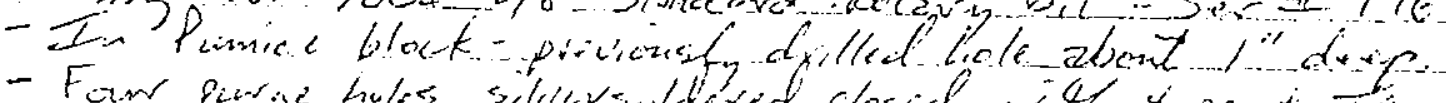

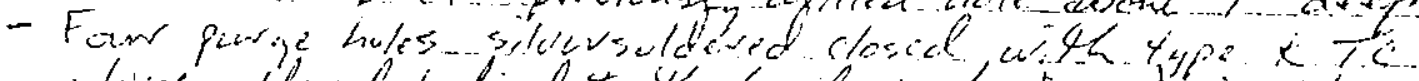

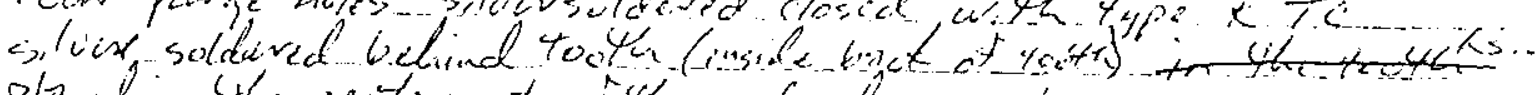

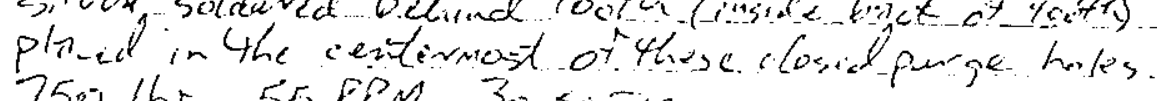

- $750.16 \%, 5589 \mathrm{M}, 30 \mathrm{sen}$

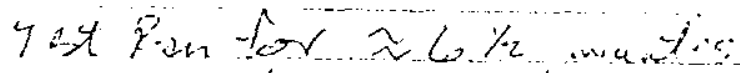

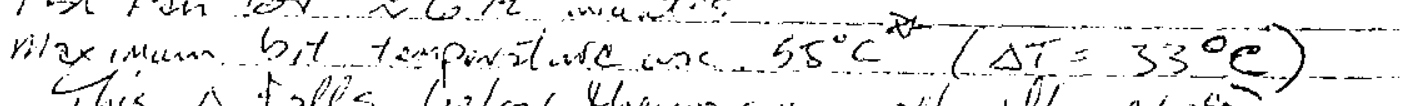

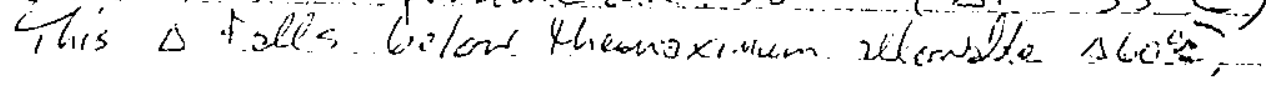

- Intryisting hew yhis bit.

$$
\text { o while purge gar fliwed. -.. }
$$

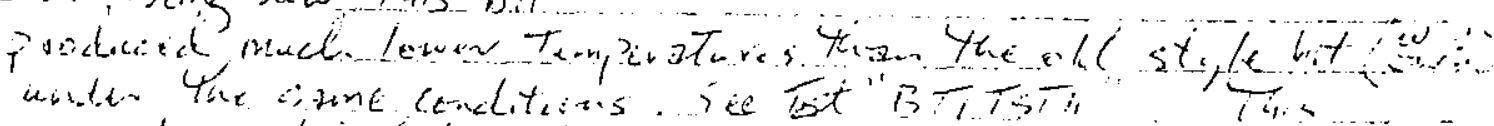

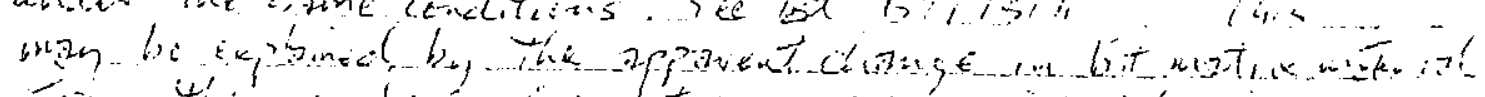

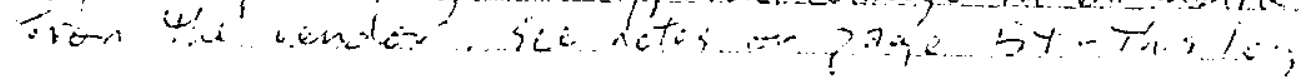


30

\section{HNF-3110}

$\therefore \therefore$ ET, $\because, 7,3$

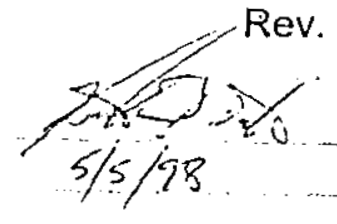

- Di:llos into A3b syicl flete

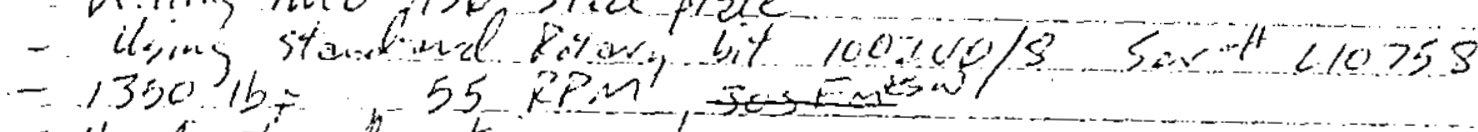

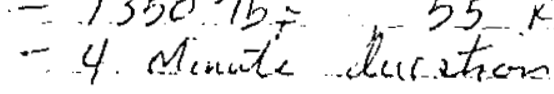

Rerifit:

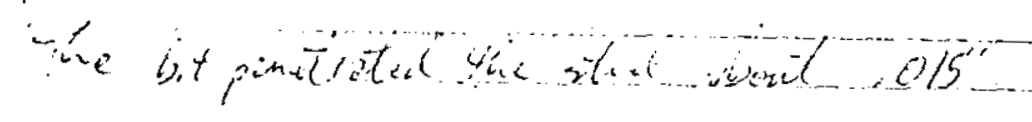

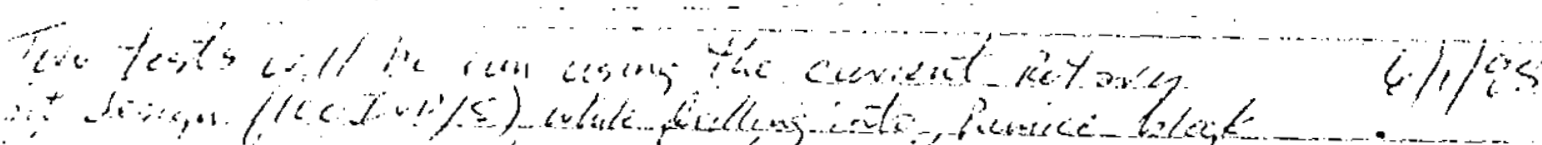

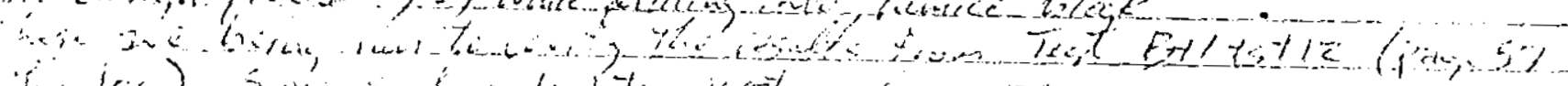

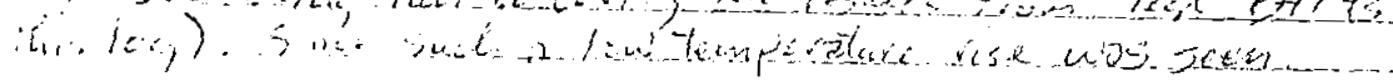

-Test BHtatiu

Pisifits

- Dielling into punice block (previoneledrilecl hole)

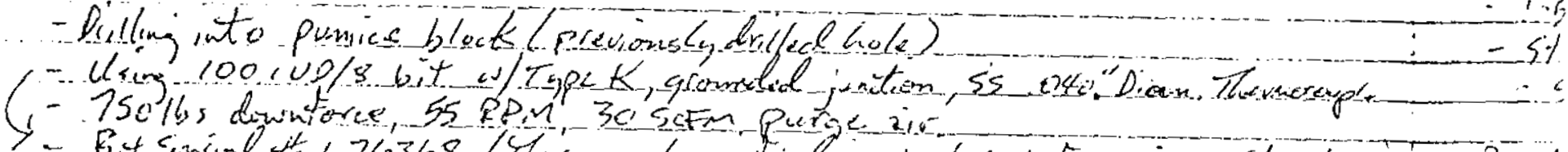

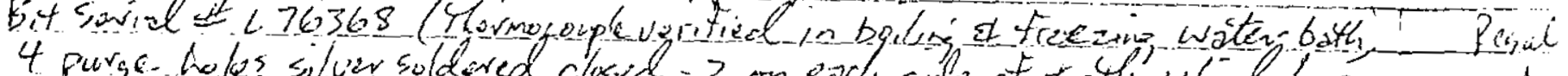

4 purge holes siluev suldared clused 2 on eacl side of tooth which has

Thernocouple nounted in it.

8csult Sit tampirature vent from $25^{\circ} \mathrm{C}$ to $69^{\circ} \mathrm{C}$ or $\Delta T=44^{\circ} \mathrm{C}$ ilis

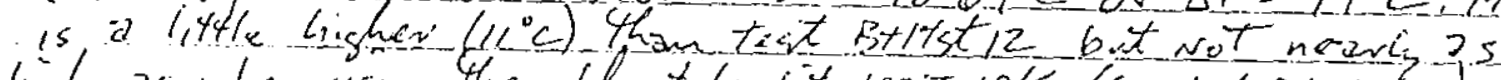

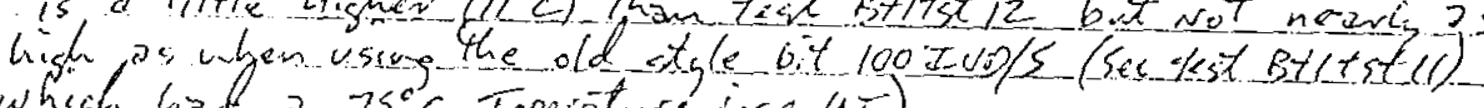
which lied 2 a $75^{\circ} \mathrm{c}$ Taperature ise $(A T)$

ist Ext istis

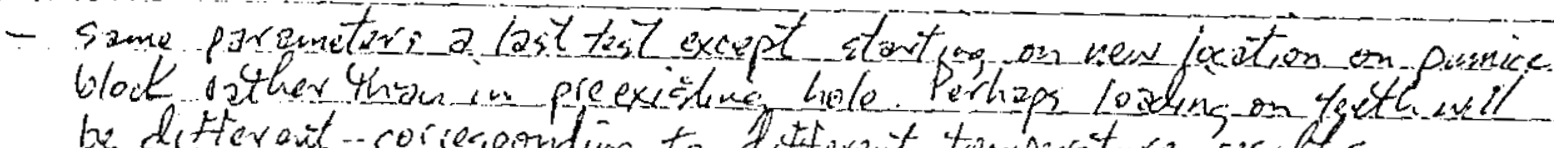

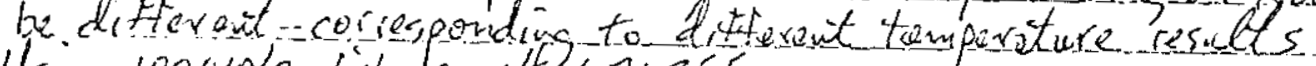

- Uciar $100 \mathrm{vo} / \mathrm{s}$ bit siv If $L 74355$

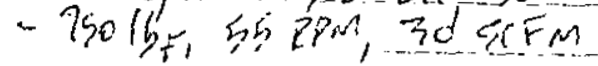

Recatt Timporature an bit went from $26^{\circ} \mathrm{C}$ to $11^{\circ} \mathrm{C}\left(\Delta T=45^{\circ} \mathrm{C}\right)$ 


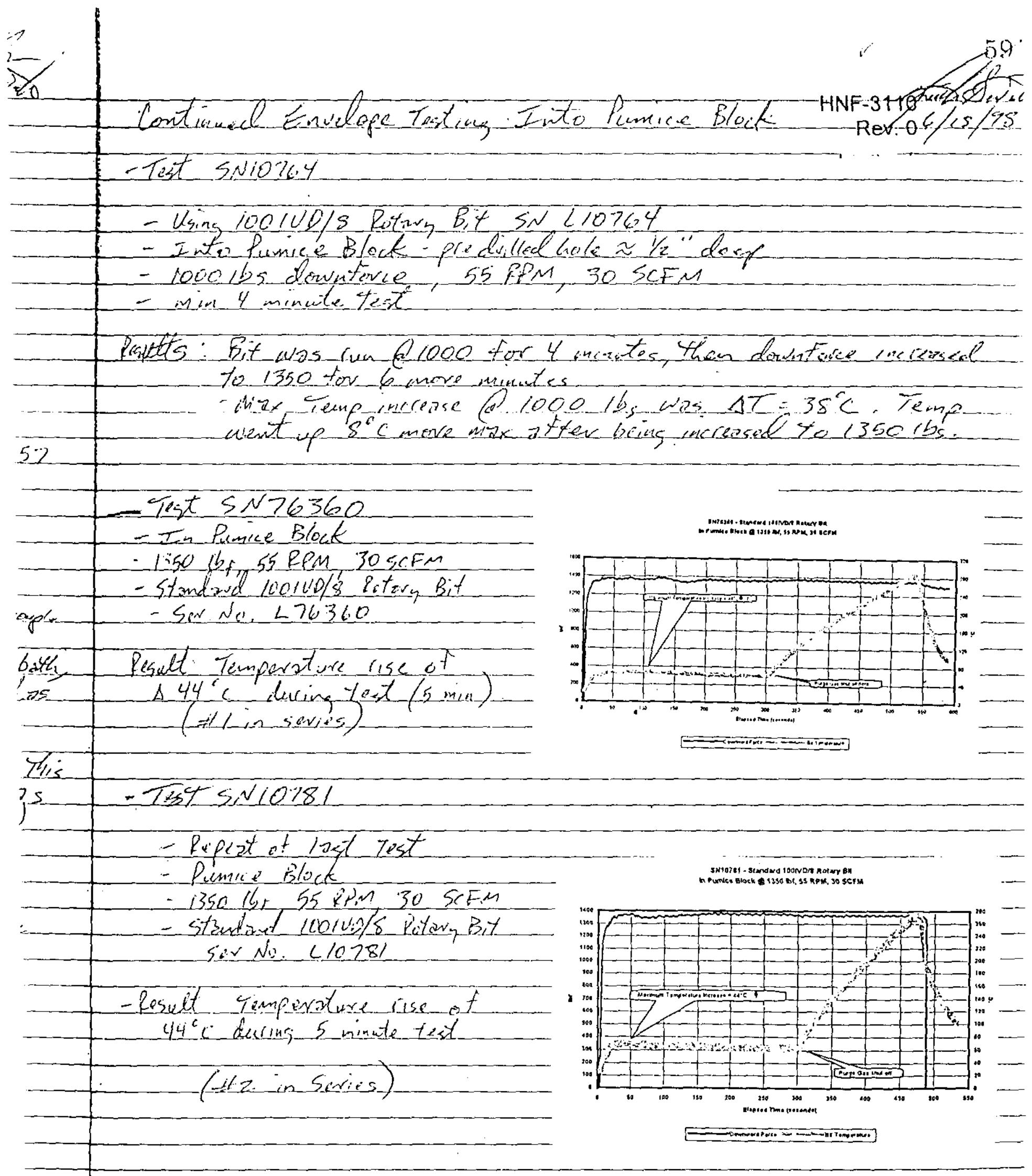


60

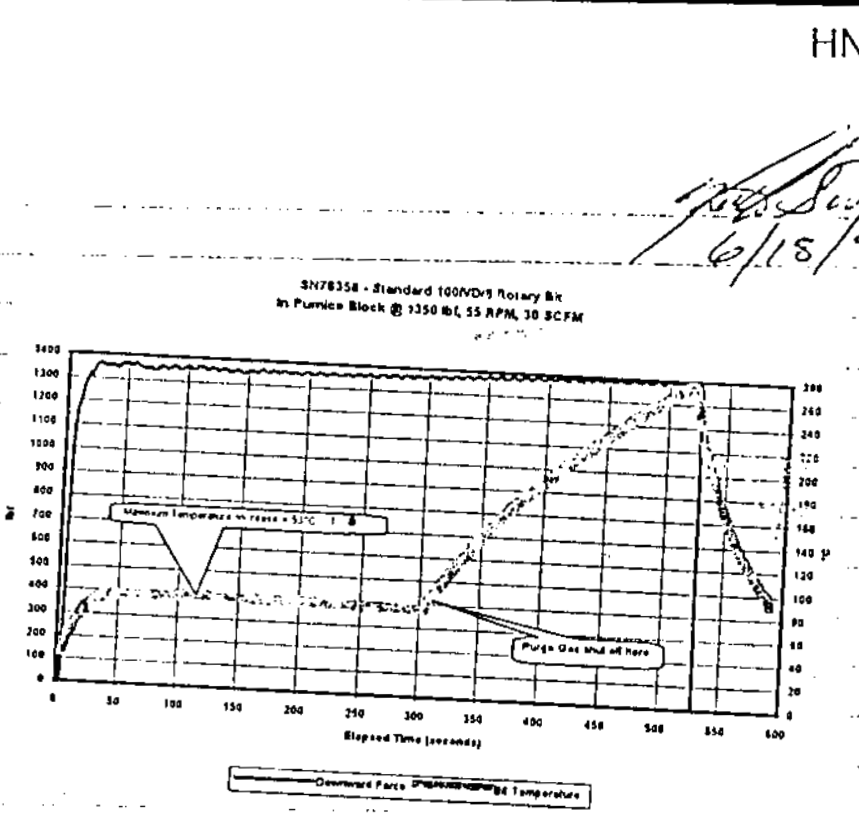

$T_{\epsilon} ; t \cdot 5 n 16.358$

- In Pumice block

- 135016 F 55 RPM 30 SCF

- Using sitidaril $1001(10) / s$ fit

- Sivigl Numbirl76358

$\therefore$ minimum 4 ininate logt

nit: Tempiriture incicased

tiom. $26^{\circ} \mathrm{C}$ to $79^{\circ} \mathrm{C}$

$-53^{c} C$ Rise (derimg smimile)

fin Serias)

HNF-3110

Rev. $\phi$.

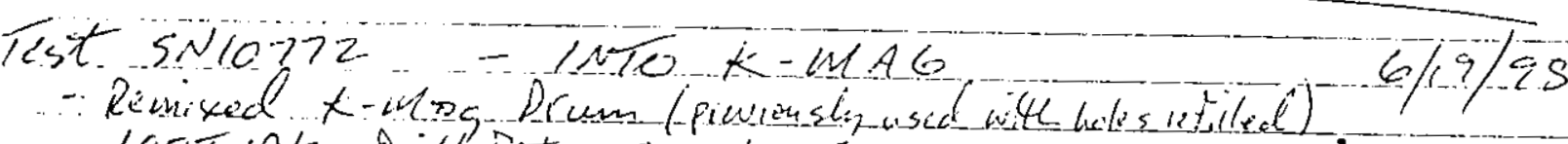

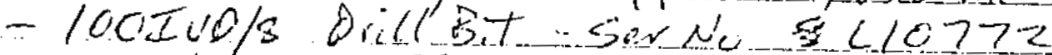

-750 l $, 5528:-30$ SCEM

isuet : Bit dillud int $K=$ wag $\left(17.5^{11}\right)$. in ibint 6.15 seconds
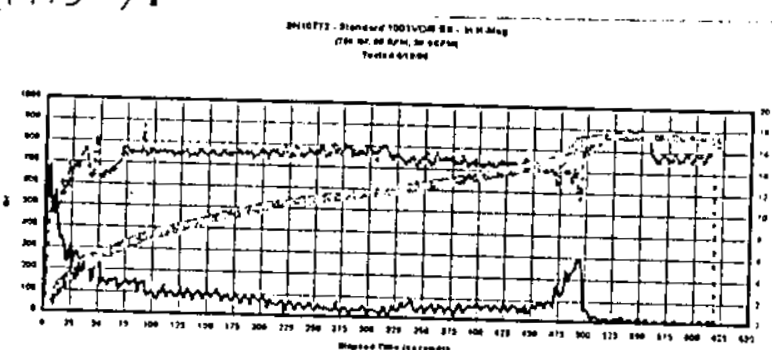

EC:

ist $\sin 10770$

- Romixed k Nog Drum G/19/98

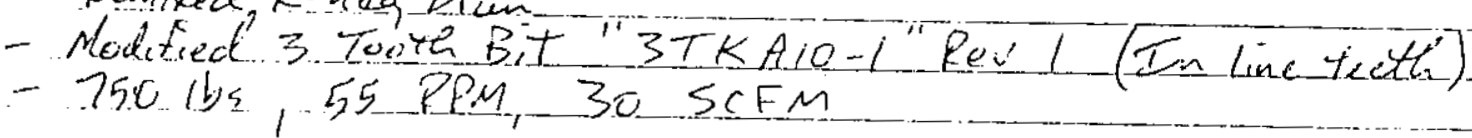

esult: bil drillool to K-mog $\left(17.5^{\prime \prime}\right)$ In $2 b_{0} \mathrm{t}$

325 siconds. Almost

halt the time it leck for the previous bit to qut

through the same bopth.
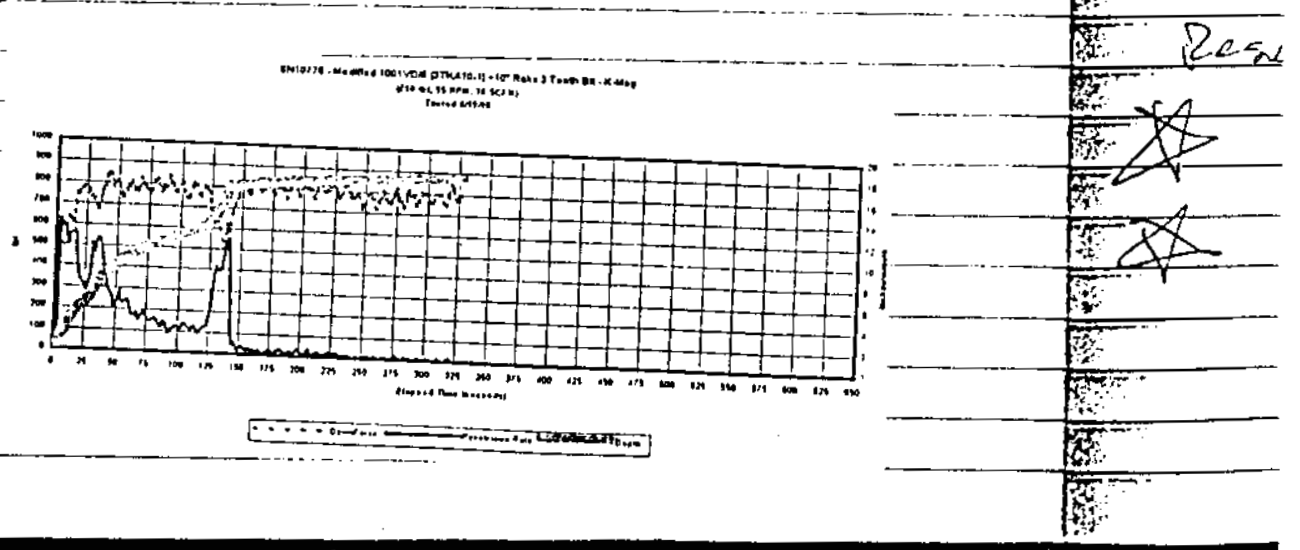
42

HNF-3110

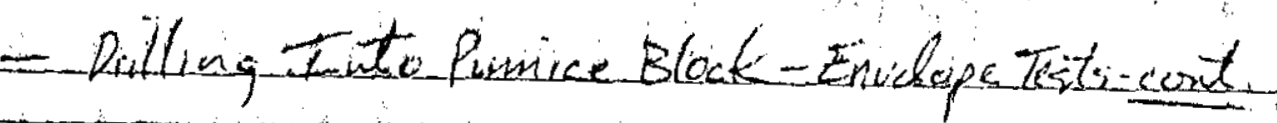
- Teat intozic

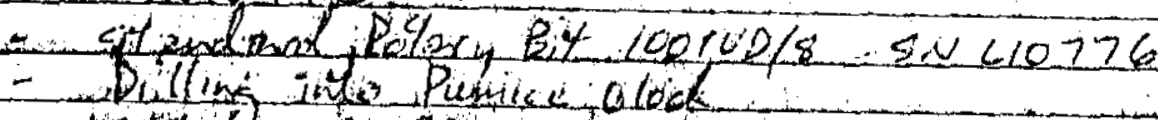

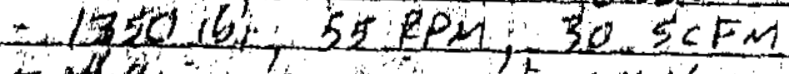

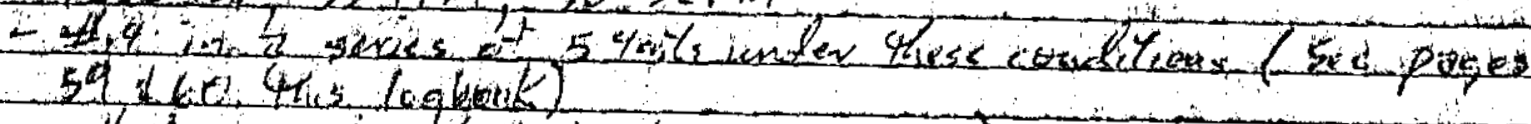

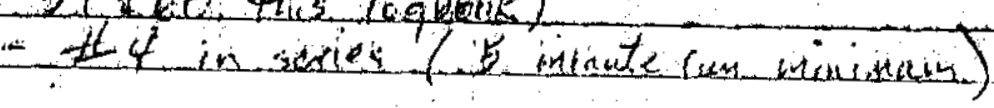

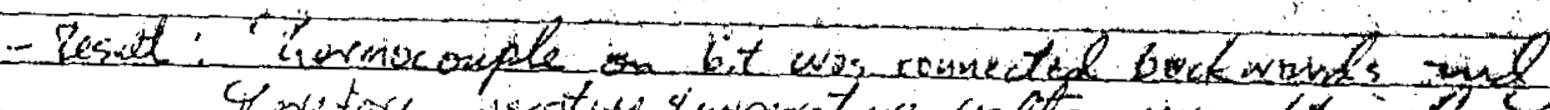

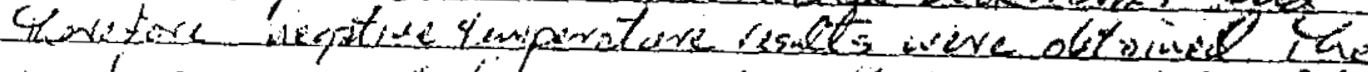

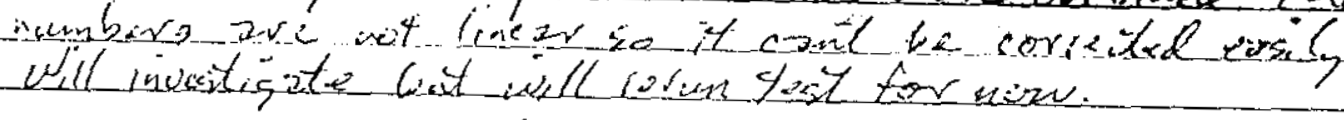

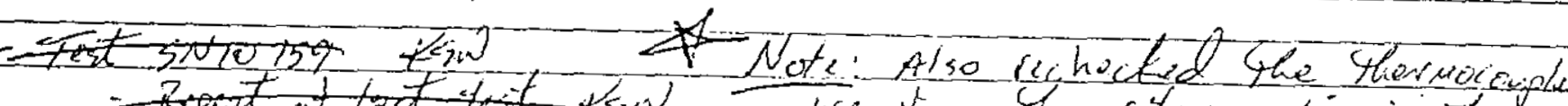

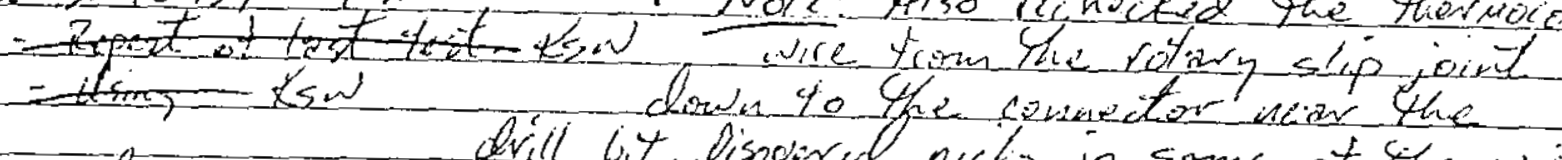

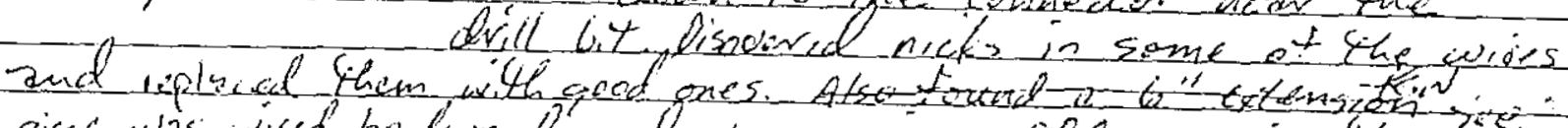

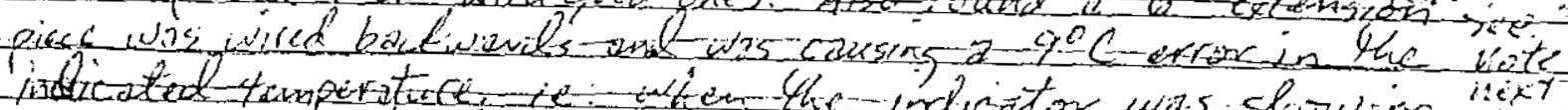

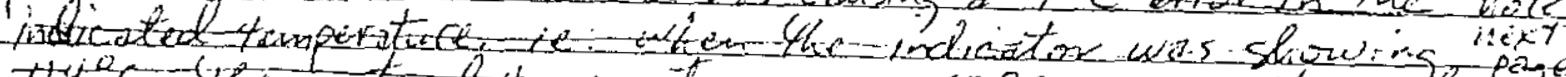

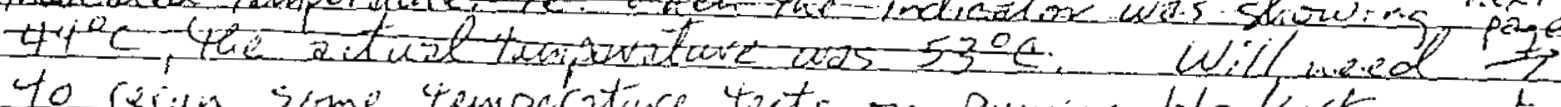

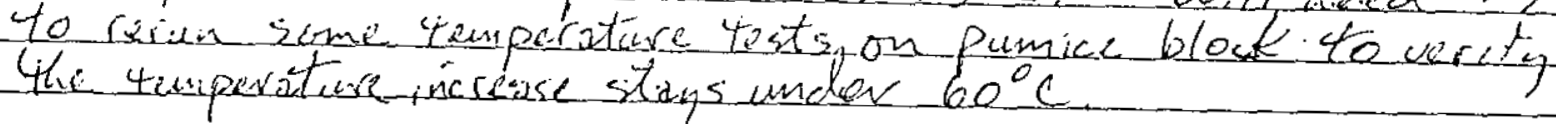

- Tet sN10759

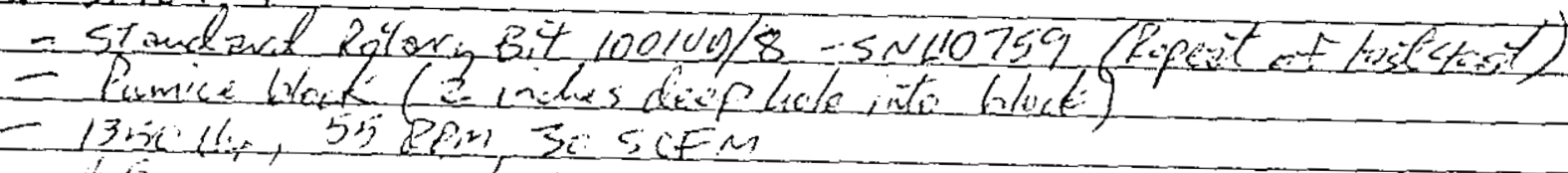

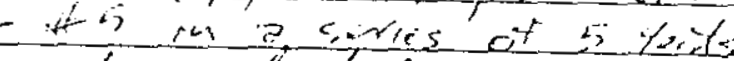

- Corp chicked

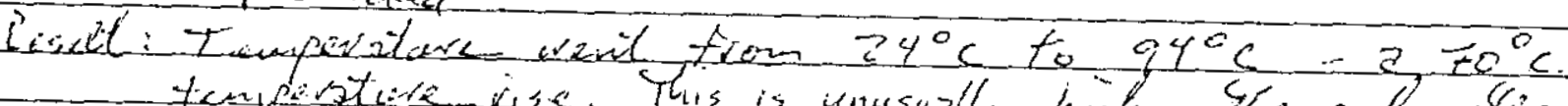

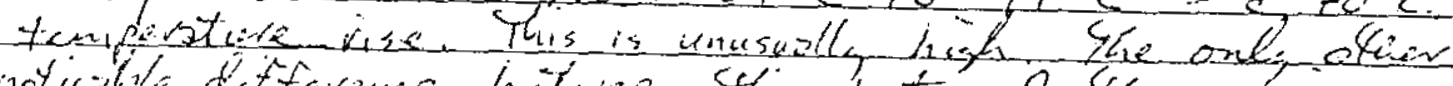

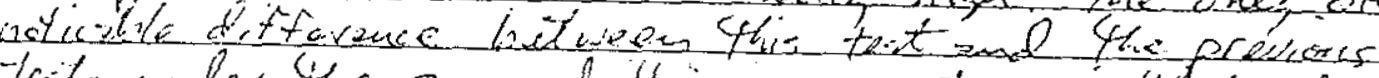

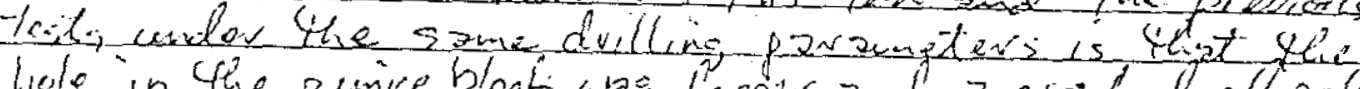

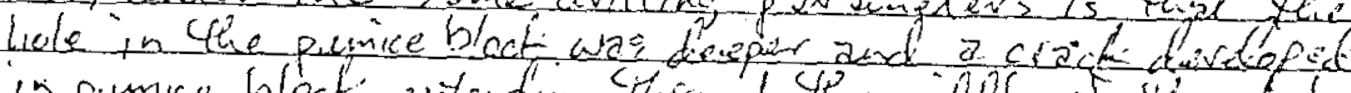

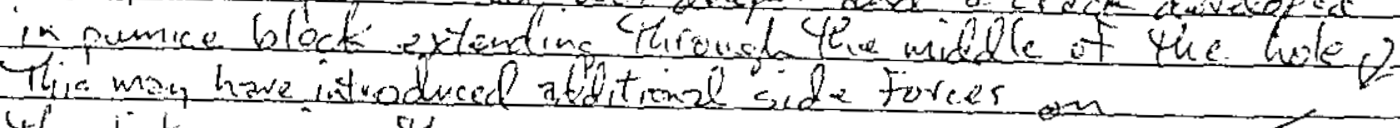
the bit ressing the ctemperature te increase.

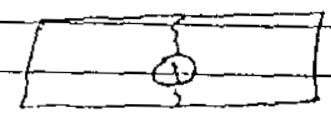




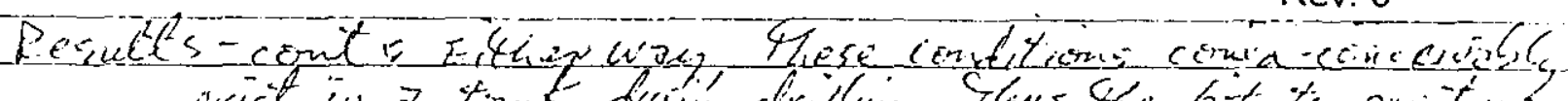

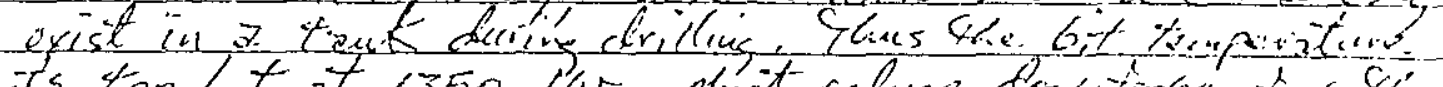

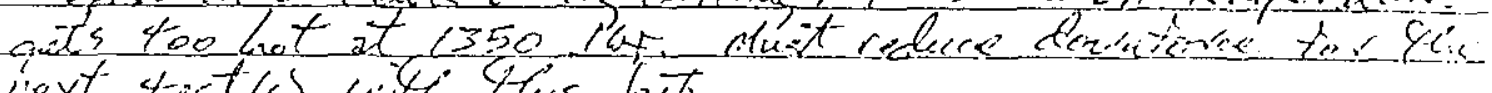
iext terts (s) witi tis bit

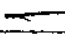

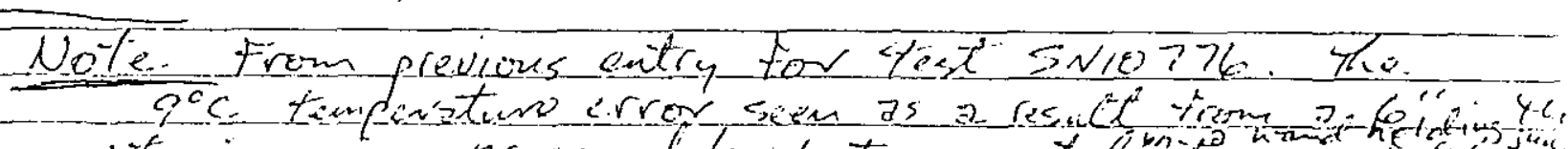

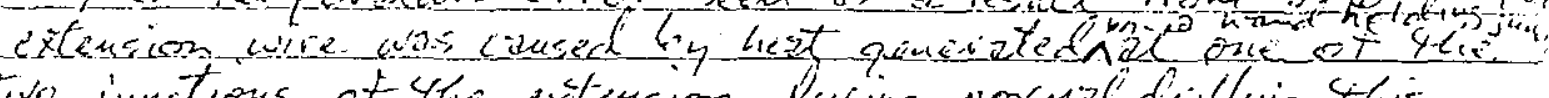

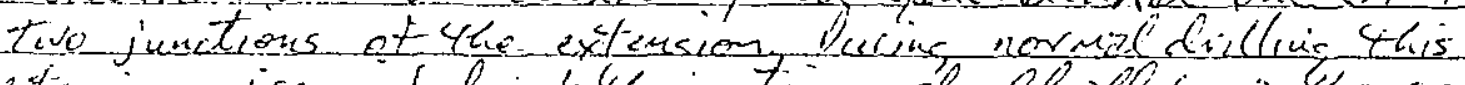

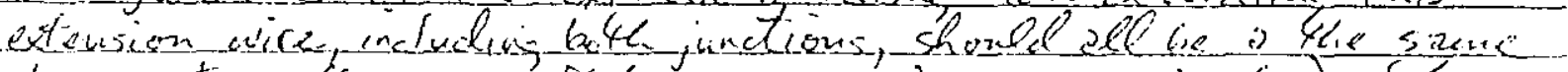

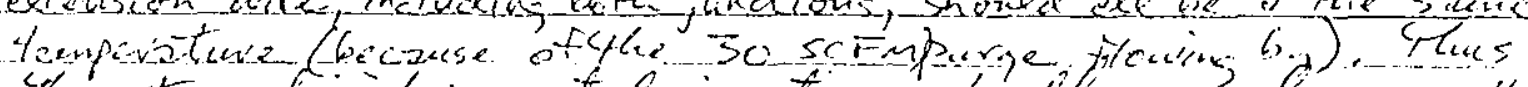

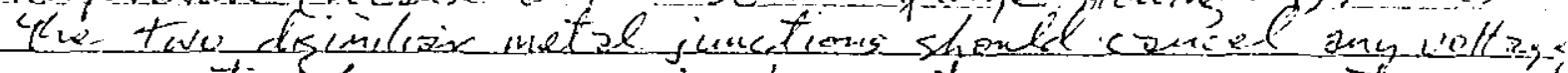

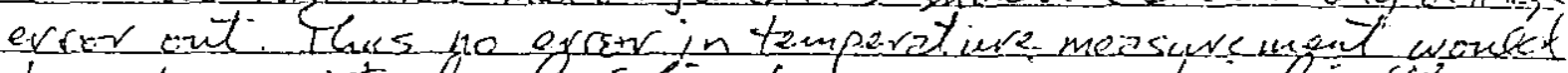
hauz heen introluced. This phenomena os explyasel in the photecopied text bulou Alsu see ihe skitch below:

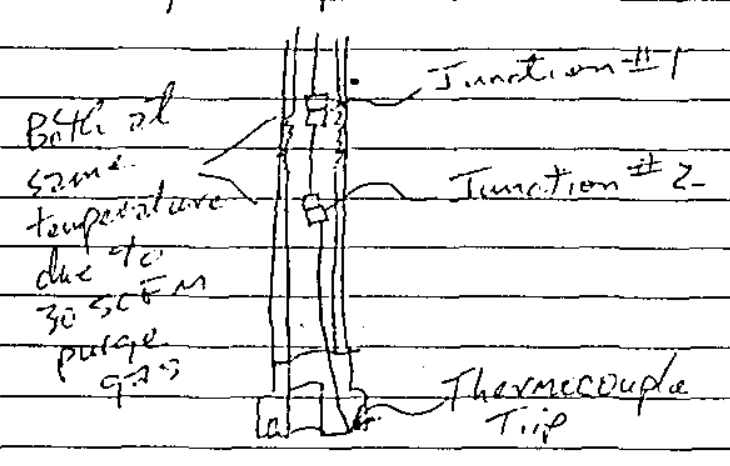

少

\section{Phatocopyed text on following piaze}

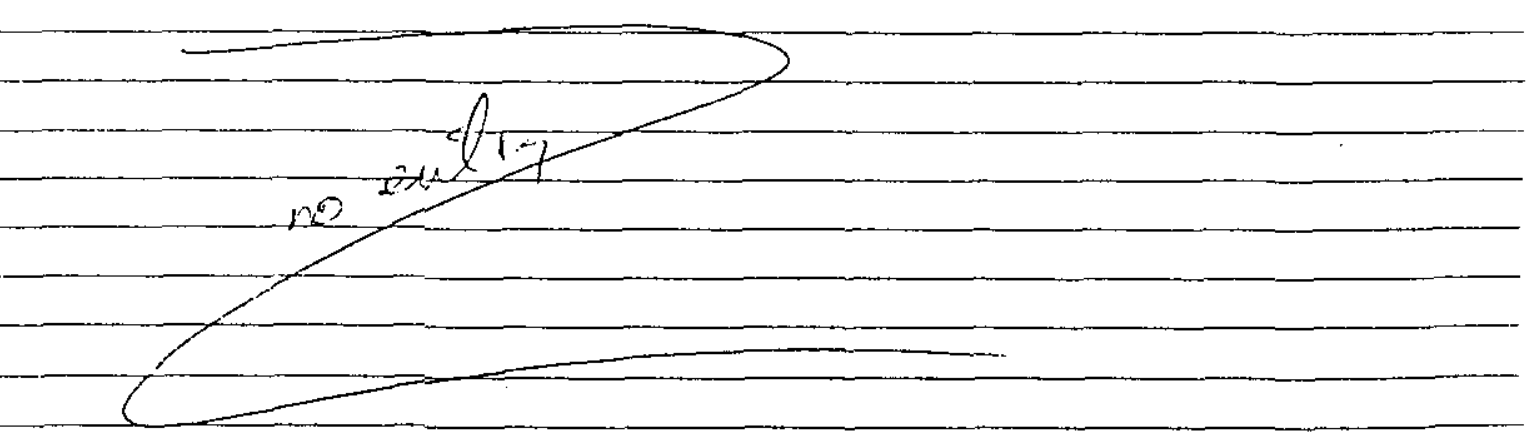




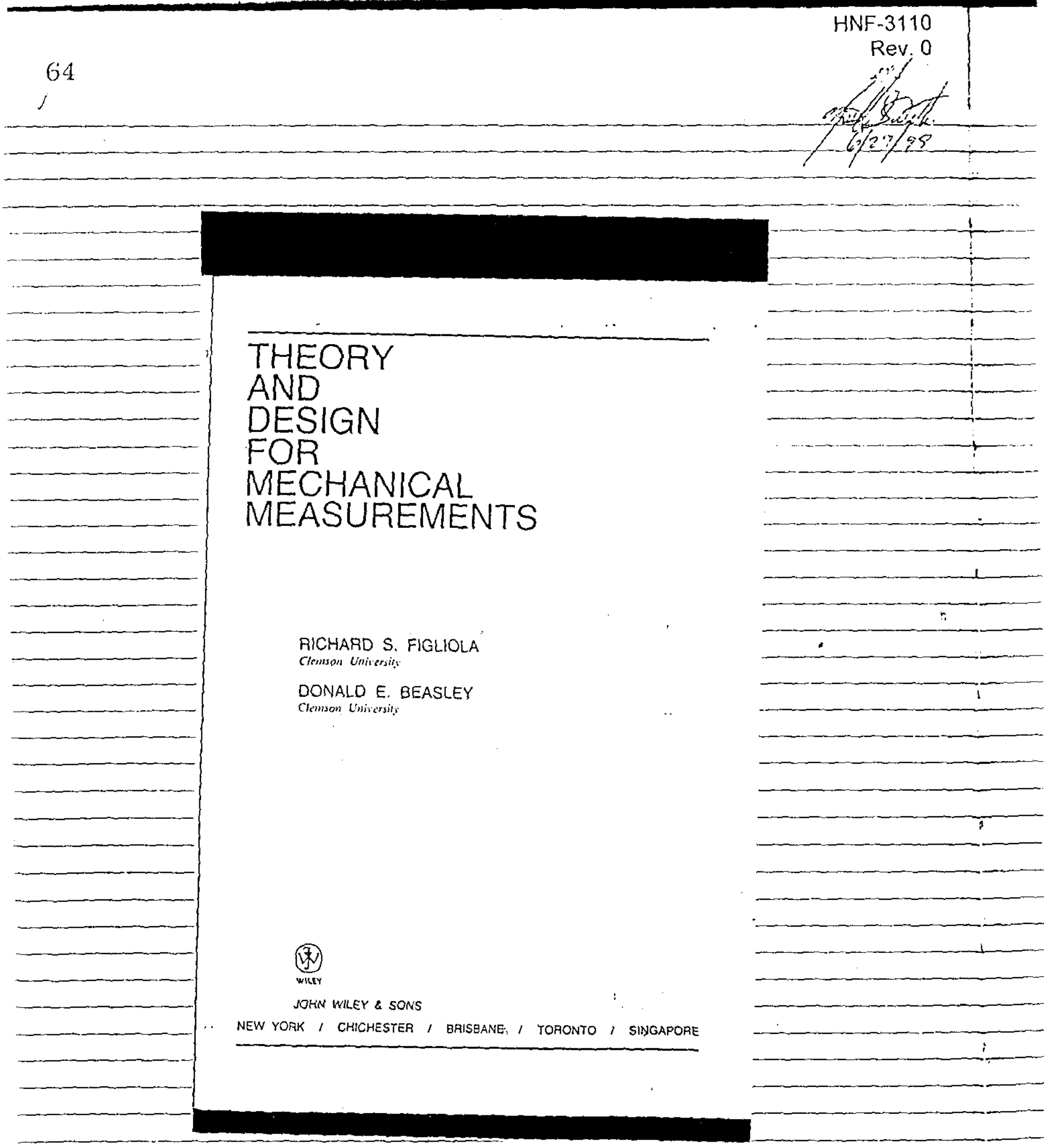

RICHARD S. FIGLIOLA

DONALD E. BEASLEY

Clemson L'miversity

AND

DESIGN

FOR

MECHANICAL

MEASUREMENTS

困 


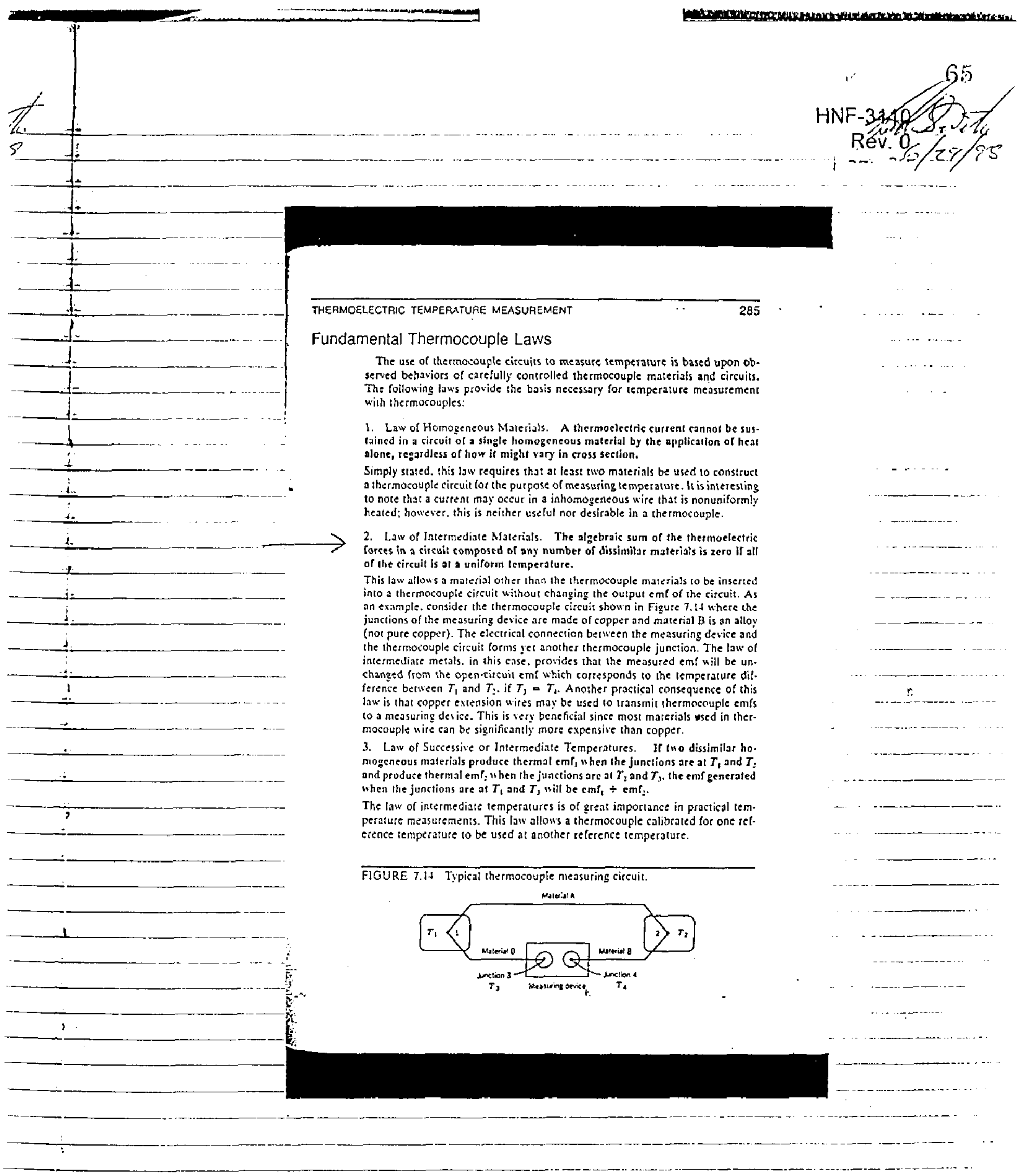

The use of thermocouple circuits to measure temperature is based upon ob. The behaviors of carefully controlled thermocouple materials and circuits. with thermocouples:

1. Law of Homogeneous Marerials. A thermoelectric current cannot be sus by the application of hea a thermocouple circuit lor the purpose of measuring lemperature. It is intetestin

2. Law of Intermediate Materials. The algebraic sum of the thermaelectric corces in a circuit compostd of any number of dissimitar materiats is zero it all of the circuit is ot a uniform temperature. . not pure copper). The electrical connection between the measuring device and the thermoiouple circuit forms yet another thesmocouple junction. The law of

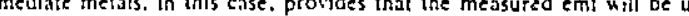
the open.ritcuir eme which cortesponds to the temperature on is aw is that copper externsion wites may be used to transmit thermocouple emfs mogeneous materials produce thermal emf, when the junctions are at $T_{1}$ and $T$ whe

The law of intermediate temperatures is of great importance in practical tem. perature measurements. This law allows a thermocouple calibrated for one retFIGURE 7.14 Typical the rmocouple neasuring circui 
66

TLet $5 \sqrt{102706}$

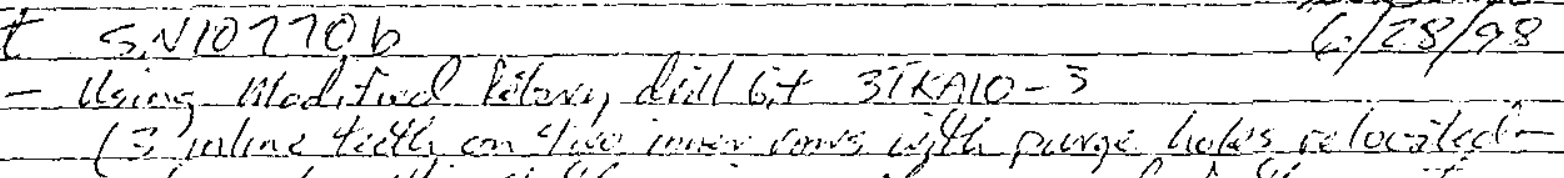

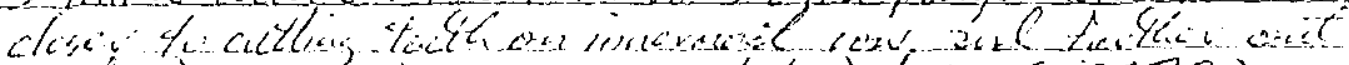

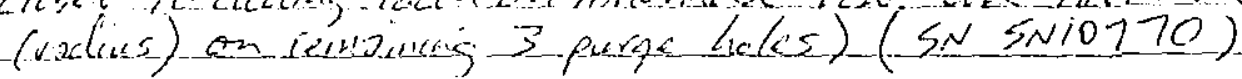

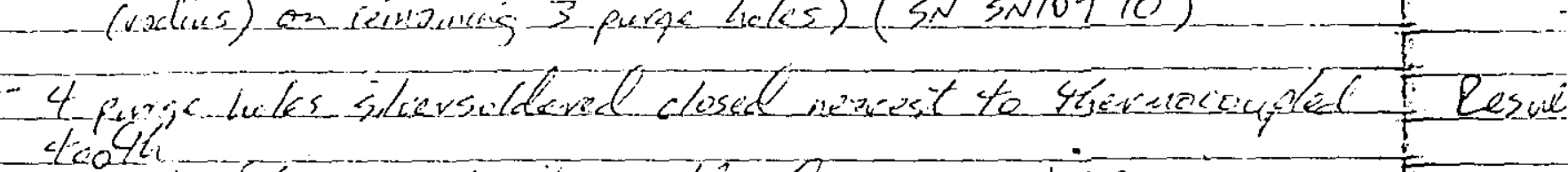

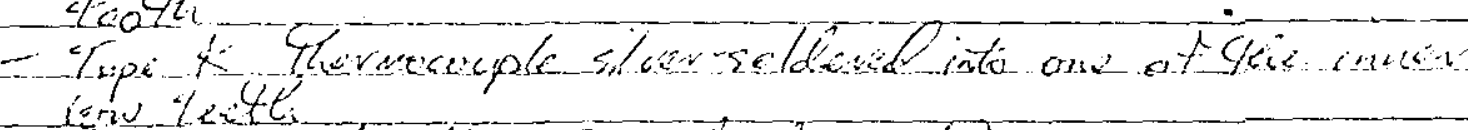

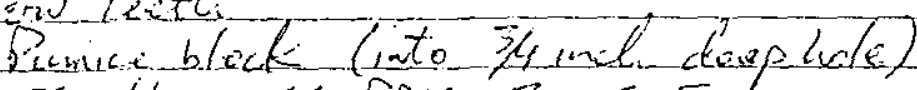
$=350,16, \ldots 5 \mathrm{RF}, 3 \mathrm{~s}, \mathrm{SCF}$

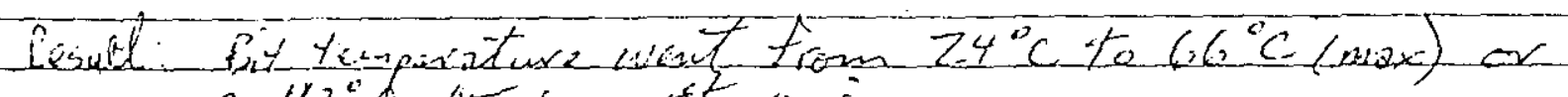

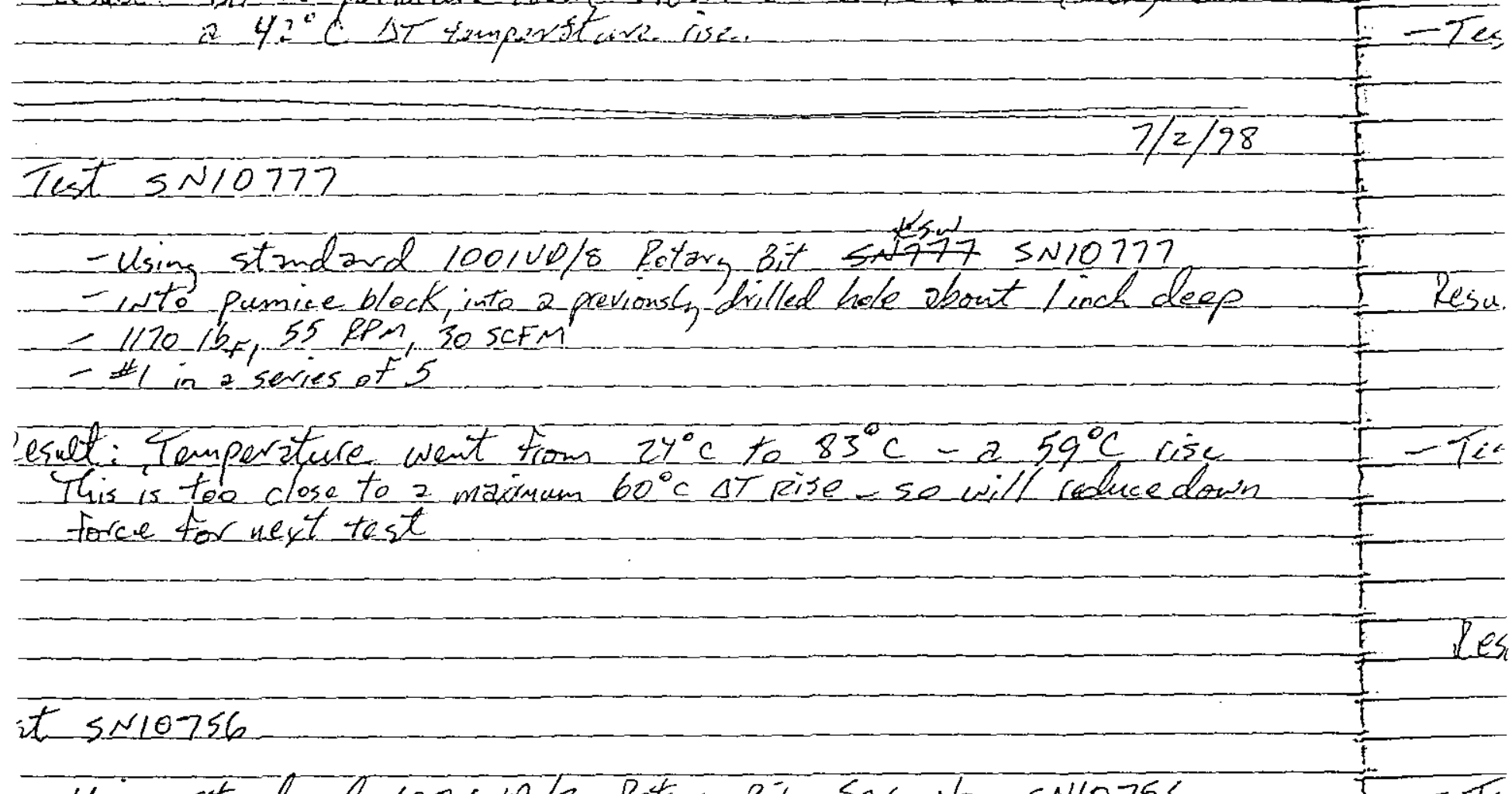

- Using standard $100100 / 8$ Rots - Into panice 6lock block 1 inch deep hole $=1000$ i5, 55 RPM, $305 C$ FM

elt: importiere went from $24^{\circ} \mathrm{C}$ to $87^{\circ} \mathrm{C}$ - a $63^{\circ} \mathrm{C} \Delta T$

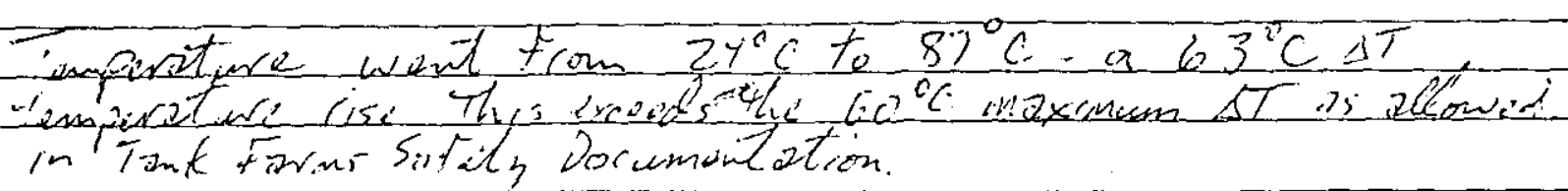




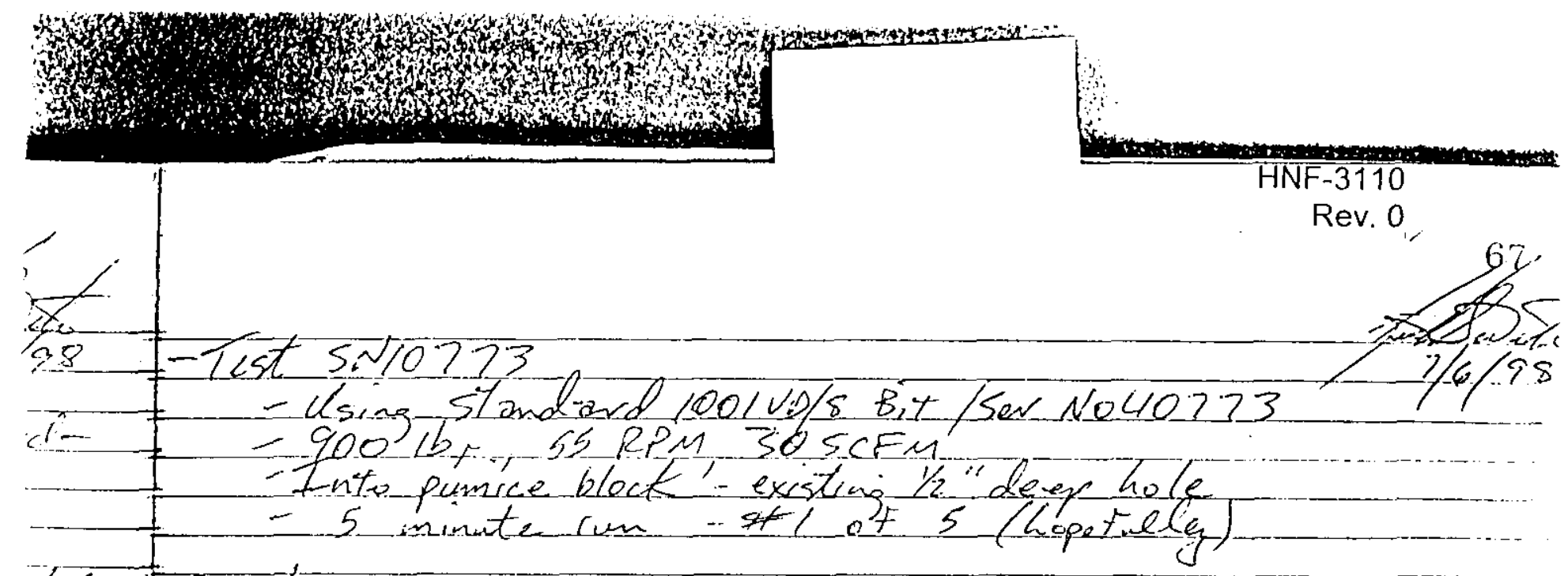

Ted Result: Temperature rose from $24^{\circ} \mathrm{C}$ to $56^{\circ} \mathrm{C}-2-32^{\circ} \mathrm{C}$ - temperature rise this, was alums halt the pauperise of the last rest where the only difference was loo lo more downferce lon the test test and the last to st sifarted ont in 2. hole " drop. Also the pumice block appeared to be soto in the tort fecit singe the hole increased in depth b. 3/1" Thus

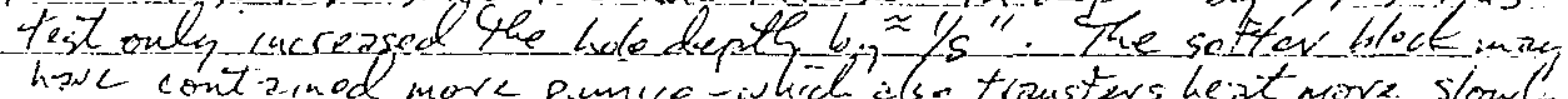

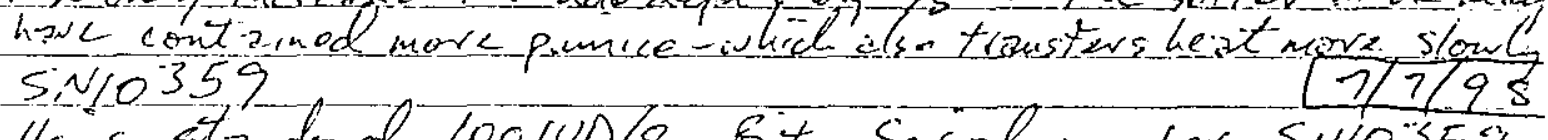
-Test $\sin 10359$

- Usia atiandand $100100 / 8$ fit serial number silos\% - $9001 \mathrm{~K}, 55 \mathrm{RPM}, 30 \mathrm{gCFM}$

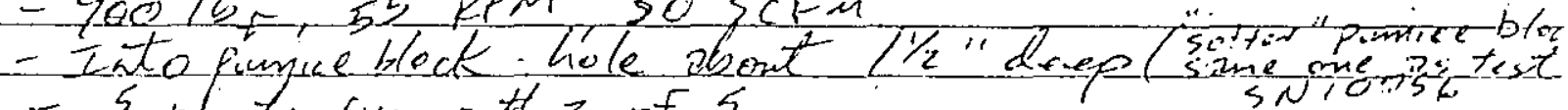
- 5 minute rim - Hz of 5

Result: Temperature rose from $24^{\circ} \mathrm{C}$ to $63^{\circ} \mathrm{C}-239^{\circ} \mathrm{C}$ youporit. rise.

- TeSt SN 1078\%

- Rupert of last test using bit isar t sim

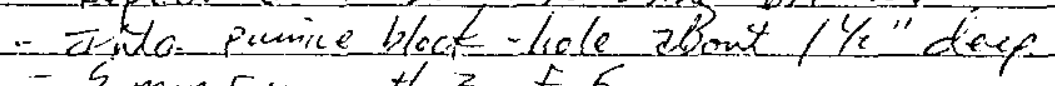
- 5 min run . 13 of 5

Result: Temperature rose from $25^{\circ} \mathrm{C}$ to $62^{\circ} \mathrm{C}-a 37^{\circ} \mathrm{C}$ temperature rise

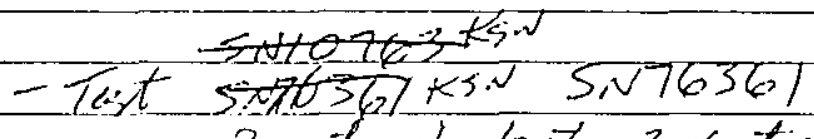

- Rupert at last 3 fests

- Into Pumice block ladle storing 1/2 deut

-5 minute rum A 4 of 5

Resect: Temperature rose from $26^{\circ} \mathrm{C}^{\circ} 1059^{\circ} \mathrm{C}-233^{\circ} \mathrm{C} \Delta \mathrm{T}$

Page $B-14$ 
68

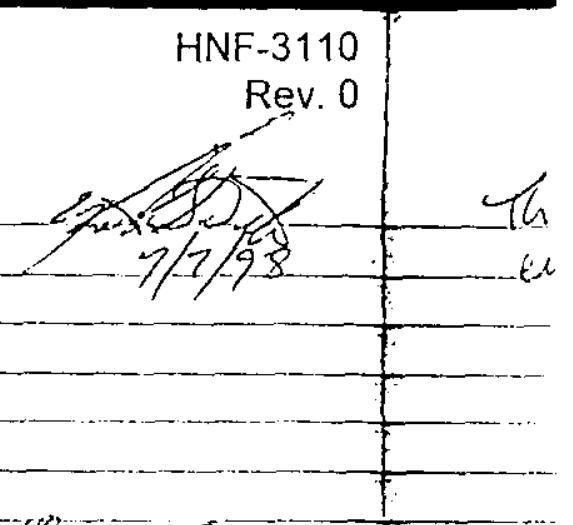

Iesuet Temperitar vos fsim $25^{\circ} \mathrm{C}$ to $59^{\circ} \mathrm{c}-234^{\circ} \mathrm{C}$ IT

10

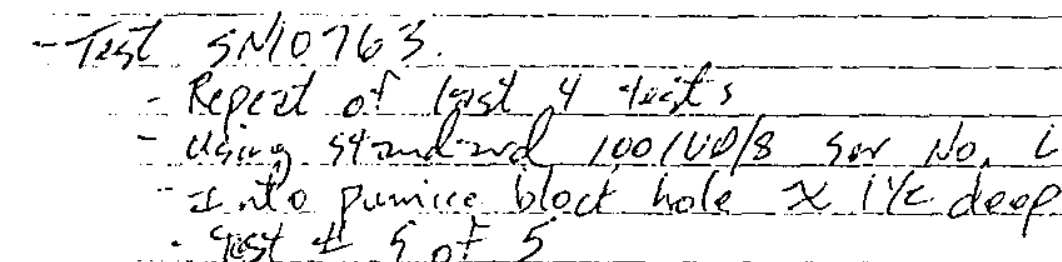

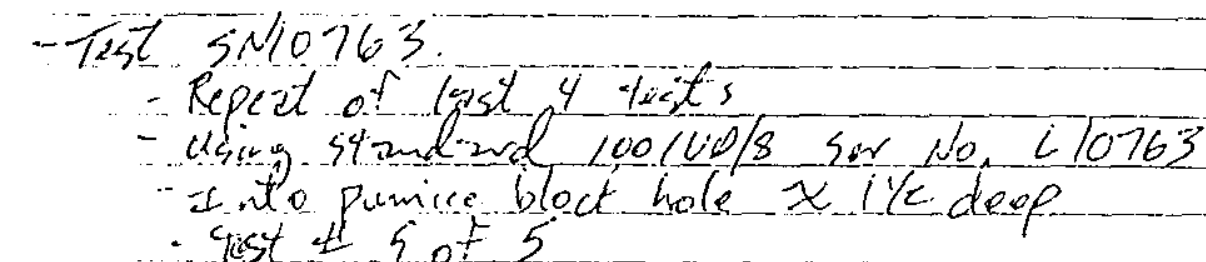

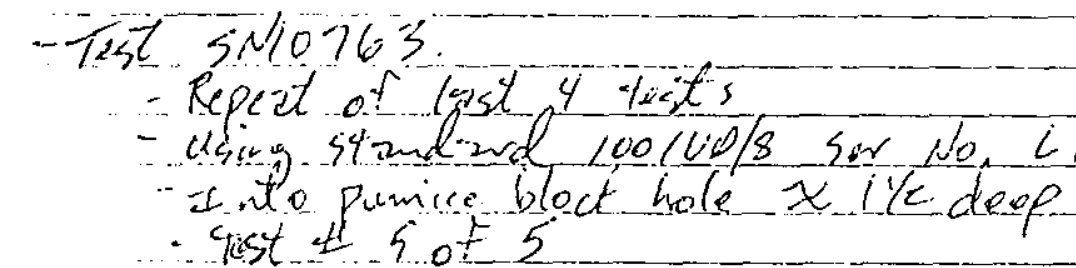




\section{This is the chavoter ization data Ine buts dith Thermorcioles}

embedded in them. specifirily the $100100 / 8$ Retan bits. Teated ofter the TCa were siluar soldered.

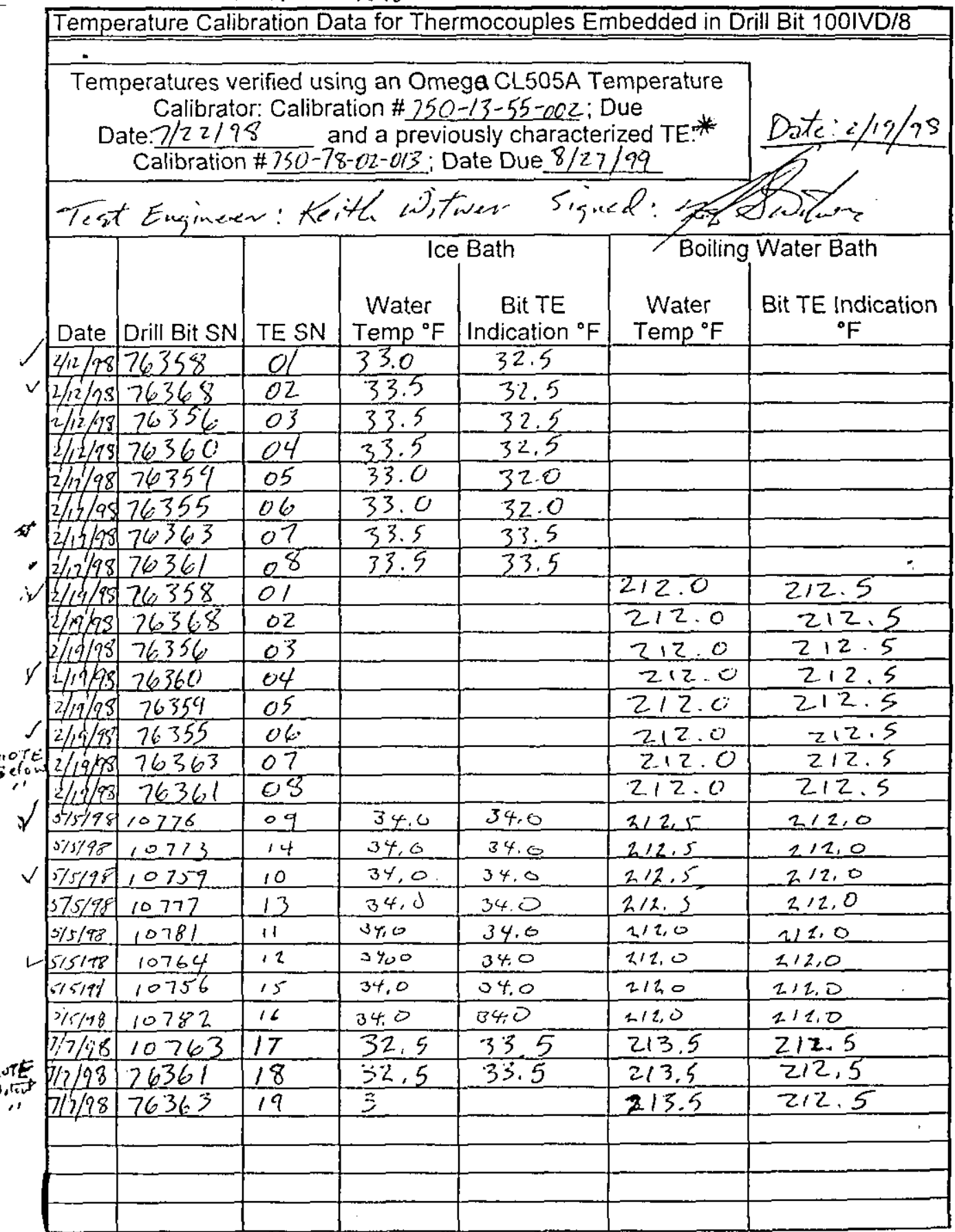

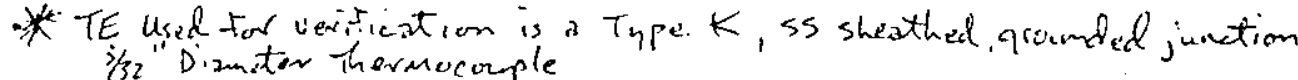
$305 \mathrm{Bldg}$ - Engineering Testing Laboratory

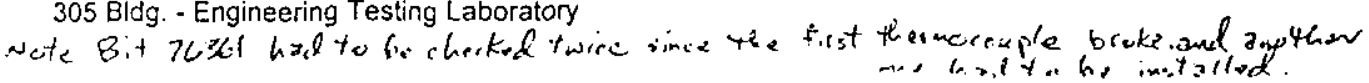


Rev. 0

\section{APPENDIX C - SIMULANT THERMAL CONDUCTIVITY RESULTS}




\section{NHC}

\section{Numatec}

From: $\quad$ Process Chemistry

Phone: $373-2162$

Date: $\quad$ February 24, 1998

Subject: THERMAL CONDUCTIVITY REMEASUREMENTS OF CINDER BLOCKS

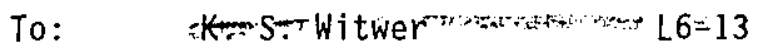

$$
\begin{array}{lll}
\text { cc: D. B. Bechtold } & T 6-07 \\
\text { M. A. Beck } & T 6-07 \\
\text { J. W. Chenault } & T 6-07 \\
\text { J. R. Jewett. } & T 6-07
\end{array}
$$

References: (1) Internal Memo, 8C510-98-003, M. A. Beck to K. S. Witwer, "Thermal Conductivity Measurements of Cinder Block," dated January 23, 1998.

(2) Chenault, J. W. et al. 1998, "Miscellaneous Laboratory Notes," WHC-N-2771, Numatec Hanford.Corporation.

(3) Internal Memo, 75764-PCS96-003, D. B. Bechtold to G. L. Ralston, "Thermal Conductivity Measurements of Core Drilling Simulant Samples," dated January I1, 1996.

(4) Bechtold, D. B., 1995, "Thermal Conductivity Measurements with the Kemtherm QTM-D3 Thermal Conductivity Meter," LT-519-184.

The thermal conductivities of two cinder block samples referred to in References (1) and (3) were remeasured on the same day by the same operator at your request. As before, the methods of measurement and reduction of results were those described in Reference (4), and the data was recorded in Reference (2).

The values of interest to you are for Ralston's Cinder Block Brick:

$$
k_{\text {th }}=0.969 \pm 0.094 \mathrm{~W} / \mathrm{m} / \mathrm{K}(95 \% \text { Confidence }) \text { at } 30^{\circ} \mathrm{C}
$$

and for Witwer's Large Cinder Block:

$$
k_{\text {th }}=0.748 \pm 0.055 \mathrm{~W} / \mathrm{m} / \mathrm{K}(95 \% \text { Confidence }) \text { at } 31^{\circ} \mathrm{C}
$$

corrected to National Institutes of Standards and Technology (NIST) standards. A spreadsheet of results is attached. In addition, Figure 1 is attached for your edification that displays all related, individual data from present and past requests. 
HNF-3110

Rev. 0

K. S. Witwer

$8 C 510-98-009$

Page 2

February 24, 1998

The major difference from recent measurements is the present use of lower heater currents for the large (Witwer) cinder block measurements. The present use of 2 Amp $^{2}$ heater setting is more in keeping with recommendations of the instrument manufacturer for better accuracy and lower temperature perturbation of the sample. This has resulted in a lower average thermal conductivity than reported in Reference (1). It appears that the two samples do indeed differ significantly in thermal conductivity.

Feel free to call me at $373-2162$ if you have any questions.

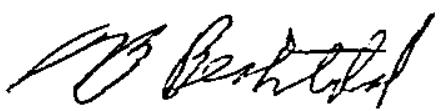

D. B. Bechtold, Scientist

Process Chemistry

fjh

Attachments (2) 
HNF-3110

Rev. 0

$8 C 510-98-009$

$8 C 510-98-009$

Attachments

Consisting of 3 pages including this cover page

Page C-4 


\begin{tabular}{|c|c|c|c|c|c|c|}
\hline \multicolumn{7}{|c|}{ CALCULATIONS FOR THERMAL CONDUCTIVITY } \\
\hline O SPECIMEN & $\begin{array}{l}1 \text { RESULT } \\
(W / m / K)\end{array}$ & $\begin{array}{l}2 \text { HEAIER } \\
\left(A^{\star \star 2} 2\right)\end{array}$ & $\begin{array}{l}3 T^{T} \text { avg } \\
(\bar{C})\end{array}$ & $\begin{array}{l}4 \mathrm{~N} \text { IST } \\
(\mathrm{W} / \mathrm{m} / \mathrm{K})\end{array}$ & 5 RATIO. & 6 DATE TIME \\
\hline $\begin{array}{l}1 \text { SID PYREX } 7740 \text {, } \\
\text { NO. } 2\end{array}$ & 1.2020 & 4 & 30 & 1.1037 & 0.9182 & 18-FEB-1998 13:46:00 \\
\hline $\begin{array}{l}2 \text { STO PYREX 7740, } \\
\text { NO. } 2\end{array}$ & 1.2040 & 4 & 30 & 1.1037 & 0.9167 & 18-FE8-1998 $14: 25: 00$ \\
\hline $\begin{array}{l}3 \text { STO PYREX } 7740 \text {, } \\
\text { NO. } 2\end{array}$ & 1.2020 & 4 & 30 & 1.1037 & 0.9182 & 18-fEB-1998 15:00:00 \\
\hline $\begin{array}{l}4 \text { STD PYROCERAM 9606, } \\
\text { NO. } 2\end{array}$ & 4.2780 & 8 & 31 & 3.9657 & 0.9270 & 18-FEB-1998 13:50:00 \\
\hline $\begin{array}{l}5 \text { STO PYROCERAM 9606, } \\
\text { NO. } 2\end{array}$ & 4.2650 & 8 & 31 & 3.9657 & 0.9298 & 1B-FEB-1988 $14: 30: 00$ \\
\hline $\begin{array}{l}6 \text { STD PYROCERAM 9606, } \\
\text { NO. } 2 \\
7 \text { MEAN RATIO }\end{array}$ & 4.2300 & 8 & 31 & 3.9657 & $\begin{array}{l}0.9375 \\
0.9246\end{array}$ & 18-FEB-1998 15:05:00 \\
\hline 8 SEM RATIO & & & & & 0.0034 & \\
\hline 9 RALSTON BLOCK & 1.0710 & 2 & 30 & 0.9902 & & 18-FEB-1998 14:35:00 \\
\hline 10 RALSTON BLOCK & 1.0710 & 2 & 30 & 0.9902 & & 18-FEB-1998 15:10:00 \\
\hline 11 RALSTON BLOCK & 1.0010 & 2 & 30 & $0.9255^{*}$ & & 18-FEB-1998 15:20:00 \\
\hline 12 RALSTON REPS & 3.0000 & & & & & \\
\hline 13 MEAN RALSTON & 1.0477 & & $\underline{30}$ & 0.9687 & & \\
\hline 14 SEM RALSTON & 0.0233 & & & 0.0219 & & \\
\hline $1595 \%$ CONF RALSTON & & & & $\underline{0.0941}$ & & $\cdot$ \\
\hline 16 WITWER BLOCX & 0.8202 & 2 & 30 & 0.7583 & & 18-FEB-1998 14:01:00 \\
\hline 17 WITWER BLOCK & 0.8243 & 2 & 31 & 0.7621 & & 18 -FEB-1998 $14: 40: 00$ \\
\hline 18 WITWER BLOCK & 0.7816 & 2 & 31 & 0.7227 & & $18-\mathrm{FEB}-1998 \quad 15: 15: 00$ \\
\hline 19 WITHER REPS & 3.0000 & & & & & \\
\hline 20 MEAN WITWER & 0.8087 & & 31 & $\underline{0.7477}$ & & \\
\hline 21 SEM WITWER & 0.0136 & & & 0.0129 & & \\
\hline 22 95\% CONF WITWER & & & & 0.0554 & & \\
\hline
\end{tabular}


HNF-3110

Rev. 0

8C510-98-009

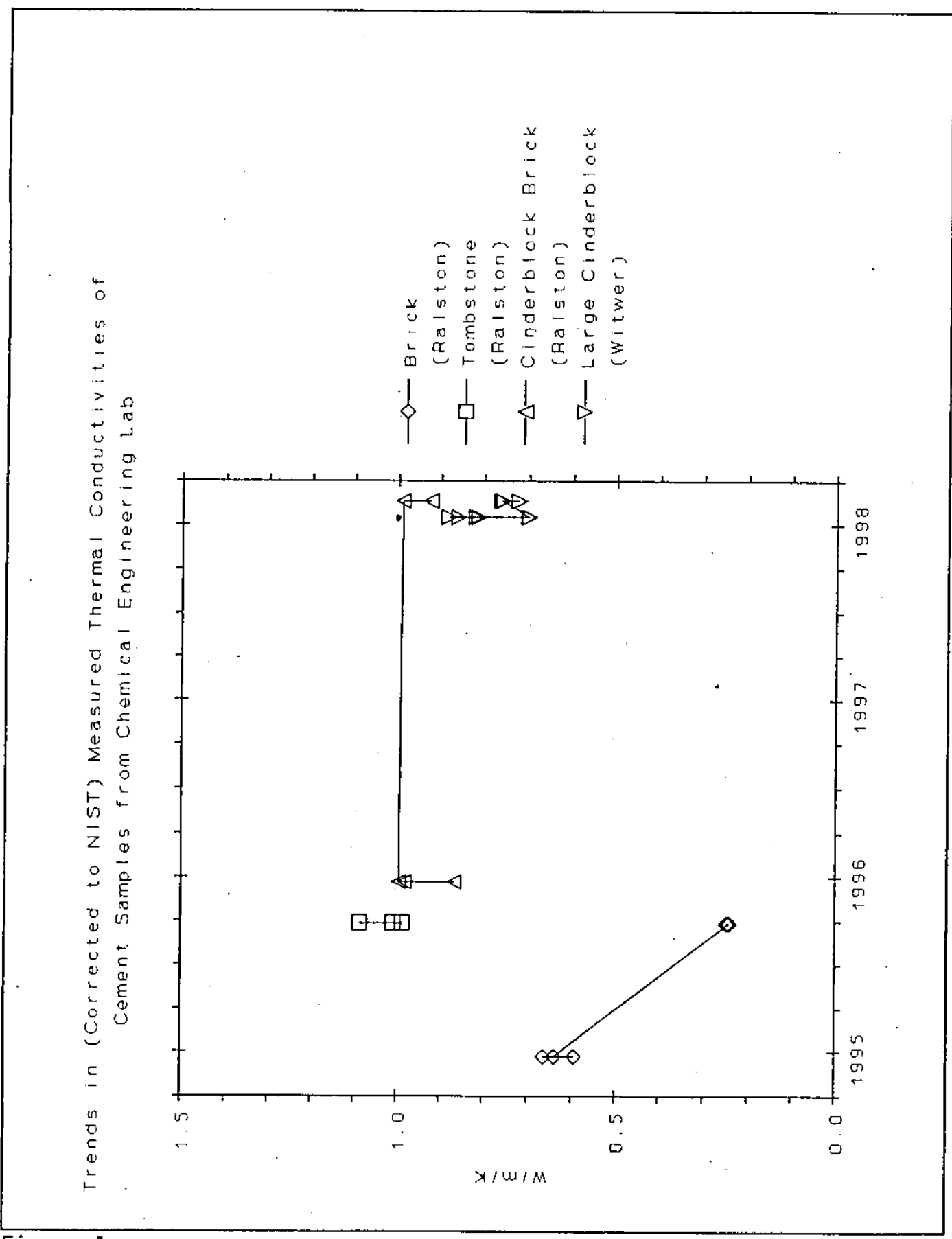

Figure 1

Page C-6 


\section{APPENDIX D - EQUIPMENT CALIBRATION DATA}

\begin{tabular}{|c|c|c|c|}
\hline Item & Hanford Std Lab \# & Range/Accuracy & Cal Expiration \\
\hline $\begin{array}{c}\text { Omega CL505A Temperature } \\
\text { Calibrator }\end{array}$ & $750-13-55-002$ & $+/-0.5^{\circ} \mathrm{C}$ & $7 / 22 / 98$ \\
\hline Celesco PT101-40A & ETL Lab Cal & $-24^{\prime \prime}+/-1 / 16^{\prime \prime}$ & Cal/Accur chk as reqd \\
\hline Toledo/Mett Mdl 8140 & $750-66-01-004$ & $0-20001 \mathrm{~b}(\mathrm{Cal})+2 \mathrm{lb}$ & $12 / 21 / 98$ \\
\hline Cole-Parmer Flowmeter & $\mathrm{N} / \mathrm{A}$ & $4-50 \mathrm{SCFM}+/-1 \mathrm{SCFM}$ & $1 / 23 / 99$ \\
\hline Type K TC & ETL Lab Cal & $0-300^{\circ} \mathrm{C} / 2^{\circ} \mathrm{C}$ & $\mathrm{N} / \mathrm{A}$ \\
\hline $\begin{array}{c}\text { Ashcroft Bourdon Tube Test } \\
\text { Pressure Gage }\end{array}$ & $752-31-04-085$ & $+/-0.5 \mathrm{PSI}$ & $12 / 30 / 98$ \\
\hline $\begin{array}{c}\text { SnapOn } \\
\text { Torque Wrench (Electronic) }\end{array}$ & $750-88-01-005$ & $15-250 \mathrm{f}-1 \mathrm{~b} / \pm \mathrm{fft}-1 \mathrm{~b}$ & $3 / 20 / 99$ \\
\hline
\end{tabular}


HNF-3110

Rev. 0

APPENDIX E - TEST DATA FILES

Page E-1 


\begin{tabular}{|c|c|c|c|c|c|c|c|c|c|c|}
\hline$T I M E$ & $\begin{array}{l}\text { Elapsed } \\
\text { Time } \\
\text { (sec) }\end{array}$ & BIT_TC1F & PURG_TC2 & PurgePSI & PurgeCFM & DEPTH(IN) & DwnFrc(lb) & $\mathrm{RPM}$ & $\begin{array}{l}\text { TORQUE } \\
\text { (FTLB) }\end{array}$ & Inches/Min \\
\hline $14: 14: 00: 43$ & 0 & 24 & 25 & $\quad 18.9$ & $\quad 56.5$ & $\begin{array}{ll}-0.8 \\
-\end{array}$ & -1 & 55.3 & 0 & -0.13 \\
\hline $14: 14: 01: 36$ & 1 & 24 & 25 & $18.9^{9}$ & 56.5 & -0.52 & $-1 !$ & 55.4 & 0 & -0.11 \\
\hline $14: 14: 02: 35$ & 2 & 24 & 25 & 18.9 & 56.5 & 0.11 & 64 & 55.4 & 5 & 6.6 \\
\hline $14: 14: 03: 34$ & 3 & 26 & 25 & 18.9 & $5 \overline{6} .5$ & $0 . \overline{13}$ & 678 & 55 & 20 & 1.2 \\
\hline $14: 14: 04: 33$ & 4 & 28 & 25 & 18.9 & 56.5 & 0.18 & 593 & 54.7 & 18 & 3 \\
\hline $14: 14: 05: 32$ & 5 & $30^{\dagger}$ & 25 & 18.9 & 56.5 & $0 . \overline{21}$ & 639 & 54.7 & 22 & 1.84 \\
\hline $14: 14: 06: 36$ & 6 & 32 & 25 & $18 . \overline{9}$ & 56.5 & 0.23 & 639 & 54.3 & 24 & 1.44 \\
\hline $14: 14: 07: 35$ & 7 & 33 & 25 & 18.9 & 56.6 & 0.26 & 659 & 54.1 & 24 & 1.44 \\
\hline $14: 14: 08: 34$ & 8 & 34 & $25^{\prime}$ & $18.9^{\dagger}$ & 56.6 & 0.27 & 642 & 54.1 & 25 & 0.93 \\
\hline $14: 14: 09: 38$ & 9 & 34 & 25 & 18.9 & 56.6 & 0.31 & 657 & 54.1 & 26 & 0.93 \\
\hline $14: 14: 10: 37$ & 10 & 36 & 25 & 18.9 & 56.6 & 0.32 & 667 & 54.2 & 27 & 0.85 \\
\hline $14: 14: 11: 36$ & 11 & 36 & 25 & 18.9 & 56.6 & 0.33 & 692 & 54.2 & 27 & 0.85 \\
\hline $14: 14: 12: 35$ & 12 & 37 & 25 & 18.9 & 56.6 & 0.33 & 709 & 54.2 & 25 & 0.04 \\
\hline $14: 14: 13: 33$ & 13 & 37 & 25 & 18.9 & 56.6 & 0.33 & 717 & 54.1 & 24 & 0.04 \\
\hline $14: 14: 14: 38$ & 14 & 38 & 25 & 18.9 & 56.6 & 0.35 & 719 & 54.1 & 24 & 0.11 \\
\hline $14: 14: 15: 37$ & 15 & 38 & 25 & 18.9 & 56.6 & 0.33 & 717 & 54.7 & 23 & 0.11 \\
\hline $14: 14: 16: 36$ & 16 & 38 & 25 & 18.9 & 56.6 & 0.35 & 729 & 54.1 & 22 & 0.11 \\
\hline $14: 14: 17: 34$ & 17 & 38 & 25 & 18.9 & 56.6 & 0.36 & 726 & 54.1 & 22 & 0.11 \\
\hline $14: 14: 18: 33$ & 18 & 38 & 25 & 18.9 & 56.6 & 0.36 & 728 & 54.2 & 21 & -0.03 \\
\hline $14: 14: 19: 32$ & $1 \overline{9}$ & 39 & 25 & 18.9 & 56.6 & 0.36 & 734 & 54.4 & 21 & -0.03 \\
\hline $14: 14: 20: 31$ & 20 & 39 & 26 & 19.1 & 56.6 & $0.3 \overline{6}$ & 741 & 54.4 & 20 & -0.14 \\
\hline $14: 14: 21: 30$ & 21 & 39 & 26 & 18.9 & 56.6 & 0.36 & 749 & 54.4 & 20 & -0.14 \\
\hline $14: 14: 22: 29$ & 22 & 40 & 25 & 19.1 & 56.6 & 0.37 & 751 & 54.4 & 19 & 0.17 \\
\hline $14: 14: 23: 28$ & 23 & 40 & 26 & 19.1 & 56.6 & 0.37 & 753 & 54.4 & 19 & 0.17 \\
\hline $14: 14: 24: 26$ & 24 & 41 & 26 & $19.1^{\dagger}$ & 56.6 & 0.36 & 758 & 54.3 & 19 & 0.05 \\
\hline $14: 14: 25: 25$ & 25 & 41 & 26 & 19.1 & 56.6 & 0.36 & 741 & 54.3 & 18 & 0.05 \\
\hline $14: 14: 26: 30$ & 26 & 41 & 26 & 19.1 & 56.6 & 0.36 & 746 & 54.3 & 18 & -0.27 \\
\hline $14: 14: 27: 29$ & 27 & 42 & $26 !$ & $1 \overline{9} .1$ & 56.6 & 0.36 & $745^{\dagger}$ & 54.4 & 18 & -0.27 \\
\hline $14: 14: 28: 27$ & 28 & 42 & 26 & 19.1 & 56.6 & 0.37 & 749 & 54.4 & 18 & -0.2 \\
\hline $14: 14: 29: 26$ & 29 & 42 & 26 & 19.1 & 56.6 & 0.37 & $756 !$ & 54.3 & 18 & -0.2 \\
\hline $14: 14: 30: 25$ & 30 & $4 \overline{2}$ & 25 & 19.1 & 56.6 & 0.37 & 763 & 54.4 & 18 & -0.04 \\
\hline $14: 14: 31: 30$ & 31 & 42 & 25 & 19.1 & 56.6 & 0.37 & 760 & 54.4 & 18 & -0.04 \\
\hline $14: 14: 32: 28$ & 32 & 42 & 25 & 19.1 & 56.6 & 0.37 & 765 & 54.4 & 18 & $-0 . \overline{04}$ \\
\hline $14: 14: 33: 27$ & 33 & 43 & 25 & 19.1 & 56.6 & 0.37 & 766 & 546 & 18 & $-0 . \overline{11}$ \\
\hline $14: 14: 34: 32$ & 34 & 43 & 25 & 19.1 ? & 56.5 & 0.37 & 763 & 54.6 & 18 & -0.11 \\
\hline $14: 14: 35: 30$ & 35 & 43 & 26 & 19.1 & 56.6 & 0.37 & 766 & 54.5 & 18 & -0.39 \\
\hline $14: 14: 36: 29$ & 36 & 43 & 26 & 19.1 & 56.6 & 0.37 & 761 & 54.5 & 18 & -0.39 \\
\hline $14: 14: 37: 28$ & 37 & 44 & 26 & 19.1 & 56.5 & 0.36 & 753 & 54.4 & 18 & 0.14 \\
\hline $14: 14: 38: 27$ & 38 & 44 & 26 & 19.1 & 56.5 & 0.37 & 752 & 54.4 & 18 & 0.14 \\
\hline $14: 14: 39: 31$ & 39 & 44 & 25 & 19.1 & 56.6 & $0 . \overline{37}$ & $755^{\prime}$ & 54.5 & 18 & -0.06 \\
\hline $14: 14: 40: 30$ & 40 & 44 & 26 & $19.1^{+}$ & 56.6 & 0.37 & 761 & 54.5 & 18 & -0.06 \\
\hline $14: 14: 41: 29$ & 41 & $4 \overline{4}$ & 26 & 19.1 & 56.6 & 0.39 & 766 & 54.5 & 18 & -0.24 \\
\hline $14: 14 \overline{4: 42: 28}$ & 42 & 44 & 26 & 19.1 & 56.6 & 0.37 & 764 & 54.5 & 17 & -0.24 \\
\hline $14: 14: 43: 27$ & 43 & 44 & 26 & 19.1 & 56.6 & 0.37 & 768 & 54.5 & 17 & -0.19 \\
\hline $14: 14: 44: 26$ & 44 & 44 & 26 & $19.1_{j}$ & 56.6 & 0.37 & 770 & 54.4 & 17 & -0.19 \\
\hline $14: 14: 45: 25$ & 45 & 44 & 26 & 19.1 & 56.6 & 0.37 & 765 & 54.4 & 17 & -0.09 \\
\hline $14: 14: 46: 23$ & 46 & 45 & 26 & 19.1 & 56.6 & 0.37 & 758 & 54.4 & 17 & -0.09 \\
\hline $14: 1 \overline{4: 47: 22}$ & 47 & 45 & 26 & 19.1 & 56.5 & 0.37 & 758 & 354.4 & 17 & -0.05 \\
\hline $14: 14: 48: 21$ & 48 & 45 & 26 & 19.1 & 56.6 & 0.39 & 763 & 54.4 & 17 & -0.05 \\
\hline
\end{tabular}




\begin{tabular}{|c|c|c|c|c|c|c|c|c|c|c|}
\hline$T I M E$ & $\begin{array}{l}\text { Elapsed } \\
\text { Time } \\
\text { (sec) }\end{array}$ & $B I T$ TCIF & PURG_TC2 & PurgePSI & PurgeCFM & DEPTH(IN) & DwnFrc $(\mid \mathrm{b})$ & RPM & $\begin{array}{l}\text { TORQUE } \\
\text { (FTLB) }\end{array}$ & Inches/Min \\
\hline $14: 14: 49: 20$ & 49 & -45 & -26 & 19.1 & $\quad 56.5$ & $\quad 0.39$ & $\quad 764$ & 54.4 & 17 & -0.36 \\
\hline $14: 14: 50: 19$ & 50 & 44 & 25 & 19.1 & 56.5 & 0.39 & 761 & 54.3 & 17 & -0.36 \\
\hline $14: 14: 51: 23$ & 51 & $\overline{44}$ & 26 & 19.1 & 56.5 & 0.39 & 767 & 54.2 & 17 & -0.1 \\
\hline $14: 14: 52: 22$ & 52 & 44 & 26 & 19.1 & 56.5 & 0.39 & 770 & 54.2 & 17 & -0.1 \\
\hline $14: 14: 53: 21$ & 53 & 45 & 26 & $19 . \overline{1}$ & 56.5 & 0.39 & 775 & 54.1 & 17 & 0.08 \\
\hline $14: 14: 54: 20$ & 54 & 45 & 26 & 19.1 & 56.5 & 0.39 & 771 & 53.9 & 17 & 0.08 \\
\hline $14: 14: 55: 19$ & 55 & 45 & 26 & 19.1 & 56.5 & 0.37 & 768 & 53.9 & 17 & -0.25 \\
\hline $14: 14: 56: 23$ & 56 & 45 & 26 & 19.1 & 56.5 & 0.39 & 772 & 53.9 & 17 & -0.25 \\
\hline $14: 14: 57: 22$ & 57 & 45 & 26 & 19.1 & 56.5 & 0.39 & 765 & 53.8 & 17 & -0.23 \\
\hline $14: 14: 58: 21$ & 58 & 45 & 26 & 19.1 & 56.5 & 0.4 & 766 & 53.8 & 17 & 0.01 \\
\hline $14: 14: 59: 25$ & 59 & 45 & 26 & 19.1 & 56.5 & 0.39 & 775 & 53.8 & 17 & $0 . \overline{01}$ \\
\hline $14: 15: 00: 24$ & 60 & 45 & 26 & 19.1 & 56.5 & 0.39 & 780 & 53.8 & 17 & -0.07 \\
\hline $14: 15: 01: 23$ & 61 & 45 & 26 & 19.1 & 56.5 & 0.39 & 777 & 53.8 & 17 & -0.07 \\
\hline $14: 15: 02: 22$ & 62 & 45 & 26 & 19.1 & 56.5 & 0.39 & 783 & 53.8 & 17 & -0.35 \\
\hline $14: 15: 03: 21$ & 63 & 45 & 26 & 19.1 & 56.5 & 0.39 & 784 & 53.8 & 17 & -0.35 \\
\hline $14: 15: 04: 25$ & 64 & 45 & 26 & 19.1 & 56.5 & 0.39 & 778 & 53.8 & 17 & -0.1 \\
\hline $14: 15: 05: 24$ & 65 & 45 & 26 & 19.1 & 56.5 & 0.37 & 762 & 53.9 & 16 & -0.1 \\
\hline $14: 15: 06: 23$ & 66 & 45 & 26 & 19.1 & 56.5 & 0.39 & 758 & 53.9 & 16 & 0.07 \\
\hline $14: 15: 07: 22$ & 67 & 45 & 26 & 19.1 & 56.5 & 0.39 & 757 & 53.9 & 16 & 0.07 \\
\hline $14: 15: 08: 21$ & 68 & 45 & 26 & 19.1 & 56.5 & 0.39 & 756 & 53.9 & 17 & -0.34 \\
\hline $14: 15: 09: 19$ & 69 & 45 & 26 & 19.1 & 56.5 & 0.41 & 761 & 53.8 & 17 & -0.34 \\
\hline $14: 15: 10: 18$ & 70 & 45 & 26 & 19.1 & 56.5 & 0.41 & 767 & 53.8 & 17 & -0.3 \\
\hline $14: 15: 11: 17$ & 71 & 45 & 25 & 19.1 & 56.5 & 0.39 & 770 & 53.7 & 17 & -0.3 \\
\hline $14: 15: 12: 16$ & 72 & 45 & 26 & 19.1 & 56.5 & 0.39 & 768 & 53.7 & $\overline{17}$ & 0.09 \\
\hline $14: 15: 13: 15$ & 73 & 45 & 26 & 19.1 & 56.5 & 0.39 & 760 & 53.7 & 17 & 0.09 \\
\hline $14: 15: 14: 14$ & 74 & 45 & 26 & 19.1 & 56.5 & 0.37 & 752 & 53.6 & 17 & -0.11 \\
\hline $14: 15: 15: 13$ & 75 & $4 \overline{5}$ & 26 & 19.1 & 56.5 & 0.39 & 753 & $\overline{53.7}$ & $1 \overline{6}$ & -0.11 \\
\hline $14: 15: 16: 17$ & 76 & 45 & 26 & 19.1 & 56.5 & 0.41 & 751 & 53.7 & 17 & -0.34 \\
\hline $14: 1 \overline{5: 17: 16}$ & 77 & 45 & 26 & 19.1 & 56.5 & 0.39 & 758 & 53.6 & 17 & -0.34 \\
\hline $14: 15: 18: 15$ & 78 & 45 & 26 & 19.1 & 56.5 & 0.41 & 765 & 53.5 & 17 & -0.02 \\
\hline $14: 15: 19: 14$ & 79 & 45 & 26 & 19.1 & $5 \overline{6.5}$ & 0.41 & 770 & 53.5 & 17 & -0.02 \\
\hline $14: 15: 20: 12$ & 80 & 45 & 26 & 19.1 & 56.5 & 0.39 & 770 & 53.6 & 17 & -0.03 \\
\hline $14: 15: 21: 17$ & 81 & 45 & 26 & 19.1 & 56.5 & 0.39 & 765 & 53.7 & 17 & -0.03 \\
\hline $14: 15: 22: 16$ & 82 & 45 & 26 & 19.1 & 56.5 & $0 . \overline{4}$ & 769 & 53.7 & 17 & -0.27 \\
\hline $14: 15: 23: 14$ & 83 & 46 & $26^{\dagger}$ & 19.1 & 56.5 & 0.37 & $76 \overline{0}$ & $53 . \overline{7}$ & 16 & -0.35 \\
\hline $14: 15: 24: 13$ & 84 & 46 & 26 & 19.1 & 56.5 & 0.4 & 754 & 53.9 & 16 & -0.35 \\
\hline $14: 15: 25: 12$ & 85 & 46 & 26 & 19.1 & 56.5 & $0 . \overline{41}$ & 755 & 53.9 & 16 & 0.02 \\
\hline $14: 15: 26: 11$ & 86 & 45 & 26 & 19.1 & 56.5 & 0.41 & 760 & 54 & 16 & 0.02 \\
\hline $14: 15: 27: 10$ & 87 & 45 & 26 & 19.1 & 56.5 & 0.39 & 766 & 54.1 & 16 & -0.1 \\
\hline $14: 15: 28: 09$ & 88 & 45 & 26 & 19.1 & 56.5 & 0.39 & 770 & 54.1 & 17 & -0.1 \\
\hline $14: 15: 29: 08$ & 89 & 45 & 26 & 19.1 & 56.5 & 0.41 & 766 & 54.2 & 17 & -0.29 \\
\hline $14: 15: 30: 07$ & 90 & 45 & 26 & 19.1 & 56.5 & 0.41 & 769 & 54.3 & 17 & -0.29 \\
\hline $14: 15: 31: 05$ & 91 & $45^{\dagger}$ & 26 & 19.1 & 56.5 & 0.39 & 767 & $5 \overline{4.3}$ & 17 & -0.13 \\
\hline $14: 15: 32: 04$ & 92 & 46 & 26 & 19.1 & 56.5 & 0.39 & 758 & 54.3 & 17 & -0.13 \\
\hline $14: 15: 33: 09$ & 93 & $\overline{46}$ & 26 & 19.1 & 56.5 & 0.39 & 752 & 54.4 & 17 & -0.08 \\
\hline $14: 15: 34: 07$ & 94 & 46 & 26 & 19.1 & 56.5 & 0.39 & 753 & 54.4 & 17 & -0.08 \\
\hline $14: 15: 35: 06$ & 95 & 45 & 26 & 19.1 & 56.5 & 0.41 & 752 & 54.4 & 17 & -0.22 \\
\hline $14: 15: 36: 05$ & 96 & 45 & 26 & 19.1 & 56.5 & 0.4 & 755 & 54.4 & 17 & -0.22 \\
\hline $14: 15: 37: 04$ & $9 \overline{7}$ & 45 & 26 & 19.1 & 56.6 & 0.41 & 760 & 54.4 & 17 & -0.3 \\
\hline
\end{tabular}




\begin{tabular}{|c|c|c|c|c|c|c|c|c|c|c|}
\hline$T I M E$ & $\begin{array}{l}\text { Elapsed } \\
\text { Time } \\
(\mathrm{sec})\end{array}$ & $B I T=T C$ & PURG_TC2 & PurgePSI & PurgeCFM & DEPTH(IN) & DwnFrc(lb) & RPM & $\begin{array}{l}\text { TORQUE } \\
\text { (FTLB) }\end{array}$ & Inches/Min \\
\hline $14: 15: 38: 03$ & $\quad 98$ & -45 & -26 & 19.1 & $\quad 56.5$ & $\quad 0.41$ & $\quad 766$ & $\begin{array}{ll}6 & 54.4\end{array}$ & $\quad 17$ & $\quad-0 . \overline{3}$ \\
\hline $14: 15: 39: 02$ & $99^{\circ}$ & 45 & 26 & 19.1 & 56.5 & 0.39 & 775 & 554.3 & 17 & 0.05 \\
\hline $14: 15: 40: 01$ & 100 & 45 & 26 & 19.1 & 56.5 & 0.41 & 773 & 3.54 .3 & 17 & 0.05 \\
\hline $14: 15: 41: 00$ & 101 & 46 & 26 & 19.1 & 56.5 & 0.39 & 765 & 554.2 & 17 & -0.1 \\
\hline $14: 15: 41: 98$ & 102 & $\overline{46}$ & 26 & 19.1 & $56 . \overrightarrow{5}$ & 0.39 & 758 & 854.3 & 16 & -0.1 \\
\hline $14: 15: 42: 97$ & 103 & 46 & 25 & 19.1 & 56.5 & 0.41 & 762 & 254.3 & 16 & $-0 . \overline{3}$ \\
\hline $14: 15: 43: 96$ & 104 & 45 & 26 & 19.1 & 56.5 & 0.41 & 758 & 854.4 & 16 & -0.3 \\
\hline $14: 15: 45: 01$ & 105 & $45 !$ & 26 & 19.1 & 56.5 & 0.39 & 759 & 9.54 & 16 & -0.23 \\
\hline $14: 15: 45: 99$ & 106 & 46 & 26 & $19 . \overline{1}$ & 56.5 & 0.41 & 765 & $5 \quad 54.4$ & 16 & -0.23 \\
\hline $14: 15: 46: 98$ & 107 & 46 & 26 & 19.1 & 56.5 & 0.41 & 771 & 154.4 & 16 & -0.1 \\
\hline $14: 15: 47: 97$ & 108 & 46 & 26 & 19.1 & 56.5 & 0.41 & 773 & 354.5 & 17 & -0.1 \\
\hline $14: 15: 48: 96$ & 109 & 46 & 26 & 19.1 & 56.5 & 0.41 & 773 & 354.5 & 17 & -0.13 \\
\hline $14: 15: 50: 00$ & 110 & 45 & 26 & 19.1 & 56.5 & 0.4 & 775 & 554.7 & 17 & -0.13 \\
\hline $14: 15: 50: 99$ & 111 & 46 & 26 & 19.1 & 56.5 & 0.41 & 774 & $4 \quad 54.9$ & 17 & -0.23 \\
\hline $14: 15: 51: 98$ & 112 & 46 & 26 & 19.1 & 56.5 & 0.39 & 766 & $6 \quad 54.9$ & 17 & -0.32 \\
\hline $14: 15: 53: 02$ & 113 & 46 & 26 & 19.1 & 56.5 & 0.39 & 757 & 7.54 .9 & 17 & -0.32 \\
\hline $14: 15: 54: 01$ & 114 & 46 & $26^{\dagger}$ & 19.1 & 56.5 & 0.41 & 756 & $6 \quad 54.9$ & 17 & -0.03 \\
\hline $14: 15: 55: 00$ & 115 & 46 & 26 & 19.1 & 56.5 & 0.39 & 756 & $6 \quad 54.9$ & 16 & -0.03 \\
\hline $14: 15: 55: 99$ & 116 & 46 & 26 & 19.1 & 56.5 & 0.41 & 756 & $6: 55$ & 16 & -0.08 \\
\hline $14: 15: 56: 98$ & 117 & 46 & 26 & 19.1 & $56 . \overline{5}$ & 0.39 & 760 & 55 & 16 & -0.08 \\
\hline $14: 15: 58: 02$ & 118 & 46 & 26 & 19.1 & 56.5 & 0.41 & 765 & 55 & 16 & -0.22 \\
\hline $14: 15: 59: 01$ & 119 & 46 & 26 & 19.1 & 56.5 & 0.41 & 773 & $3 \quad 55$ & 16 & -0.22 \\
\hline $14: 16: 00: 00$ & 120 & 46 & 26 & 19.1 & 56.5 & 0.41 & 776 & $6 \quad 54.8$ & 17 & -0.08 \\
\hline $14: 16: 00: 99$ & 121 & 46 & 26 & 19.1 & 56.5 & 0.41 & 775 & 554.8 & 17 & -0.08 \\
\hline $14: 16: 01: 98$ & 122 & $46 !$ & $26 !$ & 19.1 & 56.5 & 0.41 & 775 & $5 \quad 54.8$ & 16 & -0.22 \\
\hline $14: 16: 02: 97$ & 123 & 46 & 26 & 19.1 & 56.5 & 0.41 & 772 & 254.9 & 16 & -0.22 \\
\hline $14: 16: 03: 95$ & 124 & 46 & 26 & 19.1 & 56.5 & 0.39 & 765 & $5 \quad 54.9$ & 16 & -0.01 \\
\hline 14:16:04:94 & 125 & 46 & 26 & 19.1 & 56.5 & 0.41 & 759 & 954.8 & 16 & -0.01 \\
\hline $14: 16: 05: 93$ & 126 & 46 & 26 & 19.1 & 56.5 & 0.4 & 760 & 0.54 .8 & 16 & -0.3 \\
\hline $14: 16: 06: 92$ & 127 & 46 & 26 & 19.2 & 56.5 & 0.4 & 765 & \begin{tabular}{l|l}
5 & 54.8
\end{tabular} & 17 & -0.3 \\
\hline $14: 16: 07: 91$ & 128 & 46 & 26 & 19.2 & 56.5 & 0.41 & 771 & 1.54 .9 & 17 & -0.23 \\
\hline $14: 16: 08: 90$ & 129 & 46 & 26 & 19.2 & 56.5 & 0.41 & 765 & $5,54.9$ & 17 & -0.23 \\
\hline $14: 16: 09: 94$ & 130 & 46 & 26 & 19.2 & 56.5 & 0.41 & 771 & 154.9 & 17 & -0.15 \\
\hline $14: 16: 10: 93$ & 131 & 46 & 26 & $19 . \overline{2}$ & 56.5 & 0.41 & 775 & $5 \longdiv { 5 4 . 9 }$ & 17 & -0.15 \\
\hline $14: 16: 11: 92$ & 132 & 46 & 26 & 19.2 & 56.5 & 0.41 & 775 & $5 \quad 54.9$ & 17 & 0.02 \\
\hline $14: 16: 12: 91$ & 133 & 46 & $26^{\dagger}$ & 19.2 & 56.5 & $0 . \overline{41}$ & 770 & $0, \overline{54.9}$ & 17 & 0.02 \\
\hline $14: 16: 13: 90$ & 134 & 46 & 26 & 19.2 & 56.5 & 0.41 & 762 & 254.9 & 16 & -0.25 \\
\hline $14: 16: 14: 94$ & 135 & 46 & 26 & 19.2 & $5 \overline{6} .5$ & 0.4 & 782 & $2 \quad 55$ & 16 & -0.25 \\
\hline $14: 16: 15: 93$ & 136 & 46 & 26 & 19.2 & 56.5 & 0.41 & 798 & 55 & 17 & -0.29 \\
\hline $14: 16: 16: 92$ & 137 & 46 & 26 & 19.2 & 56.5 & $0.4 \overline{1}$ & 795 & $5 \quad 55$ & 17 & -0.09 \\
\hline $14: 16: 17: 96$ & 138 & 46 & 26 & 19.2 & $56.5^{\dagger}$ & 0.41 & 809 & 954.9 & 17 & -0.09 \\
\hline $14: 16: 18: 95$ & 139 & 46 & 26 & 19.2 & 56.4 & 0.42 & 839 & 954.9 & 18 & -0.08 \\
\hline $14: 16: 19: 94$ & 140 & 46 & 26 & 19.2 & 56.4 & $0 . \overline{41}$ & 833 & 354.9 & 17 & -0.08 \\
\hline $14: 16: 20: 93$ & 141 & 46 & 26 & 19.2 & 56.4 & 0.42 & 835 & 5.54 .8 & 18 & -0.22 \\
\hline $14: 16: 21: 92$ & 142 & $4 \overline{7}$ & 26 & 19.2 & 56.4 & 0.41 & 837 & 754.8 & 18 & $-0 . \overline{22}$ \\
\hline $14: 16: 22: 96$ & 143 & 47 & 26 & 19.2 & 56.4 & 0.41 & 837 & 754.6 & 18 & -0.3 \\
\hline $14: 16: 23: 95$ & 144 & 46 & 26 & 19.2 & 56.4 & $0 . \overline{41}$ & 834 & 4.54 .7 & 18 & -0.3 \\
\hline $14: 16: 24: 94$ & 145 & 47 & 26 & 19.2 & 56.4 & 0.41 & 824 & 454.7 & 18 & -0.08 \\
\hline $14: 16: 25: 92$ & 146 & 47 & 26 . & 19.1 & 56.4 & 0.4 & 827 & 754.8 & 18 & -0.08 \\
\hline
\end{tabular}




\begin{tabular}{|c|c|c|c|c|c|c|c|c|c|c|}
\hline$T I M E$ & $\begin{array}{l}\text { Elapsed } \\
\text { Time } \\
\text { (sec) }\end{array}$ & BIT_TC1 & PURG_TC2 & PurgePSI & PurgeCFM & DEPTH(IN) & DownFrc(lb) & $R P M$ & $\begin{array}{l}\text { TORQUE } \\
\text { (FTLB) }\end{array}$ & Inches/Min \\
\hline $14: 16: 26: 91$ & $\quad 147$ & 47 & 26 & 19.2 & $\quad 56.4$ & $\quad 0.4$ & 820 & 54.8 & 18 & $\quad-0.08$ \\
\hline $14: 16: 27: 90$ & $148^{+}$ & 47 & 26 & 19.2 & 56.4 & 0.41 & 813 & 354.8 & 17 & -0.08 \\
\hline $14: 16: 28: 89$ & 149 & 47 & 26 & 19.2 & 56.4 & 0.41 & $813 !$ & 54.8 & 18 & -0.31 \\
\hline $14: 16: 29: 88$ & 150 & 47 & $26^{\prime}$ & 19.2 & 56.4 & $0 . \overline{41}$ & $818^{\circ}$ & 354.8 & 18 & -0.31 \\
\hline $14: 16: 30: 87$ & 151 & 47 & 26 & 19.2 & 56.4 & 0.4 & 822 & 254.8 & 18 & -0.25 \\
\hline $14: 16: 31: 86$ & 152 & 47 & 26 & 19.2 & 56.4 & 0.42 & 823 & 54.8 & 17 & -0.25 \\
\hline $14: 16: 32: 85$ & 153 & $4 \overline{7}$ & 26 & 19.2 & 56.4 & 0.41 & 822 & 54.7 & 18 & -0.13 \\
\hline $14: 16: 33: 83$ & 154 & 47 & 26 & 19.2 & 56.4 & 0.41 & 814 & 54.7 & 17 & -0.13 \\
\hline $14: 16: 34: 88$ & $\overline{155}$ & 47 & 26 & $19.2^{-1}$ & 56.4 & 0.41 & 818 & 54.6 & 17 & -0.11 \\
\hline $14: 16: 35: 87$ & 156 & 47 & 26 & 19.2 & $56.4^{\dagger}$ & 0.41 & 808 & 54.6 & 17 & -0.11 \\
\hline $14: 16: 36: 85$ & 157 & 47 & 26 & 19.2 & 56.4 & 0.41 & 800 & 54.6 & 17 & -0.21 \\
\hline $14: 16: 37: 84$ & 158 & 47 & $26^{\dagger}$ & 19.2 & 56.4 & 0.41 & $798:$ & 3: 54.5 & 17 & -0.21 \\
\hline $14: 16: 38: 83$ & 159 & 47 & 26 & $19.2^{\dagger}$ & 56.4 & 0.41 & 802 & 54.5 & $17 !$ & -0.38 \\
\hline $14: 16: 39: 88$ & 160 & $47 \overrightarrow{1}$ & 26 & 19.2 & 56.4 & 0.41 & $806^{\dagger}$ & 54.5 & 18 & -0.38 \\
\hline $14: 16: 40: 86$ & 161 & 47 & 26 & 19.2 & 56.4 & 0.42 & 801 & 154.6 & 17 & 0.08 \\
\hline $14: 16: 41: 85$ & 162 & 47 & 26 & $19 . \overline{1}$ & 56.4 & 0.41 & 805 & 54.7 & 18 & -0.22 \\
\hline $14: 16: 42: 90$ & 163 & 47 & 26 & 19.2 & 56.4 & 0.42 & 808 & 54.7 & 18 & -0.22 \\
\hline $14: 16: \overline{43: 89}$ & 164 & 47 & 26 & 19.2 & 56.4 & 0.41 & 808 & 54.7 & 18 & -0.15 \\
\hline $14: 16: 44: 87$ & 165 & 47 & $26^{4}$ & $19.1^{+}$ & 56.4 & 0.41 & 802 & 54.7 & 17 & -0.15 \\
\hline $14: 16: 45: 86$ & 166 & 47 & 26 & 19.2 & 56.4 & 0.4 & $795^{\dagger}$ & 54.7 & 17 & -0.43 \\
\hline $14: 16: 46: 85$ & 167 & 47 & 26 & 19.2 & 56.4 & 0.41 & $799^{\prime}$ & $9 \quad 54.9$ & 17 & -0.43 \\
\hline $14: 16: 47: 89$ & 168 & 47 & 26 & 19.2 & 56.4 & 0.41 & 791 & 155 & 17 & 0.11 \\
\hline $14: 16: 48: 88$ & 169 & 47 & 26 & 19.2 & 56.4 & 0.41 & 787 & 55 & 17 & 0.11 \\
\hline $14: 16: 49: 87$ & 170 & 47 & 26 & 19.2 & 56.4 & 0.41 & $787 !$ & 55.1 & 17 & -0.27 \\
\hline $14: 16: 50: 86$ & 171 & 47 & 26 & 19.2 & 56.4 & 0.42 & 792 & 55.1 & 17 & -0.27 \\
\hline $14: 16: 51: 85$ & 172 & 47 & 26 & 19.2 & 56.4 & 0.41 & 797 & 75.1 & 17 & -0.22 \\
\hline $14: 16: 52: 84$ & 173 & 47 & $26^{\dagger}$ & $19.2^{\dagger}$ & 56.4 & 0.41 & 791 & $1-55$ & 17 & -0.22 \\
\hline $14: 16: 53: 83$ & 174 & 47 & 26 & 19.2 & $56.4^{\dagger}$ & 0.41 & 795 & 55 & 17 & -0.39 \\
\hline $14: 16: 54: 82$ & 175 & 47 & $26 !$ & 19.2 & 56.4 & $0 . \overline{41}$ & 798 & 55 & 17 & -0.39 \\
\hline $14: 16: 55: 80$ & 176 & 47 & 26 & 19.2 & 56.4 & 0.41 & 796 & 54.9 & 17 & -0.03 \\
\hline $14: 16: 56: 79$ & 177 & 47 & $26 \bar{t}$ & 19.2 & 56.4 & 0.41 & 788 & 54.9 & 17 & -0.03 \\
\hline $14: 16: 57: 78$ & 178 & 47 & 26 & 19.2 & 56.3 & 0.41 & 781 & 54.9 & 17 & -0.16 \\
\hline $14: 16: 58: 77$ & 179 & 47 & $26^{\prime}$ & 19.2 & 56.3 & 0.4 & 778 & 54.9 & 17 & -0.16 \\
\hline $14: 16: 59: 81$ & 180 & $47^{\prime}$ & 26 & 19.2 & $56.3^{\dagger}$ & 0.41 & 780 & 54.9 & 17 & -0.25 \\
\hline $14: 17: 00: 80$ & 181 & 47 & 26 & 19.2 & 56.3 & 0.42 & 776 & 54.8 & 17 & $-0 . \overline{25}$ \\
\hline $14: 17: 01: 79$ & 182 & 47 & 26 & 19.2 & 56.3 & 0.41 & 780 & 54.8 & 17 & -0.35 \\
\hline $14: 17: 02: 78$ & 183 & 47 & $26^{\dagger}$ & 19.2 & 56.3 & 0.41 & 785 & 554.7 & 17 & $-0 . \overline{35}$ \\
\hline $14: 17: 03: 77$ & 184 & 47 & 26 & 19.2 & 56.3 & 0.41 & 787 & 54.7 & 17 & 0.02 \\
\hline $14: 17: 04: 81$ & 185 & 47 & $26 !$ & 19.2 & 56.3 & 0.41 & $790^{\circ}$ & 54.7 & 17 & 0.02 \\
\hline$: 17: 05: 80$ & 186 & 47 & 26 & 19.2 & 56.3 & 0.41 & 788 & $\overline{54.6}$ & $\overline{17}$ & -0.16 \\
\hline $14: 17: 06: 79$ & 187 & 47 & 26 & 19.2 & $56 . \overrightarrow{3}$ & 0.41 & 787 & 54.6 & 17 & -0.26 \\
\hline $14: 17: 07: 83$ & 188 & 47 & 26 & 19.2 & 56.3 & 0.4 & 783 & 54.8 & 17 & -0.26 \\
\hline $14: 17: 08: 82$ & 189 & 47 & $26^{\prime}$ & 19.2 & 56.3 & 0.41 & 774 & 454.9 & 17 & -0.35 \\
\hline $14: 17: 09: 81$ & 190 & 47 & 26 & 19.4 & $56.3^{1}$ & 0.41 & 768 & 34.9 & 17 & -0.35 \\
\hline $14: 17: 10: 80$ & 191 & 47 & $26^{\dagger}$ & 19.4 & 56.3 & 0.4 & 768 & 54.9 & 17 & 0.04 \\
\hline $14: 17: 11: 79$ & 192 & 47 & 26 & 19.4 & $56.3^{t}$ & 0.39 & 767 & 75 & 17 & 0.04 \\
\hline $14: 17: 12: 83$ & 193 & 47 & $26^{\circ}$ & 19.4 & 56.3 & 0.41 & 768 & 55 & 17 & -0.14 \\
\hline $14: 17: 13: 82$ & 194 & $4 \overline{7}$ & 26 & 19.4 & 56.3 & 0.41 & $771^{\dagger}$ & 155.1 & 17 & -0.14 \\
\hline $14: 17: 14 \overline{4: 81}$ & 195 & 47 & $26^{\dagger}$ & 19.4 & $56.3 \mathrm{i}$ & 0.41 & 776 & 55.2 & 17 & -0.28 \\
\hline
\end{tabular}




\begin{tabular}{|c|c|c|c|c|c|c|c|c|c|c|}
\hline & $\begin{array}{l}\text { Elapsed } \\
\text { Time }\end{array}$ & & : & & & & & & TORQUE & \\
\hline$T / M E$ & $(\mathrm{sec})$ & BIT_TC1F & PURG_TC2 & PurgePSI & PurgeCFM & DEPTH(IN) & DwnFrc(lb) & $R_{P M}$ & & Inches/Min \\
\hline $14: 17: 15: 80$ & 196 & 46 & 26 & 19.4 & 56.3 & 0.41 & 778 & 55.2 & 17 & $-0 . \overline{28}$ \\
\hline $14: 17: 16: 79$ & 197 & $46 !$ & 26 & 19.4 & 56.3 & 0.39 & $780^{\circ}$ & 55.1 & 17 & -0.11 \\
\hline $14: 17: 17: 77$ & $198^{\circ}$ & 46 & 26 & 19.4 & 56.3 & 0.41 & $775 !$ & 55.1 & 17 & -0.11 \\
\hline $14: 17: 18: 76$ & 199 & 46 & 26 & 19.4 & 56.3 & 0.41 & $778^{\circ}$ & 55 & 17 & -0.16 \\
\hline $14: 17: 19: 75$ & 200 & $4 \overline{7}$ & 26 & 19.4 & 56.3 & 0.41 & 774 & 55.2 & 16 & -0.16 \\
\hline $14: 17: 20: 74$ & 201 & 47 & $26 !$ & 19.4 & 56.3 & 0.39 & 766 & 55.2 & 16 & -0.11 \\
\hline $14: 17: 21: 73$ & 202 & $46^{\prime}$ & $26:$ & 19.4 & 56.3 & 0.4 & $760^{\circ}$ & 55.4 & 16 & -0.11 \\
\hline $14: 17: 22: 72$ & 203 & 46 & 26 & 19.6 & 56.3 & 0.4 & $764^{\dagger}$ & 55.4 & 16 & -0.35 \\
\hline $14: 17: 23: 71$ & 204 & 46 & $26 !$ & 19.6 & 56.3 & 0.41 & $768^{\prime}$ & 55.4 & 16 & -0.35 \\
\hline $14: 17: 24: 75$ & 205 & 46 & 26 & 19.6 & 56.3 & 0.41 & $771^{i}$ & 55.5 & 16 & -0.06 \\
\hline $14: 17: 25: 74$ & 206 & 46 & 26 & 19.6 & 56.3 & 0.41 & 765 & 55.5 & 17 & -0.06 \\
\hline $14: 17: 26: 73$ & 207 & 46 & 26 & 19.6 & 56.3 & 0.41 & 769 & 55.5 & 17 & -0.28 \\
\hline $14: 17: 27: 72$ & 208 & $46^{\dagger}$ & 26 & 19.6 & 56.3 & 0.41 & $773^{i}$ & 55.5 & $1 \overline{7}$ & -0.28 \\
\hline $14: 17: 28: 70$ & 209 & 46 & 26 & 19.6 & 56.3 & 0.41 & 776 & 55.5 & 17 & $-0 . \overline{04}$ \\
\hline $14: 17: 29: 75$ & 210 & 46 & 26 & 196 & 56.3 & 0.41 & 773 & 55.5 & 17 & -0.04 \\
\hline $14: 17: 30: 74$ & 211 & 46 & 26 & 19.6 & 56.3 & 0.41 & 774 & 55.5 & 17 & -0.09 \\
\hline $14: 17: 31: 73$ & 212 & 46 & $26 !$ & 19.6 & 56.3 & 0.39 & 771 & $55 \overline{5}$ & 17 & -0.09 \\
\hline $14: 17: 32: 77$ & 213 ! & 46 & 26 & 19.6 & 56.3 & 0.4 & 765 & 55.5 & 16 & -0.38 \\
\hline $14: 17: 33: 76$ & 214 & 46 & $26 !$ & 19.6 & 56.3 & 0.4 & 758 & 55.5 & 16 & $-0 . \overline{27}$ \\
\hline $14: 17: 34: 75$ & 215 & 46 & 26 & 19.6 & $56.3^{\dagger}$ & 0.4 & 755 & 55.6 & 16 & -0.27 \\
\hline $14: 17: 35: 73$ & 216 & $46 !$ & 26 & 19.6 & 56.3 & 0.4 & $755^{\dagger}$ & 55.6 & 16 & -0.09 \\
\hline $14: 17: 36: 72$ & 217 & 46 & 26 & 19.8 & 56.3 & 0.42 & 760 & 55.6 & 16 & -0.09 \\
\hline $14: 17: 37: 71$ & 218 & 46 & 26 & 19.6 & 56.3 & 0.41 & 765 & 55.6 & 16 & -0.18 \\
\hline $14: 17: 38: 70$ & $219^{\circ}$ & 46 & 26 & 19.8 & 56.3 & 0.41 & $761^{\dagger}$ & $55 \overline{6}$ & 17 & -0.18 \\
\hline $14: 17: 39: 69$ & 220 & 46 & $26 !$ & 19.8 & 56.3 & 0.41 & 765 & 55.4 & 17 & -0.13 \\
\hline $14: 17: 40: 68$ & 221 & 46 & 26 & 19.8 & 56.3 & 0.41 & 768 & 55.4 & 17 & -0.13 \\
\hline $14: 17: 41: 67$ & 222 & 46 & 26 & 19.8 & $56 . \overline{3}$ & 0.41 & $768^{\dagger}$ & 55.4 & 17 & $-0 . \overline{38}$ \\
\hline $14: 17: 42: 66$ & $223 !$ & $4 \overline{6}$ & 26 & 19.8 & 56.3 & 0.41 & 765 & 55.5 & 17 & $-0 . \overline{3} 8$ \\
\hline $14: 17: 43: 64$ & 224 & 46 & 26 & 19.8 & 56.3 & 0.41 & 755 & 55.5 & 17 & -0.08 \\
\hline $14: 17: 44: 63$ & 225 & 46 & 26 & 19.8 & 56.3 & 0.4 & 748 & 55.5 & 16 & -0.08 \\
\hline $14: 17: 45: 62$ & 226 & 46 & 26 & $19.8^{\dagger}$ & 56.3 & 0.4 & $748^{\circ}$ & 55.5 & 16 & 0.02 \\
\hline $4: 17: 46: 61$ & 227 & 46 & 26 & 196 & 56.3 & 0.4 & 746 & 55.6 & 16 & 0.02 \\
\hline $14: 17: 47: 60$ & 228 & 46 & 26 & 19.6 & 56.3 & 0.41 & 746 & 55.6 & 16 & -0.42 \\
\hline $14: 17: 48: 59$ & 229 & 46 & $26 !$ & 19.6 & 56.3 & 0.41 & 747 & 55.7 & 16 & -0.42 \\
\hline $14: 17: 49: 58$ & 230 & 46 & 26 & 19.6 & 56.3 & 0.41 & 752 & 55.9 & 16 & -0.17 \\
\hline $14: 17: 50: 56$ & 231 & 46 & 26 & 19.6 & 56.3 & 0.41 & 757 & 55.9 & 17 & -0.17 \\
\hline $14: 17: 51: 55$ & 232 & 46 & 26 & 19.6 & 56.3 & $0.41^{\dagger}$ & 760 & 55.9 & 17 & -0.18 \\
\hline $14: 17: 52: 54$ & 233 & 46 & 26 & 19.6 & 56.3 & 0.4 & 761 & 55.9 & 17 & -0.18 \\
\hline $14: 17: 53: 53$ & 234 & 46 & 26 & 19.6 & 56.3 & 0.41 & $761^{\circ}$ & 55.9 & 17 & $-0 . \overline{22}$ \\
\hline $14: 17: 54: 52$ & 235 & 46 & 26 & 19.6 & 56.3 & 0.4 & 763 & 55.8 & 17 & -0.22 \\
\hline $14: 17: 55: 51$ & 236 & $46 !$ & 26 & 19.6 & 56.3 & 0.4 & 762 & 55.8 & 17 & -0.05 \\
\hline $14: 17: 56: 50$ & 237 & 46 & 26 & 19.6 & 56.3 & 0.41 & 756 & 55.8 & 16 & -0.05 \\
\hline $14: 17: 57: 49$ & 238 & 46 & 26 & 19.6 & $56.3^{\prime}$ & 0.39 & 750 & 55.8 & 16 & -0.27 \\
\hline $14: 17: 58: 47$ & 239 & 46 & $26 j$ & 19.6 & 56.3 & 0.39 & $751^{1}$ & 55.8 & 16 & -0.27 \\
\hline $14: 17: 59: 46$ & 240 & 46 & 26 & 19.6 & 56.3 & 0.4 & 753 & 55.8 & 16 & -0.21 \\
\hline $14: 18: 00: 45$ & 241 & 46 & 26 & 19.4 & 56.3 & 0.42 & 759 & 55.8 & 16 & -0.21 \\
\hline $14: 18: 01: 44$ & 242 & $4 \overline{6} !$ & 26 & 19.4 & 56.3 & 0.411 & 765 & 55.6 & 16 & 0.11 \\
\hline $14: 18: 02: 48$ & 243 & 46 & 26 & 19.4 & 56.3 & 0.41 & 761 & 55.6 & 17 & 0.11 \\
\hline $14: 18: 03: 47$ & 244 & 46 & 26 & 19.4 & 56.3 & 0.41 & 770 & $55 . \overline{4}$ & 17 & -0.18 \\
\hline
\end{tabular}




\begin{tabular}{|c|c|c|c|c|c|c|c|c|c|c|}
\hline TIME & $\begin{array}{l}\text { Elapsed } \\
\text { Time } \\
\text { (sec) }\end{array}$ & BIT_TC1 & PURG_TC2 & PurgePSI & PurgeCFM & DEPTH(IN) & DwnFrc(lb) & RPM & $\begin{array}{l}\text { TORQUE } \\
\text { (FTLB) }\end{array}$ & Inches/Min \\
\hline $14: 18: 04: 46$ & 245 & $\cdots-46$ & -26 & 19.4 & $\begin{array}{l}56.3 \\
5\end{array}$ & $\quad 0.41$ & $\quad 773$ & 55.5 & ) $\quad 17$ & $\quad-0.29$ \\
\hline $14: 18: 05: 45$ & 246 & 46 & 26 & 19.4 & 56.3 & 0.42 & 775 & 55.5 & 17 & -0.29 \\
\hline $14: 18: 06: 44$ & 247 & 46 & 26 & 19.4 & $56.3^{\dagger}$ & $0 . \overline{41}$ & 775 & 55.8 & 17 & -0.25 \\
\hline $14: 18: 07: 43$ & 248 & 46 & 26 & 19.4 & 56.3 & 0.4 & 768 & 55.9 & 17 & -0.25 \\
\hline 14:18:08:42 & 249 & 46 & $26^{\prime}$ & 19.4 & 56.3 & 0.41 & 776 & 55.9 & 17 & -0.14 \\
\hline $14: 18: 09: 40$ & 250 & 46 & 26 & 19.4 & 56.3 & 0.41 & 770 & 55.9 & 16 & -0.14 \\
\hline $14: 18: 10: 39$ & 251 & 46 & 26 & 19.4 & 56.3 & 0.4 & $76 \overline{5}$ & 55.9 & 16 & -0.04 \\
\hline 14:18:11:38 & 252 & 46 & 26 & 19.4 & $56 . \overline{3}$ & 0.39 & 768 & 55.9 & 16 & -0.04 \\
\hline $14: 18: 12: 37$ & 253 & 46 & 26 & 19.4 & 56.3 & 0.41 & 767 & 55.9 & 17 & -0.17 \\
\hline $14: 18: 13: 36$ & 254 & 46 & 26 & 19.4 & 56.3 & 0.41 & 775 & 55.8 & 17 & $-0.1 \overline{7}$ \\
\hline $14: 18: 14: 40$ & 255 & 47 & 26 & 19.4 & 56.3 & 0.42 & 781 & 55.8 & 17 & $-0 . \overline{27}$ \\
\hline $14: 18: 15: 39$ & 256 & 47 & 26 & 19.4 & 56.3 & 0.42 & 768 & 55.9 & 17 & -0.27 \\
\hline $14: 18: 16: 38$ & 257 & 47 & 26 & 19.4 & 56.3 & 0.41 & 772 & 55.9 & 17 & -0.29 \\
\hline $14: 18: 17: 37$ & 258 & 47 & $26^{\prime}$ & 19.4 & 56.3 & 0.42 & 776 & 55.9 & 17 & -0.29 \\
\hline $14: 18: 18: 36$ & 259 & 46 & 26 & 19.4 & 56.3 & 0.42 & 778 & 55.8 & 17 & -0.17 \\
\hline $14: 18: 19: 40$ & 260 & 46 & 26 & 19.4 & 56.3 & 0.41 & 776 & 55.7 & 17 & -0.17 \\
\hline $14: 18: 20: 39$ & 261 & 46 & 26 & 19.4 & 56.3 & 0.42 & 770 & 55.7 & 17 & -0.13 \\
\hline $14: 18: 21: 38$ & 262 & 46 & 26 & 19.4 & 56.3 & 0.41 & 776 & 55.6 & 17 & -0.13 \\
\hline $14: 18: 22: 42$ & 263 & 46 & $26 !$ & 19.4 & 56.3 & 0.41 & 771 & $5 \overline{5.6}$ & $\overline{16}$ & -0.04 \\
\hline $14: 18: 23: 41$ & 264 & 46 & 26 & 19.4 & 56.3 & 0.4 & 763 & 55.6 & 16 & -0.04 \\
\hline $14: 18: 24: 40$ & 265 & 46 & 26 & 19.4 & 56.3 & 0.41 & 762 & $55 . \overline{6}$ & 16 & -0.31 \\
\hline $14: 18: 25: 39$ & 266 & 46 & 26 & 19.4 & 56.3 & 0.41 & 763 & 55.4 & 16 & -0.31 \\
\hline $14: 18: 26: 38$ & 267 & 46 & 26 & 19.4 & 56.3 & 0.42 & 768 & 35.4 & 16 & -0.11 \\
\hline $14: 18: 27: 42$ & 268 & 46 & $26 !$ & 19.4 & 56.3 & 0.42 & 765 & 55.3 & 16 & -0.11 \\
\hline $14: 18: 28: 41$ & 269 & 46 & 26 & 19.4 & 56.3 & $0 . \overline{4}$ & 770 & 55.2 & 16 & -0.05 \\
\hline $14: 18: 29: 40$ & 270 & 46 & 26 & 19.4 & 56.3 & 0.42 & 775 & 55.2 & 17 & -0.13 \\
\hline $14: 18: 30: 39$ & 271 & 46 & 26 & 19.4 & 56.3 & 0.41 & 778 & 55.1 & 17 & $-\overline{0.13}$ \\
\hline $14: 18: 31: 37$ & 272 & 46 & 26 & 19.4 & 56.3 & $0 . \overline{4} 1$ & 778 & 55.2 & 17 & $-0 . \overline{21}$ \\
\hline $14: 18: 32: 36$ & 273 & 46 & 26 & 19.4 & 56.3 & 0.41 & 773 & 55.2 & 17 & -0.21 \\
\hline $14: 18: 33: 35$ & 274 & 46 & 26 & 19.4 & 56.3 & 0.41 & 767 & 55.3 & 17 & -0.38 \\
\hline $14: 18: 34: 34$ & 275 & 46 & 26 & 19.4 & 56.3 & $\overline{0.4}$ & 773 & 55.3 & $1 \overline{7}$ & -0.38 \\
\hline $14: 18: 35: 33$ & 276 & 46 & 26 & 19.4 & 56.3 & 0.41 & 766 & 55.3 & 16 & 0.11 \\
\hline $14: 18: 36: 32$ & $27 \overline{77}$ & 46 & 26 & 19.4 & 56.3 & 0.41 & 763 & 55.4 & 16 & 0.11 \\
\hline $14: 18: 37: \overline{31}$ & 278 & 46 & $26 !$ & 19.4 & 56.3 & $0 . \overline{41}$ & 763 & 55.5 & 16 & -0.25 \\
\hline $14: 18: 38: 30$ & 279 & 46 & 26 & 19.4 & 56.3 & 0.4 & 768 & 55.5 & 16 & -0.25 \\
\hline $14: 18: 39: 34$ & 280 & $4 \overline{6}$ & 26 & 19.4 & 56.3 & 0.42 & 773 & 55.6 & 16 & $-0 . \overline{24}$ \\
\hline $14: 18: 40: 33$ & 281 & 46 & 26 & 19.4 & 56.3 & 0.42 & 768 & 55.5 & $\overline{16}$ & -0.24 \\
\hline $14: 18: 41: 32$ & 282 & 47 & 26 & 19.4 & 56.3 & 0.42 & 773 & 355.5 & 17 & -0.21 \\
\hline $14: 18: 42: 30$ & 283 & 47 & 26 & 19.4 & 56.3 & 0.42 & 776 & 55.4 & 17 & -0.21 \\
\hline $14: 18: 43: 29$ & 284 & 47 & 26 & 19.4 & 56.3 & 0.42 & 779 & 755.4 & 17 & $-0 . \overline{31}$ \\
\hline $14: 18: 44: 34$ & 285 & 46 & 26 & 19.4 & 56.3 & 0.42 & 776 & 5 & 17 & $-0 . \overline{31}$ \\
\hline $14: 18: 45: 33$ & 286 & 46 & 26 & 19.4 & 56.3 & 0.41 & 770 & 55.5 & 17 & 0.08 \\
\hline $14: 18: 46: 31$ & 287 & 46 & 26 & 19.4 & 56.3 & 0.42 & 764 & +55.5 & 17 & 0.08 \\
\hline $14: 18: 47: 36$ & 288 & $46^{\dagger}$ & $26 !$ & 19.4 & 56.3 & 0.41 & 770 & 55.5 & 17 & -0.19 \\
\hline $14: 18: 48: 35$ & 289 & 47 & 26 & 19.4 & 56.3 & 0.4 & 765 & 55.6 & 16 & -0.19 \\
\hline $14: 18: 49: 34$ & 290 & 46 & 26 & $19 . \overline{4}$ & 56.3 & 0.41 & 765 & $5 \quad 55.7$ & 16 & -0.22 \\
\hline $14: 18: 50: 32$ & 291 & 46 & 26 & 19.4 & 56.3 & 0.41 & 766 & 55.7 & 16 & -0.22 \\
\hline $14: 18: 51: 31$ & 292 & 46 & $26^{\prime}$ & 19.4 & 56.3 & 0.42 & 771 & 55.7 & 16 & $-\overline{0.17}$ \\
\hline $14: 18: 52: 36$ & 293 & 46 & 26 & 19.4 & 56.3 & 0.42 & 776 & 55.7 & 16 & -0.17 \\
\hline
\end{tabular}




\begin{tabular}{|c|c|c|c|c|c|c|c|c|c|c|}
\hline$T I M E$ & $\begin{array}{l}\text { Elapsed } \\
\text { Time } \\
\text { (sec) }\end{array}$ & $B I T \_T C$ & PURG_TC2 & PurgePSI & PurgeCFM & DEPTH(IN) & DwnFrc(lb) & RPM & $\begin{array}{l}\text { TORQUE } \\
\text { (FTLB) }\end{array}$ & Inches/Min \\
\hline $14: 18: 53: 34$ & $\quad 29 \overline{4}$ & 46 & -26 & $\quad 19.4$ & 56.3 & 0.42 & 771 & 55.7 & 16 & -0.09 \\
\hline $14: 18: 54: 33$ & 295 & 46 & 26 & 19.4 & 56.3 & 0.42 & 776 & 55.7 & 16 & -0.15 \\
\hline $14: 18: 55: 32$ & 296 & 46 & 26 & $1 \overline{9.4}$ & 56.3 & 0.42 & 779 & 55.7 & 16 & -0.15 \\
\hline $14: 18: 56: 31$ & 297 & 46 & 26 & 19.4 & 56.3 & 0.41 & 781 & 55.7 & 17 & -0.19 \\
\hline $14: 18: 57: 30$ & 298 & 46 & 26 & 19.4 & 56.3 & 0.42 & 780 & 55.8 & 17 & -0.19 \\
\hline $14: 18: 58: 29$ & 299 & 46 & 26 & 19.4 & 56.3 & 0.41 & 773 & 55.8 & 17 & -0.22 \\
\hline $14: 18: 59: 28$ & 300 & 46 & 26 & 19.4 & 56.3 & 0.39 & 767 & 55.8 & 17 & -0.22 \\
\hline $14: 19: 00: 27$ & 301 & 47 & 26 & 19.4 & 56.3 & 0.4 & 773 & 55.8 & 17 & -0.28 \\
\hline & & & -.... & .... & $\ldots$ & & & & & -- \\
\hline
\end{tabular}




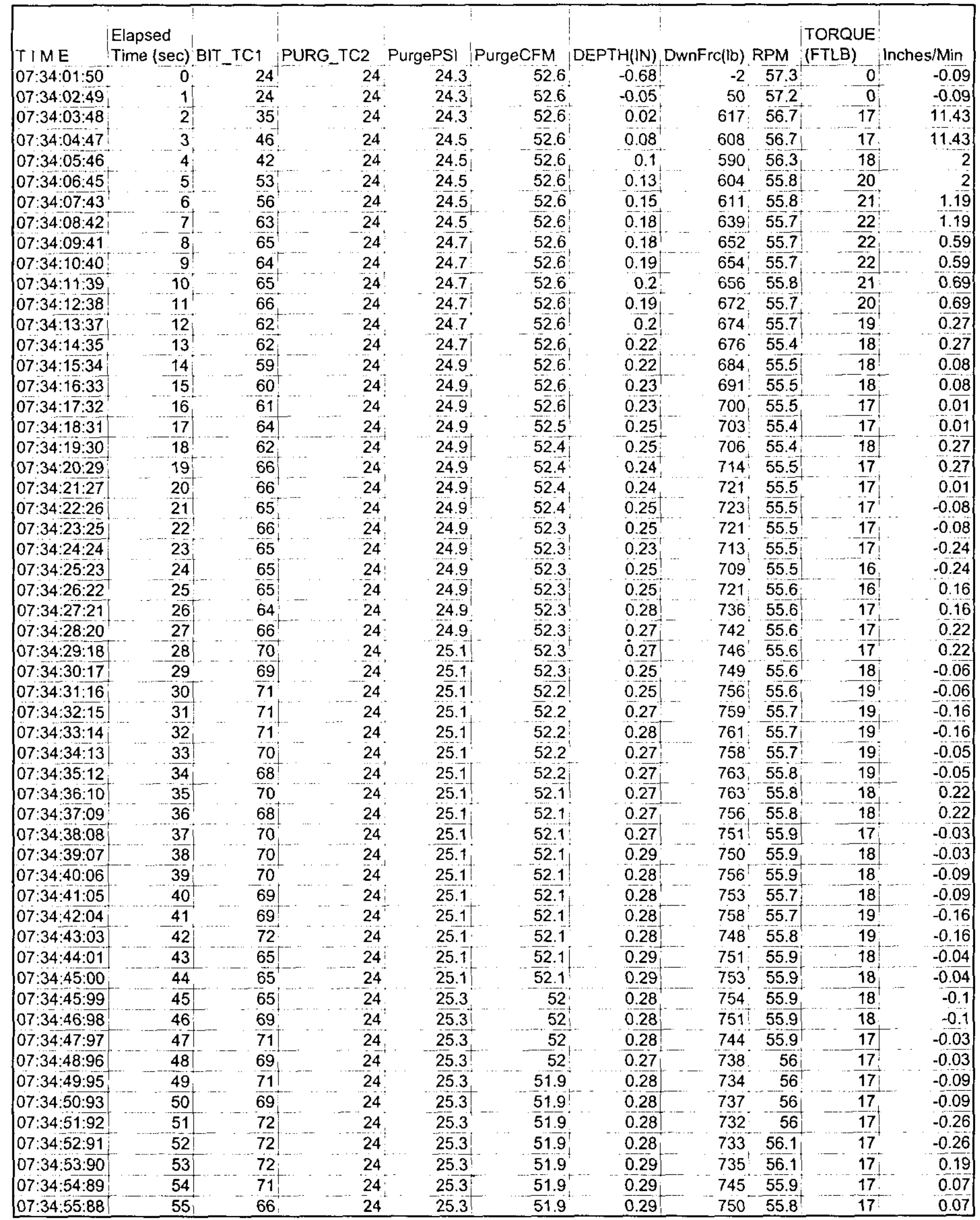




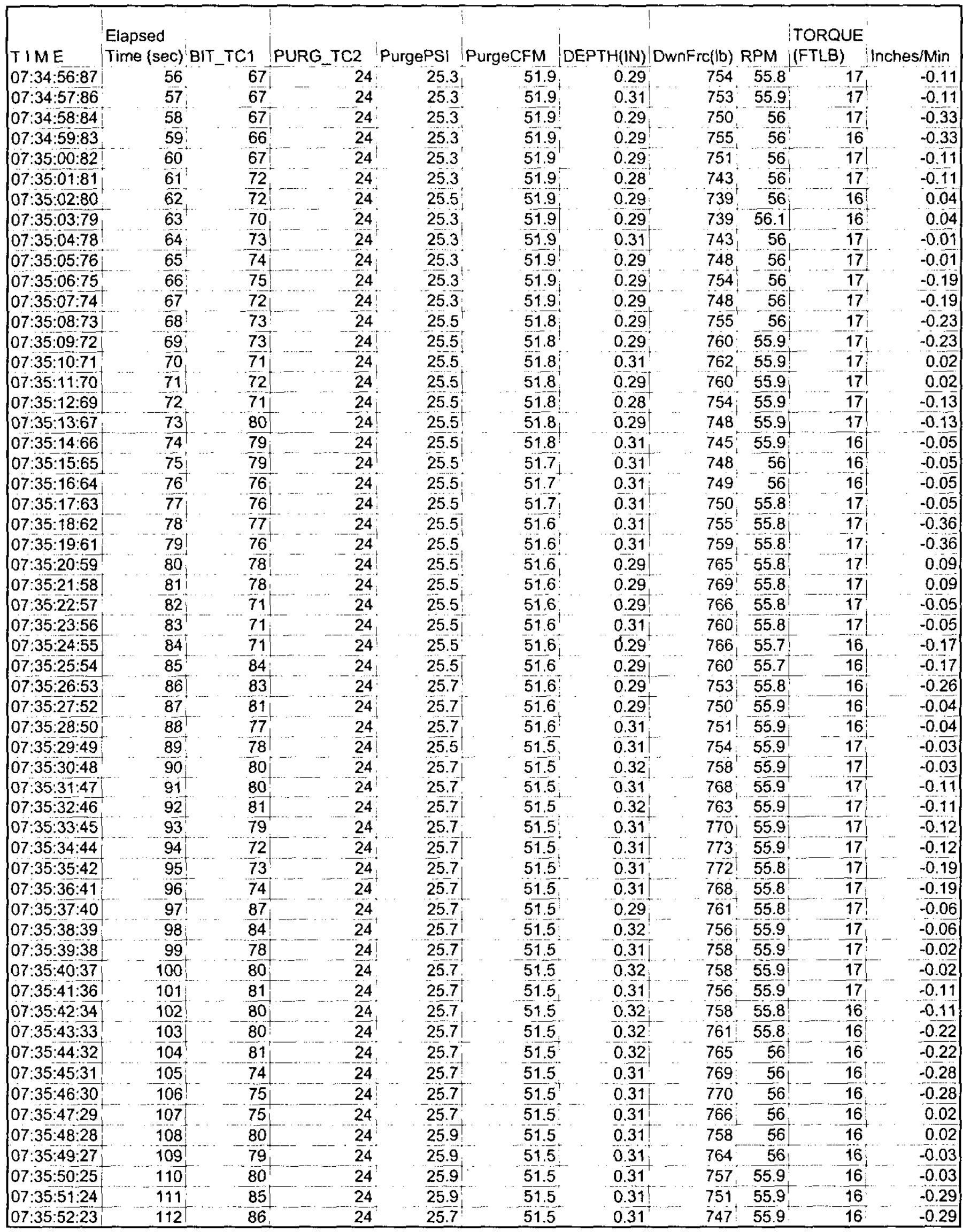




\begin{tabular}{|c|c|c|c|c|c|c|c|c|c|c|}
\hline TIME & $\begin{array}{l}\text { Elapsed } \\
\text { Time (sec)' }\end{array}$ & BIT_TC1 & PURG_TC2 & PurgePSI & PurgeCFM & DEPTH(IN) & DwnFrc(lb) & $\mathrm{RPM}$ & $\begin{array}{l}\text { TORQUE } \\
\text { (FTLB) }\end{array}$ & Inches/Min \\
\hline $07: 35: 53: 22$ & 113 & $-86^{-4}$ & 24 & 25.7 & $\quad 51.5$ & 0.31 & 746 & 55.9 & 15 & $\quad-0.18$ \\
\hline $07: 35: 54: 21$ & 114 & 86 & 24 & 25.7 & 51.5 & 0.31 & 750 & 55.9 & 15 & -0.18 \\
\hline $07: 35: 55: 20$ & 115 & 86 & 24 & 25.9 & 51.5 & 0.31 & 755 & 55.9 & 15 & -0.04 \\
\hline $07: 35: 56: 19$ & 116 & 85 & 24 & 25.7 & 51.5 & 0.32 & 760 & 55.9 & 15 & -0.04 \\
\hline $07: 35: 57: 17$ & 117 & 76 & 24 & 25.9 & 51.4 & $0 . \overline{31}$ & 753 & 55.9 & 15 & -0.09 \\
\hline $07: 35: 58: 16$ & 118 & 77 & 24 & 25.9 & 51.4 & 0.31 & 760 & $5 \overline{5.9}$ & 16 & -0.09 \\
\hline $07: 35: 59: 15$ & 119 & 76 & 24 & 25.9 & 51.4 & 0.31 & 763 & 55.9 & 16 & -0.16 \\
\hline $07: 36: 00: 14$ & 120 & 81 & 24 & 25.9 & 51.4 & 0.31 & 763 & 55.9 & 16 & -0.09 \\
\hline $07: 36: 01: 13$ & $12 \overline{1}$ & 80 & 24 & 25.9 & 51.4 & 0.31 & 760 & 55.8 & 16 & -0.09 \\
\hline $07: 36: 02: 12$ & 122 & 81 & 24 & 25.9 & 51.4 & 0.31 & 755 & 55.6 & 16 & -0.18 \\
\hline $07: 36: 03: 11^{\dagger}$ & 123 & 84 & 24 & 25.9 & 51.4 & 0.29 & 750 & 55.6 & 16 & -0.18 \\
\hline $07: 36: 04: 10$ & 124 & 83 & 24 & 25.9 & 51.4 & 0.31 & 751 & 55.6 & 16 & $-0.0 \overline{5}$ \\
\hline $07: 36: 05: 08$ & 125 & 83 & 24 & 25.9 & 51.4 & 0.29 & 746 & 55.7 & 16 & -0.05 \\
\hline $07: 36: 06: 07$ & 126 & 91 & 24 & 25.9 & 51.4 & 0.32 & 747 & 55.7 & 16 & -0.05 \\
\hline $07: 36: 07: 06$ & $12 \overline{7}$ & 92 & 24 & 25.9 & 51.4 & 0.31 & 751 & 55.8 & 16 & -0.05 \\
\hline $6: 08: 05$ & 128 & 92 & 24 & 25.9 & 51.4 & 0.32 & 755 & 55.9 & 16 & -0.15 \\
\hline $07: 36: 09: 04$ & 129 & 79 & 24 & 25.9 & 51.4 & 0.31 & 761 & 55.9 & 16 & $-0 . \overline{15}$ \\
\hline $07: 36: 10: 03$ & 130 & 80 & 24 & 25.9 & 51.4 & 0.31 & 764 & 56 & 16 & $-0 . \overline{29}$ \\
\hline $07: 36: 11: 02$ & 131 & 80 & 24 & 25.9 & 51.4 & $0.3 \overline{2}$ & 764 & 56.2 & 16 & -0.29 \\
\hline $2: 00$ & 132 & 76 & 24 & 25.9 & 51.3 & 0.32 & 760 & 56.2 & 16 & -0.2 \\
\hline $07: 36: 12: 99$ & 133 & 75 & 24 & 25.9 & 51.3 & 0.32 & 753 & 56.1 & 16 & -0.2 \\
\hline $07: 36: 13: 98$ & 134 & 73 & 24 & 25.9 & 51.3 & 0.31 & 761 & 56.1 & 16 & 0.08 \\
\hline $07: 36: 14: 97$ & 135 & 75 & 24 & 25.9 & 51.3 & 0.31 & 755 & 56.1 & 16 & 0.08 \\
\hline $07: 36: 15: 96$ & 136 & 75 & 24 & 25.9 & 51.3 & 0.31 & 748 & 56 & 16 & -0.17 \\
\hline $07: 36: 16: 95$ & 137 & 76 & 24 & 25.9 & 51.3 & 0.33 & 746 & $5 \overline{6}$ & 16 & -0.17 \\
\hline $07: 36: 17: 94$ & 138 & 86 & 24 & 25.9 & 51.3 & 0.31 & 747 & 56 & $\overline{16}$ & $-0 . \overline{2}$ \\
\hline $07: 36: 18: 93$ & 139 & 87 & 24 & 25.9 & 51.3 & 0.33 & 751 & 55.9 & 16 & -0.22 \\
\hline $19: 91$ & 140 & 87 & 24 & 25.9 & 51.3 & 0.32 & 760 & 55.9 & 15 & -0.16 \\
\hline $07: 36: 20: 90$ & 141 & $85^{1}$ & 24 & 25.9 & 51.3 & 0.31 & 764 & 55.9 & 16 & -0.16 \\
\hline $07: 36: 21: 89$ & 142 & 87 & 24 & 25.9 & 51.3 & 0.31 & 767 & 56 & 16 & -0.09 \\
\hline $22: \overline{88}$ & 143 & 88 & 24 & 25.9 & 51.3 & 0.32 & 765 & 56 & 16 & $-0.0 \overline{9}$ \\
\hline $07: 36: 23: 87$ & 144 & 76 & 24 & 25.9 & 51.3 & 0.31 & 768 & 56 & 16 & $-0 . \overline{13}$ \\
\hline $07: 36: 24: 86$ & 145 & $75 !$ & 24 & 26.1 & 51.3 & 0.32 & 766 & 56.2 & 16 & -0.13 \\
\hline $07: 36: 25: 85$ & 146 & 75 & 24 & 25.9 & 51.3 & 0.31 & 761 & 56.1 & 16 & -0.2 \\
\hline $26: 83$ & 147 & 81 & 24 & 26.1 & 51.3 & 0.31 & 754 & 56.1 & $1 \overline{6}$ & -0.2 \\
\hline $07: 36: 27: 82$ & 148 & 81 & 24 & 25.9 & 51.3 & 0.31 & 751 & 56.1 & 15 & -0.09 \\
\hline $07: 36: 28: 81$ & 149 & 82 & 24 & 26.1 & 51.3 & 0.32 & 751 & 56 & 15 & -0.09 \\
\hline $07: 36: 29: 80$ & 150 & 84 & 24 & 26.1 & 51.3 & 0.32 & 751 & 56 & 15 & -0.29 \\
\hline $30: 79$ & 151 & 83 & 24 & 25.9 & 51.3 & 0.32 & 755 & 56 & 15 & $-0 . \overline{29}$ \\
\hline $07: 36: 31: 78$ & 152 & 82 & 24 & 25.9 & 51.3 & 0.32 & 751 & 56 & 15 & -0.02 \\
\hline $32: 77$ & 153 & $85^{\dagger}$ & 24 & 26.1 & & & 756 & 56 & 15 & $-0 . \overline{02}$ \\
\hline $07: 36: 33: 76$ & 15 & 85 & 24 & 26.1 & & 32 & 765 & 55.9 & 15 & $-0.0 \overline{7}$ \\
\hline $34: 74$ & 155 & 85 & 24 & 26.1 & 51.3 & 0.32 & 772 & 55.9 & 16 & $-0 . \overline{1}$ \\
\hline $07: 36: 35: 73^{\dagger}$ & 156 & 78 & 24 & 26.1 & 51.3 & 0.32 & 778 & 55.9 & 16 & -0.1 \\
\hline $07: 36: 36: 72$ & 157 & 77 & 24 & $26.1 !$ & 51.3 & 0.31 & 766 & 55.9 & 16 & -0.29 \\
\hline $7: 71$ & 158 & $\overline{7} 9$ & 24 & 26.1 & 51.3 & 0.32 & 765 & 55.9 & 16 & -0.29 \\
\hline $38: 70$ & 159 & $7 \overline{9} \dagger$ & 24 & 26.1 & 51.3 & 0.31 & 775 & 55.7 & 16 & -0.14 \\
\hline $07: 36: 39: 69$ & 160 & 79 & 24 & 26.1 & 51.3 & 0.32 & 767 & 55.5 & 15 & -0.14 \\
\hline $07: 36: 40: 68$ & 161 & 78 & 24 & 26.1 & 51.3 & 0.32 & 763 & 55.5 & 15 & 0.02 \\
\hline $07: 36: 41: 66$ & 162 & 81 & 24 & 26.1 & 51.3 & 0.32 & 763 & 55.5 & 15 & 0.02 \\
\hline $07: 36: 42: \overline{65}$ & 163 & $80 !$ & 24 & $26.1^{\dagger}$ & 51.3 & 0.32 & 765 & 55.5 & 15 & -0.14 \\
\hline $07: 36: 43: 64$ & 164 & 80 & 24 & 26.1 & 51.3 & 0.32 & 770 & 55.5 & 15 & -0.14 \\
\hline $07: 36: 44: 63$ & 165 & 85 & 24 & 26.1 & 51.2 & 0.32 & 776 & $55 . \overline{5}$ & 15 & -0.12 \\
\hline $07: 36: 45: 62$ & 166 & 84 & 24 & 26.1 & 51.2 & 0.31 & 781 & 55.6 & 16 & -0.12 \\
\hline $07: 36: 46: 61$ & 167 & 84 & 24 & 26.1 & 51.2 & 0.33 & 780 & 55.6 & 16 & -0.25 \\
\hline $07: 36: 47: 60$ & 168 & 74 & 24 & 26.1 & 51.2 & 0.31 & 780 & 55.8 & 16 & $-0 . \overline{25}$ \\
\hline $07: 36: 48: 59$ & $16 \overline{9}$ & 74 & 24 & $26 . \overline{1}$ & 51.2 & 0.32 & 780 & 55.9 & 16 & -0.12 \\
\hline
\end{tabular}




\begin{tabular}{|c|c|c|c|c|c|c|c|c|c|c|c|}
\hline$T / M E$ & $\begin{array}{l}\text { Elapsed } \\
\text { Time (sec) }\end{array}$ & BIT_TC1 & & PURG_TC2 & PurgePSI & PurgeCFM & DEPTH(IN) & DwnFrc(lb) & RPM & $\begin{array}{l}\text { TORQUE } \\
\text { (FTLB) }\end{array}$ & Inches/Min \\
\hline $07: 36: 49: 57$ & $\quad 170$ & -7 & 5 & 24 & $\quad 26.1$ & $\quad 51.2$ & $\quad 0.31$ & $\quad 776$ & 55.9 & 16 & $\quad-0.12$ \\
\hline $07: 36: 50: 56$ & 171 & 8 & $0^{\vdots}$ & 24 & 26.1 & 51.2 & 0.31 & $770^{\circ}$ & 55.8 & 15 & -0.02 \\
\hline $07: 36: 51: 55$ & 172 & 8 & $0^{+}$ & 24 & 26.1 & 51.2 & 0.31 & $765^{\circ}$ & $55.9^{\circ}$ & 15 & -0.02 \\
\hline $07: 36: 52: 54$ & 173 & 8 & 0 & 24 & 26.1 & 51.2 & 0.31 & $763^{\circ}$ & 55.9 & 15 & -0.17 \\
\hline $07: 36: 53: 53$ & 174 & 8 & 4 & 24 & $26.1^{-1}$ & 51.2 & 0.32 & 764 & 56 & 15 & -0.17 \\
\hline $07: 36: 54: 52$ & 175 & 8 & 3 & 24 & 26.1 & 51.2 & 0.32 & 769 & 55.9 & 15 & -0.15 \\
\hline $07: 36: 55: 51$ & 176 & 8 & 5 & 24 & 26.1 & 51.2 & 0.33 & 765 & $55.9^{+}$ & 15 & -0.15 \\
\hline $07: 36: 56: 49$ & 177 & 8 & 5 & 24 & 26.1 & 51.2 & 0.32 & 770 & 55.9 & 16 & -0.22 \\
\hline 07:36:57:48 & 178 & 8 & 6 & 24 & 26.1 & 51.1 & 0.33 & 775 & 56 & 16 & -0.22 \\
\hline $07: 36: 58: 47$ & 179 & $\overline{8}$ & 7 & 24 & 26.1 & 51.1 & 0.32 & 780 & 56 & 16 & 0 \\
\hline $07: 36: 59: 46$ & 180 & 7 & 9 & 24 & 26.1 & 51.1 & 0.32 & 783 & $56 !$ & 16 & 0 \\
\hline $07: 37: 00: 45$ & 181 & & 9 & 24 & 26.1 & 51.1 & 0.32 & 780 & 56.1 & 16 & -0.1 \\
\hline 07:37:01:44 & 182 & 8 & 0 & 24 & 26.1 & $51 . \overline{1}$ & 0.32 & 775 & 56.1 & 16 & -0.1 \\
\hline $07: 37: 02: 43$ & 183 & 7 & 9 & 24 & 26.1 & 51.1 & 0.31 & 769 & 56.1 & $\overline{16}$ & -0.19 \\
\hline $07: 37: 03: 41$ & 184 & 7 & 8 & 24 & 26.1 & 51.1 & 0.31 & $775^{\circ}$ & 56 & 16 & $-0 . \overline{19}$ \\
\hline $07: 37: 04: 40$ & 185 & 8 & 7 & 24 & 26.3 & 51.1 & 0.31 & 770 & 56 & 16 & -0.35 \\
\hline 07:37:05:39 & 186 & 8 & 8 & 24 & $26.3^{7}$ & 51.1 & 0.32 & 765 & $55 \overline{9}$ & 16 & $-0 . \overline{35}$ \\
\hline $07: 37: 06: 38$ & 187 & 8 & 8 & 24 & 26.3 & 51.1 & 0.32 & 765 & 55.9 & 15 & -0.03 \\
\hline $07: 37: 07: 37$ & 188 & 8 & $7_{\mathrm{i}}^{+}$ & 24 & 26.3 & 51.1 & 0.33 & 768 & 55.9 & 15 & -0.1 \\
\hline $7: 08: 36$ & 189 & & 8 & $24^{\circ}$ & 26.3 & 51.1 & 0.32 & 773 & 55.8 & 16 & -0.1 \\
\hline $07: 37: 09: 35$ & 190 & $\overline{9}$ & 0 & 24 & 26.3 & 51.1 & $0 . \overline{33}$ & $779^{\prime}$ & 55.9 & 16 & $-0.1 \overline{6}$ \\
\hline$|07: 37: 10: 34|$ & $1 \overline{91}$ & 7 & 9 & 24 & $26.3^{\prime \prime}$ & 51.1 & 0.32 & 782 & 55.9 & 16 & -0.16 \\
\hline $07: 37: 11: 32$ & 192 & & 0 & 24 & 26.3 & 51.1 & 0.33 & 781 & 56 & 16 & -0.18 \\
\hline $07: 37: 12: 31$ & 193 & & $0^{\dagger}$ & 24 & 26.3 & 51.1 & 0.32 & $781^{\circ}$ & 55.9 & 16 & -0.18 \\
\hline $07: 37: 13: 30$ & 194 & 7 & 8 & 24 & 26.3 & 51.1 & 0.32 & 781 & 55.9 & 16 & -0.21 \\
\hline $07: 37: 14: 29$ & 195 & 7 & 7 & 24 & 26.3 & 51.1 & 0.32 & 778 & 56 & $1 \overline{6}$ & -0.21 \\
\hline $07: 37: 15: 28 \mid$ & 196 & 7 & 7 & 24 & 26.5 & 51.1 & 0.31 & 771 & 55.9 & 16 & -0.08 \\
\hline $07: 37: 16: 27$ & 197 & 8 & 1 & 24 & 26.5 & 51.1 & 0.32 & 766 & 55.9 & 16 & -0.08 \\
\hline $07: 37: 17: 26$ & 198 & 8 & 1 & 24 & 26.5 & 51.1 & 0.33 & 765 & 55.9 & 16 & -0.1 \\
\hline $07: 37: 18: 24$ & 199 & 8 & 0 & 24 & 26.5 & 51.1 & 0.33 & 763 & 55.9 & $1 \overline{6}$ & -0.1 \\
\hline $07: 37: 19: 23$ & 200 & & 3 & 24 & 26.5 & 51.1 & 0.32 & 763 & 55.9 & 16 & -0.06 \\
\hline $07: 37: 20: 22$ & 201 & & 4 & 24 & 26.5 & 51.1 & 0.32 & 759 & 55.7 & 17 & -0.06 \\
\hline $07: 37: 21: 21$ & 202 & 9 & 4 & 24 & 26.5 & 51.1 & 0.32 & 765 & 55.7 & 16 & -0.21 \\
\hline $07: 37: 22: 20$ & 203 & 8 & 1 & 24 & 26.5 & & 0.32 & $771 j$ & $55 . \overline{7}$ & 16 & -0.21 \\
\hline $07: 37: 23: 19$ & 204 & 8 & 2 & 24 & 26.6 & 51.1 & 0.33 & 773 & 55.9 & 16 & $-0 . \overline{24}$ \\
\hline $07: 37: 24: 18$ & 205 & 8 & 3 & 24 & 26.6 & 51.1 & $0.33^{\dagger}$ & 775 & 55.9 & $\overline{1} \overline{6}$ & -0.24 \\
\hline 07:37:25:17| & 206 & & 0 & 24 & 26.6 & 51.1 & 0.32 & 771 & 56.1 & 17 & 0.04 \\
\hline $07: 37: 26: 15$ & 207 & & 9 & 24 & 26.6 & & 0.32 & 763 & 56.1 & 16 & 0.04 \\
\hline $\mid 07: 37: 27: 14$ & 208 & 7 & 8 & 24 & 26.6 & 51.1 & 0.31 & 760 & 56.1 & 16 & -0.1 \\
\hline $07: 37: 28: 13$ & 209 & & $2^{+}$ & 24 & 26.6 & 51.1 & 0.32 & 765 & $56 . \overline{2}$ & 16 & -0.1 \\
\hline $07: 37: 29: 12$ & 210 & 8 & 3 & 24 & 26.6 & 51.1 & 0.32 & 760 & 56.2 & 16 & -0.16 \\
\hline 107: & 2 & & 3 & 24 & 26.6 & & 0.32 & 756 & 56.2 & 16 & -0.16 \\
\hline $07: 37: 31: 10$ & 212 & 9 & 3 & 24 & 26.8 & $51 . \overline{1}$ & 0.32 & 756 & 56.1 & 16 & -0.33 \\
\hline 07:37:32:09 & 213 & & 3 & 24 & 26.6 & 51.1 & 0.32 & 760 & 56.1 & 17 & -0.33 \\
\hline $107: 37: 33: 07$ & 214 & 9 & 2 & 24 & $26.8^{\top}$ & 51.1 & 0.33 & 764 & 56.1 & 17 & $-0.0 \overline{2}$ \\
\hline $07: 37: 34: 06$ & 215 & & 7 & 24 & 26.8 & 51.1 & 0.33 & 770 & 56 & 16 & -0.02 \\
\hline$: 35: 05$ & 216 & 9 & 8 & 24 & 26.8 & 51.1 & 0.32 & 774 & $5 \bar{b}$ & 16 & -0.05 \\
\hline$|07: 37: 36: 04|$ & 217 & & 9 & 24 & 26.8 & 51.1 & 0.33 & 773 & 56 & 16 & -0.05 \\
\hline $07: 37: 37: 03$ & 218 & 7 & 6 & 24 & 26.8 & 51.1 & 0.32 & 774 & 55.9 & 16 & -0.25 \\
\hline $07: 37: 38: 02$ & 219 & 7 & 5 & 24 & 26.8 & 51.1 & 0.32 & 773 & 55.9 & 16 & -0.25 \\
\hline |07:37:39:01 & 220 & & 5 & 24 & 27 & 51.1 & 0.32 & 770 & 55.9 & 16 & -0.09 \\
\hline $07: 37: 40: 00$ & 221 & to & $7 !$ & 24 & 27 & 51.1 & 0.31 & 763 & 56 & 16 & -0.27 \\
\hline $07: 37: 40: 98$ & 222 & 8 & 8 & 24 & 27 & 51.1 & 0.32 & 758 & 56 & 16 & -0.27 \\
\hline $07: 37: 41: 97$ & 223 & 8 & 7 & 24 & 27 & 51.1 & 0.33 & 758 & 56 & 16 & -0.04 \\
\hline $07: 37: 42: 96$ & $2 \overline{24}$ & 8 & 8 & 24 & 27 & 51.1 & 0.32 & 759 & $56 . \overline{1}$ & 16 & -0.04 \\
\hline $07: 37: 43: 95$ & 225 & & 8 & 24 & 27 & 51.1 & 0.33 & 763 & 56 & 17 & -0.11 \\
\hline $07: 37: 44: 94$ & 226 & $\underline{8}$ & 9 & 24 & 27.2 & 51.1 & 0.33 & 759 & 56 & 17 & -0.11 \\
\hline
\end{tabular}




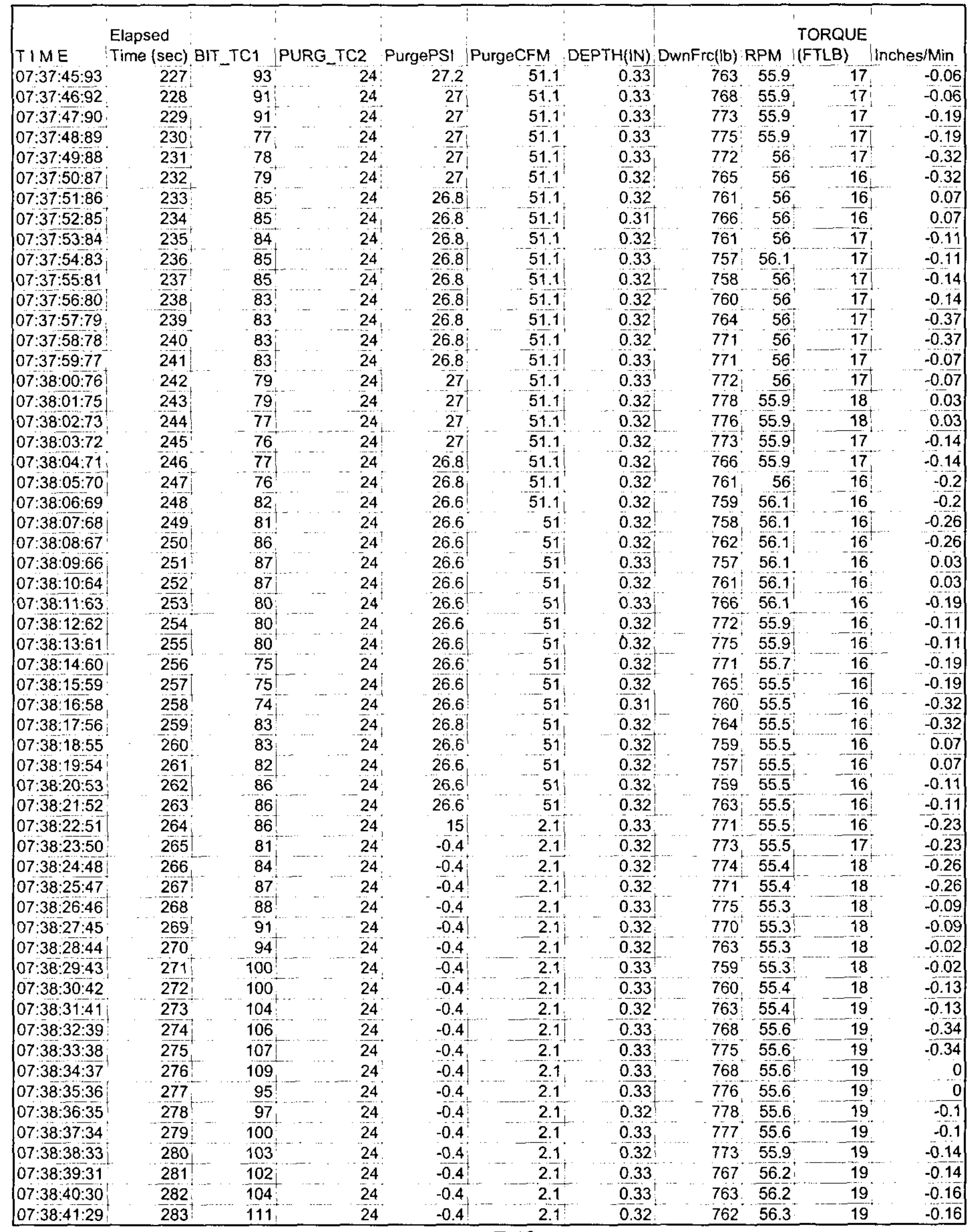




\begin{tabular}{|c|c|c|c|c|c|c|c|c|c|c|}
\hline TIME & $\begin{array}{l}\text { Elapsed } \\
\text { Time (sec) }\end{array}$ & BIT_TC1 & PURG_TC2 & ;PurgePS! & PurgeCFM & DEPTH(IN) & DwnFrc(lb) & RPM & $\begin{array}{l}\text { TORQUE } \\
\text { (FTLB) }\end{array}$ & Inches/Min \\
\hline $07: 38: 42: 28$ & 284 & $-11 \overline{3}$ & 24 & $\quad-0.4$ & 2.1 & $\quad 0.32$ & 765 & 56.4 & 19 & -0.3 \\
\hline $07: 38: 43: 27$ & 285 & 114 & 24 & -0.4 & 2.1 & 0.32 & 763 & 56.4 & 19 & -0.3 \\
\hline $07: 38: 44: 26$ & 286 & 138 & 24 & -0.4 & 2.1 & 0.32 & 763 & 56.4 & 20 & -0.01 \\
\hline $07: 38: 45: 25$ & 287 & 135 & 24 & -0.4 & 2.1 & 0.32 & 767 & 56.3 & 20 & -0.01 \\
\hline $07: 38: 46: 24$ & 288 & $133^{\dagger}$ & 24 & -0.4 & 2.1 & 0.33 & 771 & 56.3 & 20 & -0.17 \\
\hline $07: 38: 47: 22$ & 289 & 129 & 24 & -0.4 & 2.1 & 0.33 & $778^{\prime}$ & $56 . \overline{3}$ & 20 & -0.13 \\
\hline $07: 38: 48: 21$ & 290 & 132 & 24 & -0.4 & 2.1 & 0.33 & 783 & 56.3 & 20 & -0.13 \\
\hline $07: 38: 49: 20$ & 291 & $13 \overline{6}$ & 24 & -0.4 & 2.1 & 0.33 & 780 & 56.2 & 20 & -0.22 \\
\hline $07: 38: 50: 19$ & 292 & 108 & 24 & -0.4 & 2.1 & 0.32 & 776 & 56.3 & 20 & -0.22 \\
\hline $07: 38: 51: 18$ & 293 & 107 & 24 & -0.4 & 2.1 & 0.33 & $780 !$ & 56.3 & 20 & -0.23 \\
\hline $07: 38: 52: 17 \mid$ & 294 & $10 \overline{9}$ & 24 & -0.4 & 2.1 & 0.31 & 778 & 56.3 & 20 & -0.23 \\
\hline $07: 38: 53: 16$ & 295 & 131 & 24 & -0.4 & 2.1 & 0.33 & 771 & 56.4 & 20 & -0.02 \\
\hline $07: 38: 54: 14$ & 296 & 126 & 24 & -0.4 & 2.1 & 0.32 & $768^{\circ}$ & 56.4 & 20 & -0.02 \\
\hline $07: 38: 55: 13$ & 297 & 123 & 24 & -0.4 & 2.1 & 0.32 & $765^{\dagger}$ & 56.3 & 20 & -0.21 \\
\hline $07: 38: 56: 12$ & 298 & 125 & 24 & -0.4 & 2.1 & 0.32 & 765 & 56.4 & 20 & -0.21 \\
\hline $07: 38: 57: 11$ & 299 & 128 & 24 & -0.4 & 2.1 & 0.33 & 768 & 56.4 & 20 & -0.08 \\
\hline $07: 38: 58: 10$ & 300 & 131 & 24 & 0.7 & 5.3 & 0.32 & 772 & 56.4 & 20 & -0.08 \\
\hline $07: 38: 59: 09$ & 301 & 130 & 24 & 19.6 & 47.9 & 0.32 & 768 & 56.4 & 19 & -0.24 \\
\hline $07: 39: 00: 08$ & 302 & 122 & 24 & 21.8 & 50.7 & 0.33 & $771 !$ & 56.4 & 18 & -0.24 \\
\hline $01: 07$ & 303 & $117 !$ & 24 & 26.5 & 50.8 & 0.33 & 778 & 56.3 & 18 & -0.2 \\
\hline $02: 05$ & 304 & 109 & 24 & 27 & 50.8 & 0.33 & $781 !$ & 56.3 & 18 & -0.2 \\
\hline $07: 39: 03: 04$ & 305 & 107 & 24 & 27.2 & 50.8 & $0.3 \overline{2}$ & 782 & 56.3 & 18 & 0 \\
\hline $07: 39: 04: 03$ & 306 & 106 & 24 & 27.2 & 50.8 & 0.32 & 778 & 56.3 & 17 & 0 \\
\hline $05: 02$ & 307 & 102 & 24 & 27.2 & 50.8 & 0.32 & 771 & 56.4 & 17 & $-0.1 \overline{6}$ \\
\hline $07: 39: 06: 01$ & 308 & 101 & 24 & 27.2 & 50.8 & 0.33 & 765 & 56.4 & 17 & -0.16 \\
\hline $07: 39: 07: 00$ & 309 & 100 & 24 & 27.2 & 50.8 & 0.33 & 772 & 56.3 & 16 & -0.14 \\
\hline $07: 39: 07: 99$ & 310 & 97 & 24 & 27.2 & 50.8 & 0.32 & 766 & 56.2 & 16 & $-0.1 \overline{4}$ \\
\hline $8: 97$ & 311 & 95 & 24 & 27.2 & 50.8 & 0.33 & 762 & 56.2 & 16 & -0.17 \\
\hline $07: 39: 09: 96$ & 312 & 109 & 24 & 27.2 & 50.8 & 0.33 & 762 & 56.2 & 16 & -0.17 \\
\hline $07: 39: 10: 95$ & 313 & 107 & 24 & 27.2 & 50.8 & 0.32 & 764 & 56.2 & 16 & -0.24 \\
\hline $07: 39: 11: 94$ & 314 & 106 & 24 & 27 & 50.8 & 0.32 & 768 & 56.2 & 16 & -0.24 \\
\hline $07: 39$ & 315 & 104 & 24 & 27 & 50.8 & 0.32 & 773 & 56.1 & 16 & -0.06 \\
\hline $07: 39: 13: 92$ & 316 & 102 & 24 & $2 \overline{7}$ & 50.8 & 0.32 & $778^{1}$ & 55.9 & 16 & -0.06 \\
\hline $07: 39: 14: 91$ & 317 & 103 & 24 & 27 & 50.8 & 0.33 & 776 & 55.9 & 16 & -0.07 \\
\hline $15: 90$ & 318 & 93 & 24 & 27 & 50.8 & 0.33 & $777^{+}$ & 55.8 & 16 & -0.07 \\
\hline $07: 39: 16: 88$ & 319 & 92 & 24 & 27.2 & 50.8 & 0.33 & 776 & 55.8 & 16 & -0.1 \\
\hline $07: 3$ & 320 & 91 & 24 & 27.2 & 50.8 & 0.33 & 773 & 55.8 & 16 & -0.1 \\
\hline $07: 3$ & 321 & 95 & 24 & 27.2 & 50.8 & 0.32 & 766 & 55.9 & 16 & $-0 . \overline{27}$ \\
\hline $07: 39: 19: 85$ & 322 & 94 & 24 & 27.2 & 50.8 & 0.33 & 761 & 55.9 & 16 & -0.16 \\
\hline $07: 39: 20: 84$ & 323 & 93 & 24 & 27.2 & 50.8 & 0.33 & $760^{\circ}$ & 55.9 & 16 & -0.16 \\
\hline$: 83$ & 324 & 97 & 24 & 27.2 & 50.8 & 0.33 & 761 & 55.9 & 16 & 0.02 \\
\hline $22: 82$ & 325 & 96 & 24 & 27 & 50.8 & 0.33 & 765 & 56 & 16 & 0.02 \\
\hline $07: 39: 23: 80$ & 326 & 94 & 24 & 27 & 50.8 & 0.33 & $770^{\circ}$ & 56 & 16 & -0.07 \\
\hline $07: 39: 24: 79$ & 327 & 105 & 24 & 27 & 50.8 & 0.33 & 765 & 56.2 & 16 & -0.07 \\
\hline & 328 & 104 & 24 & 27 & 50.8 & & $769^{\circ}$ & 56.2 & 16 & -0.34 \\
\hline $26: 77$ & 329 & 103 & 24 & 27 & 50.8 & 0.33 & 774 & 56.2 & 16 & -0.34 \\
\hline $07: 39: 27: 76$ & 330 & 82 & 24 & 27 & 50.8 & 0.33 & 776 & 56 & 16 & -0.21 \\
\hline $07: 39: 28: 75$ & 331 & 83 & 24 & 27.2 & 50.8 & 0.33 & 773 & 55.9 & 16 & -0.21 \\
\hline $07: 39: 29: 74$ & 332 & 82 & 24 & 27.2 & 50.8 & $0 . \overline{33}$ & 768 & 55.9 & 16 & -0.02 \\
\hline $30 \overline{73}$ & 333 & 94 & 24 & 27.2 & 50.8 & 0.33 & 762 & 55.9 & 16 & -0.02 \\
\hline $07: 39: 31: 71$ & 334 & 93 & 24 & 27.2 & 50.8 & 0.33 & 758 & 55.9 & 16 & -0.18 \\
\hline $07: 39: 32: 70$ & 335 & 92 & 24 & 27.2 & 50.8 & 0.33 & $761 !$ & 55.7 & 16. & -0.18 \\
\hline $07: 39: 33: 69$ & $3 \overline{36}$ & 90 & 24 & 27.2 & 50.8 & $0 . \overline{34}$ & 758 & 55.6 & 16 & -0.02 \\
\hline $07: 39: 34: 68$ & 337 & 89 & 24 & 27 & 50.8 & 0.33 & 758 & 55.6 & 16 & -0.02 \\
\hline $07: 39: 35: 67$ & 338 & 90 & 24 & $2 \overline{7}$ & 50.8 & $0.3 \overline{3}$ & 762 & 55.6 & 16 & -0.29 \\
\hline $07: 39: 36: 66$ & 339 & 98 & 24 & 27 & 50.8 & 0.33 & $768^{\circ}$ & 55.6 & 16 & -0.29 \\
\hline $07: 39: 37: 65$ & 340 & $100^{\dagger}$ & 24 & 27.2 & $50 . \overline{8}$ & 0.33 & 773 & 55.6 & 16 & $-\overline{0.3}$ \\
\hline
\end{tabular}




\begin{tabular}{|c|c|c|c|c|c|c|c|c|c|c|}
\hline$T / M E$ & $\begin{array}{l}\text { Elapsed } \\
\text { Time (sec) }\end{array}$ & & |PURG_TC2 & PurgePS| & PurgeCFM & DEPTH(IN) & DwnFrc(lb) & RPM & $\begin{array}{l}\text { TORQUE } \\
\text { (FTLB) }\end{array}$ & Inches/Min \\
\hline $07: 39: 38: 63$ & 341 & 100 & 24 & $\quad 27.2$ & $2 \quad 50.8$ & 0.33 & 775 & 55.6 & 16 & $5 \quad-0.3$ \\
\hline $07: 39: 39: 62$ & 342 & 81 & 24 & 27.2 & 50.7 & 0.33 & 771 & 55.5 & 16 & 0.09 \\
\hline $07: 39: 40: 61$ & 343 & 79 & 24 & 27.2 & 50.7 & 0.33 & 775 & 55.5 & 16 & 0.09 \\
\hline $07: 39: 41: 60$ & 344 & 79 & 24 & 27.2 & 50.7 & 0.33 & 770 & 55.5 & 16 & -0.13 \\
\hline $07: 39: 42: 59$ & 345 & 86 & 24 & 27.2 & 50.7 & 0.33 & 763 & 55.5 & 16 & -0.13 \\
\hline $07: 39: 43: 58$ & 346 & 86 & 24 & 27.2 & 50.7 & 0.32 & 758 & $55 . \overline{5}$ & 16 & -0.33 \\
\hline $07: \overline{39: 44: 57}$ & 347 & 86 & 24 & 27.2 & 50.7 & 0.33 & 757 & $5 \overline{5} \overline{5}$ & 16 & -0.33 \\
\hline $07: 39: 45: 56$ & 348 & 89 & 24 & 27.2 & 50.7 & 0.32 & 758 & 55.5 & 16 & -0.18 \\
\hline $07: 39: 46: 54$ & 349 & 89 & 24 & 27.2 & 50.7 & 0.34 & 762 & 55.5 & 16 & -0.18 \\
\hline $07: 39: 47: 53$ & 350 & 87 & 24 & 27.2 & 50.7 & 0.33 & 768 & 55.6 & 17 & 0.1 \\
\hline $07: 39: 48: 52$ & 351 & 95 & 24 & 27.2 & 50.7 & 0.33 & 767 & 55.8 & 17 & 0.1 \\
\hline $07: 39: 49: 51$ & 352 & 93 & 24 & 27.2 & 50.7 & 0.33 & 775 & 55.8 & 17 & -0.16 \\
\hline $07: 39: 50: 50$ & 353 & 95 & 24 & 27.2 & 50.7 & 0.33 & 778 & 55.8 & 17 & -0.16 \\
\hline $07: 39: 51: 49$ & 354 & 74 & 24 & 27.2 & 50.7 & 0.32 & 780 & 56 & 17 & 0.01 \\
\hline $07: 39: 52: 48$ & 355 & 74 & 24 & 27.2 & 50.7 & 0.33 & 777 & 56 & 17 & $-0 . \overline{25}$ \\
\hline $07: 39: 53: 46$ & 356 & 74 & 24 & 27.2 & 50.7 & 0.32 & 771 & 56 & 17 & -0.25 \\
\hline$|07: 39: 54: 45|$ & 357 & 84 & 24 & 27.2 & 50.7 & 0.33 & 766 & 56 & 17 & -0.32 \\
\hline
\end{tabular}




\begin{tabular}{|c|c|c|c|c|c|c|c|c|c|c|}
\hline TIME & $\begin{array}{l}\text { Elapsed } \\
\text { Time } \\
(\mathrm{sec})\end{array}$ & $B I T \_T C 1$ & PURG_TC & PurgePSI & PurgeCFM: & DEPTH(IN) & DwnFrc(lb) & RPM & $\begin{array}{l}\text { Torque } \\
\text { (FTLB) }\end{array}$ & Inches/Min \\
\hline $12: 33: 00: 39$ & 0 & 25 & 2 & $5 \quad 21.2$ & $\quad 54$ & -1.36 & -4 & 57.5 & 0 & $\begin{array}{l}-0.07 \\
\end{array}$ \\
\hline $12: 33: 01: 38$ & 1 & 25 & 2 & 21.2 & 54 & -0.62 & -4 & 57.5 & 0 & 0.57 \\
\hline $12: 33: 02: 36$ & 2 & 25 & 2 & 21.2 & 54 & 0.04 & -2 & $56.9^{\prime}$ & 6 & $0.5 \overline{7}$ \\
\hline $12: 33: 03: 35$ & 3 & 27 & 2 & 21.6 & 54 & 0.07 & 567 & $56.9^{i}$ & 39 & 16.36 \\
\hline $12: 33: 04: 34$ & 4 & 32 & 2 & 21.8 & $54^{\dagger}$ & 0.07 & 611 & 56.2 & 32 & $16 . \overline{36}$ \\
\hline $12: 33: 05: 33$ & 5 & 35 & 2 & 21.8 & 53.9 & 0.09 & 632 & 56.1 & 28 & 1.09 \\
\hline $12: 33: 06: 32$ & 6 & 38 & 2 & 21.8 & 538 & 0.08 & $644^{+}$ & $56.1^{\circ}$ & 24 & 1.09 \\
\hline $12: 33: 07: 31$ & 7 & 41 & 2. & 22 & 53.8 & 0.1 & 656 & 56.2 & 23 & 0.24 \\
\hline $12: 33: 08: 30$ & 8 & 42 & 2 & 22 & 53.7 & 0.12 & $671^{\circ}$ & 56.3 & 23 & 0.2 \\
\hline $12: 33: 09: 29$ & 9 & 43 & 2 & 22 & 53.7 & 0.13 & $691:$ & 56.3 & 22 & 0.2 \\
\hline $12: 33: 10: 27$ & 10 & 45 & 2 & 22 & 53.7 & 0.13 & 708 & 56.2 & 23 & 0.21 \\
\hline $12: 33: 11: 26$ & 11 & 45 & 2 & 22 & $53.7^{\dagger}$ & 0.12 & $719^{\prime}$ & 56.2 & 22 & 0.21 \\
\hline $12: 33: 12: 25$ & 12 & $\overline{46}$ & 2 & 22 & 53.6 & 0.13 & $723 !$ & $56 \overline{2}$ & 22 & -0.21 \\
\hline $12: 33: 13: 24$ & 13 & $4 \overline{7}$ & 2. & 22 & 53.6 & 0.1 & 731 & 56.1 & 22 & -0.21 \\
\hline $12: 33: 14: 23$ & 14 & 48 & 2 & 22 & $53.6^{\dagger}$ & 0.12 & 736 & 56.1 & 22 & $-0 . \overline{13}$ \\
\hline $12: 33: 15: 22$ & 15 & 49 & 2 & 22.2 & 53.6 & 0.12 & 734 & $55.9^{\dagger}$ & 21 & $-0 . \overline{13}$ \\
\hline $12: 33: 16: 21$ & 16 & 50 & 2 & 22.2 & 53.6 & 0.12 & 733 & $55.9 \mathrm{i}$ & 21 & 0.22 \\
\hline $12: 33: 17: 19$ & 17 & 51 & 2 & 22.2 & $53.5^{\dagger}$ & 0.12 & 733 & 55.9 & 21 & 0.22 \\
\hline $12: 33: 18: 18$ & 18 & 51 & 2 & 22.2 & 53.5 & 0.14 & 733 & 56 & 21 & 0.2 \\
\hline $12: 33: 19: 17$ & 19 & 52 & 2 & 22.4 & 53.5 & 0.14 & 736 & 56 & 21 & 0.2 \\
\hline $20: 16$ & 20 & 52 & 2 & 22.4 & 53.5 & 0.13 & $735^{\circ}$ & 56 & 21 & -0.26 \\
\hline $12: 33: 21: 15$ & 21 & 53 & 2 & 22.4 & 53.5 & 0.14 & 738 & 55.9 & 22 & -0.26 \\
\hline $12: 33: 22: 14$ & 22 & 53 & 2 & 22.4 & 53.5 & 0.13 & $747^{!}$ & 55.7 & 22 & -0.11 \\
\hline $12: 33: 23: 13$ & 23 & 53 & 2 & 22.4 & 53.4 & 0.14 & 753 & 55.7 & 21 & $-0 . \overline{11}$ \\
\hline $12: 33: 24: 12$ & 24 & 53 & 2 & 22.4 & 53.4 & 0.14 & 758 & 55.8 & 21 & $0.0 \overline{2}$ \\
\hline $12: 33: 25: 10$ & 25 & 54 & 2 & 22.4 & 53.4 & 0.13 & $759 !$ & 55.9 & 21 & $0 . \overline{02}$ \\
\hline $12: 33: 26: 09$ & 26 & 54 & 2 & 22.4 & 53.4 & 0.13 & 755 & $55.9^{+}$ & 21 & -0.08 \\
\hline $12: 33: 27: 08$ & 27 & 54 & 2 & 22.6 & $53.4^{\dagger}$ & 0.12 & 750 & 55.9 & 20 & $-0 . \overline{0}$ \\
\hline $12 \cdot 33: 28: 07$ & 28 & 54 & 2 & 22.6 & 53.4 & 0.12 & 757 & 56 & 20 & $0 . \overline{08}$ \\
\hline $12: 33: 29: 06$ & 29 & 55 & 2 & 22.6 & 53.4 & 0.13 & 752 & 56 & 20 & 0.08 \\
\hline $12: 33: 30: 05$ & 30 & 55 & 2 & $2 \overline{2} .6$ & $53.4^{\dagger}$ & 0.14 & 749 & 56 & 20 & -0.18 \\
\hline $12: 33: 31: 04$ & $\overline{31}$ & 56 & 2 & 22.6 & 53.4 & 0.14 & 749 & $5 \overline{6}$ & 20 & -0.18 \\
\hline $12: 33: 32: 02$ & 32 & 55 & 2 & 22.8 & 53.4 & 0.14 & 752 & $5 \overline{6}$ & 20 & -0.1 \\
\hline $12: 33: 33: 01$ & 33 & 56 & 2 & 22.6 & 53.4 & 0.14 & $757^{\prime}$ & 56 & 21 & $-0 . \overline{1}$ \\
\hline $12: 33: 34: 00$ & 34 & 56 & 2 & 22.8 & 53.4 & 0.14 & 760 & 56.1 & 21 & -0.02 \\
\hline $4: 99$ & 35 & 55 & 2 & 22.8 & 53.4 & 0.14 & 765 & 56.1 & 21 & -0.02 \\
\hline $12: 33: 35: 98$ & 36 & 56 & 2 & 22.8 & $53.4^{\dagger}$ & 0.13 & 765 & 56 & 21 & 0.04 \\
\hline $12: 33: 36: 97$ & 37 & 56 & 2 & 22.8 & $53 . \overline{4}$ & 0.14 & $765^{\circ}$ & 56.1 & 20 & 0.04 \\
\hline $12: 33: 37: 96$ & 38 & 56 & 2 & 22.8 & 53.4 & 0.13 & 765 & 56.1 & 20 & -0.16 \\
\hline $12: 33: 38: 95$ & 39 & $5 \overline{6}$ & 2 & 22.8 & 53.4 & 0.14 & 763 & $56 . \overline{2}$ & 20 & -0.16 \\
\hline $12: 33: 39: 93^{\dagger}$ & 40 & $\overline{56}$ & 2 & $2 \overline{2} .9$ & 53.4 & 0.14 & 758 & 56.2 & 19 & -0.36 \\
\hline $12: 33: 40: 92$ & 41 & 57 & 2 & 22.9 & 53.4 & 0.14 & $753 i$ & 56.2 & 19 & 0.33 \\
\hline $12: 33: 41: 91$ & 42 & 57 & 2 & 22.9 & 53.4 & 0.14 & $750^{\circ}$ & 56.2 & 19 & 0.33 \\
\hline $12: 33: 42: 90$ & 43 & 57 & 2 & 22.9 & $5 \overline{3.4}$ & 0.14 & 751 & 56.2 & 19 & -0.04 \\
\hline $12: 33: 43: 89$ & 44 & 57 & 2 & 22.9 & $53.4^{\prime}$ & 0.14 & 755 & 56.2 & 19 & -0.04 \\
\hline $12: 33: 44: 88$ & 45 & 57 & 2 & 22.9 & 53.4 & 0.14 & 753 & 56.2 & 20 & -0.14 \\
\hline $12: 33: 45: 87$ & 46 & 57 & 2 & 22.9 & 53.4 & 0.14 & $757^{\circ}$ & 56.3 & 20 & -0.14 \\
\hline $12: 3 \overline{3}: 46: 85$ & 47 & $5 \overline{7}$ & 2 & 22.9 & 53.4 & 0.14 & 763 & 56.3 & 20 & -0.18 \\
\hline $3: 47: 84$ & 48 & 57 & 2 & 22.9 & $53 . \overline{4}$ & 0.14 & 767 & 56.3 & 20 & -0.18 \\
\hline $12: 33: 48: 83$ & 49 & 57 & 2 & 22.9 & 53.4 & 0.14 & 768 & 56.2 & 19 & -0.15 \\
\hline $12: 33: 49: 82$ & 50 & 57 & 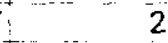 & 22.9 & 53.4 & 0.14 & $769^{\circ}$ & 56.2 & 19 & -0.15 \\
\hline $12: 33: 50: 81$ & 51 & 57 & 2 & 23.1 & 53.4 & 0.14 & 764 & 56.1 & 18 & -0.06 \\
\hline $12: 33: 51: 80$ & 52 & 57 & 2 & $2 \overline{3} .1$ & 53.4 & 0.14 & 758 & 56 & 18 & -0.06 \\
\hline $12: 33: 52: 79$ & 53 & 58 & 2 & 23.1 & 53.4 & 0.13 & $765^{\circ}$ & 56 & 18 & 0.05 \\
\hline
\end{tabular}




\begin{tabular}{|c|c|c|c|c|c|c|c|c|c|c|}
\hline$T I M E$ & $\begin{array}{l}\text { Elapsed } \\
\text { Time } \\
\text { (sec) }\end{array}$ & BIT_TC1 & PURG_TC2 & PurgePSI & PurgeCFM & DEPTH(IN) & DwnFrc(lb) & RPM & $\begin{array}{l}\text { Torque } \\
\text { (FTLB) }\end{array}$ & Inches/Min \\
\hline $12: 33: 53: 78$ & 5 & 58 & 25 & 23.1 & 53.4 & 0.14 & 760 & 56 & $\quad 18$ & 0.05 \\
\hline $12: 33: 54: 76$ & 5 & 58 & 25 & 23.1 & 53.4 & 0.14 & $75 \overline{8}$ & 56 & 18 & -0.01 \\
\hline $12: 33: 55: 75$ & 5 & 58 & 25 & 23.1 & 53.4 & 0.14 & 759 & 56 & 18 & -0.01 \\
\hline $12: 33: 56: 74$ & 5 & 58 & 25 & 23.1 & 53.4 & 0.14 & 762 & 56 & 19 & -0.13 \\
\hline $12: 33: 57: 73$ & 5 & 58 & 25 & 23.1 & 53.4 & 0.18 & 769 & 56 & 19 & -0.13 \\
\hline $12: 33: 58: 72$ & 5 & 58 & 25 & 23.1 & 53.4 & 0.15 & 772 & 55.8 & 19 & -0.31 \\
\hline $12: 33: 59: 71$ & 6 & 57 & 25 & 23.1 & 53.4 & 0.15 & 775 & 55.7 & 19 & -0.31 \\
\hline $12: 34: 00: 70$ & 6 & 58 & 25 & 23.1 & 53.4 & 0.14 & 774 & 55.7 & 19 & 0.07 \\
\hline $12: 34: 01: 68$ & 6 & 58 & 25 & 23.1 & 53.4 & 0.14 & 774 & 55.7 & 19 & 0.07 \\
\hline $12: 34: 02: 67$ & 6 & 58 & 25 & 23.1 & 53.4 & 0.14 & 772 & 55.7 & 18 & 0 \\
\hline $12: 34: 03: 66$ & 6 & 58 & 25 & 23.1 & $53.4 \mathrm{j}$ & 0.14 & 766 & 55.7 & 18 & 0 \\
\hline $12: 34: 04: 65$ & 6 & 58 & 25 & 23.1 & 53.4 & 0.13 & 759 & 55.6 & 18 & -0.16 \\
\hline $12: 34: 05: 64$ & 6 & 58 & 25 & 23.1 & 53.4 & 0.14 & $757 !$ & 55.7 & 18 & -0.16 \\
\hline $12: 34: 06: 63$ & 6 & 58 & 25 & 23.1 & 53.4 & 0.14 & 759 & 55.7 & 18 & -0.15 \\
\hline $12: 34: 07: 62$ & 6 & 58 & 25 & 23.1 & 53.4 & 0.14 & 762 & 55.8 & 18 & -0.15 \\
\hline $12: 34: 08: 60$ & 6 & 58 & 25 & 22.9 & 53.4 & 0.17 & 756 & 55.8 & 18 & -0.19 \\
\hline $12: 34: 09: 59$ & 7 & 58 & 25 & 22.9 & 53.4 & 0.14 & 760 & 558 & 18 & -0.19 \\
\hline $12: 34: 10: 58$ & 7 & 58 & 25 & 22.9 & 53.4 & 0.14 & 765 & 55.8 & 18 & 0.22 \\
\hline $12: 34: 11: 57$ & 7 & 58 & 25 & 22.9 & 53.4 & 0.14 & 769 & 55.9 & 18 & 0.22 \\
\hline $12: 34: 12: 56$ & 7 & 58 & 25 & 22.9 & 53.3 & 0.14 & 769 & 55.9 & 18 & -0.14 \\
\hline $12: 34: 13: 55$ & 7 & 58 & 25 & 22.9 & 53.3 & 0.15 & 766 & 55.9 & 18 & -0.5 \\
\hline $12: 34: 14: 54$ & 7 & 58 & 25 & 22.9 & 53.3 & 0.14 & 762 & 56 & 18 & -0.5 \\
\hline $12: 34: 15: 53$ & 7 & 58 & 25 & 23.1 & 53.3 & 0.14 & 755 & 56 & 18 & -0.08 \\
\hline $12: 34: 16: 51$ & 7 & 58 & 25 & 22.9 & 53.3 & 0.15 & 754 & 55.9 & 18 & -0.08 \\
\hline $12: 34: 17: 50$ & t & 58 & 25 & 23.1 & 53.3 & 0.15 & 755 & 55.7 & 18 & 0.09 \\
\hline $12: 34: 18: 49$ & 7 & 58 & 25 & 23.1 & 53.3 & 0.15 & 753 & 55.7 & 18 & 0.09 \\
\hline $12: 34: 19: 48$ & 8 & 58 & 25 & 23.1 & 53.3 & 0.15 & 755 & 55.8 & 18 & -0.26 \\
\hline $12: 34: 20: 47$ & 8 & 58 & 25 & 22.9 & 53.3 & 0.17 & 759 & 55.9 & 18 & -0.26 \\
\hline $12: 34: 21: 46$ & 8 & 58 & 25 & 22.9 & 53.3 & 0.15 & 764 & 55.9 & 18 & -0.15 \\
\hline $12: 34: 22: 45$ & 8 & 58 & 25 & 22.9 & 53.3 & 0.17 & 767 & 55.9 & 18 & -0.15 \\
\hline $12: 34: 23: 43^{\dagger}$ & $\underline{8}$ & 58 & 25 & 22.9 & 53.3 & 0.15 & 766 & 56 & 18 & -0.24 \\
\hline $12: 34: 24: 42$ & $\overline{8}$ & 58 & 25 & 22.9 & 53.3 & 0.15 & 762 & 56 & 18 & -0.24 \\
\hline $12: 34: 25: 41$ & 8 & 58 & 25 & 22.9 & 53.3 & 0.14 & 757 & 56 & 17 & -0.18 \\
\hline $12: 34: 26: 40$ & 8 & 58 & 25 & 22.9 & 53.3 & 0.17 & 761 & 56.1 & 17 & -0.18 \\
\hline $12: 34: 27: 39$ & 8 & 58 & 25 & 23.1 & 53.3 & 0.15 & $755 !$ & 56.1 & 17 & 0.14 \\
\hline $12: 34: 28: 38$ & 8 & 58 & 25 & 22.9 & 53.3 & 0.15 & 749 & 55.9 & 17 & 0.14 \\
\hline $12: 34: 29: 37$ & 9 & 58 & 25 & 22.9 & 53.3 & 0.14 & $747 !$ & 55.8 & 17 & -0.27 \\
\hline $12: 34: 30: 36$ & 9 & 58 & 25 & 22.9 & 53.2 & 0.17 & 749 & 55.8 & 17 & -0.27 \\
\hline $12: 34: 31: 34$ & 9 & 58 & 25 & 22.9 & 53.2 & 0.17 & 752 & 55.8 & 18 & -0.39 \\
\hline $12: 34: 32: 33$ & 9 & 58 & 25 & 22.9 & 53.2 & 0.17 & 757 & 55.9 & 18 & -0.39 \\
\hline $12: 34: 33: 32$ & 9 & 57 & 25 & 22.9 & 53.2 & 0.15 & 761 & 55.9 & 18 & -0.14 \\
\hline $12: 34: 34: 31$ & 9 & 57 & 25 & 22.9 & 53.2 & 0.15 & 756 & 55.9 & 18 & -0.14 \\
\hline $12: 34: 35: 30$ & 9 & 57 & 25 & 22.9 & 53.2 & 0.15 & 760 & 55.8 & 18 & 0.01 \\
\hline $12: 34: 36: 29$ & 9 & 57 & 25 & 22.9 & 53.2 & $0.15 !$ & 760 & 55.8 & 18 & 0.01 \\
\hline $12: 34: 37: 28$ & 9 & 57 & 25 & 22.9 & 53.2 & 0.14 & 757 & 55.7 & 18 & -0.04 \\
\hline $12: 34: 38: 26$ & 9 & 57 & 25 & 22.9 & 53.2 & 0.14 & 751 & 55.7 & 18 & -0.04 \\
\hline $12: 34: 39: 25$ & 10 & 58 & 25 & 22.9 & 53.2 & 0.15 & 753 & 55.7 & 18 & -0.35 \\
\hline $12: 34: 40: 24$ & 10 & 58 & 25 & 22.9 & 53.1 & 0.14 & 750 & 55.7 & 18 & -0.35 \\
\hline $12: 34: 41: 23$ & 10 & 58 & 25 & 22.9 & 53.1 & 0.14 & 753 & 55.7 & 18 & -0.28 \\
\hline $12: 34: 42: 22$ & 10 & 58 & 25 & 22.9 & 53.1 & 0.14 & 749 & 55.6 & 18 & -0.28 \\
\hline $12: 34: 43: 21$ & 10 & 58 & 25 & 22.9 & 53.1 & 0.17 & 752 & 55.8 & 18 & 0 \\
\hline $12: 34: 44: 20$ & 10 & 58 & $\begin{array}{r}25 \\
\end{array}$ & 22.9 & 53.1 & 0.17 & 750 & 55.8 & 18 & 0 \\
\hline $12: 34: 45: 19$ & 10 & 57 & 25 & 22.9 & 53.1 & 0.17 & 754 & 55.9 & 18 & 0.19 \\
\hline $12: 34: 46: 17$ & 10 & 57 & 25 & 22.9 & 53.1 & 0.17 & 758 & 55.9 & 18 & -0.5 \\
\hline
\end{tabular}




\begin{tabular}{|c|c|c|c|c|c|c|c|c|c|c|}
\hline$T I M E$ & $\begin{array}{l}\text { Elapsed } \\
\text { Time } \\
\text { (sec) }\end{array}$ & $B I T$ TC1 & PURG_TC2 & PurgePSI & PurgeCFM & DEPTH $(\mathbb{I N})$, & DwnFrc(lb) & RPM & $\begin{array}{l}\text { Torque } \\
\text { (FTLB) }\end{array}$ & Inches/Min \\
\hline $12: 34: 47: 16$ & $\quad 108$ & 57 & $\begin{array}{r}25 \\
\end{array}$ & $22.9^{\prime}$ & $\quad 53.1$ & $\quad 0.15$ & 764 & 55.9 & $\quad 18$ & $\begin{array}{l}-0.5 \\
\end{array}$ \\
\hline $12: 34: 48: 15$ & 109 & 57 & 25 & 22.9 & 53.1 & 0.15 & 759 & 55.8 & 18 & -0.13 \\
\hline $12: 34: 49: 14$ & 110 & 57 & 25 & 22.9 & 53.1 & 0.15 & 751 & 55.8 & 18 & -0.13 \\
\hline $12: 34: 50: 13$ & 111 & 58 & 25 & $22.9^{\circ}$ & 53.1 & 0.15 & 747 & 55.8 & 18 & -0.02 \\
\hline $12: 34: 51: 12$ & 112 & 58 & $25^{\circ}$ & 22.9 & 53.1 & 0.15 & 750 & 55.8 & $1 \overline{8}$ & -0.02 \\
\hline $12: 34: 52: 11$ & 113 & 58 & 25 & $22.9^{\circ}$ & 53.1 & 0.15 & 746 & 55.9 & 18 & $-0 . \overline{11}$ \\
\hline $12: 34: 53: 09$ & 114 & 58 & 25 & 22.9 & 53.1 & 0.17 & $7 \overline{45}$ & 55.9 & 18 & -0.11 \\
\hline $12: 34: 54: 08$ & 115 & 58 & 25 & 22.9 & 53.1 & 0.17 & 749 & 56 & 17 & $-0 . \overline{06}$ \\
\hline $12: 34: 55: 07$ & 116 & 58 & $25 !$ & 22.9 & $53 . \overline{1}$ & 0.19 & 754 & 56 & 18 & -0.06 \\
\hline $12: 34: 56: 06$ & 117 & 58 & 25 & $22.9^{\prime}$ & 53.1 & 0.17 & 757 & 56 & 18 & -0.18 \\
\hline $12: 34: 57: 05$ & 118 & 58 & 25 & 22.9 & 53.1 & 0.15 & $760^{\circ}$ & 56 & 18 & -0.18 \\
\hline $12: 34: 58: 04$ & 119 & 58 & 25 & $22.9^{\circ}$ & 53 & 0.14 & 760 & 55.9 & 18 & -0.46 \\
\hline $12: 34: 59: 03$ & 120 & 58 & 25 & $22.9^{\circ}$ & 53 & 0.15 & $760^{\circ}$ & $55.9^{\circ}$ & 18 & -0.46 \\
\hline $12: 35: 00: 02$ & 121 & 58 & 25 & 22.9 & 53 & 0.14 & $759^{\circ}$ & 56 & 18 & 0.13 \\
\hline $12: 35: 01: 00$ & 122 & 58 & 25 & $22.9^{\circ}$ & 53 & 0.15 & $755^{\dagger}$ & 55.9 & 18 & 0.13 \\
\hline $12: 35: 01: 99$ & 123 & 58 & 25 & 22.9 & 53 & $0.15^{\dagger}$ & 749 & 55.9 & 17 & 0.06 \\
\hline $12: 35: 02: 98$ & 124 & 58 & 25 & 229 & 53 & 0.17 & 740 & 558 & 17 & 0.06 \\
\hline $12: 3 \overline{5: 03: 97}$ & 125 & $5 \overline{8}$ & 25 & 22.9 & 53 & 0.14 & 739 & 55.7 & 17 & -0.29 \\
\hline $12: 35: 04: 96$ & 126 & 58 & $25 i$ & 22.9 & 53 & 0.14 & $744^{\dagger}$ & 55.7 & 17 & -0.29 \\
\hline $12: 35: 05: 95$ & 127 & 58 & $25^{t}$ & 22.9 & $53^{4}$ & 0.17 & 749 & 55.9 & 17 & -0.21 \\
\hline $12: 35: 06: 94$ & 128 & $580^{\circ}$ & 25 & $22.9^{\circ}$ & 53 & 0.18 & 744 & $55.9^{\circ}$ & 17 & -0.21 \\
\hline $12: 35: 07: 92$ & 129 & $58 \dagger$ & 25 & $22.9^{\circ}$ & 53 & 0.15 & $750^{\circ}$ & 55.9 & 17 & -0.09 \\
\hline $12: 35: 08: 91^{\dagger}$ & 130 & 58 & 25 & 22.9 & 53 & 0.15 & 754 & 55.9 & 17 & -0.09 \\
\hline $12: 35: 09: 90$ & 131 & 58 & 25 & 22.9 & 53 & 0.15 & 756 & 55.9 & 17 & -0.03 \\
\hline $12: 35: 10: 89$ & 132 & 58 & 25 & 22.9 & 53 & 0.15 & $756 !$ & 55.9 & 17 & -0.03 \\
\hline $12: 35: 11: 88$ & 133 & 58 & 25 & 22.9 & $53 !$ & 0.14 & 751 & 55.8 & 17 & -0.03 \\
\hline $12: 35: 12: 87$ & 134 & 58 & 25 & 22.9 & 53 & 0.14 & $745 !$ & 55.8 & 17 & $-0 . \overline{03}$ \\
\hline $12: 35: 13: 86$ & 135 & 58 & 25 & 22.9 & 53 & 0.14 & $743^{\circ}$ & 55.8 & 17 & -0.33 \\
\hline $12: 35: 14: 85$ & 136 & 58 & 25 & 22.9 & 53 & 0.15 & 744 & 55.7 & 17 & -0.33 \\
\hline $12: 35: 15: 83$ & 137 & 58 & 25 & $22.9^{\circ}$ & 53 & 0.17 & 744 & 55.7 & 17 & $-0 . \overline{21}$ \\
\hline $12: 35: 16: 82$ & 138 & 58 & 25 & 22.9 & 53 & 0.17 & $7466^{\dagger}$ & 55.7 & 17 & -0.21 \\
\hline $12: 35: 17: 81$ & 139 & 58 & $25 !$ & $22.9^{\circ}$ & 53 & 0.18 & 750 & 55.6 & 17 & 0.21 \\
\hline $12: 35: 18: 80$ & 140 & $58^{\dagger}$ & $25^{\circ}$ & $22.9^{4}$ & 52.9 & 0.17 & $755 !$ & 55.7 & 17 & 0.21 \\
\hline $12: 35: 19: 79$ & 141 & 58 & 25 & 22.9 & 52.9 & 0.15 & 759 & 55.7 & 17 & -0.23 \\
\hline $12: 3 \overline{5}: 20: 78$ & 142 & 57 & $25^{\circ}$ & 22.9 & 52.9 & 0.15 & $759^{\prime}$ & 55.8 & 17 & -0.3 \\
\hline $12: 35: 21: 77$ & 143 & 57 & 25 & 23.1 & 52.9 & $0 . \overline{15}$ & 758 & 55.8 & 17 & -0.3 \\
\hline $12: 35: 22: 75$ & 144 & 57 & $25^{\circ}$ & $22.9^{\circ}$ & 52.9 & 0.17 & 752 & 55.8 & 17 & -0.14 \\
\hline $12: 35: 23: 74$ & 145 & 58 & 25 & 22.9 & 52.9 & 0.17 & 758 & 55.8 & 17 & -0.14 \\
\hline $12: 35: 24: 73$ & 146 & 57 & $25^{\circ}$ & 22.9 & 52.9 & 0.14 & 752 & 55.8 & 17 & -0.24 \\
\hline $12: 35: 25: 72$ & 147. & 58 & $25^{\dagger}$ & 22,9 & 52.9 & 0.17 & 746 & $55.9^{\dagger}$ & 17 & -0.24 \\
\hline $12: 35: 26: 71$ & 148 & 58 & 25 & 22.9 & 52.9 & 0.14 & 744 & 55.9 & 17 & 0.29 \\
\hline $12: 35: 27: 70$ & 149 & 58 & 25 & 22.9 & 52.9 & 0.17 & 746 & 55.9 & 17 & 0.29 \\
\hline $12: 35: 28: 69$ & 150 & 58 & 25 & 22.9 & 52.9 & 0.18 & $751^{\prime}$ & 55.8 & 17 & -0.27 \\
\hline $12: 35: 29: 68$ & $151^{t}$ & 58 & 25 & $22.9^{\circ}$ & 52.9 & 0.18 & 756 & 55.8 & 17 & $-0 . \overline{27}$ \\
\hline $12: 35: 30: 6 \overline{6}^{\prime}$ & 152 & 57 & $25^{\circ}$ & 23.1 & 52.9 & 0.17 & 760 & 55.8 & 17 & $-\overline{0.34}$ \\
\hline $12: 35: 31: 65$ & 153 & 57 & $25^{\circ}$ & 23.1 & 52.9 & 0.18 & $756^{\circ}$ & 55.8 & 17 & -0.34 \\
\hline $12: 35: 32: 64$ & $15 \overline{4}$ & $5 \overline{7}$ & 25 & 23.1 & 52.9 & 0.17 & $759^{\circ}$ & 55.8 & 17 & 0.19 \\
\hline $12: 35: 33: 63$ & 155 & 57 & 25 & 23.1 & 52.9 & $0 . \overline{17}$ & $760^{\circ}$ & 55.8 & 17 & $0 . \overline{19}$ \\
\hline $12: 35: 34: 62$ & 156 & 57 & 25 & 23.1 & 52.9 & 0.17 & 755 & 55.6 & 17 & -0.23 \\
\hline $12: 35: 35: 61$ & 157 & $5 \overline{7}$ & $25^{i}$ & 23.1 & 52.9 & $0.15^{\dagger}$ & 750 & 55.7 & 17 & -0.23 \\
\hline $12: 35: 36: 60$ & 158 & 57 & 25 & 22.9 & 52.9 & $0 . \overline{17}$ & $745^{\circ}$ & 55.7 & 17 & -0.2 \\
\hline $12: 35: 37: 58$ & 159 & 58 & 25 & $22.9^{\circ}$ & 52.9 & 0.17 & $745^{\circ}$ & 55.7 & 17 & -0.2 \\
\hline $12: 35: 38: 57$ & 160 & 58 & 25 & 22.9 & 52.9 & 0.17 & $749^{\prime \prime}$ & 55.8 & 17 & -0.18 \\
\hline $12: 35: 39: 56$ & 161 & $5 \overline{7}$ & $25^{t}$ & $22.9^{\circ}$ & 52.9 & 0.17 & 754 & 55.8 & 17 & -0.18 \\
\hline
\end{tabular}




\begin{tabular}{|c|c|c|c|c|c|c|c|c|c|c|}
\hline$T I M E$ & $\begin{array}{l}\text { Elapsed } \\
\text { Time }\end{array}$ & $\mathrm{BIT}$ TC1 & PURG_TC2 & PurgePS! & \multicolumn{2}{|c|}{ PurgeCFM DEPTH(IN)! } & \multicolumn{2}{|c|}{ DwnFrc(lb) RPM } & $\begin{array}{l}\text { Torque } \\
\text { (FTLB) }\end{array}$ & Inches/Min \\
\hline $12: 35: 40: 55$ & $\quad 162$ & 57 & 25 & $\quad 22.9$ & 52.9 & 0.18 & $749^{\circ}$ & 55.7 & 17 & $\quad-0.36$ \\
\hline $12: 35: 41: 54$ & 163 & 57 & 25 & 23.1 & 52.9 & 0.17 & 755 & 55.6 & 17 & -0.36 \\
\hline $12: 35: 42: 53$ & $16 \overrightarrow{4}$ & 57 & 25 & 23.1 & 52.9 & 0.17 & 759 & 55.6 & 17 & 0.09 \\
\hline $12: 35: 43: 52$ & 165 & 57 & 25 & 23.1 & 52.9 & 0.17 & 761 & 55.6 & 17 & 0.09 \\
\hline $12: 35: 44: 50$ & 166 & 57 & 25 & $23.1^{\circ}$ & 52.9 & 0.17 & 760 & 55.6 & 17 & 0.03 \\
\hline $12: 35: 45: 49$ & 167 & $5 \overline{7}$ & 25 & 23.1 & 52.9 & 0.15 & 755 & $55.6^{\prime}$ & 17 & 0.03 \\
\hline $12: 35: 46: 48$ & $168^{\circ}$ & 57 & 25 & 23.1 & 52.9 & 0.17 & $749^{\circ}$ & 55.7 & 17 & -0.34 \\
\hline $12: 35: 4 \overline{7}: 47$ & 169 & 57 & 25 & $22.9^{\circ}$ & 52.9 & 0.15 & $746 !$ & $55.7^{4}$ & 17 & -0.34 \\
\hline $12: 35: 48: 46$ & 170 & 57 & 25 & 22.9 & $52.9^{9}$ & 0.15 & $748^{\circ}$ & 55.7 & 17 & -0.16 \\
\hline $12: 35: 49: 45$ & $171:$ & 57 & 25 & 22.9 & 52.9 & 0.17 & 745 & 55.8 & 17 & -0.16 \\
\hline $12: 35: 50: 44$ & 172 & 58 & $25 !$ & 22.9 & 52.9 & 0.17 & 746 & 55.9 & 17 & -0.33 \\
\hline $12: 35: 51: 43$ & 173 & 58 & 25 & 22.9 & 52.9 & $0.18^{1}$ & $750^{\circ}$ & 55.9 & 17 & $-0 . \overline{33}$ \\
\hline $12: 35: 52: 41$ & 174 & 57 & 25 & 22.9 & 52.8 & 0.17 & 755 & 56.11 & 17 & 0.07 \\
\hline $12: 35: 53: 40$ & 175 & 57 & 25 & 23.1 & 52.8 & 0.18 & $759^{\dagger}$ & 56 & 17 & -0.2 \\
\hline $12: 35: 54: 39$ & 176 & 57 & 25 & 23.1 & 52.8 & 0.17 & 761 & 56 & 17 & -0.2 \\
\hline $12: 35: 55: 38$ & 177 & 57 & 25 & 23.1 & 52.8 & 0.17 & 760 & 56 & 17 & -0.23 \\
\hline $12: 35: 56: 37$ & $17 \overline{8}$ & 57 & 25 & $23.1^{\prime}$ & 52.8 & 0.17 & 755 & 55.9 & 17 & -0.23 \\
\hline $12: 35: 57: 36$ & 179 & 57 & 25 & 23.1 & 52.8 & 0.17 & $760^{\circ}$ & 55.9 & 17 & $-0 . \overline{32}$ \\
\hline $12: 35: 58: 35$ & 180 & 57 & 25 & 23.1 & 52.8 & 0.17 & 755 & $56 !$ & 17 & -0.32 \\
\hline $12: 35: 59: 33$ & 181 & 58 & 25 & 22.9 & 52.8 & 0.17 & $7 \overline{49}$ & 55.9 & 18 & 0.1 \\
\hline $12: 36: 00: 32$ & 182 & 58 & 25 & 22.9 & 52.8 & 0.15 & 747 & $55.9^{4}$ & 18 & 0.1 \\
\hline $12: 36: 01: 31$ & $183^{\dagger}$ & 58 & 25 & 22.9 & 52.8 & 0.17 & $747^{\circ}$ & 55.8 & 18 & -0.05 \\
\hline $12: 36: 02: 30$ & 184 & 58 & 25 & 23.1 & 52.8 & 0.18 & 751 & 55.9 & 18 & -0.05 \\
\hline $12: 36: 03: 29$ & 185 & 58 & 25 & 23.1 & $52.8 !$ & 0.18 & 756 & 55.9 & 18 & -0.3 \\
\hline $12: 36: 04: 28$ & 186 & 57 & 25 & $22.9^{\prime}$ & 52.8 & 0.17 ! & 760 & 55.7 & 18 & -0.3 \\
\hline $12: 36: 05: 27$ & 187 & 57 & 25 & 23.1 & 52.8 & 0.17 & 757 & 55.6 & 17 & -0.18 \\
\hline $12: 36: 06: 26$ & 188 & 57 & 25 & 23.1 & 52.8 & $0.17 !$ & $761 !$ & 55.6 & 17 & -0.18 \\
\hline $12: 36: 07: 24$ & 189 & 57 & 25 & 23.1 & 52.8 & 0.17 & 761 & 55.4 & 17 & -0.24 \\
\hline $12: 36: 08 \cdot 23$ & $1 \overline{90}$ & 57 & 25 & 23.1 & 52.8 & 0.15 & 758 & 55.4 & 17 & -0.24 \\
\hline $12: 36: 09: 22$ & 191 & 57 & 25 & 23.1 & 52.8 & 0.17 & 752 & 55.4 & 17 & 0.04 \\
\hline $12: 36: 10: 21$ & 192 & 58 & 25 & 23.1 & 52.8 & 0.15 & 747 & 55.5 & 17 & 0.04 \\
\hline $12,36: 11: 20$ & 193 & 57 & 25 & 22.9 & 52.8 & 0.17 & 747 & 55.5 & 17 & -0.29 \\
\hline $12: 36: 12: 19$ & $19 \overline{4}$ & 58 & 25 & 22.9 & 52.8 & 0.17 & 750 & 55.7 & 17 & -0.29 \\
\hline $12: 36: 13: 18$ & 195 & 58 & 25 & 22.9 & 52.8 & 0.17 & 754 & 55.8 & 17 & -0.22 \\
\hline $12: 36: 14: 16$ & 196 & 58 & 25 & 23.1 & 52.8 & 0.18 & 750 & 55.8 & 17 & -0.22 \\
\hline $12: 3 \overline{6}: 15: 15$ & 197 & 58 & 25 & 22.9 & 52.8 & 0.17 & 756 & 55.8 & 17 & -0.15 \\
\hline $12: 36: 16: 14$ & 198 & 57 & 25 & 22.9 & 52.8 & 0.17 & 759 & 55.8 & 17 & -0.15 \\
\hline $12: 36: 17: 13$ & 199 & 57 & 25 & 23.1 & 52.7 & 0.17 & $763 i$ & $55.8^{\dagger}$ & 17 & 0.05 \\
\hline $12: 36: 18: 12$ & 200 & 57 & 25 & 23.1 & 52.7 & $0 . \overline{17}$ & 762 & 55.7 & 17 & 0.05 \\
\hline $12: 36: 19: 11$ & 201 & 57 & 25 & $23.1^{\circ}$ & 52.7 & 0.17 & $757 \mathrm{i}$ & 55.5 & 17 & -0.21 \\
\hline $12: 36: 20: 10$ & 202 & $5 \overline{7}$ & 25 & 23.1 & 52.7 & 0.17 & 750 & 55.5 & 17 & -0.21 \\
\hline $12: 36: 21: 09$ & 203 & 57 & 25 & 23.1 & 52.7 & 0.17 & 747 & 55.5 & 17 & -0.19 \\
\hline $12: 36: 22: 07$ & 204 & 58 & 25 & 23.1 & 52.7 & 0.17 & 749 & 55.6 & 17 & -0.19 \\
\hline $12: 36: 23: 06$ & 205 & 58 & 25 & 22.9 & 52.7 & 0.17 & 747 & 55.6 & 17 & $-0 . \overline{3}$ \\
\hline $12: 36: 24: 05$ & 206 & 58 & 25 & 22.9 & 52.7 & 0.18 & $749^{\circ}$ & 55.7 & 17 & -0.3 \\
\hline $12: 36: 25: 04$ & 207 & 57 & 25 & 22.9 & 52.7 & 0.19 & 753 & 55.8 & 17 & -0.05 \\
\hline $12: 36: 26: 03$ & 208 & 57 & 25 & 22.9 & 52.7 & $0 . \overline{18}$ & 758 & 55.8 & 17 & -0.18 \\
\hline $12: 36: 27: 02$ & 209 & 57 & 25 & 23.1 & 52.7 & 0.18 & 762 & 559 & 17 & $-0.1 \overline{8}$ \\
\hline $12: 36: 28: 01$ & 210 & $5 \overline{7}$ & 25 & 22.9 & 52.7 & 0.17 & 764 & 56 & 17 & -0.23 \\
\hline $12: 36: 28: 99$ & 211 & 57 & 25 & 23.1 & 52.7 & 0.17 & 763 & 56 & 17 & -0.23 \\
\hline $12: 36: 29: 98$ & 212 & 57 & 25 & 23.1 & 52.7 & 0.17 & 764 & $55 . \overrightarrow{a_{1}}$ & 17 & -0.24 \\
\hline $12: 36: 30: 97$ & 213 & 57 & 25 & 23.1 & 52.7 & 0.15 & 763 & 56 & 17 & -0.24 \\
\hline $12: 36: 3196$ & 214 & 57 & 25 & $23.1^{\circ}$ & 52.7 & 0.17 & $757^{\circ}$ & 56 & 17 & -0.19 \\
\hline $12: 36: 32: 95$ & 215 & 58 & 25 & 23.1 & 52.7 & 0.15 & $751^{t}$ & 56 & 17 & -0.19 \\
\hline
\end{tabular}




\begin{tabular}{|c|c|c|c|c|c|c|c|c|c|c|}
\hline \multirow[t]{2}{*}{$\begin{array}{l}\text { TIME } \\
12 \cdot 36.33 \cdot 94\end{array}$} & \multicolumn{2}{|l|}{$\begin{array}{l}\text { Elapsed } \\
\text { Time }\end{array}$} & \multirow{2}{*}{ PURG_TC2 } & PurgePSI: & \multicolumn{2}{|c|}{ PurgeCFMDEPTH(IN) } & \multicolumn{2}{|c|}{ DwnFrc(lb) RPM } & $\begin{array}{l}\text { 'Torque } \\
\text { (FTLB) }\end{array}$ & Inches/Min \\
\hline & $\quad 216$ & 58 & & $\quad 23.1$ & $\begin{array}{r}52.7 \\
\end{array}$ & $\quad 0.17$ & $\quad 749$ & 56 & 17 & $\quad 0.15$ \\
\hline $12: 36: 34: 93$ & 217 & 58 & 25 & 22.9 & 52.7 & 0.17 & 749 & 56 & 17 & 0.15 \\
\hline $12: 36: 35: 92$ & 218 & 58 & 25 & 23.1 & 52.7 & 0.18 & 754 & 56.1 & 17 & -0.22 \\
\hline $12: 36: 36: 90$ & 219 & 57 & 25 & 23.1 & 52.7 & 0.18 & 758 & 56 & 17 & -0.22 \\
\hline $12: 36: 37: 89$ & 220 & 57 & 25 & 22.9 & 52.7 & 0.19 & 762 & 56 & 17 & -0.21 \\
\hline $12: 36: 38: 88$ & 221 & 57 & 25 & 22.9 & 52.7 & 0.17 & 759 & 55.9 & 17 & -0.21 \\
\hline $12: 36: 39: 87$ & 222 & 57 & 25 & 23.1 & 52.7 & 0.17 & 762 & 56 & 17 & -0.37 \\
\hline $12: 36: 40: 86$ & 223 & 57 & 25 & 23.1 & 52.7 & 0.17 & 765 & 56 & 17 & -0.37 \\
\hline $12: 36: 41: 85$ & 224 & 57 & 25 & 23.1 & 52.7 & 0.17 & 764 & 56 & 17 & 0.03 \\
\hline $12: 36: 42: 84$ & 225 & 57 & 25 & 23.1 & 52.7 & 0.17 & 759 & 56 & 17 & 0.03 \\
\hline $12: 36: 43: 82$ & 226 & 57 & 25 & 23.1 & 52.7 & 0.15 & 753 & 56 & 17 & 0.07 \\
\hline $12: 36: 44: 81$ & 227 & 57 & 25 & 23.1 & 52.7 & 0.15 & 750 & 55.8 & 17 & 0.07 \\
\hline $12: 36: \overline{4} 5: 80$ & 228 & 57 & 25 & 23.1 & 52.7 & 0.15 & 750 & 55.7 & 17 & -0.37 \\
\hline $12: 36: 46: 79$ & 229 & 57 & 25 & 23.1 & 52.7 & 0.17 & 750 & $55 \overline{7}$ & 17 & -0.37 \\
\hline $12: 36: 4778$ & 230 & 57 & 25 & 23.1 & 52.6 & 0.19 & 752 & 55.7 & 17 & -0.11 \\
\hline $12: 36: 48: 77$ & 231 & 57 & 25 & 23.1 & $52 . \overline{6}$ & 0.19 & 755 & 55.7 & 17 & -0.11 \\
\hline $12: 36: 49: 76$ & 232 & 57 & 25 & 23.1 & 52.6 & 0.17 & 761 & 55.7 & 17 & -0.17 \\
\hline $12: 36: 50: 75$ & 233 & 57 & 25 & 23.1 & 52.6 & 0.17 & 765 & 55.7 & 17 & -0.17 \\
\hline $12: 36: 51: 73$ & 234 & 57 & 25 & 23.3 & 52.6 & 0.17 & 766 & 55.7 & 17 & 0.12 \\
\hline $12: 36: 52: 72$ & 235 & 57 & 25 & 23.3 & 52.6 & 0.17 & 765 & 55.8 & 17 & 0.12 \\
\hline $12: 36: 53: 71$ & 236 & 57 & 25 & 23.1 & 52.6 & 0.17 & 759 & 56 & 17 & $-0 . \overline{21}$ \\
\hline $12: 36: 54: 70$ & 237 & 57 & 25 & 23.3 & 52.6 & 0.15 & 764 & 56 & 17 & -0.21 \\
\hline $12: 36: 55: 69$ & 238 & 57 & 25 & 23.3 & 52.6 & 0.17 & 759 & 56.2 & 17 & -0.32 \\
\hline $12: 36: 56: 68$ & 239 & 57 & 25 & 23.1 & 52.6 & 0.17 & 752 & 56.1 & 17 & -0.32 \\
\hline $12: 36: 57: 67$ & 240 & 57 & 25 & 23.1 & 52.6 & 0.17 & 750 & 56.1 & 17 & -0.13 \\
\hline $12: 36: 58: 65$ & 241 & 57 & 25 & 23.1 & 52.6 & 0.17 & 751 & 55.9 & 17 & -0.12 \\
\hline $12: 36: 59: 64$ & 242 & 57 & 25 & 23.1 & 52.6 & 0.19 & 754 & 56.1 & 17 & -0.12 \\
\hline $12: 37: 00: 63$ & 243 & 57 & 25 & 23.1 & $5 \overline{2.6}$ & $0 . \overline{19}$ & 760 & 56.1 & 17 & 0.07 \\
\hline $12: 37: 01: 62$ & 244 & 57 & 25 & 23.1 & 52.6 & 0.19 & 764 & 56.2 & 17 & 0.07 \\
\hline $12: 37: 02: 61$ & 245 & 57 & 25 & 23.1 & 52.6 & 0.19 & 767 & 56.2 & 17 & -0.46 \\
\hline $12: 37: 03: 60$ & 246 & 57 & 25 & 23.1 & 52.6 & 0.17 & 764 & $5 \overline{6} .2$ & 17 & $-0 . \overline{46}$ \\
\hline $12: 37: 04: 59$ & 247 & 57 & 25 & 18.3 & 1.8 & 0.15 & 767 & $5 \overline{6} .3$ & 17 & -0.23 \\
\hline $12: 37: 05: 57$ & 248 & 58 & 25 & -0.6 & 1.9 & 0.15 & 766 & 56.1 & 18 & $-0 . \overline{23}$ \\
\hline $12: 37: 06: 56$ & 249 & 60 & 25 & -0.6 & 1.9 & 0.15 & 762 & 56.1 & 20 & -0.14 \\
\hline $12: 37: 07: 55$ & 250 & 62 & 25 & -0.8 & 1.9 & 0.17 & 757 & 56.1 & 20 & -0.14 \\
\hline $12: 37: 08: 54$ & 251 & 64 & 25 & -0.6 & 1.9 & 0.15 & 752 & 56.3 & 21 & -0.03 \\
\hline $12: 37: 09: 53$ & 252 & 66 & 25 & -0.8 & 1.9 & 0.17 & 751 & 56.3 & 21 & -0.03 \\
\hline $12: 37: 10: 52$ & 253 & 67 & 25 & -0.8 & 1.9 & 0.15 & 754 & $5 \overline{6} \overline{3}$ & 21 & -0.06 \\
\hline $12: 37: 11: 51$ & 254 & 68 & 25 & -0.8 & 1.9 & 0.19 & 752 & 56.4 & 22 & -0.06 \\
\hline $12: 37: 12: 50$ & 255 & 70 & 25 & -0.8 & 1.9 & 0.17 & 754 & 56.4 & 22 & -0.21 \\
\hline $12: 37: 13: 48$ & 256 & 71 & 25 & -0.6 & 1.9 & 0.17 & 757 & 56.2 & 22 & $-0 . \overline{21}$ \\
\hline $12: 37: 14: 47$ & 257 & 71 & 25 & -0.8 & 1.9 & 0.17 & 763 & 56.2 & 22 & -0.29 \\
\hline $12: 37: 15: 46$ & 258 & 72 & 25 & -0.6 & 1.9 & 0.17 & 768 & 56.2 & 22 & -0.29 \\
\hline $12: 37: 16: 45$ & 259 & 73 & 25 & -0.6 & 1.9 & 0.17 & 768 & 56.2 & 22 & -0.18 \\
\hline $12: 37: 17: 44$ & 260 & 73 & 25 & -0.6 & 1.9 & 0.17 & 767 & 56.1 & 22 & $-0 . \overline{18}$ \\
\hline $12: 37: 18: 43$ & 261 & 74 & 25 & -0.6 & 1.9 & 0.15 & 760 & 56.1 & 22 & $0 . \overline{12}$ \\
\hline $12: 37: 19: 42$ & 262 & 75 & 25 & -0.6 & 1.9 & 0.17 & 766 & 55.7 & 23 & 0.12 \\
\hline $12: 37: 20: 40$ & 263 & 77 & 26 & -0.6 & 1.9 & 0.17 & 760 & 55.6 & 23 & $-0 . \overline{26}$ \\
\hline $12: 37: 21: 39$ & $\overline{264}$ & 78 & 25 & -0.6 & 1.9 & 0.17 & 755 & $55 . \overline{6}$ & 23 & -0.26 \\
\hline $12: 37: 22: 38$ & 265 & 79 & $2 \overline{6}$ & -0.8 & 1.9 & 0.18 & 754 & 55.6 & 23 & -0.37 \\
\hline $12: 37: 23: 37$ & 266 & 80 & 25 & -0.6 & 1.9 & 0.17 & 757 & 55.7 & 23 & $-0.3 \overline{7}$ \\
\hline $12: 37: 24: 36$ & 267 & 81 & 26 & -0.6 & 1.9 & 0.18 & 761 & 55.7 & 23 & -0.1 \\
\hline $12: 37: 25: 35$ & 268 & 82 & 25 & -0.6 & 1.9 & 0.17 & 767 & 55.8 & 23 & -0.1 \\
\hline $12: 37: 26: 34$ & 269 & 82 & 25 & -0.6 & 1.9 & 0.19 & 770 & 55.6 & 23 & 0.05 \\
\hline
\end{tabular}




\begin{tabular}{|c|c|c|c|c|c|c|c|c|c|c|}
\hline$T I M E$ & $\begin{array}{l}\text { Elapsed } \\
\text { Time } \\
\text { (sec) }\end{array}$ & $B \mid T \_T C 1$ & |PURG_TC2 & PurgePSI & PurgeCFM & DEPTH(IN) & DwnFrc(b) & RPM & $\begin{array}{l}\text { Torque } \\
\text { (FTLB) }\end{array}$ & Inches/Min \\
\hline $12: 37: 27: 33$ & 270 & 82 & 26 & -0.6 & 1.9 & 0.17 & $\quad 770$ & 55.6 & 24 & $\quad 0.05$ \\
\hline $12: 37: 28: 31$ & 271 & 83 & 26 & -0.6 & 1.9 & 0.17 & 770 & 55.5 & 23 & -0.18 \\
\hline $12: 37: 29: 30$ & 272 & $8 \overline{4}$ & 25 & -0.6 & 1.9 & 0.15 & 770 & 55.5 & 24 & -0.18 \\
\hline $12: 37: 30: 29$ & $273^{\circ}$ & 85 & 26 & -0.6 & 1.9 & 0.17 & 765 & 55.5 & 24 & -0.29 \\
\hline $12: 37: 31: 28$ & 274 & 86 & 26 & -0.6 & 1.9 & 0.18 & 760 & 55.7 & 24 & -0.14 \\
\hline $12: 37: 32: 27$ & 275 & 87 & 26 & -0.6 & 1.9 & $0 . \overline{18}$ & 756 & 55.6 & 24 & $-0.1 \overline{4}$ \\
\hline $12: 37: 33: 26$ & 276 & 88 & 26 & -0.6 & 1.9 & 0.17 & 757 & 55.6 & 24 & -0.06 \\
\hline $12: 37: 34: 25$ & 277 & 89 & 25 & -0.6 & 1.9 & 0.17 & 760 & 55.5 & 24 & -0.06 \\
\hline $12: 37: 35: 23$ & 278 & 90 & 25 & -0.6 & 1.9 & 0.17 & 766 & 55.5 & 24 & -0.17 \\
\hline $12: 37: 36: 22$ & 279 & $\overline{91}$ & 26 & -0.6 & 1.9 & 0.18 & 761 & 55.8 & 24 & -0.17 \\
\hline $12: 37: 37: 21$ & 280 & 92 & 26 & -0.6 & 1.9 & 0.17 & 767 & 55.8 & 24 & -0.21 \\
\hline $12: 37: 38: 20$ & 281 & 91 & 26 & -0.6 & 1.9 & 0.15 & 771 & 55.8 & 24 & -0.21 \\
\hline $12: 37: 39: 19$ & 282 & 92 & 26 & -0.6 & 1.9 & 0.17 & 772 & 55.7 & 24 & -0.23 \\
\hline $12: 37: 40: 18$ & 283 & 93 & 25 & -0.6 & 1.9 & 0.17 & 770 & 55.8 & 24 & -0.23 \\
\hline $12: 37: 41: 17$ & 284 & 94 & 25 & -0.6 & 1.9 & $0 . \overline{7}$ & 765 & 55.8 & 24 & $-0 . \overline{19}$ \\
\hline $12: 37: 42: 16$ & 285 & 95 & 26 & -0.6 & 1.9 & 0.15 & 759 & 55.7 & 24 & $-0 . \overline{19}$ \\
\hline $12: 37: 43: 14$ & 286 & 95 & 26 & -0.6 & $1 . \overline{9}$ & 0.17 & 757 & $5 \overline{5} . \overline{7}$ & 24 & 0.13 \\
\hline $12: 37: 44: 13$ & 287 & 97 & 25 & -0.6 & 1.9 & $0 . \overline{17}$ & 759 & 55.7 & 24 & 0.13 \\
\hline $12: 37: 45: 12$ & 288 & 98 & 26 & -0.6 & 1.9 & 0.17 & 757 & 557 & 24 & -0.25 \\
\hline $12: 37: 46: 11$ & 289 & 98 & 26 & -0.6 & 1.9 & 0.19 & 760 & 55.7 & 24 & -0.25 \\
\hline $12237: 47: 10$ & 290 & 99 & 26 & $-0 . \overline{6}$ & 19 & 0.17 & 763 & 55.7 & 24 & -0.4 \\
\hline $12: 37: 48: 09$ & 291 & 100 & 26 & -0.6 & 1.9 & 0.19 & 769 & 55.8 & 24 & -0.4 \\
\hline $12: 37: 49: 08$ & 292 & 100 & 26 & -0.8 & 1.9 & 0.17 & 772 & 55.9 & 24 & $-0 . \overline{17}$ \\
\hline $12: 37: 50: 06$ & 293 & 100 & 26 & -0.6 & 1.9 & 0.17 & 772 & 55.9 & 24 & -0.17 \\
\hline $12: 37: 51: 05$ & 294 & 101 & 26 & -0.6 & 1.9 & 0.17 & 770 & $5 \overline{5.9}$ & 25 & 0.12 \\
\hline $12: 37: 52: 04$ & 295 & 102 & 26 & -0.8 & 1.9 & 0.17 & 764 & 55.7 & 25 & $0 . \overline{12}$ \\
\hline $12: 37: 53: 03$ & 296 & 103 & 26 & -0.6 & 1.9 & 0.15 & 770 & 55.7 & 25 & -0.18 \\
\hline $12: 37: 54: 02$ & 297 & 103 & 26 & -0.6 & 1.9 & 0.15 & 765 & 55.9 & 25 & -0.18 \\
\hline $12: 37: 55: 01$ & 298 & 104 & 25 & -0.8 & 1.9 & 0.17 & 759 & 56.1 & 25 & -0.25 \\
\hline $12: 37: 56: 00$ & 299 & 105 & 26 & -0.6 & 1.9 & 0.17 & 757 & 56.1 & 24 & -0.25 \\
\hline $12: 37: 56: 99$ & 300 & 106 & 26 & -0.6 & 7.9 & 0.17 & 759 & 56.1 & 24 & -0.25 \\
\hline $12: 37: 57: 97$ & 301 & 106 & 26 & -0.6 & 1.9 & 0.17 & 764 & 55.9 & 25 & -0.25 \\
\hline $12: 37: 58: 96$ & 302 & 107 & 26 & -0.6 & 1.9 & 0.19 & 769 & 55.9 & 25 & -0.17 \\
\hline $12: 37: 59: 95$ & 303 & 108 & 26 & -0.6 & 1.9 & 0.17 & 773 & 55.9 & 24 & -0.17 \\
\hline $12: 38: 00: 94$ & 304 & 108 & 26 & $-0.6^{\prime}$ & 1.9 & 0.17 & 769 & 55.9 & 25 & 0.16 \\
\hline $12: 38: 01: 93$ & 305 & 108 & 26 & -0.6 & $1 . \overline{9}$ & 0.17 & 773 & 55.9 & 25 & 0.16 \\
\hline $12: 38: 02: 92$ & 306 & 108 & 26 & 10.9 & 55.6 & 0.17 & 774 & 55.9 & 25 & -0.32 \\
\hline $12: 38: 03: 91$ & $\overline{3} \overline{0} \overline{7}$ & 106 & 26 & $22.9^{\prime}$ & 53.6 & 0.17 & $770^{\circ}$ & 55.9 & 22 & -0.23 \\
\hline $12: 38: 04: 89$ & 308 & 103 & 26 & 23.7 & 53.2 & 0.17 & 763 & 55.9 & 20 & -0.23 \\
\hline $12: 38: 05: 88$ & 309 & 100 & 26 & 23.7 & 53 & 0.17 & 757 & 55.9 & 19 & -0.22 \\
\hline $12: 38: 06: 87$ & 310 & 99 & $\ldots$ & 23.7 & 52.9 & 0.17 & 754 & 55.9 & 18 & -0.22 \\
\hline $12: 38: 07: 86$ & $311:$ & 97 & 26 & 23.5 & 52.8 & 0.17 & 757 & 55.9 & 18 & 0.02 \\
\hline $12: 38: 08: 85$ & 312 & 95 & 26 & 23.5 & 52.7 & 0.18 & 754 & 56 & 18 & 0.02 \\
\hline $12: 38: 09: 84$ & $313^{+}$ & 94 & 26 & 23.5 & 52.7 & 0.19 & 755 & 56.1 & 17 & -0.08 \\
\hline $12: 38: 10: 83$ & 314 & 92 & 26 & 23.5 & 52.7 & 0.18 & 758 & 56.1 & 17 & -0.08 \\
\hline $12: 38: 11: 82$ & 315 & 91 & 26 & 23.3 & 52.7 & 0.18 & 764 & 56.1 & 17 & -0.24 \\
\hline $12: 38: 1280$ & 316 & 90 & 26 & 23.5 & 52.7 & 0.19 & 767 & 56.2 & 17 & -0.24 \\
\hline $12: 38: 13: 79$ & 317 & 88 & 26 & 23.7 & 52.7 & 0.18 & 768 & 56.2 & 17 & $-0 . \overline{21}$ \\
\hline $12: 38: 14: 78$ & 318 & 87 & 26 & 23.7 & $5 \overline{2.7}$ & 0.17 & 766 & 56.3 & 16 & -0.21 \\
\hline $12: 38: 15: 77$ & 319 & 86 & 26 & 23.7 & 52.6 & 0.17 & 760 & 56.2 & 16 & 0.04 \\
\hline $12: 38: 16: 76$ & 320 & 85 & 26 & 23.7 & 52.6 & 0.17 & 767 & 56.2 & 16 & 0.04 \\
\hline $12: 38: 17: 75$ & 321 & 84 & 26 & 23.7 & 52.6 & 0.17 & 761 & 56.3 & 16 & -0.09 \\
\hline $12: 38: 18: 74$ & 322 & $8 \overline{3}$ & 26 & 23.7 & 52.6 & 0.17 & 755 & 56.3 & 16 & $-\overline{0.09}$ \\
\hline $12: 38: 19: 72$ & 323 & 82 & 26 & 23.5 & 52.6 & 0.17 & 752 & 56.2 & 16 & $-0 . \overline{24}$ \\
\hline
\end{tabular}




\begin{tabular}{|c|c|c|c|c|c|c|c|c|c|c|}
\hline TIME & $\begin{array}{l}\text { Elapsed } \\
\text { Time } \\
\text { (sec) }\end{array}$ & TC1 & PURG_TC2 & PurgePSI F & PurgeCFM & DEPTH(IN) & DwnFrc(lb) & RPM & $\begin{array}{l}\text { Torque } \\
\text { (FTLB) }\end{array}$ & Inches/Min \\
\hline $12: 38: 20: 71$ & 324 & 81 & 26 & 23.5 & $\quad 52.6$ & 0.18 & 752 & 56.1 & 16 & -0.24 \\
\hline $12: 38: 21: 70$ & 325 & 80 & 26 & 23.5 & 52.6 & 0.18 & 755 & 56.1 & 16 & -0.22 \\
\hline $12: 38: 22: 69$ & 326 & $7 \overline{9}$ & 26 & 23.5 & 52.6 & 0.18 & $761^{\circ}$ & 56.1 & 16 & -0.22 \\
\hline $12: 38: 2368$ & 327 & 78 & 26 & 23.5 & 52.6 & $0 . \overline{18}$ & 765 & 56 & 16 & -0.22 \\
\hline $12: 38: 24: 67$ & 328 & 77 & 26 & 23.5 & 52.6 & 0.19 & 768 & 56 & 16 & -0.22 \\
\hline $12: 38: 25: 66$ & 329 & $7 \overline{7}$ & 26 & 23.9 & 52.6 & 0.17 & 764 & 56 & 16 & -0.04 \\
\hline $12: 38: 26: 64$ & 330 & 76 & 26 & 23.9 & 52.6 & 0.17 & $767^{\circ}$ & 55.9 & 16 & -0.04 \\
\hline $12: 38: 27: 63$ & $331^{\dagger}$ & 75 & 26 & 23.9 & 52.6 & 0.17 & 766 & 55.9 & 16 & -0.03 \\
\hline $12: 38: 28: 62$ & 332 & 74 & 26 & 23.9 & 52.6 & 0.17 & 760 & 55.9 & 15 & -0.03 \\
\hline $12: 38: 29: 61$ & 333 & 73 & 26 & 24.1 & 52.6 & 0.17 & 754 & 55.9 & 15 & -0.31 \\
\hline $12: 38: 30: 60$ & 334 & 73 & 26 & 24.1 & 52.6 & 0.18 & 751 & 55.9 & 15 & -0.31 \\
\hline $12: 38: 31: 59$ & 335 & $73^{\dagger}$ & 26 & 23.7 & 52.6 & 0.18 & 752 & 55.8 & 16 & -0.27 \\
\hline $12: 38: 32: 58$ & 336 & 72 & 26 & 23.9 & 52.6 & 0.19 & 755 & 55.7 & 16 & -0.27 \\
\hline $12: 38: 33: 57$ & 337 & 71 & 26 & 23.9 & 52.6 & 0.19 & 753 & 55.7 & 16 & 0.08 \\
\hline $12: 38: 34: 55$ & 338 & 71 & 26 & 23.9 & 52.6 & 0.19 & $757^{\circ}$ & 55.5 & 16 & 0.08 \\
\hline $12: 38: 35: 54$ & 339 & 70 & 26 & 23.9 & 52.6 & $0 . \overline{9}$ & 762 & 55.4 & 16 & -0.13 \\
\hline $12: 38: 36: 53$ & 340 & 69 & 26 & 23.9 & 52.6 & 0.19 & 767 & 55.4 & 16 & -0.13 \\
\hline $12: 38: 37: 52$ & 341 & 69 & 26 & 24.1 & 52.6 & 0.18 & 767 & 55.6 & 15 & -0.35 \\
\hline $12: 38: 38: 51$ & $3 \overline{42}$ & 68 & 26 & 24.1 & 52.6 & 0.17 & 764 & 55.6 & 15 & $-0.2 \overline{7}$ \\
\hline $12: 38: 3950$ & $3 \overline{43}$ & 68 & 26 & 24.1 & 52.6 & 0.19 & 758 & 55.6 & 15 & -0.27 \\
\hline $12: 38: 40: 49$ & 344 & 68 & 26 & 24.1 & 52.6 & $0 . \overline{17}$ & 752 & 55.6 & 15 & -0.16 \\
\hline $12: 38: 41: 47$ & 345 & 67 & 26 & 24.1 & 52.6 & 0.18 & 758 & 55.6 & 15 & -0.16 \\
\hline $12: 38: 42: 46$ & 346 & 67 & 26 & 24.1 & 52.6 & 0.17 & 752 & 55.6 & 15 & -0.03 \\
\hline $12: 38: 43: 45$ & 347 & 67 & 26 & 23.9 & 52.6 & 0.19 & 752 & 55.6 & 16 & -0.03 \\
\hline $12: 38: 44: 44$ & 348 & 66 & 26 & 24.1 & 52.6 & 0.19 & $757 !$ & 55.5 & 16 & -0.16 \\
\hline $12: 38: 45: 43$ & $3 \overline{49}$ & 66 & 26 & $24.1_{\mathrm{i}}^{\circ}$ & 52.6 & 0.19 & 762 & $55 . \overline{5}$ & 16 & -0.16 \\
\hline $12: 38: 46: 42$ & 350 & 65 & 26 & 24.1 & 52.6 & 0.19 & 767 & 55.3 & 16 & -0.29 \\
\hline $12: 38: 47: 41$ & 351 & 65 & 26 & $24.1 j$ & $52.6^{\dagger}$ & 0.19 & 769 & 55.7 & 16 & -0.29 \\
\hline $12: 38: 48: 40$ & 352 & $65 !$ & 26 & $24.1 \dagger$ & 52.6 & 0.18 & 767 & 55.7 & 16 & -0.3 \\
\hline $12: 38: 49: 38$ & 353 & 64 & 26 & 24.3 & 52.6 & 0.18 & 763 & 55.9 & 15 & -0.3 \\
\hline $12: 3 \overline{8}: 5 \overline{0}: 3 \overline{7}$ & 354 & 64 & 26 & $24.3^{\prime}$ & 52.6 & $0 . \overline{19}$ & 768 & 56.1 & 15 & 0.01 \\
\hline $12: 38: 51: 36$ & 355 & 64 & 26 & 24.3 & 52.6 & 0.17 & 764 & $56 . \overline{1}$ & 15 & 0.01 \\
\hline $12: 38: 52: 35$ & 356 & 64 & 26 & 24.3 & 52.6 & 0.15 & 758 & 56.3 & 15 & 0.01 \\
\hline $12: 38: 53: 34$ & 357 & 64 & 26 & 24.3 & 52.6 & 0.19 & 754 & 56.3 & 16 & 0.01 \\
\hline $12: 38: 54: 33$ & 358 & 63 & 26 & $24.3^{t}$ & 52.6 & 0.19 & 752 & 56.3 & 16 & -0.32 \\
\hline $12: 38: 55: 32$ & 359 & 63 & 26 & 24.1 & 52.6 & 0.17 & $755^{\circ}$ & 56.1 & 16 & -0.32 \\
\hline $12: 38: 56: 30$ & 360 & $6 \overline{3}$ & 26 & 24.1 & 52.6 & 0.19 & 759 & 56 & 16 & -0.17 \\
\hline $12: 38: 57: 29$ & 361 & 62 & 26 & 24.1 & 52.6 & 0.19 & 764 & 56 & 16 & -0.17 \\
\hline $12: 38: 58: 28$ & 362 & 62 & 26 & 24.1 & 52.6 & 0.19 & $759 !$ & 56.1 & 16 & -0.17 \\
\hline $12: 38: 59: 27$ & 363 & 62 & 26 & 24.1 & 52.6 & 0.19 & 764 & 56.1 & 16 & -0.17 \\
\hline $12: 39: 00: 26$ & $3 \overline{64}$ & 62 & 26 & 24.1 & 52.6 & 0.19 & 768 & 56.1 & 16 & -0.02 \\
\hline $12: 39: 01: 25$ & 365 & 61 & 26 & 24.3 & 52.6 & 0.17 & 768 & 56.1 & 16 & -0.02 \\
\hline $12: 39: 02: 24$ & 366 & 61 & 26 & 24.3 & 52.6 & 0.19 & 765 & 56.1 & 16 & -0.18 \\
\hline $12: 39: 03: 23$ & 367 & 61 & 26 & 24.3 & 52.6 & 0.17 & 760 & 56.2 & 16 & -0.18 \\
\hline $12: 39: 04: 21$ & 368 & 61 & 26 & 24.3 & 52.6 & 0.17 & 754 & 56.2 & 16 & -0.21 \\
\hline $12: 39: 05: 20$ & 369 & 61 & 26 & 24.3 & 52.6 & 0.17 & 753 & 56.2 & $16 !$ & -0.21 \\
\hline $12: 39: 06: 19$ & 370 & 61 & 26 & 24.1 & 52.6 & 0.19 & 755 & 56 & 16 & -0.25 \\
\hline $12: 39: 07: 18$ & 371 & 61 & 26 & 23.9 & 52.6 & 0.19 & 752 & 56 & 16 & -0.25 \\
\hline $12: 39: 08: 17$ & 372 & 60 & 26 & 23.9 & 52.6 & 0.19 & 754 & 56 & 16. & -0.25 \\
\hline $12: 39: 09: 16_{\mathrm{j}}$ & 373 & 60 & 26 & 23.9 & 52.6 & $0 . \overline{17}$ & 757 & 56 & 16 & -0.25 \\
\hline $12: 39: 10: 15$ & 374 & 60 & 26 & 23.9 & 52.6 & 0.18 & 764 & 55.9 & 16 & 0.14 \\
\hline $12: 39: 11: 13$ & 375 & 60 & 26 & 23.9 & 52.6 & 0.19 & 767 & 55.9 & 16 & -0.21 \\
\hline $12: 39: 12: 12$ & 376 & 60 & 26 & 24.1 & 52.6 & 0.18 & 769 & 56.1 & 16 & -0.21 \\
\hline $12: 39: 13: 11$ & 377 & 59 & 26 & 24.1 & 52.6 & $0.18 \div$ & 767 & $56.1_{1}$ & 16 & -0.39 \\
\hline
\end{tabular}




\begin{tabular}{|c|c|c|c|c|c|c|c|c|c|c|}
\hline \multirow[b]{2}{*}{ TIME } & \multirow{2}{*}{$\begin{array}{l}\text { Elapsed } \\
\text { Time } \\
\text { (sec) }\end{array}$} & \multirow[b]{2}{*}{ BIT_TC1 } & \multirow{2}{*}{ PURG TC2 } & \multirow{2}{*}{ PurgePSi } & \multirow{2}{*}{ PurgeCFM } & \multirow{2}{*}{ DEPTH(IN) } & \multirow{2}{*}{ DwnFrc(lb) } & \multirow{2}{*}{ RPM } & \multirow{2}{*}{$\begin{array}{l}\text { Torque } \\
\text { (FTLB) }\end{array}$} & \multirow{2}{*}{ Inches/Min } \\
\hline & & & & & & & & & & \\
\hline $12: 39: 14: 10$ & 378 & 59 & $\quad 26$ & 24.1 & 52.6 & 0.17 & $\quad 760$ & 56.1 & 16 & $\begin{array}{r}-0.39 \\
\end{array}$ \\
\hline $12: 39: 15: 09$ & 379 & 59 & 26 & 24.1 & 52.6 & $0.19 !$ & 755 & 56.1 & 16 & -0.26 \\
\hline $12: 39: 16: 08$ & 380 & 59 & 26 & 24.1 & 52.6 & 0.17 & 760 & 56 & 16 & -0.26 \\
\hline $12: 39: 17: 07$ & 381 & $60^{\dagger}$ & 26 & 24.1 & 52.6 & 0.17 & $755^{\dagger}$ & 56 & 16 & 0.11 \\
\hline $12: 39: 18: 06$ & 382 & 59 & 26 & 23.7 & 52.6 & 0.17 & 753 & 56 & 16 & 0.11 \\
\hline $12: 39: 19: 04$ & 383 & 59 & 26 & 23.9 & 52.6 & 0.19 & 754 & 56.1 & 16 & -0.2 \\
\hline $12: 39: 20: 03$ & 384 & 59 & 26 & 23.9 & 52.6 & 0.19 & 757 & 56.1 & 16 & -0.2 \\
\hline $12: 39: 21: 02$ & 385 & 59 & 26 & 23.7 & 52.6 & 0.19 & 762 & 56.1 & 16 & -0.26 \\
\hline $12: 39: 22: 01$ & 386 & 59 & $26^{\circ}$ & 23.9 & 52.6 & 0.19 & 767 & 55.8 & 16 & -0.26 \\
\hline $12: 39: 23: 00$ & 387 & 58 & 26 & 23.7 & $52.6^{-}$ & 0.18 & 769 & 55.8 & $16 !$ & -0.27 \\
\hline $12: 39: 23: 99$ & 388 & 58 & 26 & 24.1 & 52.6 & 0.18 & 767 & 55.9 & 16 & -0.27 \\
\hline $12: 39: 24: 98$ & 389 & 58 & 26 & 24.1 & 52.6 & 0.18 & 769 & 55.9 & 16 & -0.14 \\
\hline $12: 39: 25: 96$ & 390 & 58 & 26 & 24.1 & 52.6 & 0.17 & 769 & 55.9 & 16 & -0.14 \\
\hline & & & & & - & & & & & \\
\hline
\end{tabular}




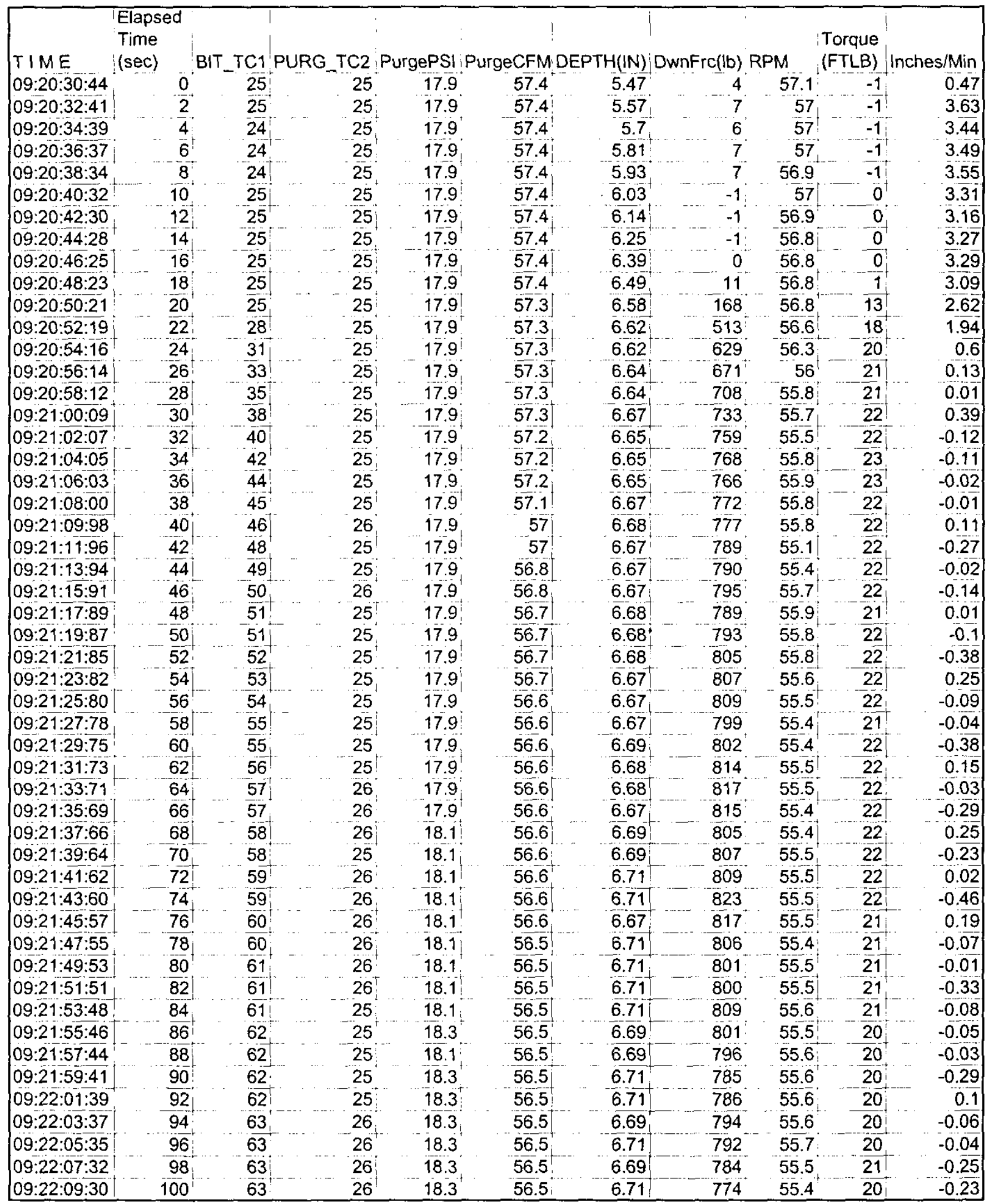




\begin{tabular}{|c|c|c|c|c|c|c|c|c|c|c|}
\hline$T I M E$ & $\begin{array}{l}\text { Elapsed } \\
\text { Time } \\
\text { (sec) }\end{array}$ & $B I T \_T C 1$ & PURG_TC2 & PurgePSI: & PurgeCFM & DEPTH(IN) & DwnFrc $(\mid \mathrm{b})$ & RPM & $\begin{array}{l}\text { Torque } \\
\text { (FTLB) }\end{array}$ & Inches/Min \\
\hline $09: 22: 1 \overline{1: 28}$ & 102 & 63 & 26 & 18.3 & $\quad 56.5$ & 6.71 & $\quad 776$ & 55.3 & 320 & $\quad 0.07$ \\
\hline $09: 22: 13: 26$ & 104 & 63 & 26 & 18.3 & 56.5 & 6.71 & 786 & 55.2 & 20 & -0.16 \\
\hline $09: 22: 15: 23$ & 106 & 64 & 26 & $18 . \overline{3}$ & 56.5 & 6.69 & 784 & 55.1 & 20 & -0.2 \\
\hline $09: 22: 17: 21$ & $10 \overline{8}$ & $64^{\circ}$ & 26 & 18.3 & 56.5 & 6.69 & 773 & 55.3 & 20 & -0.14 \\
\hline $22: 19: 19$ & 110 & 64 & 26 & 18.1 & 56.5 & 6.71 & $765^{\prime}$ & 55.6 & $20^{\circ}$ & 0.02 \\
\hline :16 & 112 & 64 & 25 & $18.1^{\dagger}$ & 56.5 & $6.71^{\dagger}$ & 770 & 55.8 & 20 & -0.14 \\
\hline $09: 22: 23: 14$ & 114 & 65 & 26 & 18.1 & 56.5 & 6.71 & 771 & 55.9 & 20 & -0.18 \\
\hline$: 12$ & 116 & 65 & 26 & 18.1. & $56 . \overline{5}$ & 6.71 & 780 & 55.7 & 20 & $-0 . \overline{2}$ \\
\hline $7: \overline{10}$ & 118 & 65 & 26 & $18.1]$ & $56.5^{\dagger}$ & 6.68 & 772 & 55.5 & 20 & 0.09 \\
\hline 07 & 120 & 65 & 26 & 18.1 & 56.5 & 6.71 & 765 & 55.6 & 19 & -0.12 \\
\hline 05 & $1 \overline{22}$ & 65 & 26 & 18.1 & 56.5 & 6.71 & 765 & 55.8 & 19 & -0.24 \\
\hline 03 & 124 & 65 & 26 & $18.1^{\prime}$ & 56.5 & 6.71 & 770 & 55.7 & 20 & $-0.2 \overline{1}$ \\
\hline 01 & 126 & 66 & 26 & $18 . \overline{1}$ & 56.5 & 6.71 & 777 & 55.6 & 20 & 0.11 \\
\hline 98 & 128 & 66 & 26 & 18.1 & 56.5 & 6.71 & 768 & 55.5 & 19 & $-0 . \overline{06}$ \\
\hline 96 & 130 & 66 & 26 & 18.1 & 56.5 & $6 . \overline{71}$ & 767 & 55.6 & 19 & -0.18 \\
\hline 94 & $\overline{132}$ & 66 & 26 & 18.1 & 56.5 & $6.7 \overrightarrow{1}$ & 760 & 55.7 & 19 & -0.3 \\
\hline 92 & 134 & 66 & 26 & 18.1 & 56.5 & 6.72 & 764 & 55.6 & 19 & $0 . \overline{03}$ \\
\hline 89 & 136 & 66 & 26 & $18.1_{\mathrm{j}}^{+}$ & 56.5 & 6.71 & 774 & 55.4 & 19 & $-0.2 \varepsilon$ \\
\hline 87 & 138 & $6 \overline{1}$ & 26 & 18.1 & 56.5 & 6.69 & 773 & 55.1 & 19 & -0.18 \\
\hline & 140 & $6 \overline{6}$ & 26 & 18.1 & 56.5 & 6.69 & 763 & 55.1 & 19 & 0.07 \\
\hline 82 & 142 & 66 & 26 & 18.1 & 56.5 & 6.71 & 758 & 55.3 & 18 & -0.07 \\
\hline & 144 & 66 & 26 & 18.1 & 56.5 & 6.71 & 767 & 55.5 & 18 & -0.25 \\
\hline & $1 \overline{46}$ & 66 & 26 & 17.9 & & 6.72 & 768 & 55.5 & 19 & $-0.2 \overline{4}$ \\
\hline & 148 & 67 & 26 & 18.1 & 56.5 & $6 . \overline{71}$ & 773 & 55.5 & 19 & -0.07 \\
\hline & 150 & 67 & 26 & 18.1 & 56.5 & 6.71 & 763 & 55.6 & 19 & 0.08 \\
\hline & 152 & 66 & 26 & 18.1 & & 6.71 & 757 & 55.7 & 19 & -0.35 \\
\hline & 154 & 67 & 26 & 18.1 & 0.5 & 6.71 & 764 & 55.7 & 19 & -0.16 \\
\hline & 156 & 67 & 26 & & & 6.71 & 765 & 55.8 & 19 & -0.04 \\
\hline & 158 & 67 & 26 & 18.1 & 56.5 & 6.71 & 771 & 55.8 & 19 & \\
\hline & 160 & 67 & 26 & 18.1 & 0.5 & 6.71 & 763 & 55.9 & 18 & -0.15 \\
\hline & $1 \overline{62}$ & 67 & 26 & 18.1 & 56.5 & 6.71 & 757 & 56.1 & 18 & -0.37 \\
\hline & 164 & 67 & 26 & 18.1 & 56.5 & 6.71 & $75 \overline{7}$ & 56.2 & 18 & -0.0 \\
\hline & 166 & $\overline{67}$ & 26 & 17.9 & 56.5 & 6.71 & 760 & 56.2 & $1 \overrightarrow{8}$ & 0.09 \\
\hline $5 \overline{3}$ & 168 & 67 & 26 & 17.9 & & 6.72 & $7 \overline{71}$ & 56.1 & 18 & $-0 . \overline{21}$ \\
\hline & 170 & 68 & 26 & 17.9 & 5 & 6.71 & 769 & 56.2 & 18 & -0.2 \\
\hline & 172 & 68 & 26 & & & 6. & 768 & 56.1 & 19 & $-0 . \overline{16}$ \\
\hline & 174 & 67 & 26 & 17.9 & & 6.7 & 758 & 55.9 & 17 & -0.13 \\
\hline & 176 & 68 & 26 & $17.9^{\circ}$ & & 6.72 & 759 & 55.9 & 18 & 0.05 \\
\hline & 178 & 68 & 26 & 17.9 & 56.5 & 6.71 & 770 & 55.8 & 18 & -0.35 \\
\hline $8: 39$ & 180 & 68 & 26 & 17.9 & 56.5 & 6.71 & 771 & 55.7 & 18 & $-0 . \overline{15}$ \\
\hline $3: 30: 37$ & 182 & 68 & 26 & 17.9 & 56.5 & 6.71 & 771 & 55.7 & 19 & 0.15 \\
\hline 35 & 184 & 68 & 26 & 17.9 & 56.5 & 6.71 & 760 & 55.6 & 18 & -0.14 \\
\hline$: 33$ & 186 & 68 & 26 & 18.1 & 56.5 & 6.72 & 762 & 55.7 & 18 & -0.16 \\
\hline & 188 & $6 \overline{8}$ & 26 & 18.1 & 56.5 & $\overline{6.71}$ & 761 & 55.9 & 18 & -0.3 \\
\hline & 190 & 68 & 26 & 18.1 & 56.5 & $6 . \overline{72}$ & 771 & $55 . \overline{7}$ & 18 & 0.05 \\
\hline $5: 26$ & 192 & 68 & 26 & $17 \overline{9}$ & 56.5 & 6.72 & 771 & 55.5 & 18 & -0.05 \\
\hline & 194 & 68 & 26 & 17.9 & 56.5 & 6.71 & 760 & $55 . \overline{5}$ & $18^{4}$ & $-0 . \overline{04}$ \\
\hline & 196 & 68 & 26 & 17.9 & 56.5 & 6.71 & 759 & 55.5 & 18 & $-0 . \overline{41}$ \\
\hline & 198 & 68 & 26 & 17.9 & 56.5 & 6.71 & 760 & 55.5 & 18 & $0 . \overline{07}$ \\
\hline $09: 23: 48: 17$ & 200 & 68 & $2 \overline{6}$ & 17.9 & 56.5 & 6.71 & 769 & 55.5 & 18 & -0.1 \\
\hline $09: 23: 50: 14$ & 202 & 68 & 26 & 17.9 & 56.5 & 6.71 & 767 & $55 . \overline{4}$ & 18 & $-0 . \overline{3 S}$ \\
\hline
\end{tabular}




\begin{tabular}{|c|c|c|c|c|c|c|c|c|c|c|}
\hline TIME & $\begin{array}{l}\text { Elapsed } \\
\text { Time } \\
\text { (sec) }\end{array}$ & BIT_TCll & PURG TC2 & PurgePSI & PurgeCFM & DEPTH(IN) & ¡DwnFrc(lb) & RPM & $\begin{array}{l}\text { Torque } \\
\text { (FTLB) }\end{array}$ & Inches/Min \\
\hline $52: 12$ & 204 & $\quad 68$ & 26 & $17.9^{\prime}$ & $\quad 56.5$ & 6.71 & $\quad \frac{755}{75}$ & 55.4 & 18 & $\quad 0.03$ \\
\hline $4: 10$ & 206 & 68 & 26 & $17.9^{\prime}$ & 56.5 & 6.71 & 755 & 55.3 & 18 & -0.05 \\
\hline & 208 & 68 & 26 & 17.9 & 56.5 & 6.72 & 758 & 55.3 & 18 & -0.09 \\
\hline 05 & 210 & 68 & 26 & 18.1 & 56.5 & $6.71^{1}$ & 770 & 55.2 & 18 & -0.33 \\
\hline & 212 & 68 & 26 & 18.1 & 56.5 & 6.71 & 763 & 55.2 & 18 & 0.01 \\
\hline & 214 & 68 & 26 & $17 . \overline{9}^{\prime}$ & 56.5 & $6 . \overline{71}$ & 762 & 55.5 & 18 & -0.06 \\
\hline & 216 & 68 & 26 & 17.9 & 56.5 & 6.71 & $75 \overline{4}$ & 55.6 & 18 & -0.03 \\
\hline & 218 & 68 & 26 & 17.9 & 56.5 & 6.72 & 759 & 55.5 & 18 & -0.38 \\
\hline & 220 & 68 & 26 & 18.1 & 56.5 & 6.71 & 769 & 55.5 & 18 & 0.18 \\
\hline 09: & 222 & 68 & 26 & 17.9 & 56.5 & 6.71 & 769 & $5 \overline{5.5}$ & 18 & -0.25 \\
\hline & 224 & 69 & 26 & 18.1 & 56.5 & 6.71 & 761 & 55.6 & 18 & -0.03 \\
\hline & 226 & 68 & 26 & 17.9 & 56.5 & 6.71 & 754 & 55.7 & 18 & $-0 . \overline{34}$ \\
\hline & 228 & 68 & 26 & 17.9 & 56.5 & 6.71 & 758 & 55.7 & 18 & -0.04 \\
\hline & 230 & 69 & 26 & 17.9 & $5 \overline{6.5}$ & 6.72 & 758 & 55.8 & 18 & -0.02 \\
\hline & 232 & 69 & 26 & $17.9 !$ & 56.5 & 71 & 769 & 55.7 & 18 & -0.07 \\
\hline & 234 & 69 & 26 & 18.1 & 56.5 & 6.71 & 762 & 55.7 & 18 & -0.25 \\
\hline & 236 & 69 & 26 & 17.9 & 56.5 & $6.71 !$ & 754 & 55.8 & 18 & -0.26 \\
\hline & 238 & 68 & 26 & 17.9 & 56.5 & 6.72 & 755 & 55.9 & 17 & 0.14 \\
\hline $7: 71$ & 240 & 69 & 26 & 17.9 & 56.5 & 6.72 & 758 & 55.9 & 17 & -0.17 \\
\hline $09: 24$ & 242 & 69 & 26 & $17.9^{\dagger}$ & 56.5 & 6.72 & 767 & 56 & $1 \overline{8}$ & -0.16 \\
\hline $09: 24: 31: 67$ & 244 & 69 & 26 & 17.9 & 56.5 & 6.72 & 765 & 55.9 & 18 & -0.16 \\
\hline & & & $\ldots$ & $\cdots$ & & & & & & $-\cdots-$ \\
\hline
\end{tabular}




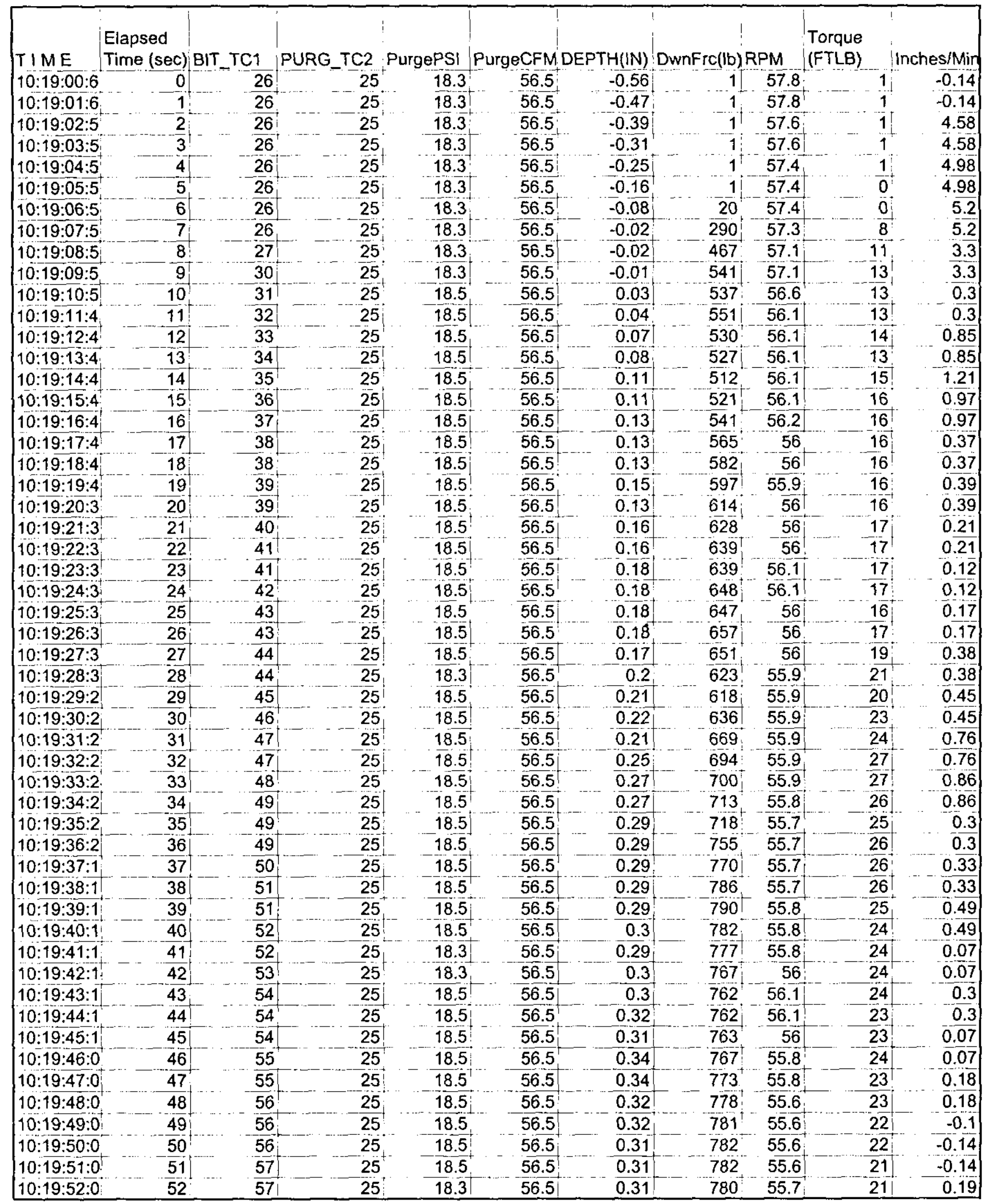




\begin{tabular}{|c|c|c|c|c|c|c|c|c|c|c|}
\hline$T I M E$ & $\begin{array}{l}\text { Elapsed } \\
\text { Time (sec) }\end{array}$ & BIT_TC1 & PURG TC2 & PurgePSI & PurgeCFM & DEPTH(IN) & DwnFic(lb) & & $\begin{array}{l}\text { Torque } \\
\text { (FTLB) }\end{array}$ & inches/Mir \\
\hline $10: 19: 53: 0$ & $\begin{array}{l}53 \\
\end{array}$ & 58 & 25 & $\quad 18.3$ & $\begin{array}{r}56.5 \\
\end{array}$ & 0.34 & $\quad 781$ & 55.7 & $\quad 22$ & $\quad 0.19$ \\
\hline $10: 19: 54: 0$ & 54 & 58 & 25 & 18.3 & 56.5 & 0.34 & $782^{\circ}$ & 55.8 & 22 & 0.01 \\
\hline $10: 19: 54: 9$ & 55 & 58 & 25 & 18.5 & 56.5 & 0.32 & 782 & 55.7 & 22 & 0.01 \\
\hline $10: 19: 55: 9$ & 56 & 59 & 25 & 18.5 & 56.5 & 0.34 & $783^{\circ}$ & 55.7 & 21 & 0.08 \\
\hline $56: 9$ & 57 & 59 & 25 & 18.3 & 56.5 & 0.34 & $787^{\circ}$ & 55.8 & 21 & 0.08 \\
\hline $10: 1$ & 58 & 59 & 25 & 18.3 & 56.5 & 0.34 & $791^{\circ}$ & 56 & 20 & -0.14 \\
\hline $10: 19: 58: 9$ & 59 & 60 & 25 & 18.5 & 56.5 & 0.34 & $793^{\circ}$ & 56 & 20 & -0.14 \\
\hline $10: 19: 59: 9$ & 60 & $60^{1}$ & 25 & 18.5 & 56.5 & 0.32 & 794 & 56.2 & 20 & -0.27 \\
\hline $00: 9$ & 61 & 61 & 25 & 18.5 & 56.5 & 0.32 & 792 & 56.2 & 20 & .0 .27 \\
\hline $1: 9$ & 62 & 61 & 25 & 18.5 & 56.5 & 0.34 & $795^{\circ}$ & 56.2 & 20 & $0 . \overline{17}$ \\
\hline $02: 9$ & 63 & 61 & 25 & 18.5 & 56.5 & 0.34 & 791 & 56.2 & 20 & 0.17 \\
\hline $10: 20: 03: 8$ & $64^{\dagger}$ & 62 & 25 & 18.5 & 56.5 & 0.35 & 785 & 56.3 & 20 & 0 \\
\hline $04: 8$ & 65 & 62 & 25 & 18.3 & 56.5 & 0.35 & 784 & $5 \overline{6} .3$ & 20 & \\
\hline $5: \overline{8}$ & 66 & $6 \overline{2}$ & 25 & 18.3 & & 34 & $780^{\circ}$ & 56.2 & 20 & -0.25 \\
\hline $66: 8$ & 67 & 62 & 25 & 18.3 & 56.5 & 0.34 & $779^{\circ}$ & 56.1 & 20 & -0.25 \\
\hline $10: 20: 07: 8$ & 68 & 62 & 25 & 18.3 & 56.5 & 0.35 & 782 & 56.1 & 20 & 0.04 \\
\hline $10: 20: 08: 8$ & 69 & 62 & 25 & 18.3 & 56.5 & 0.35 & $780^{\circ}$ & 55.9 & 20 & 0.04 \\
\hline $9: 8$ & 70 & 63 & 25 & 18.5 & 56.5 & 0.36 & 775 & 55.8 & 19 & $-0 . \overline{28}$ \\
\hline $0: 8$ & 71 & $63^{\dagger}$ & 25 & 18.5 & 56.5 & 0.34 & $775^{\circ}$ & 55.8 & 19 & -0.28 \\
\hline $10: 20: 11: 8$ & 72 & 63 & 25 & 18.5 & 56.5 & 0.34 & 773 & 55.8 & 19 & 0.07 \\
\hline $12: 7$ & 73 & 64 & 25 & 18.5 & 56.5 & 0.34 & 771 & 55.8 & $1 \overrightarrow{9}$ & 0.07 \\
\hline $3: 7$ & 74 & 64 & 25 & 18.5 & 56.5 & 0.34 & 767. & $5 \overline{5.8}$ & 19 & -0.26 \\
\hline & 75 & $64^{\circ}$ & 25 & 18.5 & & 0.35 & $763^{\circ}$ & 55.9 & 19 & -0.26 \\
\hline $10: 20: 15: 7$ & 76 & 64 & 25 & 18.5 & 56.5 & 0.35 & 762 & 55.9 & 18 & -0.4 \\
\hline $10: 20: 16: 7$ & 77 & 64 & 25 & 18.5 & 56.5 & 0.35 & 763 & 55.9 & 19 & -0.4 \\
\hline $7: 7$ & 78 & 64 & 25 & 18.5 & 56.5 & 0.35 & 766 & 55.8 & 19 & 0.2 \\
\hline $18: 7$ & 79 & 64 & 25 & 18.5 & 56.5 & 0.36 & 766 & 55.5 & 19 & 0.2 \\
\hline $199: 7$ & 80 & $6 \overline{4}$ & 25 & 18.5 & 56.5 & 0.35 & 770 & 55.5 & 19 & -0.23 \\
\hline $10: 2 \overline{0}: 2 \overrightarrow{0: 6}$ & 81 & 64 & 25 & 18.5 & 56.5 & 0.35 & 772 & 55.5 & 19 & -0.23 \\
\hline $1: 6$ & 82 & 64 & 25 & 18.3 & & 0.35 & 771 & 55.5 & 19 & -0.02 \\
\hline $2: 6$ & 83 & 65 & 25 & 18.3 & 56.5 & 0.35 & $770^{\circ}$ & 55.5 & 19 & -0.42 \\
\hline $23: 6$ & $8 \overline{4} !$ & 65 & 25 & 18.3 & 56.5 & 0.36 & 766 & 55.4 & 19 & -0.42 \\
\hline $0: \overline{24: 6}$ & 85 & 65 & 25 & 18.3 & 56.5 & 0.35 & 763 & 55.4 & 18 & \\
\hline $5: 6$ & 86 & 65 & 25 & 18 & & & & 55.4 & 18 & \\
\hline $26: 6$ & 87 & 66 & 25 & 18.3 & $56 . \overline{5}$ & 0.35 & $763^{\circ}$ & 55.4 & 19 & -0.15 \\
\hline $27: 6$ & 88 & 66 & 25 & 18.3 & $56 . \overline{5}$ & 0.3 & 764 & 55.4 & 18 & -0.15 \\
\hline $20: 28: 6$ & 89 & 66 & 25 & 18.3 & 56.5 & 0.36 & 768 & 55.3 & 19 & $-0.0 \overline{6}$ \\
\hline $10: 20: 29: 5$ & 90 & 66 & 25 & 18.3 & 56 & 0.35 & $772^{\circ}$ & 55.3 & $1 \overline{9}$ & -0.06 \\
\hline $30: 5$ & 91 & 66 & 25 & 18 & 56 & & 773 & 55.3 & 19 & -0.2 \\
\hline $31: 5$ & 92 & 66 & 25 & 18.3 & 56.5 & 0.35 & 773 & 55.4 & 19 & -0.2 \\
\hline $32: 5$ & 93 & 66 & 25 & 18.3 & 56.5 & 0.35 & 772 & $5 \overline{5.6}$ & 19 & $-0 . \overline{06}$ \\
\hline $33: 5$ & 94 & 67 & 25 & 18.3 & 56.5 & 0.35 & $770^{\circ}$ & 55.6 & 19 & -0.06 \\
\hline $34: 5$ & 95 & 67 & 25 & 18.3 & & $0 . \overline{3}$ & $772^{\circ}$ & 55.8 & 18 & \\
\hline $5: 5$ & 96 & 67 & 25 & 18.3 & & $0 . \overline{3}$ & 77 & 55.8 & 19 & \\
\hline $36: 5$ & 97 & 67 & 25 & 18.3 & 56.5 & 0.36 & 766 & 55.8 & 18 & 0.01 \\
\hline $10: 20: 37: 5$ & 98 & 67 & 25 & 18.5 & 56.5 & 0.36 & 765 & 55.8 & 18 & 0.01 \\
\hline $10: 20: 38: 4$ & $\overline{99}$ & 67 & 25 & 18.3 & 56.5 & 0.36 & 769 & 55.7 & 18 & -0.41 \\
\hline $39: 4^{\top}$ & 100 & 67 & 25 & 18.5 & 56.5 & 0.36 & 772 & 55.7 & 18 & -0.41 \\
\hline$: 40: 4$ & 101 & 67 & 25 & 18.5 & 56.5 & $0.3 \overline{6}$ & 772 & 55.6 & 18 & -0.14 \\
\hline $0: 41: 4$ & 102 & 67 & 25 & 18.3 & & 0.36 & $770^{\circ}$ & 55.6 & 18 & -0.14 \\
\hline $42: 4$ & 103 & 67 & 25 & 18.3 & 56.5 & 0.36 & 768 & 55.6 & 18 & -0.17 \\
\hline $10: 20: 43: 4$ & 104 & 67 & 25 & 18.3 & 56.5 & 0.35 & 768 & 55.5 & 18 & -0.17 \\
\hline $10: 20: 44: 4$ & 105 & $6 \overline{7}$ & 25 & 18.3 & 56.5 & 0.34 & 766 & 55.7 & 18 & -0.17 \\
\hline
\end{tabular}




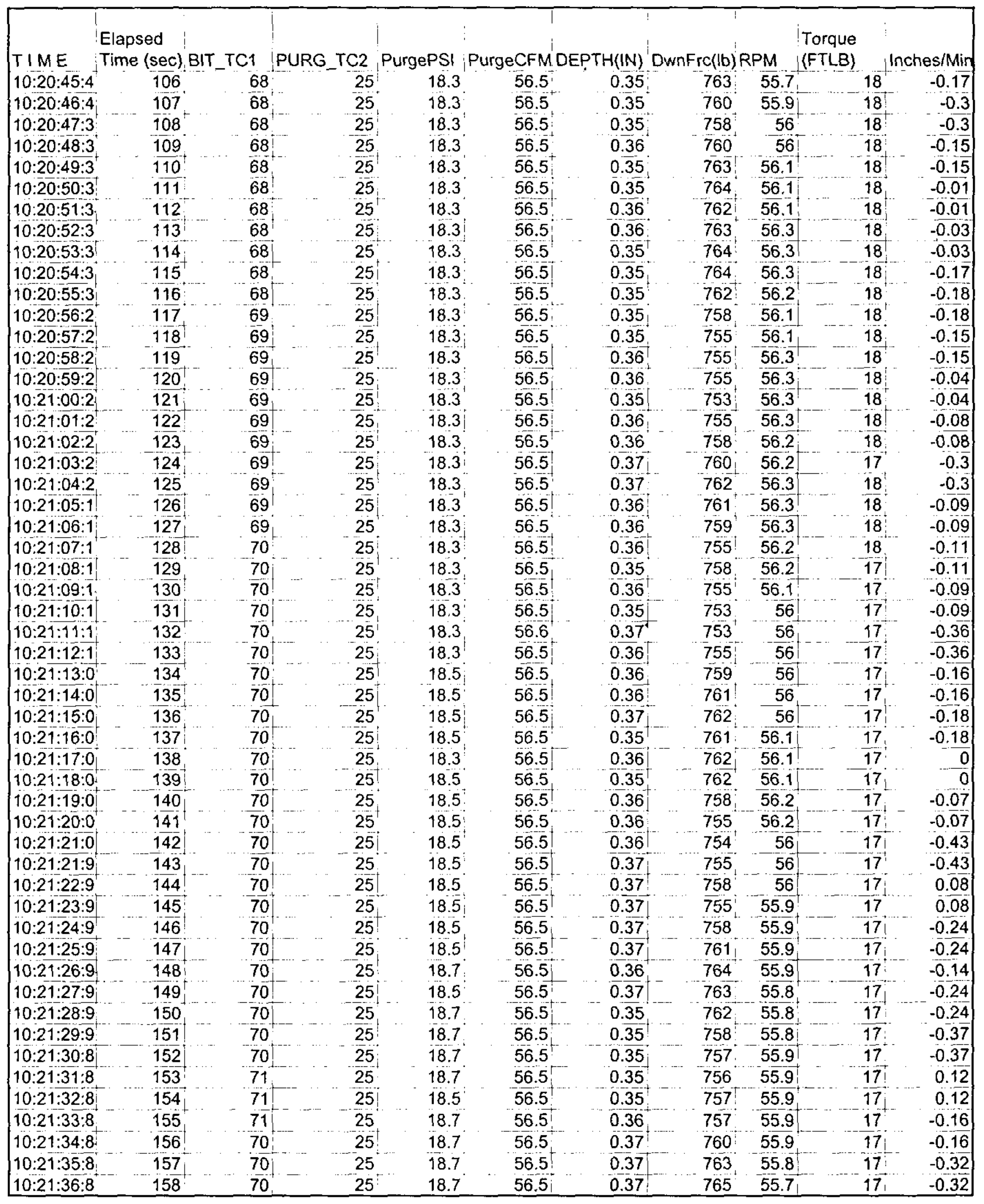




\begin{tabular}{|c|c|c|c|c|c|c|c|c|c|c|c|}
\hline$T / M E$ & $\begin{array}{l}\text { Elapsed } \\
\text { Time }(\mathrm{sec})\end{array}$ & BIT_TC1 & PURG_TC2! & PurgePS! & PurgeCFM & DEPTH $(\mathbb{I N})$ & DwnFrc(lb) & RPM & $\begin{array}{l}\text { Torque } \\
\text { (FTLB) }\end{array}$ & & Inches/Min \\
\hline $10: 21: 37: 8$ & $159^{\prime}$ & $\quad 70$ & 25 & 18.7 & 56.5 & 0.36 & $\quad 765$ & 55.7 & & 17 & -0.18 \\
\hline $10: 21: 38: 8$ & 160 & 70 & 25 & 18.7 & 56.5 & 0.36 & $763^{\circ}$ & 55.6 & & $17^{\circ}$ & -0.18 \\
\hline $10: 21: 39: 7$ & 161 & 70 & 25 & 18.7 & 56.6 & 0.36 & $760^{\circ}$ & $55 . \overline{5}$ & & 17 & -0.11 \\
\hline $10: 21: 40: 7$ & $16 \overline{2}$ & 71 & 25 & 18.7 & 56.6 & 0.35 & 762 & 55.5 & & 17 & $-0 . \overline{11}$ \\
\hline $10: 21: 41: 7$ & $163^{-1}$ & 71 & 25 & 18.7 & 56.6 & 0.37 & 759 & 55.4 & & 17 & -0.18 \\
\hline $10: 21: 42: 7$ & 164 & 71 & 25 & 18.9 & 56.6 & 0.36 & $757^{\circ}$ & 55.5 & & 17 & -0.18 \\
\hline $10: 21: 43: 7$ & 165 & 71 & 25 & 18.7 & 56.6 & 0.37 & $758 !$ & 55.5 & & 17 & -0.35 \\
\hline $10: 21: 44: 7$ & 166 & 71 & 25 & 18.7 & 56.6 & 0.36 & 760 & 55.7 & & 17 & -0.35 \\
\hline $10: 21: 45: 7$ & 167 & 71 & 25 & 18.7 & 56.6 & 0.37 & $763 !$ & 55.8 & & 17 & -0.2 \\
\hline $10: 21: 46: 7$ & 168 & 70 & 25 & 18.9 & 56.6 & 0.37 & 767 & 55.8 & & 17 & -0.2 \\
\hline $10: 21: 47: 7$ & 169 & 70 & $25^{\circ}$ & 18.7 & 56.6 & 0.37 & 767 & 55.8 & & 17 & $-0 . \overline{14}$ \\
\hline $10: 21: 48: 6$ & 170 & 70 & 25 & 18.9 & 56.6 & 0.36 & 766 & 55.8 & & 17 & -0.14 \\
\hline $10: 21: 49: 6$ & 171 & 70 & 25 & 18.9 & 56.6 & 0.36 & $767^{\circ}$ & 55.8 & & 17 & 0.03 \\
\hline $10: 21: 50: 6$ & 172 & 70 & 25 & 18.9 & 56.6 & 0.37 & $765^{\circ}$ & $5 \overline{5} .9$ & & 17 & 0.03 \\
\hline $10: 21: 51: 6$ & 173 & 70 & 25 & 18.9 & 56.6 & 0.36 & 762 & 55.9 & & 17 & -0.3 \\
\hline $10: 21: 52: 6$ & 174 & 71 & 25 & 18.7 & 56.6 & 0.36 & 759 & 56 & & 17 & -0.3 \\
\hline $3: 6$ & 175 & 71 & 25 & 18.7 & 56.6 & $\overline{0.36}$ & 758 & 56 & & 17 & -0.32 \\
\hline $54: 6$ & 176 & 70 & 25 & 18.7 & 56.6 & 0.37 & $760^{\circ}$ & 56 & & 17 & -0.32 \\
\hline $10: 21: 55: 6$ & 177 & 71 & 25 & 18.7 & $5 \overline{6.6}$ & 0.37 & 763 & $5 \overline{6}$ & & 17 & -0.03 \\
\hline $10: 21: 56: 5$ & $17 \overline{8}$ & 71 & 25 & 18.7 & 56.6 & 0.37 & 766 & 56 & & 17 & -0.03 \\
\hline $10: 21: 57: 5$ & 179 & 71 & 25 & 18.7 & 56.6 & 0.37 & $765^{\circ}$ & $5 \overline{6}$ & & 17 & $-0 . \overline{13}$ \\
\hline $8: 5$ & 180 & 71 & 25 & 18.5 & 56.6 & 0.37 & $767^{\circ}$ & 56 & & 17 & -0.13 \\
\hline $159: 5$ & 181 & 71 & 25 & 18.5 & 56.6 & 0.36 & 768 & $5 \overline{6}$ & & $\overline{17}$ & $-0 . \overline{03}$ \\
\hline $10: 22: 00: 5$ & $18 \overline{2}$ & 71 & 25 & 18.7 & 56.6 & 0.36 & 767 & 56 & & 17 & -0.03 \\
\hline $11: 5$ & 183 & 71 & 25 & 18.5 & 56.6 & 0.36 & 764 & 56.1 & & 17 & -0.29 \\
\hline $02: 5$ & 184 & 71 & 25 & 18.5 & 56.6 & 0.36 & 762 & 56.2 & & 17 & -0.29 \\
\hline $03: 5$ & 185 & 71 & 25 & 18.5 & 56.6 & 0.36 & $758^{+}$ & 56.2 & & 17 & -0.29 \\
\hline $10: 22: 04: 5$ & 186 & 71 & 25 & 18.5 & 56.6 & 0.37 & $758:$ & 56.3 & & 17 & -0.04 \\
\hline $10: 22: 05: 4$ & 187 & 71 & 25 & 18.5 & 56.6 & $0 . \overline{36}$ & $760^{\circ}$ & 56.3 & & 17 & $-0 . \overline{04}$ \\
\hline $.06: 4$ & 188 & 71 & 25 & 18.5 & 56.6 & 0.37 & $758^{\prime}$ & 56.3 & & 17 & -0.04 \\
\hline $10: 22: 07: 4$ & 189 & 71 & 25 & 18.5 & 56.6 & 0.37 & $760^{\circ}$ & 56.3 & & 17 & -0.04 \\
\hline $10: 22: 08: 4$ & 190 & 71 & 25 & 18.5 & 56.6 & 0.37 & $763^{\circ}$ & 56.3 & & 17 & -0.2 \\
\hline & $1 \overline{91}$ & 71 & 25 & 18.5 & 56.6 & & & 56.3 & & 17 & -0.2 \\
\hline $10: 4$ & 192 & 71 & $25 !$ & 18.5 & 56.6 & 0.37 & 766 & 56.3 & & 17 & $-0 . \overline{23}$ \\
\hline $1: 4$ & 193 & $\overline{7} 1$ & 25 & 18.5 & 56.6 & $0 . \overline{36}$ & 765 & 56.2 & & 17 & -0.23 \\
\hline $10: \overline{22}: 12: 4$ & 194 & 71 & 25 & 18.5 & 56.6 & $0 . \overline{36}$ & 763 & $5 \overline{6.2}$ & & 17 & -0.15 \\
\hline $10: 22: 13: 4$ & 195 & 71 & 25 & 18.5 & 56 & 0.36 & & 56.2 & & 17 & -0.15 \\
\hline $4: 3$ & $19 \overline{6}$ & 71 & 25 & 18.5 & 56.6 & 0.37 & 764 & 56.1 & & 17 & -0.03 \\
\hline $10: 22: 15: 3$ & 197 & 71 & 25 & 18.5 & $56 . \overline{6}$ & $0.37_{i}^{\dagger}$ & 760 & 56.1 & & 17 & -0.03 \\
\hline $10: 22: 16: 3$ & 198 & 71 & 26 & 18.5 & 56.6 & 0.37 & 758 & 56.1 & & 17 & -0.08 \\
\hline $10: 2 \overline{2}: 17: 3^{+}$ & 199 & 71 & 26 & 18.5 & 56.6 & 0.36 & 758 & 56.4 & & 17 & -0.08 \\
\hline $18: \overline{3}^{\dagger}$ & 200 & 71 & 25 & 18.5 & 56 & 37 & 7 & 56.4 & & $17 !$ & $-0 . \overline{3}$ \\
\hline $19: 3$ & 201 & 71 & 26 & 18.5 & 56.6 & 0.37 & 762 & 56.5 & & 17 & $-0.4 \overline{3}$ \\
\hline $10: 22: 20: 3$ & 202 & 71 & 25 & 18.5 & 56.6 & 0.37 & 763 & 56.5 & & 17 & -0.15 \\
\hline $10: 22: 21: 3$ & 203 & 71 & 26 & 18.3 & 56.6 & 0.37 & 765 & 56.5 & & 17 & -0.15 \\
\hline $10: 22: 22: 3$ & 204 & 71 & 26 & 18.3 & 56.6 & 0.37 & 765 & 56.3 & & 17 & 0.04 \\
\hline $23: 2$ & 205 & 71 & 25 & 18.3 & 56.6 & 0.37 & 765 & 56.3 & & 17 & 0.04 \\
\hline $24: 2$ & 206 & 71 & 26 & $18.5^{\prime}$ & 56.6 & $\overline{0.37}$ & 765 & 56.3 & & 17 & -0.05 \\
\hline $10: 22: 25: 2$ & 207 & 71 & 26 & 18.5 & 56.6 & 0.36 & 763 & 56.4 & & 17 & -0.05 \\
\hline $10: 22: 26: 2$ & 208 & 71 & 26 & 18.5 & 56.6 & 0.36 & 761. & 56.4 & & 17 & -0.29 \\
\hline $10: 22: 27: 2$ & 209 & 71 & 26 & 18.5 & 56.6 & 0.37 & 757 & 56.4 & & 17 & -0.29 \\
\hline $10: 22: 28: 2$ & 210 & 71 & 26 & 18.3 & 56.6 & 0.37 & 756 & 56.4 & & 17 & -0.31 \\
\hline $10: 22: 29: 2$ & 211 & 71 & 26 & 18.5 & 56.6 & 0.37 & $758^{\circ}$ & 56.3 & & 17 & -0.31 \\
\hline
\end{tabular}




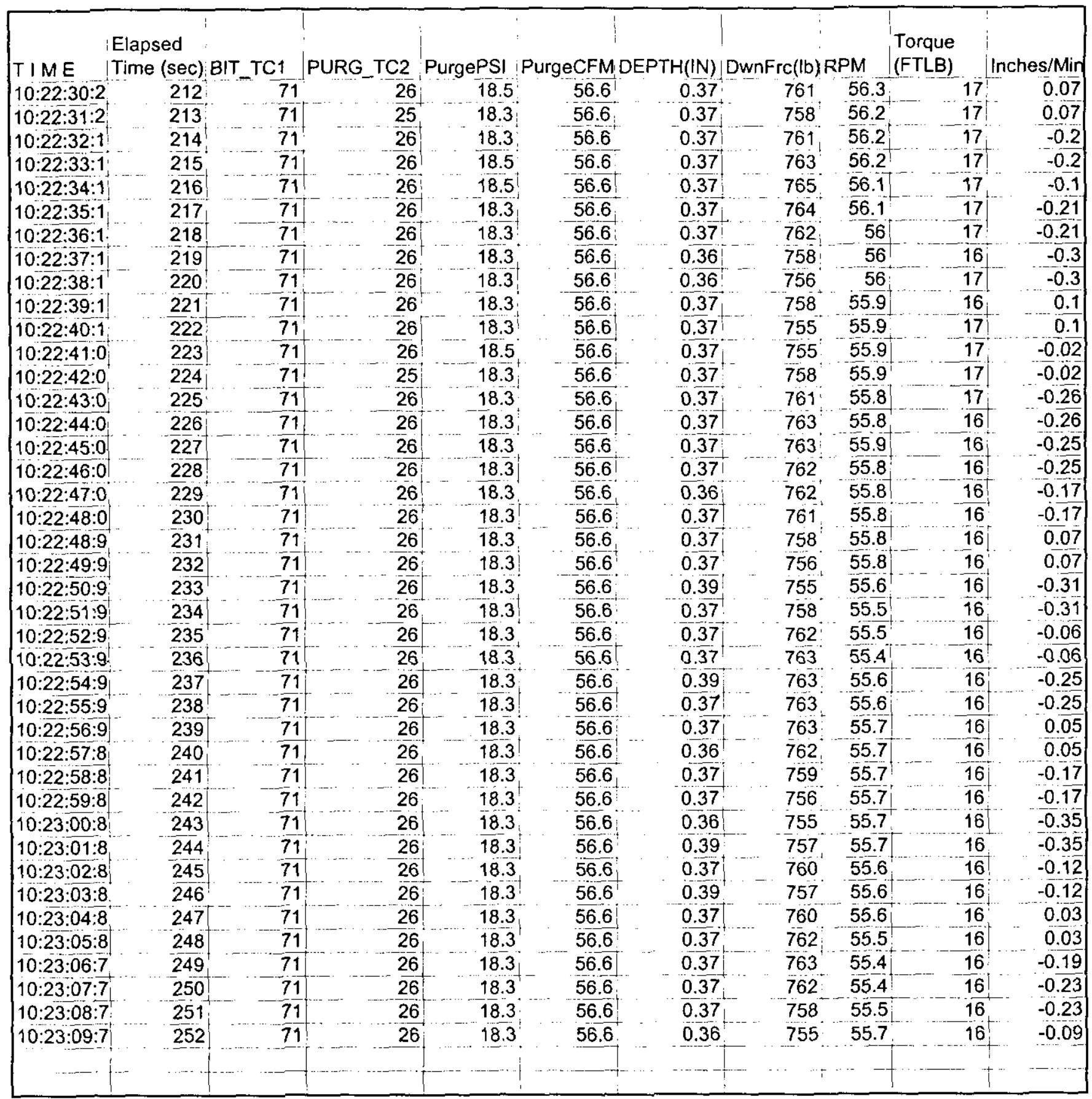




\begin{tabular}{|c|c|c|c|c|c|c|c|c|c|c|}
\hline TIME & $\begin{array}{l}\text { Elapsed } \\
\text { Time } \\
\text { (sec) }\end{array}$ & BIT_TC1 & PURG_TC2 & PurgePSI & PurgeCFM & DEPTH $(\mathbf{N})$ & & RPM & $\begin{array}{l}\text { Torque } \\
\text { (FTLB) }\end{array}$ & Inches/Min \\
\hline $09: 08: 30: 47$ & $0^{\circ}$ & 24 & 25 & 21 & $\quad 55.1^{\dagger}$ & -0.05 & 0 & 56.4 & -4 & $\begin{array}{l}0.09 \\
\end{array}$ \\
\hline $09: 08: 31: 46$ & 1 & 24 & 25 & 21 & 55.1 & -0.01 & 1 & 56.4 & -4 & -0.13 \\
\hline $09: 08: 32: 45$ & 2 & 24 & $25^{i}$ & 21 & $55.1^{\circ}$ & 0.13 & $i$ & 56.4 & -4 & -0.13 \\
\hline $09: 08: 33: 44$ & $\overline{3}$ & 24 & 25 & 21 & 55.1 & 0.25 & 71 & 56.2 & 2 & $\overline{5.42}$ \\
\hline $09: 08: 34: 43$ & 4 & 27 & 25 & 21.2 & $55.1 !$ & 0.31 & $510^{\circ}$ & 56.2 & 17 & 5.42 \\
\hline 09:08:35:42 & 5 & 31 & 25 & 21.2 & 55.1 & 0.34 & 745 & 56 & 23 & 3.69 \\
\hline $09: 08: 36: 40$ & 6 & 36 & 25 & 21.2 & $55.1^{-1}$ & 0.35 & 826 & 56 & 28 & 3.69 \\
\hline $09: 08: 37: 39$ & 7 & 39 & 25 & 21.4 & 55.1 & 0.35 & 857 & 55.6 & 29 & 0.74 \\
\hline $09: 08: 38: 38$ & 8 & 41 & 25 & 21.4 & 55.1 & $0 . \overline{37}$ & $862 !$ & 55.3 & 29 & 0.74 \\
\hline $09: 08: 39: 37$ & 9 & 43 & 25 & 21.4 & 55.1 & 0.39 & 869 & 55.3 & 28 & 0.51 \\
\hline $09: 08: 40: 36$ & 10 & 45 & 25 ? & $\overline{21.4}$ & 55.1 & $0 . \overline{39}$ & 879 & 55.1 & 28 & 0.39 \\
\hline $09: 08: 41: 35$ & 11 & $4 \overline{7}$ & 25 & 21.4 & 55.1 & 0.4 & 902 & 55.2 & 27 & 0.39 \\
\hline $09: 08: 42: 34$ & 12 & 48 & 25 & 21.4 & 55.1 & 0.4 & 917 & 55.2 & 27 & 0.33 \\
\hline $09: 08: 43: 32$ & 13 & 49 & 25 & 21.4 & 55.1 & 0.4 & 928 & 55.2 & 26 & $0.3 \overline{3}$ \\
\hline $09: 08: 44: 31$ & 14 & 49 & $2 \overline{5}$ & 21.4 & 55.1 & 0.41 & 938 & 55.2 & $2 \overline{6}$ & $-0.0 \overline{4}$ \\
\hline $09: 08: 45: 30$ & 15 & 50 & 25 & 21.6 & 55.1 & 0.4 & $960^{1}$ & 55.2 & 25 & -0.04 \\
\hline $09: 08: 46: 29$ & 16 & 51 & $25 !$ & 21.6 & 55.1 & 0.41 & 970 & 55.2 & 25 & -0.01 \\
\hline $09: 08: 47: 28$ & 17 & 52 & 25 & 21.6 & $5 \overline{5.1}$ & 0.41 & 978 & 55.1 & 25 & -0.01 \\
\hline $09: 08: 48: 27$ & 18 & 53 & 25 & 21.6 & 55.1 & 0.41 & 985 & 55.1 & 25 & 0.31 \\
\hline $09: 08: 49: 26$ & 19 & 55 & 25 & 21.6 & 55.1 & $0 . \overline{43}$ & 994 & 55 & 25 & 0.31 \\
\hline $09: 08: 50: 24$ & 20 & 55 & 25 & 21.6 & 55.1 & 0.43 & 997 & $55.1^{1}$ & 24 & -0.04 \\
\hline $09: 08: 51: 23$ & 21 & 55 & 25 & 21.6 & 55.1 & 0.44 & $999^{\prime}$ & 55.1 & 23 & -0.04 \\
\hline 09:08:52:22 & 22 & 56 & 25 & 21.6 & 55.1 & 0.44 & 1002 & 55.2 & 23 & -0.09 \\
\hline $09: 08: 53: 21$ & 23 & 57 & 25 & 21.6 & 55.1 & 0.44 & $1000^{\prime}$ & 55.2 & 24 & -0.09 \\
\hline $09: 08: 54: 20$ & 24 & 57 & 25 & 21.6 & 55.1 & 0.45 & 1004 & 55.2 & 24 & -0.16 \\
\hline $09: 08: 55: 19$ & 25 & 58 & 25 & 21.6 & 55.1 & 0.45 & 1006 & 55.3 & 24 & -0.16 \\
\hline $09: 08: 56: 18$ & 26 & 57 & 25 & 21.6 & 55.1 & 0.44 & 1004 & 55.2 & 24 & 0.23 \\
\hline $09: 08: 57: 17$ & 27 & 58 & 25 & 21.6 & 55.1 & 0.43 & 1000 & 55.2 & 24 & 0.23 \\
\hline $09: 08: 58: 15$ & 28 & 58 & 25 & 21.6 & 55.1 & 0.45 & 997 & 55.2 & 24 & 0 \\
\hline $09: 08: 59: 14$ & 29 & 59 & 25 & 21.8 & 55.1 & 0.44 & 997 & 55 & 23 & 0 \\
\hline $09: 09: 00: 13$ & 30 & 59 & 25 & 21.8 & 55.1 & 0.44 & 1000 & 55 & 23 & -0.15 \\
\hline $09: 09: 01: 12$ & $31^{t}$ & 60 & 25 & 21.8 & 55.1 & 0.44 & 1009 & 55.1 & 23 & $-0.1 \overline{5}$ \\
\hline $09: 09: 02: 11$ & 32 & 60 & 25 & 21.8 & 55.1 & 0.44 & $1007^{\dagger}$ & 55.1 & 23 & -0.11 \\
\hline $09: 09: 03: 10$ & 33 & 60 & 25 & 21.8 & $55.1^{1}$ & 0.44 & 1014 & 55.1 & 23 & -0.11 \\
\hline $09: 09: 04: 09$ & 34 & 61 & 25 & 21.8 & 55.1 & $0 . \overline{5}$ & 1017 & 55.2 & 23 & 0.05 \\
\hline $09: 09: 05: 07$ & $35^{\dagger}$ & 60 & 25 & 21.8 & 55.1 & 0.45 & $1019^{\prime}$ & $5 \overline{5} .2$ & 23 & $0 . \overline{05}$ \\
\hline $09: 09: 06: 06$ & 36 & 60 & 25 & 22 & 55.1 & 0.45 & 1016 & 55.2 & 22 & -0.05 \\
\hline $09: 09: 07: 05$ & 37 & 61 & 25 & 22 & 55.1 & 0.45 & 1009 & 55.2 & 22 & -0.05 \\
\hline $09: 09: 08: 04$ & 38 & 61 & 25 & 22 & 55.1 & 0.45 & 1008 & $55 . \overline{2}$ & 22 & -0.21 \\
\hline $09: 09: 09: 03$ & 39 & 61 & 25 & 22 & 55 & 0.44 & 1007 & 55.2 & 21 & -0.21 \\
\hline $09: 09: 10: 02$ & 40 & 61 & 25 & 22 & 55 & 0.45 & 1004 & 55.2 & 21 & -0.02 \\
\hline $09: 09: \overline{11: 01}$ & 41 & 61 & 25 & 22 & 54.9 & 0.46 & 1007 & 55.1 & 21 & -0.02 \\
\hline $09: 09: 12: 00$ & 42 & 61 & 25 & 22 & $54 . \overline{8}$ & 0.46 & 1010 & 55.1 & 21 & -0.02 \\
\hline $09: 09: 12: 98$ & 43 & 61 & 25 & 22 & 54.8 & 0.45 & 1015 & 55.1 & 21 & -0.09 \\
\hline $09: 09: 13: 97$ & 44 & 61 & $25 !$ & 22 & 54.8 & 0.45 & 1016 & 55.1 & 21 & -0.09 \\
\hline $09: 09: 14: 96$ & 45 & 61 & 25 & 22 & 54.8 & 0.45 & 1014 & 55.1 & 21 & -0.25 \\
\hline $0 \overline{9}: 09: 15: 95$ & 46 & 61 & 25 & 22 & 54.7 & 0.45 & 1009 & 55.2 & 20 & -0.25 \\
\hline $09: 09: 16: 94$ & 47 & 61 & 25 & 22 & 54.6 & 0.44 & 1009 & 55.2 & 20 & 0.01 \\
\hline 09:09:17:93 & 48 & 61 & 25 & 22.2 & 54.6 & 0.45 & 1009 & 55.2 & 20 & 0.01 \\
\hline 09:09:18:92 & 49 & 61 & 25 & 22.2 & 54.6 & 0.46 & $1010 !$ & $55.3 !$ & 20 & -0.03 \\
\hline $09: 09: 19: 90$ & 50 & 61 & 25 & 22.2 & 54.6 & 0.46 & 1012 & 55.4 & 20 & -0.03 \\
\hline $09: 09: 20: 89$ & 51 & 61 & 25 & 22.2 & 54.6 & 0.46 & 1018 & 55.4 & 20 & -0.16 \\
\hline
\end{tabular}




\begin{tabular}{|c|c|c|c|c|c|c|c|c|c|c|c|}
\hline$T I M E$ & $\begin{array}{l}\text { Elapse } \\
\text { Time } \\
\text { (sec) }\end{array}$ & & BIT_TC1 & PURG_TC2 & PurgePSI & PurgeCFM & DEPTH(IN) & DwnFic(ib) & RPM & $\begin{array}{l}\text { Torque } \\
\text { (FTLB) }\end{array}$ & Inches/Min \\
\hline $09: 09: 21: 88$ & & 52 & 62 & 25 & $\quad 22.2$ & $\quad 54.6$ & 0.48 & $\quad 1023$ & $55 . \overline{3}$ & $\quad 20$ & $-0.1 \overline{6}$ \\
\hline $9: 09: 22: 87$ & & 53 & 62 & 25 & 22.2 & 54.5 & 0.46 & 1025 & 55.3 & 20 & -0.24 \\
\hline 86 & & 54 & 62 & 25 & 22.2 & 54.5 & 0.46 & $1024^{\dagger}$ & $55 . \overline{3}$ & 20 & -0.24 \\
\hline 85 & & 55 & 61 & 25 & 22.2 & 54.5 & 0.46 & 1018 & 55.2 & 20 & -0.03 \\
\hline $09: 09: 2$ & & 56 & 61 & 25 & 22.2 & 54.5 & 0.45 & 1012 & 55.2 & 20 & -0.03 \\
\hline 83 & & 57 & 61 & 25 & 22.2 & 54.4 . & 0.46 & 1014 & 55.1 & 20 & -0.02 \\
\hline 81 & & 58 & 61 & 25 & 22.2 & 54.4 & 0.46 & 1007 & 55.2 & 20 & -0.02 \\
\hline 80 & & 59 & 61 & 25 & 22.2 & 54.4 & 0.46 & 1006 & 55.2 & 19 & -0.27 \\
\hline :79 & & 60 & 61 & 25 & 22.2 & 54.4 & 0.46 & 1007 & $55 . \overline{3}$ & 19 & -0.27 \\
\hline $5: 78$ & & 61 & 62 & 25 & 22.2 & 54.4 & 0.48 & $1010^{\circ}$ & 55.4 & 19 & -0.18 \\
\hline $09: 0$ & & 62 & 61 & 25 & 22.2 & 54.4 & $0 . \overline{4} 6$ & 1014 & 55.4 & 19 & -0.18 \\
\hline & & $63^{\dagger}$ & 61 & 25 & $22.2^{\dagger}$ & $54 . \overline{4}$ & 0.46 & 1016 & 55.3 & 19 & -0.06 \\
\hline & & 64 & 61 & 25 & 22.2 & 54 & 0.46 & 1013 & 55.2 & 19 & -0.06 \\
\hline $1: 73$ & & 65 & $6 \overline{1}$ & 25 & 22.2 & 54.3 & 0.46 & 1014 & 55.2 & 19 & 0.04 \\
\hline 72 & & 66 & $61^{\dagger}$ & 25 & 22.2 & 54.3 & 0.46 & 1011 & 55.3 & 19 & 0.04 \\
\hline & & 67 & 61 & 25 & 22.2 & & 0.46 & & 55.3 & 19 & -0.27 \\
\hline & & 68 & 60 & 25 & 22.2 & 54 & 46 & 1002 & 55.3 & 19 & -0.27 \\
\hline & & 69 & 61 & 25 & 22.2 & & & 1002 & 55.1 & 19 & -0.12 \\
\hline & & 70 & 61 ! & 25 & 22.2 & 54.3 & 0.46 & 1005 & 55.2 & 19 & -0.12 \\
\hline 67 & & $71^{t}$ & 61 & 25 & 22.2 & 54.3 & & 1010 & 55.2 & 19 & -0.12 \\
\hline & & $72 !$ & 61 & 25 & 22.2 & 54 & 0.46 & 1012 & 55.3 & 19 & -0.12 \\
\hline & & 73 & $61 j$ & 25 & $22.2^{\circ}$ & & & 1010 & 55.3 & 19 & 0.03 \\
\hline & & 74 & 61 & 25 & 22.4 & 54 & 48 & & 55.3 & 19 & 0.03 \\
\hline & & 75 & 61 & 25 & 22.4 & & 0.46 & 12 & 55.3 & 19 & -0.27 \\
\hline & & 76 & 60 & 25 & 22.4 & & & & 55.4 & 19 & -0.16 \\
\hline & & 77 & 60 & 25 & 22.4 & & & 02 & 55.4 & 19 & -0.16 \\
\hline & & 78 & 60 & 25 & 22.4 & & & & 55.4 & 19 & -0.08 \\
\hline & & 79 & 61 & 25 & 22.4 & 54.2 & 0.48 & 1000 & 55.4 & 19 & -0.08 \\
\hline & & 80 & 61 & 25 & 22.4 & 54.2 & 0.45 & 1004 & 55.4 & 19 & 0.03 \\
\hline & & 81 & 61 & 25 & 22.4 & & & & 55.5 & 19 & .03 \\
\hline & & 82 & 6 & 25 & 22.4 & & & & 55.3 & 19 & -0.29 \\
\hline & & 83 & 61 & 25 & 22.4 & 54 & 0.46 & 1005 & 55.3 & 19 & -0.29 \\
\hline & & 84 & 61 & 25 & 22.4 & 54.2 & 0.46 & 1007 & 55.4 & 19 & -0.06 \\
\hline & & 85 & $60^{\dagger}$ & 25 & 22.4 & & & & & 19 & -0.06 \\
\hline & & $86 !$ & 60 & 25 & 22.4 & 54. & 0.46 & 999 & 55.2 & 19 & -0.16 \\
\hline & & 87 & 60 & 25 & 22.4 & 54.1 & 0.46 & 997 & 55.3 & 19 & -0.16 \\
\hline & & 88 & 60 & 25 & 22.4 & 54.1 & 0.46 & 997 & 55.4 & 19 & 0.02 \\
\hline & & 89 & 60 & 25 & 22.4 & 54.1 & 0.46 & 1000 & 55.4 & 19 & 0.02 \\
\hline & & 90 & 60 & 25 & 22.4 & 54.1 & & 998 & 55.5 & 19 & -0.28 \\
\hline & & 91 & 60 & 25 & 22.4 & 54. & & & & 19 & -0.2 \\
\hline & & 92 & 60 & 25 & 22.4 & & 0.46 & 1005 & 55.5 & 19 & -0.18 \\
\hline & & 93 & 60 & 25 & 22.6 & 54.1 & 0.46 & 1009 & 55.5 & 19 & -0.18 \\
\hline & & 94 & $60 ?$ & 25 & 22.6 & 54.1 & 0.48 & 1009 & 55.4 & 19 & -0.09 \\
\hline & & 95 & 60 & 25 & 22.6 & $5 \overline{4.1}$ & 0.4 & 007 & 55.4 & 19 & -0.09 \\
\hline & & 96 & 60 & 25 & 22.6 & 54.1 & & & 55.4 & 19 & 0.02 \\
\hline & & 97 & 59 & 25 & 22.6 & 54.1 & 0.46 & 999 & 55.4 & 19 & 0.02 \\
\hline & & 98 & $59 !$ & 25 & 22.6 & $5 \overline{4.1}$ & 0.46 & 1002 & 55.4 & 19 & -0.28 \\
\hline & & $\overline{99}$ & 59 & 25 & 22.6 & 54 & & 999 & 55.5 & 19 & -0.28 \\
\hline $09: 34$ & & 100 & 60 & 25 & 22.6 & 54.1 & 0.48 & 1000 & 55.5 & 19 & -0.17 \\
\hline $09: 10: 10: 33$ & & 101 & 60 & 25 & 22.6 & 54.1 & $\overline{0.49}$ & 1004 & 55.6 & 18 & -0.17 \\
\hline & & 102 & $60^{\dagger}$ & 25 & 22.6 & 54.1 & 0.4 & 1009 & 55.6 & 19 & 0.12 \\
\hline $09: 10: 12: 30$ & & 103 & 60 & 25 & 22.6 & 54.1 & 0.48 & 1011 & 55.6 & 18 & 0.12 \\
\hline
\end{tabular}




\begin{tabular}{|c|c|c|c|c|c|c|c|c|c|c|}
\hline TIME & $\begin{array}{l}\text { Elapsed } \\
\text { Time } \\
\text { (sec) }\end{array}$ & $B I T+T C 1$ & PURG_TC2 & PurgePSI & PurgeCFM & DEPTH(IN) & OwnFrc(lo) & RPM & $\begin{array}{l}\text { Torque } \\
\text { (FTLB) }\end{array}$ & Inches/Min \\
\hline $09: 10: 13: 29$ & 104 & -60 & 25 & 22.6 & 54.1 & 0.48 & 1011 & 55.5 & $\begin{array}{r}19 \\
-\quad 19\end{array}$ & $\quad-0.13$ \\
\hline $09: 10: 14: 28$ & 105 & $60^{\dagger}$ & 25 & 22.6 & $54.1^{\circ}$ & 0.48 & 1009 & 55.6 & $19^{\circ}$ & -0.13 \\
\hline $09: 10: 1$ & 106 & 60 & 25 & 22.6 & 54.1 & 0.48 & 1005 & 55.6 & 19 & -0.24 \\
\hline $09: 10: 16: 26$ & 107 & 60 & 25 & 22.6 & $54.1^{1}$ & 0.48 & 1009 & 55.6 & 19 & -0.24 \\
\hline $09: 10: 17: 25$ & 108 & 59 & 25 & 22.6 & 54.11 & 0.48 & 1004 & 55.6 & 19 & -0.2 \\
\hline $09: 10: 18: 24$ & 109 & 59 & 25 & 22.6 & $54.1^{1}$ & 0.49 & 1001 & 55.6 & 19 & -0.08 \\
\hline $9: 22$ & $1100^{\dagger}$ & $5 \overline{9}$ & 25 & 22.6 & 54.1 & 0.48 & 1004 & 55.6 & 19 & -0.08 \\
\hline 21 & 111 & 59 & 25 & 22.6 & 54 & 0.48 & 1009 & 55.6 & 19 & -0.02 \\
\hline $29: 20$ & 112 & 60 & 25 & 22.6 & 54 & 0.49 & 1013 & 55.6 & 19 & -0.02 \\
\hline $2: 19$ & 113 & 60 & 25 & 22.6 & 54 & 0.48 & 1017 & 55.6 & 19 & -0.13 \\
\hline 18 & 114 & 60 & 25 & 22.6 & 54 & 0.48 & 1017 & 55.6 & 19 & -0.13 \\
\hline & 115 & 60 & 25 & 22.6 & 54 & 0.46 & 1017 & 55.6 & 19 & $-0 . \overline{27}$ \\
\hline & 116 & 60 & 25 & 22.6 & 54 & 0.46 & 1017 & 55.6 & 19 & -0.27 \\
\hline 14 & 117 & 59 & 25 & 22.6 & 54 & 0.46 & 1014 & 55.6 & 19 & -0.05 \\
\hline 13 & 118 & 59 & 25 & 22.6 & 54 & $0.48^{\dagger}$ & 1007 & 55.6 & 19 & -0.05 \\
\hline & 119 & $\overrightarrow{59}$ & 25 & 22.6 & 54 & $0.4 \overline{8}$ & 1006 & 55.5 & 19 & $-0 . \overline{04}$ \\
\hline & 120 & 59 & 25 & 22.6 & $54^{+}$ & 0.48 & 1006 & 55.4 & 19 & $-0 . \overline{04}$ \\
\hline & 121 & 59 & 25 & 22.6 & 54 & 0.48 & 1009 & 55.4 & 19 & -0.13 \\
\hline & $12 \overline{2}$ & 59 & 25 & $22.6^{\dagger}$ & 54 & 0.48 & 1013 & 55.4 & 19 & -0.13 \\
\hline & 123 & 60 & 25 & 22.6 & 53.9 & 0.46 & 1010 & 55.4 & 19 & -0.31 \\
\hline & 124 & 60 & 25 & 22.6 & 53.9 & $0 . \overline{4} 6$ & 1015 & 55.4 & 19 & -0.31 \\
\hline & 125 & 60 & 25 & 22.6 & & & 1017 & 55.4 & 19 & -0.02 \\
\hline & 126 & 59 & 25 & 22.6 & & 0.4 & 1017 & 55.3 & 19 & -0.02 \\
\hline & 127 & 59 & 25 & 22.6 & 53.9 & 0.48 & 1012 & 55.3 & 19 & -0.01 \\
\hline & 128 & 59 & 25 & 22.6 & & & & 55.2 & 19 & -0.01 \\
\hline & 129 & 59 & 25 & 22.8 & & & 1007 & 55.2 & 19 & -0.17 \\
\hline & 130 & 59 & 25 & 22.8 & & & 1009 & 55.2 & 19 & $-0 . \overline{17}$ \\
\hline & 131 & 59 & 25 & 22.8 & 53 & 0.49 & 1012 & 55.3 & 19 & -0.22 \\
\hline & 132 & 60 & 25 & 22.8 & 53.9 & & 1010 & 55.3 & $1 \overline{9}$ & $-0 . \overline{22}$ \\
\hline & 133 & 60 & 25 & 22.8 & 53.9 & 0.49 & 1014 & 55.3 & 18 & -0.07 \\
\hline & 134 & 60 & 25 & 22.8 & 53.9 & 0.4 & 1017 & 55.3 & 18 & -0.07 \\
\hline & 135 & 5 & 25 & 22.8 & & & 19 & 55.4 & 19 & \\
\hline & 136 & 59 & 25 & 22.8 & 53.9 & 0.48 & 1017 & 55.4 & 19 & \\
\hline & 137 & 59 & 25 & 22.8 & 53.9 & & & 55.5 & 19 & -0.17 \\
\hline & 138 & 59 & 25 & 22.8 & 53.9 & 0.48 & $1010^{\dagger}$ & 55.6 & 19 & $-0.1 \overline{7}$ \\
\hline & 139 & 59 & 25 & 22.8 & 53. & 0.48 & 1014 & 55.6 & 19 & $-0 . \overline{27}$ \\
\hline & 140 & 59 & 25 & 22.8 & 53.9 & 0.49 & 1009 & 55.6 & 18 & -0.27 \\
\hline & 141 & 59 & 25 & 22.8 & $53 . \overline{9}^{\dagger}$ & 0.49 & 1007 & 55.4 & 19 & 0.09 \\
\hline & 142 & 59 & 25 & 22.8 & & & & 55.4 & 18 & -0.16 \\
\hline & 14 & 59 & 25 & 22.8 & & & & 55.3 & 18 & -0.16 \\
\hline & 144 & 60 & 25 & 22.8 & 53. & & 1019 & 55.3 & 18 & -0.2 \\
\hline & 145 & 60 & 25 & 22.8 & 53.8 & 0.49 & 1020 & 55.3 & 18 & -0.2 \\
\hline $4: 82$ & 146 & 60 & 25 & 22.8 & 53.8 & 0.49 & 1018 & 55.3 & 18 & -0.24 \\
\hline $5: 80$ & $14 \overline{7}$ & 59 & 25 & 22.8 & 53.8 & 0.49 & 1020 & 55.3 & 18 & -0.24 \\
\hline & 148 & 59 & 25 & 22.8 & 53.8 & 0.48 & 1015 & 55.3 & 19 & -0.1 \\
\hline & 149 & 59 & 25 & 22.8 & $5 \overline{3.8}$ & 0.48 & $1011^{\dagger}$ & 55.3 & $\overline{18}$ & -0.1 \\
\hline & 15 & 59 & 25 & 22.8 & 53.8 & 0.48 & $1009^{\circ}$ & 55.3 & 18 & 0.01 \\
\hline $7: 76$ & 151 & 59 & 25 & 22.8 & 53.8 & 0.49 & $1011^{\dagger}$ & 55.3 & 18 & 0.01 \\
\hline $09: 11: 00: 75$ & 152 & 59 & 25 & 22.8 & 53.8 & 0.49 & 1015 & 55.3 & 18 & $-0 . \overline{25}$ \\
\hline $09: 11: 01: 74$ & 153 & 60 & 25 & 22.8 & 53.8 & 0.49 & 1019 & 55.3 & 18 & -0.25 \\
\hline $09: 11: 02: 73$ & 154 & 60 & 25 & 22.8 & 53.8 & 0.49 & $1021^{\dagger}$ & 55.2 & 18 & -0.19 \\
\hline $09: 11: 03: 71$ & 155 & $60_{\mathrm{i}}^{-1}$ & 25 & 22.8 & 53.8 & 0.49 & $1020^{\circ}$ & 55.1 & 18 & -0.19 \\
\hline
\end{tabular}




\begin{tabular}{|c|c|c|c|c|c|c|c|c|c|c|}
\hline$T I M E$ & $\begin{array}{l}\text { Elapsed } \\
\text { Time } \\
\text { i(sec) }\end{array}$ & BIT_TC1 & PURG_TC2 & PurgePSI & PurgeCFM! & DEPTH(IN) & DwnFrc(b) & RPM & $\begin{array}{l}\text { Torque } \\
\text { (FTLB) }\end{array}$ & Inches/Min \\
\hline $09: 11: 04: 70$ & +56 & -59 & -25 & 22.8 & $\quad 53.8$ & 0.48 & 1021 & 55.1 & 18 & $\quad-0.07$ \\
\hline $09: 11: 05: 69$ & 157 & 59 & 25 & 22.8 & 53.8 & 0.48 & 1020. & 55.1 & 18 & -0.07 \\
\hline $09: 11: 06: 68$ & 158 & 59 & 25 & 22.8 & 53.8 & 0.46 & 1015 & 55.1 & 18 & 0.01 \\
\hline $09: 11: 07: 67$ & $159^{\circ}$ & 59 & 25 & 22.8 & 53.8 & 0.48 & 1010 & 55.1 & 18 & 0.01 \\
\hline $09: 11: 08: 66$ & 160 & 59 & 25 & 22.8 & 53.8 & 0.49 & 1012 & 55 & 18 & -0.25 \\
\hline $09: 11: 09: 65$ & 161 & 59 & 25 & 22.8 & 53.8 & 0.49 & 1015 & 54.8 & 18 & -0.25 \\
\hline $09: 11: 10: 63$ & 162 & 59 & 25 & 22.8 & 53.8 & 0.49 & 1019 & 54.8 & 18 & -0.16 \\
\hline $09: 11: 11: 62$ & 163 & 59 & 25 & 22.8 & 53.8 & $0 . \overline{48}$ & 1021 & 54.8 & 18 & -0.16 \\
\hline $09: 11: 12: 61$ & 164 & 59 & 25 & $22.8^{\circ}$ & 53.8 & 0.49 & 1018 & 55 & 18 & -0.02 \\
\hline $09: 11: 13: 60$ & 165 & 59 & 25 & 22.8 & 53.8 & 0.48 & 1020 & 55 & 18 & -0.02 \\
\hline $09: 11: 14: 59$ & 166 & 59 & 25 & 22.8 & 53.8 & 0.48 & 1019 & 55.2 & 18 & -0.09 \\
\hline $09: 11: 15: 58$ & 167 & 59 & 25 & 22.8 & 53.8 & 0.48 & 1015 & 55.3 & 18 & -0.09 \\
\hline $09: 11: 16: 57$ & 168 & 58 & 25 & 22.8 & 53.8 & 0.48 & 1009 & 55.3 & 18 & -0.25 \\
\hline $09: 11: 17: 56$ & 169 & 58 & 25 & 22.8 & 53.8 & 0.49 & 1008 & 55.4 & 18 & -0.25 \\
\hline $09: 11: 18: 54$ & 170 & 58 & 25 & 22.9 & 53.8 & 0.49 & 1010 & 55.5 & 18 & -0.2 \\
\hline $09: 11: 19: 53$ & 171 & 59 & $25^{\dagger}$ & 22.8 & 53.8 & $0.49^{\dagger}$ & 1012 & 55.5 & 17 & -0.2 \\
\hline $09: 11: 20: 52$ & $17 \overline{2}$ & 59 & 25 & 22.8 & 53.8 & 0.49 & 1010 & 55.4 & 17 & 0 \\
\hline $09: 11: 21: 51$ & 173 & 59 & $25^{\dagger}$ & 22.9 & 53.8 & 0.49 & 1014 & 55.4 & 17 & 0 \\
\hline $09: 11: 22: 50$ & 174 & 59 & 25 & 22.9 & 53.7 & 0.48 & 1017 & 55.4 & 17 & -0.07 \\
\hline $09: 11: 23: 49$ & $175 !$ & 59 & 25 & 22.9 & 53.7 & 0.48 & 1018 & 55.3 & 17 & -0.28 \\
\hline $09: 11: 24: 48$ & 176 & 59 & 25 & 22.9 & 53.7 & 0.48 & 1015 & 55.3 & 17 & -0.28 \\
\hline $09: 11: 25: 46$ & 177 & 59 & 25 & 22.9 & 53.7 & 0.48 & 1011 & 55.3 & 18 & $-0 . \overline{22}$ \\
\hline $09: 11: 26: 45$ & 178 & 59 & 25 & 22.9 & 53.7 & 0.48 & 1007 & 55.5 & 18 & $-0.2 \overline{2}$ \\
\hline $09: 11: 27: 44$ & 179 & 59 & 25 & 22.9 & 53.7 & 0.48 & 1007 & 55.4 & 18 & 0 \\
\hline $09: 11: 28: 43$ & 180 & 59 & 25 & 22.9 & 53.7 & 0.48 & 1009 & 55.4 & 18 & 0 \\
\hline $09: 11: 29: \overline{42}$ & $\overline{181}$ & 59 & $25 !$ & 22.9 & 53.7 & 0.49 & 1007 & 55.3 & 18 & -0.04 \\
\hline $09: 11: 30: 41$ & 182 & 59 & 25 & 22.9 & 53.7 & 0.49 & 1010 & 55.2 & 18 & -0.04 \\
\hline $09: 11: 31: 40$ & 183 & 60 & 25 & 22.9 & 53.7 & 0.49 & 1014 & 55.2 & 18 & -0.25 \\
\hline $09: 11: 32: 38$ & 184 & 59 & 25 & 22.9 & 53.7 & 0.48 & 1017 & 55.1 & 18 & -0.25 \\
\hline $09: 11: 33: 37$ & 185 & 60 & 25 & 22.9 & 53.7 & 0.48 & 1017 & 55.1 & 18 & -0.21 \\
\hline $09: 11: 34: 36$ & 186 & 59 & 25 & 22.9 & 53.7 & 0.49 & 1014 & 55.3 & 18 & -0.21 \\
\hline $09: 11: 35: 35$ & 187 & 59 & $25 !$ & 22.9 & 53.7 & 0.46 & 1009 & 55.4 & 18 & 0.02 \\
\hline $09: 11: 36: 34$ & 188 & 59 & 25 & 22.9 & 53.7 & 0.48 & 1007 & 55.4 & 18 & 0.02 \\
\hline $09: 11: 37: 33$ & 189 & 58 & 25 & 22.9 & 53.7 & 0.49 & 1008 & 55.2 & 18 & -0.1 \\
\hline $09: 11: 38: 32$ & 190 & 58 & 25 & 22.9 & 53.7 & 0.49 & 1007 & 55.2 & 18 & -0.1 \\
\hline $09: 11: 39: 31$ & 191 & 58 & 25 & 22.9 & 53.7 & 0.49 & 1009 & 55.2 & 18 & -0.2 \\
\hline $09: 11: 40: 29$ & 192 & 59 & 25 & 22.9 & 53.7 & 0.5 & 1013 & 55.3 & 18 & -0.2 \\
\hline $09: 11: 41: 28$ & 193 & 59 & 25 & 22.9 & 53.7 & 0.5 & 1016 & 55.3 & 18 & $-0.2 \overline{3}$ \\
\hline $09: 11: 42: 27$ & 194 & 59 & 25 & 22.9 & 53.7 & 0.49 & 1017 & 55.3 & 18 & -0.23 \\
\hline $09: 11: 43: 26$ & 195 & 59 & 25 & 22.9 & 53.7 & 0.49 & 1017 & $55 . \overline{4}$ & 18 & 0.04 \\
\hline $09: 11: 44: 25$ & 196 & 59 & 25 & 22.9 & 53.7 & 0.49 & 1012 & 55.5 & 18 & 0.04 \\
\hline $09: 11: 45: 24$ & 197 & 58 & 25 & 22.9 & 53.7 & 0.49 & 1007 & 55.5 & 18 & $-0 . \overline{16}$ \\
\hline $09: 11: 46: 23$ & 198 & 58 & 25 & 22.9 & 53.7 & 0.49 & 1010 & 55.5 & 18 & -0.16 \\
\hline $09: 11: 47: 21$ & 199 & 58 & 25 & 22.9 & 53.7 & 0.49 & 1007 & 55.6 & 18 & -0.21 \\
\hline $09: 11: 48: 20$ & 200 & 58 & 25 & $22.9^{7}$ & 53.7 & 0.5 & 1007 & 55.6 & 18 & -0.21 \\
\hline $09: 11: 49: 19$ & 201 & 58 & 25 & 22.9 & 53.7 & 0.5 & 1009 & 55.6 & 18 & -0.23 \\
\hline $09: 11: 50: 18$ & 202 & 58 & 25 & 22.9 & $53 . \overline{7}$ & 0.5 & 1012 & 55.6 & 18 & -0.23 \\
\hline $09: \overline{11: 51: 17}$ & 203 & 59 & 25 & 22.9 & 53.7 & 0.49 & 1015 & 55.6 & 17 & $0 . \overline{6}$ \\
\hline $09: 11: 52: 16$ & 204 & 59 & 25 & $22 . \overline{9}$ & $53 . \overline{7}$ & $0 . \overline{49}$ & 1017 & 55.6 & 17 & 0.06 \\
\hline $09: 11: 53: 15$ & 205 & 59 & 25 & 22.9 & 53.7 & 0.49 & 1016 & 55.5 & 17 & -0.11 \\
\hline $09: 11: 54: 14$ & 206 & 59 & 25 & 22.9 & 53.6 & 0.49 & 1017 & 55.5 & 17 & -0.11 \\
\hline $09: 11: 55: 12$ & 207 & 58 & 25 & 22.9 & 53.6 & 0.48 & 1015 & 55.5 & $1 \overline{8}$ & -0.25 \\
\hline
\end{tabular}




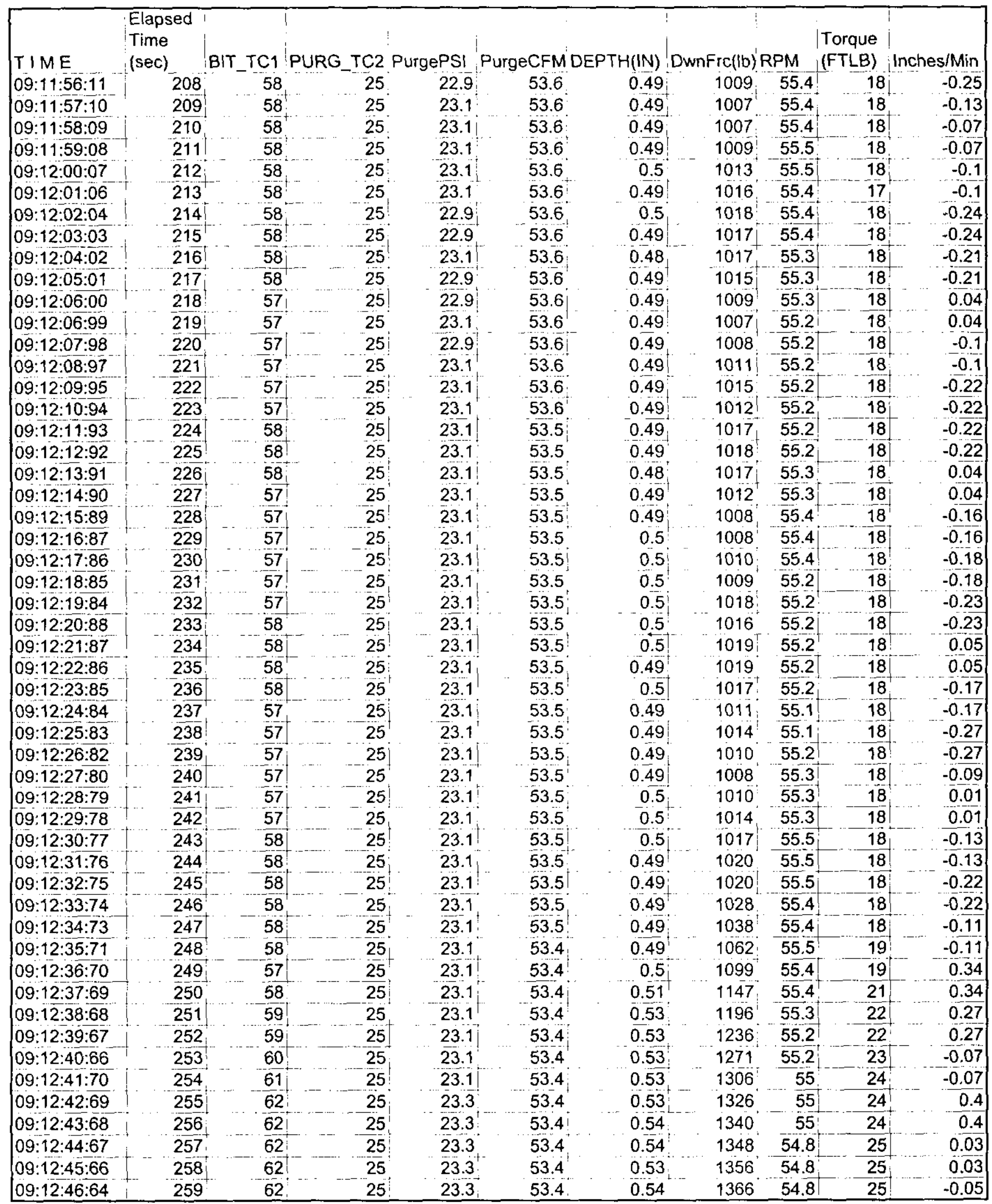




\begin{tabular}{|c|c|c|c|c|c|c|c|c|c|c|}
\hline TIME & $\begin{array}{l}\text { Elapsed } \\
\text { Time } \\
\text { (sec) }\end{array}$ & BIT_TC1 & PURG_TC2 & PurgePS! & PurgeCFM & DEPTH(IN) & DwnFrc(b) & RPM & $\begin{array}{l}\text { Torque } \\
\text { (FTLB) }\end{array}$ & es/Min \\
\hline $09: 12: 47: 63$ & 260 & -62 & 25 & $\quad 23.3$ & 53.4 & $\quad 0.55$ & 1376 & 54.7 & 25 & -0.05 \\
\hline $09: 12: 48: 62$ & 261 & 63 & 25 & 23.1 & 53.4 & 0.54 & 1376 & 54.6 & 25 & -0.12 \\
\hline $9: 61$ & 262 & 64 & 25 & 23.1 & 53.4 & 0.55 & 1380 & 54.6 & 25 & -0.12 \\
\hline$: 60$ & 263 & 64 & 25 & 23.3 & 53.4 & 0.54 & 1382 & 54.5 & 25 & -0.07 \\
\hline$: 59$ & 264 & 65 & 25 & 23.3 & 53.4 & 0.54 & 1381 & 54.5 & 25 & -0.07 \\
\hline $2: 58$ & 265 & 65 & 25 & 23.3 & 53.4 & 0.55 & 1377 & 54.5 & 25 & -0.02 \\
\hline 56 & 266 & 65 & 25 & 23.3 & 53.4 & 0.54 & 1371 ? & 54.5 & 25 & -0.02 \\
\hline 55 & $\overline{26} 7$ & 64 & 25 & 23.3 & 53.4 & 0.54 & 1367 & 54.5 & 25 & -0.29 \\
\hline 54 & 268 & 64 & 25 & 23.3 & 53.4 & 0.54 & 1368 & 54.5 & 25 & -0.29 \\
\hline & 269 & 65 & 25 & 23.3 & 53.4 & 0.55 & 1365 & 54.6 & 25 & -0.18 \\
\hline & 270 & 65 & 25 & 23.3 & 3.4 & 0.55 & $1367 !$ & 54.7 & 25 & $-0 . \overline{18}$ \\
\hline & 271 & 65 & 25 & $23 . \overline{3}$ & 53.4 & 0.54 & 1370 & 54.7 & 25 & 0.06 \\
\hline & 272 & 65 & 25 & 23.3 & 53.4 & 0.54 & 1371 & 54.7 & 25 & -0.19 \\
\hline & 273 & 66 & 25 & 23.5 & 53.4 & 0.54 & 1370 & 54.7 & 25 & -0.19 \\
\hline & 274 & 66 & 25 & 23.5 & 53.4 & 0.55 & 1367 & 54.7 & 25 & -0.28 \\
\hline & 275 & 66 & 25 & 23.5 & & 54 & & 54.9 & 25 & -0.28 \\
\hline & 276 & $65^{\dagger}$ & 25 & 23.5 & 53. & 0.5 & & 55 & 25 & -0.07 \\
\hline & 277 & 66 & 25 & 23.5 & 53.4 & 0. & & 55 & 25 & -0.07 \\
\hline & 278 & 65 & 25 & 23.5 & 53.4 & 0.54 & 1357 & 55.1 & 25 & -0.01 \\
\hline & 279 & 66 & 25 & 23.3 & & 0.54 & & 55 & 25 & $-0 . \overline{1}$ \\
\hline & 280 & 66 & 25 & 23.3 & 53. & $0.5 \overline{5}$ & & 55 & 25 & -0.15 \\
\hline & $2 \overline{81}$ & 66 & 25 & 2 & & & & 55 & 25 & $-0 . \overline{15}$ \\
\hline & 282 & 67 & 25 & 23.5 & 53 & & & 55.1 & 25 & -0.27 \\
\hline & 283 & 67 & 25 & 23.5 & 53.4 & & & 55.1 & 25 & -0.27 \\
\hline & 284 & 67 & 25 & 23.5 & 53.4 & & & 0.1 & 25 & -0.02 \\
\hline & 285 & 66 & 25 & 23.5 & & & & 55.2 & 25 & -0.02 \\
\hline & 286 & 66 & 25 & 23.5 & 53 & & & 55.2 & 25 & -0.03 \\
\hline & 287 & 66 & 25 & 23.5 & 53. & 0.54 & & 55.3 & 25 & -0.03 \\
\hline & 288 & $6 \overline{6}$ & 25 & 23.5 & 53 & 0.54 & 1355 & 55.3 & 25 & -0.18 \\
\hline & 289 & 66 & 25 & & & & & 55.3 & 25 & -0.18 \\
\hline & 290 & 66 & 25 & 23.5 & 53 & 0.5 & & 55.3 & 25 & -0.23 \\
\hline & 291 & 67 & 25 & 23.5 & 53.4 & 0.9 & & 55.3 & 25 & -0.23 \\
\hline & 292 & 67 & 25 & & 53.4 & & & 55.4 & 25 & -0.04 \\
\hline & 293 & 6 & 25 & & & & & & 25 & -0.04 \\
\hline & 294 & 67 & 25 & 23.7 & & & & 55 & 24 & \\
\hline & 295 & 67 & 25 & 23.7 & & 0. & & 55 & 25 & 0 \\
\hline & 296 & 67 & 25 & 23.7 & 53.3 & 0. & & 55.2 & 25 & -0.25 \\
\hline & 297 & 66 & 25 & 23.5 & 53.3 & & 53 & 55 & 25 & -0.25 \\
\hline & 298 & 66 & 25 & 23. & & & & & 25 & -0.21 \\
\hline & 299 & 66 & 25 & 23. & & & & 55 & 25 & -0.21 \\
\hline 23 & 300 & 67 & 25 & 23.5 & 53 & & 13 & 55.3 & 25 & -0.03 \\
\hline 22 & 301 & 67 & 25 & 23.7 & 53.3 & 0.54 & 1361 & 55.2 & 25 & -0.03 \\
\hline $9: 21$ & 302 & 67 & 25 & 23.7 & 53.3 & 0.54 & 1363 & 55.3 & 25 & -0.03 \\
\hline & 303 & 67 & 25 & 23.7 & 53.3 & & & 55.3 & 25 & -0.24 \\
\hline & 304 & 67 & 25 & 23.7 & & & & & 25 & -0.24 \\
\hline & 305 & 67 & 25 & 23.7 & & & & 55.1 & 25 & -0.19 \\
\hline & 306 & 66 & 25 & 23.7 & 53 & & & 55.1 & 25 & -0.19 \\
\hline $34: 15$ & 307 & 66 & 25 & 23.7 & 53.3 & 0.55 & 1353 & 55.1 & 25 & 0.01 \\
\hline $09: 13: 35: 14$ & 308 & 66 & 25 & 23.7 & 53.3 & 0.55 & 1358 & 55.1 & 25 & 0.01 \\
\hline $09: 13: 36: 13$ & 309 & 66 & 25 & 23.7 & 53.3 & 0.55 & 1355 & 55.1 & 25 & -0.04 \\
\hline $3: 37: 12$ & 310 & 66 & 25 & 23.7 & 53.3 & 0.55 & 1358 & 55 & $25 !$ & -0.04 \\
\hline $09: 13: 38: 11$ & 311 & 66 & 25 & 23.7 & 53.3 & 0.55 & 1362 & 55.1 & 25 & -0.28 \\
\hline
\end{tabular}




\begin{tabular}{|c|c|c|c|c|c|c|c|c|c|c|}
\hline$T \backslash M E$ & $\begin{array}{l}\text { Elapsed } \\
\text { Time } \\
\text { (sec) }\end{array}$ & BIT_TC1 & PURG_TC2 & PurgePSI & PurgeCFM & DEPTH $(\mathbb{N})_{j}$ & DwnFrc(lb) & RPM & $\begin{array}{l}\text { Torque } \\
\text { (FTLB) }\end{array}$ & Inches/Min \\
\hline $09: 13: 39: 10$ & 312 & $-\overline{67}$ & -25 & $\quad 23.7$ & $\quad 53.3$ & 0.55 & 1360 & 55.1 & 25 & $\quad-0.28$ \\
\hline $09: 13: 40: 09$ & 313 & 67 & $25^{t}$ & 23.7 & 53.3 & 0.55 & $1358^{\circ}$ & 55.2 & 25 & -0.21 \\
\hline $9: 13: 41: 08$ & 314 & 67 & 25 & 23.7 & 53.3 & 0.54 & 1355 & 55.2 & 25 & -0.21 \\
\hline $9: 13: 42: 06$ & 315 & 66 & 25 & 23.7 & 53.3 & 0.54 & 1351 & 55.2 & 25 & 0.06 \\
\hline $09: 13: 43: 05$ & 316 & 66 & 25 & 23.7 & 53.3 & 0.54 & $1351^{\circ}$ & 55.1 & 25 & 0.06 \\
\hline $09: 13: 44: 04$ & 317 & 66 & 25 & $23.9^{\prime}$ & 53.3 & 0.55 & 1350 & 55.1 & 25 & -0.12 \\
\hline 03 & 318 & 66 & 25 & 23.9 & 53.3 & 0.55 & 1352 & 55.1 & 25 & -0.12 \\
\hline $09: 13: 46: 02$ & 319 & 66 & 25 & 23.9 & 53.2 & 0.55 & 1356 & 54.9 & 25 & -0.17 \\
\hline$: 01$ & $320^{\prime}$ & 66 & 25 & $23 . \overline{7}$ & 53.2 & 0.55 & $1359^{\circ}$ & 55 & 25 & $-\overline{0.17}$ \\
\hline $13: 48: 05$ & 321 & 67 & 25 & 23.9 & 53.1 & $0.5 \overline{5}$ & 1361 & 55 & 25 & -0.16 \\
\hline 04 & 322 & 67 & 25 & 23.9 & 53.1 & 0.55 & 1359 & 54.8 & 25 & -0.16 \\
\hline & 323 & 67 & 25 & 23.9 & 53.1 & 0.55 & $1355^{*}$ & 54.8 & 25 & 0.04 \\
\hline & 324 & 66 & 25 & 23.9 & 53.1 & 0.55 & 1357 & 54.8 & 24 & $0 . \overline{04}$ \\
\hline & 325 & 66 & 25 & 23.9 & 53.1 & 0.54 & 1353 & 54.9 & 25 & -0.18 \\
\hline $3: 52: 99$ & 326 & 66 & 25 & 23.9 & 53.1 & 0.54 & $1350_{i}^{\circ}$ & 54.9 & 24 & $-0 . \overline{18}$ \\
\hline & 327 & 66 & 25 & 23.9 & 53.1 & 0.55 & $1351^{\circ}$ & 54.9 & 25 & -0.26 \\
\hline & 328 & 66 & 25 & 23.9 & 53. & 0.54 & 1355 & $5 \overline{4.9}$ & 25 & $-0 . \overline{26}$ \\
\hline & 329 & 66 & 25 & 23.9 & 53 & 0.54 & 1358 & 54.9 & 25 & 0.12 \\
\hline & 330 & 67 & 25 & $23.9^{1}$ & 53 & 0.55 & 1360 & 54.9 & 25 & 0.12 \\
\hline $7: 94$ & 331 & 67 & 25 & 23.9 & 53.1 & $0 . \overline{55}$ & $1359^{\circ}$ & 54.9 & 25 & -0.14 \\
\hline & 332 & 67 & 25 & 23.9 & 53 & 0.55 & & 54.9 & 25 & $-0.1 \overline{4}$ \\
\hline & 333 & 66 & 25 & 23.9 & 53 & 0.54 & & 54.9 & 25 & $-0.2 \overline{3}$ \\
\hline & 334 & 66 & 25 & 24.1 & 53 & 0.5 & & $54 . \overline{9}$ & 25 & -0.23 \\
\hline & $335 !$ & 66 & 25 & 24.1 & 53 & 0.54 & & 54.8 & 25 & -0.23 \\
\hline & 336 & $6 \overline{6}$ & 25 & 24.1 & 53 & 0.54 & & 54.8 & 25 & 0.01 \\
\hline & $3 \overline{37}$ & 66 & 25 & 23.9 & $53^{-1}$ & 0.55 & 13 & 54.8 & 25 & 0.01 \\
\hline & 338 & 66 & 25 & 23.9 & 53 & 0.55 & 1358 & 54.8 & 25 & $-0.0 \overline{3}$ \\
\hline & $339 !$ & 67 & 25 & 23.9 & 53 & 0.55 & & 54.8 & 25 & -0.03 \\
\hline & 340 & 67 & 25 & 24.1 & 53 & 0.54 & & 54.7 & 25 & -0.28 \\
\hline $4: \overline{07: 82}$ & 341 & 67 & $25^{\dagger}$ & $24.1^{\dagger}$ & 53 & 0.54 & & 54.7 & 25 & -0.28 \\
\hline & 342 & $\overline{67}$ & 25 & 24.1 & 53 & 0.55 & & 54.7 & 25 & -0.2 \\
\hline & 343 & 66 & 25 & $24.1^{1}$ & 53 & 0.54 & 1353 & 54.8 & 25 & -0. \\
\hline & 344 & 66 & 25 & 24.1 & 53 & 0.5 & & 54.8 & 25 & 0.1 \\
\hline 78 & 345 & 66 & 25 & 24.1 & 53 & 0.54 & 1351 & $\overline{54.7}$ & 25 & $0 . \overline{11}$ \\
\hline & 346 & 66 & 25 & & 53 & & & 54.8 & 25 & $-0 . \overline{15}$ \\
\hline & 347 & 66 & 25 & & 52.9 & & & 54.8 & 25 & -0.1 \\
\hline & 348 & 67 & 25 & $24.1^{-1}$ & $52.9^{+}$ & 0.55 & $1359^{\circ}$ & $\overline{54.9}$ & 25 & -0.2 \\
\hline & 349 & 67 & 25 & 24.1 & 52.9 & 0.54 & 1358 & 55 & 25 & $-0 . \overline{2}$ \\
\hline $4: 16: 72$ & 350 & 67 & 25 & 24.1 & 52.9 & 0.55 & 1359 & 55 & 25 & -0.15 \\
\hline $7: 71$ & 351 & 67 & 25 & 24.1 & 52.9 & 0.55 & & 55 & 25 & $-0.1 \overline{5}$ \\
\hline & 352 & 67 & 25 & & & & & 55 & 25 & 0.0 \\
\hline & 353 & & 2 & & $52.9^{-1}$ & & & 55 & 25 & 0.0 \\
\hline$\overline{68}$ & 354 & 67 & 25 & 24.1 & 52.9 & 0.55 & 1349 & 55 & 25 & $-0 . \overline{14}$ \\
\hline $21: 67$ & 355 & 67 & 25 & 24.1 & 52.9 & 0.55 & $1353^{\circ}$ & 54.9 & 25 & -0.14 \\
\hline $09: 14: 22: 65$ & 356 & 67 & 25 & 24.3 & 52.9 & 0.55 & 1357 & $5 \overline{4.9}$ & 25 & -0.27 \\
\hline $09: 1 \overline{4: 23: 64}$ & 357 & 67 & 25 & 24.1 & 52.9 & $0 . \overline{55}$ & 13 & 54.9 & 25 & $-0 . \overline{27}$ \\
\hline & 358 & 67 & 25 & 24.3 & 52.9 & 0.55 & & 54.9 & 25 & $-0 . \overline{06}$ \\
\hline $5: 62$ & 359 & 68 & 25 & 24.1 & 52.9 & 0.55 & 1358 & 54.9 & 25 & -0.06 \\
\hline & 360 & 68 & 25 & 24.3 & $52.9^{\dagger}$ & $0 . \overline{55}$ & $1358^{\circ}$ & 55 & 25 & 0 \\
\hline $14: 27: 60$ & 361 & 68 & 25 & 24.3 & 52.9 & 0.55 & $1355^{\circ}$ & 54.9 & 25 & 0 \\
\hline $09: 14: 28: 59$ & 362 & 68 & 25 & 24.3 & 52.9 & 0.55 & 1350 & 54.9 & 25 & -0.22 \\
\hline $09: 14: 29: 57$ & 363 & 67 & 25 & 24.1 & 52.9 & 0.54 & $1349^{\circ}$ & $5 \overline{5_{i}}$ & 25 & -0.22 \\
\hline
\end{tabular}




\begin{tabular}{|c|c|c|c|c|c|c|c|c|c|c|}
\hline$T I M E$ & $\begin{array}{l}\text { Elapsed } \\
\text { Time } \\
\text { (sec) }\end{array}$ & $B \mid T_{-} T C 1_{i}$ & PURG_TC2 & PurgePSI & PurgeCFMI & DEPTH(IN) & DwnFrc(ib) & RPM & $\begin{array}{l}\text { Torque } \\
\text { (FTLB) }\end{array}$ & Inches/Min \\
\hline $09: 14: 30: 56$ & $\quad 364$ & -67 & -25 & 24.1 & $\quad 52.8$ & 0.55 & $\quad 1353$ & 55.1 & $\begin{array}{l}25 \\
\end{array}$ & 5. $\quad-0.17$ \\
\hline $09: 14: 31: 55$ & 365 & 67 & $25^{\circ}$ & 24.1 & 52.8 & $0.55^{\dagger}$ & 1356 & 55.1 & 25 & -0.17 \\
\hline $09: 14: 32: 54$ & 366 & 67 & 25 & 24.1 & 52.8 & 0.55 & 1358 & 55.2 & 25 & -0.05 \\
\hline $09: 14: 33: 58$ & 367 & 67 & 25 & 24.1 & 52.8 & 0.55 & 1356 & 55.1 & 25 & -0.05 \\
\hline $09: 14: 34: 57$ & 368 & 67 & 25 & 24.1 & 52.8 & 0.55 & 1359 & 55.1 & 25 & -0.03 \\
\hline $09: 14: 35: 56$ & 369 & 68 & 25 & 24.3 & $52.8^{-}$ & 0.55 & 1360 & 55 & 25 & -0.24 \\
\hline $6: 55$ & 370 & 68 & 25 & $24.3^{2}$ & 52.8 & 0.55 & 1356 & 55 & 25 & -0.24 \\
\hline $09: 14$ & 371 & 68 & 25 & 24.3 & 52.8 & 0.55 & 1352 & 55 & 25 & -0.17 \\
\hline $09: 14: 38: 53$ & $3 \overline{72}+$ & 67 & $25^{\circ}$ & $24.3^{4}$ & 52.8 & 0.55 & 1350 & 55.1 & $25 \dagger$ & -0.97 \\
\hline $09: 14: 39: 52$ & 373 & 67 & 25 & 24.3 & 52.8 & 0.54 & 1351 & 55.1 & 25 & -0.02 \\
\hline $09: 14: 40: 50$ & 374 & $6 \overrightarrow{7_{i}}$ & 25 & 24.3 & 52.8 & 0.55 & 1350 & 55.1 & 26 & -0.02 \\
\hline$: 49$ & 375 & 67 & 25 & 24.1 & 52.8 & 0.55 & 1353 & 55.2 & 25 & -0.07 \\
\hline & $37 \overline{1}$ & 67 & 25 & $24.3^{\circ}$ & 52.8 & 0.57 & $135 \overline{7}$ & 55.1 & 25 & $-0.0 \overline{7}$ \\
\hline $3: 47$ & 377 & $67^{\dagger}$ & $25^{\circ}$ & $24.3^{4}$ & 52.8 & 0.55 & 1359 & 55.1 & 25 & -0.27 \\
\hline $09: 14: 44: 46$ & 378 & 68 & 25 & 24.3 & $52.8^{\circ}$ & 0.55 & 1360 & 55 & 25 & -0.27 \\
\hline $09: 14: 45: \overline{45}$ & 379 & 68 & 25 & 24.3 & 52.8 & 0.55 & 1358 & $5 \overline{5}$ & 25 & -0.17 \\
\hline$\overline{44}$ & 380 & 68 & 25 & $24.3^{\circ}$ & 52.7 & 0.55 & 1353 & 55 & 25 & -0.17 \\
\hline 43 & 381 & 67 & 25 & 24.3 & 52.7 & 0.55 & 1356 & 54.9 & 25 & 0.1 \\
\hline $3: 41$ & $3 \overline{82}$ & 67 & $25^{\circ}$ & $24 . \overline{3}$ & 52.7 & 0.55 & 1351 & 54.8 & 25 & 0.1 \\
\hline 40 & 383 & 67 & 25 & 24.3 & 52.7 & 0.55 & 1350 & 54.8 & 25 & $-0 . \overline{15}$ \\
\hline & 384 & 67 & 25 & $24.3^{1}$ & 52.7 & 0.55 & 1353 & 54.7 & 25 & -0.15 \\
\hline & 385 & 67 & 25 & 24.3 & 52.7 & 0.57 & 13 & 54.8 & 25 & -0.25 \\
\hline & 386 & 67 & $25^{\circ}$ & $24.3^{\circ}$ & 52.7 & $0.55^{\dagger}$ & $1360^{\circ}$ & 54.8 & 26 & -0.25 \\
\hline & $38 \overrightarrow{4}$ & 68 & $25^{\circ}$ & 24.5 & 52.7 & 0.55 & 1360 & 54.8 & 25 & -0.11 \\
\hline & $3 \overline{8} \overline{8}$ & 68 & 25 & $24.5^{\circ}$ & 52.7 & $0.55^{\circ}$ & $1358^{\circ}$ & 54.8 & 25 & -0.11 \\
\hline & 389 & 68 & 25 & $24.5^{!}$ & 52.7 & 0.55 & & $54.9^{\prime}$ & 25 & 0.03 \\
\hline & 390 & $6 \overline{8}$ & $25^{4}$ & 24.5 & 52.7 & 0.55 & 1356 & 55 & 25 & 0.03 \\
\hline & 391 & $67 !$ & $25^{\prime}$ & $24.5^{\dagger}$ & 52.7 & 0.55 & 1353 & 55 & $25^{\dagger}$ & $-0 . \overline{16}$ \\
\hline$: 36$ & 392 & 67 & 25 & 24.5 & 52.7 & 0.55 & 1351 & 55 & 25 & $-0 . \overline{16}$ \\
\hline & 393 & 67 & 25 & $24.3^{3}$ & 52.7 & $0.55^{\dagger}$ & 1353 & 54.8 & 25 & -0.21 \\
\hline & 394 & 67 & 25 & 24.3 & $52.7^{+}$ & 0.55 & 1357 & $54.8^{\dagger}$ & 25 & $-0 . \overline{21}$ \\
\hline & 395 & 67 & $25^{\circ}$ & 24.3 & $52.7^{\dagger}$ & $0.55^{t}$ & 1361 & 54.8 & 25 & -0.09 \\
\hline & 396 & 67 & 25 & $24.3^{\circ}$ & $52 . \overline{7}$ & 0.55 & $35 \overline{9}^{-1}$ & 54.8 & 25 & $-0 . \overline{09}$ \\
\hline & 397 & 68 & 25 & 24.3 & 52.7 & $0 . \overline{55}$ & 1362 & 54.8 & 25 & -0.02 \\
\hline & 398 & $6 \overline{8}$ & 25 & 24 & 52.7 & 0.55 & & 54.8 & 25 & -0.26 \\
\hline & 399 & $6 \overline{7}$ & 25 & 24.7 & 52.7 & 0.55 & & 54.8 & 25 & -0.26 \\
\hline$: 26$ & $\overline{400}$ & 67 & 25 & 24.7 & 52.7 & 0.55 & 1353 & 54.8 & $25^{\dagger}$ & $-0.1 \overline{5}$ \\
\hline $7: 25$ & 401 & 67 & 25 & 24.7 & 52.7 & 0.55 & 1351 & 54.8 & 25 & -0.15 \\
\hline $8: 24$ & 402 & 67 & $25^{\prime}$ & $24.7^{\circ}$ & 52.7 & 0.55 & & 54.9 & 25 & \\
\hline & $403 i$ & 67 & 25 & 24.7 & 52.7 & 0.5 & & 54.9 & 25 & \\
\hline & $404^{+}$ & 67 & 25 & 24.7 & 52.7 & 0.57 & & 55 & $25^{\top}$ & -0.09 \\
\hline & 405 & 68 & $26^{\circ}$ & 24.7 & 52.7 & 0.57 & 1359 & $5 \overline{5}$ & 25 & -0.09 \\
\hline $12: 20$ & 406 & 68 & 26 & 24.7 & $52 . \overline{7_{i}}$ & 0.55 & 1362 & 55 & 25 & -0.24 \\
\hline $09: 15: 13: \overline{19}$ & 407 & 68 & $25 !$ & 24.7 & 52.7 & 0.55 & 1362 & 54.9 & 25 & -0.24 \\
\hline $09: 15: 14: 17$ & 408 & 68 & 25 & $24.7^{\circ}$ & 52.7 & 0.55 & 1360 & 55 & 25 & -0.16 \\
\hline $15: 22$ & 409 & $6 \overrightarrow{8}$ & $25^{\prime}$ & 24.7 & $52 . \overline{7}$ & 0.55 & 1355 & 55 & 25 & -0.16 \\
\hline $6: 21$ & 410 & 68 & 25 & 24.7 & 52.7 & 0.55 & 1358 & 55 & 25 & 0.07 \\
\hline $17: 20$ & 411 & 68 & 26 & $24.7 i^{\circ}$ & 52.7 & 0.55 & 1353 & 54.9 & 25 & 0.07 \\
\hline $09: 15: 18: 18$ & 412 & 67 & $25^{\dagger}$ & 24.5 & $52 . \overline{6}$ & 0.55 & 1352 & 54.9 & 25 & -0.15 \\
\hline $09: 15: 19: 17$ & 413 & 67 & 25 & 24.7 & 52.6 & $0 . \overline{55}$ & 1355 & 54.9 & 25 & -0.15 \\
\hline $09: 15: 20: \overline{16}$ & 414 & 67 & 25 & 24.7 & $52.6^{\dagger}$ & 0.55 & 1360 & 54.8 & 25 & -0.27 \\
\hline $09: 15: 21: 15$ & $\overline{415}$ & 68 & 25 & 24.7 । & 52.6 & 0.55 & 1362 & 54.8 & 25 & -0.27 \\
\hline
\end{tabular}




\begin{tabular}{|c|c|c|c|c|c|c|c|c|c|c|}
\hline$T I M E$ & $\begin{array}{l}\text { Elapsed } \\
\text { Time } \\
\text { (sec) }\end{array}$ & BIT_TC1 & & PurgePS! & PurgeCFMD & DEPTH $(\mathrm{N})$ & DwnFrc(lb) & RPM & $\begin{array}{l}\text { Torque } \\
\text { (FTLB) }\end{array}$ & Inches/Min \\
\hline $09: 15: 22: 14$ & 416 & 68 & $\quad 26$ & 24.7 & $\begin{array}{l}52.6 \\
\end{array}$ & 0.55 & 1363 & 54.8 & $\quad 25$ & $\quad-0.07$ \\
\hline $09: 15: 23: 13$ & 417 । & 68 & 25 & 24.7 & 52.6 & $0.55^{\circ}$ & 1361. & 54.8 & 25 & -0.07 \\
\hline $4: 12$ & 418 & 68 & 25 & 24.9 & 52.6 & 0.55 & 1356 & 54.8 & 25 & 0.0 \\
\hline & $419^{\circ}$ & 68 & 26 & 24.9 & 52.6 & 0.55 & 1359 & 54.8 & 25 & 0.01 \\
\hline 09 & 420 & 67 & 26 & 24.9 & 52.6 & 0.55 & 1354 & 54.7 & 25 & -0.21 \\
\hline $15: 27: 08$ & 421 & 67 & 26 & 24.9 & 52.6 & 0.55 & 1353 & 54.7 & 25 & $-0 . \overline{21}$ \\
\hline $3: 07$ & 422 & 67 & 26 & 24.9 & 52.6 & 0.55 & 1356 & 54.6 & 25 & -0.21 \\
\hline 06 & 423 & 67 & 26 & $24.9^{!}$ & 52.6 & 0.57 & 1361 & 54.7 & 26 & -0.2 \\
\hline & 424 & 67 & 26 & 24.9 & 52.6 & 0.57 & 1363 & $54.7^{\circ}$ & 25 & -0.01 \\
\hline & 425 & 67 & 25 & 24.9 & 52.6 & 0.55 & 1363 & $54.7 !$ & 25 & -0.01 \\
\hline & 426 & 67 & 25 & 18.9 & 7.3 & 0.55 & 1362 & 54.7 & 25 & -0.04 \\
\hline & 427 & 69 & 25 & -0.6 & 1.8 & 0.55 & $1363^{\circ}$ & 54.7 & 26 & -0.04 \\
\hline & 428 & 72 & 25 & -0.6 & 1.8 & 0.55 & & 54.6 & 26 & -0.2 \\
\hline & $429^{+}$ & 75 & 25 & -0.6 & 1.8 & 0.55 & 1355 & 54.6 & 27 & $-\overline{0.2}$ \\
\hline & 430 & 77 & 25 & -0.6 & 1.8 & 0.55 & 1354 & 54.6 & 27 & -0.2 \\
\hline & 431 & 79 & 25 & -0.6 & 1.8 & 0.57 & 1358 & 54.5 & 28 & 0.1 \\
\hline & 432 & 82 & 25 & -0.6 & 1.8 & 0.57 & 1364 & 54.5 & 28 & 0. \\
\hline & 433 & 84 & 25 & -0.6 & $1.8^{1}$ & 0.57 & 6 & 54.5 & 28 & -0.19 \\
\hline & $4 \overline{34}$ & 87 & 25 & $-0.6^{\prime}$ & 1.8 & 0. & 1366 & 54.5 & 29 & $-0.1 \mathrm{~s}$ \\
\hline & 435 & 89 & 25 & -0.6 & 1.8 & 0.55 & 1367 & 54.6 & 30 & -0.28 \\
\hline & 436 & 90 & 25 & $-0.6 !$ & 1.8 & 0.54 & 1366 & 54.6 & 30 & -0.28 \\
\hline & $437^{\dagger}$ & 91 . & 25 & -0.6 & 1.8 & & & 54.6 & 30 & -0.0 \\
\hline & 438 & 92 & 25 & -0.6 & 1.8 & & & 54.6 & & -0.0 \\
\hline & 439 & 94 & 25 & -0.6 & 1.8 & & $1360 !$ & 54.6 & 31 & -0.04 \\
\hline & $440 !$ & 96 & 25 & -0.6 & $1.8 !$ & 0.57 & 1364 & 54.6 & 31 & -0.04 \\
\hline & 441 & 98 & 25 & -0.6 & 1.8 & & $1368^{\prime}$ & 54.6 & 31 & -0.2 \\
\hline & 442 & 100 & 26 & -0.6 & 1.81 & & & 54.6 & 32 & -0.2 \\
\hline & 443 & 102 & 25 & -0.6 & 1.8 & & 369 & 54.5 & 31 & -0.1 \\
\hline & 444 & 103 & 25 & -0.6 & 1.8 & & 1371 & 54.5 & 31 & -0.18 \\
\hline & 445 & 104 & 25 & $-0.6 !$ & 1.8 & 0.55 & & 54.4 & 32 & 0.05 \\
\hline & 446 & 106 & 25 & -0.6 & $1.8^{\dagger}$ & & & & 32 & 0.0 \\
\hline & 447 & 106 & 26 & $-0.6 !$ & $1.8 !$ & & & 54.3 & 32 & -0.1 \\
\hline & 448 & 108 & 26 & -0.6 & 1.8 & & & 54.3 & 32 & -0.1 \\
\hline & 449 & 110 & 25 & -0.6 & $1.8 !$ & 0.57 & 1369 & 54.3 & 33 & -0.26 \\
\hline & 450 & 112 & 26 & -0.6 & 1.8 & & 72 & 54.3 & 33 & -0.26 \\
\hline & 451 & 113 & 26 & -0.6 & 1.8 & & & & 33 & -0 \\
\hline & 452 & 11 & 25 & -0.6 & 1.8 & & 1373 & 54.5 & 33 & -0 \\
\hline & 453 & 116 & 25 & -0.6 & 1.8 & 0.5 & 1373 & 54.5 & 33 & -0.01 \\
\hline & 454 & 11 & 26 & -0.6 ! & 1.8 & 0.5 & 1369 & 54.5 & 33 & -0.01 \\
\hline & 455 & 119 & 26 & -0.6 & 1.8 & 0.55 & & 54.6 & 33 & -0.25 \\
\hline & 456 & 120 & $26^{\dagger}$ & -0.6 & 1.8 & & & 54.6 & 33 & -0.2 \\
\hline & 45 & 12 & 26 & -0.6 & 1.8 & & 1368 & 54.5 & 34 & -0.2 \\
\hline & 458 & 12 & 26 & -0.6 & 1.8 & & 1367 & 54.4 & 34 & -0.2 \\
\hline 71 & 459 & 124 & 26 & -0.6 & 1.8 & 0.5 & 1372 & 54.4 ! & 34 & 0.12 \\
\hline & 460 & 126 & 25 & $-0.6 !$ & 1.8 & 0.5 & 1375 & 54.3 & 34 & 0.12 \\
\hline $06: 68$ & 461 & 127 & 26 & -0.6 & 1.8 & 0.57 & 1376 & 54.2 & 34 & -0.16 \\
\hline & 462 & 129 & 26 & -0.6 & 1.8 & & 1375 & 54.2 & 34 & -0.2 \\
\hline & 463 & 130 & 26 & -0.6 & 1.8 & & & 54.4 & 34 & -0.2 \\
\hline & 464 & 131 & 26 & -0.6 & $1.8^{+}$ & & & 54.7 & 34 & -0.1 \\
\hline & 465 & 132 & 26 & -0.6 & 1.8 & 0.55 & 1367 & 54.7 & 34 & -0.16 \\
\hline $09: 16: 11: 63$ & 466 & 133 & 26 & -0.6 & 1.8 & 0.55 & 1372 & 54.8 & 35 & 0.01 \\
\hline $09: 16: 12: 61$ & 467 & 134 & 26 & -0.6 & $1.8^{\prime}$ & 0.57 & 1369 & 54.9 & 35 & 0.01 \\
\hline
\end{tabular}




\begin{tabular}{|c|c|c|c|c|c|c|c|c|c|c|}
\hline$T / M E$ & $\begin{array}{l}\text { Elapsed } \\
\text { Time } \\
\text { ¡(sec) }\end{array}$ & BIT TC1 & PURG TC2 & & PurgeCFM & DEPTH $(|N\rangle$ & DwnFrc(lb) & RPM & $\begin{array}{l}\text { Torque } \\
\text { (FTLB) }\end{array}$ & Inches/Min \\
\hline $09: 16: 13: 60$ & 468 & -136 & $\begin{array}{r}-26 \\
\end{array}$ & $-0.6^{\dagger}$ & 1.8 & $\quad 0.55$ & 1373 & 54.9 & $\quad 35$ & $\quad-0.17$ \\
\hline $09: 16: 14: 59$ & 469 & 138 & 26 & -0.6 & 1.8 & 0.57 & 1377 & 54.8 & 35 & -0.17 \\
\hline 58 & $4 \overline{70}$ & 139 & 26 & -0.6 & 1.8 & 0.57 & 1378 & $54.7^{\dagger}$ & 35 & $-0 . \overline{24}$ \\
\hline $5: 57$ & 47 & 141 & 26 & -0.6 & 1.8 & 0.57 & 1377 & 54.7 & 36 & -0.24 \\
\hline $09: 16$ & 472 & 142 & 26 & -0.6 & 1.8 & 0.55 & 1374 & 54.8 & 36 & -0.07 \\
\hline $09: 1$ & 473 & 143 & 26 & -0.6 & 1.8 & 0.55 & 1370 & 54.7 & 36 & $-0.0 \overline{7}$ \\
\hline 54 & 47 & 144 & 26 & -0.6 & 18 & $0.55^{!}$ & 1372 & 54.7 & 36 & -0.04 \\
\hline 52 & 475 & 145 & 26 & $-0.6]$ & 1.8 & 0.55 & 1371 & $54 \overline{7}$ & 36 & -0.04 \\
\hline & 476 & 146 & 26 & -0.6 & 1.8 & 0.57 & 1373 & 54.7 & 36 & -0.25 \\
\hline 50 & 477 & 147 & 26 & -0.6 & 1.8 & 0.57 & 1378 & 54.7 & 36 & -0.25 \\
\hline 09:1 & 478 & 149 & 26 & $-0 . \overline{6}$ & 1.8 & 0.57 & 1380 & 54.7 & 36 & $-0 . \overline{18}$ \\
\hline 48 & 475 & 151 & 26 & -0.6 & 1.8 & 0.57 & 1382 & 54.6 & 36 & -0.18 \\
\hline 47 & 480 & 151 & 26 & -0.6 & 1.8 & 0.55 & 1380 & 54.6 & 36 & -0.01 \\
\hline & 481 & $15 \overline{3}$ & 26 & -0.6 & 1.8 & 0.55 & 1375 & 54.6 & 36 & -0.01 \\
\hline$: 44$ & 482 & 154 & 26 & -0.6 & 1.8 & $0.55 !$ & 1372 & 54.6 & 36 & -0.11 \\
\hline & 483 & 155 & 26 & -0.6 & 1.8 & 0.55 & 73 & 54.6 & 37 & -0.11 \\
\hline & 484 & 155 & 26 & -0.6 & 1.8 & 0.57 & 1378 & 54.6 & 37 & -0.22 \\
\hline & 485 & 157 & 26 & -0.6 & 1.8 & 0.57 & 1375 & 54.4 & 37 & -0.22 \\
\hline & 486 & 159 & 26 & -0.6 & 1.8 & 0.55 & 1380 & 54.4 & 37 & -0.17 \\
\hline & 487 & 160 & 26 & -0.6 & 1.8 & 0.57 & 83 & 54.3 & 37 & -0.17 \\
\hline & 488 & 162 & 26 & 6 & 1.8 & 0.55 & & 54.2 & 37 & 0.02 \\
\hline & $48 \bar{S}$ & 163 & 26 & $-0.6 !$ & 1.8 & 0.55 & 1380 & 54.2 & 37 & 0.02 \\
\hline & 490 & 16 & 26 & -0.6 & 1.8 & 0.55 & 75 & 54.5 & 37 & -0.18 \\
\hline & 49 & 16 & 26 & $-0.6 !$ & 1.8 & 0.55 & & 54.5 & 37 & -0.18 \\
\hline & 492 & 165 & 26 & -0.6 & 1.8 & 0.55 & & 54.5 & 37 & -0.27 \\
\hline & 493 & 167 & 26 & -0.6 & 1.8 & 0.57 & & 54.6 & 38 & -0.27 \\
\hline & 494 & 168 & 26 & -0.6 & 1.8 & 0.57 & & 54.6 & 38 & -0.02 \\
\hline & 495 & 170 & 26 & -0.6 & 1.8 & 0.57 & 1383 & 54.6 & 37 & -0.04 \\
\hline & 496 & 171 & 26 & -0.6 & 1.8 & 0.57 & 1385 & 54.6 & 37 & -0.04 \\
\hline & 497 & 173 & 26 & & 1.8 & 0.55 & & $5 \overline{4.6}$ & 37 & -0.26 \\
\hline & 498 & 173 & 26 & -0.6 & 1.8 & 0.57 & 1380 & 54.6 & 37 & -0.26 \\
\hline & 499 & 174 & 26 & -0.6 & 1.8 & 0.57 & & 54.5 & 38 & -0.15 \\
\hline & 500 & 17 & 26 & -0.6 & 1.8 & 0.57 & 1376 & 54.5 & 38 & -0.15 \\
\hline & 501 & 175 & 26 & -0.6 & 1.8 & 0.55 & & 54.3 & 38 & \\
\hline & 50 & & 26 & $-0.6 !$ & 1. & 55 & & 54.1 & 38 & \\
\hline & 503 & $17 \overline{8}$ & 26 & -0.6 & 1.8 & 0.57 & 1381 & 54.1 & 38 & -0.14 \\
\hline & 504 & 180 & 25 & -0.6 & 1.8 & 0.57 & $13 \overline{8}$ & 54.1 & 38 & -0.14 \\
\hline 09: & 505 & 182 & 26 & -0.6 & 1.8 & 0.55 & 1384 & 54.2 & 38 & -0.27 \\
\hline & 506 & 183 & $26^{\dagger}$ & $-0.6^{-1}$ & 1.8 & 0.57 & & 54.2 & 38 & $-0 . \overline{27}$ \\
\hline & 50 & 18 & 26 & -0 & 1. & & & 54.3 & 38 & -0.02 \\
\hline & 508 & & 26 & $-0.6 !$ & 1. & & 1380 & 54.3 & 39 & -0.02 \\
\hline & 509 & 185 & 26 & -0.6 & 1.8 & 0.57 & 1375 & 54.3 & 39 & -0.03 \\
\hline 18 & 510 & 6 & 26 & -0.6 & 1.8 & 0.57 & 1375 & 54.4 & 39 & -0.03 \\
\hline $6: 17$ & 511 & 187 & 26 & -0.6 & 1.8 & 0.57 & 1379 & 54.4 & 39 & -0.29 \\
\hline & 512 & 188 & 26 & -0.6 & 1.8 & 0.57 & & $54 . \overline{4}$ & 39 & -0.29 \\
\hline & 51 & 190 & 26 & -0.6 & 1.8 & & & 54 & 39 & -0.16 \\
\hline & 51 & 19 & 26 & -0.6 & 1.8 & & & 54.5 & 39 & -0.16 \\
\hline & & 19 & 26 & -0.6 & 1.8 & 0.55 & 1385 & 54.5 & 39 & 0.05 \\
\hline $7: 01: 11$ & 516 & 194 & 26 & -0.6 & 1.8 & $0.55 !$ & 1383 & 54.5 & 40 & 0.05 \\
\hline $09: 17: 02: 10$ & 517 & 194 & 26 & -0.6 & 1.8 & 0.55 & 1378 & 54.5 & 40 & -0.18 \\
\hline $09: 17: 03: 09$ & 518 & 195 & 26 & -0.6 & 1.8 & 0.55 & 1375 & 54.5 & 39 & -0.18 \\
\hline 09:17:04:08 & 519 & 195 & 26. & -0.6 & 1.8 & 0.57 & 1377 & 54.5 & 40 & -0.28 \\
\hline
\end{tabular}




\begin{tabular}{|c|c|c|c|c|c|c|c|c|c|c|}
\hline TIME & $\begin{array}{l}\text { Elapsed } \\
\text { Time } \\
\text { (sec) }\end{array}$ & $B I T$ TC1 & PURG_TC2 & PurgePSI & PurgeCFMD & EPTH $(I N)$ & DwnFrc(b) & RPM & $\begin{array}{l}\text { Torque } \\
\text { (FTLB) }\end{array}$ & Inches/Min \\
\hline $09: 17: 05: 07$ & 520 & -197 & 26 & -0.6 & 1.8 & 0.57 & 1382 & 54.3 & 40 & $\quad-0.28$ \\
\hline$: 17: 06: 0 \overline{6}$ & 521 & 198 & 26 & -0.6 & 1.8 & 0.57 & 1385 & 54.3 & 40 & -0.06 \\
\hline $9: 17: 07: 10$ & 522 & 199 & 26 & -0.6 & 1.8 & $0.5 \overline{7}$ & 1387 & 54.3 & 40 & -0.06 \\
\hline $9: 17: 08: 09$ & 523 & 202 & 26 & -0.6 & 1.8 & 0.57 & 1386 & 54.3 & 40 & \\
\hline $9: 08$ & 524 & 202 & 26 & -0.6 & 1.8 & 0.57 & 1386 & 54.3 & 40 & $-0.2 \overline{6}$ \\
\hline $0: 07$ & 525 & 204 & 26 & -0.6 & 1.8 & 0.57 & 1382 & 54.4 & 40 & $-0 . \overline{26}$ \\
\hline & 526 & $20 \overline{3}$ & 26 & -0.6 & 1.8 & $0.55^{\circ}$ & $1377^{\dagger}$ & 54.6 & 41 & -0.2 \\
\hline 04 & 527 & 204 & 26 & -0.6 & $1 . \overline{8}$ & 0.55 & 1377 & 54.6 & 41 & -0.2 \\
\hline $3: 03$ & 528 & 204 & 26 & -0.6 & 1.8 & 0.57 & 1379 & 54.7 & 49 & 0.06 \\
\hline 02 & 529 & 205 & 26 & -0.6 & 1.8 & 0.57 & 1383 & 54.8 & 41 & 0.06 \\
\hline & 530 & 206 & 26 & -0.6 & 1.8 & 0.57 & 1381 & 54.8 & 41 & -0.13 \\
\hline & 531 & 208 & 26 & -0.6 & 1.8 & $0.5 \overline{8}$ & 1385 & 54.8 & 41 & -0.13 \\
\hline & 532 & 210 & 26 & -0.6 & 1.8 & 0.55 & 1387 & 54.8 & 41 & -0.25 \\
\hline $17: 98$ & 533 & 211 & 26 & -0.6 & 1.8 & 0.55 & 1386 & 54.8 & 41 & -0.25 \\
\hline 96 & 534 & 212 & $26^{+}$ & -0.6 & 1.8 & 0.57 & $1383^{4}$ & 54.7 & 41 & -0.14 \\
\hline & 535 & 212 & 26 & -0.6 & 1.8 & $0 . \overline{55}$ & 1378 & 54.6 & 41 & -0.14 \\
\hline & 536 & 213 & 26 & $-0 . \overline{6}$ & 1.8 & $0 . \overline{55}$ & 1377 & 54.6 & 41 & -0.02 \\
\hline & 537 & 214 & 26 & -0.6 & 1.8 & $0 . \overline{57}$ & 1381 & 54.7 & 41 & .0 .02 \\
\hline 92 & 538 & 215 & 26 & -0.6 & 1.8 & 0.57 & 1378 & 54.7 & 41 & -0.18 \\
\hline & 539 & 216 & 26 & -0.6 & 1.8 & $0 . \overline{57}$ & 1382 & 54.7 & 42 & -0.18 \\
\hline & 540 & 217 & 26 & -0.6 & 1 & & 88 & 54.7 & 42 & -0.27 \\
\hline & 541 & 221 & 26 & $-0 . \bar{\epsilon}$ & 1. & & 1390 & 54.7 & 42 & -0.27 \\
\hline & 542 & 222 & 26 & -0.6 & 1.8 & 0.55 & 1389 ] & 54.7 & 42 & -0.03 \\
\hline & $5 \overline{43}$ & 223 & $2 \ddot{6}^{\dagger}$ & -0.6 & 1.8 & $0.5 \overline{7}$ & 1384 & 54.7 & $4 \overline{2}$ & -0.03 \\
\hline & $5 \overline{44}$ & 223 & 26 & -0 & 1.8 & 0.55 & 1378 & 54.7 & 42 & $-0 . \overline{03}$ \\
\hline & 545 & 224 & 26 & -0.6 & 1. & 0.5 & 1382 & 54.7 & $4 \overline{3}$ & $-0 . \overline{03}$ \\
\hline & 546 & 225 & 26 & -0.6 & 1.8 & 0.57 & 1378 & 54.8 & 43 & -0.23 \\
\hline & 547 & 225 & 26 & -0.6 & 1.8 & $0.5 \overline{7}$ & 1378 & 54.9 & 43 & -0.23 \\
\hline & 548 & 226 & 26 & -0.6 & 1.8 & & 1382 & 54.9 & 43 & -0.18 \\
\hline & 549 & 227 & 26 & -0. & & 0.57 & 1386 & 54.9 & 43 & -0.18 \\
\hline & 550 & 231 & 26 & -0.6 & 1. & 0.57 & 1387 & 54.9 & 43 & 0.03 \\
\hline & 551 & 232 & 26 & $-0 . \overline{6}$ & 1 & & 87 & 54 & 43 & 0.03 \\
\hline & 552 & 233 & 26 & -0.6 & 1. & 0.5 & & 54.8 & $4 \overline{3}$ & -0.12 \\
\hline & 553 & & 26 & -0. & & & & & 43 & -0.12 \\
\hline & 554 & 233 & 26 & -0.6 & 1. & 0.5 & 1382 & 54.8 & 43. & -0.22 \\
\hline & 555 & 234 & 26 & -0.6 & 1.8 & 0.55 & $1377^{1}$ & 54.8 & 44 & -0.15 \\
\hline$: 77$ & 556 & 233 & 26 & -0.6 & 1.8 & 0.57 & 1378 & 54.8 & 44 & -0.15 \\
\hline 76 & 557 & $23 \overline{5}$ & 26 & -0.6 & 1.8 & 0.57 & 1382 & 54.8 & 44 & 0.01 \\
\hline & 558 & 236 & 26 & $-0 . \overline{6}$ & 1.8 & 0.57 & 1385 & 54.9 & 44 & 0.01 \\
\hline & 559 & 240 & 26 & -0.6 & 1.8 & 0.57 & & 54.9 & $44 !$ & -0.16 \\
\hline & 560 & 241 & 26 & -0.6 & $1 . \overline{8}$ & & 1386 & 55 & 45 & -0.16 \\
\hline & 561 & 243 & 26 & -0.6 & 1.8 & 0.5 & 1383 & 54.9 & 44 & -0.23 \\
\hline $6: 70$ & 562 & 243 & 26 & -0.6 & 1.8 & 0.55 & $1385^{\circ}$ & 54.9 & 45 & -0.23 \\
\hline $17: 47: 69$ & $563^{\dagger}$ & 244 & 26 & -0.6 & 1.8 & 0.55 & 1381 & 54.9 & $\overline{45}$ & -0.05 \\
\hline & 564 & $24 \overline{5}$ & 26 & -0.6 & 1.8 & & 1377 & 55 & 45 & -0.05 \\
\hline & 565 & 243 & 26 & -0.6 & 1.8 & 0.5 & $1375 !$ & 55 & 45 & -0.03 \\
\hline & 566 & 244 & 26 & -0.6 & 1.8 & 0.57 & 1379 & 54.9 & 45 & -0.03 \\
\hline & 567 & 245 & 26 & -0.6 & 1.8 & 0.57 & 1383 & 54.6 & 46 & $-0 . \overline{23}$ \\
\hline $09: 17: 52: 63$ & 568 & 249 & 26 & -0.6 & 1.8 & 0.57 & 1385 & 54.6 & 45 & -0.23 \\
\hline $09: 17: 53: 62$ & 569 & 250 & 26 & -0.6 & 1.8 & 0.55 & 1384 & 54.5 & 45 & -0.19 \\
\hline $09: 17: 54: 61$ & 570 & 251 & 26 & -0.6 & 1.8 & 0.55 & 1385 & 54.5 & 45 & -0.19 \\
\hline $09: 17: 55: 60$ & 571 & 251 & 26 & -0.6 & 1.8 & 0.55 & 1383 & 54.5 & 45 & -0.01 \\
\hline
\end{tabular}




\begin{tabular}{|c|c|c|c|c|c|c|c|c|c|c|}
\hline$T I M E$ & $\begin{array}{l}\text { Elapsed } \\
\text { Time } \\
\text { (sec) }\end{array}$ & $\mathrm{BIT}$ TC1 & |PURG_TC2 & PurgePSI & PurgeCFM & DEPTH $(\mathrm{IN})$ & DwnFrc(ib) & RPM & $\begin{array}{l}\text { Torque } \\
\lfloor\text { (FTLB) }\end{array}$ & Inches/Min \\
\hline $09: 17: 5 \overline{6}: 59$ & 572 & 252 & 26 & $\quad-0.6$ & 1.8 & $\quad 0.55$ & 1378 & 54.7 & $\quad 46$ & $\quad-0.01$ \\
\hline $09: 17: 57: 58$ & 573 & 253 & 26 & -0.6 & 1.8 & 0.55 & 1374 & 55 & 46 & -0.09 \\
\hline $09: 17:$ & $5 \overline{7} 4$ & 251 & 26 & -0.6 & 1.8 & 0.57 & 1374 & 55 & 46 & -0.09 \\
\hline $09: 17:$ & 575 & 252 & 26 & -0.6 & 1.8 & 0.55 & 1377 & $55 !$ & 47 & -0.21 \\
\hline $09: 1$ & 576 & 253 & 26 & -0.6 & 1.8 & 0.57 & 1381 & 54.9 & 47 & -0.21 \\
\hline $09: 18$ & 577 & 257 & 26 & -0.6 & 1.8 & 0.55 & 1383 & 54.9 & 46 & -0.2 \\
\hline $09: 1$ & 578 & 257 & 26 & -0.6 & $1.8 !$ & 0.55 & 1382 & 54.8 & 46 & -0.2 \\
\hline $09: 18$ & 579 & 259 & 26 & -0.6 & 1.8 & 0.57 & 1383 & 54.6 & 46 & 0.08 \\
\hline $09: 1$ & 580 & $260 !$ & 26 & -0.6 & 1.8 & 0.55 & 1380 & 54.6 & 46 & 0.08 \\
\hline $09: 1$ & 581 & 260 & 26 & $-0.6 !$ & 1.8 & 0.55 & 1375 & 54.6 & 46 & -0.17 \\
\hline 48 & 582 & 262 & 26 & -0.6 ! & 1.8 & 0.55 & 1372 & $54.6 !$ & 46 & -0.17 \\
\hline 46 & 583 & 260 & 26 & -0.6 & 1.8 & 0.55 & 1373 & 54.6 & 46 & -0.27 \\
\hline$: 45$ & 584 & 261 & 26 & -0.6 & 1.8 & 0.55 & $1376^{\prime}$ & 54.7 & 46 & -0.27 \\
\hline 44 & 585 & 262 & 26 & -0.6 & $1.8^{\dagger}$ & 0.57 & 1379 & 54.7 & 46 & -0.04 \\
\hline & 586 & 265 & 26 & -0.6 & 1.8 & 0.57 & 1381 & 54.7 & 46 & -0.04 \\
\hline & 587 & 267 & 26 & $-0.6 !$ & 1.8 & 0.55 & 138 & 54.7 & 46 & -0.04 \\
\hline 09:1 & 588 & 268 & 26 & -0.6 & 1.8 & 0.57 & 1380 & 54.6 & 46 & -0.04 \\
\hline 45 & 589 & 268 & 26 & -0.6 & 1.8 & 0.55 & 1378 & 54.6 & 46 & -0.22 \\
\hline$: 44$ & 590 & 269 & 26 & -0.6 & 1.8 & 0.55 & 1373 & 54.6 & 46 & -0.21 \\
\hline & 591 & 269 & 26 & -0.6 & 1.8 & 0.57 & 1370 & 54.7 & 46 & -0.21 \\
\hline 42 & 592 & 268 & 26 & -0.6 & 1.8 & 0.55 & 1371 & $54 . \overline{7}$ & 47 & 0 \\
\hline$: 41$ & 593 & 268 & 26 & -0.6 & 1.8 & 0.57 & 1369 & 54.7 & 47 & \\
\hline & 594 & 269 & 26 & -0.6 & 1.8 & 0.57 & 1372 & 54.8 & 47 & $-0 . \overline{07}$ \\
\hline & 595 & 273 & 26 & -0.6 & $1.8 !$ & 0.55 & 1375 & 54.8 & 47 & -0.07 \\
\hline & 596 & $274^{!}$ & 26 & -0.6 & 1.8 & 0.57 & 1378 & 54.7 & 47 & $-0 . \overline{24}$ \\
\hline $09: 1$ & 597 & 276 & 26 & -0.6 & $1.8^{t^{-}}$ & 0.57 & & 54.5 & $4 \overline{7}$ & -0.24 \\
\hline & 598 & 276 & 26 & $-0.6 !$ & 1.8 & 0.55 & 1375 & 54.5 & 47 & $0 . \overline{14}$ \\
\hline & 599 & 277 & 26 & -0.6 & 1.8 & 5 & 1371 & 54.4 & 47 & -0.14 \\
\hline & 600 & 277 & 26 & -0.6 & 1.8 & 0.55 & 368 & 54.4 & 47 & $-0 . \overline{01}$ \\
\hline & 601 & 276 & 26 & -0.6 & $1.8 \mathrm{i}$ & 0.55 & & 54.5 & 47 & -0.01 \\
\hline & 602 & $276 !$ & 26 & -0.6 & 1.8 & 0.57 & & 54.4 & 48 & $-0.1 \overline{6}$ \\
\hline & 603 & 277 & 26 & -0.6 & 1.8 & 0.55 & 1373 & 54.4 & 48 & -0.16 \\
\hline & 604 & 280 & 26 & -0.6 & 1.8 & 0.57 & & 54.2 & 48 & -0.26 \\
\hline & 605 & 280 & 26 & -0.6 & 1.8 & & & 54.1 & 47 & -0.26 \\
\hline & 606 & 282 & 26 & -0.6 & 1.8 & 0.57 & 1376 & 54.1 & 47 & \\
\hline & 607 & 281 & 26 & $-0.6 i$ & 1.8 & 0.57 & 13 & 54.2 & 48 & \\
\hline 24 & $60 \overline{8}$ & $282 !$ & 26 & -0.6 & 1.8 & 0.57 & 1368 & 54.2 & 48 & -0.09 \\
\hline & 609 & 283 & 26 & -0.6 & 1.8 & 0.55 & & 54.2 & 48 & -0.09 \\
\hline & 610 & 28 & 26 & -0.6 & 1. & & & 54.2 & 49 & -0.23 \\
\hline & 611 & 284 & 26 & -0.6 & 12.3 & 0.55 & 1370 & 54.1 & 49 & -0.23 \\
\hline & 612 & 280 & 26 & 1.1 & 1.9 & 0.58 & 1375 & 54.1 & 47 & -0.06 \\
\hline & 613 & 282 & 26 & -0.6 & 1.9 & 0.57 & 1376 & 54 & 46 & -0.06 \\
\hline & 614 & $28 \overline{3}$ & 26 & -0.6 & 1.9 & 0.57 & 1375 & 54.2 & 46 & $-0 . \overline{07}$ \\
\hline & 615 & 284 & 26 & -0.6 & 1.9 & 0.55 & 1372 & 54.2 & 46 & -0.07 \\
\hline $0: 14$ & 616 & 283 & 26 & -0.6 & 1.9 & 0.55 & & 54.3 & 46 & -0.18 \\
\hline $1: 13$ & 617 & 284 & 26 & -0.6 & 1.9 & 0.5 & 1365 & 54.3 & 46 & $-0 . \overline{18}$ \\
\hline $8: 42: 12$ & 618 & 284 & 26 & -0.6 & 1.8 & 0.55 & 1368 & 54.3 & 46 & -0.26 \\
\hline $3: 11$ & 619 & 284 & 26 & -0.6 & 1.9 & 0.55 & 1365 & 54.3 & 46 & -0.26 \\
\hline & 620 & 285 & 26 & $1.5^{+}$ & 31.8 & 0.57 & 1369 & 54.3 & 46 & 0.04 \\
\hline & 621 & & 26 & & $48 !$ & & & 54.3 & 44 & -0.1 \\
\hline $09: 18: 46: 08$ & 622 & 269 & 26 & 23.5 & 51.1 & 0.57 & 13 & 54.4 & 43 & -0.1 \\
\hline $09: 18: 47: 07$ & 623 & 253 & 26 & 26.8 & $51.1_{1}$ & 0.57 & 1368 & 54.4 & 42 & -0.24 \\
\hline
\end{tabular}




\begin{tabular}{|c|c|c|c|c|c|c|c|c|c|c|}
\hline$T I M E$ & $\begin{array}{l}\text { Elapsed } \\
\text { Time } \\
\text { (sec) }\end{array}$ & $B I T+T C 1$ & PURG_TC2 & PurgePSI & PurgeCFM & DEPTH(IN) & DwnFrc(ib) & RPM & $\begin{array}{l}\text { Torque } \\
\text { (FTLB) }\end{array}$ & Inches/Min \\
\hline $09: 18: 48: 05$ & 624 & 240 & $\quad 26$ & 27.4 & 51.1 & 0.57 & 1360 & $\quad 54.4$ & $4 \quad 40$ & $\quad-0.24$ \\
\hline $09: 18: 49: 04$ & 625 & 231 & 26 & 27.4 & 51.1 & 0.57 & 1354 & 54.5 & 38 & -0.12 \\
\hline $09: 18: 50: 03$ & 626 & 222 & 26 & 27.4 & $51.1 !$ & 0.57 & 1352 & 54.6 & 36 & -0.12 \\
\hline $1: 02$ & 627 & 214 & 26 & 27.4 & 51.1 & 0.57 & 1348 & 54.6 & 34 & 0 \\
\hline $09: 18: 52: 01$ & 628 & 209 & 26 & 27 & 51.1 & $0.57^{\prime}$ & 1348 & 54.8 & 33 & 0 \\
\hline $09: 1$ & 629 & 202 & 26 & 27 & 51.1 & 0.57 & 1350 & 55 & 32 & -0.15 \\
\hline 09:1 & 630 & 196 & 26 & 27 & 51.1 & 0.57 & 1353 & 55 & 31 & -0.15 \\
\hline $09: 18$ & $\overline{63} 1^{1}$ & 193 & $26 !$ & 27 & 51.1 & 0.57 & 1352 & 55 & 29 & $-0 . \overline{28}$ \\
\hline $09: 18$ & 632 & 188 & 26 & 27 & 51.1 & 0.58 & 1348 & 55 & 28 & -0.28 \\
\hline $09: 1$ & 633 & 182 & 26 & 27 & 51.1 & 0.57 & 1342 & 55 & 27 & -0.04 \\
\hline $09: 1$ & 634 & 178 & 26 & 27.2 & 51.1 & 0.57 & 1336 & 55.1 & 27 & 0.04 \\
\hline & 635 & 173 & 26 & 27.2 & 51.1 & 0.55 & 1337 & 55.1 & 26 & -0.06 \\
\hline $09: 1$ & 636 & 169 & 26 & 27.2 & 51.1 & 0.57 & 1333 & 55.1 & 26 & -0.06 \\
\hline$: 96$ & 637 & 164 & 26 & 27.2 & 51.1 & 0.57 & 1332 & 55.2 & 25 & -0.13 \\
\hline $51: 95$ & 638 & 160 & 26 & 27.2 & 51.1 & 0.57 & 1334 ! & 55.2 & 25 & -0.13 \\
\hline 94 & 639 & $15 \overline{6}$ & 26 & 27.2 & 51 & 0.5 & 1336 & 55.2 & 24 & -0.26 \\
\hline 09: & 640 & 155 & 26 & 27 & 51.1 & $0 . \overline{58}$ & $1337^{+}$ & $5 \overline{5} .3$ & 24 & $-\overline{0.26}$ \\
\hline$: 92$ & 641 & 151 & 26 & 27 & 51.1 & 0.58 & 1334 & 55.2 & 24 & -0.05 \\
\hline $5: 90$ & 642 & 148 & 26 & $27.2^{\dagger}$ & 51.1 & 0.57 & $1335^{\circ}$ & 55.2 & 24 & -0.05 \\
\hline $06: 89$ & 643 & 145 & 26 & 27.2 & $51.1^{\prime}$ & 0.58 & 1333 & 55.3 & 23 & -0.02 \\
\hline & $6 \overline{44}$ & 141 & $26 !$ & 27.2 & 51.1 & 0.57 & 1328 & 55.3 & 23 & -0.02 \\
\hline 87 & 645 & 139 & 26 & $27.2^{1}$ & 51. & 0.57 & 1323 & 55.3 & 23 & -0.17 \\
\hline & 646 & $1 \overline{35}$ & 26 & 27.2 & 51.1 & 0.57 & $1319^{\circ}$ & 55.4 & 23 & -0.17 \\
\hline & $6 \overline{7}$ & 132 & 26 & 27.2 & 51.1 & 0.5 & $1320^{\circ}$ & 55.3 & 23 & -0.2 \\
\hline & 648 & 129 & 26 & $27.2^{!}$ & 51. & 0.58 & 1323 & 55.3 & 23 & -0.2 \\
\hline $8 \overline{3}$ & 649 & 128 & 26 & 27.2 & 51.1 & 0.57 & 1325 & 55.3 & 22 & $-0.0 \overline{9}$ \\
\hline & 650 & 126 & $26 !$ & 27.2 & 51.1 & 0.57 & 1322 & 55.3 & 22 & -0.09 \\
\hline & 651 & 124 & 26 & 27.2 & 51.1 & 0.57 & 1324 & 55.3 & 22 & -0.02 \\
\hline & 652 & 123 & 26 & 27.4 & $51.1^{\dagger}$ & & 1325 & 55.2 & 22 & -0.18 \\
\hline $6: 78$ & 653 & 120 & $26 !$ & 27.4 & 51.1 & 0.57 & 1322 & 55.2 & 22 & -0.18 \\
\hline $7: 77$ & 654 & 118 & 26 & 27.6 & 51.1 & 0.57 & 1316 & $55.2]$ & 22 & -0.25 \\
\hline $8: \overline{76}$ & 655 & 115 & 26 & $27.6 !$ & $51.1^{\prime}$ & 0.55 & 1312 & 55.1 & 22 & -0.25 \\
\hline & 656 & 113 & $26 !$ & 27.6 & 51.1 & 0.57 & 1311 & $55.1^{\dagger}$ & 22 & $-0 . \overline{01}$ \\
\hline $0: \overline{79}$ & 657 & $\uparrow 11 \mid$ & 26 & 27.6 & & 0.5 & $1309^{\circ}$ & 55.2 & 22 & -0.01 \\
\hline $09: 19: 21: 78$ & 658 & 109 & 26 & $27.6 !$ & 51.1 & 0.58 & 1310 & 55.2 & 22 & -0.03 \\
\hline $09: 19: 22: 77$ & 659 & 108 & 26 & 27.2 & 51.1 & 0.58 & 1313 & 55.2 & $2 \overrightarrow{2}$ & -0.03 \\
\hline & & & & & & $\cdots \cdot$ & & & & \\
\hline
\end{tabular}




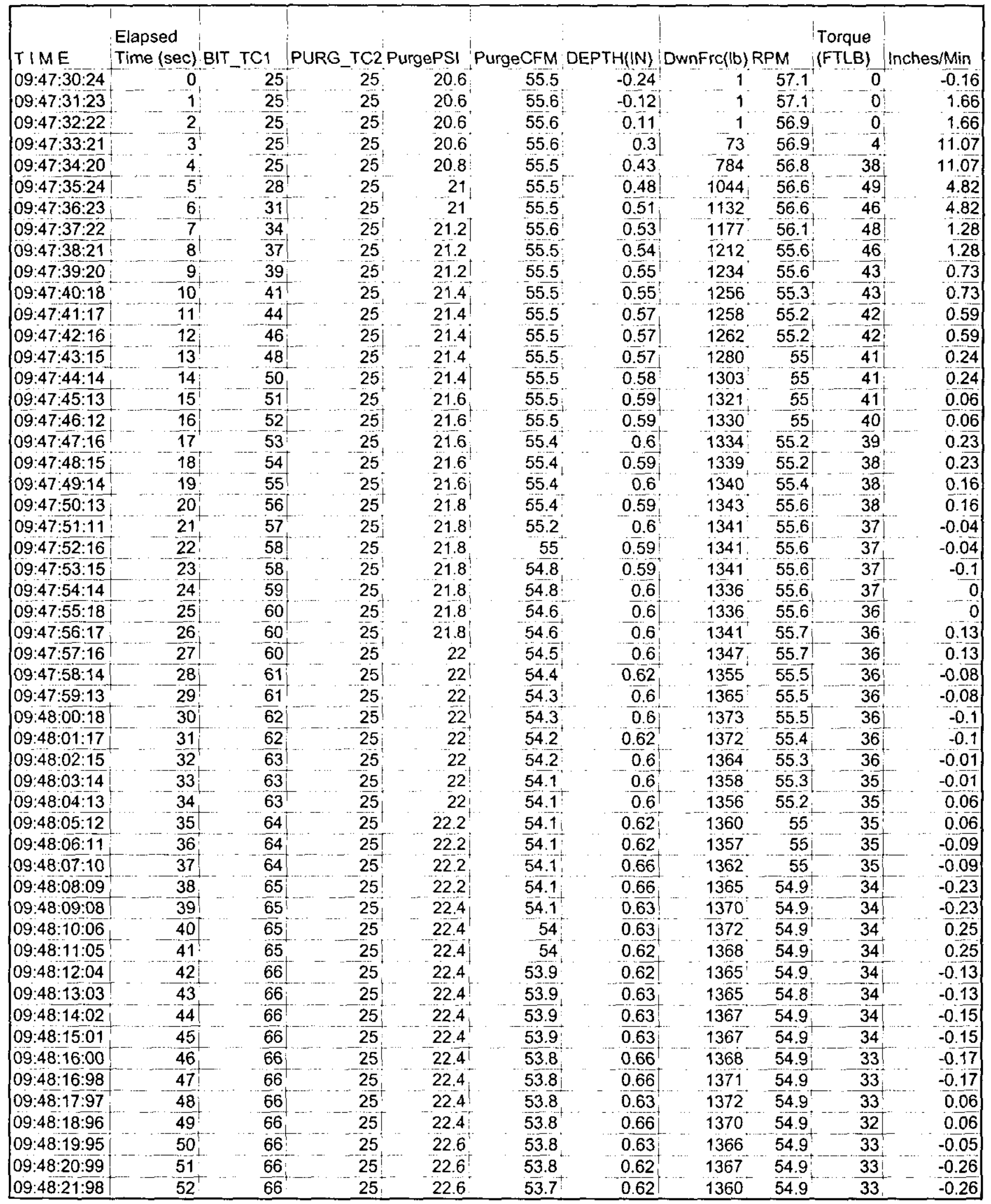




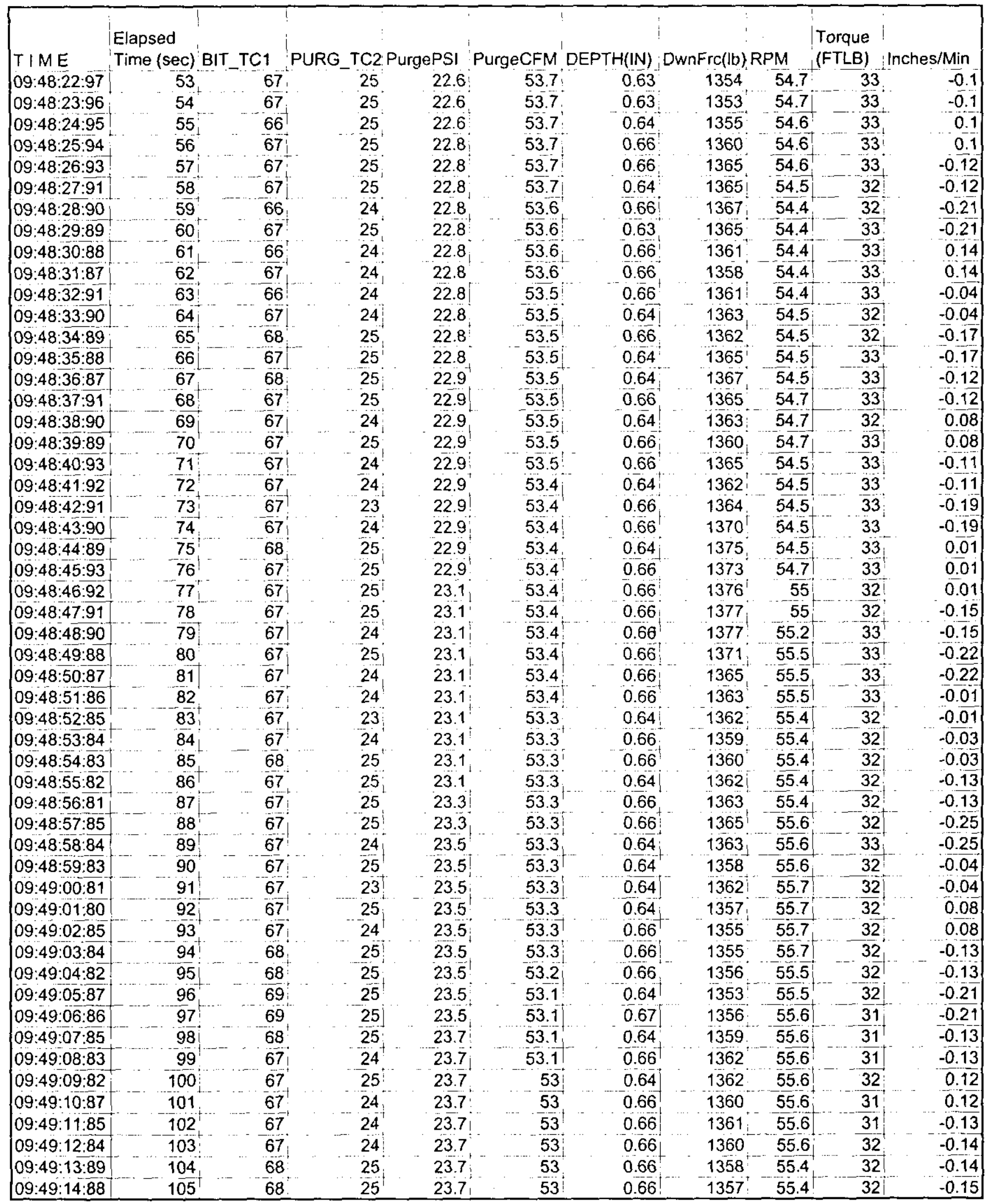




\begin{tabular}{|c|c|c|c|c|c|c|c|c|c|c|}
\hline$T / M E$ & $\begin{array}{l}\text { Elapsed } \\
\text { Time (sec) }\end{array}$ & BIT_TC1 & PURG_TC2 & PurgePSI & PurgeCFM & DEPTH(IN) & DwnFrc $(\mathrm{lb})$ & RPM & $\begin{array}{l}\text { Torque } \\
\text { (FTLB) }\end{array}$ & Inches/Min \\
\hline $09: 49: 15: 86$ & $\quad 106$ & 68 & 25 & 23.7 & 53 & 0.64 & 1356 & 55.4 & $\quad 32$ & -0.15 \\
\hline $09: 49: 16: 85$ & 107 & 68 & 25 & 23.7 & $52.9^{\circ}$ & $0.6 \overline{7}$ & 1358 & 55.3 & 32 & 0.05 \\
\hline $09: 49: 17: 84$ & 108 & 67 & 25 & 23.7 & 52.9 & 0.67 & 1356 & 55.3 & 31 & 0.05 \\
\hline $09: 49: 18: 83$ & 109 & 67 & 24 & 23.9 & $52 . \overline{9}$ & 0.67 & 1361 & $5 \overline{5.1}$ & 31 & -0.11 \\
\hline $9: 49: 19: 82$ & 110 & 68 & 25 & 23.9 & $52.9^{\prime}$ & 0.66 & 1363 & 55.1 & 31 & -0.11 \\
\hline $09: 49: 20: 81$ & 111 & 67 & 24 & 24.1 & 52.8 & 0.66 & 1366 & 55.1 & 31 & -0.24 \\
\hline $09: 49: 21: 80$ & 112 & 67 & 24 & 24.1 & 52.8 & 0.66 & 1365 & 55.1 & 31 & -0.24 \\
\hline $09: 49: 22: 79$ & 113 & 66 & 24 & 24.1 & $52.8 \dagger$ & 0.66 & 1359 & 55.1 & 31 & -0.07 \\
\hline $9: 49: 23: 77$ & 114 & 67 & 25 & 24.1 & 52.8 & 0.66 & 1355 & 55.1 & 31 & -0.07 \\
\hline $09: 49: 24: 76$ & 115 & 67 & 25 & 23.9 & 52.8 & 0.64 & 1357 & 55.1 & 31 & 0.03 \\
\hline $49: 25: 75$ & 116 & 67 & 25 & 23.9 & $52.8^{\dagger}$ & $0.64^{-1}$ & 1360 & $5 \overline{5.1}$ & 31 & 0.03 \\
\hline $6: \overline{74}$ & $1 \overline{1} 7$ & 67 & 24 & 24.1 & $52 . \overline{8}$ & 0.67 & 1363 & 55.1 & 31 & -0.09 \\
\hline 778 & 118 & $\overline{67}$ & 25 & 24.1 & 52.8 & $0 . \overline{6} 7$ & 1368 & 55 & 31 & -0.09 \\
\hline $28: 77$ & 119 & 67 & 25 & 24.1 & 52.8 & 0.67 & $1 \overline{3} 73$ & 55 & 31 & -0.27 \\
\hline $09: 49: 29: 76$ & 120 & 67 & 25 & 24.1 & 52.8 & 0.67 & 1377 & 55 & 31 & -0.27 \\
\hline 80 & 121 & 66 & 23 & 24.1 & 52.8 & 0.67 & 1377 & 55 & 31 & 0.1 \\
\hline & 122 & 66 & 24 & 24.3 & 52.8 & $0.67 !$ & $13 \varepsilon$ & 55 & 32 & 0.1 \\
\hline $32: 78$ & 123 & 66 & 24 & 24.3 & 52.7 & 0.66 & 1381 : & 55.1 & 32 & -0.08 \\
\hline $33: 77$ & 124 & 66 & $25^{\dagger}$ & 24,3 & 52.7 & 0.67 & 1378 & 55.2 & 32 & -0.08 \\
\hline $4: 76$ & 125 & 67 & 25 & 24.3 & 52.7 & $0.66^{\prime}$ & & 55.2 & 32 & -0.1 \\
\hline $5: 80$ & 126 & $6 \overline{7}$ & 25 & 24.3 & 52.7 & $0.68 \mathrm{i}$ & 13 & 55.2 & 32 & -0.1 \\
\hline $94: 36: 79$ & 127 & 68 & 25 & 24.3 & 52.7 & 0.67 & $1377 !$ & 55.2 & 32 & -0.3 \\
\hline 778 & 128 & 67 & 25 & 24.3 & 52.7 & 0.67 & 1377 & 55.2 & 32 & -0.3 \\
\hline 82 & 129 & $66^{\dagger}$ & 24 & 24.5 & 52.7 & 0.66 & $1379^{\dagger}$ & 55.1 & 31 & 0.14 \\
\hline 81 & 130 & 66 & 25 & 24.5 & 52.7 & 0.68 & 1380 & 55 & 31 & -0.09 \\
\hline $40: 80$ & 131 & 66 & 24 & 24.5 & 52.7 & 0.68 & 1384 & 55 & 31 & -0.09 \\
\hline$: \overline{79}$ & 132 & 66 & 24 & 24.5 & 52.7 & $0 . \overline{67}$ & $1383^{\circ}$ & 54.9 & 31 & -0.18 \\
\hline 78 & $13 \overline{3}$ & 66 & 23 & 24.5 & 52.7 & 0.68 & 1377 & 55 & 31 & -0.18 \\
\hline $3: 82$ & 134 & $6 \overline{6}$ & 25 & 24.7 & 52.7 & 0.67 & 1377 & 55 & 31 & -0.22 \\
\hline $44: 81$ & 135 & 67 & 25 & 24.7 & 52.6 & 0.67 & 13 & 55 & 31 & $-0 . \overline{22}$ \\
\hline 50 & 136 & 67 & 25 & 24.5 & 52.6 & 0.66 & 13 & 55 & 31 & 0.02 \\
\hline $6: \overline{79}$ & 13 & 67 & 25 & 24.5 & 52.6 & $0.67 i$ & 13 & 55 & 31 & 0.02 \\
\hline $47: 78$ & 138 & 67 & 25 & 24.5 & 52.6 & 0.67 & 1365 & 55 & 31 & -0.12 \\
\hline & 139 & 66 & 25 & 24.5 & 52.6 & & & 55 & 31 & -0.12 \\
\hline $9: \overline{75}$ & 140 & 66 & 25 & 24.5 & $52 . \overline{6}$ & 0.67 & 1361 & 55 & 30 & -0.27 \\
\hline $0: 74$ & 141 & 65 & 24 & 24.5 & 52.6 & 0.68 & 1363 & $55 . \overline{1}$ & 30 & -0.27 \\
\hline $1: 73$ & 142 & 65 & 24 & 24.7 & 52.6 & $0 . \overline{67}$ & 136 & 55 & 30 & -0.13 \\
\hline & $14 \overline{3}$ & 65 & 24 & 24 & 52.6 & & & 55 & 30 & -0.13 \\
\hline$\overline{71}$ & 144 & 66 & 25 & 24.9 & 52.6 & 0.66 & 135 & 55 & 30 & $0 . \overline{02}$ \\
\hline $4: \overline{70}$ & 145 & 65 & 24 & 24.9 & $5 \overline{2.6}$ & 0.67 & 1351 & 55 & 30 & 0.02 \\
\hline $5: 74$ & $\overline{146}$ & 66 & 25 & 24.9 & $52 . \overline{6}$ & 0.67 & $13 \overline{4} 8^{\circ}$ & 55 & 30 & -0.14 \\
\hline & 147 & 66 & 25 & & 52.6 & & 13 & 55.1 & 30 & $-0 . \overline{14}$ \\
\hline & $14 \overline{8}$ & 66 & 25 & & $52.5^{\dagger}$ & 0.68 & 1345 & 55.2 & 30 & $-0 . \overline{23}$ \\
\hline $8: 71$ & 149 & 66 & 25 & 24.7 & 52.4 & 0.68 & 1345 & 55.2 & 30 & -0.23 \\
\hline $09: 49: 59: 70$ & 150 & 65 & 25 & 24.7 & 52.4 & 0.67 & 1346 & 55.3 & 30 & -0.13 \\
\hline$\overline{74}$ & 151 & 64 & 23 & 24.9 & 52.3 & 0.67 & 1349 & 55.4 & 29 & -0.13 \\
\hline & 152 & 64 & $24^{i}$ & 24.9 & 52.3 & 0.67 & & 55.4 & 29 & 0.02 \\
\hline & 15 & 64 & 24 & 24.9 & 52.3 & 0.68 & & 55.3 & 29 & 0.02 \\
\hline $09: 50: 03: 76$ & 154 & 65 & 25 & 24.9 & $52.2^{\dagger}$ & 0.67 & 1347 & 55.5 & 29 & -0.1 \\
\hline $09: 50: 04: 75$ & 155 & 65 & 25 & 24.9 & 52.2 & 0.67 & 1345 & 55.5 & 29 & $-0 . \overline{23}$ \\
\hline & 156 & 65 & 25 & 24.9 & 52.2 & 0.67 & 1340 & 55.6 & 29 & -0.23 \\
\hline $09: 50: \overline{06}: \overline{73}$ & $15 \overline{7}$ & 65 & 25 & 24.9 & 52.2 & 0.67 & $13 \overline{35}$ & 55.6 & 29 & -0.13 \\
\hline $09: 50: 07: 71$ & 158 & 65 & $\quad 25$ & 24.9 & 52.21 & 0.67 & 1334 & 55.6 & $5 \quad 29$ & -0.13 \\
\hline
\end{tabular}




\begin{tabular}{|c|c|c|c|c|c|c|c|c|c|c|}
\hline TIME & $\begin{array}{l}\text { Elapsed } \\
\text { Time }(\mathbf{s e c})\end{array}$ & & PURG TC2 & PurgePSI & PurgeCFM & DEPTH(IN) & DwnFrc(lb) & RPM & $\begin{array}{l}\text { Torque } \\
\text { (FTLB) }\end{array}$ & Inches/Min \\
\hline $09: 50: 08: 76$ & $\quad 159$ & 65 & 25 & $\quad 24.9$ & 52.2 & $\quad 0.67$ & 1333 & 55.6 & 29 & $\quad 0.01$ \\
\hline $09: 50: 09: 75$ & 160 & 64 & 24 & 24.9 & 52.2 & 0.68 & 1333 & $5 \overline{5} .6$ & 29 & 0.01 \\
\hline $09: 50: 10: 74$ & 161 & 64 & 24 & 24.9 & 52.1 & 0.67 & 1335 & 55.6 & 29 & -0.13 \\
\hline $09: 50: 11: 72$ & 162 & 64 & 24 & 25.1 & 52.1 & 0.68 & 1338 & 55.6 & 29 & -0.13 \\
\hline $09: 50: 12: 71$ & 163 & 64 & 24 & 25.1 & 52.1 & 0.67 & $1340^{\circ}$ & 55.6 & 29 & -0.28 \\
\hline 09:50:13:70 & 164 & 64 & 25 & 25.1 & 52 & 0.67 & 1341 & 55.6 & 29 & -0.28 \\
\hline $09: 50: 14: 69$ & 165 & 64 & 24 & 25.1 & 52 & 0.67 & $1338^{4}$ & 55.6 & 29 & -0.12 \\
\hline $09: 50: 15: 68$ & 166 & 65 & 25 & 25.3 & 52 & 0.67 & 1340 & 55.6 & 29 & -0.12 \\
\hline $09: 50: 16: 67$ & 167 & 64 & 25 & 253 & 519 & 0.68 & 1336 & 55.6 & 29 & 0.05 \\
\hline $09: 50: 17: 66$ & 168 & 65 & 25 & 25.1 & 51.9 & 0.67 & 1332 & 55.5 & 29 & 0.05 \\
\hline $09: 50: 18: 64$ & 169 & 64 & 25 & 25.1 & 51.9 & 0.68 & 1330 & 55.5 & 29 & -0.12 \\
\hline $09: 50: 19: 63$ & 170 & 64 & 24 & 25.1 & 51.9 & 0.68 & 1329 & 55.5 & 29 & -0.12 \\
\hline $09: 50: 20: 68$ & 171 & 63 & 23 & 25.1 & 519 & 0.68 & 1331 & 55.6 & 28 & $-\overline{0.3}$ \\
\hline $09: 50: 21: 67$ & 172 & 63 & 24 & 25.1 & 51.9 & 0.68 & 1330 & 55.7 & 28 & -0.3 \\
\hline $9: 50: 22: 65$ & 173 & 64 & 24 & 25.1 & 51.9 & 0.68 & 1332 & 55.7 & 28 & -0.13 \\
\hline $09: 50: 23: 64$ & 174 & 64 & 25 & 25.1 & 51.9 & 0.68 & 1336 & 55.7 & 28 & -0.13 \\
\hline $09: 50: 24: 63$ & 175 & 64 & 25 & 25.3 & 51.9 & 0.67 & 1338 & 55.6 & 28 & 0.03 \\
\hline $50: 25: 62$ & 176 & 64 & $25^{1}$ & 25.3 & 51.8 & 0.68 & 1340 & 55.6 & 28 & 0.03 \\
\hline $09: 50: 26: 61$ & 177 & 64 & 25 & 25.3 & 51.8 & 0.67 & 1340 & 55.7 & 28 & -0.04 \\
\hline $09: 50: 27: 60$ & 178 & 65 & 25 & 25.5 & 51.8 & 0.67 & 1337 & 55.7 & 28 & -0.04 \\
\hline 09:50:28:59 & 179 & 64 & 25 & 25.5 & 51.8 & 0.67 & 1335 & 55.7 & 28 & -0.17 \\
\hline $9: 50: 29: 57$ & 180 & 63 & 24 & 253 & 51.8 & 0.66 & 1335 & 55.6 & 28 & -0.17 \\
\hline $09: 50: 30: 56$ & 181 & 64 & 25 & 25.3 & 51.8 & 0.67 & 1340 & 55.6 & 28 & -0.19 \\
\hline $09: 50: 31: 55$ & 182 & 63 & 24 & 25.3 & 51.8 & 0.68 & 1343 & 55.6 & $2 \overline{8}$ & 0.05 \\
\hline 54 & 183 & 63 & 25 & 25.3 & 51.8 & 0.68 & 1345 & 55.6 & 28 & 0.05 \\
\hline $09: 50: 33: 53$ & 184 & 63 & 24 & $25.3 !$ & $51.7 !$ & 0.68 & 1350 & 55.6 & 28 & -0.13 \\
\hline $09: 50: 34: 52$ & 185 & 63 & 25 & 25.31 & 51.7 & 0.68 & 1354 & $5 \overline{5.6}$ & 28 & $-0 . \overline{13}$ \\
\hline $5: 51$ & 186 & 64 & $25 !$ & 25.5 & 51.7 & 0.68 & 1355 & 55.6 & 28 & -0.18 \\
\hline $09: 50: 36: 50$ & 187 & 63 & 25 & 25.5 & 51.6 & 0.68 & 1354 & $55 . \overline{6}$ & 28 & -0.18 \\
\hline $09: 50: 37: 48$ & 188 & 64 & 25 & 25.5 & $51.6 !$ & 0.67 & 1350 & 55.6 & 28 & -0.27 \\
\hline $09: 50: 38: 47$ & 189 & 63 & $25 !$ & 25.5 & 51.6 & 0.67 & 1355 & 55.5 & 28 & -0.27 \\
\hline $09: 50: 39: 46$ & 190 & 63 & 25 & 25.5 & 51.6 & 0.67 & 1351 & 55.3 & 28 & 0.11 \\
\hline $09: 50: 40: 45$ & 191 & 63 & 25 & 25.5 & 51.6 & 0.67 & 1350 & 55.3 & 28 & 0.11 \\
\hline $09: 50: 41: 44$ & 192 & 63 & 23 & 25.5 & 51 & 0.68 & 1353 & 55.1 & 28 & -0.08 \\
\hline $09: 50: 42: 43$ & 193 & 63 & 24 & 25.5 & 51.6 & 0.67 & 1356 & 55 & 28 & $-0 . \overline{08}$ \\
\hline 09:50:43:42 & 194 & 63 & 24 & 25.5 & 51.6 & 0.68 & 1361 & 55 & 28 & -0.24 \\
\hline $09: 50: 44: 40$ & 195 & 63 & $25 !$ & 25.7 & 51.6 & 0.68 & 1363 & 54.9 & 28 & -0.24 \\
\hline $09: 50: 45: 39$ & 196 & 63 & 25 & 25.7 & 51.6 & 0.68 & 1362 & 54.9 & 28 & -0.16 \\
\hline $09: 50: 46: 38$ & 197 & 63 & 25 & 25.7 & 51.5 & 0.68 & 1359 & 54.9 & 28 & -0.16 \\
\hline $47: 37$ & 198 & 63 & 25 & 25.7 & 51.5 & 0.67 & 1363 & $5 \overline{5.1}$ & 29 & $0 . \overline{01}$ \\
\hline $09: 50: 48: 36$ & 199 & 63 & 25 & 25.5 & 51.5 & 0.67 & & 55.1 & 29 & 0.01 \\
\hline $09: 50: 49: 35$ & 200 & 63 & 25 & 25.5 & 51.5 & 0.68 & 1357 & 55.1 & 29 & -0.1 \\
\hline 09:50:50:39 & 201 & 62 & 24 & 25.7 & 51.5 & 0.68 & 1358 & 55 & 29 & -0.1 \\
\hline $51: 38$ & 202 & 63 & 25 & 25.7 & 51 & 0.69 & 1362 & $54 . \overline{9}$ & 29 & -0.23 \\
\hline 09:50:52:37 & 203 & 62 & 24 & 25.7 & 51.5 & 0.68 & 1366 & 54.9 & 28 & -0.23 \\
\hline $09: 50: 53: 41$ & 204 & 62 & 24 & $25.7 !$ & 51.5 & 0.68 & 1363 & 54.9 & 28 & -0.15 \\
\hline $09: 50: 54: 40$ & 205 & 63 & 25 & 25.9 & 51.5 & 0.69 & 1366 & 54.9 & 28 & $-0 . \overline{15}$ \\
\hline $09: 50: 55: 39$ & 206 & 63 & 25 & $25.9^{\circ}$ & 51.5 & 0.69 & $1368^{\circ}$ & 54.9 & 29 & 0.04 \\
\hline $09: 50: 56: 38$ & 207 & 64 & 25 & 25.9 & 51.5. & 0.68 & 1365 & 54.8 & 28 & 0.04 \\
\hline $09: 50: 57: 37$ & 208 & 63 & 25 & 25.9 & 51.5 & 0.68 & 1359 & 54.7 & 28 & -0.16 \\
\hline $\mid 09: 50: 58: 41$ & 209 & 64 & 25 & 25.9 & 51.4 & 0.68 & 1362 & 54.7 & 28 & -0.16 \\
\hline $09: 50: 59: 40$ & 210 & 63 & 25 & 25.9 & 51.4 & 0.67 & 1356 & 54.6 & 28 & -0.29 \\
\hline $09: 51: 00: 39$ & 211 & 63 & 24 & 25.9 & $51 . \overline{4}$ & 0.68 & 1355 & 54.6 & 28 & 0.04 \\
\hline
\end{tabular}




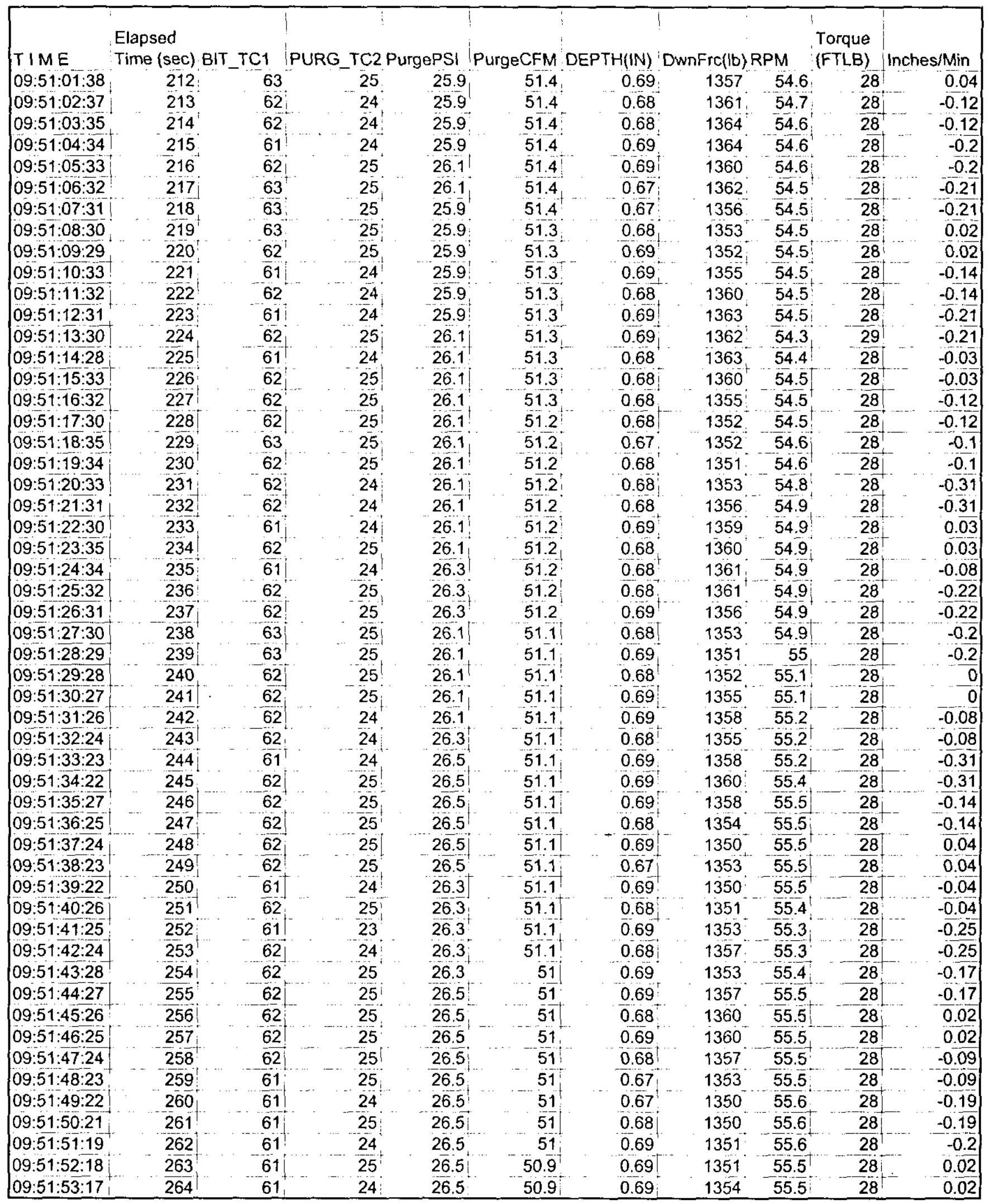




\begin{tabular}{|c|c|c|c|c|c|c|c|c|c|c|}
\hline TIME & $\begin{array}{l}\text { Elapsed } \\
\text { |Time (sec)' }\end{array}$ & BIT_TC1 & PURG_TC2 & PurgePSI & PurgeCFM & DEPTH $(I N)$ & DwnFrc(lb) & RPM & $\begin{array}{l}\text { Torque } \\
\text { (FTLB) }\end{array}$ & Inches/Min \\
\hline $09: 51: 54: 16$ & 265 & 62 & -25 & $26.5^{-1}$ & 50.9 & $\quad 0.69$ & 1357 & 55.5 & $\quad 28$ & -0.06 \\
\hline $09: 51: 55: 15$ & 266 & 62 & 25 & 26.5 & 50.9 & $0.69^{\circ}$ & $1360^{\circ}$ & 55.5 & 28 & -0.06 \\
\hline $09: 51: 56: 14$ & 267 & 62 & 25 & 26.5 & 50.9 & 0.69 & $1360^{\circ}$ & 55.6 & 28 & -0.21 \\
\hline $09: 51: 57: 18$ & 268 & 62 & $25 !$ & 26.6 & 50.9 & 0.69 & 1356 & 55.6 & 28 & -0.21 \\
\hline $09: 51: 58: 17$ & 269 & 61 & 25 & 26.6 & $50.9^{\prime}$ & 0.69 & 1359 & 55.6 & 28 & -0.21 \\
\hline $09: 51: 59: 16$ & $2 \overline{7} 0^{\prime}$ & 62 & 25 & 26.5 & 50.9 & $0.69^{\circ}$ & 1355 & 55.6 & 28 & -0.21 \\
\hline $09: 52: 00: 20$ & 271 & 61 & 24 & $26.5^{i}$ & 50.9 & 0.68 & 1351 & 55.6 & 28 & 0.02 \\
\hline $09: 52: 01: 19$ & 272 & 61 & 24 & 26.6 & 50.9 & 0.68 & 1350 & 55.6 & 28 & 0.02 \\
\hline $09: 52: 02: 18$ & 273 & 62 & $25 !$ & 26.6 & 50.9 & 0.68 & 1350 & 55.5 & 28 & -0.06 \\
\hline $09: 52: 03: 17$ & 274 & 61 & 24 & 26.6 & 50.8 & $0.68^{+}$ & 1353 & 55.5 & 28 & -0.06 \\
\hline $09: 52: 04: 16$ & 275 & 62 & 25 & 26.6 & 50.8 & 0.68 & 1352 & 55.5 & 28 & -0.23 \\
\hline $09: 52: 05: 20$ & 276 & $6 \overline{2}$ & 25 & 26.6 & 50.8 & $0.69^{-1}$ & 1355 & 55.4 & 28 & $-0.2 \overline{3}$ \\
\hline $09: 52: 06: 19$ & 277 & 61 & 25 & 26.8 & 50.8 & 0.68 & 1358 & $5 \overline{5} .4$ & 28 & -0.21 \\
\hline $09: 52: 07: 18$ & 278 & 61 & 25 & 26.8 & 50.8 & 0.69 & 1361 & 55.5 & 28 & -0.21 \\
\hline $09: 52: 08: 22$ & 279 & 61 & 25 & 26.8 & 50.8 & 0.68 & 1360 & 55.5 & 28 & 0.01 \\
\hline $09: 52: 09: 21$ & 280 & 61 & 24 & 26.8 & 50.8 & 0.69 & 1360 & 55.5 & 28 & 0.01 \\
\hline $09: 52: 10: 20$ & 281 & 61 & 24 & 26.8 & 50.8 & 0.68 & 1359 & 55.5 & 28 & $-0.0 \overline{7}$ \\
\hline $09: 52: 11: 19$ & 282 & 61 & 24 & 26.8 & 50.8 & $0 . \overline{6} 8$ & 1354 & 55.5 & 28 & -0.07 \\
\hline $09: 52: 12: 18$ & 283 & 61 & 25 & 26.6 & 50.8 & 0.69 & $1350 !$ & 55.5 & 28 & -0.2 \\
\hline $09: 52: 13: 22$ & 284 & 62 & $25^{\circ}$ & 26.6 & 50.8 & 0.68 & 1350 & 55.5 & 28 & -0.2 \\
\hline $09: 52: 14: \overline{2} 1$ & 285 & 62 & 25 & 26.6 & 50.8 & 0.69 & 1352 & 55.5 & 28 & -0.23 \\
\hline $09: 52: 15: 20$ & 286 & 62 & 25 & 26.6 & 50.7 & 0.71 & $1350^{\circ}$ & 55.5 & 27 & -0.23 \\
\hline $09: 52: 16: 18$ & 287 & 62 & 25 & 26.6 & 50.7 & 0.69 & 1355 & 55.4 & 28 & 0.11 \\
\hline $09: 52: 17: 17$ & 288 & 61 & 25 & 26.6 & 50.7 & $0.69 !$ & 1359 & 55.3 & 28 & -0.13 \\
\hline $09: 52: 18: 16$ & 289 & 60 & 24 & 26.6 & 50.7 & 0.69 & 1362 & 55.3 & 27 & -0.13 \\
\hline $09: 52: 19: 15$ & 290 & 61 & 25 & $27 !$ & $50 . \overline{7}$ & $0.69^{\prime}$ & 1362 & 55.3 & 27 & -0.21 \\
\hline $09: 52: 20: 14$ & 291 & 60 & 24 & 27 & $50.7 !$ & 0.68 & $1359^{\dagger}$ & 55.3 & 27 & -0.21 \\
\hline $09: 52: 21: 13$ & 292 & 61 & 25 & 27 & 50.7 & 0.68 & 1355 & 55.3 & $\overline{2} \overline{7}$ & -0.2 \\
\hline $09: 52: 22: 12$ & 293 & 61 & 24 & 27 & 50.7 & $0.69^{\dagger}$ & 1352 & 55.3 & 27 & -0.2 \\
\hline $09: 52: 23: 11$ & 294 & 62 & $25 !$ & 27 & 50.7 & 0.69 & 1353 & 55.3 & 27 & 0.05 \\
\hline $09: 52: 24: 09$ & 295 & 62 & 25 & 27 & $50 . \overline{7}$ & $0 . \overline{69}$ & 1352 & 55.3 & 27 & 0.05 \\
\hline $09: 52: 25: 14$ & 296 & 62 & $25^{\prime}$ & 27 & 50.7 & 0.69 & 1353 & 55.3 & 27 & -0.11 \\
\hline $09: 52: 26: 1 \overline{3}$ & 297 & 62 & 25 & 27 & 50.7 & 0.69 & $1356:$ & 55.3 & 27 & -0.19 \\
\hline $09: 52: 27: 12$ & 298 & 61 & 25 & $27 !$ & 50.6 & $0 . \overline{6} 9$ & 1360 & 55.3 & 27 & $-0 . \overline{22}$ \\
\hline $09: 52: 28: 10$ & 299 & 61 & 23 & 27 & $50 . \overline{6}$ & 0.69 & $1363^{t}$ & $55 . \overline{3}$ & $2 \overline{7}$ & -0.22 \\
\hline $09: 52: 29: 09$ & 300 & 61 & 24 & 27 & 50.6 & 0.69 & 1361 & 55.1 & 27 & $-0 . \overline{18}$ \\
\hline $09: 52: 30: 14$ & 301 & 60 & 24 & $27.2 !$ & 50.6 & 0.69 & 1362 & 55.1 & 27 & -0.18 \\
\hline $09: 52: 31: 12$ & 302 & 61 & 25 & 27.2 & 50.6 & 0.69 & $1360^{\circ}$ & 55 & 27 & 0.04 \\
\hline $09: 52: 32: 11$ & 303 & 61 & 25 & 25.3 & 1.8 & 0.68 & 1357 & 54.9 & 27 & 0.04 \\
\hline $09: 52: 33: 16$ & 304 & 62 & 25 & 0.2 & 19 & 0.68 & $1353^{\circ}$ & 54.9 & 28 & -0.12 \\
\hline $09: 52: 34: 15$ & 305 & 65 & $25^{t}$ & -0.6 & 1.9 & 0.69 & $1354^{\dagger}$ & 54.9 & 29 & -0.12 \\
\hline $09: 52: 35: 13$ & 306 & 67 & 25 & -0.6 & 1.9 & $0.69^{7}$ & $1353^{\circ}$ & 55.1 & 30 & -0.32 \\
\hline $09: 52: 36: 12$ & 307 & 69 & 25 & -0.6 & 1.8 & 0.69 & 1354 & 55.1 & 30 & $-0 . \overline{32}$ \\
\hline $09: 52: 37: 11$ & 308 & 70 & $25 !$ & -0.6 & 1.9 & 0.69 & 1356 & 55.2 & $30^{\circ}$ & $-0 . \overline{13}$ \\
\hline $09: 52: 38: 16$ & $309^{\dagger}$ & 71 & 24 & $-0 . \overline{6}$ & 1.9 & $0.69^{\circ}$ & 1362 & 55.2 & 31 & -0.13 \\
\hline $09: 52: 39: 14$ & 310 & 74 & 25 & -0.6 & 1.9 & 0.69 & 1364 & 55.2 & 31 & 0 \\
\hline $09: 52: 40: 13$ & $311 i$ & 74 & 24 & -0.6 & 1.9 & $0.69^{9}$ & $13 \overline{63}$ & 55.2 & 31 & $\overline{0}$ \\
\hline $09: 52: 41: 12$ & 312 & 77 & 25 & -0.6 & 1.9 & 0.69 & 1365 & 55.2 & 32 & -0.14 \\
\hline $09: 52: 42: 11$ & 313 & 78 & 25 & -0.6 & 1.9 & 0.68 & $1363^{!}$ & 55.2 & 32 & -0.27 \\
\hline $09: 52: 43: 10$ & 314 & 80 & 25 & -0.6 & 1.9 & 0.68 & $1359^{\circ}$ & 55 & 32 & -0.27 \\
\hline $09: 52: 44: 0 \overline{9}$ & 315 & 81 & 25 & -0.6 & 1.9 & $0 . \overline{6} 9^{1}$ & 1357 & 54.9 & 32 & $-0 . \overline{13}$ \\
\hline $09: 52: 45: 08$ & 316 & 82 & 25 & -0.6 & 1.9 & 0.69 & 1358 & $54 . \overline{9}$ & $3 \overline{3}$ & $-0 . \overline{13}$ \\
\hline $09: 52: 46: 06$ & 317 & 84 & 25 & $-0.6 !$ & 1.9 & 0.69 & 1361 & 54.9 & 33 & -0.01 \\
\hline
\end{tabular}




\begin{tabular}{|c|c|c|c|c|c|c|c|c|c|c|}
\hline$T I M E$ & $\begin{array}{l}\text { Elapsed } \\
\text { Time (sec) }\end{array}$ & & PURG_TC2 & & PurgeCFM & DEPTH(IN) & DwnFrc(lb) & RPM & $\begin{array}{l}\text { Torque } \\
\text { (FTLB) }\end{array}$ & Inches/Min \\
\hline $09: 52: 47: 05$ & 318 & -84 & $\quad 24$ & -0.6 & 18 & $\quad 0.69$ & 1365 & 54.8 & 33 & $\quad-0 . \overline{01}$ \\
\hline $52: 48: 04$ & 319 & 86 & 25 & -0.6 & 1.9 & $0.69^{\circ}$ & 1368 & 54.8 & 34 & -0.13 \\
\hline $9: 52: 49: 03$ & 320 & 87 & 24 & -0.6 & 1.9 & 0.69 & 1367 & 54.8 & 34 & -0.13 \\
\hline $0: 07$ & 321 & 89 & 25 & -0.6 & 1.9 & 0.69 & $1368^{\dagger}$ & 54.8 & 34 & -0.32 \\
\hline $1: 06$ & 322 & 90 & 24 & -0.6 & 1.9 & 0.69 & 1367 & 54.8 & 34 & -0.32 \\
\hline $2: 05$ & 323 & 92 & $26^{\dagger}$ & -0.6 & 1.9 & 0.69 & 1363 & 54.9 & 34 & 0.06 \\
\hline $3: 04$ & 324 & 93 & 26 & -0.6 & 1.9 & 0.68 & 1360 & 54.9 & 34 & 0.06 \\
\hline 03 & 325 & 94 & 26 & -0.6 & 1.9 & $0 . \overline{69}$ & 1359 & 54.9 & 35 & -0.13 \\
\hline & 326 & 96 & 26 & -0.6 & 1.9 & 0.69 & 1360 & 54.9 & 35 & -0.13 \\
\hline 01 & 327 & 97 & 26 & -0.6 & 1.9 & 0.69 & 1364 & $54 . \overline{9}$ & 35 & -0.17 \\
\hline 99 & 328 & 98 & 26 & -0.6 & 1.9 & 0.69 & 1364 & 54.9 & 35 & -0.17 \\
\hline 98 & $\overline{329}$ & 99 & 26 & -0.6 & 1.9 & $0.69^{+}$ & 1368 & 54.9 & 35 & -0.24 \\
\hline & 330 & 100 & 26 & -0.6 & 19 & $0.69 !$ & 1371 & 55 & 35 & -0.24 \\
\hline & 331 & 102 & 26 & -0.6 & 1.9 & 0.71 & $1370^{\circ}$ & 55 & 35 & 0.07 \\
\hline 95 & 332 & 103 & 26 & -0.6 & 1.9 & $0.6 \overline{9}$ & 1366 & 55 & 35 & 0.07 \\
\hline & $3 \overline{3} \overline{3}$ & 105 & 26 & -0.6 & 1.9 & 0.68 & 1363 & 55 & 35 & -0.09 \\
\hline & 334 & 106 & 26 & -0.6 & 1.8 & 0.68 & 1363 & 55 & 36 & -0.09 \\
\hline & 335 & 107 & 26 & -0.6 & 1.9 & 0.68 & 1363 & 55.2 & 36 & -0.28 \\
\hline & 336 & 109 & 26 & -0.6 & 1.9 & 0.69 & 1363 & 55.4 & 36 & -0.28 \\
\hline & 337 & 110 & 26 & -0.6 & 1.9 & 0.69 & 1365 & 55.4 & 36 & -0.15 \\
\hline & $338^{\prime}$ & $11 \overline{1}$ & 26 & -0.6 & 1.9 & 9 & 1368 & 55.5 & 36 & -0.15 \\
\hline & 339 & $11 \overline{2}$ & 26 & -0.6 & 1.9 & 0.69 & 1372 & 55.5 & 36 & \\
\hline & 340 & 113 & 26 & -0.6 & 1.9 & 0.69 & 1373 & 55.5 & 36 & \\
\hline & 341 & 114 & 26 & $-0.6 !$ & 1.9 & 0.69 & 1371 & 55.5 & 36 & $-0.0 \overline{6}$ \\
\hline & 34 & 115 & 26 & -0.6 & 1.9 & & $1367^{\dagger}$ & 55.4 & 36 & -0.06 \\
\hline $11: 82$ & 343 & 117 & 26 & -0.6 & 1.9 & 0.68 & 1370 & 55.4 & 36 & -0.25 \\
\hline & 34 & $1 \overline{18}$ & 26 & -0.6 & 1.9 & & 1365 & 55.2 & 36 & -0.06 \\
\hline & 34 & 119 & 26 & -0.6 & 1.9 & 0.69 & 1364 & 55.1 & 37 & -0.06 \\
\hline & 34 & $1 \overline{21}$ & 26 & $-0.6 !$ & 1.9 & 0.69 & 1365 & $5 \overline{5} . \overline{1}$ & 37 & -0.13 \\
\hline & 34 & 121 & $26 \mid$ & -0.6 & 1.9 & 0.69 & 1367 & 55.1 & 37 & -0.13 \\
\hline & 34 & 123 & 26 & -0.6 & 1.9 & 0.69 & 1372 & 55.1 & 37 & -0.02 \\
\hline$: 76$ & 34 & 124 & 26 & $-\overline{-0.6}$ & 1.9 & 0.69 & 1375 & 55.2 & 37 & 0.02 \\
\hline & 35 & $\overline{124}$ & 26 & -0.6 & 1.9 & 0.69 & 1374 & 55.2 & 37 & -0.32 \\
\hline & 351 & 126 & 26 & -0.6 & 1.8 & & & 55.2 & 37 & -0.32 \\
\hline & 35 & 127 & 26 & -0.6 & 1.9 & 0.68 & $1373^{\circ}$ & 55.2 & 37 & -0.11 \\
\hline $1: 71$ & 35 & 128 & 26 & -0.6 & 1.8 & 0.68 & 1369 & 55.3 & 37 & -0.11 \\
\hline $2: 70$ & 35 & 130 & 26 & $-0.6^{1}$ & 1.9 & 0.68 & 1365 & 55.3 & 37 & -0.02 \\
\hline & 35 & 131 & 26 & -0.6 & 1.9 & & & 55.4 & 37 & -0.02 \\
\hline & 35 & 132 & 26 & -0 & 1.9 & 0.69 & 1367 & 55.3 & 37 & -0.09 \\
\hline & 35 & 133 & 26 & -0.6 & 1.9 & 0.69 & 1370 & 55.3 & 38 & $-0 . \overline{09}$ \\
\hline 65 & 35 & $\overline{134}$ & 26 & -0.6 & 1.9 & 0.69 & 1374 & 55.4 & 37 & -0.31 \\
\hline $7: 64$ & 359 & 135 & 26 & -0.6 & 1.9 & 0.71 & 1372 & 55.5 & 37 & -0.31 \\
\hline & 36 & 136 & 26 & -0. & 1.9 & & 374 & 55.5 & 37 & -0.14 \\
\hline & 36 & 137 & 26 & -0.6 & 1.9 & 0.69 & 1375 & 55.6 & 37 & -0.14 \\
\hline $30: 61$ & 362 & 138 & 26 & -0.6 & 1.9 & $0.69^{1}$ & 1372 & 55.5 & 37 & 0 \\
\hline $31: 60$ & 363 & 139 & $26^{\dagger}$ & -0.6 & 1.9 & 0.68 & 1368 & $55 . \overline{5}$ & 38 & \\
\hline & 364 & 141 & 26 & -0.6 & 1.9 & & 1365 & 55.4 & 38 & -0.08 \\
\hline & 365 & 142 & 26 & -0.6 & 1.9 & 0.69 & 1365 & 55.3 & 38 & $-0 . \overline{08}$ \\
\hline & 366 & 143 & 26 & -0.6 & 1.9 & 0.69 & $1367^{\dagger}$ & 55.3 & 38 & -0.32 \\
\hline $35: 55$ & 367 & 144 & 26 & -0.6 & 1.9 & 0.69 & 1365 & 55.3 & 39 & -0.32 \\
\hline $09: 53: 36: 54$ & 368 & 144 & 26 & -0.6 & 1.9 & 0.71 i & 1367 & 55.3 & 38 & -0.09 \\
\hline $37: 53$ & $36 \overline{9}$ & 145 & 26 & $-0.6 !$ & 1.9 & 0.69 & 1372 & 55.3 & 39 & -0.09 \\
\hline $09: 53: 38: 52$ & $370^{\dagger}$ & 146 & 26 & $-\overline{0.6}$ & 1.9 & 0.69 & 1375 & 55.2 & 38 & -0.02 \\
\hline
\end{tabular}




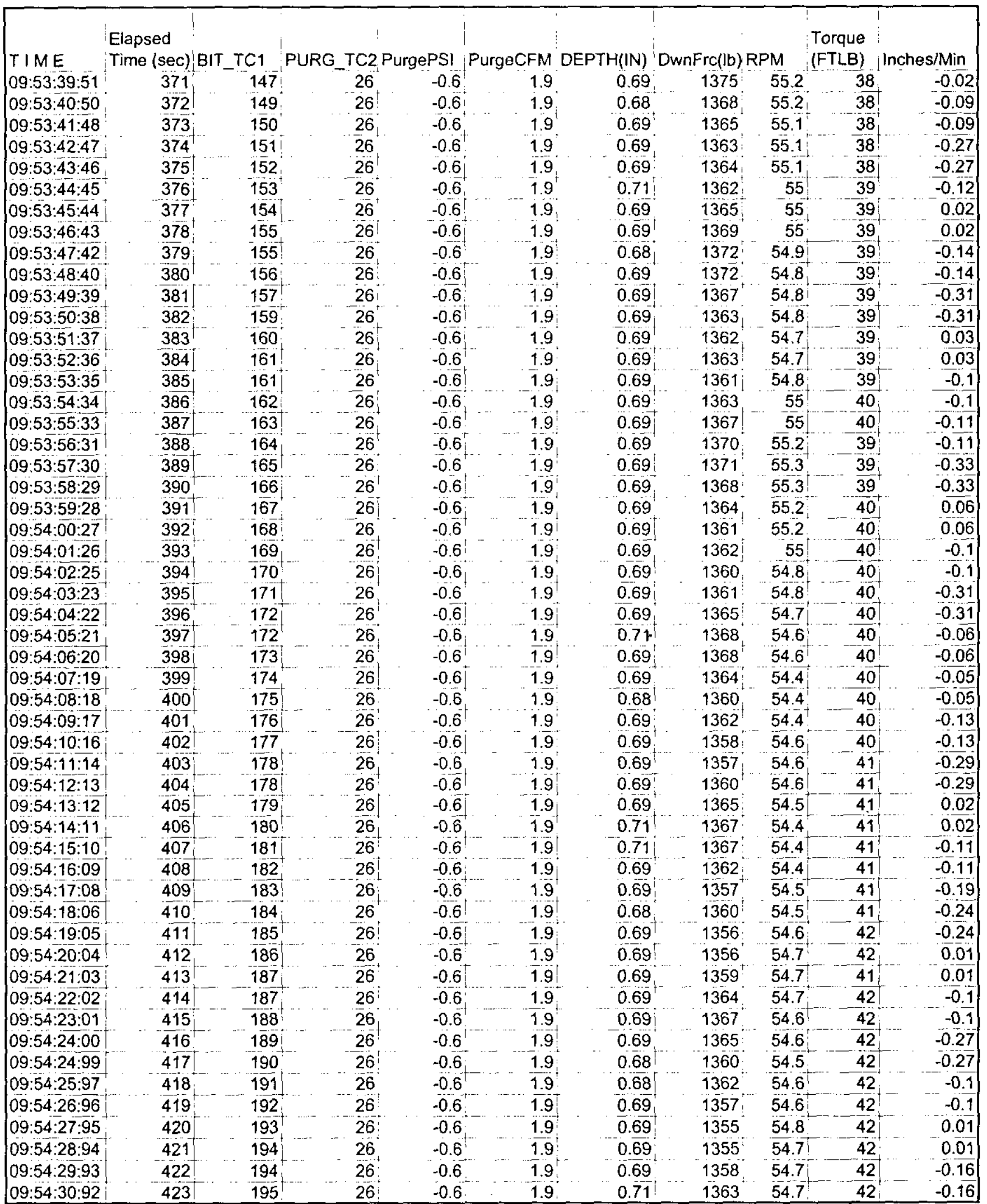




\begin{tabular}{|c|c|c|c|c|c|c|c|c|c|c|}
\hline TIME & $\begin{array}{l}\text { Elapsed } \\
\text { Time (sec) }\end{array}$ & BIT_TC1 & PURG_TC2 & PurgePSI & PurgeCFM & DEPTH(IN) & DwnFrc(lb) & RPM & $\begin{array}{l}\text { Torque } \\
\text { (FILB) }\end{array}$ & Inches/Min \\
\hline $09: 54: 31: 91$ & 424 & 196 & 26 & -0.6 & 1.9 & $\quad 0.69$ & 1365 & 54.7 & $\quad 42$ & $\quad-0.32$ \\
\hline $09: 54: 32: 89$ & 425 & 197 & 26 & -0.6 & 1.9 & 0.69 & 1363 & $54 . \overline{7}$ & $4 \overline{2}$ & $-0.3 \overline{2}$ \\
\hline $09: 54: 33: 88$ & 426 & 197 & 26 & -0.6 & 1.9 & $0.69^{\circ}$ & 1365 & 54.7 & 42 & $0 . \overline{06}$ \\
\hline $09: 54: 34: 87$ & 427 & 199 & 26 & -0.6 & 1.9 & 0.69 & 1361 & 54.7 & 43 & 0.06 \\
\hline $09: 54: 35: 86$ & 428 & 200 & 26 & -0.6 & $1 . \overline{9}$ & 0.68 & 1356 & 54.7 & 43 & -0.07 \\
\hline $09: 54: 36: 85$ & 429 & 201 & $26 !$ & -0.6 & 1.9 & $0.69^{\circ}$ & 1354 & 54.7 & 43 & -0.07 \\
\hline $09: 54: 37: 84$ & 430 & 201 & 26 & -0.6 & 1.9 & 0.69 & 1355 & 54.8 & 43 & -0.21 \\
\hline $09: 54: 38: 83$ & $43 \overline{1}$ & 202 & 26 & $-0.6 !$ & 1.9 & $0.69^{\top}$ & 1358 & 54.8 & 43 & -0.21 \\
\hline $9: 82$ & $\overline{432}$ & 203 & $2 \overline{6}$ & -0.6 & 1.9 & 0.69 & 1362 & 54.8 & 43 & -0.23 \\
\hline $09: 54: 4 \overline{0: 80}$ & 433 & 203 & 26 & $-0 . \overline{6}$ & 1.9 & 0.69 & 1364 & 54.9 & 43 & -0.23 \\
\hline $09: 54: 4179$ & 434 & 204 & $26^{\prime}$ & -0.6 & 1.9 & 0.69 & 1362 & $5 \overline{4.9}$ & 44 & $\overline{0}$ \\
\hline 78 & 435 & 205 & $26 !$ & -0.6 & 1.9 & 0.69 & 1363 & 55 & 43 & 0 \\
\hline $3: 77$ & $43 \overrightarrow{6}$ & 206 & 26 & -0.6 & 1.9 & 0.68 & $1360^{\circ}$ & 55 & 44 & -0.06 \\
\hline$: 44: 7 \overline{6}$ & 437 & 207 & 26 & -0.6 & 1.9 & 0.69 & 1355 & 55 & 44 & -0.06 \\
\hline $4: 45: 75$ & 438 & 208 & 26 & -0.6 & 1.9 & 0.68 & 1353 & 55 & 44 & -0.32 \\
\hline 74 & 439 & 209 & 26 & -0.6 & 1.9 & 0.69 & 13 & 55 & 44 & $-0 . \overline{32}$ \\
\hline $7: 72$ & 440 & 210 & 26 & -0.6 & 1.9 & 0.69 & 1355 & 55 & 43 & -0.05 \\
\hline $4: 48: 71$ & 441 & 210 & 26 & -0.6 & 1.9 & 0.69 & 1360 & 54.9 & 44 & -0.05 \\
\hline 70 & 442 & 211 & 26 & $-0 . \overline{6}$ & 1.9 & & & 54.9 & 44 & -0.09 \\
\hline 69 & 443 & 212 & 26 & -0.6 & 1.8 & 0.69 & & 55 & 44 & -0.09 \\
\hline 68 & 444 & 213 & 26 & $-0 . \overline{6}$ & 1.9 & 0.69 & 13 & 55.2 & $\overline{44}$ & $-0 . \overline{08}$ \\
\hline $2: 67$ & 445 & 213 & 26 & -0.6 & 1.9 & 0.69 & 1361 & 55.2 & 44 & -0.33 \\
\hline 66 & 446 & 214 & 26 & -0.6 & 1.9 & 0.69 & & 552 & 44 & -0.33 \\
\hline & $4 \overline{447}$ & $21 \overline{6}$ & 26 & -0.6 & 1.9 & 0.69 & 13 & 55.2 & 45 & -0.09 \\
\hline $5: 63$ & 448 & 217 & 26 & -0.6 & 1.9 & 0.69 & 1351 & $5 \overline{5.2}$ & 45 & -0.09 \\
\hline $6: 62$ & 449 & 218 & 26 & -0.6 & 1.9 & 0.69 & 13 & 55.1 & 45 & $-0 . \overline{03}$ \\
\hline 61 & 450 & 218 & 26 & -0.6 & 1.9 & & 13 & 55 & 45 & -0.03 \\
\hline & 45 & 21 & $26^{\dagger}$ & -0.6 & 1.9 & 0.71 & 135 & $5 \overline{5}$ & 45 & -0.13 \\
\hline 959 & 452 & 220 & 26 & -0.6 & 1.9 & 0.69 & & 54.9 & 45 & -0.13 \\
\hline & $4 \overline{5}$ & 220 & 26 & $-\overline{0}$ & 1.9 & & & $5 \overline{5.1}$ & $\overline{45}$ & $-\overline{0.31}$ \\
\hline & 45 & $\overline{221}$ & $26^{\dagger}$ & -0.6 & 1.9 & & & 55.1 & 45 & -0.31 \\
\hline 55 & 455 & 222 & 26 & -0.6 & 1.9 & 0.69 & 1356 & 55.1 & 45 & $-0 . \overline{0} 7$ \\
\hline & $4 \overline{56}$ & 224 & 26 & $-0 . \overline{6}$ & 1.9 & 0.69 & & 55.2 & $\overline{45}$ & -0.07 \\
\hline & 45 & & 26 & -0.6 & & & & & & 0.03 \\
\hline & 45 & 225 & $26 !$ & -0.6 & 1.9 & & & 55.2 & 45 & -0.03 \\
\hline 6.51 & 459 & 226 & 26 & -0.6 & 1.9 & 0.69 & $1355^{\circ}$ & 55.3 & 46 & -0.16 \\
\hline & 46 & 2 & 26 & -0 . & 1.9 & & & 55.3 & $\overline{46}$ & $-0 . \overline{16}$ \\
\hline & 46 & & $26\rceil$ & -0. & 1.9 & & & 55.2 & & -0.32 \\
\hline 47 & 46 & 228 & 26 & -0.6 & 1.9 & & 13 & 55.1 & 46 & -0.32 \\
\hline 46 & 463 & 229 & 26 & -0.6 & 1.9 & 0.71 & 1361 & $5 \overline{5.1}$ & $4 \overline{6}$ & 0.11 \\
\hline & 464 & 230 & 26 & -0.6 & 1.9 & 0.6 & & 55 & & $0 . \overline{11}$ \\
\hline & 46 & 23 & 26 & -0 & 1.9 & & & 54.9 & & -0.17 \\
\hline & 466 & 232 & 26 & $-0.6^{\dagger}$ & 1.9 & $0 . \bar{\epsilon}$ & $1350^{\circ}$ & 54.9 & 46 & -0.17 \\
\hline $1 \overline{4}: 42$ & 467 & 233 & $26^{\prime}$ & -0.6 & 1.9 & 0.69 & $1350^{\circ}$ & 55 & 46 & -0.12 \\
\hline 41 & 468 & 233 & 26 & -0.6 & 1.9 & 0.6 & & 55.1 & 46 & -0.12 \\
\hline & 469 & $\overline{234}$ & 26 & -0.6 & 1.9 & & & 55.1 & 47 & -0.33 \\
\hline & $\overline{470}$ & 235 & 26 & -0.6 & 1.9 & 0.69 & & 55.1 & 47 & -0.33 \\
\hline $5: 18: 37$ & $471^{\prime}$ & 235 & 26 & -0.6 & 1.9 & 0.69 & 1358 & 55.1 & 47 & $0 . \overline{05}$ \\
\hline $09: 55: 19: 36$ & 472 & $\overline{236}$ & 26 & -0.6 & 1.9 & 0.69 & 1360 & 55.1 & 47 & $0 . \overline{05}$ \\
\hline $09: 55: 20: 35$ & $47 \overline{3}$ & 237 & 26 & -0.6 & 1.9 & $0.6 \overline{9}$ & 1358 & 55.1 & 47 & -0.09 \\
\hline & 474 & 238 & 26 & -0.6 & 1.9 & 0.69 & 1354 & 54.9 & 46 & -0.09 \\
\hline & $4 \overline{7} 5^{\dagger}$ & 239 & 26 & -0.6 & 1.9 & & 13 & 54.9 & 47 & $-0 . \overline{23}$ \\
\hline $09: 55: 23: 32$ & $476 !$ & 240 & $26^{\prime}$ & -0.6 & 1.9 & 0.69 & 1353 & 55 & 47 & -0.23 \\
\hline
\end{tabular}




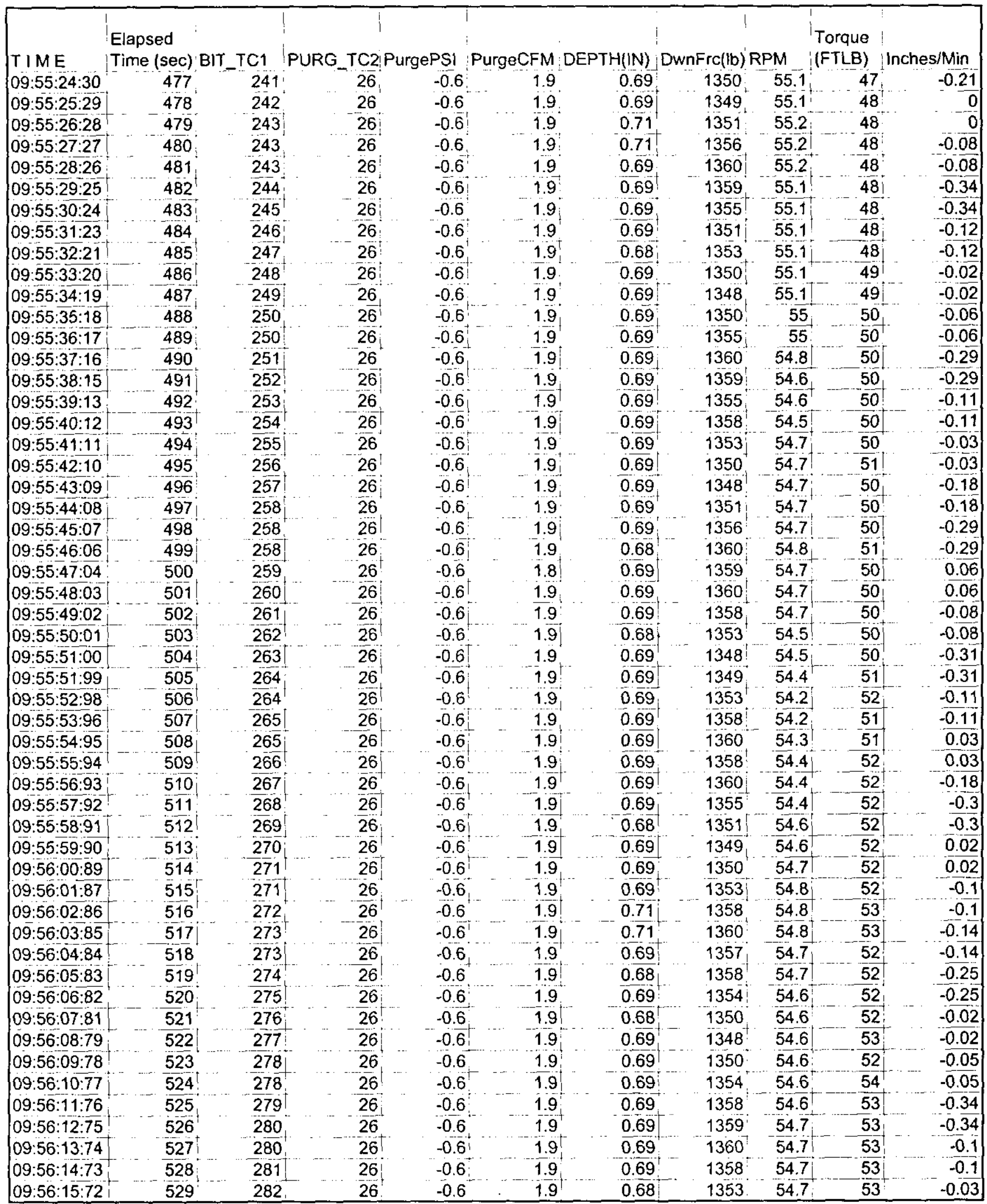




\begin{tabular}{|c|c|c|c|c|c|c|c|c|c|c|}
\hline$T I M E$ & $\begin{array}{l}\text { Elapsed } \\
\text { !Time (sec) }\end{array}$ & & PURG_TC2F & PurgePSI & PurgeCFM & EPTH(IN) & DwnFrc(lb) & RPM & & Inches/Min \\
\hline $09: 56: 16: 70$ & 530 & -283 & 26 & -0.6 & 19 & 0.68 & $\quad 1350$ & 54.7 & 53 & -0.03 \\
\hline $09: 56: 17: 69$ & 531 & 284 & $26^{\dagger}$ & -0.6 & 1.9 & 0.69 & 1348 & 54.8 & 53 & -0.18 \\
\hline $09: 56: 18: 68$ & 532 & 285 & 26 & -0.6 & 1.9 & 0.69 & 1350 & 54.9 & 53 & -0.18 \\
\hline $9: 56: 19: 67$ & 533 & 285 & 26 & -0.6 & 1.9 & 0.69 & 1354 & 54.9 & 53 & -0.3 \\
\hline $20: 66$ & 534 & 286 & $26 !$ & -0.6 & 1.9 & $0 . \overline{71}$ & 1358 & 54.9 & 53 & $-0 . \overline{3}$ \\
\hline $21: 65$ & 535 & 286 & $26^{\circ}$ & -0.6 & 1.8 & 0.69 & 1356 & 54.9 & 53 & 0.02 \\
\hline $56: 22: 64$ & 536 & 287 & 26 & $-0.6^{\dagger}$ & 2.3 & 0.69 & $1360^{\circ}$ & 54.9 & 53 & 0.02 \\
\hline $3: 62$ & 537 & 286 & 26 & 0.6 & 5.6 & 0.69 & 1358 & 55 & 52 & -0.08 \\
\hline & 538 & 284 & 26 & 0.6 & 5.7 & 0.69 & 1353 & 55 & 51 & $-0 . \overline{08}$ \\
\hline $5: 60$ & 539 & 283 & 26 & 2.9 & 25 & 0.69 & 1348 & $5 \overline{5}$ & 50 & -0.14 \\
\hline $6: 59$ & 540 & $279^{\dagger}$ & 26 & 6.2 & 25.7 & 0.69 & 1345 & 55 & 49 & -0.14 \\
\hline $7: 58$ & $5 \overline{4} \overline{1}$ & 274 & 26 & 8.1 & 28.7 & 0.69 & 344 & 55.1 & 47 & -0.32 \\
\hline & 542 & 270 & 26 & 8.3 & 28.8 & 0.69 & 1346 & 55.1 & 47 & -0.32 \\
\hline 29.56 & 543 & 265 & 26 & 9.5 & 31.2 & 0.69 & 1343 & 55.2 & 46 & 0.05 \\
\hline $0: 54$ & 544 & 260 & 26 & $16.1^{\dagger}$ & $442^{\dagger}$ & 0.69 & 346 & 55.2 & 45 & -0.11 \\
\hline & 545 & 255 & $26^{\prime}$ & 23.5 & 46.6 & 0.69 & 1348 & 55.2 & 44 & -0.11 \\
\hline & $5 \overline{46}$ & 246 & 26 & 26.5 & 46.9 & 0.69 & 13 & 55 & $\overline{42}$ & -0.22 \\
\hline $33: 51$ & 547 & 238 & 26 & 27.8 & 46.9 & 0.69 & 1341 & 54.9 & 40 & -0.22 \\
\hline & 548 & 232 & $26 !$ & 27.8 & 47 & $0 . \overline{6} 9$ & & 54.9 & 40 & -0.2 \\
\hline & 549 & 225 & 26 & 2 & $47^{4}$ & & & 5.1 & 38 & -0.2 \\
\hline 48 & 550 & 218 & $26 !$ & 28 & 47 & 0.71 & 328 & 55.3 & 36 & $0.0 \overline{2}$ \\
\hline $77: 47$ & 551 & 213 & 26 & 28 & 47 & 0.69 & 1325 & 55.3 & 35 & 0.02 \\
\hline & 552 & 207 & 26 & 28 & 47 & 0.71 & & 55.6 & 33 & -0.1 \\
\hline & 553 & 201 & $26 !$ & 27.8 & 47 & & & 55.7 & 32 & -0.1 \\
\hline & 554 & 196 & 26 & 28 & 47 & 0.68 & & 55.7 & 32 & -0.19 \\
\hline & 555 & 191 & $\overline{26}$ & 28 & 47 & 0.69 & 1330 & 55.6 & 31 & -0.19 \\
\hline & 556 & 186 & 26 & 28 & 47.1 & 0.69 & & 55.7 & 30 & -0.33 \\
\hline & 557 & $18 \overline{2}$ & 26 & 28 & 47.1 & 0.69 & & 55.7 & 29 & -0.33 \\
\hline & 558 & 177 & 26 & 28 & 47.1 & 0.69 & 1321 & 55.7 & 28 & 0.07 \\
\hline & 559 & 172 & $26 !$ & 27.8 & $\overline{47} .1$ & 0.68 & & 5.7 & 28 & 0.07 \\
\hline & 560 & 169 & 26 & 278 & 47.1 & 0.69 & & 55.7 & 27 & -0.11 \\
\hline & 561 & 165 & 26 & 27.8 & 47.2 & 0.69 & 309 & 55.6 & 27 & -0.11 \\
\hline & 562 & 161 & 26 & 27.8 & 47.2 & 0.71 & & 55.4 & 26 & -0.11 \\
\hline & 56 & 158 & 26 & & 47.2 & 0.69 & & 55.4 & 26 & -0.11 \\
\hline & 564 & $154^{\dagger}$ & $26^{\prime}$ & 27.8 & 47.2 & 0.69 & & 55.4 & 25 & -0.33 \\
\hline & 56 & 150 & $26 !$ & 27.8 & $4 \overline{7.2}$ & 0.71 & & 55.5 & 26 & -0.33 \\
\hline & 566 & 147 & 26 & 27.8 & 47.2 & 0.69 & & 55.5 & $\overline{25} \dagger$ & 0.07 \\
\hline & 567 & $14 \overline{4}$ & 26 & & 47.2 & & & 55.6 & 25 & 0.07 \\
\hline & 568 & 140 & $26 !$ & 27.8 & 47.3 & 0.69 & & 55.6 & 25 & -0.16 \\
\hline 526 & 569 & 138 & 26 & 27.6 & 47.3 & 0.69 & & 55.6 & 25 & -0.16 \\
\hline $56: 25$ & 570 & 135 & 26 & 27.6 & 47.4 & 0.68 & $1303 !$ & 55.5 & 24 & -0.08 \\
\hline & 571 & 132 & 26 & 27.6 & 47.4 & 0.69 & & 55.5 & 24 & -0.08 \\
\hline 23 & 572 & 130 & 26 & 27.6 & 47.5 & 0.69 & 12 & 55.4 & 24 & -0.35 \\
\hline $59: \overline{22}$ & 573 & 127 & 26 & 27.6 & 47.5 & 0.69 & 1299 & 55.4 & 24 & -0.35 \\
\hline $00: 20$ & 574 & 125 & 26 & 27.6 & $47.5^{\dagger}$ & 0.71 & 1304 & $55 . \overline{4}$ & $\overrightarrow{23}$ & 0.02 \\
\hline $01: 19$ & 575 & $\overline{122}$ & 26 & 27.6 & 47.5 & 0.71 & & 55.4 & $2 \overline{3}$ & 0.02 \\
\hline & 576 & 120 & $26 !$ & 276 & 47.5 & & & 55.4 & 23 & -0.1 \\
\hline & 577 & 118 & $26^{\prime}$ & 27.6 & 47.5 & 0. & 130 & 55.4 & 23 & -0.1 \\
\hline $09: 57: 0 \overline{4}: 16$ & 578 & 116 & 26 & 27.6 & 47.5 & 0.69 & 1302 & 55.4 & 23 & -0.14 \\
\hline $09: 57: 05: 15$ & $5 \overline{79}$ & 113 & 26 & 27.6 & 47.6 & 0.69 & 12 & 55.4 & 23 & -0.31 \\
\hline $09: 57: 06: 14$ & 580 & 111 & 26 & 27.6 & $47.6 !$ & 0.69 & 1293 & 55.4 & 22 & -0.31 \\
\hline $07: 13$ & 581 & 110 & 26 & 27.6 & 47.6 & 0.71 & & 55.5 & 22 & 0.02 \\
\hline $09: 57: 08: 11$ & $5 \overline{8} 2$ & 108 & 26 & 27.6 & 47.6 & 0.69 & $1289^{\circ}$ & 55.5 & 22 & 0.02 \\
\hline
\end{tabular}




\begin{tabular}{|c|c|c|c|c|c|c|c|c|c|c|}
\hline TIME & $\begin{array}{l}\text { Elapsed } \\
\text { Time (sec) }\end{array}$ & BIT_TC1 & PURG_TCZ & PurgePS| & PurgeCFM & DEPTH(IN) & DwnFrc $(\mathrm{bb})$ & RPM & $\begin{array}{l}\text { Torque } \\
\text { (FTLB) }\end{array}$ & Inches/Min \\
\hline $09: 10$ & 583 & 106 & 26 & 27.6 & 47.6 & $\quad 0.71$ & 1291 & 55.5 & 22 & -0.1 \\
\hline $09: 57: 10: 09$ & 584 & 105 & 26 & 27.6 & 47.6 & 0.71 & 1295 & $5 \overline{5.4}$ & 22 & -0.1 \\
\hline 09.5 & 585 & 103 & 26 & 27.6 & 47.6 & 0.71 & 1291 & 55.4 & 22 & -0.13 \\
\hline $09: 57: 1$ & 586 & 102 & 26 & 27.6 & 47.6 & 0.69 & 1294 & 55.4 & 22 & -0.13 \\
\hline $09: 57: 13: 06$ & 587 & 100 & $26 i$ & 27.6 & 47.6 & 0.69 & 1296 & 55.5 & 22 & -0.36 \\
\hline $09: 57: 14: 05$ & 588 & 99 & 26 & 27.6 & 47.6 & 0.69 & 1294 & 55.5 & 22 & -0.36 \\
\hline & & & & & ...- & & & & & \\
\hline
\end{tabular}




\begin{tabular}{|c|c|c|c|c|c|c|c|c|c|c|}
\hline $\mathrm{TIME}$ & $\begin{array}{l}\text { Elapsed } \\
\text { Time } \\
\text { (sec) }\end{array}$ & $B \mid T \_T C 1$ & PURG_TC2 & PurgePSI ! & PurgeCFM L & DEPTH $($ IN $)$ & DwnFrc(lb) & RPM & $\begin{array}{l}\text { Torque } \\
\text { (FTLB) }\end{array}$ & Inches/Min \\
\hline $10: 56: 15: 26$ & 0 & 26 & $\quad 25$ & 18.9 & $\quad 55.3$ & $\quad-0.72$ & 2 & 57.2 & 1 & -0.11 \\
\hline $10: 56: 16: 30$ & 1 & 26 & 25 & 18.9 & 55.3 & -0.59 & 3 & 57 & 1 & 0.76 \\
\hline $10: 56: 17: 29$ & 2 & 26 & 25 & 18.9 & $5 \overline{5.3}$ & $-0 . \overline{3} 9^{\prime}$ & 2 & 56.7 & 1 & 0.76 \\
\hline $10: 56: 18: 28$ & 3 & 26 & 25 & $18.9^{+}$ & 55.3 & -0.18 & 12 & 56.7 & 2 & 10.41 \\
\hline $10: 56: 19: 32$ & 4 & 26 & - & 19.1 & $55 . \overline{4}$ & -0.03 & 412 & 56.5 & $19 !$ & 10.41 \\
\hline $10: 56: 20: 31$ & 5 & 29 & 25 & $19.2 !$ & 55.4 & 0.01 & 908 & 56.2 & 46 & 6.57 \\
\hline $10: 56: 21: 30$ & $\overline{6}$ & 33 & $25^{\circ}$ & 19.2 & 55.4 & 0.05 & 1057 & 56.2 & 50 & $6.5 \overline{7}$ \\
\hline $10: 56: 22: 29$ & 7 & 37 & 25 & 19.4 & 55.4 & 0.08 & 1137 & 55.4 & $499^{\dagger}$ & 1.87 \\
\hline $10: 56: 23: 28$ & 8 & 42 & 25 & 19.4 & 55.4 & 0.1 & $11 \overline{72}$ & 55.1 & 48 & 1.87 \\
\hline $10: 56: 24: 32$ & 9 & 45 & 25 & 19.4 & 55.4 & 0.11 & 1202 & $55.1^{\dagger}$ & $47^{\dagger}$ & $0 . \overline{83}$ \\
\hline $10: 56: 25: 31$ & 10 & 47 & & $19.6^{\dagger}$ & 55.4 & 0.14 & 1235 & $54.9^{\prime}$ & 46 & 0.83 \\
\hline $10: 56: 26: 30$ & 11 & 49 & $\ldots$ & 19.6 & 55.4 & 0.15 & $125 \overline{7}$ & 54.8 & $45]$ & 0.67 \\
\hline $10: 56: 27: 29$ & 12 & 51 & 25 & 19.6 & 55.4 & 0.14 & 1274 & 54.8 & 43 & $0.6 \overline{7}$ \\
\hline $10: 56: 28: 27$ & 13 & 51 & 25 & 19.6 & 55.4 & 0.14 & $12811^{\dagger}$ & 54.6 & 46 & 0.53 \\
\hline $10: 56: 29: 32$ & 14 & 52 & & 19.8 & 55.4 & 0.15 & 1274 & 54.5 & 45 & 0.53 \\
\hline $10: 56: 30: 31$ & 15 & 54 & & 19.8 & 55.4 & 0.15 & 1279 & 54.5 & 47 & 0.13 \\
\hline $10: 56: 31: 30$ & 16 & 56 & 25 & 19.8 & $55 . \overline{4}$ & 0.17 & 1292 & 54.6 & 46 & 0.52 \\
\hline $10: 56: 32: 34$ & 17 & 60 & 25 & 20 & 55.4 & $0.19^{\dagger}$ & $1306^{\dagger}$ & 54.7 & 47 & 0.52 \\
\hline $10: 56: 33: 33$ & 18 & 63 & & 20 & 55.4 & 0.19 & 1304 & 54.7 & 47 & 0.6 \\
\hline $10: 56: 34: 32$ & 19 & 65 & 25 & 20 & 55.4 & 0.2 & $1310^{\circ}$ & 54.7 & 46 & 0.6 \\
\hline $10: 56: 35: 36$ & 20 & $65^{\dagger}$ & 25 & $20^{\dagger}$ & 55.4 & 0.21 & 1322 & 54.4 & $47^{\dagger}$ & 0.17 \\
\hline $10: 56: 36: 35$ & 21 & 65 & - . & 20.2 & 55.4 & 0.21 & $1335^{\circ}$ & 54.4 & 46 & $0 . \overline{17}$ \\
\hline $7: 34$ & 22 & 64 & 25 & 20.2 & 55.4 & $\underline{0} .2$ & 1343 & 54 & 45 & 0.17 \\
\hline $10: 56: 38: 33$ & 23 & 65 & 25 & 20.2 & $55 . \overline{3}$ & 0.21 & 1348 & 54 & 44 & 0.17 \\
\hline $10: 56: 39: 31$ & 24 & 65 & 25 & 20.4 & 55.3 & 0.2 & 1357 & 54 & 44 & 0.23 \\
\hline $40: 36$ & 25 & 65 & 25 & 20.4 & 55.3 & 0.21, & 1347 & $54.1\}$ & 44 & 0.23 \\
\hline $1: 35$ & 26 & 66 & $25_{j}^{\circ}$ & 20.4 & 55.3 & 0.23 & 1341 & 54.1 & 43 & -0.04 \\
\hline $2: 34$ & 27 & 69 & 25 & 20.6 & 55.2 & $0 . \overline{23}$ & $133 \overline{8}$ & 54.1 & 41 & $-0 . \overline{04}$ \\
\hline $10: 56: 43: 38$ & 28 & 70 & 25 & 20.6 & 55.2 & 0.24 & 1341 & 54.1 & 41 & -0.17 \\
\hline $10: 56: 44: 37$ & 29 & 71 & 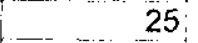 & 20.6 & 55.2 & 0.24 & 1353 & 54.2 & 41 & -0.17 \\
\hline $45: 36$ & 30 & 67 & 25 & $20.6 !$ & 55.1 & 0.24 & 1362 & 54.2 & 40 & 0.27 \\
\hline $10: 56: 46: 35$ & 31 & 67 & 25 & 20.8 & 54.8 & 0.23 & 1364 & 54.3 & 39 & 0.27 \\
\hline 7:33! & 32 & 67 & 25 & 20.8 & 54.8 & 0.23 & 1363 & 54.3 & 38 & -0.02 \\
\hline $10: 56: 48: 38$ & 33 & 67 & 25 & 20.8 & 54.6 & 0.24 & $1362 !$ & $54.3^{\dagger}$ & 38 & -0.02 \\
\hline 37 & 34 & $6 \overline{8}$ & 25 & 21 & 54.6 & 0.23 & $1360^{\circ}$ & $54.4 !$ & 40 & -0.38 \\
\hline$: 35$ & 35 & 68 & 25 & 21.2 & $54 . \overline{5}$ & 0.24 & 1362 & $54 . \overrightarrow{4}$ & 38 & -0.38 \\
\hline 51:40 & 36 & 69 & $\quad 25$ & 21.6 & 54.5 & 0.24 & 1359 & 54.4 & 37 & -0.02 \\
\hline $10: 56: 52: 39$ & 37 & 70 & 25 & 21.6 & 54.5 & 0.24 & $1 \overline{364}$ & 54.6 & 37 & 0.02 \\
\hline $10: 56: 53: 38$ & 38 & 71 & 25 & 21.8 & 54.5 & 0.26 & 1370 & 54.7 & 37 & 0.02 \\
\hline $10: 56: 54: 36$ & 39 & 70 & 25 & 21.8 & 54.5 & 0.25 & 1372 & 54.7 & 37 & -0.11 \\
\hline$: 55: 35$ & 40 & 70 & 25 & 21.8 & 54.4 & 0.25 & 1369 & $5 \overline{4.7}$ & 37 & -0.11 \\
\hline $56: 40$ & 41 & 68 & 25 & 21.8 & 54.4 & 0.24 & 1372 & 54.9 & 37 & -0.24 \\
\hline $57: 39$ & 42 & 68 & 25 & 21.8 & 54.4 & 0.24 & 1371 & $54 . \overline{9}$ & 37 & -0.24 \\
\hline $58: 37$ & 43 & 69 & 25 & 21.8 & 54.4 & 0.24 & 1367 & 54.7 & 36 & $-0 . \overline{11}$ \\
\hline $10: 56: 59: 36$ & 44 & 69 & 25 & 22 & 54.4 & 0.24 & 1364 & 54.7 & 36 & -0.11 \\
\hline $10: 57: 00: 35$ & 45 & 69 & 25 & 22 & 54.3 & 0.24 & 1364 & 54.7 & 36 & 0.02 \\
\hline 1:39 & 46 & 69 & 25 & 22 & 54.3 & 0.25 & 1368 & $54.8^{\dagger}$ & 36 & 0.02 \\
\hline $10: 57: 02: 38$ & 47 & 70 & 25 & 22.2 & 54.2 & 0.25 & 1367 & 54.9 & 35 & -0.19 \\
\hline $10: 57: 03: 37$ & 48 & 71 & 25 & 22.2 & 54.2 & 0.26 & $137 \overline{0}$ & 54.9 & 35 & -0.19 \\
\hline $10: 57: 04: 42$ & 49 & 70 & 25 & 22.2 & 54.2 & 0.25 & 1377 & 55 & 36 & -0.25 \\
\hline $10: 57: 05: 40$ & 50 & 69 & 25 & 22.2 & 54.1 & 0.26 & 1378 & $54.9^{\dagger}$ & 35 & -0.25 \\
\hline $10: 57: 06: 39$ & 51 & 67 & 26 & 22.4 & 54.1 & 0.26 & 1375 & 54.9 & 35 & 0.08 \\
\hline
\end{tabular}




\begin{tabular}{|c|c|c|c|c|c|c|c|c|c|c|}
\hline TIME & $\begin{array}{l}\text { Elapsed } \\
\text { Time } \\
\text { (sec) }\end{array}$ & $B \mid T \_T C 1$ & |PURG_TC2! & PurgePS! & PurgeCFM & DEPTH(IN) & DwnFrc(lb) & RPM & $\begin{array}{l}\text { Torque } \\
\text { (FTLB) }\end{array}$ & s/Min \\
\hline $10: 57: 07: 38$ & 52 & -67 & 126 & 22.4 & 54.1 & 0.26 & 1372 & 54.8 & $\quad 35$ & 0.08 \\
\hline $0: 57: 08: 37$ & 53 & 67 & $25^{\prime}$ & 22.4 & 54.1 & 0.26 & 1369 & 54.8 & 35 & -0.09 \\
\hline $10: 57: 09: 41$ & 54 & 67 & 26 & 22.4 & 54.1 & 0.25 & 1363 & 54.8 & 35 & -0.09 \\
\hline $10: 57: 10: 40$ & 55 & 67 & 26 & 22.6 & $54.1^{1}$ & 0.26 & 1358 & 54.9 & 35 & -0.32 \\
\hline $10: 57: 11: 39$ & 56 & 68 & 25 & 22.4 & 54 & 0.26 & 1354 & 55 & 34 & -0.32 \\
\hline$: 43$ & 57 & 68 & 25 & 22.4 & 54 & 0.26 & 355 & 55 & 34 & $-0 . \overline{09}$ \\
\hline $10: 57: 13: 42$ & 58 & 68 & 25 & 22.6 & 53.9 & 0.25 & 1351 & 54.9 & 34 & -0.09 \\
\hline $10: 57: 14: 41$ & 59 & 69 & $25 !$ & 22.6 & 53.9 & $0 . \overline{28}$ & 1353 & 54.9 & 33 & -0.17 \\
\hline $0: 57: 15: 40$ & $60^{!}$ & 69 & 25 & 22.6 & 53.9 & 0.26 & 1355 & 54.9 & 33 & 0.04 \\
\hline 39 & $61 \mathrm{i}$ & 69 & 25 & 22.6 & 53.8 & 0.26 & 1354 & 55 & 33 & 0.04 \\
\hline 38 & 62 & 67 & $25 !$ & 22.8 & 53.8 & 0.26 & 1351 & 54.9 & 34 & -0.34 \\
\hline 37 & 63 & 67 & 25 & 22.8 & 53.8 & 0.25 & 1347 & 54.9 & 33 & -0.34 \\
\hline $9: 41$ & 64 & 67 & 25 & 22.8 & 53.7 & 0.26 & $1343^{\dagger}$ & 54.9 & 32 & -0.09 \\
\hline$: 45$ & 65 & 67 & 26 & 22.8 & 53.7 & 0.26 & 1342 & 54.8 & 32 & -0.09 \\
\hline & 66 & 67 & 26 & 22.8 & 53.7 & 0.26 & $134 \vec{i}$ & 54.8 & 32 & $-0 . \overline{02}$ \\
\hline 43 & 67 & 68 & 26 & 22.8 & 53.6 & 0.28 & $42^{+}$ & 54.8 & 32 & $-0 . \overline{02}$ \\
\hline $23: 42$ & 68 & 68 & 26 & 22.9 & 53.6 & 0.26 & $1345^{1}$ & 55 & 32 & $-0 . \overline{22}$ \\
\hline $24: 41$ & 69 & 70 & 26 & 22.8 & 53.6 & 0.26 & 1348 & 55 & 33 & -0.22 \\
\hline $5: 45$ & 70 & 70 & 26 & 22.9 & 53.6 & 0.26 & $46^{7}$ & 55.1 & 33 & -0.21 \\
\hline & $71 i$ & 69 & 26 & 22.9 & 53.5 & 0.29 & $1350^{\dagger}$ & 55.1 & 32 & -0.21 \\
\hline & $72^{\dagger}$ & 66 & 26 & 22.9 & & 0.2 & 1348 & 55.1 & 32 & -0.1 \\
\hline $8: \overline{42}$ & $73 !$ & 66 & 26 & 23.1 & $53.5^{\dagger}$ & $0.26]$ & 1345 & 55 & 32 & -0.1 \\
\hline 41 & $74 i$ & 66 & 26 & 23.3 & 53.5 & 0.26 & $1343^{\dagger}$ & 54.8 & 33 & -0.04 \\
\hline & 75 & 66 & 26 & 23.5 & 3.5 & 0.25 & $1341 \mid$ & 54.8 & 33 & -0.04 \\
\hline & $7 \overline{6}$ & 65 & 26 & 23.5 & 53.5 & 0.25 & 1342 & 54.9 & 32 & -0.18 \\
\hline & 77 & 65 & 26 & 23.9 & 53.5 & 0.26 & 1341 & 54.8 & 32 & $-0 . \overline{18}$ \\
\hline $3: 47$ & 78 & 65 & 26 & 23.9 & 53.5 & 0.26 & 1343 & 54.8 & 32 & -0.29 \\
\hline $4: 46$ & 79 & 68 & 26 & 23.9 & 53.5 & 0.29 & 48 & 54.8 & 32 & -0.29 \\
\hline 45 & 80 & 68 & 26 & 23.9 & 53.5 & 0.29 & & 54.8 & 32 & 0.05 \\
\hline & 81 & 68 & 26 & 23.9 & 53.5 & 0.29 & $1350^{\dagger}$ & 54.8 & 32 & 0.05 \\
\hline & 82 & 65 & 26 & $23.9^{\dagger}$ & 53.5 & 0.26 & $1353 !$ & 54.9 & 32 & 0.06 \\
\hline 47 & 83 & 65 & 26 & 24.1 & $53.5^{\dagger}$ & 0.26 & $1353 !$ & 54.8 & 32 & -0.37 \\
\hline & 84 & 65 & 26 & & & & & & 32 & -0.37 \\
\hline & 85 & 65 & 26 & 4.1 & & & $1345^{\dagger}$ & 54 & 34 & -0.16 \\
\hline & 86 & 65 & 26 & 24.3 & 3.5 & 0.29 & 1344 & 54.7 & 31 & -0.16 \\
\hline $42: 48$ & 87 & 65 & 26 & 24.3 & 53.4 & 0.29 & 1346 & 54.7 & $31 \mid$ & 0.04 \\
\hline $3: 47$ & 88 & 65 & 26 & 24.3 & 53.4 & 0.29 & $1346 !$ & 54.7 & 31 & 0.04 \\
\hline & 89 & 66 & 26 & 24.3 & 3.4 & 0.29 & 1350 & 54.7 & 31 & $-0 . \overline{14}$ \\
\hline & 90 & 67 & $26^{\dagger}$ & 24.3 & 53.4 & & & 54.7 & 31 & -0.14 \\
\hline$: 49$ & $\overline{91}$ & 66 & 26 & 13 & 53.4 & 0.28 & 1357 & 54 & 31 & -0.28 \\
\hline $47: 48$ & 92 & 66 & 26 & 24.5 & 53.4 & 0.28 & 1359 & 54.5 & 31 & $-0 . \overline{28}$ \\
\hline $10: 57: 48: 47$ & $93 \uparrow$ & 64 & 26 & 24.5 & 53.4 & 0.26 & 1357 & 54.5 & 32 & -0.19 \\
\hline $9: 51$ & 94 & 65 & 26 & 24.5 & 53.4 & & & 54 & 32 & -0.19 \\
\hline & 95 & 65 & 26 & $24.5^{\top}$ & & & & 55 & 32 & 0.05 \\
\hline & 96 & 65 & 26 & 24.5 & 3.4 & & 1350 & 55 & 31 & 0.05 \\
\hline $52: 4 \overline{8}$ & 97 & 65 & 26 & 24.5 & 53.4 & $0 . \overline{28}$ & 1349 & 54.9 & 30 & $-0 . \overline{22}$ \\
\hline $10: 57: 53: 46$ & $\overline{98}$ & 65 & $26 !$ & 24.5 & $53 . \overrightarrow{4}$ & 0.28 & 1349 & 55 & 30 & -0.22 \\
\hline $10: 57: 54: 51$ & $99^{\dagger}$ & 66 & 26 & 24.5 & 53.4 & 0.29 & 1351 & 55 & 30 & -0.3 \\
\hline $10: 57: 55: 50$ & $100 !$ & 67 & 26 & 24.5 & $53.4 !$ & 0.29 & $1355^{\prime}$ & 55 & $31 \mid$ & -0.3 \\
\hline $56: 48$ & 101 & 66 & 26 & 24.5 & 53.4 & 0.29 & 1358 & 55 & 31 & -0.03 \\
\hline & 102 & 66 & 26 & 24.7 & 53.4 & 0.28 & 1358 & 55 & 31 & -0.03 \\
\hline $10: 57: 58: 46$ & $103^{\dagger}$ & 64 & 26 & 24.5 & 53.4 & $0.29 !$ & 1356 & $54.9^{+}$ & 31 & -0.07 \\
\hline
\end{tabular}




\begin{tabular}{|c|c|c|c|c|c|c|c|c|c|c|}
\hline TIME & \begin{tabular}{l|} 
Elapsed \\
Time \\
$(\mathrm{sec})$
\end{tabular} & $B \mid T-T C 1$ & PURG_TC2 & PurgePSI & PurgeCFM & DEPTH(IN) & DwnFrc(lb) & RPM I & $\begin{array}{l}\text { Torque } \\
\text { [(FTLB) }\end{array}$ & Inches/Min \\
\hline $10.57: 59: 51$ & 104 & $\quad 64$ & 26 & $\quad 24.9^{\prime}$ & $\quad 53.4$ & $\quad 0.29$ & 1358 & 54.8 & 31 & -0.07 \\
\hline $10: 58: 00: 49$ & 105 & 65 & 26 & 24.9 & 53.3 & 0.28 & 1355 & 54.8 & 31 & -0.26 \\
\hline $10: 58: 01: 48$ & 106 & 65 & 26 & $24.9^{t}$ & 53.3 & 0.26 & 1351 ! & 54.7 & 31 & -0.15 \\
\hline 53 & 107 & 65 & 26 & 24.9 & 53.3 & 0.29 & 1351 & $54.6^{\dagger}$ & 31 & -0.15 \\
\hline 03.52 & 108 & 65 & 26 & 24.9 & 53.3 & 0.28 & $1353^{\dagger}$ & 54.6 & 31 & -0.05 \\
\hline 50 & 109 & $6 \overline{5}$ & 26 & 24.9 & 53.3 & 0.28 & 1357 & $54.7^{+}$ & 31 & -0.05 \\
\hline $5: 49$ & 110 & 67 & 26 & 24.9 & 53.3 & 0.29 & 1354 & 54.8 & 31 & \\
\hline 48 & 111 & $66^{\dagger}$ & 26 & $24.9^{!}$ & 53.3 & 0.29 & 1360 & 54.8 & 31 & \\
\hline$: 47 !$ & 112 & 66 & 26 & 24.9 & 53.2 & 0.26 & 1363 & 54.8 & 31 & -0.33 \\
\hline 46 & 113 & 64 & 26 & 24.9 & 53.2 & 0.29 & 1362 & 54.9 & 31 & -0.33 \\
\hline & 114 & 64 & 26 & 24.9 & 53.1 & 0.28 & 1358 & 54.9 & 32 & -0.23 \\
\hline 44 & 115 & 64 & 26 & 24.9 & 53.1 & 0.26 & & 54.9 & 32 & -0.23 \\
\hline & 116 & 65 & 26 & 24.9 & 53.1 & 28 & & 54.9 & 31 & 0.07 \\
\hline & 117 & 65 & 26 & 24.9 & 53.1 & 29 & & 54.9 & 31 & 0.07 \\
\hline & 118 & 65 & 26 & 24.9 & 53 & 0.29 & & 54.9 & 31 & -0.2 \\
\hline & 119 & 68 & 26 & 24.9 & 53 & 0.28 & & 55 & 31 & -0.2 \\
\hline & 120 & 68 & 26 & 24.9 & 53 & 0.29 & 1363 & 55 & 31 & -0.26 \\
\hline 42 & 121 & 67 & 26 & 24.9 & 53 & 29 & 1366 & 55 & 31 & -0.26 \\
\hline & 122 & 67 & 26 & 24.9 & 53 & 0.29 & 1365 & 55.1 & 31 & -0.05 \\
\hline $40^{+}$ & 123 & 64 & $26 !$ & 25.1 & 52.9 & 0.29 & 363 & 55.1 & 31 & -0.05 \\
\hline & 124 & 64 & 26 & 25.3 & 52.9 & 0.28 & & 55 & 31 & -0.03 \\
\hline 43 & 125 & 64 & 26 & 25.3 & 52.9 & 0.28 & 3 & 54.9 & 31 & -0.03 \\
\hline & 126 & 65 & 26 & & 9 & & & 54.9 ? & 30 & -0.15 \\
\hline & 127 & 65 & 26 & & 52.9 & 0.28 & & 54.9 & 30 & -0.15 \\
\hline & 128 & 65 & $26^{5}$ & 25.3 & 52.9 & 0.29 & $361^{\dagger}$ & 55 & 30 & -0.33 \\
\hline $4: 44$ & 129 & 68 & 26 & 25.3 & 52.8 & 0.3 & 1366 & 55 & 30 & -0.33 \\
\hline 25:43 & 130 & 67 & 26 & 25.3 & 52.8 & 0.3 & & $55.1^{\dagger}$ & 30 & 0.07 \\
\hline & 13 & 67 & 26 & & 52.8 & 0.3 & 368 & 55.1 & 31 & -0.07 \\
\hline 46 & 132 & 67 & 26 & 5.1 & 52.8 & 0.29 & & 55.1 & 31 & -0.07 \\
\hline & 133 & 63 & 26 & $25.1]$ & 52.8 & 0.29 & & 55.1 & 30 & -0.17 \\
\hline & 13 & 63 & 26 & $5.3^{4}$ & 52.8 & 0.29 & 1365 & $55.2 !$ & 30 & -0.17 \\
\hline & 13 & 63 & 26 & & & 28 & & 55.2 & 30 & -0.27 \\
\hline & $13 \overline{6}^{\dagger}$ & 64 & 26 & & 52.8 & 0.28 & & 55.2 & 30 & -0.27 \\
\hline & 137 & 64 & 26 & 25.3 & 52.8 & 28 & & $55.1 !$ & 30 & 0.03 \\
\hline $3: 45$ & 138 & 64 & 26 & & 2.8 & 29 & & 55.1 & 30 & 0.03 \\
\hline & 139 & 64 & 26 & 25.5 & 52.8 & 0.26 & & 55.2 & 30 & -0.07 \\
\hline & 140 & 67 & 26 & 25.5 & 52.8 & 0.28 & 1367 & 55.4 & 30 & -0.07 \\
\hline$: 47$ & 141 & 68 & 26 & 25.5 & 52.8 & 0.28 & 1365 & 55.4 & 30 & -0.19 \\
\hline & $\overline{142}$ & 68 & $\ldots 26$ & 25.5 & 2.8 & 0.29 & & 55.5 & 30 & -0.19 \\
\hline & 14 & 68 & 26 & & & 0.3 & & 5.6 & 30 & -0.27 \\
\hline & 14 & 63 & 26 & & 52.7 & $0 . \overline{28}$ & & 55.6 & 31 & -0.27 \\
\hline & $1 \overline{45}$ & 63 & 26 & & 52.7 & 0.3 & 1372 & 55.5 & 31 & 0.04 \\
\hline $41: 47$ & 146 & 63 & 26 & 25.7 & 52.7 & 0.3 & 1373 & 55.5 & 31 & 0.04 \\
\hline $42: 46$ & 147 & 64 & 26 & $25 . \overline{7}$ & 52.7 & 0.28 & $1371^{+}$ & 55.5 & 30 & -0.05 \\
\hline $43: 50$ & 148 & 65 & 26 & & 52.7 & 0.29 & 8 & 55.4 & -30 & -0.05 \\
\hline & 149 & 64 & 26 & 25 & 52.7 & 0.29 & 5 & 55.3 & 30 & -0.1 \\
\hline & 150 & 66 & 26 & & 52.7 & 0.29 & & 55.3 & 30 & -0.19 \\
\hline$: 46: 47 !$ & 151 & 67 & 26 & 25.5 & 52.7 & 0.29 & 1368 & 55.1 & 30 & -0.28 \\
\hline $10: 58: 47: 46$ & 152 & 67 & 26 & 25.5 & 52,7 & 0.3 & 1367 & 55.2 & 30 & -0.28 \\
\hline $10: 58: 48: 50$ & 153 & 68 & 26 & 25.5 & 52.7 & 0.31 & $1370^{\circ}$ & 55.2 & 30 & $\overline{0.01}$ \\
\hline $10: 58: 49: 49$ & 154 & 64 & 26 & 25.5 & 52.7 & 0.31 & 1375 & 55.1 & 30 & -0.04 \\
\hline $10: 58: 50: 48$ & 155 & 63 & 26 & $25.7 i$ & 52.7 & 0.29 & $1377^{\circ}$ & 55.1 & 30 & -0.04 \\
\hline
\end{tabular}




\begin{tabular}{|c|c|c|c|c|c|c|c|c|c|c|}
\hline$T I M E$ & $\begin{array}{l}\text { |Elapsed } \\
\text { 'Time } \\
\text { |(sec) }\end{array}$ & $B I T$ TC1 & PURG_TC2 & PurgePSi & & & DwnFrc(lb) & RPM & $\begin{array}{l}\text { Torque } \\
\text { (FTLB) }\end{array}$ & Inches/Min \\
\hline $10: 58: 5 \overline{1}: 4 \overline{7}$ & $1 \quad 156$ & 63 & 26 & $\quad 25.7$ & $\quad 52.7$ & $\quad 0.3$ & $\quad 1376$ & 55.1 & $\quad 30$ & -0.32 \\
\hline $10: 58: 52: 45$ & 157 & 64 & 26 & 25.7 & 52.7 & 0.29 & 1372 & 55 & 30 & -0.32 \\
\hline $3: 53: 50$ & 158 & 64 & 26 & 25.9 & 52.7 & $0 . \overline{28}$ & 1375 & 55.1 & 30 & -0.12 \\
\hline$: 49$ & 159 & 64 & 26 & 25.9 & $52.7 !$ & 0.29 & 1372 & $55.1^{1}$ & 30 & -0.12 \\
\hline 47 & $1 \overline{60}$ & 64 & 26 & 25.9 & 52.7 & 0.29 & 1369 & 55.1 & 30 & $-0 . \overline{03}$ \\
\hline 3:52 & 161 & 65 & 26 & 25.9 & 52.6 & 0.29 & 1368 & 54.9 & 30 & -0.03 \\
\hline 51 & 162 & $66^{\dagger}$ & 26 & 25.9 & $5 \overline{2.6}$ & 0.3 & 1372 & 54.9 & 29 & $-0 . \overline{12}$ \\
\hline & 163 & 66 & 26 & 25.9 & 52.6 & 0.31 & 1378 & 55 & 29 & $-0 . \overline{12}$ \\
\hline & $1 \overline{64}$ & 64 & 26 & 25.9 & 52.6 & 0.3 & 1376 & $54 . \overline{8}$ & 29 & -0.31 \\
\hline$: \overline{4}$ & 165 & 63 & 26 & 25.9 & 52.6 & 0.3 & 1380 & 54.8 & 30 & -0.31 \\
\hline & 166 & 62 & 26 & 25.9 & 52.6 & 0.28 & & 54.7 & 30 & -0.2 \\
\hline & 167 & 62 & 26 & 25.9 & 52.6 & 0.3 & & 54.6 & 30 & -0.2 \\
\hline & $16 \overline{8}$ & $63 i$ & 26 & 25.9 & .6 & 0.29 & $1 \overline{3}$ & 4.6 & 30 & 0 \\
\hline & 169 & 64 & 26 & 26.1 & 52.6 & 0.29 & 13 & 54.6 & 29 & 0 \\
\hline & 170 & 64 & 26 & 26.1 & 52.6 & 0.29 & $1 \overline{3}$ & $54.6 !$ & $30 i$ & -0.13 \\
\hline & 171 & $6 \overline{6}$ & 26 & 26.1 & 52.6 & 0.3 & & 54.6 & 30 & -0.13 \\
\hline & 172 & 67 & 26 & 25.7 & 52.5 & 0.3 & & 54.3 & 29 & 0.33 \\
\hline & 173 & 67 & 26 & & & 0.29 & & 54.2 & 29 & -0.33 \\
\hline & 174 & 62 & 26 & 25.9 & 52.4 & 0.3 & & 54.2 & 29 & 0.05 \\
\hline & 175 & 62 & 26 & 259 & 52.3 & 0.3 & & 54 & 30 & 0.05 \\
\hline & 176 & 62 & 26 & & & & & 54 & 30 & 0.1 \\
\hline & $177 !$ & 62 & 26 & & & & & 54 & 29 & -0.1 \\
\hline & 178 & $6 \overline{3}$ & 26 & $9 !$ & & & & 54.1 & 29 & -0.39 \\
\hline & 179 & 63 & 26 & 25.9 & & 29 & & $54.1^{\dagger}$ & 29 & -0.09 \\
\hline & 180 & 63 & 26 & 25.9 & & 29 & & 54.1 & 29 & -0.09 \\
\hline & 181 & $6 \overline{7}$ & 26 & $25.9^{\dagger}$ & 5 & 0 & & 54.2 & 29 & $0 . \overline{06}$ \\
\hline & 182 & 66 & 26 & 25 & & 0.31 & & 54.2 & 29 & -0.06 \\
\hline & 983 & 66 & 26 & 5.9 & 52.2 & 0.3 & & 54.2 & 29 & -0.24 \\
\hline & 184 & 64 & 26 & $25.9^{9}$ & 52.2 & 0.31 & & 54.2 & 29 & -0.24 \\
\hline & 185 & 62 & 26 & $25.9^{\dagger}$ & & & & & 30 & 0.17 \\
\hline & 186 & 62 & 26 & & 52. & & & 54.1 & 29 & 0.17 \\
\hline & 187 & 62 & 26 & & & & & 4.1 & 29 & 0.02 \\
\hline & 188 & 63 & 26 & 26.3 & 52.1 & 0.3 & & $5 \overline{4.2}$ & 29 & 0.02 \\
\hline & 189 & 63 & 26 & & & & & $5 \overline{4.2}$ & 29 & -0.17 \\
\hline & 190 & 64 & 26 & & & & & 54.4 & 29 & $0 . \overline{17}$ \\
\hline & 191 & 64 & 26 & 26.3 & 2.1 & & & 54.7 & 29 & -0.27 \\
\hline & 192 & 65 & 26 & 26.1 & 52.1 & $0 . \overline{3}$ & 1 & 54.7 & 30 & -0.27 \\
\hline 3:54 & 193 & 64 & 26 & 26.1 & 52 & 0.31 & 1380 & 54.7 & 30 & -0.17 \\
\hline & 194 & 63 & 26 & & 52 & & & 54.7 & $\overline{30}$ & -0.17 \\
\hline & & 62 & 26 & & 52 & & & & 30 & 0.04 \\
\hline & 196 & 62 & 26 & $26.3 !$ & 52 & & & 54.8 & 30 & -0.04 \\
\hline & 197 & 62 & 26 & 26.3 & 51.9 & 0.29 & & 54.9 & 30 & -0.3 \\
\hline & 198 & 63 & 26 & 26.3 & 51.9 & 0.29 & $1 \overline{37}$ & 54.9 & 30 & -0.3 \\
\hline & 199 & 63 & $26 !$ & 26.3 & 51.9 & 0.28 & 1372 & 54.9 & 30 & -0.36 \\
\hline & 200 & 63 & 26 & 26.3 & & 0.31 & 1 & 54.9 & 29 & $-0.0 \overline{3}$ \\
\hline & 201 & 64 & 26 & 26.3 & $51.9^{\dagger}$ & $0.3^{\top}$ & & 54.9 & 29 & -0.03 \\
\hline & 202 & 65 & 26 & 26.3 & 51.9 & $0.31 \dagger$ & 13 & 55 & 29 & -0.04 \\
\hline $8: 59$ & 203 & 65 & $26^{i}$ & $26.3^{!}$ & 51.9 & $0 . \overline{31}$ & 138 & 55.1 & 29 & $-0 . \overline{04}$ \\
\hline $39: 58$ & 204 & 64 & 26 & 26.3 & 51.9 & 0.31 & 1380 & 55.1 & 30. & -0.3 \\
\hline $59: 40: 57$ & $20 \overline{5}$ & 63 & 26 & 26.3 & 51.8 & 0.3 & 1382 & 55 & 30 & -0.3 \\
\hline & 206 & 62 & 26 & $26.3^{+}$ & 51.8 & 0.29 & 13 & 54.9 & 30 & -0.28 \\
\hline $10: 59: 42: 60$ & 207 & 62 & 26 & 26.5 & 51.8 & 0.29 & 1376 & 55 & 29 & -0.28 \\
\hline
\end{tabular}




\begin{tabular}{|c|c|c|c|c|c|c|c|c|c|c|}
\hline TIME & $\begin{array}{l}\text { Elapsed } \\
\text { Time } \\
\text { (sec) }\end{array}$ & BIT_TC1 & PURG_TC2 & PurgePSI & PurgeCFM & DEPTH(IN) & $D w n F r c(b)$ & & $\begin{array}{l}\text { Torque } \\
\text { (FTLB) }\end{array}$ & Inches/Min \\
\hline $10: 59: 43: 59$ & $\quad 208$ & 62 & $\quad 26$ & $\quad 26.5$ & $\quad 51.8$ & 0.28 & $\quad 1372$ & 55 & $3 \quad 29$ & -0.05 \\
\hline $10: 59: 44: 58$ & 209 & 63 & 26 & 26.5 & 51.8 & 0.29 & 1375 & 54.9 & 29 & -0.05 \\
\hline $5: 57$ & 210 & 63 & 26 & 26.6 & 51.8 & 0.3 & 1372 & 54.9 & 29 & 0.03 \\
\hline 61 & $211^{\prime}$ & 63 & 26 & 26.6 & $51 . \overline{8}$ & 0.3 & 1371 & 54.9 & 29 & 0.03 \\
\hline 60 & 212 & 63 & 26 & 26.5 & 51.8 & 0.3 & 1374 & 54.9 & 29 & -0. \\
\hline & 213 & 64 & 26 & 26.5 & 51.7 & 0.31 & $1379^{\circ}$ & 54.9 & 30 & -0. \\
\hline & 214 & 64 & 26 & 26.5 & & 0.31 & & 54.9 & 30 & -0.16 \\
\hline & 215 & 63 & $\overline{26}$ & 26.5 & $51.7^{\prime}$ & 0.28 & 1379 & 54.9 & 30 & -0.16 \\
\hline 61 & 216 & 62 & 26 & 26.5 & $51.7 !$ & 0.31 & 1380 & 54.9 & 30 & -0.03 \\
\hline & 217 & 62 & 26 & 26.5 & 51.6 & 0.29 & 1378 & 55 & 30 & -0.03 \\
\hline & 218 & 62 & 26 & 26.5 & 51.6 & 0.3 & 1375 & 55 & 30 & -0.22 \\
\hline & 219 & 63 & 26 & 26.6 & 51. & 0.3 & 32 & 55 & 30 & -0.22 \\
\hline & 220 & 63 & 26 & 26.6 & & 0.3 & 1372 & 54.9 & 30 & -0.27 \\
\hline & 221 & 63 & 26 & 26.5 & 51.6 & 0.3 & 1374 & 54.8 & 29 & -0.27 \\
\hline & 222 & 63 & $\overline{26}$ & 26.5 & 51.6 & 0.3 & $1373^{\dagger}$ & 54.9 & 29 & -0.11 \\
\hline & 223 & 65 & 26 & 26.5 & & $0 . \overline{3}$ & 6 & 54.9 & 29 & 0.01 \\
\hline & 224 & 65 & 26 & 26.5 & & 0.3 & & 55 & 29 & 0.01 \\
\hline & 225 & 64 & 26 & $2 \overline{6} .5$ & & $0.3^{7}$ & & $55.1 \mathrm{i}$ & 29 & -0.2 \\
\hline & 226 & 62 & 26 & 26.5 & $6^{\dagger}$ & 0.29 & & 55.1 & 30 & -0.2 \\
\hline & 227 & 62 & 26 & 26.8 & 51.6 & 0.3 & $76^{+}$ & 55.1 & 30 & -0.37 \\
\hline & 228 & 62 & 26 & 26.8 & & 0.3 & & 55.1 & 30 & -0.37 \\
\hline & 229 & 62 & 26 & 26.8 & 51 & 0.28 & & 55.2 & 29 & -0.09 \\
\hline & 230 & 63 & 26 & 26.8 & & 0.3 & & 55.1 & 29 & -0.09 \\
\hline & 231 & 63 & 26 & 26.8 & & 0.29 & & 55.1 & 29 & -0.09 \\
\hline & 232 & 63 & 26 & 26.8 & & $0.31^{1}$ & & 55.1 & 29 & -0.09 \\
\hline & 233 & 63 & 26 & 26.8 & & 0.3 & & 55.1 & 29 & $-0 . \overline{24}$ \\
\hline & 23 & 66 & 26 & & & & 13 & 55.1 & 30 & -0.24 \\
\hline & 235 & 65 & 26 & 26.6 & & & & 55.1 & 30 & -0.32 \\
\hline & 236 & 65 & 26 & 26.6 & & $0.31^{\prime}$ & & 55.1 & 30 & -0.32 \\
\hline & 237 & 61 & 26 & 26.6 & & & & 55.1 & 30 & 0.01 \\
\hline & 238 & 61 & 26 & 26.6 & & & & 55.1 & 30 & 0.0 \\
\hline & 239 & 62 & 26 & 26.6 & & & & 55.1 & 30 & -0.05 \\
\hline & 240 & 63 & 26 & 26.8 & & 0. & & 55.1 & 30 & -0.05 \\
\hline & 241 & 63 & 26 & 26.8 & & 0.3 & & 5.1 & 29 & -0.31 \\
\hline & 242 & 63 & 26 & 26.8 & & 0.31 & 1370 & $55.2^{\prime}$ & 29 & $-0 . \overline{31}$ \\
\hline & 243 & 63 & 26 & 26.8 & & & & 55.1 & 29 & -0.24 \\
\hline & 244 & 66 & 26 & 26.8 & & 0.31 & 1372 & 55.1 & 29 & -0.24 \\
\hline & $24 \overline{5}$ & 65 & 26 & 26.8 & 51 & 0.31 & & 55 & 29 & -0.11 \\
\hline & 246 & 65 & 26 & 26.6 & & & & 54.9 & 29 & 0.03 \\
\hline & 247 & 61 & 26 & 26.6 & & & & 54.9 & 29 & 0.0 \\
\hline & 248 & 61 & 26 & 27 & & 0.3 & & 54.8 & 29 & -0.34 \\
\hline & 249 & 61 & 26 & $27 . \overline{2}$ & 51 & 0.3 & $1380^{\circ}$ & 54.9 & 29 & $-0 . \overline{34}$ \\
\hline & 250 & 64 & 26 & 27.2 & 51.4 & 0.3 & 1378 & 54.9 & 29 & -0.15 \\
\hline & 251 & 64 & 26 & 27.2 & 51 & 0.28 & 1375 & 55 & 29 & -0.15 \\
\hline & 252 & 64 & $2 \overline{6}$ & 27.2 & & 0.3 & & 55 & 29 & -0.01 \\
\hline & 253 & 64 & 26 & 27.2 & & & & 55 & 29 & -0.01 \\
\hline & 254 & $\overline{6} \overline{6}$ & 26 & 27.2 & & 1 & 13 & 55 & 29 & -0.18 \\
\hline$\overline{66}$ & $255^{\dagger}$ & 65 & 26 & 26.8 & 51 & 0.31 & 1373 & 54.9 & 29 & $-0 . \overline{18}$ \\
\hline $0: 31: 70$ & 256 & 65 & 26 & 26.8 & 51.3 & 0.31 & $1378^{\circ}$ & 54.9 & 29 & -0.32 \\
\hline $00: 32: 69$ & 257 & 65 & 26 & 26.8 & 51.3 & 0.31 & 1380 & 54.8 & 30 & -0.32 \\
\hline $11: 00: 33: 68$ & 258 & 61 & 26 & 26.8 & 51. & 0.31 & 1380 & 54.6 & 30 & 0.05 \\
\hline $11: 00: 34: 67$ & 259 & 61 & 26 & 27 & $51 . \overline{3}$ & 0.31 & 1377 & 54.6 & 6 $\quad 30$ & 0.0 \\
\hline
\end{tabular}




\begin{tabular}{|c|c|c|c|c|c|c|c|c|c|c|}
\hline$T I M E$ & $\begin{array}{l}\text { Elapsed } \\
\text { Time } \\
\text { (sec) }\end{array}$ & $B \mid T \_T C 1$ & PURG_TC2 & iPurgePSI ! & PurgeCFM & IDEPTH $(\mathbb{I N})$ & DwnFrc(lb) & RPM I & $\begin{array}{l}\text { Torque } \\
\text { (FTLB) }\end{array}$ & es/Min \\
\hline $11: 00: 35: 66$ & 260 & -61 & 26 & $\quad 27$ & $\quad 51.3$ & $\quad 0.3^{3}$ & $\quad 1375$ & 54.6 & $\quad 29$ & -0.16 \\
\hline $11: 00: 36: 70$ & 261 & 64 & 26 & 27 & 51.2 & 0.29 & $1377^{i}$ & 54.8 & 29 & -0.16 \\
\hline 69 & 262 & 64 & 26 & 27 & 51.2 & 0.3 & 1373 & 54.8 & 29 & -0.28 \\
\hline $68^{+}$ & 263 & 63 & 26 & 27 & 51.2 & $0.3^{\prime}$ & 1372 & 54.8 & 29 & -0.28 \\
\hline 72 & 264 & 63 & 26 & 27 & 51.2 & 0.31 & 1373 & 54.9 & 29 & -0.3 \\
\hline & 265 & 65 & 26 & 27 & 51.2 & 0.3 & $1378^{1}$ & 54.9 & 29 & $-0 . \overline{3}$ \\
\hline & 266 & 65 & 26 & 27 & 51.2 & 0.31 & 1381 & 55 & 29 & $0.0 \overline{2}$ \\
\hline 69 & 267 & 65 & 26 & 27 & 51.2 & & 1380 & 55 & 29 & 0.02 \\
\hline & 268 & 61 & 26 & $27^{!}$ & 51.1 & 1 & $1382^{i}$ & 55 & 29 & -0.04 \\
\hline 72 & 269 & 61 & 26 & 27.4 & 51.1 & 0.3 & 1380 & 55 & 29 & -0.29 \\
\hline 71 & 270 & 61 & 26 & 27.4 & 51.1 & $0.31^{\dagger}$ & 1378 & 55.1 & 29 & -0.29 \\
\hline & $271^{\dagger}$ & 64 & 26 & 27.4 & 51.1 & 0.3 & $1375^{t}$ & 55.1 & 29 & -0.23 \\
\hline 69 & 272 & 64 & 26 & 27.6 & 51.1 & 0.29 & $1379^{:}$ & 55.1 & 29 & -0.23 \\
\hline & 273 & 64 & 26 & $27 . \overline{6}$ & 51.1 & & 1374 & 55 & 29 & -0.04 \\
\hline & 274 & 64 & 26 & 27.6 & 51.1 & 0.31 & 1372 & 55 & 29 & -0.04 \\
\hline $71^{+}$ & 275 & 65 & 26 & 27.6 & 51.1 & 0.3 & 1374 & 54.9 & 29 & 0.02 \\
\hline & 276 & $6 \overline{6}$ & 26 & 27.2 & 51.1 & 0.31 & $1379^{\dagger}$ & $54.9^{\dagger}$ & 29 & 0.02 \\
\hline & 277 & 65 & 26 & 27.2 & 51 & 0.3 & 1382 & 54.9 & 29 & -0.30 \\
\hline & 278 & 61 & 26 & 27.2 & 51 & 0. & 30 & 54.7 & 29 & -0.39 \\
\hline & 279 & 61 & 26 & 27.2 & 51 & & 1381 & 54.7 & 30 & -0.17 \\
\hline & 280 & 61 & 26 & 27.2 & 51 & 0.3 & 1379 & 54.7 & 30 & -0.17 \\
\hline & 281 & 61 & 26 & 27.4 & 51 & & 1374 & 54.8 & 29 & 0.01 \\
\hline & 282 & 64 & 26 & & 51 & & 1370 & 54.8 & 29 & $0 . \overline{01}$ \\
\hline & 283 & 64 & 26 & 27.4 & & & 1373 & 54.8 & 29 & -0.15 \\
\hline & 284 & 64 & 26 & 27.4 & 9 & & 1372 & 54.9 & 29 & -0.15 \\
\hline & 285 & 66 & 2 & 27.4 & & & & $4.9 !$ & 29 & 0.25 \\
\hline & 286 & 65 & 2 & & & & 72 & 54.9 & 29 & -0.2 \\
\hline & 287 & $6 \overrightarrow{5}$ & 26 & $27 . \overline{4}$ & 50.9 & 0. & 1377 & 54.9 & 29 & -0.04 \\
\hline & 288 & 65 & 26 & $27.4 !$ & 50.9 & & 1378 & 54.8 & 29 & -0.04 \\
\hline $771^{t}$ & 289 & 61 & 26 & 27.6 & 0.9 & & 1376 & 54.8 & 29 & -0.05 \\
\hline & 290 & 61 & 26 & 27.6 & 50.9 & & 1376 & 54.9 & 29 & -0.05 \\
\hline & 291 & 61 & 26 & 27.8 & 50.8 & $0.3^{\top}$ & 1374 & 54.9 & 29 & $-0 . \overline{2}$ \\
\hline & 292 & 64 & 26 & 27.8 & 5 & 0.3 & 1371 & 54.9 & 29 & $-0.3 \overline{8}$ \\
\hline & 293 & 64 & 26 & 27.8 & & 0 & 1367 & 54.9 & 29 & -0.38 \\
\hline & 294 & 64 & 26 & 27.8 & & 0. & & 54.9 & 29 & 0.02 \\
\hline & 295 & 65 & 26 & 27.8 & & 0.3 & 1369 & 54 & 29 & 0.02 \\
\hline & 296 & 65 & 26 & 27.8 & & 0 & 1367 & 54.9 & 29 & -0.0 \\
\hline & 297 & 65 & 26 & 27.4 & 50.8 & & 1370 & 54.9 & 29 & -0.0 \\
\hline & 298 & 65 & 26 & 27 & & & 373 & $54.9 !$ & 29 & -0.35 \\
\hline 777 & 299 & 61 & 26 & 27.4 & & & & 55 & 29 & -0.35 \\
\hline & 300 & 61 & 26 & 27.4 & & & 1372 & 55.1 & 29 & -0.17 \\
\hline & 301 & 61 & 26 & 27.4 & 50.7 & 0.3 & $1372^{\prime}$ & 55.1 & 29 & -0.17 \\
\hline $7: 73$ & $302^{\dagger}$ & 64 & 26 & 27 & 50.2 & 0 & 1370 & 55.2 & 29 & -0.04 \\
\hline $8: 78$ & $30 \overline{3}$ & 64 & 26 & 18.7 & 1.9 & 0.31 & 1366 & 55.2 & 29 & -0.04 \\
\hline $1: 19: 76$ & 304 & 68 & 26 & -0.4 & 1.9 & 0.3 & 1363 & 55.2 & 29 & -0.06 \\
\hline & 305 & 72 & 26 & -0.6 & 1.9 & & 1360 & 55.2 & 30 & -0.0 \\
\hline & 306 & 74 & 26 & -0. & 1.9 & & 1363 & 55.4 & 30 & -0.3 \\
\hline & 307 & 77 & 26 & -0 & 1.9 & & & 55.4 & 31 & -0.3 \\
\hline $1: 23: 77$ & 308 & 81 & 26 & -0.6 & 1.9 & 0.3 & 1363 & 55.4 & $\overline{3 i}$ & -0.1 \\
\hline $1: 24: 76$ & 309 & 83 & 26 & -0.6 & 1.9 & 0.31 & 1369 & 55.3 & 32 & -0.15 \\
\hline $01: 25: 75$ & 310 & 80 & 26 & -0.6 & 1.9 & 0.31 & $1371 \mathrm{i}$ & 55.3 & 33 & -0.03 \\
\hline $11: 01: \overline{26}: \overline{79}$ & 311 & 82 & 26 & -0.6 & $1 . \overline{9}$ & 0.31 & 1370 & 55.2 & 33 & -0.03 \\
\hline
\end{tabular}




\begin{tabular}{|c|c|c|c|c|c|c|c|c|c|c|}
\hline$T I M E$ & $\begin{array}{l}\text { Elapsed } \\
\text { Time } \\
\text { (sec) }\end{array}$ & BIT_TC1 & PURG_TC2 & PurgePSI If & PurgeCFM & DEPTH(IN) & DwnFrc(b) & RPM & $\begin{array}{l}\text { Torque } \\
\text { (FTLB) }\end{array}$ & Inches/Min \\
\hline $11: 01: 27: 78$ & $\quad 312$ & 84 & $\quad 26$ & -0.6 & 1.9 & $\quad 0.31$ & $\quad 1370$ & 55.3 & 34 & -0.04 \\
\hline $11: 01: 28: 77$ & 313 & 88 & 26 & -0.6 & 1.9 & $0.3^{+}$ & 1369 & 55.3 & 34 & $-0 . \overline{04}$ \\
\hline $11: 01: 29: 82$ & 314 & 90 & 26 & -0.6 & 1.9 & 0.3 & 1365 & 55.4 & 34 & -0.38 \\
\hline $30: 80$ & 315 & 93 & 26 & -0.6 & 1.9 & 0.3 & 1361 & 55.5 & 34 & -0.13 \\
\hline $11: 01: 31: 79$ & 316 & 92 & 26 & -0.6 & 1.9 & 0.3 & 1360 & 55.5 & 35 & -0.13 \\
\hline $11: 01: 32: 78$ & 317 & 94 & 26 & -0.6 & 1.9 & 0.31 & 1362 & 55.6 & 34 & -0.05 \\
\hline 77 & 318 & 96 & 26 & -0.6 & 1.9 & 0.31 & 1360 & 55.5 & 35 & -0.05 \\
\hline 81 & 319 & 98 & 26 & -0.6 & 1.9 & 0.31 & 1363 & 55.5 & 35 & -0.03 \\
\hline & 320 & 101 & 26 & -0.6 & 1.9 & 0.31 & 1368 & 55.4 & $36^{\dagger}$ & $-0 . \overline{03}$ \\
\hline & 321 & 101 & 26 & -0.6 & 1.9 & & $370^{\prime}$ & 55.3 & 36 & -0.31 \\
\hline & 322 & 103 & 26 & -0.6 & 1.9 & 31 & 1370 & 55.3 & 36 & -0.31 \\
\hline & 323 & $10 \underline{3}$ & 26 & -0.6 & 1.9 & 0.31 & 1372 & 55.3 & 36 & $-0 . \overline{15}$ \\
\hline & 324 & 104 & 26 & -0.6 & 1.9 & 0.3 & 1370 & 55.2 & 36 & -0.15 \\
\hline$: 80$ & 325 & 106 & 26 & -0.6 & 1.9 & 0.3 & 1366 & 55.2 & 36 & -0.04 \\
\hline $1: 79$ & 326 ! & 108 & 26 & -0.6 & 1.9 & 0.3 & 1363 & 55.1 & 36 & -0.04 \\
\hline $2: 78$ & 327 & 110 & 26 & -0.6 & $1 . \overline{9}$ & 0.3 & 1361 & 55.1 & 37 & -0.07 \\
\hline$: 77$ & 328 & 111 & 26 & -0.6 & $1 . \overline{9}$ & 0.31 & 1361 & 55.1 & 37 & -0.07 \\
\hline & 329 & 112 & 26 & -0.6 & 1.9 & 0.31 & 13 & 55 & 37 & -0.29 \\
\hline & 330 & 116 & 26 & -0.6 & 1.9 & 0.3 & $3^{\top}$ & 54.9 & 37 & $-0.2 \overline{9}$ \\
\hline 84 & 331 & 117 & 26 & -0.6 & 1.9 & 0.31 & & 54.9 & 37 & -0.21 \\
\hline & 332 & 118 & 26 & -0.6 & 1.9 & & & 54.8 & 38 & -0.21 \\
\hline & 333 & 118 & 26 & $-0.6 !$ & 1.9 & & & 54.8 & 38 & .08 \\
\hline & 334 & 118 & 26 & -0.6 & 1.9 & 0.2 & & 8 & 39 & .08 \\
\hline & 335 & 120 & 26 & $-0.6^{\dagger}$ & 1.9 & 0.3 & 0 & 54.8 & 39 & -0.16 \\
\hline & 336 & 122 & 26 & -0.6 & 1.9 & 3 & & 4.8 & 39 & -0.32 \\
\hline & 337 & 124 & 26 & -0.6 & 1.9 & & & 54.8 & 39 & -0.32 \\
\hline & 338 & 125 & 26 & -0.6 & 1.9 & 0 & 1360 & 54.7 & 38 & -0.02 \\
\hline & 339 & 126 & 26 & -0.6 & $1.9^{\dagger}$ & 0.31 & & 54.5 & 39 & -0.02 \\
\hline & 340 & 130 & 26 & -0.6 & 1.9 & 0. & & 54.5 & 39 & -0.04 \\
\hline & 341 & 130 & 26 & -0.6 & 1.9 & & & & 39 & -0.04 \\
\hline & 342 & 129 & 26 & & 1.9 & & & 54.2 & 39 & -0.36 \\
\hline & 343 & 130 & 26 & $-0 . \overline{6^{\dagger}}$ & 1.9 & & & 54.2 & 39 & -0.36 \\
\hline & 34 & & 26 & -0.6 & 1.9 & 0. & & 54.2 & 39 & -0.17 \\
\hline & 345 & 135 & 26 & -0.6 & 1.9 & 0.28 & 3 & 54.3 & 39 & -0.17 \\
\hline & 346 & 37 & 26 & -0.6 & 1.9 & & & 4.3 & 39 & 0.05 \\
\hline & 347 & & 26 & -0.6 & 1.9 & & & & 39 & .05 \\
\hline & 348 & 139 & 26 & -0.6 & 1.9 & & & 54.3 & 39 & -0.19 \\
\hline & 349 & 141 & 26 & -0.6 & 1.9 & & 363 & 54.3 & 39 & -0.19 \\
\hline 85 & 350 & 144 & 26 & -0.6 & 1.9 & & 1369 & 54.2 & 39 & -0.33 \\
\hline & 351 & 140 & 26 & -0.6 & 1.9 & & $371:$ & 54.2 & 40 & -0.33 \\
\hline & 35 & & 26 & -0 & 1.9 & & & 54.2 & 40 & -0.01 \\
\hline & 35 & & 26 & -0.6 & & & & 54.2 ! & 40 & -0.01 \\
\hline & 354 & 144 & 26 & -0.6 & $1 . \overline{9}$ & 0 & 1 & 54.2 & 40 & -0.05 \\
\hline$: 84$ & 355 & 148 & 26 & -0.6 & 1.9 & 0.3 & 1362 & 54.2 & 40 & -0.05 \\
\hline $1: 89$ & 356 & 149 & 26 & -0.6 & 1.9 & 0.3 & 1360 & 54.2 & 41 & -0.36 \\
\hline $12: 88$ & 357 & 150 & 26 & -0.6 & 1.9 & 0.3 & 1362 & 54.1 & 40 & -0.36 \\
\hline & 358 & 154 & 26 & -0.6 & 1.9 & & & 54.1 & 41 & -0.16 \\
\hline & 359 & 155 & 26 & -0.6 & 1.9 & & 1365 & 54.1 & 41. & -0.16 \\
\hline & $360^{\circ}$ & & 26 & -0.6 & $1 . \overrightarrow{9}$ & & & 54.1 & 41 & 0.03 \\
\hline & 361 & 152 & 26 & -0.6 & 1.9 & 0.31 & 1371 & 54.1 & 41 & -0.27 \\
\hline $2: 17: 88$ & 362 & 154 & 26 & -0.6 & 1.9 & 0.3 & 1368 & 54.1 & 41 & -0.27 \\
\hline $11: 02: 18: 86$ & 363 & 156 & 26 & -0.6 & 1.9 & 0.29 & 1365 & 54.1 & 41 & -0.3 \\
\hline
\end{tabular}




\begin{tabular}{|c|c|c|c|c|c|c|c|c|c|c|}
\hline$T / M E$ & $\begin{array}{l}\text { Elapsed } \\
\text { Time } \\
\text { (sec) }\end{array}$ & BIT TC1 & |PURG TC2; & PurgePS! & PurgeCFM & DEPTH(IN) & DwnFrcllb & RPM & $\begin{array}{l}\text { Torque } \\
\text { (SFTLB) }\end{array}$ & Inches/Min \\
\hline $11: 02: 19: 91$ & $\quad 364$ & 158 & -26 & -0.6 & 1.9 & $\quad 0.3$ & $\quad 1366$ & 54.1 & 41 & $\begin{array}{l}-0.3 \\
-\end{array}$ \\
\hline $11: 02: 20: 90$ & 365 & 158 & 26 & -0.6 & 1.9 & 0.3 & 1362 & 54.1 & 41 & $0 . \overline{01}$ \\
\hline $11: 02: 21: 88$ & 366 & 159 & 26 & -0.6 & 1.9 & 0.31 & $1360 !$ & 54.1 & 41 & $0 . \overline{01}$ \\
\hline $11: 02: 22: 87$ & $3 \overline{6} 7$ & 161 & 26 & -0.6 & 1.9 & 0.31 & 1364 & 54.1 & 41 & -0.06 \\
\hline $23: 86$ & 368 & 163 & 26 & -0.6 & 1.9 & 0.31 & 1370 & 54.1 & 41 & -0.06 \\
\hline $1102: 24: 91$ & 369 & 164 & 26 & -0.6 & 1.9 & 0.31 & 1372 & 54.2 & 41 & -0.33 \\
\hline $11: 02: 25: 89$ & 370 & 164 & 26 & -0.6 & 1.9 & 0.31 & 1372 & 54.2 & 41 & $-0 . \overline{33}$ \\
\hline $11: 02: 26: 88$ & 371 & 166 & 26 & -0.6 & 1.9 & 0.29 & 1371 & 54.3 & $4 \overline{1}$ & -0.15 \\
\hline $27: 93$ & 372 & 168 & 26 & -0.6 & 1.9 & 0.3 & 1368 & 54.4 & 41 & -0.15 \\
\hline $11: 02: 28: 92$ & 373 & 170 & 26 & -0.6 & 1.9 & 0.3 & 1365 & 54.4 & 41 & 0.01 \\
\hline $11: 02: 29: 90$ & 374 & 171 & 26 & -0.6 & 1.9 & 0.3 & 1362 & 54.6 & 41 & 0.01 \\
\hline $11: 02: 30: 89$ & 375 & $170^{\top}$ & $2 \overline{6}$ & -0.6 & 1.9 & $0.3^{4}$ & $1363^{\prime}$ & 54.6 & $4 \overline{2}$ & -0.22 \\
\hline $1: 88$ & 376 & 171 & 26 & -0.6 & 1.9 & $0.3^{\dagger}$ & 1361 & 54.6 & 42 & -0.22 \\
\hline $32: 87$ & 377 & 173 & 26 & -0.6 & 1.9 & 0.31 & 1363 & $54.6^{\dagger}$ & 41 & -0.3 \\
\hline $11: 02: 33: 86$ & 378 & 174 & 26 & -0.6 & 1.9 & 0.31 & 1369 & 54.6 & 42 & -0.3 \\
\hline $11: 02: 34: 90$ & 379 & 173 & 26 & -0.6 & 1.9 & 0.31 & 1372 & 54.6 & 42 & 0.04 \\
\hline $35: 95$ & 380 & 174 & 26 & -0.6 & 1.9 & 0.3 & 1371 & 54.5 & 42 & 0.04 \\
\hline $6: 93$ & 381 & 175 & $26 !$ & -0.6 & 1.9 & 0.31 & 1372 & 54.5 & 43 & -0.12 \\
\hline $11: 02: 37: 92$ & 382 & 180 & 26 & -0.6 & 1.9 & $0.3^{1}$ & $1370^{\prime}$ & 54.5 & 42 & -0.12 \\
\hline $11: 02: 38: 91$ & 383 & 181 & 26 & -0.6 & 1.9 & 0.29 & 1367 & 54.5 & 42 & -0.32 \\
\hline $39: 90$ & 384 & 182 & 26 & $-0.6^{+}$ & 1.9 & 0.3 & 1363 & 54.5 & 42 & -0.17 \\
\hline$: 94$ & 385 & 181 & 26 & -0.6 & $1.9^{\dagger}$ & 0.28 & 1362 & 54.5 & 42 & -0.17 \\
\hline $11: 02: 41: 93$ & 386 & 183 & 26 & -0.6 & 1.9 & 0.31 & 1362 & 54.5 & 43 & $\overline{0}$ \\
\hline $11: 02: 42: 92$ & $3 \overline{87}$ & $185^{\dagger}$ & 26 & -0.6 & 1.9 & 0.31 & 1370 & 54.5 & 43 & $\frac{0}{0}$ \\
\hline $11: 02: 43: 96$ & 388 & 188 & 26 & $-0.6^{\dagger}$ & 1.9 & 0.31 & 1368 & 54.5 & 43 & -0.28 \\
\hline $1: 02: 44: 95$ & 389 & 189 & 26 & -0.6 & 1.9 & 0.31 & 1372 & 54.5 & 43 & -0.28 \\
\hline$: 94$ & 390 & 184 & 26 & -0.6 & 1.9 & 0.31 & 1372 & 54.5 & 43 & -0.34 \\
\hline $46: 93$ & 391 & 185 & 26 & $-0.6^{\dagger}$ & 1.9 & 0.3 & 1368 & 54.5 & 44 & -0.34 \\
\hline $11: 02: 47: 92$ & 392 & 191 & 26 & -0.6 & 1.9 & $0.3^{\dagger}$ & 1365 & 54.5 & 44 & -0.02 \\
\hline $48: 96$ & 393 & 192 & 26 & -0.6 & 1.9 & 0.3 & 1367 & 54.5 & 44 & -0.02 \\
\hline $49: 95$ & 394 & 193 & 26 & -0.6 & 1.9 & 0.28 & 1362 & 54.5 & 44 & 0.01 \\
\hline $50: 94$ & 395 & 194 & 26 & -0.6 & 1.9 & 0.31 & 1361 & $54.5^{\dagger}$ & 4 & 0.01 \\
\hline 93 & 396 & 196 & 26 & -0.6 & 1.9 & 0.3 & 1364 & 54.5 & 44 & -0.39 \\
\hline $2: 92$ & 397 & 198 & 26 & -0.6 & 1.9 & 0.31 & 1370 & 54.5 & 44 & -0.39 \\
\hline 11:02:53:96 & 398 & 199 & $26 !$ & $-0 . \overline{6}$ & 1.9 & 0.31 & 1367 & 54.5 & 44 & $-0 . \overline{14}$ \\
\hline & 399 & 200 & 26 & -0.6 & 1.9 & 0.31 & 1371 & 54.6 & 44 & -0.14 \\
\hline $5: 94$ & 400 & 195 & 26 & -0.6 & 1.9 & 0.31 & 1372 & 54.6 & 44 & -0.01 \\
\hline $56: 98$ & 401 & 196 & 26 & -0.6 & 1.9 & 0.3 & 1370 & 54.6 & 44 & -0.01 \\
\hline 57:97! & 402 & 198 & 26 & $-\overline{0.6}$ & 1.9 & 0.29 & 1366 & 54.6 & 4 & -0.15 \\
\hline $8: 96$ & 403 & 203 & 26 & -0.6 & 1.9 & 0.29 & 1362 & $5 \overline{4.6}$ & 45 & -0.15 \\
\hline $59: 95$ & 404 & 204 & 26 & -0.6 & 1.9 & 0.3 & 1363 & 54.7 & 45 & -0.29 \\
\hline $0: 94$ & 405 & 204 & 26 & $-0.6^{+}$ & 1.9 & $0.3^{4}$ & 1362 & 54.6 & 45 & -0.29 \\
\hline $01: 98$ & 406 & 208 & 26 & -0.6 & 1.9 & 0.31 & 1363 & 54.6 & 46 & $-0 . \overline{11}$ \\
\hline $11: 03: 02: 97$ & 407 & 209 & 26 & -0.6 & 1.9 & 0.3 & 1368 & 54.6 & 46 & .0 .06 \\
\hline $11: 03: 03: 96$ & 408 & 209 & 26 & -0.6 & 1.9 & 0.31 & 1372 & 54.8 & $\overline{46}$ & -0.06 \\
\hline $11: 03: 05: 00$ & 409 & 210 & 26 & $-0.6^{\dagger}$ & 1.9 & 0.31 & 1372 & 54.8 & 46 & -0.22 \\
\hline $05: 99$ & 410 & 206 & 26 & -0.6 & 1.9 & 0.31 & 1372 & 54.7 & 46 & $-0 . \overline{22}$ \\
\hline $6: 98$ & 411 & 207 & 26 & -0.6 & 1.9 & 0.3 & 1371 & 54.7 & 46 & -0.32 \\
\hline $11: 03: 07: 97$ & 412 & 209 & 26 & -0.6 & 1.9 & 0.29 & 1366 & 54.7 & & .0 .32 \\
\hline $11: 03: 08: 96$ & 413 & 215 & 26 & -0.6 & 1.9 & 0.3 & $13 \overline{6}$ & 54.8 & 46 & 0.03 \\
\hline 11:03:10:00 & 414 & 215 & 26 & -0.6 & 1.9 & 0.3 & 1360 & 54.8 & 46 & $0 . \overline{03}$ \\
\hline $11: 03: 10: 99$ & 415 & 216 & 26 & -0.6 & 1.9 & 0.3 & 1362 & 54.8 & 47 & 0 \\
\hline
\end{tabular}




\begin{tabular}{|c|c|c|c|c|c|c|c|c|c|c|}
\hline TIME & $\begin{array}{l}\text { Elapsed } \\
\text { Time } \\
\text { (sec) }\end{array}$ & BIT_TC1 & :PURG_TC2 & PurgePSI P & PurgeCFM & DEPTH(IN) & DwnFrc(ib) & ) RPM & $\begin{array}{l}\text { Torque } \\
\text { (FTLB) }\end{array}$ & Inches/Min \\
\hline $11: 03: 11: 98$ & 416 & 218 & 26 & -0.6 & $\begin{array}{r}1.9 \\
\end{array}$ & $\quad 0.3$ & $\quad 1361$ & 54.8 & 47 & $1 \quad 0$ \\
\hline 11:03:13:02 & $417^{\circ}$ & 219 & $26^{\dagger}$ & -0.6 & 1.9 & $0.31 !$ & 1365 & 54.7 & 48 & -0.36 \\
\hline $11: 03: 14: 01$ & 418 & 220 & 26 & -0.6 & 1.9 & 0.31 & 1369 & 54.7 & 47 & -0.36 \\
\hline $11: 03: 15: 00$ & 419 & 220 & 26 & -0.6 & 1.9 & 0.31 & 1373 & 54.6 & 47 & -0.11 \\
\hline $11: 03: 15: 99$ & 420 & 216 & $26^{\top}$ & -0.6 & 1.9 & 0.31 & 1371 & 54.5 & 47 & $-0 . \overline{11}$ \\
\hline $11: 03: 16: 98$ & 421 & 218 & 26 & -0.6 & 1.9 & 0.3 & 1367 & 54.5 & 47 & 0.09 \\
\hline $11: 03: 18: 02$ & 422 & $220^{\circ}$ & 26 & -0.6 & 1.9 & 0.3 & 1368 & $54.5^{\dagger}$ & 48 & 0.09 \\
\hline 11:03:19:01 & 423 & 222 & 26 & -0.6 & 1.9 & 0.3 & 1365 & 54.5 & 48 & -0.22 \\
\hline $11: 03: 20: 00$ & 424 & 225 & $26 !$ & -0.6 & 1.9 & 0.3 & 1362 & 54.5 & 48 & $-0 . \overline{22}$ \\
\hline $11: 03: 20: 98$ & 425 & 225 & 26 & -0.6 & 1.9 & $0.31 !$ & 1362 & 54.6 & 48 & -0.31 \\
\hline $11: 03: 21: 97$ & 426 & 228 & 26 & -0.6 & 1.9 & 0.31 & 1365 & 54.7 & 48 & -0.31 \\
\hline $11: 03: 22: 96$ & 427 ! & 229 & 26 & -0.6 & 1.9 & 0.31 & 1370 & 547 & 49 & -0.1 \\
\hline $1103: 23: 95$ & 428 & 230 & 26 & $-0.6 !$ & 1.9 & 0.31 & 1372 & 54.8 & 49 & -0.1 \\
\hline $11: 03: 24: 99$ & 429 & $231 \dagger$ & 26 & -0.6 & 1.9 & 0.31 & 1372 & 54.8 & 49 & 0.04 \\
\hline $11: 03: 26: 04$ & 430 & 231 & 26 & -0.6 & 1.9 & 0.31 & 1372 & 54.8 & 49 & -0.28 \\
\hline $11: 03: 27: 03$ & $431 i$ & 229 & 26 & -0.6 & 1.9 & $0.3^{+}$ & 1368 & 54.7 & 49 & -0.28 \\
\hline $11: 03: 28: 02$ & 432 & 231 & 26 & -0.6 & 1.9 & $0.3^{\top}$ & 1365 & 54.6 & 50 & -0.28 \\
\hline $11: 03: 29: 00$ & 433 & 234 & 26 & $-0 . \overline{6}^{1}$ & 1.9 & 0.3 & 1367 & 54.6 & 50 & -0.28 \\
\hline $11: 03: 29: 99$ & 434 & 235 & 26 & $-0.6 j$ & 1.9 & $0.3^{\dagger}$ & 1363 & 54.5 & 50 & $-0.0 \overline{3}$ \\
\hline $11: 03: 31: 04$ & 435 & 236 & 26 & -0.6 & 1.9 & 0.31 & 1361 & 54.6 & 50 & -0.03 \\
\hline $11: 03: 32: 02$ & 436 & 236 & 26 & $-0.6^{\dagger}$ & 1.9 & 0.3 & 1364 & 54.6 & 50 & -0.03 \\
\hline $11: 03: 33: 01$ & 437 & 240 & 26 & -0.6 & 1.9 & 0.31 & 1370 & $54.7^{\dagger}$ & 50 & -0.03 \\
\hline $11: 03: 34: 06$ & 438 & 240 & 26 & -0.6 & 1.9 & 0.31 & 1366 & 54.6 & 50 & $-0 . \overline{35}$ \\
\hline $11: 03: 35: 05$ & 439 & 241 & 26 & -0.6 & 1.9 & 0.3 & 1371 & 54.6 & 50 & -0.35 \\
\hline$|11: 03: 36: 03|$ & $440^{\circ}$ & 241 & $26 !$ & $-0.6 !$ & 1.9 & $0.29^{+}$ & 1372 & 54.7 & 51 & -0.16 \\
\hline $11: 03: 37: 02$ & 441 & 241 & 26 & -0.6 & 1.9 & 0.3 & 1370 & 54.7 & 50 & -0.16 \\
\hline $11: 03: 38: 01$ & 442 & 244 & 26 & -0.6 & 1.9 & 0.31 & 1367 & 54.7 & 50 & 0.02 \\
\hline $11: 03: 39: 06$ & 443 & 246 & 26 & -0.6 & 1.9 & 0.3 & 1362 & 54.7 & 51 & 0.02 \\
\hline $11: 03: 40: 04$ & 444 & 248 & $26 !$ & -0.6 & 1.9 & 0.29 & 1364 & 54.6 & 51 & -0.25 \\
\hline $11: 03: 41: 03$ & 445 & 246 & 26 & -0.6 & 1.9 & 0.3 & 1362 & 54.6 & 50 & -0.25 \\
\hline $11: 03: 42: 08$ & 446 & 247 & 26 & -0.6 & 1.9 & 0.3 & 1363 & 54.6 & 51 & -0.29 \\
\hline $11: 03: 43: 06$ & 447 ! & 248 & 26 & -0.6 & 1.9 & 0.31 & 1367 & 54.7 & 51 & -0.29 \\
\hline $11: 03: 44: 05$ & 448 & 251 & 26 & -0.6 & 1.9 & $0.31 \mathrm{j}$ & 1371 & 54.7 & 51 & 0.02 \\
\hline $11: 03: 45: 04$ & 449 & 251 & $26 !$ & -0.6 & 1.9 & 0.3 & 1370 & 54.8 & 52 & 0.02 \\
\hline $11: 03: 46: 03$ & 450 & 251 & 26 ! & -0.6 & 1.9 & 0.3 & 1370 & 54.9 & 52 & 0.01 \\
\hline $11: 03: 47: 07$ & 451 & 251 & 26 & -0.6 & 1.9 & 0.3 & 1372 & 54.9 & 52 & -0.25 \\
\hline $11: 03: 48: 06$ & 452 & 254 & 26 & -0.6 & 1.9 & 0.3 & 1367 & 54.8 & 52 & -0.25 \\
\hline $11: 03: 49: 05$ & 453 & 256 & 26 & -0.6 & 1.9 & 0.3 & 1364 & 54.7 & 52 & -0.22 \\
\hline $11: 03: 50: 04$ & 454 & 258 & $26 j$ & -0.6 & 1.9 & 0.3 & 1362 & 54.7 & 52 & -0.22 \\
\hline $11: 03: 51: 03$ & 455 & 256 & 26 & -0.6 & 1.9 & $0 . \overline{3}$ & $1363 !$ & 54.7 & 52 & -0.04 \\
\hline $11: 03: 52: 07$ & $456^{\dagger}$ & 257 & 26 & -0.6 & 1.9 & 0.31 & 1361 & 54.7 & 53 & -0.04 \\
\hline $11: 03: 53: 06$ & 457 & 258 & 26 & -0.6 & 1.9 & 0.31 & 1365 & 54.7 & 53 & -0.03 \\
\hline $11: 03: 54: 05$ & 458 & 260 & 26 & -0.6 & 1.9 & $0 . \overline{31}$ & 1370 & 54.6 & 54 & -0.03 \\
\hline $11: 03: 55: 09$ & 459 & 259 & 26 & -0.6 & 1.9 & 0.3 & 1373 & 54.5 & 53 & -0.36 \\
\hline $11: 03: 56: 08$ & 460 & 258 & 26 & -0.6 & 1.9 & 0.31 & 1372 & $5 \overline{4.4}$ & 53 & -0.36 \\
\hline $11: 03: 57: 07$ & 461 & 263 & 26 & -0.6 & 1.9 & 0.3 & 1367 & 54.4 & 53 & -0.15 \\
\hline $11: 03: 58: 06$ & 462 & 265 & 26 & -0.6 & 1.9 & 0.3 & 1369 & 54.4 & 54 & -0.15 \\
\hline $11: 03: 59: 05$ & 463 & 267 & 26 & -0.6 & 1.9 & 0.3 & 1365 & 54.4 & 54 & 0.04 \\
\hline $11: 04: 00: 09$ & 464 & 269 & 26 & -0.6 & 1.9 & 0.3 & 1362 & 54.4 & 54 & 0.04 \\
\hline $11: 04: 01: 08$ & 465 & 270 & $26^{\top}$ & -0.6 & 1.9 & 0.3 & 1363 & 54.4 & 54 & -0.26 \\
\hline $11: 04: 02: 07$ & 466 & 268 & 26 & -0.6 & 1.9 & 0.3 & 1367 & 54.4 & 55 & -0.26 \\
\hline $11: 04: 03: 11$ & 467 & 271 & 26 & -0.6 & 1.9 & 0.31 & 1364 & 54.4 , & 54 & -0.24 \\
\hline
\end{tabular}




\begin{tabular}{|c|c|c|c|c|c|c|c|c|c|c|c|}
\hline TIME & $\begin{array}{l}\text { Elapsed } \\
\text { Time } \\
\text { (sec) }\end{array}$ & $\mathrm{BIT} \mathrm{TC1}_{1}$ & PURG & LTC2 & PurgePSI & ¡PurgeCFM & DEPTH(IN) & DwnFrc(lb) & RPM & $\begin{array}{l}\text { Torque } \\
\text { (FTLB) }\end{array}$ & Inches/Min \\
\hline $11: 04: 04: 10$ & 468 & $27 \overline{3}$ & & 26 & $\quad-0.6$ & 1.9 & $\quad 0.31$ & $\quad 1370$ & 54.4 & $\begin{array}{l}55 \\
\end{array}$ & $\quad-0.24$ \\
\hline $11: 04: 05: 09$ & $469^{\circ}$ & 267 & & 26 & -0.6 & 1.9 & 0.31 & 1373 & 54.4 & 55 & 0.08 \\
\hline 11:04:06:08 & 470 & 268 & & 26 & -0.6 & 1.9 & 0.31 & 1371 & 54.5 & 54 & 0.08 \\
\hline $11: 04: 07: 07$ & 471 & $270^{\prime}$ & & $26 !$ & -0.6 & 1.9 & 0.3 & 1367 & 54.6 & 54 & -0.07 \\
\hline $11: 04: 08: 11$ & 472 & 276 & & 26 & -0.6 & 1.9 & 0.3 & 1363 & 54.6 & $55^{\dagger}$ & -0.07 \\
\hline 11:04:09:10 & 473 & 278 & & 26 & -0.6 & 1.9 & 0.3 & 1367 & 54.8 & 55 & -0.35 \\
\hline $11: 04: 10: 09$ & 474 & 279 & & 26 & -0.6 & 1.9 & 0.28 & 1362 & 54.8 & 55 & -0.35 \\
\hline $11: 04: 11: 13$ & 475 & 280 & & 26 & $-0 . \overline{6}$ & $1.9^{\dagger}$ & 0.3 & 1362 & 54.7 & 55 & -0.12 \\
\hline $11: 04: 12: 12$ & 476 & 279 & & 26 & -0.6 & 1.9 & 0.31 & 1365 & $54 . \overline{7}$ & 56 & 0.08 \\
\hline $11: 04: 13: 11$ & 477 & 281 & & 26 & -0.6 & 1.9 & 0.31 & $1370^{+}$ & $54 . \overline{7}$ & 56 & 0.08 \\
\hline $11: 04: 14: 10$ & 478 & 283 & & 26 & -0.6 & 1.9 & 0.3 & 1367 & 54.7 & 56 & -0.21 \\
\hline $11: 04: 15: 09$ & 479 & 276 & & 26 & 3.7 & 37.2 & 0.31 & 1371 & $54 . \overline{7}$ & $55 !$ & -0.21 \\
\hline $11: 04: 16: 13$ & 480 & 262 & & 27 & 12 & 37.3 & 0.31 & 1372 & 54.7 & $\overrightarrow{51}$ & -0.27 \\
\hline $11: 04: 17: 12$ & 481 & 249 & & 27 & 14.4 & $42^{\dagger}$ & 0.3 & 1367 & 54.7 & 49 & -0.27 \\
\hline $11: 04: 18: 11$ & 482 & 241 & & 27 & 25.3 & 48.5 & 0.31 & 1362 & 54.7 & 46 & -0.04 \\
\hline $11: 04: 19: 10$ & 483 & 227 & & $26^{\circ}$ & 27.4 & 48.6 & 0.3 & 1355 & 54.8 & 44 & -0.04 \\
\hline $11: 04: 20: 08$ & 484 & $2 \overline{15}$ & & 26 & 28.6 & 48.6 & 0.3 & 1352 & 54.8 & 42 & 0 \\
\hline $11: 04: 21: 13$ & 485 & 206 & & 27 & 28.6 & 48.6 & 0.29 & 1348 & 54.8 & 40 & 0 \\
\hline $11: 04: 22: 12$ & 486 & 199 & & 26 & 28.6 & $48.6^{\circ}$ & 0.31 & 1345 & 54.8 & 38 & -0.26 \\
\hline $11: 04: 23: 11$ & 487 & 192 & & $27 !$ & 28.6 & 48.6 & -0.02 & 1336 & 54.8 & 36 & -0.26 \\
\hline $11: 04: 24: 15$ & 488 & 185 & & 26 & 19.1 & 53.3 & -1.92 & 3 & 55 & 1 & -6.04 \\
\hline $11: 04: 25: 14$ & 489 & 185 & & 26 & 18.7 & 54 & -2.25 & $2 !$ & 54.7 & 1 & -6.04 \\
\hline $11: 04: 26: 13$ & 490 & 184 & & 26 & 18.7 & 54.1 & -2.17 & 2 & 55.4 & 1 & -3.73 \\
\hline $11: 04: 27: 11$ & 491 & 181 & & 26 & 18.7 & $54.1 !$ & -2.17 & 2 & 55.4 & 1 & -3.73 \\
\hline $11: 04: 28: 10$ & 492 & 179 & & 27 & 18.5 & 54.1 & -2.17 & 2 & 56.8 & 1 & $0 . \overline{18}$ \\
\hline $11: 04: 29: 15$ & $493 i$ & 176 & & 26 & 18.7 & 54.2 & -2.17 & 2 & 57.3 & 1 & 0.18 \\
\hline $11: 04: 30: 14$ & 494 & $17 \overline{3}$ & & 27 & 18.7 & 54.3 & -2.18 & 2 & 57.3 & 1 & -0.11 \\
\hline $11: 04: 31: 12$ & 495 & 170 & & $27 !$ & 18.7 & 54.4 & -2.17 & 2 & 57.5 & 1 & -0.11 \\
\hline $11: 04: 32: 17$ & 496 & 168 & & 27 & 18.7 & 54.5 & -2.18 & 2 & $5 \overline{7.2}$ & 1 & -0.2 \\
\hline $11: 04: 33: 16$ & 497 & 165 & & 27 & 18.7 & 54.6 & -2.18 & 2. & 57.2 & 1 & -0.12 \\
\hline $11: 04: 34: 15$ & 498 & 162 & & 27 & 18.7 & 54.6 & -2.18 & 2 & 48.5 & $1 !$ & -0.12 \\
\hline $11: 04: 35: 13$ & 499 & $160^{\dagger}$ & & 27 & 18.7 & 54.6 & -2.17 & 2 & 23.6 & 1 & $-0.1 \overline{6}$ \\
\hline $11: 04: 36: 12$ & 500 & 157 & & $26^{t}$ & 18.7 & 54.6 & -3.52 & 2 & 23.6 & 1 & -0.16 \\
\hline $11: 04: 37: 17$ & 501 & $15 \overline{5}$ & & 26 & 18.7 & 54.6 & -5.47 & 2 & 10.9 & 1 & -15.1 \\
\hline $11: 04: 38: 16$ & 502 & 153 & & 27 & 18.7 & 54.6 & -7.44 & 2 & 5.3 & 1. & $-15 . \overline{1}$ \\
\hline $11: 04: 39: 14$ & 503 & 151 & & 26 & 18.7 & 54.6 & -7.97 & 2 & 5.3 & 1 & -14.73 \\
\hline $11: 04: 40: 19$ & 504 & 149 & & $2 \overline{7}$ & $18.7^{+}$ & 54.6 & $-7.97 !$ & 2 & $2 . \overline{9}$ & 1 & -14.73 \\
\hline $11: 04: 41: 18$ & 505 & 146 & & 27 & 18.7 & 54.6 & -7.97 & 2 & $\overrightarrow{1.8}$ & 1 & -0.16 \\
\hline $11: 04: 42: 16$ & 506 & 144 & & 27 & 18.7 & 54.6 & -7.99 & 2 & 1.8 & 1 & $-0.1 \overline{6}$ \\
\hline $11: 04: 43: 15$ & 507 & 143 & & 27 & 18.7 & $54.6^{\dagger}$ & -7.99 & 2 & 1.3 & 1 & -0.13 \\
\hline $11: 04: 44: 14$ & 508 & 140 & & 27 & 18.7 & 54.6 & -7.99 & 2 & 1.1 & 1 & -0.13 \\
\hline $11: 04: 45: 19$ & 509 & 138 & & 27 & 18.7 & 54.6 & -7.97 & 2 & 1.1 & 1 & -0.14 \\
\hline $11: 04: 46: 17$ & 510 & 136 & & 27. & 18.7 & 54.6 & -7.99 & 2 & $1.1^{\dagger}$ & 1 & -0.14 \\
\hline $11: 04: 47: 16$ & 511 & 135 & & 27 & 18.7 & 54.6 & -7.97 & 2. & 1.4 & 1 & $-0 . \overline{14}$ \\
\hline $11: 04: 48: 15$ & 512 & 133 & & 27 & 18.7 & 54.6 & -7.99 & 2 & 1.4 & 1 & -0.14 \\
\hline $11: 04: 49: 14$ & 513 & 131 & & 26 & 18.7 & 54.6 & -7.99 & 2 . & 1.1 & 1 & -0.12 \\
\hline $11: 04: 50: 18$ & 514 & 129 & & 27 & 18.7 & 54.6 & -8 & 2 & 1 & 1. & -0.12 \\
\hline $11: 04: 51: 17$ & 515 & 128 & & 27 & 18.7 & $54.6^{-t}$ & -8 & 2 & 1 & 1 & -0.13 \\
\hline $11: 04: 52: 16$ & 516 & 126 & & 27 & $18.7^{\circ}$ & 54.6 & -7.99 & 2 & 1 & 1 & -0.13 \\
\hline $11: 04: 53: 20$ & 517 & 124 & & 27 & 18.7 & 54.6 & -7.97 & 2 & 0.9 & 1 & -0.13 \\
\hline $11: 04: 54: 19$ & 518 & 122 & & 27 & $18.7^{\circ}$ & 54.6 & -7.97 & 2 & 0.9 & 1 & -0.13 \\
\hline $11: 04: 55: 18$ & $519 !$ & 121 & & $27^{\prime}$ & $18.7^{\circ}$ & 54.6 & $-7.97 i$ & 2 & 0.9 & 1 & -0.13 \\
\hline
\end{tabular}




\begin{tabular}{|c|c|c|c|c|c|c|c|c|c|c|}
\hline TIME & $\begin{array}{l}\text { Elapsed } \\
\text { Time } \\
\text { Isec) }\end{array}$ & BIT_TC1 & PURG_TC2 & PurgePS1 & PurgeCFM & DEPTH(IN) & DwnFrc(lb) RPM & & $\begin{array}{l}\text { Torque } \\
\mid \text { (FTLB) }\end{array}$ & \\
\hline $11: 04: 56: 17$ & 520 & {$[119$} & 27 & 18.7 & $5 \overrightarrow{4.6}$ & -7.99 & 2 & 0.9 & 1 & -0.13 \\
\hline & 521 & 118 & 27 & 18.7 & 54.6 & -7.97 & 2 & 0.9 & 1 & -0.13 \\
\hline & 522 & 116 & 27 & 18.7 & 54.6 & -7.97 & 2 & 0.9 & 1 & -0.13 \\
\hline & 523 & 115 & 26 & 18.7 & 54.6 & -7.97 & 2 & 0.9 & 1 & -0.13 \\
\hline & 524 & 113 & 27 & 18.7 & 54.6 & -7.99 & 2 & $0 . \overline{9}$ & 1 & -0.13 \\
\hline & 525 & 112 & 27 & 18.7 & 54.6 & $-7.9 \overline{7}$ & 2 & 0.9 & 1 & -0.13 \\
\hline & 526 & 110 & 27 & 18.7 & $54 . \overline{6}$ & -7.99 & 2 & $0 . \overline{9}$ & 1 & -0.13 \\
\hline & 527 & 109 & 27 & 18.7 & 54.6 & -7.99 & 2 & 0.9 & 1 & $-0 . \overline{13}$ \\
\hline & 528 & 108 & 27 & 18.7 & $54.6^{\dagger}$ & $-\overline{7.99} \overline{9}^{\dagger}$ & 2 & 0.9 & $\overline{1}$ & -0.13 \\
\hline & 529 & 106 & 27 & 18.7 & 54.6 & -7.97 & 2 & $0 . \overline{9}$ & 1 & $-\overline{0 . \overline{13}}$ \\
\hline & 530 & 105 & 27 & $18 . \overline{7}$ & 54.6 & -7.97 & 2 & 0.9 & 1 & -0.13 \\
\hline & 531 & 104 & $2 \overline{7}$ & 18.7 & $54 . \overline{6}$ & -7.99 & 2 & 0.9 & 1 & -0.13 \\
\hline & 532 & 102 & 27 & 18.7 & 54.6 & -7.97 & $2]^{-}$ & 0.9 & 1 & -0.13 \\
\hline $11: 05: 09: 24$ & 533 & 101 & 27 & 18.7 & 54.6 & -7.99 & 2 & 0.9 & 1. & -0.13 \\
\hline & & & $\ldots$ & & & & $\begin{array}{lll} & \\
\cdots & - & \end{array}$ & - & - & - \\
\hline
\end{tabular}




\begin{tabular}{|c|c|c|c|c|c|c|c|c|c|c|}
\hline TIME & $\begin{array}{l}\text { Elapsed } \\
\text { Time } \\
\text { (sec) }\end{array}$ & BIT_TC1 & PURG_TC2 & PurgePSI & & & & & $\begin{array}{l}\text { Torque } \\
\text { (FTLB) }\end{array}$ & Inches/Min \\
\hline $12: 18: 00: 38$ & $\overrightarrow{0}$ & 26 & 25 & 21.4 & $\quad 53.8$ & -0.53 & $\therefore \quad 3$ & -56.7 & -2 & $2 \quad-0.22$ \\
\hline $12: 18: 01: 37$ & 1 & 26 & 25 & 21.4 & 53.8 & $-0 . \overline{42}$ & 3 & 56.7 & -2 & 1.82 \\
\hline $12: 18: 02: 36$ & 2 & 26 & 25 & 21.4 & 53.8 & $-0.26^{+}$ & 7 & 56.8 & -2 & 1.82 \\
\hline $12: 18: 03: 40$ & 3 & 26 & 25 & 21.4 & 53.8 & -0.11 & 9 & 56.8 & -1 & 6.25 \\
\hline : & 4 & 26 & 25 & 21.4 & 53.8 & 0.03 & $63^{-1}$ & 56.5 & 2 & 6.25 \\
\hline 43 & 5 & 28 & 25 & 21.8 & 53.8 & 0.15 & 484 & 56.5 & 32 & 5.29 \\
\hline$: 42$ & 6 & 31 & 25 & 22 & 53.8 & 0.2 & 885 & 56.5 & 39 & 5.29 \\
\hline $7: 41$ & 7 & 35 & 25 & 22.2 & 53.8 & 0.23 & $1065^{\dagger}$ & 56.1 & 47 & 2.79 \\
\hline 40 & 8 & 39 & 25 & 22.2 & 53.8 & 0.24 & 1112 & 55.5 & 49 & 2.79 \\
\hline 44 & 9 & 43 & 25 & 22.4 & 53.8 & .25 & 1166 & 55.1 & 48 & 1.43 \\
\hline $43^{\prime}$ & $10^{\dagger}$ & 45 & 25 & 22.2 & 53.8 & 0.28 & 1775 & 55.1 & 49 & $1 . \overline{43}$ \\
\hline$: 42$ & 11 & 46 & 25 & 22.2 & 53.8 & $0 . \overline{3}$ & 1196 & 55 & 48 & 0.59 \\
\hline 41 & 12 & 49 & 25 & 22.2 & 53.8 & 0.31 & 1222 & 55 & 48 & 0.59 \\
\hline & 13 & 50 & 25 & 22.2 & 53.8 & 0.33 & 1247 & 55 & 48 & 0.55 \\
\hline & 14 & 53 & 25 & 22.2 & 53.8 & 0.33 & $1264^{\dagger}$ & 54.9 & 47 & 0.55 \\
\hline & 15 & 55 & 25 & 22.2 & 3.8 & 0.34 & 1283 & 54.8 & 46 & 0.48 \\
\hline & 16 & 57 & 25 & 22.4 & 53.8 & 0.31 & 1292 & 54.8 & $\overline{46}$ & 0.48 \\
\hline$: 46$ & 17 & 58 & 25 & 22.4 & 3.7 & 34 & 1306 & 54.9 & 44 & 0.23 \\
\hline$: 45$ & 18 & 60 & 25 & 22.4 & 53.7 & 33 & $1320^{t}$ & $54.9^{\circ}$ & 44 & -0.02 \\
\hline & 19 & 61 & 25 & 22.4 & & & 1314 & 54.9 & $4 \overline{3}$ & -0.02 \\
\hline & $20^{\dagger}$ & 63 & 25 & 22.4 & 53.7 & 35 & 1323 & 54.9 & $\overline{42}$ & 0.17 \\
\hline & 21 & 64 & 25 & 22.4 & & & 1331 & 54.9 & 40 & 0.17 \\
\hline & 22 & 65 & $25 !$ & 22.6 & & & 1344 & 54.9 & 40 & $0.2 \overline{9}$ \\
\hline & 23 & 66 & 25 & 22.6 & & & 1355 & 55 & $\overline{42}$ & 0.29 \\
\hline & 24 & 67 & 25 & 22.4 & & & 358 & 55.1 & 42 & -0.18 \\
\hline & 25 & 67 & 25 & 22.6 & & & $360^{\circ}$ & 55.1 & 40 & -0.18 \\
\hline & 26 & $6 \overline{8}$ & 25 & $2 \overline{2.6}$ & & & $1363^{1}$ & 55 & 39 & $-0 . \overline{06}$ \\
\hline & 27 & 69 & 25 & 22.6 & 5 & & 1363 & 54.9 & 39 & -0.06 \\
\hline & 28 & $7 \overline{0}$ & 25 & 22.6 & & & 1359 & 54.9 & 40 & 0.13 \\
\hline & 29 & 71 & 25 & 22.6 & & & 355 & 54.9 & 39 & 0.13 \\
\hline & 30 & 72 & 25 & 22.6 & & & 355 & 54.9 & 37 & 0.13 \\
\hline & 31 & 72 & 25 & 22.6 & & & 1355 & 54.9 & 37 & 0.13 \\
\hline & 32 & 73 & 25 & & & & & 54.8 & 37 & -0.25 \\
\hline & 33 & 73 & 25 & 22.6 & & $3 \overline{8}$ & 1356 & $5 \overline{4.7}$ & 37 & -0.25 \\
\hline & 34 & $\overline{74}$ & 25 & 22.6 & & & & 54.7 & 37 & -0.16 \\
\hline & 35 & 74 & 25 & $22 . \overline{8}$ & & & & 54.8 & 36 & -0.16 \\
\hline & 36 & $\overline{75}$ & 25 & 22.8 & & & 1356 & 55 & 35 & 0.13 \\
\hline & $3 \overline{7}$ & 75 & 25 & 22.8 & 53.5 & & 1351 & $55^{\dagger}$ & 35 & 0.13 \\
\hline & 38 & 76 & 25 & 22.8 & 53.5 & $0 . \overline{38}$ & $1355^{i}$ & 55 & 34 & -0.05 \\
\hline & 39 & 76 & 25 & 22.8 & 5 & $3 \overline{8}$ & 1351 & 55 & $3 \overline{4}$ & -0.05 \\
\hline & $40 \mathrm{j}$ & 76 & 25 & 22.8 & 53.5 & 38 & $348^{\circ}$ & 55 & 34 & $-0.2 \overline{9}$ \\
\hline & 41 & 76 & $25^{\dagger}$ & 22.8 & 53 & & $1349^{\dagger}$ & $54 . \overline{9}$ & 34 & -0.02 \\
\hline & 42 & 76 & 25 & 22.8 & & & & 55 & 34 & -0.02 \\
\hline & 43 & 76 & 25 & 22.8 & 53.4 & & 1353 & 55 & 34 & 0.11 \\
\hline & 44 & 76 & 25 & $22 . \overline{8}$ & 53.4 & & 1360 & 54.6 & 33 & 0.11 \\
\hline & $\overline{45}$ & 76 & 25 & 22.8 & & & 1364 & 54.7 & 33 & -0.18 \\
\hline 52 & 46 & 76 & 25 & 22.8 & 53.4 & 0.39 & 1365 & 54.7 & 33 & -0.18 \\
\hline$\overline{77}: 50$ & 47 & 77 & 25 & 22.8 & 53.4 & $0.3 \overline{9}$ & 1367 & 55 & 33 & $-0 . \overline{23}$ \\
\hline $18: 48: 49$ & 48 & $\overline{77}$ & 25 & 22.8 & 53.4 & $\overline{0.38}$ & $1361^{\circ}$ & $55.1 !$ & $3 \overline{3}$ & $-0.2 \overline{3}$ \\
\hline & 49 & 77 & 25 & 22.8 & $53 . \overline{4}$ & 0.38 & 1365 & 55.1 & 33 & -0.03 \\
\hline & 50 & 77 & 25 & 22.8 & & $0.3 \overline{8}$ & 1362 & 55.1 & 33 & -0.03 \\
\hline $12: 18: 51: 46$ & $51 !$ & 77 & 25 & 22.8 & 53.4 & 0.39 & $1359^{\circ}$ & 55.1 & $3 \overline{3}$ & $0.1 \overline{2}$ \\
\hline
\end{tabular}




\begin{tabular}{|c|c|c|c|c|c|c|c|c|c|c|}
\hline TIME & $\begin{array}{l}\text { ¿Elapsed } \\
\text { Time } \\
\text { (sec) }\end{array}$ & & PURG_TC2 & & PurgeCFM & $1 \mathrm{DEPTH}(\mathrm{IN})$ & DwnFrc(ib) & RPM & $\begin{array}{l}\text { Torque } \\
\text { (FTLB) }\end{array}$ & Inches/Min \\
\hline $12: 18: 52: 45$ & $\quad 52$ & $\quad-77$ & $\quad-25$ & $\quad 22.8$ & i $\quad 53.4$ & $\quad 0.39$ & 1360 & 55.1 & $\quad 33^{\prime}$ & $\quad 0 . \overline{12}$ \\
\hline $12: 18: 53: 49$ & 53 & 77 & 25 & 22.9 & 53.4 & 0.4 & 1366 & 55.1 & 33 & -0.2 \\
\hline $12: 18: 54: 53$ & 54 & 77 & 25 & 22.9 & 53.4 & 0.4 & 1362 & 55.1 & 33 & -0.2 \\
\hline $12: 18: 55: 52$ & 55 & 78 & 25 & 22.9 & 53.4 & 0.39 & 1367 & 55.1 & 33 & -0.2 \\
\hline $12: 18: 56: 51$ & 56 & 78 & 25 & 22.9 & 53.4 & 0.39 & 1368 & 55 & 33 & -0.2 \\
\hline $12: 18: 57: 50$ & 57 & 78 & 25 & 22.9 & 53.4 & 0.39 & 1367 & 55 & 32 & -0.02 \\
\hline $12: 18: 58: 49$ & 58 & 78 & 25 & 22.9 & 53.4 & 0.39 & 1362 & 55 & 32 & -0.02 \\
\hline $12: 18: 59: 53$ & $59^{\dagger}$ & 78 & 25 & 22.9 & $53.3^{+}$ & 0.39 & 1355 & 55 & 32 & 0.04 \\
\hline $00: 52$ & $60^{\circ}$. & 78 & 25 & 22.9 & 53.3 & 0.38 & 1356 & 54.9 & 31 & 0.04 \\
\hline $12: 19: 01: 51$ & $\overline{61}$ & 78 & 25 & 22.9 & 53.3 & 0.38 & 1351 & 54.9 & 32 & -0.3 \\
\hline $12: 19: 02: 55$ & 62 & 78 & 25 & 22.9 & 53.3 & 0.38 & 1346 & 55 & $32^{4}$ & -0.3 \\
\hline $12: 19: 03: 54$ & 63 & 78 & 25 & 22.9 & 53.3 & 0.39 & 1346 & 55.1 & 31 & -0.15 \\
\hline $12: 19: \overline{04}: 53$ & 64 & 78 & 25 & 22.9 & 53.3 & 0.38 & 1351 & 55.1 & 31 & -0.15 \\
\hline $55: 52$ & 65 & 78 & 25 & 22.9 & 53.3 & 0.39 & 1353 & 55 & $31 j$ & $0 . \overline{07}$ \\
\hline $12: 19: 06: 51$ & $66_{4}$ & 78 & 25 & 22.9 & $53 . \overline{3}$ & $0 . \overline{3}$ & 1350 & 54.9 & 31 & -0.07 \\
\hline $7: 55$ & 67 & 78 & 25 & 22.9 & 53.3 & 0.39 & 1353 & 54.9 & 31 & $-0.0 \overline{7}$ \\
\hline $12: 19: 0 \overline{8: 54}$ & 68 & 78 & 25 & 22.9 & 53.3 & 0.4 & 1351 & 54.8 & 31 & -0.28 \\
\hline $12: 19: 09: 53$ & 69 & 78 & 25 & 22.9 & 53.3 & 0.39 & 1347 & 54.8 & 31 & $-0.2 \overline{8}$ \\
\hline $10: 52$ & 70 & 78 & 25 & 22.9 & 53.3 & 0.38 & 1343 & 54.8 & $31 T$ & -0.11 \\
\hline $9: 11: 51$ & 71 & 78 & 25 & 22.9 & 53.3 & 0.4 & 1341 & 54.9 & 31 & -0.11 \\
\hline $12: 19: 12: 55$ & 72 & 78 & 25 & 22.9 & 53.3 & 0.39 & 1344 & 54.9 & 31 & 0.12 \\
\hline$: 1 \overline{3}: 54$ & $73 !$ & 78 & 25 & $22 . \overline{9}$ & 3.3 & 0.4 & 1343 & 54.9 & 31 & 0.12 \\
\hline $12: 19: 14: \overline{53}$ & 74 & 78 & 25 & 22.9 & 53.3 & 0.4 & 1348 & 54.9 & 31 & -0.13 \\
\hline $12: 19: 15: 57$ & 75 & 78 & 25 & 22.9 & 53.3 & 0.39 & 1355 & 55 & 31 & -0.13 \\
\hline $12: 19: 16: 56$ & 76 & 78 & 25 & 22.9 & 53.3 & 0.42 & 1352 & 55 & 31 & -0.27 \\
\hline $17: 55$ & 77 & 77 & 25 & 22.9 & 53.3 & 0.4 & 1357 & 55 & 31 & -0.27 \\
\hline $3: 54$ & 78 & 77 & 25 & 22.9 & 53.3 & 0.4 & 1360 & 54.9 & 31 & 0.11 \\
\hline $9: 19: 53$ & $7 \overline{9}$ & 78 & 25 & 22.9 & 53.3 & 0.4 & 1359 & 54.9 & $3 \overline{1}$ & 0.11 \\
\hline $12: 19: 20: 57$ & 80 & 78 & 25 & 22.9 & 53.3 & 0.4 & 1356 & 54.8 & 31 & -0.03 \\
\hline $12: 19: 21: 56$ & 81 & 78 & 25 & 22.9 & 53.3 & 0.39 & 1353 & 54.8 & 31 & -0.03 \\
\hline 22.55 & 82 & 78 & 25 & 22.9 & 53.3 & 0.39 & 1350 & 54.8 & 31 & -0.25 \\
\hline $23: 59$ & 83 & 78 & 25 & 22.9 & 53.3 & 0.4 & 1352 & 54.9 & 31 & -0.25 \\
\hline $4: 58$ & 84 & 78 & 25 & 22.9 & $5 \overline{3} .3$ & 0.35 & 1352 & 55 & 31 & -0.22 \\
\hline$: 25: 57$ & 85 & 78 & 25 & 22.9 & 53.3 & 0.4 & 1356 & $5 \overline{5}$ & $31^{1}$ & -0.22 \\
\hline $12: 19: 26: 56$ & 86 & 78 & 25 & 22.9 & 3.3. & 0.42 & 1358 & 55 & 31 & 0.06 \\
\hline $7: 55$ & 87 & 78 & $\overline{25}$ & 23.1 & & $0 . \overline{39}$ & 1360 & 55 & 31 & 0.06 \\
\hline $8: 59$ & 88 & 78 & 25 & 23.1 & 53.3 & 0.42 & 1359 & 55 & 31 & 0.03 \\
\hline $9: 58$ & 89 & $\overline{78}$ & 25 & 23.1 & 53.3 & 0.4 & 1360 & 55 & 31 & -0.28 \\
\hline $30: 57$ & 90 & 78 & 25 & 22.9 & $5 \overline{3} .3]$ & 0.4 & 1358 & 54.8 & 31 & -0.28 \\
\hline $12: 19: 31: 61$ & 91 & 78 & 25 & 23.1 & 53.3 & 0.39 & 1354 & 54.8 & 31 & -0.13 \\
\hline $12: 19: 32: 60$ & 92 & 78 & 25 & 23.1 & 53.2 & 0.4 & 1350 & 54.9 & 31 & -0.13 \\
\hline $12: 19: 33: 59$ & 93 & 78 & 25 & 22.9 & $5 \overline{3} .2$ & 0.39 & $13 \overline{49}$ & 54.9 & 31 & 0.06 \\
\hline & 94 & 78 & 25 & 23.1 & 53.2 & 0.4 & $1350^{\circ}$ & 54.9 & 31 & 0.06 \\
\hline & 95 & 78 & 25 & 23.1 & 53.1 & 0.39 & 1351 & 55 & 31 & -0.09 \\
\hline $12: 19: 36: 61$ & 96 & 79 & 25 & 23.1 & 53.1 & 0.4 & 1355 & 55.1 & 30 & $-0.0 \overline{9}$ \\
\hline $12: 19: 37: 60$ & 97 & 78 & 25 & 23.1 & 53.1 & 0.42 & 1360 & 55.1 & 30 & -0.32 \\
\hline $12: 19: 38: 59$ & 98 & 78 & 25 & 23.1 & 53.1 & 0.42 & 1363 & 54.9 & 31 & -0.32 \\
\hline $12: 19: 39: 57$ & 99 & 78 & 25 & 23.1 & 53.1 & 0.4 & 1362 & 54.9 & 31 & -0.11 \\
\hline $12: 19: 40: 56$ & 100 & 79 & 25 & 23.1 & $5 \overline{3.1}$ & $0 . \overline{4}$ & 1357 & 54.9 & 31 & -0.11 \\
\hline $12: 19: 41: 55$ & 101 & $7 \overline{9}$ & 25 & 23.1 & 53.1 & 0.4 & 1360 & 54.9 & 31 & 0.15 \\
\hline $12: 19: 42: 54$ & 102 & 79 & 25 & 23.1 & 53.1 & 0.39 & 1356 & 55 & 30 & 0.15 \\
\hline $12: 19: 43: 58$ & 103 & 78 & 25 & 23.1 & 53.1 & 0.4 & 1352 & 55 & 31 & $-0 . \overline{18}$ \\
\hline
\end{tabular}




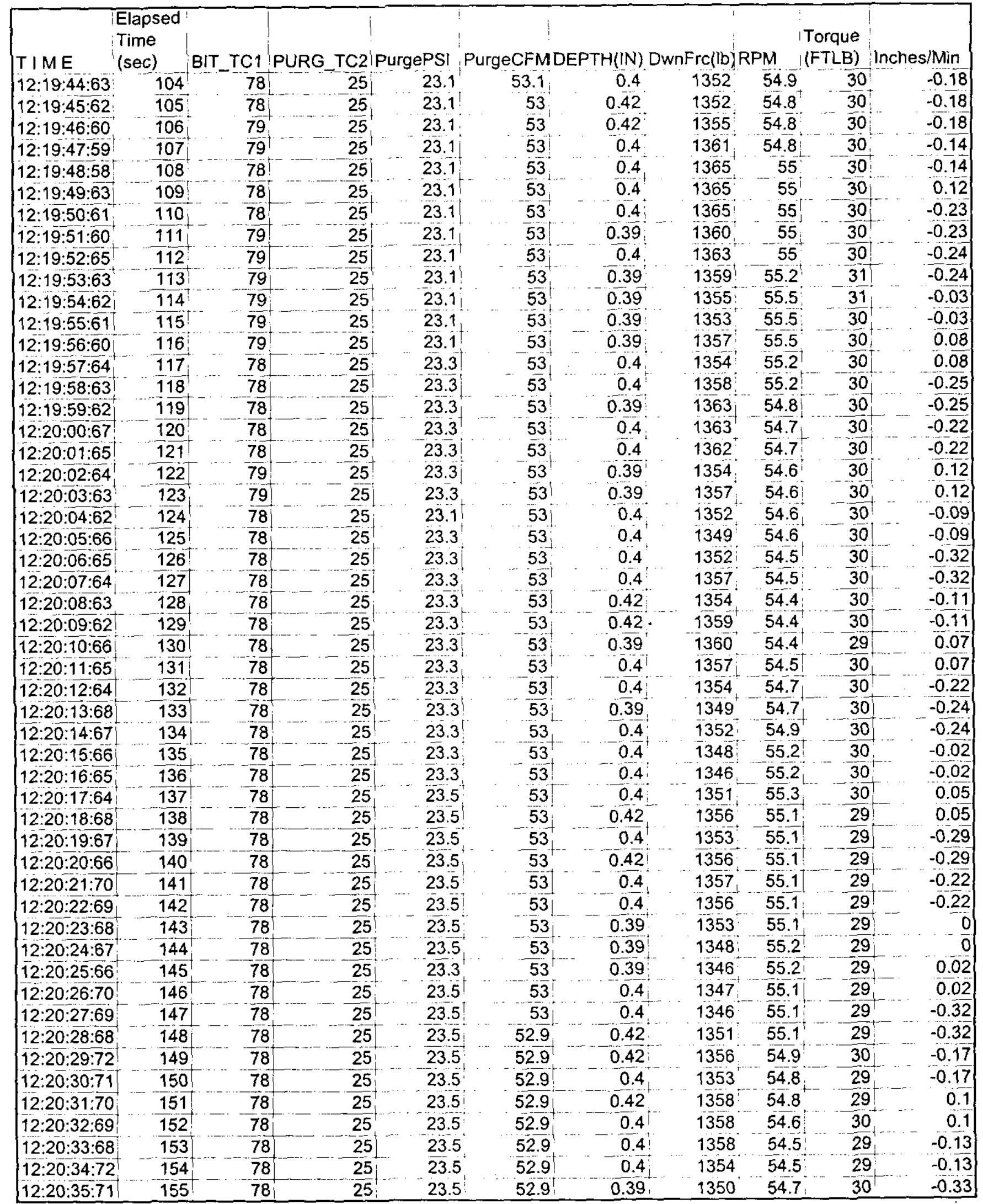




\begin{tabular}{|c|c|c|c|c|c|c|c|c|c|c|}
\hline TIME & $\begin{array}{l}\text { Elapsed } \\
\text { Time } \\
(\mathrm{sec})\end{array}$ & BIT_TC1 & PURG_TC2 & PurgePSI & PurgeCFM & DEPTH $(I N)$ & DwnFrc(lb) & RPM & $\begin{array}{l}\text { Torque } \\
\text { (FTLB) }\end{array}$ & Inches/Min \\
\hline $12: 20: 36: 70$ & 156 & $\quad 78$ & 25 & 23.5 & $\quad 52.9$ & 0.4 & 1351 & 54.7 & $\quad 29$ & $\quad-0.33$ \\
\hline $12: 20: 37: 68$ & 157 & 78 & 26 & 23.5 & 52.9 & 0.42 & 1350 & 54.7 & 29 & -0.08 \\
\hline $12: 20: 38: 67$ & 158 & 78 & $26 !$ & 23.7 & 52.9 & 0.42 & $1353^{\prime}$ & 54.5 & 29 & 0.07 \\
\hline $12: 20: 3972$ & 159 & 78 & 26 & 23.7 & 52.9 & 0.4 & 1359 & 54.4 & 29 & 0.07 \\
\hline $12: 20: 40: 71$ & $160 \mathrm{O}$ & 78 & 26 & 23.7 & 52.9 & 0.42 & 1363 & 54.4 & 29 & -0.31 \\
\hline $12: 20: 41: 69$ & 161 & 77. & $26^{1}$ & $23.7^{\dagger}$ & 52.9 & 0.4 & 1361 & 54.3 & 29 & -0.31 \\
\hline $12: 20: 42: 74$ & 162 & 78 & 26 & 23.7 & 52.8 & 0.4 & 1363 & 54.1 & 29 & -0.19 \\
\hline $12: 20: 43: 73$ & 163 & 78 & 26 & 23.7 & 52.8 & 0.4 & 1360 & 54.1 & 29 & -0.19 \\
\hline $12: 20: 44: 72$ & $16 \overrightarrow{4}$ & 77 & 26 & 23.7 & 52.8 & 0.4 & 1355 & 54.1 & 29 & 0.05 \\
\hline $12: 20: 45: 70$ & 165 & 77 & 26 & 23.7 & 52.8 & 0.4 & 1351 & 54.2 & $29^{1}$ & 0.05 \\
\hline $12: 20: 46: 69$ & 166 & 77 & 25 & 23.7 & 52.8 & 0.42 & 1353 & 54.2 & 29 & -0.16 \\
\hline $12: 20: 47: 74$ & 167 & 77 & $26 !$ & 23.7 & 52.8 & 0.42 & 1358 & 54.2 & 29 & -0.16 \\
\hline $12: 20: 48: 7 \overline{3}$ & 168 & 77 & $26 !$ & 23.7 & 52.8 & 0.42 & 1355 & $54.1^{-t}$ & 28 & -0.31 \\
\hline $12: 20: 4971$ & 169 & 77 & 26 & 23.7 & 52.8 & 0.42 & $1 \overline{362}$ & 54.1 & 29 & -0.31 \\
\hline $12: 20: 50: 76$ & $170^{\circ}$ & 77 & 26 & 23.7 & 52.8 & 0.4 & 1363 & 54 & 29 & -0.06 \\
\hline $12: 20: 51: 75$ & 171 & 77 & 26 & 23.7 & 52.8 & 0.42 & 1362 & 54 & 29 & -0.06 \\
\hline $12: 20: 52: 73$ & 172 & 78 & $26 !$ & 23.5 & $52 . \overline{8}$ & 0.42 & 1356 & 54 & 29 & 0.08 \\
\hline $12: 20: 53: 72$ & 173 & $77^{\dagger}$ & 25 & 23.5 & 52.8 & 0.4 & 1359 & $54 \overrightarrow{4}$ & 29 & 0.08 \\
\hline $12: 20: 54: 71$ & 174 & 77 & 25 & 23.5 & 52.8 & 0.4 & 1353 & 54.1 & 29 & -0.34 \\
\hline $12: 20: 55: 76$ & 175 & 77 & 26 & 23.5 & 52.8 & 0.4 & 1351 . & 54.1 & 29 & -0.34 \\
\hline $12: 20: 56: 74$ & 176 & $77_{1}^{\circ}$ & 26 & 23.7 & 52.8 & 0.42 & 1354 & $54.1^{1}$ & 29 & -0.14 \\
\hline $12: 20: 57: 73$ & 177 & 77 & 26 & 23.7 & 52.8 & 0.42 & 1360 & $54.1^{t}$ & 29 & -0.14 \\
\hline $12: 20: 58: 7 \overline{8}$ & 178 & 77 & 26 & 23.7 & 52.8 & 0.42 & $1363^{-}$ & 54.1 & 29 & 0.07 \\
\hline $12: 20: 59: 77$ & $17 \overline{9}$ & 77 & $26^{+}$ & 23.7 & 52.8 & 0.42 & 1363 & 54 & 29 & -0.2 \\
\hline $12: 21: 00: 75$ & 180 & 78 & 26 & 23.5 & $5 \overline{2} .8$ & 0.4 & 1363 & 53.9 & 29 & $-0 . \overline{2}$ \\
\hline $12: 21: 01: 74$ & 181 & 781 & 26 & 23.7 & 52.8 & 0.4 & 1360 & 53.9 & 29 & -0.27 \\
\hline $12: 21: 02: 73$ & 182 & 77 & $26 !$ & $23.5^{\dagger}$ & 52.8 & $0.4^{1}$ & 1355 & 54 & 29 & $-0.2 \overline{7}$ \\
\hline $12: 21: 03: 77$ & $18 \overline{3}^{\dagger}$ & 77 & 26 & 23.5 & 52.8 & 0.42 & 1352 & 54.1 & 29 & -0.01 \\
\hline $12: 21: 04: 76$ & 184 & 77 & 26 & 23.5 & 52.8 & 0.42 & 1353 & 54.1 & 30 & -0.01 \\
\hline $12: 21: 05: 75$ & 185 & 77 & 26 & 23.5 & 52.8 & $0 . \overline{42}$ & 1360 & 54.1 & 29 & -0.03 \\
\hline $12: 21: 06: 74$ & 186 & 77 & 26 & 23.7 & 52.8 & 0.43 & 1356 & 54.2 & 29 & -0.03 \\
\hline $12: 21: 07: 73$ & 187 & 77 & 26 & 23.7 & 52.8 & 0.42 & 1362 & 54.2 & 29 & -0.33 \\
\hline $12: 21: 08: 77$ & 188 & 77 & 26 & 23.7 & 52.8 & 0.42 & 1363 & 54.1 & 29 & $-0 . \overline{3} 3$ \\
\hline $12: 21: 09: 76 !$ & 189 & 77 & 26 & 23.7 & 52.8 & $0.42 !$ & 1360 & 53.9 & 29 & -0.1 \\
\hline $12: 21: 10: 75$ & 190 & 77 & 26 & $23.7 j$ & 52.8 & 0.4 & 1355 & 53.9 & 29 & -0.1 \\
\hline $12: 21: 11: 79$ & 191 & 77 & 26 & 23.7 & 52.8 & 0.42 & 1358 & 53.9 & 29 & 0.08 \\
\hline $12: 21: 12: 78$ & 192 & 77 & $26^{\dagger}$ & 23.7 & 52.8 & 0.42 & 1353 & 54 & 30 & 0.08 \\
\hline $12: 21: 13: 77$ & 193 & 77 & 26 & 23.7 & 52.8 & 0.42 & $1350^{\dagger}$ & 54 & 29 & -0.28 \\
\hline $12: 21: 14: 76$ & 194 & 77 & 26 & 23.7 & 52.8 & 0.42 . & 1354 & 54.2 & 29 & -0.28 \\
\hline $12: 21: 15: 75$ & 195 & 77 & $26 !$ & 23.7 & 52.8 & $0.42 !$ & 1359 & $54.5^{i}$ & 29 & -0.22 \\
\hline $12: 21: 16: 79$ & 196 & 77 & 26 & 23.7 & 52.8 & 0.42 & 1362 & $54.5 !$ & 29 & -0.22 \\
\hline $12: 21: 17: 78$ & 197 & 77 & 26 & 23.7 & 52.8 & 0.42 & 1362 & 54.7 & 29 & 0.03 \\
\hline 12:21:18:77 & 198 & $7 \overline{1}$ & 26 & $23 . \overline{7}$ & 52.8 & 0.42 & 1363 & 54.7 & 29 & 0.03 \\
\hline $12: 21: 19: 81$ & 199 & 78 & $26^{+}$ & 23.7 & 52.8 & 0.42 & 1360 & 54.7 & 29 & -0.02 \\
\hline $12: 21: 20: 80$ & 200 & 78 & $26 !$ & $23.7^{\top}$ & 52.8 & 0.4 & 1357 & 54.8 & 29 & -0.02 \\
\hline $12: 21: 21: 79$ & 201 & 77 & 26 & $23 . \overline{7}$ & 52.8 & 0.4 & 1352 & $54 . \overline{9}$ & 29 & -0.29 \\
\hline $12: 21: 22: 78$ & 202 & $77^{\dagger}$ & 26 & 23.7 & 52.8 & 0.4 & 1354 & 54.9 & 29 & -0.07 \\
\hline $12: 21: 23: 77$ & 203 & 77 & 26 & 23.7 & 52.8 & 0.42 & 1351 & 54.8 & 29 & -0.07 \\
\hline $12: 21: 24: 81$ & 204 & 77 & 26 & $23 . \overline{7}$ & 52.8 & 0.42 & 1353 & 54.8 & 29 & 0.06 \\
\hline $12: 21: 25: 80$ & 205 & 77 & 26 & 23.7 & 52.8 & 0.4 & 1356 & 54.8 & 29 & 0.06 \\
\hline $12: 21: 26: 79$ & 206 & 78 & 26 & 23.7 & $52.8 !$ & 0.42 & 1362 & 55 & 29 & $-0.1 \overline{8}$ \\
\hline $12: 21: 27: 83$ & $207 !$ & 78 & $26 !$ & 23.7 & 52.8 & 0.42 & 1363 & 54.9 & 29 & -0.18 \\
\hline
\end{tabular}




\begin{tabular}{|c|c|c|c|c|c|c|c|c|c|c|}
\hline TIME & $\begin{array}{l}\text { Elapsed } \\
\text { Time } \\
\text { (sec) }\end{array}$ & BIT_TC1 & PURG_TC2 & PurgePSI & PurgeCFM & DEPTH(IN) & DwnFrc(lb) & RPM & $\begin{array}{l}\text { Torque } \\
\text { (FTLB) }\end{array}$ & Inches/Min \\
\hline $12: 21: 28: 82$ & 208 & $\quad-77$ & 26 & $\quad 23.7$ & $\quad 52.7$ & 0.43 & 1363 & 54.9 & $9 \quad 28$ & -0.3 \\
\hline $12: 21: 29: 81$ & 209 & 78 & 26 & 23.7 & 52.7 & 0.43 & 1363 & 55 & 29 & -0.3 \\
\hline $12: 21: 30: 80$ & 210 & 78 & 26 & 23.7 & 52.7 & 0.4 & 1361 & 55 & 29 & -0.1 \\
\hline $12: 21: 31: 79$ & $211^{\dagger}$ & 77 & 26 & 23.7 & $52.7^{\dagger}$ & 0.42 & 1356 & 55 & 29 & -0.1 \\
\hline $12: 21: 32: 83$ & 212 & 77 & 26 & 23.7 & 52.7 & 0.42 & 1352 & 55 & 29 & $0 . \overline{08}$ \\
\hline $12: 21: 33: 82$ & 213 & 77 & 26 & 23.7 & 52.7 & 0.42 & 1355 & 55 & 29 & 0.08 \\
\hline $12: 21$ & 214 & 77 & 26 & 23.9 & 52.7 & 0.42 & 1356 & 55 & 28 & -0.22 \\
\hline 12:21: & 215 & 77 & 26 & 23.9 & 52.7 & 0.42 & 1354 & 54.9 & 28 & -0.22 \\
\hline $2: 21: 36: 78$ & 216 & 77 & $26^{\dagger}$ & 23.9 & 52.7 & 0.43 & 1359 & 54.9 & 28 & $-0 . \overline{24}$ \\
\hline $12: 21: 37: 83$ & 217 & 77 & 26 & 23.9 & $5 \overline{2} .7$ & 0.42 & $1363^{\dagger}$ & 54.9 & 28 & -0.24 \\
\hline $12: 21: 38: 82$ & 218 & 77 & 26 & 23.9 & 52.7 & 0.42 & 1363 & 54.9 & 28 & -0.03 \\
\hline $39: 81$ & $219 !$ & 77 & 26 & 23.9 & $5 \overline{2.7}$ & $0 . \overline{42}$ & 1362 & 54.8 & 28 & $-0 . \overline{03}$ \\
\hline 85 & 220 & $\overline{77}$ & 26 & 23.9 & 52.7 & $0 . \overline{42}$ & 1363 & 54.8 & 29 & 0.04 \\
\hline 84 & 221 & 77 & 26 & 23.9 & 52.7 & 0.42 & 1361 & 54.8 & 29 & 0.04 \\
\hline $12: 21: 42: 83$ & 222 & 77 & 26 & 23.7 & 52.7 & 0.4 & 1356 & 54.9 & 28 & $-0 . \overline{32}$ \\
\hline $12: 21: 43: 82$ & 223 & 77 & 26 & 23.7 & 52.7 & 0.42 & $1353^{+}$ & 54.9 & 28 & -0.32 \\
\hline $4: 80$ & 224 & 77 & 26 & 23.7 & 52.7 & 0.42 & 1354 & 54.9 & 29 & -0.2 \\
\hline $5: 85$ & 225 & 77 & 26 & 23.7 & 52.7 & 0.4 & 1358 & $54 . \overline{9}$ & 28 & 0.03 \\
\hline $8: 84$ & 226 & 77 & 26 & 23.7 & 52.7 & 0.42 & 1355 & 54.9 & 29 & 0.03 \\
\hline 82 & 227 & 77 & 26 & 23.9 & 52.7 & 0.43 & 1362 & 54.9 & 28 & -0.03 \\
\hline$\overline{8} 7$ & $2 \overline{2 B}^{t}$ & $7 \overline{7}$ & 26 & 23.9 & $5 \overline{2} .7$ & $0 . \overline{43}$ & 1364 & 54.8 & 28 & $-0 . \overline{03}$ \\
\hline $9: 86$ & 229 & 77 & 26 & 23.9 & $52.6^{\prime}$ & 0.42 & 1364 & 54.8 & 28 & -0.3 \\
\hline 85 & $230^{-}$ & 77 & 26 & 23.9 & 52.6 & 0.42 & 1361 & 54.8 & 28 & -0.3 \\
\hline 83 & 231 & 77 & 26 & 23.9 & 52.6 & 0.42 & & 54.8 & 29 & $-0.1 \overline{4}$ \\
\hline $82 !$ & 232 & 77 & 26 & 23.9 & 52.6 & 0.42 & 1360 & 54.8 & 28 & -0.14 \\
\hline 87 & 233 & 77 & 26 & 23.9 & $5 \overline{2.6}$ & 0.42 & 1355 & 54.9 & 29 & 0.11 \\
\hline$: 86$ & 234 & $\overline{7} \overline{7}$ & 26 & 23.9 & 52.6 & 0.42 & 1353 & 55 & 29 & 0.11 \\
\hline $5: 84$ & 235 & 77 & 26 & 23.9 & 52.6 & $4 \overline{2}$ & & 55 & 28 & -0.2 \\
\hline $5: 89$ & 236 & 77 & 26 & 23.9 & 52.6 & 0.42 & 1361 & 55 & 28 & $-\overline{0.2}$ \\
\hline 88 & 237 & 77 & 26 & 23.9 & 52.6 & 0.42 & & 55 & 28 & -0.29 \\
\hline 86 & $23 \overline{8}$ & 77 & 26 & 23.9 & 52.6 & 0. & $363^{i}$ & 55 & 28 & -0.29 \\
\hline 85 & 239 & 77 & 26 & 23.9 & 52.6 & 0.42 & 1365 & 55 & 28 & -0.06 \\
\hline 84 & 240 & 77 & 26 & 23.9 & 52.6 & 0.42 & 1364 & 54.9 & 28 & -0.06 \\
\hline & 241 & 77 & 26 & & & & & 54.9 & 28 & .03 \\
\hline & 242 & 77 & 26 & 23.9 & 52.6 & 0.42 & & 54.6 & 29 & 0.03 \\
\hline & $2 \overline{43}$ & 76 & 26 & 23.9 & 52.6 & 0.4 & $1359^{\circ}$ & 54.6 & 28 & -0.22 \\
\hline 85 & 244 & 76 & 26 & 23.9 & 52.6 & $0 . \overline{42}$ & 355 & 54.6 & 29 & $-0 . \overline{22}$ \\
\hline $12: 22: 05: 84$ & 245 & 76 & 26 & 23.9 & 52.6 & $0 . \overline{4}$ & 1355 & 54.6 & $\overline{28}$ & -0.24 \\
\hline 88 & 246 & 76 & 26 & 23.9 & 52.6 & 0.43 & 1360 & 54.5 & 28 & -0.24 \\
\hline $7: 87$ & $24 \overline{7}$ & $7 \overline{6}$ & 26 & 23.9 & 52.6 & $0 . \overline{43}$ & 1365 & 54.5 & 28 & 0.03 \\
\hline & 248 & 76 & $26 !$ & 23.9 & 52.6 & 0.42 & & 54.5 & 28 & -0.04 \\
\hline & 249 & 77 & 26 & 23.9 & 52.6 & 0.43 & 1366 & 54.4 & 28 & -0.04 \\
\hline $0: 89$ & 250 & 77 & 26 & 23.9 & 52.6 & 0.42 & $1365^{\circ}$ & 54.4 & 28 & -0.29 \\
\hline $12: 22: 11: 88$ & 251 & 77 & 26 & 23.9 & 52.6 & 0.42 & $1 \overline{3} 63$ & 54.4 & 28 & -0.29 \\
\hline $12: 22: 12: 87$ & 252 & 77 & 26 & 23.9 & $52 . \overline{6}$ & 0.42 & 1358 & 54.5 & 28 & -0.15 \\
\hline $12: 22: 13: 86$ & 253 & 76 & 26 & 23.9 & 52.6 & $0 . \overline{4}$ & 1355 & 54.5 & 28 & -0.15 \\
\hline $4: \overline{90}$ & 254 & 76 & 26 & 23.9 & 52.6 & 0.42 & 1357 & 54.6 & 28 & 0.09 \\
\hline & 255 & 76 & 26 & 23.9 & 52.6 & 0.42 & 13 & 54.7 & 28 & 0.09 \\
\hline $6: 8 \overline{8}$ & 256 & 76 & 26 & 24.1 & 52.6 & $0 . \overline{3}$ & & 54.7 & 28 & -0.2 \\
\hline $7: 92$ & 257 & 76 & 26 & & 52.6 & 0.43 & & 54.7 & 28 & -0.2 \\
\hline & 258 & 76 & 26 & 24 & 52.6 & 0.44 & 1367 & 54.7 & 28 & -0.27 \\
\hline $12: 22: 19: 90$ & 259 & 76 & 26 & 24.1 & 52.6 & 0.43 & 1367 & 54.7 & 28 & -0.27 \\
\hline
\end{tabular}

Page E-72 


\begin{tabular}{|c|c|c|c|c|c|c|c|c|c|c|}
\hline TIME & $\begin{array}{l}\text { Elapsed } \\
\text { Time } \\
\text { (sec) }\end{array}$ & $B I T=T C 1$ & PURG_TC2 & PurgePSI & PurgeCFM & DEPTH(IN) & DwnFrc(ib) & RPM : & $\begin{array}{l}\text { Torque } \\
\text { (FTLB) }\end{array}$ & Inches/Min \\
\hline $12: 22: 20: 89$ & $\quad 260$ & $\quad-76$ & 26 & $\quad 24.1$ & 52.6 & 0.43 & 1367 & 54.8 & 27 & $\quad 0.01$ \\
\hline $12: 22: 21: 88$ & 261 & 76 & 26 & $24.1^{7}$ & 52.6 & 0.42 & 1366 & 54.8 & 28 & 0.01 \\
\hline $12: 22: 22: 92$ & 262 & 76 & $26 j$ & 23.9 & 52.6 & 0.42 & 1362 & 54.8 & 28 & $0 . \overline{04}$ \\
\hline $12: 22: 23: 91$ & 263 & 76 & 26 & 23.9 & 52.6 & 0.42 & 1357 & 54.8 & 28 & 0.04 \\
\hline $12: 22: 24: 90$ & 264 & 76 & 26 & $23.9^{\prime}$ & 52.6 & 0.43 & 1355 & 54.9 & 28 & -0.25 \\
\hline $12: 22: 25: 89$ & 265 & 76 & 26 & 24.1 & 52.6 & 0.43 & 1360 & 54.9 & 28 & -0.25 \\
\hline $12: 22: 26: 88$ & 266 & 76 & $26^{\prime}$ & $24.1^{\dagger}$ & 52.6 & $0.43^{\circ}$ & 1365 & 54.9 & 28 & -0.16 \\
\hline $12: 22: 27: 92$ & 267 & 76 & $26:$ & 24.1 & 52.6 & $0.43^{4}$ & 1362 & 54.8 & 28 & $-0 . \overline{16}$ \\
\hline$|12: 22: 28: 91|$ & 268 & 76 & 26 & 24.1 & 52.6 & $0 . \overline{42}$ & 1367 & 54.9 & 28 & 0.03 \\
\hline $12: 22: 29: 90$ & 269 & 76 & 26 & 24.1 & 52.6 & 0.43 & 1368 & 54.9 & 28 & 0.03 \\
\hline $12: 22: 30: 94$ & 270 & 76 & 26 & 24.1 & 52.6 & $0.42^{\circ}$ & 1367 & 54.9 & 28 & -0.06 \\
\hline $12: 22: 31: 93$ & 271 & 76 & 26 & 24.1 & 52.6 & 0.42 & 1367 & 54.9 & 27 & $-0 . \overline{21}$ \\
\hline $12: 22: 32: 92$ & $2 \overline{7} 2$ & 76 & $26 !$ & 24.1 & 52.6 & 0.42 & 1366 & 54.9 & 27 & -0.21 \\
\hline $12: 22: 33: 96$ & 273 & 76 & 26 & 24.1 & 52.6 & 0.42 & 1362 & 54.8 & 28 & -0.19 \\
\hline $12: 22: 34: 95$ & 274 & 75 & 26 & 23.9 & 52.6 & 0.4 & 1357 & 54.8 & 28 & -0.19 \\
\hline $12: 22: 35: 94$ & 275 & 75 & 26 & 24.1 & 52.6 & 0.42 & 1357 & 54.8 & 28 & 0.1 \\
\hline $12: 22: 36: 93$ & 276 & 75 & 26 & 24.1 & 52.6 & 0.42 & 1356 & 54.9 & 28 & 0.1 \\
\hline $12: 22: 37: 92$ & 277 & 76 & $26^{\prime}$ & 24.3 & 52.6 & 0.43 & 1367 & 54.9 & 27 & -0.18 \\
\hline $12: 22: 38: 96$ & 278 & 76 & $26 !$ & 24.3 & 52.6 & 0.43 & 1365 & 54.8 & 27 & -0.18 \\
\hline $12: 22: 39: 95$ & 279 & $7 \overline{6}$ & 26 & 24.3 & 52.6 & 0.42 & 1368 & 54.8 & 27 & -0.27 \\
\hline $12: 22: 40: 94$ & 280 & 76 & 26 & 24.3 & 52.6 & 0.43 & 1368 & 54.8 & 28 & -0.27 \\
\hline $12: 22: 41: 93$ & 281 & 76 & 26 & 24.3 & 52.6 & 0.42 & 1367 & 54.8 & 28 & -0.07 \\
\hline $12: 22: 42: 92$ & 282 & 76 & 26 & 24.3 & 52.6 & 0.4 & 1362 & 54.8 & 28 & -0.07 \\
\hline $12: 22: 43: 96$ & 283 & 76 & 26 & $24.1^{\dagger}$ & 52.5 & 0.42 & 1365 & 54.9 & 28 & 0.04 \\
\hline $12: 22: 44: 95$ & 284 & 75 & 26 & 24.1 & 52.5 & $0.43^{\dagger}$ & 1361 & 54.9 & 28 & 0.04 \\
\hline $12: 22: 45: 94$ & 285 & 76 & 26 & 24.1 & 52.5 & 0.42 & 1357 & 55 & 28 & -0.2 \\
\hline $12: 22: 46: 98$ & 286 & 76 & $26 !$ & 24.1 & 52.5 & 0.43 & 1359 & 55.1 & 28 & -0.2 \\
\hline $12: 22: 47: 97$ & $28 \overline{7}$ & 75 & $26 i^{\circ}$ & 24.1 & 52.4 & 0.43 & 1357 & 55.1 & 28 & -0.23 \\
\hline $12: 22: 48: 96$ & 288 & 76 & 26 & 24.1 & 52.4 & 0.43 & 1368 & 55.1 & 28 & -0.23 \\
\hline $12: 22: 49: 95$ & 289 & 76 & 26 & 24.1 & 52.4 & 0.43 & 1365 & 55.3 & 28 & -0.04 \\
\hline $12: 22: 50: 93$ & $29 \overline{0}$ & 76 & 26 & 24.1! & 52.4 & 0.42 & 1368 & 55.3 & 28 & -0.04 \\
\hline $12: 22: 51: 98$ & 291 & 76 & 26 & 24.1 & 52.4 & 0.42 & 1369 & 55.2 & 28 & 0.02 \\
\hline $12: 22: 52: 97$ & 292 & 76 & 26 & 24.1 & 52.4 & 0.43 & 1367 & 54.9 & 28 & -0.25 \\
\hline $12: 22: 53: 96$ & 293 & 76 & 26 & 24.1 & $52 . \overline{4}$ & 0.42 & 1363 & 54.9 & 28 & -0.25 \\
\hline $12: 22: 55: 00$ & 294 & 75 & 26 & 24.1 & 52.4 & 0.42 & $1365^{+}$ & 54.7 & 28 & -0.21 \\
\hline $12: 22: 55: 99$ & 295 & 75 & 26 & 24.1 & 52.4 & 0.42 & $1360^{\circ}$ & 54.5 & 28 & -0.21 \\
\hline $12: 22: 56: 98$ & 296 & 75 & 26 & 24.1 & 52.4 & 0.4 & $1358^{\circ}$ & 54.5 & 28 & 0.04 \\
\hline $12: 22: 57: 96$ & 297 & 75 & 26 & 24.3 & 52.4 & 0.43 & 1363 & 54.5 & 28 & 0.04 \\
\hline $12: 22: 58: 95$ & 298 & 75 & 26 & 24.3 & 52.4 & 0.43 & 1368 & 54.5 & 27 & -0.09 \\
\hline $12: 23: 00: 00$ & 299 & 75 & 26 & 24.3 & 52.4 & 0.43 & 1371 & 54.5 & 27 & -0.09 \\
\hline 12:23:00:99 & 300 & 75 & 26 & 24.3 & 52.4 & 0.42 & 1369 & 54.7 & 27 & -0.31 \\
\hline $12: 23: 01: 97$ & 301 & 75 & 26 & 24.3 & 52.4 & 0.42 & 1371 & 55.2 & 27 & $-0 . \overline{31}$ \\
\hline $12: 23: 03: 02$ & 302 & 76 & 26 & 24.3 & 52.4 & $0 . \overline{42}$ & 1370 & 55.2 & 27 & -0.16 \\
\hline $12: 23: 04: 01$ & 303 & 76 & 26 & 1.3 & 1.8 & 0.42 & 1368 & 55.4 & 28 & -0.16 \\
\hline $12: 23: 05: 00$ & 304 & 77 & 26 & -0.6 & 1.8 & 0.42 & 1361 & 55.3 & 28 & 0.09 \\
\hline 12:23:05:98 & 305 & 79 & 26 & -0.6 & 1.8 & 0.43 & 1365 & 55.3 & 28 & $\overline{0.09}$ \\
\hline $12: 23: 06: 97$ & 306 & 82 & 26 & -0.8 & 1.8 & 0.43 & 1360 & 55.2 & 29 & -0.14 \\
\hline $12: 23: 08: 02$ & 307 & 83 & 26 & -0.6 & 1.8 & 0.42 & 1359 & 55.1 & 30 & $-0 . \overline{14}$ \\
\hline $12: 23: 09: 00$ & 308 & 85 & 26 & -0.6 & 1.8 & 0.42 & 1360 & 55.1 & 30 & -0.29 \\
\hline $12: 23: 09: 99$ & 309 & 87 & 26 & -0.6 & 1.8 & 0.43 & $1369^{\circ}$ & 55.1 & 31 & -0.29 \\
\hline $12: 23: 10: 98$ & $310^{\dagger}$ & 88 & 26 & -0.6 & 1.8 & 0.43 & 1373 & 55 & 30 & -0.13 \\
\hline $12: 23: 11: 97$ & 311 & 89 & 26 & -0.6 & $1.8 !$ & 0.43 & 1374 & 55 & 31 & -0.13 \\
\hline
\end{tabular}




\begin{tabular}{|c|c|c|c|c|c|c|c|c|c|c|}
\hline TIME & $\begin{array}{l}\text { Elapsed } \\
\text { Time } \\
\text { (sec) }\end{array}$ & & & & PurgeCFM & DEPTH(IN) & DwnFrc(Ib) & RPM & $\begin{array}{l}\text { Torque } \\
\text { (FTLB) }\end{array}$ & Inches/Min \\
\hline $12: 23: 13: 01$ & $\quad 312$ & 91 & -26 & -0.6 & $\quad 1.8$ & 0.43 & $\quad 1373$ & 55 & $\quad 32$ & $2 \quad 0.12$ \\
\hline $12: 23: 14: 00$ & 313 & 92 & 26 & -0.6 & 1.8 & 0.42 & 1372 & 55 & 31 & 0.12 \\
\hline $12: 23: 14: 99$ & 314 & $9 \overline{94}$ & 26 & -0.6 & 1.8 & 0.42 & 1369 & 55 & 31 & -0.25 \\
\hline $12: 23: 15: 98$ & 315 & 95 & 26 & -0.6 & 1.8 & 0.4 & 1365 & 55 & 32 & -0.25 \\
\hline $12: 23: 16: 97$ & $\overline{316}$ & 96 & 26 & -0.6 & 1.8 & $0 . \overline{42}$ & 1363 & 54.8 & 32 & -0.28 \\
\hline $12 \overline{2} 3: 18: 01$ & 317 & 98 & 26 & -0.6 & 1.8 & 0.42 & 1363 & 54.8 & $3 \overline{2}$ & -0.04 \\
\hline $12: 23: 19: 00$ & $318^{\prime}$ & 99 & 26 & -0.6 & 1.8 & 0.42 & 1363 & 54.7 & 32 & -0.04 \\
\hline $12: 23: 19: 99$ & 319 & 100 & 26 & -0.6 & 1.8 & 0.43 & 1370 & 54.6 & 33 & 0 \\
\hline $12: 23: 21: 03$ & 320 & 102 & 26 & -0.6 & 1.8 & 0.43 & 1374 & 54.6 & 33 & 0 \\
\hline $12: 23: 22: 02$ & 321 & 103 & 26 & -0.6 & 1.8 & 0.43 & 1377 & 54.4 & 33 & -0.3 \\
\hline $12: 23: 23: 01$ & 322 & 105 & 26 & -0.6 & 1.8 & 0.43 & 1375 & 54.4 & 33 & -0.3 \\
\hline $12: 23: 24: 05$ & 323 & 106 & 26 & -0.6 & 1.8 & $0 . \overline{42}$ & 1377 & 54.4 & 34 & -0.18 \\
\hline $12: 23: 25: 04$ & 324 & 108 & 26 & -0.6 & 1.8 & 0.43 & 1373 & 54.4 & 34 & $-0.1 \overline{8}$ \\
\hline $12: 23: 26: 03$ & 325 & 108 & 26 & -0.6 & 1.8 & 0.42 & 1368 & 54.4 & 34 & 0.09 \\
\hline $12: 23: 27: 02$ & 326 & 109 & 26 & -0.6 & 1.8 & $0 . \overline{42}$ & 1365 & 54.4 & 34 & 0.09 \\
\hline $12: 23: 28: 01$ & 327 & 111 & 26 & -0.6 & 1.8 & 0.43 & 1368 & 54.4 & 34 & -0.2 \\
\hline $12: 23: 29: 05$ & 328 & $1 \overline{11}$ & 26 & -0.6 & 1.8 & 0.43 & 1366 & 54.4 & 35 & -0.2 \\
\hline $12: 23: 30: 04$ & 329 & 114 & 26 & -0.6 & 1.8 & 0.43 & 1371 & 54.4 & 34 & -0.33 \\
\hline $12: 23$ & 330 & 115 & 26 & -0.6 & 1.8 & 0.43 & 1377 & 54.4 & 35 & $-0.3 \overline{3}$ \\
\hline $12: 23$ & 331 & 116 & 26 & -0.6 & 1.8 & 0.44 & 1378 & 54.2 & 35 & -0.07 \\
\hline $12: 23: 33: 06$ & 332 & 118 & 26 & -0.6 & $1.8^{i}$ & 0.42 & 1377 & 54.2 & 34 & -0.07 \\
\hline $12: 23: 34: 05$ & 333 ! & 119 & 26 & -0.6 & 1.8 & 0.42 & 1373 & 54.3 & 34 & 0 \\
\hline $12: 23: 35: 04$ & 334 & 121 & 26 & $-0.6 !$ & 1.8 & 0.42 & 1375 & 54.3 & 35 & 0 \\
\hline $12: 23: 36: 03$ & 335 & 121 & 26 & -0.6 & 1.8 & 0.42 & 1370 & 54.3 & 35 & -0.32 \\
\hline $12: 23: 37: 07$ & 336 & 122 & 26 & -0.6 & 1.8 & 0.42 & 1367 & 54.2 & 35 & -0.32 \\
\hline $12: 23$ & 337 & 124 & 26 & -0.6 & 1.8 & 0.43 & $13 \overline{7} 1$ & 54.1 & 35 & -0.17 \\
\hline $12: 23: 39: 05$ & 338 & 125 & 26 & -0.6 & 1.8 & 0.43 & 1377 & 54.1 & 35 & -0.17 \\
\hline $12: 23: 40: 04$ & 339 & 126 & 26 & -0.6 & 1.8 & 0.42 & 1374 & 54.2 & 35 & 0.09 \\
\hline $12: 23: 41: 03$ & 340 & 127 & 26 & -0.6 & 1.8 & 0.43 & 1379 & 54.2 & 35 & $-0.2 \overline{7}$ \\
\hline $12: 23: 42: 07$ & 341 & 129 & 26 & -0.6 & 1.8 & $0 . \overline{43}$ & 1380 & 54.2 & 35 & -0.21 \\
\hline $12: 23: 4$ & 3427 & 130 & 26 & -0.6 & 1.8 & 0.42 & 1377 & 54.2 & 35 & -0.28 \\
\hline $12: 23: 44: 05$ & 343 & 131 & 26 & -0.6 & 1.8 & 0.43 & 1373 & 54.2 & 35 & -0.28 \\
\hline $12: 23: 45: 09$ & 344 & 132 & 26 & -0.6 & 1.8 & $0 . \overline{42}$ & 1368 & 54.2 & 35 & 0 \\
\hline $12: 23: 46: 08$ & 345 & 134 & 26 & -0.6 & 1.8 & 0.42 & 13 & 54.1 & 35 & 0 \\
\hline $12: 2$ & 346 & 135 & 26 & -0.6 & 1.8 & 0.43 & 1368 & 54.1 & 36 & -0.03 \\
\hline $12: 23: 48: 06$ & 347 & $\overline{136}$ & 26 & -0.6 & 1.8 & 0.43 & 1373 & 54.1 & 36 & -0.03 \\
\hline $12: 23: \overline{49: 05}$ & 348 & 137 & 26 & -0.6 & 1.8 & 0.43 & 1378 & 54 & 36 & -0.32 \\
\hline $12: 23: 50: 09$ & 349 & 138 & 26 & -0.6 & 1.8 & 0.42 & 1380 & 53.9 & 36 & -0.32 \\
\hline $12: 23: 51: 08$ & 350 & 140 & 26 & -0.6 & 1.8 & 0.42 & 1381 & 53.9 & 36 & -0.16 \\
\hline $12: 23: 52: 07$ & 351 & 141 & 26 & -0.6 & 1.8 & $0 . \overline{42}$ & 1375 & 53.8 & 36 & -0.16 \\
\hline $12: 23$ & 352 & 142 & 26 & -0.6 & 1.8 & 0.42 & 1377 & 53.9 & 36 & 0.06 \\
\hline $12: 2$ & 353 & 144 & 26 & -0.6 & 1.8 & 0.42 & 1372 & 53.9 & 36 & 0.06 \\
\hline $55: 09$ & 354 & 144 & 26 & -0.6 & 1.8 & $0 . \overline{43}$ & 1369 & 54.1 & 36 & -0.24 \\
\hline $12: 23: 56: 08$ & 355 & 145 & 26 & -0.6 & 1.8 & 0.42 & 1372 & 54.4 & 36 & -0.24 \\
\hline $12: 23: 57: 06$ & 356 & 146 & 26 & -0.6 & $1.8 !$ & 0.43 & 1378 & 54.4 & 37 & -0.22 \\
\hline $12: 23: 58: 11$ & 357 & 147 & 26 & -0.6 & 1.8 & 0.43 & 1375 & 54.5 & 37 & -0.22 \\
\hline $12: 23: 59: 10$ & 358 & $\overline{14} 8$ & 26 & -0.6 & 1.8 & 0.43 & 1380 & 54.5 & 37 & 0 \\
\hline $12: 24: 00: 09$ & 359 & 150 & 26 & -0.6 & 1.8 & 0.43 & 1382 & 54.5 & 36 & 0 \\
\hline $12: 24: 01: 13$ & 360 & 151 & 26 & -0.6 & 1.8 & 0.42 & 1380 & 54.6 & 36 & -0.04 \\
\hline $12: 24: 02: 12$ & 361 & 152 & 26 & -0.6 & 1.8 & $0 . \overline{43}$ & 1382 & 54.7 & 36 & -0.28 \\
\hline $12: 24: 03: 11$ & 362 & 153 & 26 & -0.6 & $1 . \overline{8}$ & 0.42 & 1372 & 54.7 & 36 & -0.28 \\
\hline $12: 24: \overline{04: 09}$ & 363 & 155 & 26 & -0.6 & 1.8 & 0.42 & 1375 & 54.8 & 36 & -0.14 \\
\hline
\end{tabular}




\begin{tabular}{|c|c|c|c|c|c|c|c|c|c|c|}
\hline TIME & $\begin{array}{l}\text { Elapsed } \\
\text { Time } \\
\text { (sec) }\end{array}$ & BIT_TC1 & PURG_TC2 & PurgePSI & PurgeCFM & DEPTH(IN) & DwnFrc(lb) & RPM & $\begin{array}{l}\text { Torque } \\
\text { (FTLB) }\end{array}$ & Inches/Min \\
\hline $12: 24: 05: 08$ & $\quad 364$ & 155 & 26 & -0.6 & $\quad 18$ & 0.43 & $\quad 1370$ & 54.8 & $\quad 36$ & -0.14 \\
\hline $12: 24: 06: 07$ & 365 & 156 & 26 & -0.6 & 1.8 & 0.43 & 1372 & 54.8 & 36 & 0.08 \\
\hline $12: 24: 07: 06$ & 366 & 157 & 26 & -0.6 & 1.8 & 0.44 & 1375 & 54.8 & 37 & 0.08 \\
\hline $8: 10$ & 367 & 158 & 26 & -0.6 & 1.8 & $0.4 \overrightarrow{3}$ & 1381 & 54.9 & 37 & -0.17 \\
\hline $12: 24: 09: 09$ & 368 & $15 \overline{9}$ & 26 & -0.6 & 1.8 & 0.43 & 1378 & $54 . \overline{9}$ & 37 & -0.17 \\
\hline $12: 24: 10: 08$ & 369 & 160 & 26 & -0.6 & 1.8 & 0.421 & 1382 & 55 & 37 & -0.3 \\
\hline $12: 24: 11: 13$ & 370 & 162 & 26 & -0.6 & 1.8 & 0.42 & 1383 & 54.9 & 37 & -0.3 \\
\hline $12: 2$ & 371 & 163 & 26 & -0.6 & 1.8 & 0.43 & 1381 & 54.9 & 37 & -0.09 \\
\hline $12: 2$ & 372 & $16 \overline{4}$ & 26 & -0.6 & $1.8{ }^{1}$ & $0 . \overline{42}$ & 1377 & $54 . \overline{8}$ & 37 & -0.09 \\
\hline 15 & 373 & 165 & 26 & -0.6 & 1.8 & 0.42 & 1373 & 54.8 & 37 & 0.09 \\
\hline $5: 14$ & 374 & 165 & 26 & -0.6 & 1.8 & 0.42 & 1375 & 54.8 & 37 & 0.09 \\
\hline$: 12$ & 375 & 166 & 26 & $-0.6^{\dagger}$ & 1.8 & 0.42 & 1373 & 54.8 & 37 & -0.27 \\
\hline :11 & $3 \overline{76}$ & 167 & 26 & -0.6 & 1.8 & 0.43 & 1375 & 54.7 & 38 & -0.27 \\
\hline & $37 \overline{7}$ & 168 & 26 & -0.6 & $1.8 !$ & 0.44 & 1380 & 54.7 & 38 & -0.24 \\
\hline & 378 & $\overline{169}$ & 26 & -0.6 & 1.8 & 0.43 & 1384 & 54.7 & 38 & -0.24 \\
\hline $12: 2$ & 379 & 170 & 26 & $-0 . \overline{6}$ & 1.8 & 0.43 & 1384 & 54.5 & 38 & 0.01 \\
\hline $12: 2$ & 380 & 171 & 26 & -0.6 & 1.8 & 0.42 & 1385 & 54.5 & 37 & 0.01 \\
\hline 17 & 381 & $1 \overline{72}$ & 26 & -0.6 & 1.8 & 0.42 & 84 & 54.5 & 37 & -0.05 \\
\hline & 382 & 173 & 26 & -0.6 & 1.8 & $0.43 !$ & 1380 & 54.6 & 37 & -0.05 \\
\hline & 383 & 174 & 26 & -0.6 & 1.8 & 0.42 & 1376 & 54.6 & 37 & -0.27 \\
\hline $12: 2$ & 384 & 174 & 26 & -0.6 & 1.8 & 0.42 & 1374 & 54.7 & 37 & -0.17 \\
\hline $26: 12$ & 385 & 175 & 26 & -0.6 & $1.8 !$ & 0.43 & 1377 & 54.7 & 37 & -0.17 \\
\hline 16 & 386 & 176 & 26 & -0.6 & 1.8 & $\overline{0.43}$ & 1375 & 54.7 & 37 & 0.12 \\
\hline & 387 & 177 & $2 \overline{6}$ & -0.6 & 1.8 & 0.43 & 1380 & 54.7 & 38 & 0.12 \\
\hline & 388 & 178 & 26 & -0.6 & 1.8 & 0.43 & 1384 & 54.7 & 38 & -0.19 \\
\hline & 389 & 179 & 26 & -0.6 & 1.8 & 0.42 & 1386 & 54.7 & 38 & -0.19 \\
\hline & 390 & 180 & 26 & -0.6 & 1.8 & 0.43 & 1385 & 54.7 & 38 & -0.3 \\
\hline $12: 2$ & 391 & 181 & 26 & -0.6 & 1.8 & 0.42 & 1380 & 54.7 & 38 & -0.3 \\
\hline $12:$ & 392 & 182 & 26 & -0.6 & 1.8 & 0.42 & 1382 & 54.7 & 37 & -0.06 \\
\hline & 393 & 183 & $26 !$ & -0.6 & 1.8 & 0.42 & 1378 & 54.5 & 38 & -0.06 \\
\hline 18 & 394 & $18 \overline{3}$ & 26 & -0.6 & 1.8 & 0.43 & 1375 & 54.6 & 38 & 0.01 \\
\hline & 395 & 184 & 26 & -0.6 & 1.8 & 0.42 & 1376 & $5 \overline{4.6}$ & 39 & 0.01 \\
\hline 16 & 396 & $1 \overline{85}$ & $26 !$ & -0.6 & 1.8 & 0.43 & 1381 & 54.7 & 39 & -0.25 \\
\hline & 397 & 186 & 26 & -0.6 & 1.8 & $0.43^{\dagger}$ & 1378 & 54.7 & 39 & -0.25 \\
\hline & 398 & $1 \overline{8} \overline{6}$ & 26 & -0.6 & 1.8 & & & 54.7 & 39 & -0.18 \\
\hline & 399 & 187 & 26 & -0.6 & 1.8 & 0.43 & 1387 & 54.4 & 38 & -0.15 \\
\hline & 400 & 188 & 26 & -0.6 & 1.8 & 0.4 & 1386 & 54.3 & 38 & 0.01 \\
\hline $2: 16$ & 401 & 190 & 26 & -0.6 & 1.8 & 0.42 & 1383 & 54.3 & 38 & 0.01 \\
\hline 20 & $\overline{402}$ & 190 & $26 !$ & -0.6 & $1.8 !$ & 0.43 & 1378 & 54.4 & 38 & -0.12 \\
\hline $4: 19$ & 403 & 191 & 26 & -0.6 & 1.8 & $0 . \overline{43}$ & 1380 & 54.4 & 38 & -0.12 \\
\hline $5: 18$ & 404 & 192 & 26 & -0.6 & 1.8 & & & 54.4 & 38 & -0.33 \\
\hline $6: 17$ & 405 & 192 & 26 & -0.6 & 1.8 & & & 54.4 & 38 & -0.33 \\
\hline & 406 & 193 & 26 & -0.6 & 1.8 & 0.4 & 1382 & 54.3 & 39 & $-0.0 \mathrm{~s}$ \\
\hline & 407 & 194 & 26 & -0.6 & 1.8 & 0.4 & 1387 & 54.3 & 39 & -0.09 \\
\hline $9: 19$ & 408 & 195 & 26 & -0.6 & 1.8 & 0.43 & 1385 & 54.4 & 38 & 0.07 \\
\hline $50: 18$ & 409 & 196 & 26 & -0.6 & 1.8 & 0.43 & 1387 & 54.4 & 38 & -0.29 \\
\hline $12: 24: 51: 22$ & 410 & 197 & 26 & -0.6 & 1.8 & 0.42 & 1387 & $54 . \overline{4}$ & 38 & -0.29 \\
\hline $12: 24: 52: 21$ & 411 & 198 & 26 & -0.6 & 1.8 & 0.43 & 1384 & 54.5 & 38 & -0.21 \\
\hline $12: 24: 53: 20$ & 412 & 199 & 26 & -0.6 & 1.8 & 0.42 & 1379 & 54.5 & 38 & $-0 . \overline{21}$ \\
\hline $4: 19$ & 413 & 199 & 26 & -0.6 & 1.8 & 0.42 & 1377 & 54.5 & 39 & 0.06 \\
\hline & 414 & 200 & 26 & -0.6 & 1.8 & 0.42 & 1380 & 54.5 & 40 & 0.06 \\
\hline $12: 24: 56: 16$ & 415 & 201 & 26 & -0.6 & 1.8 & $0 . \overline{42}$ & 1378 & 54.4 & 39 & -0.14 \\
\hline
\end{tabular}




\begin{tabular}{|c|c|c|c|c|c|c|c|c|c|c|}
\hline TIME & $\begin{array}{l}\text { Elapsed } \\
\text { Time } \\
\text { (sec) }\end{array}$ & BIT_TC1 & PURG_TC2 & & PurgeCFM & DEPTH(IN) & & & $\begin{array}{l}\text { 'Torque } \\
\text { (FTLB) }\end{array}$ & Inches/Min \\
\hline $12: 24: 57: 15$ & 416 & 202 & 26 & -0.6 & 1.8 & 0.43 & $\quad 1382$ & 54.4 & $\quad 39$ & $\quad-0.14$ \\
\hline $12: 24: 58: 20$ & 417 & 203 & 26 & -0.6 & 1.8 & 0.42 & 1387 & 54.4 & 40 & -0.3 \\
\hline $12: 24: 59: 24$ & 418 & 204 & 26 & -0.6 & $1.8^{r}$ & 0.43 & 1388 & 54.5 & 40 & -0.3 \\
\hline $12: 25: 00: 23$ & 419 & 205 & 26 & -0.6 & $1.8 !$ & 0.43 & 1388 & 54.5 & 40 & -0.12 \\
\hline $12: 25: 01: 22$ & 420 & 206 & 26 & -0.6 & 1.8 & $0.43^{3}$ & 1387 & 54.7 & 40 & -0.12 \\
\hline $12: 25: 02: 21$ & 421 & 207 & 26 & -0.6 & 1.8 & 0.42 & 1385 & 54.7 & 40 & 0.02 \\
\hline $12: 25: 03: 19$ & 422 & 207 & 26 & -0.6 & 1.8 & 0.42 & 1381 & 54.7 & 40 & 0.02 \\
\hline $12: 25: 04: 24$ & 423 & 208 & 26 & -0.6 & 1.8 & $0 . \overline{4}$ & 1377 & 54.8 & $4 \overline{0}$ & $\overline{-0 . \overline{2}}$ \\
\hline $5: 23$ & 424 & 208 & 26 & -0.6 & 1.8 & 0.42 & 1378 & 54.8 & $40^{\circ}$ & -0.2 \\
\hline $5: 06: 22$ & 425 & 209 & 26 & -0.6 & 1.8 & 0.42 & $138 \overline{3}$ & 54.8 & 40 & -0.26 \\
\hline $12: 25: 07: 20$ & 426 & 210 & 26 & -0.6 & 1.8 & 0.43 & 1381 & 54.8 & $41 \mathrm{i}$ & -0.26 \\
\hline $12: 25: 08: 19$ & 427 & 211 & 26 & -0.6 & 1.8 & $0 . \overline{4} 4$ & 1385 & 54.6 & 41 & -0.02 \\
\hline $12: 25: 09: 24$ & 428 & 212 & 26 & -0.6 & 1.8 & 0.43 & 1389 & 54.6 & 39 & -0.02 \\
\hline $0: 23$ & 429 & 213 & 26 & -0.6 & $1.8^{\dagger}$ & 0.42 & 1388 & 54.7 & $3 \overline{9}$ & 0 \\
\hline $12: 2$ & 430 & 213 & 26 & -0.6 & 1.8 & $0 . \overline{42}$ & 1386 & 54.7 & 39 & 0 \\
\hline $12: 25: 12: 26$ & 431 & 214 & 26 & -0.6 & 1.8 & 0.42 & 1388 & 54.7 & 40 & -0.3 \\
\hline $12: 25: 13: 25$ & 432 & 215 & 26 & -0.6 & 1.8 & $0.42^{\dagger}$ & 1385 & 54.5 & 40 & -0.21 \\
\hline $12: 25: 14: 23$ & 433 & $21 \overline{5}$ & 26 & -0.6 & 1.8 & 0.42 & 1379 & 54.5 & 40 & $-0 . \overline{21}$ \\
\hline $12: 25: 15: 22$ & 434 & 216 & 26 & -0.6 & 1.8 & 0.43 & 1378 & 54.5 & 40 & 0.11 \\
\hline $6: 21$ & 435 & 217 & 26 & -0.6 & 1.8 & 0.44 & 1383 & 54.5 & 41 & 0.11 \\
\hline 26 & 436 & $217^{\dagger}$ & 26 & -0.6 & 1.8 & & 1388 & 54.4 & 41 & -0.15 \\
\hline $12: 25: 18: 24$ & 437 & 218 & 26 & -0.6 & 1.8 & $0.43^{+}$ & 1386 & 54.4 & 41 & -0.15 \\
\hline 23 & 438 & 219 & 26 & $-0.6 !$ & 1.8 & 0.43 & 1389 & 54.3 & 41 & -0.3 \\
\hline $0: 28$ & 439 & 220 & 26 & -0.6 & 1.8 & $0 . \overline{4} 3$ & 1390 & 54.3 & 41 & $-0 . \overline{3}$ \\
\hline$: 27$ & $4 \overline{40}$ & $2 \overline{21}$ & 26 & -0.6 & 1.8 & 0.43 & 1387 & 54.3 & 41 & -0.07 \\
\hline 25 & 441 & $2 \overline{2} 2$ & 26 & -0.6 & 1.8 & 2 & 1383 & 54.5 & 41 & $-0 . \overline{07}$ \\
\hline $12: 25: 2$ & 442 & 223 & 26 & $-0 . \overline{6}$ & 1.8 & 0.42 & 1387 & 54.7 & 41 & 0.02 \\
\hline $4: 23$ & $4 \overline{43}$ & $2 \overline{23}$ & 26 & -0.6 & 1.8 & $0 . \overline{42}$ & 1380 & 54.7 & $\overline{42}$ & 0.02 \\
\hline $5: 27$ & 444 & 224 & 26 & -0.6 & 1.8 & $0.43^{\prime}$ & 1379 & 54.7 & 42 & -0.25 \\
\hline $6: 26$ & 445 & 224 & 26 & -0.6 & 1.8 & & 1383 & $5 \overline{4} .8$ & $\overline{42}$ & $-0.2 \overline{5}$ \\
\hline $7: 25$ & 446 & 225 & 26 & -0.6 & 1.8 & 0.43 & 1387 & 54.8 & 42 & -0.23 \\
\hline 8:30 & 447 & 226 & 26 & -0.6 & 1.8 & 0.43 & 1391 & 54.8 & 42 & -0.23 \\
\hline $12: 25: 29: 28$ & 448 & 227 & $2 \overline{6}$ & -0.6 & 1.8 & 0.43 & 1389 & 54.7 & 42 & 0.06 \\
\hline $0: 27$ & 449 & 228 & 26 & -0.6 & 1.8 & 0.43 & 1392 & 54.7 & 41 & 0.06 \\
\hline $1: 26$ & 450 & 229 & 26 & -0.6 & 1.8 & 0.42 & 1390 & 54.7 & $4 \overline{2}$ & -0.07 \\
\hline & 451 & 230 & 26 & -0.6 & 1.8 & 0.4 & 1386 & 54.6 & 42 & -0.07 \\
\hline $3: 29$ & 452 & 230 & 26 & $-0.6 !$ & 1.8 & 0.42 & 1381 & 54.6 & 42 & -0.31 \\
\hline $1: 28$ & 453 & $23 \overrightarrow{1}$ & 26 & -0.6 & 1.8 & $0 . \overline{4}$ & 1383 & 54.5 & 42 & -0.12 \\
\hline $5: 27$ & 454 & 231 & 26 & -0.6 & 1.8 & 0.43 & 1383 & 54.6 & $4 \overline{2}$ & -0.12 \\
\hline $6: 26$ & 455 & 232 & 26 & -0.6 & 1.8 & $0.4 \overline{2}$ & 1382 & 54.6 & 42 & 0.08 \\
\hline $12: 25: 37: 25$ & 456 & 233 & 26 & -0.6 & 1.8 & 0.43 & 1386 & 54.6 & 42 & 0.08 \\
\hline $12: 25: 38: 29$ & 457 & 234 & 26 & -0.6 & 1.8 & 0. & 1390 & 54.5 & 42 & -0.19 \\
\hline$: 28$ & 458 & 234 & 26 & -0.6 & 1.8 & 0. & 1392 & 54.5 & 42 & -0.19 \\
\hline $40: 2 \overline{7}$ & 459 & 235 & 26 & -0.6 & 1.8 & 0.42 & 1389 & 54.5 & 42 & -0.26 \\
\hline $1: 31$ & 460 & 237 & 26 & -0.6 & 1.8 & 0.42 & 1392 & 54.6 & 43 & -0.26 \\
\hline $12: 25: 42: 30$ & 461 & 237 & 26 & -0.6 & 1.8 & 0.42 & 1388 & 54.6 & 42 & -0.05 \\
\hline $12: 25: 43: 29$ & $4 \overline{62}$ & 238 & 26 & -0.6 & 1.8 & 0.42 & $1385^{\prime}$ & 55 & 42 & -0.05 \\
\hline $12: 25: 44: 28$ & 463 & 239 & 26 & -0.6 & 1.8 & 0.42 & 1381 & $55.1^{\top}$ & 44 & 0.01 \\
\hline $12: 25: 45: 27$ & 464 & $23 \overline{9}$ & 26 & -0.6 & 1.8 & 0.42 & 1382 & 55.1 & 44 & 0.01 \\
\hline $12: 25: 46: 26$ & 465 & 240 & 26 & -0.6 & 1.8 & 0.43 & 1386 & 55.1 & 44 & -0.25 \\
\hline & 466 & 240 & 26 & -0.6 & 1.8 & 0.43 & 1383 & 55.2 & 44 & -0.25 \\
\hline $12: 25: 4 \overline{8}: 29$ & $46 \overline{7}$ & 241 & 26 & -0.6 & 1.8 & 0.42 & 1387 & 55.2 & 45 & $-0.2 \overline{3}$ \\
\hline
\end{tabular}




\begin{tabular}{|c|c|c|c|c|c|c|c|c|c|c|}
\hline TIME & $\begin{array}{l}\text { Elapsed } \\
\text { Time } \\
\text { (sec) }\end{array}$ & BIT_TC1 & PURG_TC2 & & PurgeCFM & DEPTH $(I N)$ & DwnFrc(lby) & RPM & $\begin{array}{l}\text { Torque } \\
\text { (FTLB) }\end{array}$ & Inches/Min \\
\hline $12: 25: 49: 33$ & 468 & 242 & 26 & -0.6 & 1.8 & 0.43 & 1391 & 55.3 & 45 & $\quad-0.23$ \\
\hline $12: 25: 50: 32$ & 469 & 243 & 26 & -0.6 & 1.8 & 0.43 & 1393 & 55.3 & 45 & 0 \\
\hline $12: 25: 51: 31$ & 470 & 244 & 26 & -0.6 & 1.8 & 0.42 . & 1391 & 55.3 & 43 & 0 \\
\hline $12: 25: 52: 30$ & 471 & 245 & 26 & -0.6 & $1.8 !$ & 0.43 & $1393^{\circ}$ & 55.2 & 43 & 0.01 \\
\hline $12: 25: 53: 29$ & 472 & 246 & $26^{i}$ & -0.6 & 1.8 & 0.42 & 1390 & 55.1 & 44 & $0 . \overline{01}$ \\
\hline $12: 25: 54: 33$ & 473 & 246 & 26 & -0.6 & $1.8^{\dagger}$ & 0.43 & 1385 & 55.1 & $\overline{44}$ & -0.25 \\
\hline $12: 25: 55: 32$ & 474 & 247 & 26 & -0.6 & 1.8 & 0.42 & 1382 & 54.8 & 44 & $-0 . \overline{25}$ \\
\hline $12: 25: 56: 31$ & 475 & 247 & 26 & -0.6 & 1.8 & 0.42 & 1383 & 54.8 & 44 & $-0.2 \overline{3}$ \\
\hline $12: 25: 57: 35$ & 476 & 248 & 26 & -0.6 & 1.8 & 0.42 & 1388 & 54.8 & 44 & 0.08 \\
\hline $12: 25: 58: 34$ & 477 & 248 & 26 & -0.6 & 1.8 & 0.43 & 1385 & 54.8 & 44 & $0.0 \overline{8}$ \\
\hline $12: 25: 59: 33$ & 478 & 249 & 26 & -0.6 & 1.8 & 0.43 & 1390 & 54.8 & 44 & -0.07 \\
\hline $12: 26: 00: 32$ & 479 & 250 & $26 !$ & -0.6 & 18 & 0.42 & 1393 & 54.8 & $4 \overline{4}^{\dagger}$ & -0.07 \\
\hline $12: 26: 01: 31$ & 480 & 250 & 26 & -0.6 & 1.8 & $0 . \overline{42}$ & $1393^{\prime}$ & 54.8 & 45 & $-0 . \overline{3}$ \\
\hline $12: 26: 02: 35$ & 481 & 252 & 26 & -0.6 & $1.8^{\dagger}$ & 0.42 & 1390 & 54.7 & 44 & -0.3 \\
\hline $12: 26: 03: 34$ & 482 & 253 & 26 & -0.6 & 1.8 & 0.42 & 1393 & 54.7 & 44 & -0.06 \\
\hline $12: 26: 04: 33$ & 483 & 253 & 26 & -0.6 & 1.8 & 0.42 & 1388 & 54.6 & $43\}$ & -0.06 \\
\hline $12: 26: 05: 32$ & 484 & 254 & 26 & -0.6 & 1.8 & 0.42 & 1384 & 54.5 & $45 !$ & 0.04 \\
\hline $12: 26: 06: 30$ & 485 & 254 & 26 & -0.6 & 1.8 & 0.43 & 1383 & 54.5 & 45 & 0.04 \\
\hline $12: 26: 07: 35$ & $486 !$ & 255 & 26 & -0.6 & $1.8 !$ & 0.43 & $1387 !$ & 54.5 & 46 & -0.28 \\
\hline $12: 26: 08: 34$ & 487 & 255 & 26 & -0.6 & 1.8 & 0.43 & 1392 & 54.5 & 46 & -0.28 \\
\hline $12: 26: 09: 33$ & 488 & 256 & 26 j & -0.6 & 1.8 & 0.44 & 1395 & 54.5 & $4 \overline{5}$ & -0.27 \\
\hline $12: 26: 10: 37$ & 489 & 257 & 26 & -0.6 & 1.8 & $0.43^{-1}$ & 1393 & 54.6 & $45 i$ & $-0.2 \overline{7}$ \\
\hline $12: 26: 11: 36$ & $490]$ & 257 & $26 !$ & -0.6 & 1.8 & 0.43 & 1395 & 54.9 & 45 & $0 . \overline{03}$ \\
\hline $12: 26: 12: 35$ & 491 . & 259 & $26 !$ & $-0.6 !$ & 1.8 & 0.42 & 1392 & 54.9 & 45 & 0.03 \\
\hline $12: 26: 13: 33$ & 492 & 259 & 26 & -0.6 & 1.8 & 0.42 & 1388 & 54.8 & $44 !$ & -0.04 \\
\hline $12: 26: 14: 32$ & 493 & 260 & 26 & -0.6 & 1.8 & 0.42 & 1384 & 54.5 & 45 & -0.04 \\
\hline $12: 26: 15: 37$ & 494 & 260 & $26 !$ & -0.6 & $1 . \overline{8}$ & 0.43 & 1386 & 54.5 & 44 & -0.28 \\
\hline $12: 26: 16: 36$ & 495 & 261 & $26 !$ & -0.6 ! & 1.8 & 0.42 & 1384 & 54.5 & 45 & -0.28 \\
\hline $7: 34$ & 496 & 261 & 26 & -0.6 & $1.8^{t}$ & 0.44 & 1386 & 54.4 & 45 & -0.17 \\
\hline $12: 26: 18: 39$ & 497 & 262 & 26 & -0.6 & 1.8 & 0.43 & 1392 & 54.4 & $4 \overline{5}$ & -0.17 \\
\hline $12: 26: 19: 38$ & 498 & 263 & $26 !$ & -0.6 & 1.8 & 0.43 & 1395! & 54.4 & 45 & 0.11 \\
\hline $12: 26: 20: 37$ & 499 & 263 & 26 & -0.8 & 1.81 & 0.43 & 1395 & 54.4 & 45 & -0.23 \\
\hline $12: 26: 21: 35$ & 500 & 264 & 26 & -0.6 & 1.8 & 0.42 & $1395^{\dagger}$ & 54.4 & 44 & -0.23 \\
\hline $12: 26: 22: 34$ & 501 & 265 & $26^{\dagger}$ & -0.6 & $1.8^{\dagger}$ & $0.43^{-1}$ & 1394 & 54.3 & 44 & $-0 . \overline{3}$ \\
\hline $12: 26: 23: 39$ & 502 & 266 & $26 !$ & -0.6 & 1.8 & 0.42 & 1389 & 542 & 44 & -0.3 \\
\hline $12: 26: 24: 37$ & 503 & 266 & 26 & -0.6 & 1.8 & 0.42 & 1385 & 54.1 & 44 & 0 \\
\hline $12: 26: 25: 36$ & 504 & 267 & 26 & -0.6 & 1.8 & 0.43 & $1386^{\dagger}$ & 54.1 & 46 & 0 \\
\hline $12: 26: 26: 41$ & 505 & 267 & 26 & -0.6 & 1.8 & 0.44 & 1385 & 54 & 45 & -0.04 \\
\hline $12: 26: 27: 40$ & 506 & 268 & 26 & $-0.6^{\dagger}$ & 1.8 & 0.43 & 1388 & 54.1 & $4 \overrightarrow{6}$ & -0.04 \\
\hline $12: 26: 28: 38$ & 507 & 268 & 26 & $-0.6 !$ & 1.8 & 0.43 & 1394 & 54.1 & 46 & -0.33 \\
\hline $12: 26: 29: 37$ & 508 & $269^{\circ}$ & 26 & -0.6 & 1.8 & 0.43 & 1396 & 54.1 & 46 & $-0.3 \overline{3}$ \\
\hline $12: 26: 30: 36$ & 509 & 270 & 26 & -0.6 & 1.8 & 0.42 & 1395 & 54.1 & 46 & -0.15 \\
\hline $12: 26: 31: 41$ & 510 & 271 & 26 & -0.6 & 1.8 & 0.42 & 1391. & 54 & 46 & -0.15 \\
\hline $12: 26: 32: 39$ & 511 & 272 & 26 & -0.6 & 1.8 & 0.43 & 130 & 54 & 46 & 0.05 \\
\hline $12: 26: 33: 38$ & 512 & 271 & 26 & -0.6 & 1.8 & 0.4 & 1388 & 54 & 46 & 0.05 \\
\hline $12: 26: 34: 37$ & 513 & 272 & 26 & -0.6 & 1.8 & 0.43 & 1385 & 54 & 46 & -0.27 \\
\hline $12: 26: 35: 36$ & $5 \overline{14}$ & 272 & 26 & $-0 . \overline{6}$ & 1.8 & $0 . \overline{43}$ & 1389 & 54.1 & 47 & -0.27 \\
\hline $12: 26: 36: 35$ & 515 & 273 & $26 !$ & -0.6 & 1.8 & $0 . \overline{44}$ & 1395 & $54.1^{\dagger}$ & 47 & $-0 . \overline{18}$ \\
\hline $12: 26: 37: 34$ & 516 & 274 & 26 & -0.6 & 1.8 & 0.43 & 1398 & 53.9 & 46 & -0.18 \\
\hline $12: 26: 38: 38$ & 517 & 274 & 26 & -0.6 & 1.8 & 0.42 & 1397 & 54 & $4 \overline{6}$ & $0.0 \overline{7}$ \\
\hline $12: 26: 39: 42$ & 518 & 275 & 26 & -0.6 & 1.8 & 0.42 & 1397 & 54.1 & 46 & $0.0 \overline{7}$ \\
\hline $12: 26: 40: 41$ & 519 & 276 & $26 !$ & -0.6 & 15.9 & 0.44 & 1395 & 54.1 & 45 & -0.14 \\
\hline
\end{tabular}




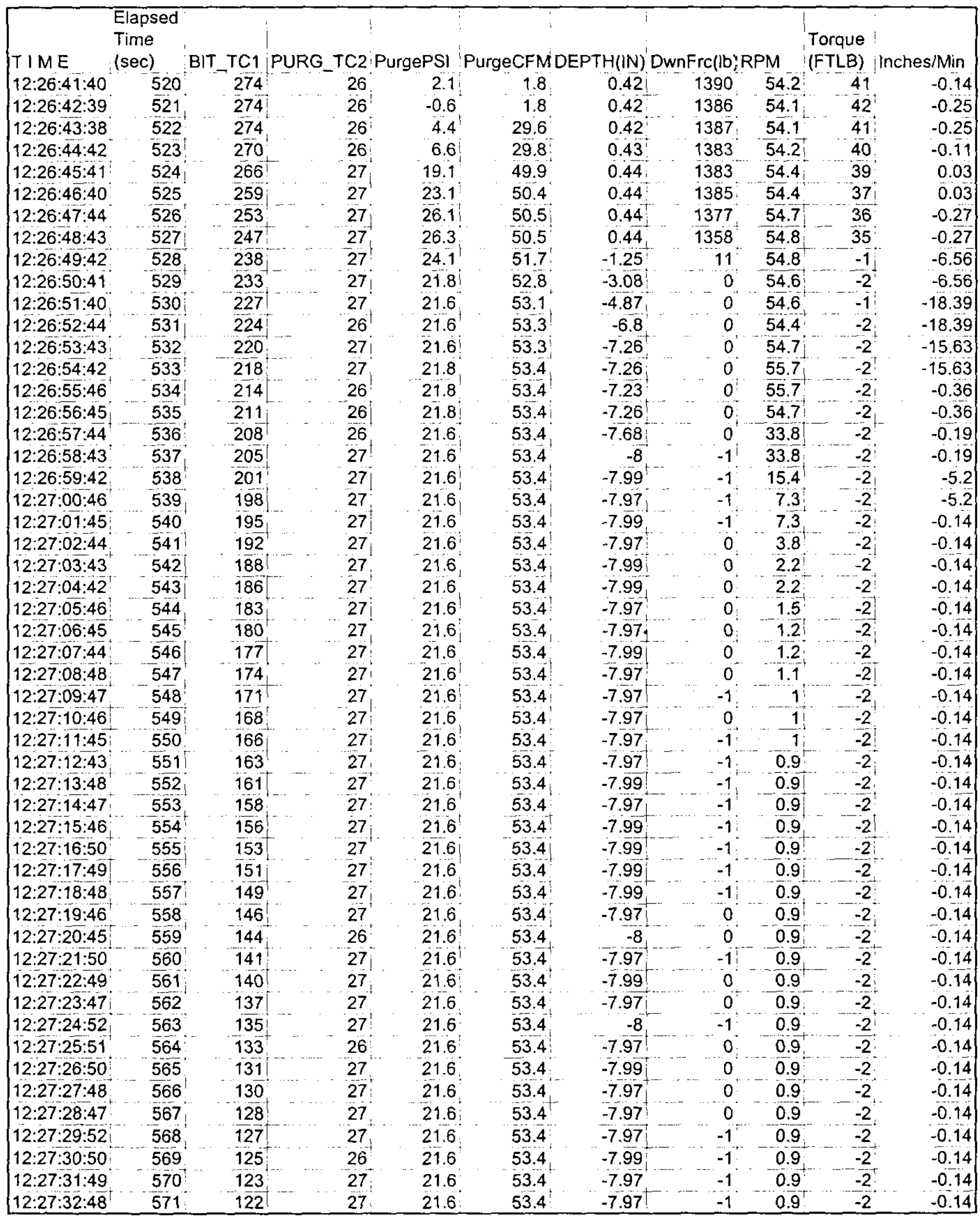




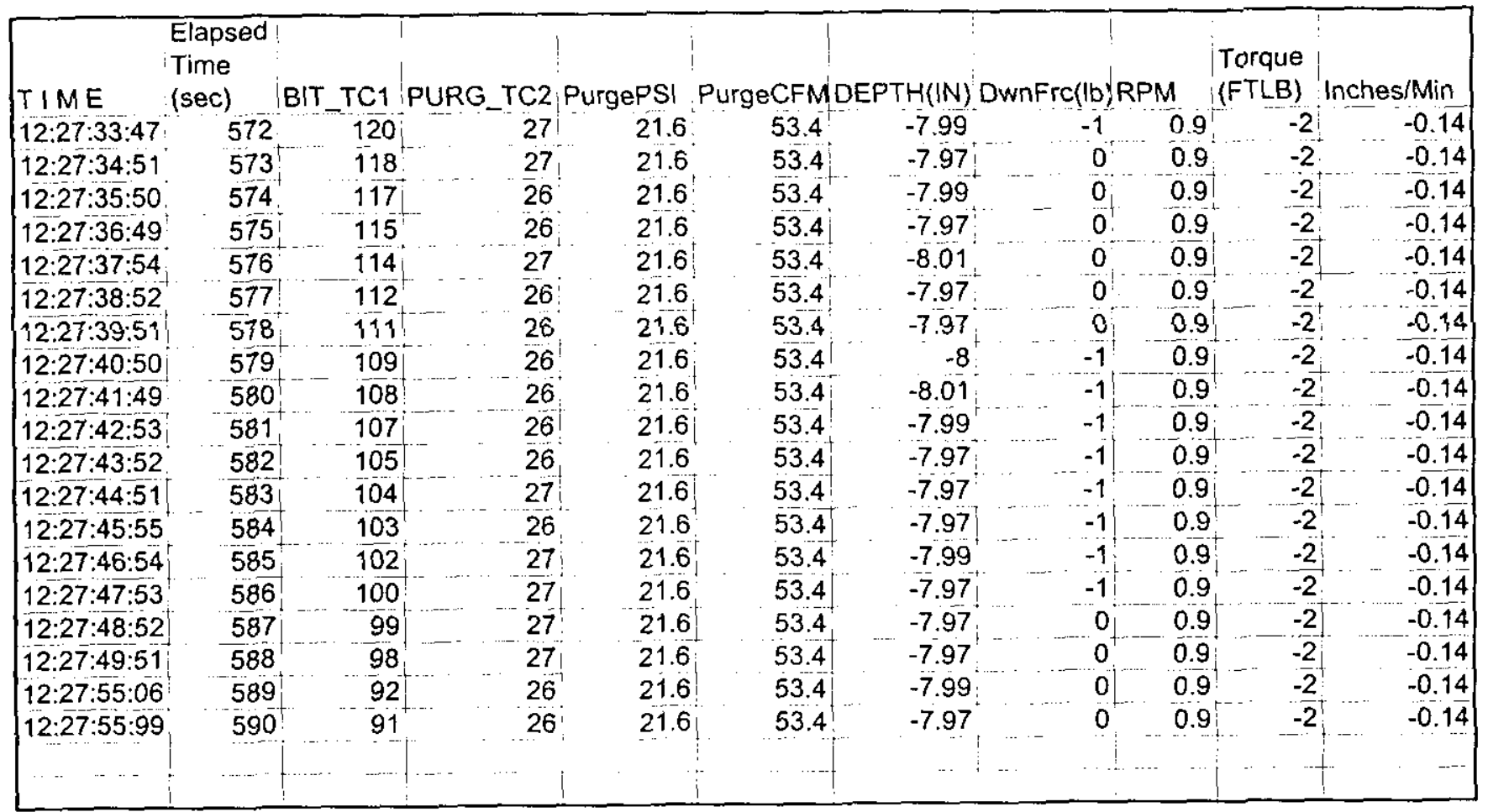




\begin{tabular}{|c|c|c|c|c|c|c|c|c|c|c|}
\hline $\mathrm{T} I \mathrm{ME}$ & \begin{tabular}{|l} 
Elapsed \\
Time \\
(sec)
\end{tabular} & BIT_TC1 & PURG_TC2 & PurgePSI & PurgeCFM & DEPTH $(I N)$ & $D w n F r c(l b)$ & RPM & $\begin{array}{l}\text { |Torque } \\
\text { (FTLB) }\end{array}$ & es/Min \\
\hline $14: 09: 45: 85$ & 0 & -24 & $\quad 24$ & $\quad 212$ & $\quad 54$ & $\quad 2.41$ & -761 & 56.5 & 0 & -0.07 \\
\hline & & 24 & 24 & 21.2 & 54 & 3.04 & .759 & 56.5 & 0 & -0.13 \\
\hline 88 & & 24 & 24 & 21.4 & 54 & 429 & -721 & 56 & 31 & $-0 . \overline{3}$ \\
\hline & 3 & 34 & 24 & 218 & 54 & 4.42 & $428]$ & 56 & 88 & 13.16 \\
\hline & 4 & 36 & $24 i$ & 226 & 54 & 4.45 & 464 & 546 & 77 & 4.27 \\
\hline & 5 & 39 & 24 & 22.8 & 54 & 4.5 & 481 & 543 & 72 & 4.27 \\
\hline & & 45 & 24 & 229 & 54 & 4.54 & 491 & 54.3 & 71 & 1.96 \\
\hline & 7 & 51 & 24 & 22.9 & 54 & $4.5 \overline{5}$ & 503 & 54.2 & 68 & 1.96 \\
\hline & & 54 & 24 & 22.9 & 54 & 4.59 & 617 & 54.2 & $\overline{75}$ & 2.03 \\
\hline & 9 & 58 & 24 & 23.1 & 54 & 464 & 737 & 54.3 & 77 & $2 . \overline{03}$ \\
\hline & 10 & 64 & 24 & 23.1 & 54 & 4.64 & 712 & 54.2 & 78 & 1.6 \\
\hline & 11 & 67 & 24 & 23.5 & 53.9 & 4.68 & 703 & 54.2 & 79 & $1 \overline{6}$ \\
\hline & $-\overline{12}$ & 69 & 24 & 23.7 & 53.8 & 4.68 & $67 \overline{7}$ & 542 & 80 & 0.82 \\
\hline & 13 & 71 & 24 & 23.7 & 53.8 & 4.69 & 666 & 54.1 & 80 & 0.82 \\
\hline & & 74 & 24 & 23.7 & 53.7 & 4.7 & 673 & 54.1 & 82 & 0.54 \\
\hline & 15 & 76 & 24 & 23.7 & 53.7 & 4.7 & 643 & 54.2 & 80 & .54 \\
\hline & 16 & 80 & 24 & 23.9 & 53.6 & 4.69 & 580 & 54.1 & 78 & 0.29 \\
\hline & 17 & 84 & & 23.9 & 53.6 & 4.69 & 520 & 54.2 & 74 & 0.29 \\
\hline & 18 & 87 & 24 & 24.1 & 53.5 & 4.72 & 528 & 54.2 & 73 & $0 . \overline{6}$ \\
\hline & 19 & 90 & 24 & 24.3 & 53.4 & 4.72 & 533 & 54.3 & 70 & $0.1 \overline{6}$ \\
\hline & 20 & 88 & 24 & 24.7 & 53.4 & 4.72 & $54 \overline{9}$ & 54.3 & 67 & 0.24 \\
\hline & & 87 & 24 ! & 24.7 & 53.3 & 4.73 & 545 & $54.3_{+}^{+}$ & 61 & $0 . \overline{24}$ \\
\hline & 22 & 88 & 24 & 24.7 & 53.2 & 4.74 & 556 & 54.4 & 58 & -0.02 \\
\hline & & 90 & 24 & 24.7 & 53.2 & 4.74 & 600 & 54.6 & 56 & $-0.0 \overline{2}$ \\
\hline & & 91 & 24 & $2 \overline{4} .7$ & 53.1 & 4.74 & 597 & 54.6 & 54 & $0.2 \overline{7}$ \\
\hline & 25 ? & 93 & 24 & 24.9 & 53 & 4.74 & 595 & 54.8 & 51 & 0.27 \\
\hline & 261 & 93 & 24 & $24.9]$ & 53 & 4.73 & 592 & 54.8 & 49 & -0.06 \\
\hline & 27 & 94 & 24 & 24.9 & 53 & 4.73 & 586 & 54.8 & 48 & $-0 . \overline{23}$ \\
\hline & 28 & $94 !$ & $24{ }^{\dagger}$ & 24.9 & 52.9 & 4.73 & 584 & 54.8 & 47 & -0.23 \\
\hline & 29 & 94] & 24 & 24.9 & 52.9 & 4.73 & 578 & 54.8 & 46 & -0.09 \\
\hline & 30 & 94 & 24 & 249 & 52.9 & & & 54.8 & 45 & -0.09 \\
\hline & 31 & 93 & 24 & & & & $57 \overline{2}$ & 54.8 & $45^{\dagger}$ & 0.06 \\
\hline & 32 & 94 & 24 & & 52.8 & 4.74 & 572 & 54.8 & 44 & \\
\hline & 33 & 94 & 24 & & 2.8 & & & 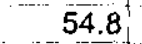 & 44 & -0.07 \\
\hline & 34 & 94 & 24 & & & & & 54.7 & 43 & -0.07 \\
\hline & 35 & 9 & 24 & 25.1 & 52.8 & 4.74 & & 4.6 & 42 & -0.2 \\
\hline & 36 & 9 & 24 & & & & & 4.6 & $4 \overline{2}$ & -0.2 \\
\hline & 37 & 9 & -24 & & & & 575 & 4.6 & 42 & -0.01 \\
\hline & 38 & 9 & 24 & & 2.8 & 4.73 & & 54.6 & 41 & -0.01 \\
\hline & 39 & 92 & 24 & 25.3 & 52.8 & 4.73 & 573 & 54.6 & 41 & -0.02 \\
\hline & 40 & 92 & 24 & & & & 56 & $5 \overline{4} .5$ & 40 & -0.02 \\
\hline & 41 & 92 & 24 & & 52.7 & & & & 40 & $-0 . \overline{19}$ \\
\hline & 42 & 93 & 24 & 5.3 & 2.7 & & & & 40 & -0.19 \\
\hline & 43 & 9 & 24 & & 2.7 & & $570^{\circ}$ & 54.6 & 40 & -0.2 \\
\hline & 44 & 9 & 24 & & & & & 4.7 & 40 & -0.2 \\
\hline & 45 & 92 & $24]$ & & & & & 54.7 & 39 & 0.1 \\
\hline & 46 & 91 & 24 & 25.3 & 52.7 & 4.74 & 575 & 54.8 & 39 & 0.1 \\
\hline & 47 & 91 & 24 & & 52.7 & & 576 & 54.6 & 39 & -0.07 \\
\hline & 48 & 91 & 24 & & & & & & & -0.07 \\
\hline & 49 & 91 & $24 !$ & 25.3 & 52.7 & & & & 399 & -0.24 \\
\hline & 50 & 90 & 24 & 25.3 & 52.6 & 4.75 & 569 & 54.8 & 39 & 0.04 \\
\hline & 51 & 90 & 24 & & 52.6 & 4.14 & 569 & 54.8 & 38 & 0.04 \\
\hline & 52 & 90 & 24 & 25.3 & 52.6 & & & 54.3 & 38 & -0.1 \\
\hline $14: 10: 38: 96$ & 53 & 90 & 24 & 25.3 & 52.6 & 4.75 & $57 \overrightarrow{2}$ & 54.7 & 38 & -0.1 \\
\hline
\end{tabular}




\begin{tabular}{|c|c|c|c|c|c|c|c|c|c|c|}
\hline TIME & $\begin{array}{l}\text { Elapsed } \\
\text { Time } \\
\text { (sec) }\end{array}$ & BIT_TC1 & PURG_TC2 & PurgePSI & PurgeCFM & DEPTH(IN) & DwnFrc(lb) & RPM & $\begin{array}{l}\text { Torque } \\
\text { (FTLB) }\end{array}$ & es/Min \\
\hline $14: 10: 40: 00$ & $-54 i$ & $\quad \overline{9} 1$ & 24 & $\quad 25.3$ & $\quad 52.6$ & $\begin{array}{l}4.75 \\
\end{array}$ & 575 & 54.7 & $\quad 38$ & -0.09 \\
\hline $1: 10: 40: 99$ & 55 & 90 & 24 & 25.3 & 52.6 & 4.74 & 578 & 54.8 & 38 & $-0 . \overline{09}$ \\
\hline $1: \overline{98}$ & 56 & 90 & 24 & 25.3 & 52.6 & 4.74 & 579 & $54.9^{2}$ & $38^{\prime}$ & -0.25 \\
\hline $42: 97$ & 57 & 90 & 24 & 25.5 & 52.6 & 4.75 & 579 & 549 & 38 & -0.25 \\
\hline $14: 10: 43: 96$ & 58 & 90 & 24 & 25.5 & 52.6 & 4.74 & $580^{+}$ & 55 & 37 & -0.02 \\
\hline $5: 00$ & 59 & 89 & 24 & 25.5 & 52.6 & 4.74 & 577 & 55 & 37 & $-0 . \overline{02}$ \\
\hline & 60 & 89 & 24 & 25.5 & 52.6 & $\overline{4} . \overline{74}$ & 572 & 55 & 37 & 0.01 \\
\hline 98 & 61 & 89 & 24 & 25.5 & 52.6 & $4 . \overline{74}$ & 571 & 55 & 37 & 0.01 \\
\hline $3: 02$ & 62 & 89 & 24 & 25.5 & 52.6 & 4.74 & 572 & 55 & 36 & $-0 . \overline{13}$ \\
\hline 01 & 63 & 89 & 24 & 25.7 & 52.6 & 4.74 & 572 & 55 & 37 & -0.13 \\
\hline & 64 & 89 & 24 & 25.7 & 52.6 & 4.74 & 573 & 55 & 36 & -0.17 \\
\hline 99 & 65 & 89 & 24 & 25.5 & 52.6 & $4 . \overline{74}$ & 577 & 54.9 & 37 & -0.17 \\
\hline 98 & 66 & $8 \overline{9}$ & 24 & 25.5 & 52.6 & 4.74 & 581 & $54 . \overline{9}$ & 37 & 0.03 \\
\hline & 67 & 89 & 24 & 25.7 & 52.6 & 4.75 & $581 !$ & 54.9 & 37 & 0.03 \\
\hline & 68 & 89 & 24 & 25.7 & 52.5 & 4.75 & 582 & 54.9 & 37 & -0.08 \\
\hline 00 & 69 & 88 & 24 & 25.7 & 52.4 & 4.74 & 581 & 54.9 & 37 & -0.08 \\
\hline & 70 & 88 & 24 & 25.7 & 52.4 & 4.74 & 578 & 55.1 & 37 & -0.18 \\
\hline & 71 & 88 & 24 & 25.7 & $5 \overline{2.3} 3^{\dagger}$ & 4.74 & 575 & 55.1 & 36 & $-0 . \overline{18}$ \\
\hline$: 02$ & 72 & 88 & 24 & 25.7 & 52.3 & 4.74 & 573 & $5 \overline{5} .1\}$ & 36 & -0.24 \\
\hline & $7 \overline{3}$ & 88 & 24 & $25 . \overline{7}$ & 52.2 & 4.74 & 575 & 55 & 36 &.$\overline{08}$ \\
\hline & 74 & 88 & 24 & 25.7 & 52.2 & 4.74 & 578 & 54.9 & 36 & 0.08 \\
\hline & 75 & 88 & 24 & 25.7 & 52.2 & 4.75 & 577 & 54.9 & 36 & -0.04 \\
\hline & 76 & 88 & 24 & 25.7 & 52.2 & $4 . \overline{74}$ & 80 & 55 & 37 & -0.04 \\
\hline & 77 & 88 & & 25.7 & 52.2 & 4.75 & 583 & 55.1 & $36{ }^{\dagger}$ & -0.18 \\
\hline & 78 & 87 & $24 !$ & 25.9 & 52.1 & 4.77 & 589 & 55.1 & $37 !$ & -0.18 \\
\hline & 79 & 87 & 24 & 25.9 & 52.1 & 4.75 & 58 & 55 & 36 & -0.05 \\
\hline & 80 & 87 & 24 & 25.9 & 52.1 & 4.75 & & 54.9 & 36 & -0.05 \\
\hline & 81 & 87 & 24 & 26.1 & 52 & 4.74 & 592 & 54.9 & 36 & -0.02 \\
\hline & 82 & 87 & 24 & 26.1 & 52 & 4.74 & 590 & 55 & 36 & -0.02 \\
\hline & 83 & 87 & 24 & 26.1 & 52 & 4.74 & 590 & 55 & 36 & -0.07 \\
\hline & 84 & 87 & 24 & 26.1 & 52 & 4.75 & 592 & 55 & 36 & -0.07 \\
\hline & 85 & 87 & & 26.1 & 51.9 & $4.77^{\dagger}$ & 592 & 54.9 & 37 & -0.25 \\
\hline & 86 & 87 & 24 & 25.9 & & 4.77 & 595 & $54 . \overline{8}$ & $366^{\dagger}$ & -0.25 \\
\hline & 87 & 87 & 24 & 26.1 & & $4 . \overline{7} 7$ & 598 & 54.8 & 36 & -0.13 \\
\hline & 88 & 87 & 24 & 26.1 & 51.9 & 4.77 & 602 & 54.9 & 36 & -0.13 \\
\hline & 89 & 86 & 24 & & 9 & 5 & & 55 & $3 \overline{6}^{2}$ & 08 \\
\hline & 90 & 86 & 24 & 26.1 & 51.9 & $4 . \overline{74}$ & $600^{\dagger}$ & 55 & 36 & 0.08 \\
\hline & 91 & 86 & 24 & 26.1 & 51.9 & 4.75 & 603 & 55.1 & 36 & -0.1 \\
\hline & 92 & 86 & 24 & 26.1 & & $\overline{4.74}$ & & $55.2^{\dagger}$ & 36 & -0.1 \\
\hline & 93 & 86 & 24 & & $51.8 !$ & 4.74 & & 55.2 & 35 & -0.24 \\
\hline & 94 & 86 & $\quad 24$ & 26.3 & 51.8 & 4.74 & 595 & 55 & 35 & -0.1 \\
\hline & 95 & 86 & $24 !$ & 26.1 & 51.8 & 4.75 & 596 & 54.8 & 35 & -0.1 \\
\hline & 96 & 85 & 24 & & & 4.75 & & & 35 & .07 \\
\hline & $9 \overline{7}$ & 86 & 24 & 26.1 & 51.7 & 4.77 & 597 & 54.7 & 35 & 0.07 \\
\hline & 98 & 85 & 24 & 26.3 & $51.7 !$ & 4.77 & 600 & 54.6 & 35 & -0.13 \\
\hline & 99 & 85 & 24 & 26.3 & $5 \overline{1.7}$ & 4.75 & 602 & 54.6 & 35 & -0.13 \\
\hline & 100 & 85 & 24 & 26.3 & 51.7 & 4.75 & & 54.5 & 35 & -0.23 \\
\hline 08 & 101 & 85 & 24 & 26.3 & 51.7 & $4 . \overline{74}$ & 600 & 54.4 & 35 & -0.23 \\
\hline & 102 & 85 & 24 & 26.3 & $5 \overline{1.7}$ & 4.75 & 596 & 54.4 & 35 & 0.02 \\
\hline & 10 & 85 & 24 & 26.3 & 51.6 & 4.75 & 598 & 54.5 & 35 & 0.02 \\
\hline & 104 & 85 & 24 & & & 4.74 & & 54.6 & 34 & -0.03 \\
\hline $1: 31: 08$ & 105 & 85 & $2 \overline{4}$ & 26 & 51.6 & 4.75 & 595 & 54.6 & 34 & -0.03 \\
\hline & 106 & 85 & 24 & 26.3 & 51.6 & 4.74 & 598 & 54.6 & 35 & $-0 . \overline{2}$ \\
\hline $14: 11: 33: 06$ & 107 & 85 & 24 & 26.3 & 51.5 & 4.77 & 600 & 54.6 & 35 & -0.2 \\
\hline
\end{tabular}




\begin{tabular}{|c|c|c|c|c|c|c|c|c|c|c|}
\hline TIME & $\begin{array}{l}\text { Elapsed } \\
\text { Time } \\
\text { (sec) }\end{array}$ & ${ }_{L} \mathrm{BIT}_{2} \mathrm{TC}_{1}$ & PURG_TC2 & PurgePsI & PurgeCFM & DEPTH(IN) & DwnFrc(lb) & RPM & $\begin{array}{l}\text { Torque } \\
\perp \text { (FTLB) }\end{array}$ & es/Min \\
\hline $14: 11: 34: 05$ & 108 & 85 & 24 & 26.3 & 51.5 & $\quad 4.77$ & $\quad 602$ & 54.6 & $\quad 35$ & -0.08 \\
\hline $14: 11: 35: 09$ & 109 & 85 & 24 & 26.5 & 51.5 & 4.75 & 602 & 54.7 & 35 & -0.08 \\
\hline $14: 11: 36: \overline{08}$ & 110 & 85 & 24 & 26.5 & 51.5 & 4.77 & 604 & 54.9 & 36 & -0.02 \\
\hline $14: 11: 37: 07$ & 111 & 85 & 24 & 26.5 & $51.5^{\prime}$ & 4.75 & $602^{\circ}$ & 54.9 & 35 & -0.02 \\
\hline $14: 11: 38: 12$ & 112 & 85 & 24 & 26.5 & 51.5 & 4.75 & 598 & 54.9 & 35 & -0.11 \\
\hline $14: 11: 39: 10$ & 113 & 85 & 24 & $26.5^{\prime}$ & 51.5 & 4.75 & 595 & 54.8 & 35 & -0.11 \\
\hline $14: 11: 40: 09$ & 114 & 84 & 24 & 26.5 & 51.5 & 4.75 & 597 & 54.8 & 35 & -0.22 \\
\hline $14: 11: 41: 08$ & 115 & 84 & 24 & 26.5 & 51.5 & 4.77 & 595 & 54.9 & 34 & -0.22 \\
\hline $14: 11: 42: 07$ & 116 & 84 & 24 & 26.5 & 51.5 & 4.77 & 596 & 54.9 & 35 & 0.03 \\
\hline $14: 11: 43: 11$ & 117 & 84 & 24 & 26.5 & 51.5 & 4.75 & 599 & 54.9 & 35 & 0.03 \\
\hline $14: 11: 44: 10$ & 118 & 85 & 24 & 26.5 & 51.5 & 4.77 & 603 & 54.7 & 35 & -0.07 \\
\hline & 119 & 85 & 24 & $26.5^{-}$ & 51.5 & 4.77 & 604 & 54.6 & 35 & -0.12 \\
\hline $14: 11: 46: 13$ & 120 & 84 & 24 & 26.6 & 51.5 & 4.75 & 603 & 54.6 & 35 & -0.12 \\
\hline $14: 11: 47: 12$ & 121 & 84 & 24 & 26.6 & 51.5 & 4.75 & 604 & 54.6 & 34 & -0.26 \\
\hline 11 & 122 & 84 & 24 & 26.6 & 51.5 & $4.7 \overline{5}$ & 602 & 54.8 & 35 & -0.26 \\
\hline $14: 11: 49: 10$ & 123 & 84 & 24 & 26.6 & 51.4 & $4.7 \overline{4}$ & 598 & 54.8 & 34 & -0.01 \\
\hline 09 & 124 & 84 & 24 & 26.6 & 51.4 & 4.75 & 597 & 54.8 & 34 & -0.01 \\
\hline $1: 13$ & 125 & 84 & 24 & $26.6^{\dagger}$ & 51.3 & 4.77 & 597 & 54.7 & 34 & 0.01 \\
\hline $14: 11: 52: 12$ & 126 & 84 & 24 & $2 \overline{6} .6$ & 51.3 & 4.77 & 597 & 54.7 & 34 & 0.01 \\
\hline 11 & 127 & 84 & 24 & 26.6 & 51.3 & 4.75 & 600 & 54.7 & 35 & -0.17 \\
\hline $14: 11: 54: 10$ & 128 & 84 & 24 & $26.6 !$ & 51.3 & 4.75 & 602 & 54.7 & 35 & -0.17 \\
\hline 09 & 129 & 84 & 24 & 26.6 & 51.2 & 4.77 & 605 & 54.7 & 35 & -0.23 \\
\hline $14: 11: 5$ & 130 & 84 & 24 & 26.6 & 51.2 & 4.77 & $606^{4}$ & 54.7 & 35 & -0.23 \\
\hline $14: 11: 57: 12$ & 131 & 84 & 24 & 26.6 & 51.2 & 4.75 & 605 & 54.8 & 35 & 0.09 \\
\hline 14: & 132 & 84 & 24 & 26.6 & 51.2 & 4.75 & $605^{\dagger}$ & 54.8 & $3 \overline{5}$ & 0.09 \\
\hline $14: 11: 5$ & 133 & 84 & 24 & 26.8 & 51.2 & 4.75 & 602 & 54.7 & 35 & -0.07 \\
\hline $14: 12: 00: 14$ & $13 \overline{4}$ & 83 & 24 & 26.8 & 51.2 & 4.77 & 598 & 54.7 & 34 & -0.07 \\
\hline $2: 01: 13$ & 135 & 83 & 24 & 26.8 & 51.2 & 4.77 & 598 & 54.7 & 34 & -0.18 \\
\hline$\overline{12}$ & $\overline{136}$ & 83 & 24 & $26.8^{\dagger}$ & 51.1 & 4.77 & 597 & $54.6^{\dagger}$ & 34 & $-0 . \overline{18}$ \\
\hline $14: 12: 03: 11$ & 137 & 83 & 24 & 26.8 & 51.1 & 4.77 & 602 & $54.5]$ & 34 & -0.18 \\
\hline & 138 & 83 & 24 & 26.8 & $51.1^{\dagger}$ & 4.77 & 601 & 54.5 & 34 & -0.18 \\
\hline $14: 12: 05: 14$ & 139 & 84 & 24 & 26.8 & 51.1 & 4.77 & 604 & 54.6 & 35 & 0.05 \\
\hline & 140 & 84 & 24 & 26.8 & 51.1 & 4.77 & 606 & 54.7 & 35 & 0.05 \\
\hline$: 17$ & 141 & 84 & 24 & 27 & 51.1 & 4.75 & 604 & 54.7 & 34 & -0.09 \\
\hline 16 & 142 & 83 & 24 & 27 & 51 & 4.75 & 607 & $54.7 !$ & $\overline{34}$ & -0.24 \\
\hline $14:$ & 143 & 83 & 24 & 27 & 51 ? & 4.75 & 604 & 54.6 & 34 & -0.24 \\
\hline $10: 14$ & 144 & 83 & 24 & $27 !$ & 51 & 4.75 & 600 & 54.6 & 34 & -0.05 \\
\hline $12: 11: 13$ & 145 & 83 & 24 & 27 & 51 & 4.75 & 598 & 54.6 & 34 & -0.05 \\
\hline $2: 12: 17$ & 146 & 83 & 24 & 27 & $51^{\dagger}$ & 4.75 & 598 & 54.8 & 34 & 0.01 \\
\hline 16 & 147 & 83 & 24 & 27 & 51 & 4.77 & 602 & 54.8 & 34 & 0.01 \\
\hline $4: 15$ & 148 & 83 & 24 & 27 & 51 & $\overline{4.7 \overline{7}}$ & 605 & 54.8 & 35 & $-0 . \overline{17}$ \\
\hline $14: 12: 15: 19$ & 149 & 83 & 24 & 27 & 51 & 4.77 & 602 & 54.7 & 35 & -0.17 \\
\hline $14: 12: 16: 18$ & 150 & 83 & 24 & 27 & 50.9 & 4.77 & $606^{\prime}$ & 54.7 & 35 & -0.21 \\
\hline $14: 12: 17: 17$ & 151 & 83 & 24 & 27 & 50.9 & 4.77 & 607 & 54.5 & 35 & -0.21 \\
\hline $2: 18: 16$ & 152 & 83 & 24 & 27 & 50.9 & $4.7 \overline{7}$ & 604 & 54.5 & 35 & 0.05 \\
\hline $14: 12: 19: 14$ & 153 & 83 & 24 & 27 & 50.9 & $4 . \overline{75}$ & $600^{\circ}$ & 54.5 & 35 & 0.05 \\
\hline $12: 20: 19$ & 154 & 83 & 24 & 27 & 50.9 & 4.77 & 602 & 54.6 & 35 & -0.05 \\
\hline $21: 18$ & 155 & 83 & 24 & 27 & 50.9 & $4 . \overline{77}$ & 600 & $5 \overline{4.7}$ & 34 & -0.05 \\
\hline $14: 12: 22: 17$ & 156 & 83 & 24 & 27 & 50.9 & $4 . \overline{7}$ & 598 & 54.7 & 34 & -0.22 \\
\hline $14: 12: \overline{23}: \overline{21}$ & 157 & 83 & 24 & 27 & 50.9 & 4.77 & 600 & 54.8 & 34 & -0.22 \\
\hline $14: 12: 24: 20$ & 158 & 83 & 24 & 27.2 & 50.9 & 4.77 & 603 & $5 \overline{4.8}$ & 34 & -0.02 \\
\hline $14: 12: 25: 19$ & 159 & 83 & 24 & 27.2 & 50.9 & 4.77 & 607 & 54.8 & 35 & -0.02 \\
\hline $14: 12: 26: 17$ & 160 & 84 & 24 & 27.2 & 50.9 & 4.77 & 605 & 54.7 & 34 & -0.06 \\
\hline $14: 12: 27: 16$ & 161 & 83 & 24 & 27.2 & 50.9 & 4.75 & 607 & 54.8 & 34 & -0.06 \\
\hline
\end{tabular}




\begin{tabular}{|c|c|c|c|c|c|c|c|c|c|c|}
\hline$T I M E$ & $\begin{array}{l}\text { Tlapsed } \\
\text { Time } \\
\text { (sec) }\end{array}$ & BIT_TC1 & PURG_TC2 & PurgePSI & PurgeCFM & DEPTH $(\mathbb{N})$ & DwnFrc(lb) & RPM & $\begin{array}{l}\text { Torque } \\
\text { (FTLB) }\end{array}$ & Inches/Min \\
\hline $14: 12: 28: 21$ & 162 & -83 & 24 & $\quad 27.2$ & 50.8 & $\quad 4.75$ & 608 & $3 \quad 54.8$ & $\quad 34$ & $\quad-0.09$ \\
\hline $14: 12: 29: 20$ & $163^{\circ}$ & 83 & 24 & 27.4 & 50.8 & 4.74 & $604^{\circ}$ & 54.9 & 34 & -0.27 \\
\hline $14: 12 ; 30: 18$ & 164 & 83 & 24 & 27.4 & 50.8 & 4.75 & $601^{\circ}$ & 55 & 34 & $-0.2 \overline{7}$ \\
\hline $14: 12: 31: 1 \overline{7}$ & 165 & $83]$ & 24 & 27.4 & 50.8 & 4.77 & $602^{+}$ & 55 & 34 & 0.05 \\
\hline $14: 12: 32: 16$ & 166 & 83 & 24 & 27.4 & 50.8 & 4.74 & 599 & $55^{\top}$ & 34 & 0.05 \\
\hline $14: 12: 33: 21$ & 167 & 83 & 24 & 27.4 & 50.8 & $4.7 \overrightarrow{7}$ & $600^{\circ}$ & 55 & 33 & -0.1 \\
\hline $14: 12: 34: 19$ & 168 & 83 & 24 & 27.4 & 50.8 & 4.77 & 602 & 55 & 33 & -0.1 \\
\hline $14: 12: 35: 18$ & $169^{\circ}$ & 83 & $24 !$ & $27.4 !$ & 50.8 & 4.78 & 604 & 55 & 35 & -0.1 \\
\hline $14: 12: 36: 23$ & 170 & 84 & 24 & 27.4 & 50.8 & 4.77 & 607 & 55.1 & 34 & -0.1 \\
\hline $14: 12: 37: 21$ & 171 & 83 & 24 & 27.4 & 50.7 & 4.78 & 605 & 55.1 & 35 & -0.27 \\
\hline $14: 12: 38: 20$ & 172 & 83 & 24 & 27.4 & 50.7 & 4.77 & $609^{\circ}$ & 55 & 35 & -0.27 \\
\hline $14: 12: 39: 19$ & 173 & 83 & 24 & 27.4 & 50.7 & 4.77 & 609 & 55.1 & 35 & $-0.0 \overline{3}$ \\
\hline $14: 12: 40: 18$ & 174 & 83 & 24 & 27.4 & $5 \overline{0.7}$ & $4 . \overline{77}$ & 605 & 55.1 & 34 & -0.03 \\
\hline $14: 12: 41: 22$ & 175 & 83 & 24 & 27.4 & 50.7 & 4.75 & 600 & 55.1 & 34 & 0.02 \\
\hline $14: 12: 42: 21$ & 176 & 83 & 24 & 27.6 & 50.7 & 4.77 & $59 \overline{9}$ & 55 & 33 & 0.02 \\
\hline $14: 12: 43: 20$ & 177 & 82 & 24 ! & 27.6 & 50.6 & 4.77 & 599 & 55 & 33 & -0.14 \\
\hline $14: 12: 44: 19$ & $178 !$ & 82 & 24 & 27.6 & 50.7 & 4.77 & 600 & $54 . \overline{7}$ & 33 & -0.14 \\
\hline $14: 12: 45: 18$ & $179^{\circ}$ & 82 & 24 & 27.6 & 50.6 & 4.77 & 601 & 54.9 & 34 & -0.24 \\
\hline $14: 12: 46: 22$ & $180^{\circ}$ & 83 & 24 & 27.6 & 50.6 & 4.77 & 603 & 54.9 & 33 & -0.24 \\
\hline $14: 12: 47: 21$ & 181 & 83 & 24 & 27.6 & 50.6 & 4.75 & 607 & 55.1 & 34 & 0.09 \\
\hline $14: 12: 48: 20$ & 182 & 83 & 25 & $27.6^{\prime}$ & $50.6^{\circ}$ & 4.77 & 608 & 55 & 34 & 0.09 \\
\hline $14: 12: 49: 24$ & 183 & 83 & 25 & 27.6 & 50.6 & $4.77 !$ & 609 & 55 & 34 & -0.08 \\
\hline $14: 12: 50: 23$ & 184 & 83 & 24 & 27.6 & 50.6 & 4.75 & 608 & 54.9 & 33 & -0.08 \\
\hline $14: 12: 51: 22$ & 185 & 82 & 25 & 27.8 & 50.6 & 4.74 & $604^{\circ}$ & 54.9 & 33 & $-0 . \overline{17}$ \\
\hline $14: 12: 52: 26$ & 186 & 82 & 24 & $27.8_{\mathrm{f}}^{+}$ & 50.6 & 4.75 & 601 & 54.9 & 33 & -0.21 \\
\hline $14: 12: 53: 25$ & 187 & 82 & $\cdots$ & 27.8 & 50.6 & 4.75 & $600^{\circ}$ & 54.8 & 33 & -0.21 \\
\hline $14: 12: 54: 24$ & 188 & 82 & 24 & 27.8 & 50.6 & 4.77 & 600 & 54.7 & 33 & 0.04 \\
\hline $14: 12: 5 \overline{5}: 23$ & $189^{\circ}$ & 82 & 24 & 27.8 & 50.6 & 4.77 & $600^{\circ}$ & 54.7 & 34 & $0 . \overline{04}$ \\
\hline $14: 12: 56: 22$ & $190 !$ & 83 & 25 & 27.8 & 50.6 & 4.77 & 601 & $54 . \overline{7}^{\dagger}$ & 34 & -0.07 \\
\hline $14: 12: 57: 26$ & 191 & 82 & 25 & 27.8 & 50.6 & 4.77 & 605 & 54.6 & 34 & -0.07 \\
\hline $14: 12: 58: 25$ & 192 & 82 & 25 & $27.8^{\dagger}$ & 50.6 & 4.77 & 609 & 54.6 & 34 & -0.25 \\
\hline $14: 12: 59: 24$ & 193 & 82 & 24 & $27.8 !$ & 50.6 & 4.78 & 608 & 54.5 & 34 & -0.25 \\
\hline $14: 13: 00: \overline{23}$ & 194 & 82 & 24 & 28 & 50.6 & 4.77 & 609 & 54.6 & 34 & -0.1 \\
\hline $14: 13: 01: 22$ & 195 & 82 & 25 & 28 & 50.6 & 4.77 & 606 & 54.6 & 34 & -0.1 \\
\hline $14: 13: 02: 26$ & 196 & 82 & 24 & 28 & 50.6 & 4.77 & 602 & 54.6 & 33 & 0.01 \\
\hline $14: 13: 03: 25$ & 197 & 81 & 24 & 28 & 50.6 & 4.77 & 600 & 54.5 & 33 & 0.01 \\
\hline $14: 13: 04: 24$ & 198 & 81 & 24 & 28.2 & 50.6 & 4.77 & 601 & 54.5 & 33 & -0.1 \\
\hline $14: 13: 05: 28$ & 199 & 81 & 24 & 28.2 & 50.6 & 4.78 & 600 & 54.5 & 33 & -0.1 \\
\hline $14: 13: 06: 27$ & 200 & 81 & 25 & 28.2 & 50.6 & 4.78 & 600 & 54.6 & 33 & -0.26 \\
\hline $14: 13: 07: 26$ & 201 & 82 & 24 & 28.2 & 50.6 & 4.78 & 603 & 54.6 & 33 & -0.26 \\
\hline $14: 13: 08: 25$ & 202 & 82 & 25 & 28.2 & 50.6 & 4.78 & 606 & 54.5 & 33 & 0.01 \\
\hline $14: 13: 09: 24$ & 203 & $8 \overline{2}$ & 25 & 28.4 & 50.6 & 4.77 & 606 & 54.5 & 33 & 0.01 \\
\hline $14: 13: 10: 28$ & 204 & 82 & 25 & 28.4 & 50.6 & 4.77 & 602 & 54.5 & 33 & 0 \\
\hline $14: 13: 11: \overline{27}$ & 205 & 81 & 25 & 28.4 & 50.6 & 4.77 & 603 & 54.5 & 33 & 0 \\
\hline $14: 13: 12: 26$ & 206 & 81 & 25 & 28.6 & 50.5 & 4.77 & 599 & 54.3 & 33 & -0.2 \\
\hline $14: 13: 13: \overline{30}$ & 207 & 81 & 25 & 28.6 & $5 \overline{0.5}$ & 4.75 & 595 & 54.3 & 33 & -0.2 \\
\hline $14: 13: 14: 29$ & 208 & 81 & 25 & 28.6 & 50.5 & 4.77 & 594 & 54.3 & 32 & -0.18 \\
\hline $14: 13: 15: 28$ & 209 & 81 & 25 & $28 . \overline{6}$ & 50.5 & 4.77 & 596 & 54.3 & 32 & 0.04 \\
\hline $14: 13: 16: 2 \overline{7}$ & 210 & $8 \overline{1}$ & 25 & 28.6 & 50.5 & 4.77 & 593 & 54.3 & 32 & 0.04 \\
\hline $14: 13: 17: 26$ & 211 & 81 & 25 & 28.6 & 50.5 & 4.78 & 602 & 54.4 & 34 & -0.09 \\
\hline $14: 13: 18: 30$ & 212 & 81 & 25 & $28 . \overline{6}$ & 50.5 & 4.78 & 600 & 54.4 & 34 & -0.09 \\
\hline $14: 13: 19: 29$ & 213 & 81 & 25 & 28.6 & 50.5 & $4 . \overline{77}$ & 600 & 54.4 & 34 & -0.31 \\
\hline $14: 13: 20: 28$ & 214 & 81 & 25 & $28 . \bar{B}$ & 50.5 & 4.77 & 598 & 54.3 & 33 & -0.31 \\
\hline $14: 13: 21: 32$ & 215 & 80 & 25 & 28.8 & 50.5 & $4.7 \overline{7}$ & 593 & $54 . \overline{5}$ & 33 & -0.03 \\
\hline
\end{tabular}




\begin{tabular}{|c|c|c|c|c|c|c|c|c|c|c|}
\hline TIME & $\begin{array}{l}\text { Elapsed } \\
\text { Time } \\
\text { (sec) }\end{array}$ & BIT_TC1 & PURG_TC2 & PurgePSI & PurgeCFM & DEPTH(IN) & DwnFrc(lb) & RPM & $\begin{array}{l}\text { Torque } \\
\text { (FTLB) }\end{array}$ & inches/Min \\
\hline $14: 13: 22: 31$ & 216 & -80 & -25 & $\quad 28.8$ & 50.5 & 4.77 & 595 & 54.5 & 33 & -0.03 \\
\hline $14: 13: 23: 30$ & 217 & 80 & 25 & 28.8 & 50.5 & 4.77 & 590 & 54.7 & 33 & -0.01 \\
\hline $14: 13: 24: 29$ & 218 & 80 & 25 & 28.8 & 50.5 & $4 . \overline{78}$ & 588 & 54.7 & 33 & -0.01 \\
\hline $14: 13: 25: 27$ & 219 & 80 & 25 & 29 & 50.5 & 4.77 & 590 & 54.7 & 33 & -0.12 \\
\hline $14: 13: 26: 32$ & 220 & 80 & 25 & 29 & 50.5 & 4.78 & $592^{\circ}$ & 54.7 & 33 & -0.12 \\
\hline $14: 13: 27: 31$ & 221 & 80 & 25 & 29 & 50.5 & 4.78 & 595 & 54.8 & 33 & -0.26 \\
\hline $14: 13: 28: 30$ & 222 & 80 & 25 & $29^{4}$ & 50.5 & 4.78 & 595 & 54.8 & 33 & -0.26 \\
\hline $14: 13: 29: 28$ & 223 & 80 & 25 & 29 & 50.5 & $4.77^{\dagger}$ & 594 & 54.9 & 33 & $0 . \overline{04}$ \\
\hline $14: 13: 30: 27$ & 224 & 80 & 25 & 29 & $50.5^{\prime}$ & 4.77 & 595 & 55 & 32 & 0.04 \\
\hline $14: 13: 31: 32$ & 225 & $80^{-1}$ & 24 & 29.2 & 50.5 & $4.77^{\circ}$ & 592 & 55 & 32 & -0.02 \\
\hline $14: 13: 32: 31$ & 226 & 80 & 25 & 29.2 & 50.5 & 4.77 & 588 & 55 & 32 & -0.02 \\
\hline $14: 13: 33: 29$ & 227 & 80 & 25 & 29.2 & 50.5 & $4.77 i$ & 584 & 55.1 & 32 & -0.2 \\
\hline $14: 13: \overline{34: 28}$ & 228 & 80 & 25 & 29.4 & 50.5 & $4.77^{\circ}$ & 585 & 55.1 & 32 & -0.2 \\
\hline $14: 13: 35: 27$ & 229 & 80 & 25 & 29.4 & 50.5 & $4.77 j$ & 585 & 55 & 32 & -0.18 \\
\hline $14: 13: 36: 31$ & 230 & 80 & 25 & 29.4 & 50.5 & 4.77 & $585^{\dagger}$ & 55 & 32 & $-0 . \overline{18}$ \\
\hline $14: 13: 37: 30$ & 231 & 80 & 25 & 29.4 & 50.5 & 4.78 & $588 !$ & 55 & 34 & 0.03 \\
\hline $14: 13: 38: 29$ & 232 & 80 & 25 & 29.4 & 50.5 & 4.78 & 591 & 55 & 33 & 0.03 \\
\hline $14: 13: 39: 34$ & 233 & 80 & 25 & 29.4 & 50.5 & 4.78 & 592 & 54.9 & 34 & -0.05 \\
\hline $14: 13: 40: 32$ & 234 & $80^{4}$ & 25 & 29.4 & 50.5 & $4.77 !$ & 592 & 54.9 & 34 & -0.22 \\
\hline $14: 13: 41: 31$ & 235 & 80 & 24 & 29.4 & 50.5 & $4.77 j^{\circ}$ & 591 & 54.9 & 34 & -0.22 \\
\hline $14: 13: 42: 36$ & $2 \overline{3} 6$ & 80 & 25 & 29.4 & 50.5 & $4.77^{+}$ & 588 & 54.9 & 34 & -0.19 \\
\hline $14: 13: 43: 35$ & 237 & 80 & 24 & 29.4 & 50.5 & 4.77 & 583 & $54 . \overline{9}$ & 33 & -0.19 \\
\hline $14: 13: 44: 33$ & 238 & 80 & 25 & 29.4 & 50.5 & 4.77 & 582 & 54.9 & 33 & 0.05 \\
\hline $14: 13: 45: 32$ & 239 & $79^{\circ}$ & 25 & 29.4 & 50.5 & 4.78 & 582 & $5 \overrightarrow{4.9}$ & 33 & 0.05 \\
\hline $14: 13: 46: 31$ & 240 & 79 & 25 & 29.4 & 50.5 & 4.78 & 580 & 54.9 & 33 & -0.09 \\
\hline $14: 13: 4 \overline{7}: 35$ & 241 & 80 & 25 & 29.4 & 50.5 & 4.78 & 583 & 54.8 & $3 \overline{3}$ & -0.09 \\
\hline $14: 13: 48: 34$ & 242 & 80 & 25 & 29.4 & 50.5 & 4.77 ! & 586 & 54.6 & 33 & -0.23 \\
\hline $14: 13: 49: 33$ & 243 & 80 & 25 & 29.4 & 50.5 & 4.78 & 588 & 54.6 & 33 & -0.23 \\
\hline $14: 13: 50: 38$ & 244 & 80 & 25 & 29.4 & 50.5 & $4 . \overline{7} 8$ & 589 & 54.7 & 33 & $-0 . \overline{09}$ \\
\hline $14: 13: 51: 36$ & 245 & 80 & 25 & 29.4 & 50.5 & 4.77 & 589 & 54.9 & 32 & $-0 . \overline{09}$ \\
\hline $14: 13: \overline{52: 35}$ & 246 & $8 \overline{0}$ & 25 & 29.4 & 50.4 & 4.77 & 588 & 54.9 & 32 & 0.04 \\
\hline $14: 13: 53: 34$ & 247 & 80 & 25 & $29.4]$ & 50.4 & 4.77 & $5 \overline{85}$ & 54.9 & 32 & 0.04 \\
\hline $14: 1 \longdiv { 3 : 5 4 : \overline { 3 3 } }$ & 248 & 79 & 25 & 29.4 & 50.4 & 4.75 & $581]$ & 54.9 & 32 & -0.11 \\
\hline $14: 13: 55: 37$ & 249 & 79 & 25 & 29.4 & 50.4 & 4.77 & 580 & 54.8 & 32 & -0.11 \\
\hline $14: 13: 56: 36$ & 250 & 79 & 25 & 29.4 & 50.3 & 4.78 & 583 & 54.8 & 32 & $-0 . \overline{2} \overline{7}$ \\
\hline $14: 13: 57: 35$ & 251 & $7 \overline{9}$ & 25 & 29.4 & 50.3 & $4.77 j$ & 587 & 54.8 & 33 & $-0 . \overline{27}$ \\
\hline $14: 13: 58: 34$ & 252 & 79 & 25 & $29.4 !$ & 50.3 & 4.78 & 584 & 54.9 & 33 & 0.02 \\
\hline $14: 13: 59: 33$ & 253 & 80 & 25 & 29.4 & 50.2 & 4.78 & 588 & 54.9 & 33 & 0.02 \\
\hline $14: 14: 00: 37$ & 254 & 79 & 25 & 29.4 & 50.2 & $4 . \overline{7} 7$ & 590 & 54.9 & 33 & 0 \\
\hline $14: 14: 01: 36$ & 255 & 80 & 25 & 29.4 & 50.2 & 4.77 & 590 & 54.9 & 33 & -0.16 \\
\hline $14: 14: 02: 35$ & 256 & 80 & 25 & 29.4 & 50.2 & $4.78 !$ & 588 & $55^{\dagger}$ & 33 & -0.16 \\
\hline $14: 1 \overline{4: 03: 39}$ & 257 & 80 & 25 & 29.4 & 50.2 & 4.77 & 590 & 55 & 33 & -0.27 \\
\hline $14: 14: 04: 38$ & 258 & 80 & 25 & 29.4 & 50.2 & 4.77 & 586 & 55 & 33 & -0.27 \\
\hline $14: 14: 05: \overline{37}$ & 259 & 79 & 25 & 29.6 & 50.1 & 4.77 & 582 & 55 & 32 & 0.06 \\
\hline $14: 14: 06: 36$ & $260 !$ & 79 & 25 & 29.6 & 50.1 & 4.77 & 582 & 54.9 & 32 & 0.06 \\
\hline $14: 14: \overline{07}: 35$ & 261 & 79 & 25 & 29.6 & 50.1 & 4.77 & 583 & 54.9 & 32 & $-0 . \overline{02}$ \\
\hline $14: 14: 08: 39$ & 262 & 79 & 25 & 29.6 & 50.1 & 4.78 & 586 & 54.8 & 32 & -0.02 \\
\hline $14: 14: 09: 38$ & 263 & 80 & 25 & 29.6 & 50 & 4.77 & $584^{\circ}$ & 54.8 & 32 & -0.21 \\
\hline $14: 14: 10: 37$ & 264 & 80 & 25 & 29.6 & 50 & 4.77 & 587 & 54.8 & 32 & -0.21 \\
\hline $14: 14: 11: 41$ & 265 & 80 & 25 & 29.6 & 50 & $\overline{4.77 i}$ & 590 & 54.8 & 33 & -0.21 \\
\hline $14: 14: 12: 40$ & $26 \overline{6}$ & 79 & 25 & 29.6 & 50 & 4.77 & 589 & 54.7 & 32 & -0.21 \\
\hline $14: 14: 13: 39$ & 267 & $\overrightarrow{79}$ & 25 & 29.6 & 49.9 & 4.77 & 585 & $54.7^{\dagger}$ & 32 & 0.09 \\
\hline $14: 14: \overline{14}: \overline{38}$ & 268 & $79^{+}$ & 25 & 29.6 & 49.9 & 4.77 & 588 & 54,7 & 32 & $0 . \overline{09}$ \\
\hline $14: 14: \overline{15}: 37$ & 269 & 79 & 25 & 29.6 & $\overline{49.9}$ & 4.77 & 583 & 54.6 & 32 & -0.05 \\
\hline
\end{tabular}




\begin{tabular}{|c|c|c|c|c|c|c|c|c|c|c|}
\hline$T / M E$ & $\begin{array}{l}\text { Elapsed } \\
\text { Time } \\
\text { ( }(\mathrm{sec})\end{array}$ & BIT_TC1 & PURG_TC2 & PurgePS1 & PurgeCFM & DEPTH(IN) & DwnFrc(b) & RPM & $\begin{array}{l}\text { Torque } \\
\text { (FTLB) }\end{array}$ & Inches/Min \\
\hline $14: 14: 16: 41$ & $\quad 270$ & -79 & -25 & $\quad 29.6$ & $\quad 49.9^{\prime}$ & $\begin{array}{l}1.78 \\
\end{array}$ & $\quad 581$ & 54.6 & 32 & -0.05 \\
\hline $14: 14: 17: 40$ & 271 & 79 & 25 & 29.6 & 49.9 & 4.77 & 582 & 54.6 & 32 & -0.27 \\
\hline $14: 14: 18: 39$ & 272 & 79 & $25^{\dagger}$ & 29.6 & 49.9 & $4.77^{4}$ & 583 & 54.6 & 33 & -0.27 \\
\hline $4: 14: 19: 43$ & 273 & 79 & 25 & 29.6 & 49.9 & 4.78 & 587 & 54.6 & 33 & -0.09 \\
\hline $14: 14: 20: 42$ & 274 & 79 & 25 & 29.6 & 49.9 & $4.77^{\circ}$ & 585 & 54.6 & 33 & -0.09 \\
\hline $14: 14: 21: 41$ & 275 & 79 & 25 & $29.6 !$ & 49.9 & $4.77^{\dagger}$ & 588 & 54.6 & 33 & 0.03 \\
\hline $2: 40$ & 276 & 79 & 25 & 29.6 & 49.9 & 4.78 & 589 & 54.7 & 33 & $0.0 \overline{3}$ \\
\hline $3: 39$ & 277 & 79 & 25 & 29.8 & 49.9 & 4.77 & 587 & 54.7 & 33 & -0.16 \\
\hline $14: 14: 24: 37$ & $27 \overline{8}$ & 79 & 25 & 29.8 & 49.9 & 4.75 & 583 & 54.6 & 33 & -0.16 \\
\hline $5: 36$ & 279 & 79 & 25 & 29.8 & 49.9 & 4.77 & 580 & 54.6 & 32 & -0.28 \\
\hline $4 \overline{1}$ & 280 & 79 & 25 & 29.8 & 49.9 & $4.7 \overline{5}$ & 581 & 54.6 & 32 & 0.05 \\
\hline $27: 40$ & 281 & $\overline{79}$ & 25 & 29.8 & 49.9 & 4.77 & 580 & 54.6 & 32 & 0.0 \\
\hline $14: 28: 38$ & $28 \overline{2}$ & 79 & 25 & $29.8 \mathrm{j}$ & 49.8 & 4.77 & 582 & 54.6 & 32 & -0.02 \\
\hline 43 & 283 & 79 & 25 & 29.8 & 49.8 & 4.78 & 585 & 54.6 & 33 & -0.02 \\
\hline & 284 & 79 & 25 & 29.8 & & 4.78 & 588 & 54.6 & 32 & -0.21 \\
\hline 1:41 & 285 & 79 & 25 & 29.8 & 49 & $4.78^{\circ}$ & 588 & 54.5 & 32 & -0.21 \\
\hline 45 & 286 & 79 & 25 & 29.8 & 49.8 & 4.78 & 588 & 54.5 & 32 & -0.2 \\
\hline & 287 & 79 & $25:$ & 30 & & 4.77 & 587 & 54.5 & 32 & -0 \\
\hline & 288 & 79 & 25 & 30 & $49.7^{\prime}$ & 4.78 & 583 & 54.5 & 32 & 0.07 \\
\hline 41 & 289 & $7 \overline{9}$ & 25 & 30 & 49.7 & 4.75 & 580 & 54.5 & 32 & 0.07 \\
\hline & 290 & 79 & 25 & 30 & & 4.77 & 80 & 54.5 & 32 & -0 \\
\hline & 291 & 79 & 25 & 30 & & 4.77 & 80 & 54.5 & 32 & -0.1 \\
\hline & 292 & $\overline{79}$ & 25 & $30^{+}$ & 49 & $4.7 \overline{7}$ & 580 & 54.5 & 32 & -0.27 \\
\hline & 293 & 79 & 25 & $30\rceil$ & & 4.78 & 83 & 54.5 & 33 & -0.27 \\
\hline & 294 & 79 & 25 & 30 & 49. & 4.77 & 587 & 54.5 & 33 & $-0 . \overline{04}$ \\
\hline & 295 & 79 & 25 & 30 & 49.7 & 4.78 & 588 & 54.5 & 33 & -0.04 \\
\hline & 296 & 79 & 25 & 30 & & $4.78^{\dagger}$ & 586 & 54.5 & $\overline{33}$ & $0 . \overline{01}$ \\
\hline & 297 & 79 & 25 & $30 \mathrm{i}$ & & $4 \overline{77}$ & 587 & 54.4 & 32 & 0.01 \\
\hline & 298 & 78 & $25 !$ & $30 \dagger$ & & 4.77 & 583 & 54.4 & 32 & $-0 . \overline{18}$ \\
\hline & 299 & 78 & 25 & 30 & & 4.77 & 580 & 54.4 & 32 & -0.18 \\
\hline & 300 & 78 & 25 & 30 & 49.7 & 4.77 & 579 & 54.4 & 32 & -0.24 \\
\hline 44 & 301 & $7 \overline{9}$ & $25 !$ & 30 & 49.7 & 4.77 & 580 & 54.4 & 32 & 0.06 \\
\hline & 302 & 79 & 25 & 30 & & 4.78 & & $54.6 !$ & 32 & 0.06 \\
\hline & 303 & 79 & 25 & 30 & & & & & 32 & -0.05 \\
\hline & 304 & $7 \overline{9}$ & $25^{1}$ & 30.2 & & 4.77 & 584 & 54.6 & 32 & -0.0 \\
\hline & 305 & 79 & 25 & 30.2 & 49.7 & $4.7 \overline{7}$ & 588 & 54.5 & 33 & -0.26 \\
\hline & 306 & 79 & 25 & & & $4 . \overline{77}$ & 5 & $5 \overline{4.6}$ & 33 & $-0 . \overline{26}$ \\
\hline & 307 & 79 & 25 & $30 . \overline{2}$ & & 4.77 & 583 & 54.6 & 32 & -0.13 \\
\hline & 308 & 79 & 25 & 30.2 & 49.7 & $4 . \overline{75}$ & 585 & 54.8 & 31 & -0.13 \\
\hline & 309 & 78 & 25 & 30.4 & 49.7 & 4.77 & 582 & 54.7 & 32 & 0.05 \\
\hline & 310 & 78 & 25 & & & 4.77 & & & 32 & $\overline{0.0}$ \\
\hline & 311 & 78 & 25 & 30.4 & 49.7 & 4.77 & 580 & $54.6 \dagger$ & 32 & -0.0 \\
\hline $8: 48$ & 312 & 78 & 25 & 30.4 & 49.6 & 4.77 & 582 & 54.5 & 31 & -0.09 \\
\hline & 313 & 78 & 25 & 30.4 & 49.6 & 4.78 & 585 & 54.5 & 31 & -0.28 \\
\hline & 314 & 78 & 25 & 4 & & 4.77 & & 54.6 & 33 & -0.28 \\
\hline & 315 & 79 & 25 & 30.4 & 49 & 4.78 & 586 & 54.6 & 33 & 0.0 \\
\hline $15: 02: 49$ & 316 & 79 & 25 & 30.4 & 49.6 & 4.77 & 587 & 54.6 & 32 & 0.05 \\
\hline & 317 & 78 & 25 & 30.4 & 49.6 & 4.77 & 584 & 54.5 & 32 & -0.09 \\
\hline & 318 & 78 & 25 & 30.4 & 49.5 & 4.77 & $580^{\circ}$ & 54.5 & 32 & -0.0 \\
\hline & 319 & 78 & 25 & $30.5^{+}$ & 49.5 & 4.77 & 578 & 54.5 & 32 & -0.18 \\
\hline $14: 15: 06: 50$ & 320 & 78 & 25 & 30.5 & 49.5 & 4.77 & 580 & 54.5 & 32 & -0.18 \\
\hline $14: 15: 07: 49$ & 321 & 78 & 25 & 30.4 & 49.5 & 4.77 & $579^{\circ}$ & 54.5 & 32 & -0.21 \\
\hline & 322 & 78 & 25 & 30.5 & 49.5 & 4.78 & $580^{\circ}$ & 54.5 & 32 & -0.21 \\
\hline $14: 15: 09: 52$ & 323 & 78 & 25 & $30.5^{\dagger}$ & 49.5 & 4.78 & 583 & $54.5^{+}$ & 32 & 0.0 \\
\hline
\end{tabular}




\begin{tabular}{|c|c|c|c|c|c|c|c|c|c|c|}
\hline T I ME & \begin{tabular}{l|} 
Elapsed \\
Time \\
|(sec)
\end{tabular} & BIT_TC1 & PURG_TC2 & PurgePSI & PurgeCFM & DEPTH $(I N)$ & DwnFrc(lb) & RPM & $\begin{array}{l}\text { Torque } \\
\text { (FTLB) }\end{array}$ & Inches/Min \\
\hline $14: 15: 10: 51$ & 324 & 78 & $\quad 25$ & $\quad 30.5$ & $\quad 49.5$ & $\quad 4.78$ & $\quad 586$ & $\quad 54.5$ & 32 & -0.08 \\
\hline $14: 15: 11: 50$ & 325 & 78 & 25 & 30.5 & 49.5 & 4.78 & 587 & 54.5 & 33 & -0.08 \\
\hline 2449 & 326 & 78 & 25 & 30.5 & 49.5 & 4.78 & 586 & 54.6 & 33 & -0.27 \\
\hline $14: 15: 13: 48$ & 327 & 78 & 25 & 307 & 49.5 & 4.77 & 585 & 54.8 & 32 & -0.27 \\
\hline $14: 1$ & 328 & 78 & 25 & 30.7 & $4 \overline{9} .5$ & 4.77 & 582 & 54.8 & 31 & -0.1 \\
\hline $14: 1$ & $329 !$ & 78 & 25 & 30.7 & 49.5 & 4.77 & 578 & 54.8 & 32 & -0.1 \\
\hline $14: 1$ & 330 & 78 & 25 & 30.7 & 49.5 & 4.77 & 578 & 54.7 & 31 & $0 . \overline{4}$ \\
\hline $14: 15$ & 331 & 78 & 25 & 30.7 & 49.5 & 4.77 & 578 & 54.7 & 31 & 0.04 \\
\hline $14: 1$ & 332 & 78 & 25 & 30.7 & 49.5 & 4.78 & 578 & 54.8 & $3 \overline{2}$ & $-0 . \overline{13}$ \\
\hline & 333 & 78 & 25 & $30.7 !$ & 49.5 & $4.77 !$ & 580 & 54.8 & 32 & -0.13 \\
\hline $14: 1$ & 334 & 78 & 25 & 30.7 & 49.5 & 4.78 & 583 & 54.8 & 32 & -0.17 \\
\hline $14: 1$ & 335 & 78 & 25 & 30.7 & 49.5 & 4.77 & 586 & 54.6 & 32 & -0.17 \\
\hline & 336 & 78 & 25 & 30.7 & 49.5 & 4.77 & 585 & 54.7 & 32 & $-0 . \overline{06}$ \\
\hline $14: 1$ & 337 & 78 & 25 & $30.7 !$ & 49.4 & 4.77 & 586 & 54.7 & 32 & 0.06 \\
\hline $14: 1$ & 338 & 78 & 25 & 30.7 & 49.4 & 4.77 & 584 & & 32 & 0.04 \\
\hline & 339 & 78 & 25 & 30.7 & 49.4 & 4.77 & 581 & 54.8 & 32 & -0.04 \\
\hline & $34 \overline{0}$ & 77 & 25 & 30.9 & 49.4 & 4.77 & 579 & 54.8 & 32 & -0.16 \\
\hline 54 & 341 & 77 & 25 & 30.9 & 49.4 & $4.7 \overline{7}$ & 578 & 54.8 & 32 & -0.16 \\
\hline & $342^{\dagger}$ & 77 & 25 & 30.7 & 49.4 & 4.77 & 580 & 54.8 & 32 & -0.14 \\
\hline 52 & 343 & 78 & 25 & 30.7 & 49.4 & 4.78 & 579 & 54.8 & 32 & 0.14 \\
\hline 56 & 344 & 78 & 25 & 30.7 & 49.4 & 4.78 & 582 & 54.8 & 33 & -0.02 \\
\hline & $345 !$ & 78 & 25 & 30.7 & 49.4 & 4.78 & 585 & 54.8 & 33 & -0.02 \\
\hline 54 & 346 & 78 & 25 & 30.7 & 49 & 4.78 & 586 & 54.8 & 33 & -0.07 \\
\hline & 347 & 78 & 25 & 30.9 & 49.3 & 4.78 & 584 & 54 & 33 & -0.24 \\
\hline & 348 & 78 & 25 & 30.9 & 49.3 & 4.78 & 81 & 54 & 32 & -0.24 \\
\hline & 349 & 78 & 25 & 30.9 & 49.3 & 4.77 & 583 & 54.7 & 32 & -0.18 \\
\hline & 350 & 77 & 25 & 30.9 & 49.3 & 4.77. & $5 \overline{8} 0$ & 54.7 & 31 & -0.18 \\
\hline & 351 & 77 & $25 !$ & 3 & 49.3 & 4.78 & 578 & & 32 & \\
\hline & & 77 & 25 & & & 4. & & 9 & 32 & 0.11 \\
\hline & 353 & 77 & 25 & 31. & & 4.78 & 582 & 54. & 32 & $-0 . \overline{09}$ \\
\hline & 35 & 78 & 25 & 31.1 & 49 & 4.7 & 580 & 54 & 32 & -0.09 \\
\hline & 35 & 78 & 25 & 31.1 & $49.3 !$ & 4.78 & 582 & 54.9 & 32 & -0.28 \\
\hline & 356 & 78 & $\ldots 25$ & 30.9 & 49.3 & 4.78 & 585 & 55.1 & 33 & $-0 . \overline{28}$ \\
\hline & 357 & 78 & - $\quad 25^{\dagger}$ & 31. & & & & 55 & 32 & -0.11 \\
\hline & 358 & 78 & 25 & & & & 584 & 55 & 33 & .11 \\
\hline & 359 & 78 & 25 & & 49.3 & 4.77 & 581 & 55 & 32 & 0.04 \\
\hline & $360^{\circ}$ & 78 & 25 & & & & & $55^{+}$ & 32 & 0.04 \\
\hline & 361 & 77 & 25 & $1.1^{\prime}$ & & 4.77 & 580 & 55 & 32 & \\
\hline & & 77 & 25 & $31.1\}$ & $49 \overline{2}$ & 4.77 & 578 & 54.9 & 32 & -0.09 \\
\hline & 363 & 77 & 25 & & & & & 54 & 33 & $-0 . \overline{3}$ \\
\hline & 36 & 78 & 25 & & 49 & 4.7 & 582 & 54 & 32 & -0.3 \\
\hline & 365 & 78 & -25 & 31.1 & 49.2 & 4.77 & 580 & 54.9 & 32 & \\
\hline & & 78 & 25 & & $4 \mathrm{~S}$ & 4.78 & 583 & 54.9 & 32 & 0 \\
\hline & 36 & 78 & 25 & 31.1 & & 4.77 & & 54.9 & 33 & \\
\hline & 368 & 78 & 25 & 31.1 & 49 & 4.77 & & 9 & 33 & \\
\hline & 369 & 78 & 25 & 31.3 & 49.2 & 4.78 & 584 & 54.8 & 32 & -0.16 \\
\hline & 37 & 78 & 25 & & & $4 . \overline{7}$ & 581 & 54.8 & 31 & -0.27 \\
\hline & 3711 & 78 & 25 & 31.3 & & 4.78 & & & 31 & -0.27 \\
\hline & 372 & 78 & 25 & 31.3 & 49.2 & 4.78 & 580 & 54.9 & 31 & 0.1 \\
\hline & 373 & 78 & 25 & 31.3 & 49.1 & 4.78 & 578 & 54.9 & 31 & 0.1 \\
\hline $00: 60$ & 374 & 78 & 25 & $31.3^{\dagger}$ & & & & 54.9 & 31 & -0.1 \\
\hline & 375 & 78 & 25 & 31.3 & 49.1 & 4.78[ & 583 & 54.8 & 31 & -0.1 \\
\hline & 376 & 78 & 25 & 31.3 & 49.1 & 4.78 & 580 & 54.8 & 32 & -0.2 \\
\hline 4:16:03:57 & 377 & 78 & 25 & 31.3 & 49.1 & 4.78 & 587 & 55 & 33 & -0.2 \\
\hline
\end{tabular}




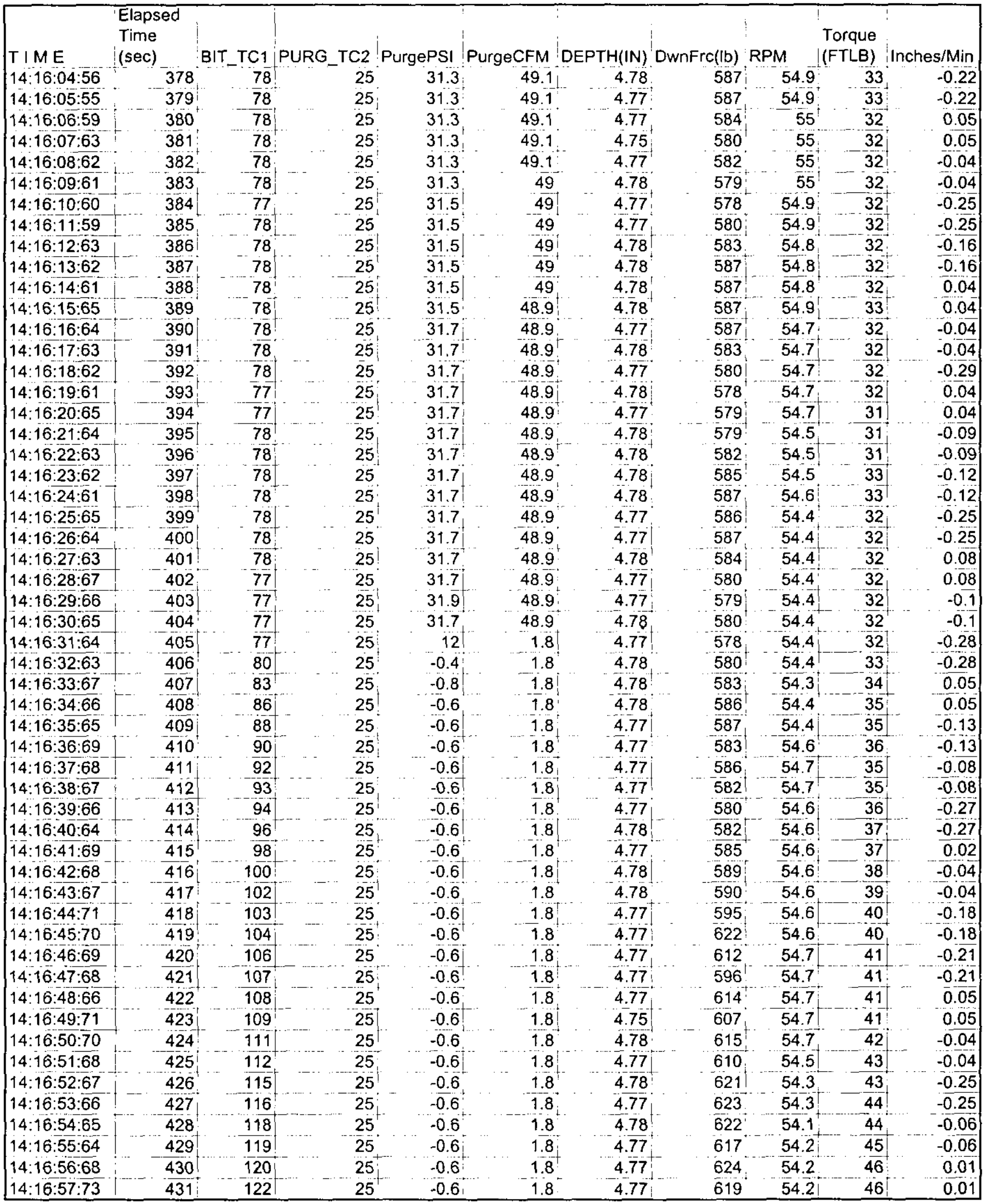




\begin{tabular}{|c|c|c|c|c|c|c|c|c|c|c|}
\hline$T / M E$ & $\begin{array}{l}\text { Elapsed } \\
\text { Time } \\
\text { (sec) }\end{array}$ & BIT_TC1 & PURG_TC2 & & PurgeCFM & DEPTH(IN) & DwnFrc(lb) & RPM & $\begin{array}{l}\text { Torque } \\
\text { (FTLB) }\end{array}$ & ies/Min \\
\hline $14: 16: 58: 72$ & $\quad 432$ & $\quad 123$ & 25 & -0.6 & 1.8 & 4.77 & $\quad 609$ & $\quad 54.1$ & 44 & -0.19 \\
\hline $14: 16: 59: 70$ & 433 & 124 & 25 & $-0.6 !$ & 1.8 & 4.78 & 592 & 54.1 & 45 & -0.19 \\
\hline $00: 69$ & 434 & 126 & 25 & -0.6 & $1 . \overline{8}$ & 4.78 & $5 \overline{9} 2$ & 54.1 & 46 & -0.15 \\
\hline $14: 17: 01: 68$ & 435 & 128 & 25 & -0.6 & 1.8 & 4.77 & 594 & 54 & 46 & -0.15 \\
\hline $14: 17: 02: 72$ & 436 & 130 & 25 & -0.6 & 1.8 & 4.78 & 598 & 54 & 471 & -0.04 \\
\hline $3: 71$ & 437 & 131 & $25^{\prime}$ & -0.6 & 1.8 & $4.78^{+}$ & 602 & 53.9 & 48 & -0.04 \\
\hline 470 & 438 & 133 & $25 !$ & $-0 . \overline{6}$ & 1.8 & 4.78 & 602 & 54 & 47 & -0.05 \\
\hline $14: 17: 05: 75$ & 439 & 134 & 25 & -0.6 & 1.8 & 4.77 & 599 & 54 & 47 & -0.32 \\
\hline $14: 17: 06: 73$ & 440 & 135 & 25 & -0.6 & 1.8 & 4.77 & 601 & 54.1 & 48 & -0.32 \\
\hline $7 \overline{72}$ & $441 !$ & 137 & 25 & -0.6 & 1.8 & 4.77 & 597 & 54.2 & 48 & 0.07 \\
\hline 71 & 442 & 138 & 25 & -0.6 & 1.8 & 4.77 & 594 & 54.2 & 48 & 0.07 \\
\hline $9: 70^{\circ}$ & 443 & 140 & 25 & -0.6 & 1.8 & 4.77 & 595 & 54.2 & 49 & -0.1 \\
\hline 74 & 444 & 142 & 25 & -0.6 & 1.8 & 4.78 & 599 & 54.1 & 49 & -0.1 \\
\hline & 445 & 144 & 25 & -0.6 & 1.8 & 4.78 & 607 & 54.1 & 50 & -0.18 \\
\hline $2: 72$ & 446 & $1 \overline{46}$ & 25 & -0.6 & 1.8 & 4.78 & 605 & 54.1 & 51 & -0.18 \\
\hline $3: 76$ & 447 & $\overline{146}$ & 25 & -0.6 & 1.8 & $4.78^{\dagger}$ & 609 & 54 & 51 & -0.22 \\
\hline & 448 & 148 & $25 !$ & -0.6 & 1.8 & 4.77 & 609 & 54 & 51 & -0.22 \\
\hline & 449 & 150 & 25 & $-0 . \overline{6}$ & $\overline{1} .8$ & 4.77 & 605 & 54 & 51 & $0.0 \overline{6}$ \\
\hline $8: 73$ & $45 \overline{0}$ & 151 & 25 & $-0 . \overline{6}$ & 1.8 & 4.77 & 602 & 54 & 51 & 0.06 \\
\hline 72 & 451 & 152 & 25 & -0.8 & 1.8 & 4.77 & 602 & 54 & 52 & -0.1 \\
\hline & 452 & 153 & $25^{\dagger}$ & -0.6 & 1.8 & 4.77 & 602 & 54.2 & 52 & -0.1 \\
\hline & 453 & 1551 & $25 !$ & -0.6 & 1.8 & $4.77^{+}$ & 603 & 54.3 & 53 & -0.26 \\
\hline & 454 & 158 & 25 & -0.6 & 1.8 & 4.78 & 606 & 54.3 & 54 & -0.26 \\
\hline & 455 & 160 & 25 & $-0 . \overline{6}$ & 1.8 & 4.78 & 610 & 54.6 & 54 & -0.05 \\
\hline & 456 & 162 & 25 & -0.6[ & 1.8 & 4.78 & 608 & $54.8^{\circ}$ & 54 & -0.05 \\
\hline & 457 & 162 & 25 & -0.6 & 1.8 & 4.77 & 606 & 54.8 & 55 & 0.01 \\
\hline & 458 & 164 & 25 & $-0.6^{+}$ & 1.8 & 4.77 & 608 & 55.1 & 55 & 0.01 \\
\hline & 459 & 166 & 25 & -0.6 & 1.8 & 4.78 & $\overline{6} 06$ & 55.3 & 54 & -0.12 \\
\hline & 460 & 167 & 25 & -0.6 & 1. & 4.77 & 603 & 55.3 & 55 & -0.12 \\
\hline & 461 & 168 & 25 & -0.6 & 1. & 4.77 & 601 & 55.4 & $5 \overline{5}^{\dagger}$ & -0.23 \\
\hline & 462 & $170^{\prime}$ & 25 & -0.6 & 1.8 & 4.78 & 600 & 55.4 & 56 & -0.07 \\
\hline & 463 & 172 & 25 & -0.6 & 1.8 & 4.78 & 601 & 55.4 & 56 & -0.07 \\
\hline & 464 & 174 & 25 & -0.6 & 1.8 & $4 . \overline{78}$ & 601 & 55.5 & 57 & -0.01 \\
\hline & 465 & $176 !$ & $25^{\dagger}$ & -0.6 & 1.8 & $4.78^{\prime}$ & 603 & 55.5 & 57 & -0.01 \\
\hline & 466 & 178 & 25 & -0.6 & 1. & 4.78 & $606^{\prime}$ & 5.5 & 59 & -0.09 \\
\hline & 46 & 17 & 25 & $-0.6 !$ & 1 & 4. & & & & -0.09 \\
\hline & 468 & 181 & 25 & -0.6 & 1. & 4.78 & 607 & 55.5 & 60 & -0.25 \\
\hline & 469 & $\overline{183}$ & 25 & -0.61 & 1.8 & 4.78 & 610 & 55.5 & 61 & -0.25 \\
\hline & 470 & 185 & 25 & -0.6 & 1. & & 612 & 55.5 & 62 & -0.1 \\
\hline & 471 & 187 & 25 & -0.6 & 1. & $4 . \overline{7} 8^{\dagger}$ & 610 & 55.5 & 62 & $-0 . \overline{1}$ \\
\hline & 472 & 188 & 25 & -0.6 & 1.8 & 4.77 & 606 & 55.5 & 63 & \\
\hline & 473 & 189 & $25 !$ & -0.6 & 1. & 4.78 & 604 & 55.5 & 62 & \\
\hline & 47 & 19 & 25 & -0. & 1 & 4. & & 55.3 & & -0.09 \\
\hline & 47 & 1 & 25 & $-\overline{0.6}$ & 1. & & $604^{\circ}$ & 55.3 & 63 & -0.09 \\
\hline 82 & 476 & 195 & 25 & -0.6 & 1. & $4.7 \overline{8}$ & 605 & 55 & 65 & -0.3 \\
\hline & 477 & $1 \overline{98}$ & 25 & -0.6 & 1. & 4.79 & 609 & 55 & 66 & -0.3 \\
\hline & 478 & 20 & 25 & -0.6 & 1. & 4.79 & 612 & 55 & 67 & 0.09 \\
\hline & 479 & 20 & $2 \overline{5}$ & -0.6 & 1 & & $610^{\circ}$ & 54.9 & 68 & $0.0 \overline{9}$ \\
\hline $17: 46: 77$ & $480^{-1}$ & 203 & 25 & -0.6 & 1. & 4.78 & 613 & 54.8 & 68 & -0.07 \\
\hline & 481 & 205 & 25 & -0.6 & 1 & 4.77 & 615 & 54.8 & 69 & -0.07 \\
\hline & 482 & 206 & 25 & -0.6 & 1 & 4.78 & 612 & 54.8 & 70 & -0.17 \\
\hline & 483 & $20 \overline{8}$ & 25 & -0.6 & 1. & $4 . \overline{8}$ & $609^{\circ}$ & 54.7 & 71 & -0.17 \\
\hline $7: 50: 84$ & 484 & 210 & 25 & -0.6 & 1.8 & 4.78 & 607 & 54.7 & 71 & -0.22 \\
\hline $14: 17: 51: 83$ & 485 & $212^{\dagger}$ & $25^{\dagger}$ & -0.6 & 1.8 & 4.78 & 609 & 54.6 & 72 & 0.08 \\
\hline
\end{tabular}




\begin{tabular}{|c|c|c|c|c|c|c|c|c|c|c|c|}
\hline TIME & $\begin{array}{l}\text { Elapsed } \\
\text { Time } \\
\text { (sec) }\end{array}$ & BIT_TC1 & PURG_TC2 & PurgePSI & PurgeCFM & DEPTH(IN) & DwnFrc(lb) & RPM & \multirow{3}{*}{$\begin{array}{l}\text { Torque } \\
\text { (FTLB) } \\
73 \\
\text { stenfs }\end{array}$} & \multirow{3}{*}{\multicolumn{2}{|c|}{$\begin{array}{r}\text { Inches/Min } \\
0.08\end{array}$}} \\
\hline $4: 175282$ & $\quad 486$ & 214 & 25 & $\quad-0.6$ & 1.8 & 4.78 & $\begin{array}{r}609 \\
0\end{array}$ & 545 & & & \\
\hline & & & & & tas & ode $=6$ & Squ & ing 4 & & & \\
\hline & & $=21 v^{2}$ & elope & $5 \mathrm{Q} 9$ & -53 & Revas & matter & Hanf & 77 & & -0.07 \\
\hline 58 & & & & 0 & 1.8 & 4.79 & 617 & 55.1 & 77 & & -0.24 \\
\hline $4: 17: 56: 83$ & Compa & स्यूकी & Nas & tgh. & 1.8 & 4.79 & $\overline{6} 19$ & 55.1 & 79 & & -0.24 \\
\hline $7: \overline{82}$ & $\begin{array}{l}1 \\
-491\end{array}$ & 226 & $\quad 25$ & $\quad-0.6$ & $\quad 1.8$ & 4.78 & 617 & 552 & 80 & & -0.12 \\
\hline 8 & on, $\bar{G} .192$ & 1528 & Witwer25 & $1996,-8.8 t$ & tandard .8 & Rotary $\$$ & mpers & turesst & ings & & -0.12 \\
\hline 79 & Supo 483 & $t 29$ & Self Asse & ssmerftof & for Rotáry & Mode $C$ & Sapto & lings $\mathrm{a} R$ & Flampa & able & 0.01 \\
\hline 84 & & & & $R^{2} \beta^{6}$ & Reg & Nês] & uŝ̉ं & Hanfol & Cogit & & $y_{1}^{0.01}$ \\
\hline 82 & Gas 459 & Went & finaton 25 & & & & & & & & $\begin{array}{l}3 \\
-0.02\end{array}$ \\
\hline 81 & & & & -0.6 & 1.8 & 4.77 & 612 & 55.1 & 84 & & -0.02 \\
\hline 86 & $\quad 497$ & 239 & 25 & -0.6 & 1.8 & 4.78 & 612 & 55.1 & 85 & & -0.24 \\
\hline $4: 18 \mathrm{n}^{4} 8 \mathrm{~s}$ & Tant & 242 & sis 位 & am, 1990 & 6. "A Saf & ety Asses & entods & Pusha & de 85 & ind & -0.24 \\
\hline $06: 82$ & Rotado & Modêt & Ftammab & ple Gas. & Single $\$$ t & Tank & NHE & D-W & $A D_{88}^{33}$ & & $\begin{array}{c}-0.1 \\
-0.1\end{array}$ \\
\hline & Rev 50! & 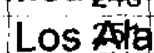 & mos Natho & al Labic & oratory & ios Alamo & Newab & 1exis:? & $\frac{00}{90}$ & & -0.01 \\
\hline & 502 & 252 & 25 & -0.6 & 1.8 & 4.78 & 622 & 55.2 & 91 & & -0.01 \\
\hline 84 & 503 & 255 & 25 & -0.6 & 1.8 & 4.77 & 623 & $55 . \overline{2}$ & 92 & & $-0.0 \overline{4}$ \\
\hline & 504 & 258 & 25 & -0.6 & 1.8 & 4.77 & 622 & 55.3 & 92 & & -0.0 \\
\hline & 505 & 281 & 25 & -0.6 & 1.8 & 4.78 & 619 & 55.1 & 93 & & 3.2 \\
\hline & 50 & 262 & 25 & -0.6 & 1.8 & 4.78 & 616 & 55.1 & 94 & & -0.14 \\
\hline & 507 & 265 & 25 & -0.6 & 1.8 & 4.77 & 616 & 55 & 94 & & -0.14 \\
\hline & 508 & 268 & 25 & -0.6 & 1.8 & 4.78 & 617 & 55 & 95 & & .04 \\
\hline & 509 & 272 & 25 & -0.6 & 1.8 & 4.78 & 617 & 55 & 96 & & 0.04 \\
\hline & 510 & $2 \overline{7} 4$ & 25 & -0.6 & 1.8 & 4.78 & 620 & 54.9 & 97 & & -0.06 \\
\hline & 511 & 277 & 25 & -0.6 & 1.8 & 4.78 & 624 & 54.9 & 98 & & -0.06 \\
\hline & 512 & 279 & 25 & -0.6 & 1.8 & 4.77 & 625 & 54.9 & 98 & & -0.22 \\
\hline & 513 & 282 & 25 & -0.6 & 1.8 & $4 . \overline{78}$ & 624 & 54.9 & 99 & & -0.22 \\
\hline & 514 & & 2 & -0.6 & 1.8 & 4.78 & 625 & 55.1 & 99 & & \\
\hline & 515 & 28 & 2 & $-0 . \bar{\epsilon}$ & 1.8 & 4.77 & 623 & 55.1 & 97 & & \\
\hline & 516 & 289 & 25 & -0.6 & 1.8 & 4.77 & 620 & 55.1 & 98 & & -0.04 \\
\hline & 517 & 292 & 25 & -0.6 & 1.8 & 4.78 & 617 & 55.1 & 99 & & -0.0 \\
\hline & 518 & 295 & 25 & -0.6 & 1.8 & 4.78 & 617 & 55 & $\overline{99}$ & & -0.07 \\
\hline & 519 & 298 & 25 & -0.6 & 1.8 & 4.78 & 619 & 55 & 99 & & $-0 . \overline{07}$ \\
\hline & 520 & & 25 & $-0.6^{-t}$ & 1.8 & 4.78 & 617 & 55 & 100 & & -0.27 \\
\hline & 521 & 304 & 25 & 9.1 & 49 & 4.78 & 617 & 55 & 97 & & -0.27 \\
\hline & 522 & 2 & 25 & 23.5 & 49.6 & 4.37 & 528 & 55 & 90 & & -0.08 \\
\hline & 523 & 280 & 25 & 22.4 & 51.9 & 2.39 & -759 & 55.2 & 2 & & -0 \\
\hline & 524 & & & & & & -7 & 55.2 & 1 & & 14.68 \\
\hline & 525 & 260 & 25 & & 54 & -1.41 & -759 & 55.8 & 1 & & -14.65 \\
\hline & 526 & $253^{\dagger}$ & 25 & $2 \overline{1.6}$ & 54 & -1.79 & -759 & 56.3 & 1 & & 39 \\
\hline & 527 & & 25 & & 54 & & -759 & 56.3 & 1 & 1 & \\
\hline & 528 & & 25 & 2 & $54 . \overline{2}$ & -1.76 & -759 & 57.3 & 1 & & \\
\hline 88 & 529 & $23 \overline{7}$ & 25 & 1.6 & 54.2 & -1.77 & -759 & 49.7 & 1 & & $-0.1 \overline{5}$ \\
\hline & 530 & 232 & 25 & 1.6 & $54 . \overline{2}$ & -1.77 & -759 & 49.7 & 1 & & -0.15 \\
\hline & & 2 & 25 & & 54.2 & -1.77 & -759 & 24.5 & $\overline{1}$ & & -0.14 \\
\hline & & & & & $5 \overline{4.2}$ & -1.77 & -759 & 11.3 & 1 & & \\
\hline$\overline{9} 89$ & 5 & 218 & 25 & 21.6 & 54.2 & -1.76 & -759 & 11.3 & 1 & & -0.11 \\
\hline 40.93 & 534 & 213 & 25 & 21.4 & 54.2 & -1.77 & -759 & 5.6 & 1 & & -0.11 \\
\hline & 5 & 208 & 25 & 214 & 54.2 & $-1 . \overline{7} 7$ & -759 & 3.2 & 1 & & -0.1 \\
\hline & & & & & 54.2 & -1.77 & .759 & 3.2 & 1 & & \\
\hline $3: 90$ & 537 & 200 & 25 & 21.4 & 54.2 & -1.76 & -759 & 1.9 & 1 & & -0.11 \\
\hline & 538 & 198 & 25 & 21.4 & 54.2 & -1.76 & 759 & 1.4 & 1 & & -0.11 \\
\hline $4: 18: 45: 93$ & 539 & 192 & 25 & 21.4 & 54.2 & -1.76 & -759 & 1.4 & 1 & & -0.11 \\
\hline
\end{tabular}




\begin{tabular}{|c|c|c|c|c|c|c|c|c|c|c|}
\hline$T I M E$ & $\begin{array}{l}\text { Elapsed } \\
\text { Time } \\
(s e c)\end{array}$ & BIT_TC1 & PURG_TC2 & PurgePsI & PurgeCFM & DEPTH(IN) & DwnFrc(lb) & $R P M$ & $\begin{array}{l}\text { Torque } \\
\text { (FTLB) }\end{array}$ & ches/Min \\
\hline $14: 18: 46: 92$ & $\quad 540$ & 189 & -25 & $\quad 21.4$ & $\quad 54.2$ & $\begin{array}{r}-1.76 \\
\end{array}$ & $\quad-759$ & $\quad 1.1$ & 1 & -0.11 \\
\hline $14: 18: \overline{47: 91}$ & 541 & 185 & 25 & 21.4 & 54.2 & -1.76 & -759 & 1 & 1 & -0.11 \\
\hline $14: 18: 48: 90$ & 542 & 182 & 25 & 21.4 & 54.2 & -1.76 & $-\overline{75} \overline{9}$ & 1 & 1 & -0.11 \\
\hline $14: 18: 49: 88$ & 543 & 179 & 25 & 21.4 & 54.2 & -1.77 & -759 & 1 & 1 & -0.11 \\
\hline $14: 18: 50: 93$ & 544 & 175 & $2 \overline{5}$ & 21.2 & 54.2 & -1.77 & -759 & 0.9 & 1 & -0.11 \\
\hline $14: 18: 51: 92$ & 545 & 172 & 25 & 21.2 & 54.2 & -1.79 & -759 & 0.9 & 1 & -0.11 \\
\hline $14: 18: 52: 91$ & 546 & 169 & 25 & 21.2 & 54.2 & -1.76 & -759 & 0.9 & 1 & -0.11 \\
\hline $14: 18: 53: 95$ & 547 & 166 & 25 & 21.2 & 54.2 & -1.77 & -759 & 0.9 & 1 & -0.71 \\
\hline $14: 18: 54: 94$ & 548 & 163 & 25 & 21.2 & 54.2 & -1.77 & -759 & 0.9 & 1 & $-0 . \overline{11}$ \\
\hline $14: 18: \overline{55: 93}$ & 549 & 160 & 25 & 21.2 & 54.2 & -1.77 & -759 & 0.9 & 1 & -0.11 \\
\hline $14: 18: 56: 91$ & 550 & 157 & 25 & 21.2 & 54.2 & -1.77 & -760 & 0.9 & 1 & -0.11 \\
\hline 90 & 551 & 156 & 25 & 21,2 & 54.2 & -1.77 & -760 & 0.9 & 1 & -0.11 \\
\hline $14: 18: 58: 95$ & 552 & 153 & 25 & 21.2 & 54.2 & -1.79 & -759 & 0.9 & 1 & $-0 . \overline{11}$ \\
\hline $14: 18: 59: 94$ & 553 & 150 & 25 & 21.2 & 54.2 & -1.76 & $-75 \overline{9}$ & 0.9 & 1 & -0.11 \\
\hline $14: 19: 00: 92$ & 554 & 147 & 25 & 21.21 & 54.2 & -1.77 & $-75 \overline{9}$ & 0.9 & 1 & -0.11 \\
\hline $14: 19: 01: 97$ & 555 & 145 & 25 & $21.2 i$ & 54.2 & -1.77 & -759 & 0.9 & 1 & -0.11 \\
\hline $14: 19: 02: 96$ & 556 & 142 & 25 & 21.2 & 54.2 & -1.76 & -759 & 0.9 & 1 & -0.11 \\
\hline $14: 19: 03: 95$ & 557 & 139 & 25 & 21.2 & 54.2 & -1.77 & -759 & 0.9 & $\overline{1}$ & -0.11 \\
\hline $14: 19: 04: 93$ & 558 & 137 & 25 & 21.2 & 54.2 & -1.76 & -759 & 0.9 & 1 & -0.11 \\
\hline $14: 19: 05: 92$ & 559 & 135 & 25 & 21.2 & 54.2 & -1.77 & -759 & 0.9 & 1 & -0.11 \\
\hline $14: 19: 06: 97$ & 560 & 132 & 25 & 21.2 & 54.2 & -1.79 & -759 & 0.9 & 1 & -0.11 \\
\hline $14: 19: 07: 95$ & 561 & 131 & 25 & 21.2 & 54.2 & -1.76 & -759 & 0.9 & 1 & -0.11 \\
\hline $14: 19: 08: 94$ & 562 & 128 & 25 & 21.2 & 54.2 & -1.77 & -759 & $0 . \overline{9}$ & 1 & -0.11 \\
\hline $14: 19: 09: 99$ & 563 & 126 & 25 & $21.2^{\dagger}$ & 54.2 & -1.76 & -759 & 0.9 & 1 & -0.11 \\
\hline $14: 19: 10: 98$ & 564 & 124 & 25 & $21.2^{\prime}$ & 54.2 & -1.76 & .759 & 0.9 & 1 & -0.11 \\
\hline $14: 19: 11: 96$ & 565 & 122 & 25 & 21.2 & 54.2 & -1.77 & -759 & 0.9 & 1 & -0.11 \\
\hline $14: 19: 12: 95$ & 566 & 120 & 25 & 21.2 & 54.2 & -1.76 & -759 & 0.9 & 1 & -0.11 \\
\hline $14: 19: 13: 94$ & $567 \vec{j}$ & 198 & 25 & 21.2 & 54.2 & -1.77 & -759 & 0.9 & 1 & -0.11 \\
\hline $14: 19: 14: 99$ & 568 & 116 & 25 & $21.2 !$ & 54.2 & -1.77 & -759 & 0.9 & 1 & -0.11 \\
\hline $14: 19: 15: 97$ & 569 & 114 & 25 & 21.2 & 54.2 & $-1.7 \overline{6}$ & -759 & 0.9 & 1 & -0.11 \\
\hline $14: 19: 16: 96$ & 570 & 113 & 25 & 21.2 & 54.2 & -1.77 & -759 & 0.9 & 1 & -0.11 \\
\hline $14: 19: 18: 01$ & 571 & 111 & 25 & 21.2 & 54.2 & -1.77 & -759 & 0.9 & 1 & -0.11 \\
\hline $14: 19: 18: \overline{99}$ & 572 & 109 & 25 & 21 & $54 . \overline{2}$ & -1.77 & -759 & $0 . \overline{9}$ & 1. & -0.11 \\
\hline $14: 19: 19: \overline{98}$ & 573 & 108 & 25 & 21 & 54.2 & -1.77 & -759 & 0.9 & 1 & -0.11 \\
\hline $14: 19: 20: 97$ & 574 & 106 & 25 & 21.2 & 54.2 & -1.76 & -759 & 0.9 & 1 & -0.11 \\
\hline $14: 19: 21: 96$ & $5 \overline{75}$ & 105 & 25 & 21.2 & 54.2 & $-1.7 \overline{7}$ & -759 & 0.9 & 1 & -0.11 \\
\hline $14: 19: 23: 00$ & 576 & 103 & 25 & 21 & 54.2 & -1.77 & -759 & 0.9 & 1 & -0.11 \\
\hline $14: 19: 23: 99$ & $57 \overline{7}$ & 101 & 25 & $21^{t+}$ & 54.2 & -1.77 & -759 & 0.9 & 1 & -0.11 \\
\hline $14: 19: 24: 98$ & 578 & 100 & 25 & 21 & 54.2 & -1.77 & $-760 !$ & 0.9 & 1 & $-0 . \overline{11}$ \\
\hline $14: 19: 25: 97$ & 579 & 98 & 25 & 21 & 54.2 & -1.76 & -759 & 0.9 & 0 & -0.11 \\
\hline & & & & & & & & & & \\
\hline
\end{tabular}




\begin{tabular}{|c|c|c|c|c|c|c|c|c|c|c|}
\hline TIME & $\begin{array}{l}\text { Elapsed } \\
\text { Time } \\
\text { (sec) }\end{array}$ & BIT_TC1 & PURG_TC2 & PurgePSI & PurgeCFM & DEPTH $(\mathrm{IN})$ & $w n F r c(\mid b)$ & RPM & $\begin{array}{l}\text { Torque } \\
\text { (FTLB) }\end{array}$ & Inches/Min \\
\hline $10: 13: 30: 40$ & 0 & -24 & $\quad 24$ & 21.2 & $\quad 55.2$ & -1.29 & 0 & 57.1 & 0 & -0.13 \\
\hline $10: 13: 31: 34$ & 1 & 24 & 24 & 21.2 & 55.2 & -1.1 & 0 & 57.1 & 0 & -0.13 \\
\hline $32: 32$ & 2 & 24 & 24 & 21.2 & 55.2 & -0.75 & 0 & 57.1 & 0 & 8.74 \\
\hline $3: 31$ & 3 & 24 & 24 & 21.2 & 55.2 & -0.37 & 2 & 56.6 & 1 & 8.74 \\
\hline $10: 13: 34: 30$ & 4 & $\overline{24}$ & 24 & 21.2 & 55.2 & -0.05 & 59 & 56.6 & $\overline{6}$ & 13.78 \\
\hline $10: 13: 35: 29$ & 5 & 32 & 24 & 22 & 55.2 & 0.05 & 861 & 56.4 & 47 & 13.78 \\
\hline $10: 13: 36: 28$ & 6 & 43 & 24 & 22.4 & 55.2 & 0.07 & 1003 & 55.9 & 48 & 4.43 \\
\hline $7: 27$ & 7 & 49 & 24 & 22.6 & 54.9 & 0.07 & 1044 & 55.9 & 44 & 4.43 \\
\hline $8: 26$ & 8 & 57 & 24 & 22.6 & 54.5 & 0.07 & 1066 & 55.4 & 45 & 0.68 \\
\hline $9: 24$ & 9 & 62 & 24 & 22.8 & 54.4 & 0.07 & 1086 & 55.2 & $\overline{43}$ & 0.68 \\
\hline$\overline{40: 23}$ & 10 & 60 & 24 & 22.8 & 54.3 & 0.07 & 1105 & 55.2 & 40 & $0 . \overline{67}$ \\
\hline $11: 22$ & 11 & 61 & 24 & 22.9 & 54.3 & 0.1 & $1121^{t}$ & 55.1 & 36 & 0.67 \\
\hline $2: 21$ & 12 & 62 & 24 & 22.9 & 54.1 & 0.1 & 1125 & 55.1 & 34 & 0.15 \\
\hline $3: 20$ & 13 & 64 & 24 & 22.9 & 54.1 & 0.11 & 1133 & 55.1 & 32 & $0.1 \overline{5}$ \\
\hline & 14 & 66 & 24 & 23.1 & 54.1 & 0.13 & 1153 & 55 & 33 & -0.17 \\
\hline $45: 18$ & 15 & 66 & 24 & 23.1 & 54 & 0.14 & 1168 & 55 & 33 & -0.17 \\
\hline $6: 17$ & 16 & 67 & 24 & 23.1 & 54 & 0.13 & 1174 & 55 & 32 & 0.02 \\
\hline$: 15$ & 17 & 68 & 24 & 23.1 & 54 & 0.11 & 1173 & 55 & 3 & 0.02 \\
\hline $8: 14$ & 18 & 68 & 24 & 23.1 & 53.9 & 0.11 & 1172 & 55 & 31 & 0.13 \\
\hline $0: 13$ & 19 & 69 & 24 & 23.1 & 53.9 & 0.13 & 1169 & 55 & 31 & 0.13 \\
\hline & 20 & 69 & 24 & 23.1 & 53.9 & 0.1 & $1 \overline{162}$ & 55 & 30 & -0.2 \\
\hline $1: 11$ & 21 & 71 & 24 & 23.1 & 53.9 & 0.13 & 1158 & 55 & 30 & -0.2 \\
\hline & 22 & 71 & 24 & 23.3 & 53.9 & 0.13 & 1159 & 55 & 29 & -0.15 \\
\hline & 23 & 72 & 24 & 23.3 & 53.9 & 0.14 & 1161 & 54.9 & 29 & -0.15 \\
\hline & 24 & 73 & 24 & 23.3 & 53.9 & 0.14 & 1158 & 54.9 & 28 & 0.08 \\
\hline & 25 & $7 \overline{3}$ & 24 & 5 & 53.9 & 0.14 & 1162 & 54.9 & 28 & 0.08 \\
\hline$: 05$ & 26 & $\overline{74}$ & 24 & 23.5 & 53.9 & 0.13 & 1168 & 54.9 & 27 & 0.01 \\
\hline $7: 04$ & 27 & 74 & 24 & 23.5 & 53.8 & 0.14 & 1173 & 54.9 & 26 & -0.21 \\
\hline & 28 & 74 & 24 & 23.5 & 53.8 & 0.13 & 1174 & 54.9 & 26 & -0.21 \\
\hline & 29 & 74 & 24 & 23.7 & 53.8 & 0.13 & 1172 & 54.8 & 26 & -0.23 \\
\hline & 30 & 75 & 24 & 23.7 & 53.8 & 0.13 & 1169 & 54.9 & 26 & $-0 . \overline{33}$ \\
\hline & 31 & 75 & 24 & $23 . \overline{7}$ & 53.8 & 0.13 & 1171 & 54.9 & 26 & 0.04 \\
\hline & 32 & 76 & 24 & 23.7 & 53.8 & 0.13 & 1171 & 54.9 & 26 & 0.04 \\
\hline & 33 & 76 & 24 & 23.7 & 3.8 & 14 & 1174 & 54.9 & 26 & 0.06 \\
\hline & 34 & $\overline{7} 6$ & 24 & 23.9 & 3.7 & & 1178 & 54.9 & 26 & 0.06 \\
\hline & 35 & 77 & 24 & 23.9 & 53.7 & 0.14 & 1182 & 55 & 26 & -0.22 \\
\hline 94 & 36 & 77 & 24 & 23.9 & 53.7 & 0.14 & 1184 & 55 & 25 & -0.22 \\
\hline $6: 93$ & 37 & $7 \overline{7}$ & 24 & 23.9 & 53.7 & 0.14 & 1184 & 55 & 25 & -0.19 \\
\hline 92 & $3 \overline{8}$ & 77 & 24 & 23.9 & 53.7 & $\overline{0.13}$ & 1181 & 55 & 25 & -0.19 \\
\hline & 39 & 77 & 24 & 24.1 & $53 . \overline{7}$ & 13 & 1176 & 55.1 & 25 & 0.07 \\
\hline & 40 & 77 & 24 & 24.1 & 53.7 & & 1175 & 55.1 & 24 & 0.07 \\
\hline & 41 & 78 & 24 & 24.1 & 53.7 & & 1175 & 55.2 & 24 & -0.05 \\
\hline & 42 & 79 & 24 & 24.1 & 53.7 & & 1174 & 55.2 & 24 & -0.05 \\
\hline $2: 8 \overline{6}$ & $\overline{43}$ & 79 & 24 & 24.1 & 53.7 & 0.15 & 1178 & 55.1 & 25 & -0.21 \\
\hline $13: 85$ & $\overline{44}$ & 79 & 24 & 24.1 & 53.7 & 0.15 & 1183 & 55.1 & 24 & -0.21 \\
\hline $4: 14: 84$ & 45 & 79 & 24 & 24.3 & 53.7 & 0.15 & 1186 & 55.1 & 25 & -0.25 \\
\hline $1: 15: 82$ & 46 & $\overline{79}$ & 24 & 24.3 & 53.7 & $0 . \overline{14}$ & 1186 & 55.2 & 25 & -0.25 \\
\hline & $4 \overline{7}$ & 80 & 24 & $24 . \overline{3}$ & 53.7 & 0.14 & 1184 & 55.3 & 25 & 0.09 \\
\hline & 48 & 79 & 24 & 24. & 53.7 & $0.1 \overrightarrow{4}$ & 1181 & 55.3 & 25 & 0.09 \\
\hline & 49 & 79 & 24 & 24.3 & .7 & & 1178 & 55.3 & 25 & -0.01 \\
\hline & 50 & 79 & 24 & 24.3 & 53.7 & & 1181 & 55.2 & 25 & -0.01 \\
\hline $10: 14: 20: 77$ & 51 & 80 & 24 & 24.3 & 53.7 & 0.14 & 1179 & 55.2 & 25 & -0.27 \\
\hline
\end{tabular}




\begin{tabular}{|c|c|c|c|c|c|c|c|c|c|c|}
\hline TIME & $\begin{array}{l}\text { Elapsed } \\
\text { Time } \\
\text { (sec) }\end{array}$ & $\mathrm{BIT}+\mathrm{TC} 1$ & PURG_TC2 & PurgePSI & PurgeCFM & DEPTH $(I N)$ & DwnFrc(lb) & RPM & $\begin{array}{l}\text { Torque } \\
\text { (FTLB) }\end{array}$ & Inches/Min \\
\hline $10: 14: 21: 76$ & $\begin{array}{l}52 \\
1\end{array}$ & -80 & 24 & $\quad 24.3$ & 53.7 & 0.15 & 1181 & 55.2 & $\quad 25$ & -0.27 \\
\hline $10: 14: 22: 75$ & 53 & $8 \overline{1}$ & 24 & 24.3 & 53.7 & 0.15 & 1186 & 55.1 & 25 & -0.21 \\
\hline $10: 14: 23: 73$ & 54 & 81 & 24 & 24.5 & 53.7 & 0.16 & 1189 & 55.1 & 25 & -0.21 \\
\hline$: 14: 24: 72$ & 55 & 81 & 24 & 24.5 & 53.7 & 0.15 & 1190 & $55.1^{\circ}$ & 24 & 0.1 \\
\hline $10: 14: 25: 71$ & 56 & 81 & 24 & 24.7 & 53.7 & 0.14 & 1188 & $55.1^{t}$ & 24 & 0.1 \\
\hline $10: 14: 26: 70$ & 57 & 81 & 24 & 24.7 & 53.7 & 0.14 & 1183 & 55.1 & 24 & -0.03 \\
\hline $10: 14: 27: 69$ & 58 & 81 & 24 & 24.7 & 53.7 & 0.14 & 1185 & $55.1 !$ & 24 & -0.03 \\
\hline $28: 68$ & 59 & 81 & 24 & 24.7 & 53.7 & 0.14 & 1180 & 55.11 & 24 & -0.31 \\
\hline 67 & 60 & 81 & 24 & 24.7 & 53.7 & 0.14 & 1174 & 55.1 & 23 & -0.31 \\
\hline $0: \overline{65}$ & $61^{-1}$ & 81 & 24 & $24.7^{\prime}$ & 53.7 & $0 . \overline{14}$ & $1173^{+}$ & 55.2 & 24 & -0.15 \\
\hline $10: 14: 31: 64$ & 62 & $8 \overline{1}$ & 24 & 24.7 & 53.7 & 0.15 & 1174 & 55.2 & 24 & 0.09 \\
\hline $10: 14: 32: 63$ & 63 & $\overline{8} 2$ & 24 & 24.7 & 53.7 & 0.15 & $1179^{\dagger}$ & 55.2 & 24 & 0.09 \\
\hline$: 62$ & 64 & 82 & 24 & $24.7 \mid$ & 53.7 & 0.15 & 1181 & $55.2^{-4}$ & 24 & -0.1 \\
\hline 61 & $65^{\circ}$ & 82 & 24 & $24.7 !$ & $53 . \overrightarrow{7}$ & 0.15 & 1182 & $55.1^{+}$ & $23 !$ & -0.1 \\
\hline 60 & 66 & 82 & 24 & 24.7 & 53.7 & 0.15 & 1181 & 55.1 & 23 & -0.23 \\
\hline $5: 59$ & $\overline{67 !}$ & $8 \overline{2}$ & 24 & 24.7 & 53.7 & 0.15 & 1180 & 55 & $23 !$ & $-0 . \overline{2} 3$ \\
\hline $77: 58$ & 68 & 82 & 24 & 24.7 & 53.7 & 0.14 & 1178 & 55.1 & 23 & -0.14 \\
\hline $3: 56$ & 69 & 81 & 24 & 24.7 & 53.7 & 0.14 & 1173 & 55.1 & 23 & -0.14 \\
\hline 55 & $70 !$ & 81 & 24 & 24.7 & 53.7 & 0.14 & $70^{\dagger}$ & 55.2 & 23 & 0.13 \\
\hline & 71 & 81 & 24 & $24.7^{\circ}$ & 53.7 & 0.15 & $1170^{\circ}$ & 55.2 & $22 !$ & 0.13 \\
\hline & 72 & 82 & 24 & 24.7 & 53.7 & 0.15 & 1173 & $55.2^{\prime}$ & $23 !$ & -0.15 \\
\hline $42: 52$ & 73 & 82 & 24 & 24.7 & 53.7 & 0.15 & 1177 & 55.1 & 23 & -0.15 \\
\hline $3: 51$ & 74 & 82 & 24 & 24.7 & 53.7 & 0.16 & 1178 & 55.1 & 23 & -0.27 \\
\hline $1: 50$ & $75^{\circ}$ & $\overline{82}$ & 24 & $24.7^{1}$ & 53.7 & 0.15 & 1176 & $55.1 !$ & 22 & -0.27 \\
\hline 48 & $76^{\dagger-}$ & 82 & 24 & 24.7 & 53.71 & 0.15 & 1176 & 55.2 & 22 & -0.12 \\
\hline 47 & 77 & 81 & 24 & 24.7 & 53.7 & 0.15 & 1174 & 55.3 & 22 & -0.12 \\
\hline 46 & $\overline{78}$ & 81 & 24 & 24.5 & 53.6 & 0.14 & $1169^{\circ}$ & 55.3 & 22 & 0.09 \\
\hline $4 \overline{5}$ & $7 \overline{9}$ & 81 & 24 & 24.7 & 53.6 & 0.14 & 1164 & 55.3 & 23 & 0.09 \\
\hline 9.44 & 80 & 81 & 24 & 24.5 & 53.6 & 0.14 & 1163 & 55.3 & 22 & -0.12 \\
\hline $0: 43$ & $81 j$ & 82 & $24^{\dagger}$ & 24.5 & 53.6 & $0 . \overline{14}$ & $1164^{\prime}$ & $5 \overline{5.3}$ & 22 & -0.12 \\
\hline & 82 & 82 & 24 & 24.5 & 53.6 & 0.15 & 1167 & $55.3^{+}$ & 22 & $-0 . \overline{25}$ \\
\hline 41 & 83 & 82 & 24 & 24.5 & 53.6 & 0.15 & 1163 & 55.2 & $2 \overline{1}$ & -0.25 \\
\hline & $8 \overline{4}$ & 82 & 24 & 24.7 & 53.5 & 0.14 & 1167 & 55.2 & 22 & -0.15 \\
\hline 38 & 85 & 82 & 24 & 24.7 & 53.5 & 0.15 & $1170 !$ & 55.2 & 22 & -0.15 \\
\hline & 86 & 81 & $24^{i}$ & $24.7^{7}$ & 53.5 & 0.15 & 1171 & 55.2 & 22 & 0.11 \\
\hline & 87 & 81 & $24 i$ & 24 & & & & 55.3 & $2 \overrightarrow{3}$ & 0.11 \\
\hline & 88 & 81 & 24 & & 53.4 & & & 55.3 & 22 & -0.13 \\
\hline $3: \overline{34}$ & 89 & $8 \overline{1}$ & 24 & $24.7^{\prime}$ & $5 \overline{3} .4$ & 0.14 & $1160^{\circ}$ & 55.3 & 22 & -0.13 \\
\hline 33 & 90 & 81 & 24 & $24.7^{\prime}$ & $53.4 !$ & 0.14 & $1159 \mathrm{i}$ & 55.4 & 23 & -0.3 \\
\hline 31 & 91 & 81 & 24 & 24.7 & 53.4 & 0.15 & 1161 & 55.5 & 23 & -0.3 \\
\hline & 92 & 82 & 24 & 24.7 & 53.4 & 0.15 & 1162 & 55.5 & 23 & -0.1 \\
\hline & 93 & 82 & 24 & 24.7 & & $0 . \overline{15}$ & & 55.4 & $23 j$ & -0.1 \\
\hline & $9 \overline{4}$ & 82 & 24 & 24.7 & 53.4 & 0. & 11 & 55.2 & 23 & 0.11 \\
\hline & 95 & 82 & 24 & 24 & 53.4 & 0.15 & 1174 & 55.2 & 23 & -0.14 \\
\hline 26 & 96 & 82 & 24 & 24.7 & 53.4 & $\overline{0.14}$ & 1176 & 55.2 & 22 & -0.14 \\
\hline $06: 25$ & 97 & 82 & 24 & 24.7 & 53.4 & 0.14 & 1176 & 55.3 & 22 & -0.27 \\
\hline $07: 24$ & 98 & 81 & 24 & 24.7 & 53.4 & 0.15 & 1172 & 55.3 & 22 & -0.27 \\
\hline $10: 15: 08: 22$ & $99^{\top}$ & 81 & 24 & 24.7 & 53.4 & 0.14 & 1169 & 55.4 & 22 & -0.12 \\
\hline $10: 15: 09: 21$ & 100 & 81 & 24 & 24.7 & 53.4 & 0.14 & 1173 & 55.6 & 22 & -0.12 \\
\hline $5: 10: 20$ & 101 & 82 & 24 & 24.7 & 53.3 & 0.15 & 1169 & 55.6 & 22 & 0.1 \\
\hline $1: 19$ & 102 & 82 & 24 & $24 . \overline{7}$ & 53.3 & 0.15 & 1170 & 55.6 & 23 & 0.1 \\
\hline $10: 15: 12: 18$ & 103 & 81 & 24 & $24.7^{\dagger}$ & 53.3 & $0 . \overline{15}$ & $1173 i$ & $5 \overline{5.7}$ & 23 & -0.05 \\
\hline
\end{tabular}




\begin{tabular}{|c|c|c|c|c|c|c|c|c|c|c|}
\hline TIME & $\begin{array}{l}\text { Elapsed } \\
\text { Time } \\
\text { (sec) }\end{array}$ & BIT_TC1 & PURG_TC2 & PurgePSI & PurgeCFM & DEPTH(IN) & DwnFrc(lb) & $R P M$ & $\begin{array}{l}\text { Torque } \\
\text { (FTLB) }\end{array}$ & Inches/Min \\
\hline $10: 15: 13: 17$ & $\quad 104$ & 82 & 24 & $\quad 24.7$ & $\quad 53.3$ & 0.16 & 1177 & 55.7 & 23 & $\quad-0.05$ \\
\hline $10: 15: 14: 16$ & 105 & 82 & $24^{\circ}$ & 24.7 & 53.3 & 0.16 & $1179^{\circ}$ & 55.6 & 23 & $-\overline{0.26}$ \\
\hline $10: 15: 15: \overline{14}$ & 106 & 82 & 24 & 24.7 & 53.3 & 0.16 & 1180 & 55.6 & 23 & $-0 . \overline{26}$ \\
\hline $10: 15: 16: 13$ & 107 & 82 & 24 & 24.7 & 53.3 & 0.16 & 1179 & 55.6 & 23 & 0.01 \\
\hline $10: 15: 17: 12$ & $108^{\circ}$ & 82 & 24 & 24.7 & 53.3 & 0.15 & 1180 & 55.4 & 22 & 0.01 \\
\hline 10:15:18:11 & 109 & $82^{-1}$ & 24 & 24.7 & 53.3 & 0.15 & $1178^{\circ}$ & 55.4 & 22 & -0.08 \\
\hline $10: 15: 19: 10$ & $110^{\circ}$ & 81 & 24 & 24.7 & 53.3 & 0.14 & 1174 & 55.4 & 22 & $-0 . \overline{08}$ \\
\hline $10: 15: 20: 09$ & 111 & 81 & 24 & 24.7 & 53.2 & 0.15 & 1171 & 55.4 & 22 & -0.06 \\
\hline $10: 15: 21: 08$ & 112 & 81 & 24 & 24.7 & 53.2 & 0.15 & 1171 & 55.4 & 22 & -0.06 \\
\hline $10: 15: 22: 06$ & 113 & 82 & 24 & 24.7 & 53.2 & 0.15 & 1174 & 55.4 & 22 & -0.24 \\
\hline $10: 15: 23: 05$ & 114 & 82 & 24 & 24.9 & 53.2 & 0.16 & 1179 & 55.4 & 22 & -0.24 \\
\hline $10: 15: 24: 04$ & 115 & 82 & 24 & 24.9 & 53.2 & 0.16 & 1182 & 55.3 & 22 & -0.18 \\
\hline $10: 15: 25: 03$ & 116 & 83 & 24 & 24.9 & 53.2 & 0.15 & 1181 & 55.3 & 22 & -0.18 \\
\hline $10: 15: 26: 02$ & 117 & 83 & 24 & 24.9 & 53.2 & 0.15 & 1183 & 55.3 & 22 & 0.1 \\
\hline $10: 15: 27: 01$ & 118 & 83 & 24 & 24.9 & 53.2 & 0.15 & 1183 & 55.3 & 22 & 0.1 \\
\hline $10: 15: 28: 00$ & 119 & 81 & 24 & 24.9 & $5 \overline{3} .2$ & 0.15 & 1180 & 55.3 & 22 & -0.02 \\
\hline $10: 15: 28: 99$ & 120 & $\overline{81}$ & 24 & 24.7 & 53.2 & 0.15 & 1176 & 55.2 & 22 & -0.02 \\
\hline $10: 15: 29: 97$ & 121 & 81 & 24 & 24.7 & 53.2 & 0.16 & 1173 & 55.2 & 22 & $-0 . \overline{32}$ \\
\hline $10: 15: 30: 96$ & 122 & 82 & 24 & 24.5 & 53.2 & 0.15 & 1174 & 55.2 & 22 & -0.32 \\
\hline $10: 15: 31: 95$ & 123 & 82 & 24 & 24.5 & 53.2 & 0.16 & 1178 & 55 & 22 & -0.13 \\
\hline $32: 94$ & 124 & 82 & 24 & 24.5 & 53.1 & 0.16 & 1181 & 55 & 22 & -0.13 \\
\hline $10: 15: 33: 93$ & 125 & 83 & 24 & 24.7 & 53.1 & 0.16 & 1179 & 55 & 22 & 0.08 \\
\hline $10: 15: 34: 92$ & 126 & 83 & $24 !$ & 24.7 & 53 & 0.16 & 1181 & 55 & 22 & 0.08 \\
\hline $5: 35: 91$ & 127 & 82 & 24 & 24.7 & 53 & 0.15 & 1183 & 55 & 22 & -0.05 \\
\hline $5: 36: 89$ & 128 & 81 & 24 & 24.7 & 53 & 0.14 & 1179 & 55 & 22 & -0.32 \\
\hline $77: 88$ & 129 & 81 & 24 & 24.7 & 53 & 0.14 & 1175 & 55 & 21 & -0.32 \\
\hline $5: 38: 87$ & 130 & 81 & 24 & 24.7 & 53 & 0.14 & 1170 & 55 & 21 & -0.13 \\
\hline $10: 15: 39: 86$ & 131 & 81 & 24 & 24.7 & 53 & 0.16 & 1170 & 54.9 & 21 & -0.13 \\
\hline $10: 15: 40: 85$ & 132 & 81 & 24 & 24.7 & 53 & 0.15 & 1173 & 54.9 & 22 & 0.13 \\
\hline $41: 84$ & 133 & 81 & 24 & 24.7 & 52.9 & 0.16 & 1171 & 54.9 & 22 & $0 . \overline{13}$ \\
\hline $42: 83$ & 134 & 82 & 24 & 24.7 & 52.9 & 0.18 & 1174 & 54.9 & 22 & -0.18 \\
\hline $5: 43: 82$ & $1 \overline{35}$ & 82 & 24 & 24.7 & 52.9 & 0.16 & 1179 & 54.8 & 22 & -0.18 \\
\hline $10: 15: 44: 80$ & 136 & 82 & 24 & 24.7 & 52.9 & 0.16 & 1178 & 54.8 & 22 & -0.27 \\
\hline $10: 15: 45: 79$ & 137 & 81 & 24 & 24.7 & 52.9 & 0.16 & 1176 & 54.8 & 22 & -0.27 \\
\hline $10: 15: 46: 78$ & 138 & 81 & 24 & 24.7 & 52.9 & 0.16 & 1171 & 54.9 & 21 & -0.09 \\
\hline $10: 15: 47: 77$ & 139 & 81 & 24 & 24.7 & 52.8 & 0.15 & 1166 & 54.9 & 21 & -0.09 \\
\hline $10: 15: 48: 76$ & 140 & 81 & 24 & 24.7 & 52.8 & 0.15 & 1164 & 54.9 & 21 & 0.13 \\
\hline $10: 15: 49: 75$ & 141 & 81 & 24 & 24.7 & 52.8 & 0.15 & 1163 & 54.8 & 21 & 0.13 \\
\hline $10: 15: 50: 74$ & 142 & 81 & 24 & 24.7 & 52.8 & 0.16 & 1164 & 54.8 & 21 & -0.27 \\
\hline $10: 15: 51: \overline{72}$ & 143 & 81 & 24 & 24.9 & 52.8 & 0.16 & 1168 & 54.7 & 21 & -0.27 \\
\hline $10: 15: 52: 71$ & 144 & $8 \overline{1}$ & 24 & 24.9 & 52.8 & 0.16 & 1172 & 54.6 & 21 & -0.21 \\
\hline $53: 70$ & 145 & 81 & 24 & 24.9 & 52.8 & 0.16 & 1173 & 54.6 & 21 & -0.21 \\
\hline $4: 69$ & 146 & 81 & 24 & 24.9 & 52.8 & $0.1 \overline{6}$ & 1171 & 54.8 & 21 & -0.07 \\
\hline $5: 55: 68$ & 147 & 81 & 24 & 24.9 & 52.8 & 0.14 & 1168 & 55 & 21 & $-0 . \overline{07}$ \\
\hline $10: 15: 56: 67$ & 148 & 81 & 24 & 24.9 & 52.8 & 0.15 & 1164 & 55 & 21 & 0.06 \\
\hline $10: 15: 57: 66$ & 149 & 80 & 24 & 24.7 & 52.8 & 0.15 & 1161 & 55.1 & 21 & 0.06 \\
\hline $10: 15: 58: 65$ & 150 & 80 & 24 & 24.7 & 52.8 & $0 . \overline{15}$ & 1162 & 55.2 & 21 & -0.27 \\
\hline $10: 15: 59: 63$ & 151 & 80 & 24 & 24.7 & 52.8 & 0.16 & 1160 & 55.2 & 21 & -0.27 \\
\hline $10: 16: 00: 62$ & 152 & 81 & 24 & 24.7 & 52.8 & 0.16 & 1164 & 55.3 & 22 & -0.17 \\
\hline $10: 16: 01: 61$ & 153 & 81 & 24 & 24.7 & 52.8 & 0.16 & 1168 & 55.2 & 21 & -0.17 \\
\hline $10: 16: 02: 60$ & 154 & 81 & 24 & 24.7 & 52.8 & 0.16 & 1173 & 55.2 & 21 & -0.02 \\
\hline $10: 16: 03: 59$ & 155 & 81. & 24 & 24.9 & 52.8 & 0.15 & 1174 & 55.2 & 21 & -0.02 \\
\hline
\end{tabular}




\begin{tabular}{|c|c|c|c|c|c|c|c|c|c|c|}
\hline TIME & $\begin{array}{l}\text { iElapsed } \\
\text { Time } \\
\text { (sec) }\end{array}$ & BIT_TC1 & PURG_TC2 & PurgePSI & PurgeCFM & DEPTH(IN) & DwnFrc(lby & RPM & $\begin{array}{l}\text { Torque } \\
\text { (FTLB) }\end{array}$ & Inches/Min \\
\hline $10: 16: 04: 58$ & $\quad 156$ & $\quad 81$ & 24 & 24.9 & 52.8 & 0.16 & 1176 & 55.2 & $\quad 21$ & $\quad 0.04$ \\
\hline $10: 16: 05: 57$ & 157 & 81 & 24 & 24.9 & $52.8^{\dagger}$ & 0.14 & $1173^{\prime}$ & 55.2 & 21 & 0.04 \\
\hline $10: 16: 06: 55$ & 158 & 80 & 24 & 24.9 & 52.8 & 0.15 & $1177^{\circ}$ & 55.3 & 21 & -0.29 \\
\hline $10: 16: 07: 54$ & $159^{\circ}$ & 80 & 24 & 24.9 & 528 & 0.15 & 1173 & 55.3 & 21 & -0.29 \\
\hline $10: 16: 08: 53$ & 160 & 80 & 24 & 24.9 & 52.8 & 0.15 & 1171 & 55.3 & 21 & -0.23 \\
\hline $10: 16: 09: 52$ & 161 & 80 & 24 & 24.9 & 52.8 & 0.16 & $1171:$ & 55.3 & 21 & 0.01 \\
\hline $10: 16: 10: 51$ & 162 & 80 & 24 & 24.9 & 52.8 & 0.16 & 1175 & 55.3 & 21 & 0.01 \\
\hline $1: 50$ & 163 & 80 & 24 & 24.9 & $52.8^{1}$ & 0.18 & 1179 & 55.3 & 21 & $0.0 \overline{4}$ \\
\hline$\overline{49}$ & $\overline{164}$ & 81 & 24 & 24.9 & 52.8 & 0.16 & 1183 & 55.2 & 21 & 0.04 \\
\hline 48 & 165 & 81 & 24 & 24.9 & 52.8 & 0.16 & 1183 & 55 & 21 & -0.23 \\
\hline$: 16: 14: 46$ & 166 & 80 & 25 & 24.9 & $52.8^{i}$ & 0.16 & $11 \overline{4}$ & 55 & 21 & $-0 . \overline{23}$ \\
\hline $10: 16: 15: 45$ & 167 & 80 & 25 & 24.9 & 52.7 & 0.15 & 1184 & 55.1 & 21 & -0.24 \\
\hline $10: 16: 16: 44$ & 168 & 80 & 25 & 24.9 & 52.7 & 0.16 & 1183 & 55.1 & 21 & -0.24 \\
\hline $7: 43$ & $169^{\circ}$ & 80 & 24 & 24.9 & $52.7 i$ & 0.16 & 1177 & 55.1 & 21 & 0.03 \\
\hline 42 & 770 & 80 & 25 & 24.9 & 52.7 & 0.16 & 1175 & 55.1 & 21 & 0.03 \\
\hline 41 & 171 & 80 & 25 & 24.9 & 52.7 & 0.16 & 1179 & 55.1 & 21 & 0.01 \\
\hline $2 \overline{0: 40}$ & $1 \overline{72}$ & 80 & 25 & 24.9 & 52.7 & 0.16 & 1182 & 55.1 & $\overline{21}$ & $0 . \overline{01}$ \\
\hline $0: 16: 21: 38$ & 173 & 80 & 25 & 24.9 & 52.7 & 0.18 & 1185 & 55.1 & 21 & -0.3 \\
\hline $22: 37$ & 174 & 80 & 25 & 24.9 & 52.7 & 0.16 & 1187 & 55.1 & 21 & -0.3 \\
\hline $3: 36$ & 175 & $8 \overline{1}$ & 24 & 24.9 & 52.7 & 0.16 & $1185^{\dagger}$ & 55.1 & $21 i$ & -0.05 \\
\hline 35 & 176 & 81 & 25 & 24.9 & 52. & 0.16 & 1187 & 55.1 & 21 & -0.05 \\
\hline 34 & 177 & 80 & 24 & 24.9 & & 0.15 & 1185 & 55.1 & 21 & 0.03 \\
\hline$: 16: 26: 33$ & 178 & 79 & 25 & 24.9 & 52.6 & 0.15 & 1182 & 55 & 21 & $0.0 \overline{3}$ \\
\hline$: 27: 32$ & 179 & 79 & 25 & 24.9 & 52.6 & 0.16 & 1178 & 55 & 22 & -0.12 \\
\hline $8: 31$ & 180 & 79 & 24 & 24.9 & 52.6 & 0.16 & 1178 & 55 & 22 & -0.12 \\
\hline & 181 & 80 & 25 & 24.9 & 52.6 & 0.16 & 1182 & 55.1 & 22 & -0.27 \\
\hline 28 & 182 & 80 & 24 & 24.9 & $52.6^{\circ}$ & 0.18 & 1186 & 55.2 & 22 & $-0 . \overline{27}$ \\
\hline & $18 \overline{3}$ & 80 & 24 & 24.9 & 52.6 & 0.16 & 1184 & 55.2 & 22 & -0.2 \\
\hline 26 & 184 & 81 & 24 & 24.9 & 52.6 & 0.18 & $11 \overline{88}$ & 55.2 & 22 & -0.2 \\
\hline $3: 25$ & 185 & 81 & 24 & 24.9 & 52.6 & 0.16 & 1189 & $55.1^{\dagger}$ & 21 & 0.1 \\
\hline$: 24$ & $1 \overrightarrow{86}$ & 81 & 24 & 24.7 & 5 & 0.15 & 1189 & 55.1 & 21 & 0.1 \\
\hline 23 & 187 & 80 & 24 & 24.7 & 52. & 0.15 & 1186 & 55.1 & 21 & -0.09 \\
\hline 21 & 188 & 80 & 24 & 24.7 & 52.6 & 0.14 & 1181 & 55.1 & 21 & $-0 . \overline{09}$ \\
\hline 20 & 189 & 80 & 24 & 24.7 & 52.6 & 0.16 & 1179 & 55.1 & $2 \overrightarrow{1}$ & -0.32 \\
\hline 3:19 & 190 & 80 & 24 & 24.7 & 52.6 & 0.16 & $1181 !$ & 55.1 & 21 & -0.32 \\
\hline & 191 & 80 & 24 & 24.7 & 52.4 & 0.18 & $1180^{\circ}$ & 55 & 22 & -0.03 \\
\hline & $1 \overline{92}$ & 80 & 24 & 24.7 & & 0.18 & & 55 & 22 & -0.03 \\
\hline & 193 & 81 & 25 & 24.7 & & & 1186 & $54 . \overline{9}$ & 22 & 0.03 \\
\hline $42: 15$ & $19 \overline{4}$ & 81 & 25 & 24.7 & 52.2 & 0.18 & 1191 & 55 & 21 & $-\overline{0.16}$ \\
\hline $43: 14$ & 195 & 81 & 24 & 24.7 & 52.2 & 0.16 & 1188 & 55 & 21 & -0.16 \\
\hline $6: 44: 12$ & 196 & 80 & 25 & 24.7 & 52.2 & 0.16 & 1186 & 55.1 & 21 & $-0 . \overline{29}$ \\
\hline $5: 11$ & 197 & 80 & 24 & 24.7 & 52.1 & $0 . \overline{1} 6$ & 1181 & 55.1 & 21 & -0.29 \\
\hline & 198 & 80 & 24 & 24.7 & 52.1 & 0.1 & 1177 & 55.1 & 21 & -0.12 \\
\hline & 199 & 80 & 24 & 24.7 & 52 & 0.1 & & 55.1 & 21 & -0.12 \\
\hline & 200 & 80 & 24 & & 52. & & 117 & 55.1 & 21 & 0.1 \\
\hline $9: 07$ & 201 & 80 & 24 & 24.7 & 2.1 & 0.18 & 1175 & 55.1 & 21 & 0.1 \\
\hline $50: 06$ & 202 & 80 & 24 & 24.7 & 52.1 & 0.18 & 1179 & 55.1 & 21 & -0.16 \\
\hline $6: 51: 04$ & 203 & 80 & 24 & 24.9 & $52.1^{\dagger}$ & 0.18 & 1182 & 55.1 & 21 & $-0.1 \overline{6}$ \\
\hline $10: 16: 52: 03$ & 204 & 80 & 24 & 24.9 & 52.1 & 0.16 & 1184 & 55.1 & 21 & -0.29 \\
\hline $10: 16: 53: 02$ & 205 & 80 & 24 & 24.9 & $5 \overline{2.1}$ & $0.15^{\dagger}$ & 1183 & 55.1 & 20 & -0.29 \\
\hline $10: 16: 54: 01$ & 206 & 80 & 24 & 24.9 & 52 & $0.16^{\dagger}$ & $1179^{\circ}$ & 55.1 & 20 & $-0 . \overline{09}$ \\
\hline $10: 16: 55: 00$ & 207 & 80 & 24 & 24.9 & 52 & 0.18 & 1173 & 55.1 & 20 & -0.09 \\
\hline
\end{tabular}




\begin{tabular}{|c|c|c|c|c|c|c|c|c|c|c|}
\hline TIME & $\begin{array}{l}\text { Elapsed } \\
\text { Time } \\
\text { (sec) }\end{array}$ & BIT_TC1 & PURG_TC2 & PurgePSI & PurgeCFM & DEPTH(IN) & DwnFre (lb) & RPM & $\begin{array}{l}\text { !Torque } \\
\text { (FTLB) }\end{array}$ & Inches/Min \\
\hline $10: 16: 55: 99$ & $\quad 208$ & 79 & -25 & 24.9 & $\quad 51 . \overline{9}$ & 0.16 & $\quad 1176$ & 55.1 & $\quad 21$ & $\quad 0.08$ \\
\hline $10: 16: 56: 98$ & 209 & 79 & 24 & 24.7 & $51.9^{\circ}$ & 0.18 & 1171 & 55 & 21 & 0.08 \\
\hline $10: 16: 57: 96$ & 210 & 79 & 24 & 24.7 & 51.9 & $0.16^{-1}$ & 1170 & 55 & 21 & -0.2 \\
\hline$: 16: 58: 95$ & 211 & 80 & 24 & 24.7 & 51.9 & 0.18 & 1173 & 55.1 & 21 & -0.2 \\
\hline $10: 16: 59: 94$ & $21 \overline{2}$ & 80 & 24 & 24.7 & 51.9 & 0.18 & 1177 & 55.2 & 21 & $-0 . \overline{27}$ \\
\hline $10: 17: 00: 93$ & 213 & 80 & 24 & $24.7 i$ & $51.9^{\dagger}$ & 0.18 & 1179 & 55.2 & 21 & -0.27 \\
\hline $10: 17: 01: 92$ & 214 & 81 & 25 & 24.7 & 51.9 & 0.16 & 1179 & 55.3 & 21 & -0.09 \\
\hline $10: 17: 02: 91$ & 215 & 81 & 25 & 24.9 & $51.9^{+}$ & 0.16 & 1176 & 55.3 & 20 & -0.09 \\
\hline 390 & 216 & 81 & 24 & 24.9 & 51.9 & 0.16 & 1176 & 55.3 & 20 & 0.09 \\
\hline $4: 89$ & 217 & $79^{\dagger}$ & $25 !$ & 24.9 & $51.9^{\circ}$ & 0.16 & 1174 & 55.3 & 20 & 0.09 \\
\hline $8: 87$ & 218 & 79 & 24 & 24.9 & 51.9 & 0.16 & $1169^{\circ}$ & $55.3^{1}$ & 20 & $-0 . \overline{2} 2$ \\
\hline$\overline{06: 86}$ & $21 \overline{9}$ & 79 & 24 & 24.9 & 51.9 & 0.16 & 1164 & 55.3 & 20 & -0.22 \\
\hline $10: 17: 07: 85$ & 220 & 80 & 24 & 24.9 & 51.9 & 0.16 & 1164 & 55.2 & 21 & -0.31 \\
\hline 84 & 221 & 80 & 24 & $24.9^{\circ}$ & 51.9 & 0.18 & 1168 & 55.2 & 20 & -0.31 \\
\hline 83 & 222 & 80 & 25 & 24.9 & 51.9 & 0.18 & $1171^{\circ}$ & 55.1 & $20^{\circ}$ & $-0 . \overline{08}$ \\
\hline 82 & 223 & 80 & 25 & 24.9 & $51 . \overline{9}$ & 0.16 & 1173 & 55 & 20 & -0.08 \\
\hline$: 81$ & 224 & 80 & 25 & 24.9 & 51.9 & $0 . \overline{16}$ & 1172 & 55 & 20 & $0 . \overline{14}$ \\
\hline :12:79 & 225 & 80 & 25 & 24.9 & 51.8 & 0.16 & 1173 & 54.9 & 20 & 0.14 \\
\hline $7: 13: 78$ & 226 & 79 & 24 & 24.9 & 51.8 & 0.16 & & 54.7 & 20 & -0.27 \\
\hline 77 & 227 & 79 & 25 & 24.9 & 51. & 0.16 & & 54.7 & 20 & $-0 . \overline{27}$ \\
\hline 76 & 228 & 79 & 25 & $24.9^{\dagger}$ & 51.8 & 0.15 & 11 & 54.8 & 20 & -0.26 \\
\hline $7 \overline{5}$ & 229 & 79 & $\overline{25}$ & 24.9 & 51.8 & 0.15 & $1161^{\circ}$ & $54.9^{-4}$ & $20^{\dagger}$ & $-0 . \overline{01}$ \\
\hline$: 17: 74$ & $230^{\dagger}$ & 79 & 25 & 24.9 & 51.8 & 0.16 & 1163 & 54.9 & 20 & -0.01 \\
\hline $7: 18: 73$ & 231 & 80 & 25 & 24.9 & 51.8 & 0.16 & 11 & 55 & 20 & 0.08 \\
\hline & 232 & 80 & 25 & 25 & & .16 & & 55.2 & 20 & $0 . \overline{08}$ \\
\hline 70 & 233 & 80 & 25 & 25.1 & 51.8 & 0.16 & & 55.2 & 20 & -0.29 \\
\hline $6 \overline{9}$ & 234 & 80 & 25 & 24.9 & 51.8 & 0.16 & 11 & $55.3^{\prime}$ & 20 & -0.29 \\
\hline $2: 68$ & 235 & $80^{\dagger}$ & 25 & 24.9 & 51.8 & 0.16 & $1173^{\circ}$ & 55.3 & 20 & -0.22 \\
\hline$: 17: 23: 67$ & 236 & 80 & 25 & 25.1 & $5 \overline{1} .7$ & 0.16 & 1170 & 55.3 & 20 & -0.22 \\
\hline $4: 66$ & 237 & 79 & 25 & 24.9 & 51.7 & 0.15 & & 55.4 & $19+$ & -0.01 \\
\hline & 238 & $79^{\dagger}$ & 25 & 24.9 & & & & 55.4 & 20 & -0.01 \\
\hline $6: 64$ & 239 & 79 & 25 & 24.9 & 51.7 & $0.18^{-}$ & 11 & 55.4 & 20 & 0.05 \\
\hline $27: 62$ & 240 & 79 & 25 & 24.9 & 51.7 & 0.18 & $1164^{\circ}$ & $55.3^{4}$ & 20 & 0.05 \\
\hline $8: 61$ & 241 & 79 & 25 & 24.9 & 51.7 & 0.18 & 1170 & 55.1 & 20 & $-0 . \overline{27}$ \\
\hline $99: 60$ & 242 & 79 & 25 & 24.9 & $51.7 !$ & 0.18 & & 55.1 & 20 & -0.27 \\
\hline & 243 & 80 & 25 & 24 & & & & 55.1 & 20 & $-0 . \overline{25}$ \\
\hline & 244 & 80 & 25 & 25 & 1.6 & 0.16 & & 55.2 & 19 & -0.25 \\
\hline $2: 57$ & 245 & 80 & 25 & 25. & $51.6 !$ & $0.16^{\dagger}$ & & 55.2 & 19 & 0.02 \\
\hline 56 & $2 \overline{46}$ & 78 & 25 & 25.1 & $51 . \overline{6}$ & 0.15 & $1168^{\circ}$ & 55.2 & 19 & 0.02 \\
\hline $7: 34: 55$ & 247 & 78 & 25 & 25.1 & 51.6 & 0.15 & $1163^{\circ}$ & 55.2 & 19 & 0.06 \\
\hline $35: 53$ & 248 & $78^{+}$ & $25^{-1}$ & 25.1 & 51.6 & 0.15 & 1161 & $55.2^{+}$ & 19 & 0.06 \\
\hline$: 36: 52$ & $2 \overline{49}$ & 79 & $25 \vdots$ & $25 . \overline{1}$ & 51.6 & 0.16 & 1163 & 55.1 & 19 & -0.31 \\
\hline & 250 & 79 & 25 & 24.9 & 51. & $0.16^{\prime}$ & 1162 & 55.1 & 20 & -0.31 \\
\hline & $251^{\circ}$ & 79 & $25 !$ & 25.1 & & 0.1 & & $55.1^{\dagger}$ & 20 & $-0 . \overline{23}$ \\
\hline & 252 & 79 & 24 & & & 0. & 11 & 55.2 & 20 & -0.23 \\
\hline 48 & 253 & 79 & 25 & 25.1 & 51.6 & 0.18 & 11 & 55.1 & 20 & 0.03 \\
\hline $7: 41: 47$ & 254 & 80 & 25 & 25.1 & 51.6 & $0.16^{+}$ & 1173 & $55.1^{\dagger}$ & 20 & 0.03 \\
\hline $17: 42: 45$ & 255 & 79 & 25 & 25.1 & 51.5 & $0.16^{\prime}$ & 1171 & 55.2 & 20 & 0.02 \\
\hline$: 17: 43: \overline{44}$ & 256 & 79 & 25 & 24.9 & 51.5 & 0.16 & 1168 & 55.2 & 20 & 0.02 \\
\hline$: 17: 44: 43$ & 257 & 79 & 24 & 25.1 & 51.5 & 0.16 & 116 & 55.2 & 20 & $-0 . \overline{29}$ \\
\hline$: 45: 42$ & 258 & 78 & 25 & 24.9 & 51.5 & 0.16 & 1164 & 55.3 & 20 & $-0 . \overline{29}$ \\
\hline $10: 17: 46: 41$ & 259 & 79 & $25^{\top}$ & 24.9 & 51.5 & 0.16 & 1164 & 55.3 & 20 & -0.22 \\
\hline
\end{tabular}




\begin{tabular}{|c|c|c|c|c|c|c|c|c|c|c|}
\hline TIME & $\begin{array}{l}\text { Elapsed } \\
\text { Time } \\
\text { (sec) }\end{array}$ & BIT_TC1 & PURG_TC2 & PurgePSI & PurgeCFMD & EPTH(IN) & DwnFrc(lb) & RPM & $\begin{array}{l}\text { Torque } \\
\text { (FTLB) }\end{array}$ & Inches/Min \\
\hline $10: 17: 47: 40$ & 260 & $\begin{array}{l}79 \\
79\end{array}$ & $\quad 25$ & 24.9 & 51.5 & 0.18 & 1164 & 55.3 & $\quad 20$ & $\quad-0.22$ \\
\hline $10: 17: 48: 39$ & 261 & 79 & 25 & 25.1 & 51.5 & 0.18 & $1169^{\circ}$ & 55.3 & 20 & 0.02 \\
\hline $10: 17: 49: 38$ & 262 & 79 & 25 & 25.1 & 51.5 & 0.18 & 1173 & 55.3 & 20 & 0.02 \\
\hline $7: 50: 36$ & 263 & 79 & 25 & $25.1^{\dagger}$ & 51.5 & 0.18 & 1175 & $55.4^{\dagger}$ & 20 & 0.02 \\
\hline$: 35$ & 264 & 80 & 25 & $25.1 !$ & 51.5 & 0.18 & 1175 & 55.3 & 20 & -0.27 \\
\hline 34 & 265 & 80 & 25 & 25.1 & 51.5 & 0.16 & 1173 & $55.3^{+}$ & 20 & -0.27 \\
\hline 33 & 266 & 80 & 25 & 25.1 & 51.5 & 0.16 & 1169 & 55.3 & 20 & -0.12 \\
\hline 32 & $267 !$ & $\overline{7} \overline{8}$ & 25 & 25.1 & 51.5 & 0.16 & 1173 & 55.2 & 20 & -0.12 \\
\hline 31 & 268 & 78 & 25 & 24.9 & 51.5 & 0.16 & 1168 & 55.2 & 20 & -0.07 \\
\hline 30 & 269 & 78 & 25 & 25.1 & 51.5 & 0.16 & 1165 & 55.2 & 20 & -0.07 \\
\hline 28 & 270 & 79 & 25 & 25.1 & 51.5 & 0.18 & 1168 & 55.2 & 20 & 0.02 \\
\hline $8: 27$ & 271 & 79 & 25 & 25.1 & 51.5 & 0.18 & 1171 & 55.2 & 20 & 0.02 \\
\hline 26 & 272 & 79 & $25^{i}$ & 25.1 & 51.5 & 0.18 & 1175 & 55.3 & 20 & -0.28 \\
\hline & 273 & 80 & 25 & 25.1 & $51.5^{\prime}$ & 18 & 1176 & 55.3 & 20 & -0.28 \\
\hline & 274 & 80 & 25 & 25.3 & 51.5 & $1 \overline{8}$ & 1175 & 55.3 & 20 & -0.22 \\
\hline 23 & 275 & 80 & 25 & 25.3 & 51.5 & 0.16 & 76 & 55.2 & 19 & -0.22 \\
\hline 22 & 276 & 79 & 25 & $25 \overline{3}$ & 51.5 & 0.16 & 1176 & $55.3^{\dagger}$ & $20^{+}$ & 0.04 \\
\hline 21 & $277^{\dagger}$ & 79 & 25 & 25.3 & $51.5 !$ & $0.16 !$ & 1172 & 55.3 & 20 & 0.04 \\
\hline & 278 & 78 & 25 & 25.3 & 51.5 & 0.16 & & 55.3 & 20 & -0.01 \\
\hline & 279 & 79 & 25 & 25.1 & 1.5 & & & 55.3 & 21 & -0.01 \\
\hline & 280 & 79 & 25 & 25.1 & 51.5 & 0.18 & 1168 & 55.3 & 21 & $-0.2 \overline{9}$ \\
\hline & 281 & 79 & 25 & 25.1 & 1.5 & 0.18 & 11 & 55.3 & 21 & -0.29 \\
\hline & 282 & 79 & 25 & 25.1 & & 0.16 & & 55.3 & 20 & -0.19 \\
\hline & 283 & 79 & 25 & 25.1 & & 0.18 & 1176 & 55.3 & 21 & -0.19 \\
\hline & 284 & 79 & 25 & 25.1 & & 18 ! & & 55.3 & 21 & \\
\hline & 285 & 79 & 25 & 25.3 & & 16 & 1176 & 55.3 & 21 & \\
\hline & 286 & 79 & 25 & $25.3^{1}$ & & 0.96 & 1174 & 55.3 & 20 & 0.01 \\
\hline & 287 & 79 & 25 & 25. & & .16 & & 55.3 & 20 & 0.01 \\
\hline & 288 & 78 & 25 & 25.3 & 1.4 & 0.16 & 166 & 55.3 & 20 & -0.29 \\
\hline & 289 & 79 & 25 & 25.3 & 1.4 & 0.16 & & 55.3 & 20 & -0.29 \\
\hline & $290 !$ & 79 & 25 & 25.3 & 51.4 & 0.16 & & 55.4 & 20 & -0.23 \\
\hline $3: 05$ & 291 & 80 & 25 & 25.3 & 51.4 & 0.18 & 1173 & 55.3 & 20 & -0.23 \\
\hline & 292 & 79 & 25 & 25.3 & $51.4 !$ & 0.18 & & 55.3 & 20 & $0 . \overline{17}$ \\
\hline & 293 & 79 & 25 & 25.3 & 51.4 & 0.18 & 1173 & 55.2 & 20 & 0.17 \\
\hline 01 & 294 & 80 & 25 & 25.3 & & 0.18 & & 55.1 & 20 & -0.12 \\
\hline & 295 & 80 & 25 & 25.3 & & 0.18 & & 55.1 & 20 & $-0 . \overline{28}$ \\
\hline & 296 & 80 & 25 & 25.3 & & 0.15 & 1173 & 55.3 & 20 & -0.28 \\
\hline & 297 & 79 & 25 & 25.3 & $5 \overline{4}$ & 0.16 & 1169 & 55.4 & 20 & -0.23 \\
\hline ::97 & 298 & 78 & 25 & 25.3 & 51.4 & 0.16 & 1166 & 55.4 & 20 & -0.23 \\
\hline & 299 & 79 & 25 & 25.3 & 51.4 & 0.16 & 1166 & 55.4 & 20 & 0.05 \\
\hline 5:94 & 300 & 79 & 25 & $25.3^{-1}$ & 51.4 & 0.18 & 1165 & 55.4 & 20 & 0.05 \\
\hline & 301 & 79 & 25 & 25.3 & 51.4 & 0.18 & 1166 & 55.4 & 20 & 0.02 \\
\hline $8: 92$ & 302 & 80 & 25 & $25.3 !$ & 51.4 & 0.18 & 1170 & 55.3 & 20 & 0.02 \\
\hline & 303 & 80 & 25 & 25.3 & 51.4 & 0.18 & 11 & 55.3 & 20 & -0.27 \\
\hline & 304 & 80 & 25 & 25.5 & & & 1176 & 55.3 & 20 & $-0 . \overline{27}$ \\
\hline & 305 & 80 & 25 & 25.5 & 51.4 & 0.16 & 1174 & 55.3 & 19 & -0.1 \\
\hline $32: 88$ & 306 & 80 & 25 & 25.5 & 51.4 & 0.15 & 1173 & 55.3 & 19 & -0.1 \\
\hline $10: 18: 33: 86$ & 307 & 79 & 25 & 25.5 & 51.4 & 0.16 & 1167 & 55.2 & 19 & -0.13 \\
\hline $10: 18: 34: 85$ & 308 & 79 & 25 & 25.5 & 51.4 & 0.16 & 1164 & 55.2 & 19 & -0.13 \\
\hline $10: 18: 35: 84$ & 309 & 79 & 25 & $23.5^{\dagger}$ & 1.8 & 0.16 & 1166 & 55.2 & 20 & \\
\hline $10: 18: 36: 83$ & 310 & 81 & 25 & $-0.4 !$ & 1.8 & 0.18 & 1164 & 55.2 & 21 & \\
\hline $10: 18: 37: 82$ & 311 & 83 & 25 & -0.8 & 1.8 & 0.16 & 1166 & 55.3 & 21 & -0.27 \\
\hline
\end{tabular}




\begin{tabular}{|c|c|c|c|c|c|c|c|c|c|c|}
\hline TIME & $\begin{array}{l}\text { Elapsed } \\
\text { Time } \\
\text { (sec) }\end{array}$ & BIT_TC1 & & PurgePSI & PurgeCFMD & EPTH $(I N)$ & DwnFrc(lb) & & $\begin{array}{l}\text { Torque } \\
\text { (FTLB) }\end{array}$ & Inches/Min \\
\hline $10: 18: 38: 81$ & $\quad 312$ & -85 & -25 & $\quad-0.8$ & 1.8 & 0.18 & 1171 & $55 . \overline{3}$ & $3 \quad 22$ & -0.27 \\
\hline $10: 18: 39: 80$ & 313 & 86 & 24 & -0.8 & 1.8 & 0.16 & 1174 & 55.2 & 22 & -0.23 \\
\hline $40: 79$ & 314 & 88 & 25 & -0.8 & 1.8 & 0.16 & 1172 & $55 . \overline{1}$ & 22 & -0.23 \\
\hline $1: \overline{77}$ & 315 & 90 & $25^{\circ}$ & -0.8 & 1.8 & 0.16 & 1171 & 55.1 & 21 & 0.03 \\
\hline $10: 18: 42: 76$ & 316 & $\overline{91}$ & 25 & -0.8 & 1.8 & 0.16 & 1167 & 55.1 & 22 & 0.03 \\
\hline $10: 18: 43: 75$ & 317 & 90 & 25 & -0.8 & 1.8 & 0.16 & 1171 & 55 & 22 & -0.01 \\
\hline $44: 74$ & 318 & 91 & 25 & -0.8 & 1.8 & 0.16 & 1167 & 55 & 22 & -0.01 \\
\hline $45: 73$ & 319 & 92 & 25 & -0.8 & 1.8 & 0.16 & 1163 & 55 & 22 & -0.24 \\
\hline $6: 72$ & 320 & 94 & 25 & -0.8 & 1.8 & 0.16 & $1164^{\dagger}$ & 54.9 & $\overline{23}$ & $-0 . \overline{24}$ \\
\hline $7: 71$ & 321 & 95 & 25 & -0.8 & 1.8 & $0 . \overline{1}$ & 1169 & 54.9 & $2 \overline{3}$ & -0.22 \\
\hline $8: 69$ & 322 & 96 & $2 \overline{5}$ & -0.8 & 1.8 & 0.18 & 1173 & 55 & 23 & $-0 . \overline{22}$ \\
\hline $9: 68$ & 323 & 98 & $25 !$ & -0.8 & 1.8 & 0.16 & $1176^{-t}$ & 55.1 & 23 & 0.09 \\
\hline 67 & 324 & $\overline{9} \overline{9}$ & 25 & -0.8 & 1.8 & 0.16 & 1175 & 55.1 & 23 & 0.09 \\
\hline & 325 & 100 & 25 & -0.8 & 1.8 & 0.16 & 1176 & 55.1 & 23 & -0.1 \\
\hline :65 & 326 & 99 & 25 & -0.8 & 1.8 & 0.16 & 1183 & 55.1 & 23 & -0.1 \\
\hline $3: 64$ & 327 & 100 & 25 & -0.8 & 1.8 & 0.16 & 1188 & 55.1 & 24 & -0.29 \\
\hline $4: 63$ & 328 & 101 & 25 & -0.8 & $1.8 !$ & 0.15 & 1169 & 55.1 & 24 & $-0.1 \overline{7}$ \\
\hline 62 & 329 & 102 & 25 & -0.8 & 1.8 & 0.16 & 1168 & 55.1 & 24 & -0.17 \\
\hline & 330 & 103 & 25 & -0.8 & 1.8 & 0.18 & $1171^{*}$ & 55.1 & $2 \overline{4}$ & 0.09 \\
\hline & $331^{\dagger}$ & 105 & 25 & -0.8 & $1.8^{-\dagger}$ & 0.18 & $1175^{\circ}$ & 54.9 & 25 & 0.09 \\
\hline & 332 & 107 & $25^{\circ}$ & -0.8 & $1.8 !$ & 0.16 & 1178 & 54.9 & 25 & -0.13 \\
\hline$: 57$ & $333^{\dagger}$ & $10 \overline{8}$ & 25 & -0.8 & 1.8 & 0.16 & $1179^{\circ}$ & 54.9 & 24 & -0.13 \\
\hline 56 & 334 & $1 \overline{09}$ & 25 & -0.8 & 1.8 & 0.18 & 1179 & 55 & 24 & -0.24 \\
\hline & 335 & 109 & $25^{-1}$ & -0.8 & $1.8^{+}$ & 0.16 & 1179 & 55.1 & 24 & -0.24 \\
\hline & 336 & 109 & 25 & -0. & 1.8 & 0.15 & 1177 & 55.1 & 24 & -0.17 \\
\hline & 337 & 110 & 25 & -0.8 & 1.8 & 0.15 & 1173 & 55.1 & 24 & -0.17 \\
\hline & 338 & 111 & 25 & -0.6 & 1.8 & 0.18 & $1170^{\dagger}$ & 55.2 & 24 & 0.12 \\
\hline 50 & 339 & 112 & $25 !$ & -0.8 & 1.8 & 0.16 & 1171 & $5 \overline{5.2}$ & 26 & 0.12 \\
\hline & 340 & 113 & 25 & -0.8 & 1.8 & 18 & 174 & 55.2 & 26 & -0.19 \\
\hline & 341 & 11 & 25 & -0.6 & 1.8 & 16 & & 55.3 & 26 & -0.19 \\
\hline$: 47$ & 342 & 116 & 25 & -0.8 & 1.8 & 0.16 & 1175 & 55.3 & 26 & $-0.2 \overline{6}$ \\
\hline & 343 & 117 & 25 & -0.8 & 1.8 & 0.18 & 1178 ! & 55.3 & 26 & -0.26 \\
\hline & 344 & 119 & 25 & -0.8 & 1.8 & 0.16 & $1181 j$ & 55.3 & 26 & -0.18 \\
\hline & 345 & 120 & 25 & -0.8 & 1.8 & 0.16 & $1180^{+}$ & 55.3 & 25 & -0.18 \\
\hline & 346 & 121 & 25 & -0. & 1.8 & 0.16 & $1177^{\circ}$ & $55 . \overline{3}$ & $\overline{26}$ & 0.12 \\
\hline & 347 & 119 & $25^{\dagger}$ & -0.8 & 1.8 & 16 & & 55.2 & 26 & 0.12 \\
\hline & 348 & 12 & 25 & -0.8 & 1.8 & 0.15 & 11 & 55.2 & 26 & -0.16 \\
\hline & 349 & 122 & 25 & -0.6 & 1.8 & 0.18 & 1173 & 55.2 & 26 & -0.16 \\
\hline 38 & 350 & 124 & $25^{\dagger}$ & -0.8 & 1.8 & 0.18 & 1171 & 55.1 & 26 & -0.27 \\
\hline $17: 37$ & 351 & 125 & 25 & -0.8 & 1.8 & 0.18 & 1174 & 55.1 & 27 & -0.27 \\
\hline & 352 & 126 & 25 & -0.8 & 1.8 & 0.18 & 1178 & 55.1 & 27 & -0.13 \\
\hline & 353 & 128 & 25 & -0.8 & 1.8 & 0.18 & 1 & 55.1 & 26 & -0.13 \\
\hline & 354 & 129 & $25^{\dagger}$ & -0 & 1.8 & 0.16 & 1183 & 55.1 & 26 & 0.11 \\
\hline & 355 & 130 & 25 & -0.8 & 1.8 & 0.18 & 1181 & 55.2 & 26 & 0.11 \\
\hline $22: 31$ & $3 \overline{56}$ & 129 & 25 & -0.8 & 1.8 & 0.16 & $1177^{\circ}$ & $5 \overline{5.2}$ & 27 & -0.21 \\
\hline $10: \overline{9}: \overline{23}: \overline{30}$ & 357 & 130 & 25 & -0.8 & 1.8 & 0.16 & $1173^{t}$ & 55.2 & 27 & -0.21 \\
\hline $10: 19: 24: 29$ & 358 & 130 & 25 & -0.8 & 1.8 & 0.16 & 1173 & 55.2 & 27 & -0.27 \\
\hline $10: 19: 25: 28$ & 359 & 132 & 25 & -0.8 & 1.8 & 0.16 & $1173^{\circ}$ & 55.2 & 27 & -0.27 \\
\hline $26: 26$ & $360^{\dagger}$ & 133 & 25 & -0.8 & 1.8 & 0.18 & 1174 & 55.2 & 27 & -0.13 \\
\hline $27: 25$ & 361 & $13 \overrightarrow{4}$ & 25 & -0.8 & $1 . \overline{8}$ & $0 . \overline{18}$ & $1178^{+}$ & 55.2 & $\overline{2} \overline{7}$ & -0.13 \\
\hline & 362 & 135 & 25 & -0.8 & 1.8 & 0.18 & 1181 & 55.2 & 27 & 0.12 \\
\hline $10: 19: 29: 23$ & 363 & 136 & 25 & -0.8 & 1.8 & 0.18 & 1184 & 55.2 & 27 & -0.22 \\
\hline
\end{tabular}




\begin{tabular}{|c|c|c|c|c|c|c|c|c|c|c|}
\hline TIME & $\begin{array}{l}\text { Elapsed } \\
\text { Time } \\
\text { (sec) }\end{array}$ & BIT_TC1 & PURG_TC2 & PurgePSI & PurgeCFM & DEPTH(IN) & DwnFrc(lb) & & $\begin{array}{l}\text { Torque } \\
\text { (FTLB) }\end{array}$ & $\begin{array}{l}\text { Inches/Min } \\
\text { I }\end{array}$ \\
\hline $10: 19: 30: 22$ & 364 & -137 & 25 & -0.8 & 1.8 & 0.16 & 1184 & 55 & 27 & $\quad-0.22$ \\
\hline $10: 19: 31: 21$ & 365 & $137^{\prime}$ & 25 & -0.8 & 1.8 & 0.16 & 1181 & $55^{\circ}$ & 27 & -0.26 \\
\hline $10: 19: 32: 20$ & 366 & 138 & $25 !$ & -0.8 & 1.8 & 0.16 & 1176 & 55 & 27 & -0.26 \\
\hline $10: 19: 33: 18$ & 367 & 138 & 25 & -0.8 & 1.8 & 0.16 & $1180^{\dagger}$ & 55 & 27 & -0.1 \\
\hline $10: 19: \overline{34}: 17$ & 368 & 139 & 25 & -0.8 & 1.8 & 0.16 & 1175 & 55 & 28 & -0.1 \\
\hline $10: 19: 35: 16$ & 369 & 140 & $25^{4}$ & -0.8 & 1.8 & 0.18 & 1174 & 55 & 28 & $0.0 \overline{8}$ \\
\hline $10: 19: 36: 15$ & $370^{\circ}$ & 141 & 25 & -0.8 & 1.8 & $0.18^{\circ}$ & 1177 & 55 & 28 & 0.08 \\
\hline $37: 14$ & 371 & 142 & 25 & -0.8 & 1.8 & $0.18^{\circ}$ & 1181 & 55 & 28 & -0.25 \\
\hline $8: 13$ & 372 & 143 & 25 & -0.8 & 1.8 & 0.18 & 1184 & 55 & 28 & -0.25 \\
\hline $9: 12$ & 373 & 145 & 25 & -0.8 & 1.8 & 0.16 & 1184 & 55 & 28 & $-0.1 \overline{7}$ \\
\hline $10: 19: 40: 10$ & 374 & $146^{t}$ & 25 & $-0 . \overline{8}$ & 1.8 & 0.16 & 1183 & 55 & 28 & -0.17 \\
\hline 09 & 375 & 147 & 25 & -0.8 & 1.8 & 0.18 & 1184 & 54.9 & 28 & -0.08 \\
\hline 08 & 376 & 145 & 25 & $-0 . \overline{8}$ & 1.8 & 0.16 & 1181 & 54.8 & 28 & -0.08 \\
\hline 07 & $37 \overline{7}$ & 146 & 25 & -0.8 & 1.8 & 0.1 & 1176 & 54.8 & 28 & 0.02 \\
\hline $4 \overline{4: 06}$ & 378 & 147 & 25 & $-0 . \overrightarrow{8}$ & 1.8 & 0.16 & 1173 & 54.8 & 28 & 0.02 \\
\hline $10: 19: \overline{45: 05}$ & 379 & 149 & 25 & -0.6 & 1.8 & 0.18 & 1176 & 54.8 & 29 & .0 .26 \\
\hline $6: 04$ & 380 & 150 & 25 & -0.8 & 1.8 & 0.19 & $1180^{\prime}$ & 54.8 & 29 & -0.26 \\
\hline 03 & $381^{\dagger}$ & 151 & 25 & -0.8 & 1.8 & 0.18 & 1183 & $54.8^{1}$ & 29 & -0.28 \\
\hline 01 & 382 & 153 & 25 & -0.8 & 1.8 & 0.16 & 1184 & 55 & 29 & -0.28 \\
\hline 00 & 383 & 154 & 25 & -0.8 & 1.8 & 0.1 & $1183^{\dagger}$ & 55 & 29 & 0.06 \\
\hline 99 & 384 & 155 & 25 & -0.8 & 1.8 & $0.18^{\prime}$ & $1184^{\dagger}$ & 55.1 & 29 & 0.06 \\
\hline $0: 98$ & $385^{\dagger}$ & 153 & 25 & -0.8 & 1.8 & 0.16 & 1182 & $55.2^{i}$ & 29 & -0.04 \\
\hline $1: 97$ & 386 & $154^{\dagger}$ & $25 !$ & -0.8 & 1.8 & 0.18 & 1178 & 55.2 & $3 \overline{0}$ & -0.04 \\
\hline 96 & 387 & 155 & 25 & -0.6 & 1.8 & 0.16 & 1174 & 55.1 & 30 & -0.23 \\
\hline 95 & 388 & 157 & 25 & -0.8 & 1.8 & $0.18^{1}$ & $1175^{\dagger}$ & 54.9 & 30 & -0.23 \\
\hline $4: 93$ & 389 & 158 & $\overline{25}$ & -0.6 & 1.8 & 0.16 & 1178 & 54.9 & 30 & -0.22 \\
\hline $5: 92$ & 390 & 158 & $25^{1}$ & -0.8 & 1.8 & 0.18 & 1182 & 55 & 30 & -0.22 \\
\hline 91 & 391 & 160 & 25 & -0.8 & $1.8^{-4}$ & 0.18 & 1184 & 55.2 & 30 & 0.05 \\
\hline :90 & 392 & $1 \overline{6} 1$ & 25 & -0.8 & 1.8 & & 1183 & 55.2 & 29 & 0.05 \\
\hline & 393 & 162 & 25 & -0.8 & 1.8 & $0.16 !$ & 1184 & 55.2 & 30 & -0.06 \\
\hline 88 & 394 & 162 & 25 & -0.8 & 1.8 & 0.18 & 1183 & 55.2 & 30 & -0.06 \\
\hline $00: 87$ & 395 & 162 & 25 & -0.8 & 1.8 & 0.18 & 1181 & 55.2 & 30 & -0.29 \\
\hline 186 & 396 & 163 & 25 & -0.8 & 1.8 & 0.1 & 1176 & 55.2 & 30 & -0.2 \\
\hline & 397 & $16 \bar{t}$ & 25 & $-0 . \overline{8}$ & 1.8 & 0. & 1173 & 55.2 & 30 & -0.2 \\
\hline & 398 & 164 & 25 & -0.8 & 1.8 & 0.16 & $117 \overrightarrow{6}$ & 55.3 & 31 & 0.12 \\
\hline $4: 82$ & 399 & 166 & 25 & -0.8 & 1.8 & 0.18 & 1179 & 55.1 & 31 & $0 . \overline{2}$ \\
\hline 81 & 400 & 167 & 25 & -0.8 & 1.8 & 0.18 & 1183 & 55.1 & 31 & $-0 . \overline{13}$ \\
\hline 80 & 401 & 168 & 25 & -0.8 & 1.8 & 0.1 & 1180 & 55.1 & 31 & $-0.1 \overline{3}$ \\
\hline $7: 79$ & 402 & $169^{\circ}$ & 25 & -0.8 & 1.8 & & 1183 & 55.2 & 31 & -0.27 \\
\hline & 403 & 170 & 25 & -0.8 & 1.8 & 0. & 1184 & 55.2 & 31 & -0.27 \\
\hline & 404 & 170 & 25 & -0.8 & 1.8 & & 1183 & 55.2 & 30 & -0.26 \\
\hline & 405 & 171 & 25 & -0.8 & 1.8 & 0.16 & 1179 & 55.2 & 30 & -0.26 \\
\hline 74 & 406 & 170 & 25 & -0.8 & 1.8 & 0.16 & 1174 & 55.2 & 30 & 0.11 \\
\hline $20: 12: 73$ & 407 & 171 & 25 & -0.8 & 1.8 & 0.18 & 1174 & 55 & 30 & 0.11 \\
\hline $0: 13: 72$ & 408 & 172 & 25 & -0.8 & 1.8 & 0.18 & 1177 & 54.9 & 30 & -0.13 \\
\hline $0: 14: 71$ & 409 & 173 & 25 & -0.8 & $1.8^{1}$ & 0.16 & 1174 & 54.9 & 30 & $-0 . \overline{13}$ \\
\hline $10: 20: 15: 70$ & 410 & 174 & 25 & -0.8 & 1.8 & 0.19 & $1178^{-1}$ & 54.8 & $30^{-}$ & -0.28 \\
\hline $16: 69$ & 411 & 175 & 25 & -0.8 & 1.8 & 0.1 & 1183 & 54.8 & 30 & -0.28 \\
\hline $17: 67$ & 412 & 177 & 25 & -0.6 & 1.8 & 0.18 & 1184 & 54.8 & 30 & $-0 . \overline{18}$ \\
\hline $18: 66$ & 413 & 177 & $25^{i}$ & -0.8 & 1.8 & 0.18 & 1183 & 54.8 & 30 & -0.18 \\
\hline 65 & $\overline{414}$ & 178 & 25 & -0.8 & 1.8 & 0.1 & $1179^{\circ}$ & 54.9 & 31 & 0.13 \\
\hline $10: 20: 20: 64$ & 415 & $17 \overline{6}$ & 25 & -0.8 & 1.8 & 0.16 & $1175^{\circ}$ & 54.9 & 31 & 0.13 \\
\hline
\end{tabular}




\begin{tabular}{|c|c|c|c|c|c|c|c|c|c|c|}
\hline TIME & $\begin{array}{l}\text { 'Elapsed } \\
\text { Time } \\
\text { (sec) }\end{array}$ & BIT_TC1 & PURG_TC2 & PurgePSI & PurgeCFM & DEPTH(IN) & DwnFrc(lb) & RPM & $\begin{array}{l}\text { Torque } \\
\text { (FTLB) }\end{array}$ & Inches/Min \\
\hline $10: 20: 21: 63$ & 416 & 177 & 25 & -0.8 & $3 \quad 1.8$ & 0.18 & 1173 & 54.9 & 9. $\quad 31$ & -0.22 \\
\hline $10: 20: 22: 62$ & 417 & 178 & 25 & -0.8 & 1.8 & 0.18 & 1176 & 55 & 31 & $-0.2 \overline{2}$ \\
\hline $10: 20: 23: 61$ & 418 & 180 & 25 & -0.6 & 1.8 & 0.16 & 1174 & 55 & 31 & -0.3 \\
\hline $10: 20: 24: 59$ & 419 & 180 & 25 & -0.8 & 1.8 & 0.18 & 1177 & 55.1 & 31 & -0.3 \\
\hline $10: 20: 25: 58$ & 420 & 181 & 25 & -0.8 & 1.8 & 0.18 & 1181 & 55.2 & 32 & 0.08 \\
\hline $10: 20: 26: 57$ & $4 \overline{42} 1$ & 183 & 25 & -0.8 & 1.8 & 0.18 & 1184 & 55.2 & 31 & 0.08 \\
\hline $10: 20: 27: 56$ & 422 & 184 & 25 & -0.8 & 1.8 & 0.18 & 1184 & 55 & 31 & -0.06 \\
\hline $10: 20: 28: 55$ & 423 & 185 & 25 & -0.8 & 1.8 & 0.18 & 1182 & 55.1 & 31 & -0.06 \\
\hline $10: 20: 29: 54$ & 424 & 183 & 25 & -0.8 & 1.8 & 0.16 & $1177^{\prime}$ & 55.1 & 31 & -0.2 \\
\hline $10: 20: 30: 53$ & 425 & $184 !$ & $25 !$ & -0.8 & 1.8 & 0.18 & 1174 & 55.3 & 31 & -0.2 \\
\hline $1: 52$ & $426^{\prime}$ & 184 & 25 & -0.8 & 1.8 & $0.16^{+}$ & 1176 & 55.3 & 31 & $-0 . \overline{29}$ \\
\hline $10: 20: 32: 50$ & $427 !$ & 186 & 25 & -0.8 & 1.8 & 0.16 & 1173 & 55.3 & 31 & -0.29 \\
\hline $10: 20: 33: 49$ & 428 & 187 & 25 & -0.8 & 1.8 & 0.18 & 1174 & 55.3 & 32 & -0.1 \\
\hline $10: 20$ & 429 & 188 & 25 & -0.8 & 9.8 & 0.16 & $1178^{\prime}$ & 55.3 & 32 & 0.09 \\
\hline $10: 2$ & 430 & $18 \overline{8}$ & $25 !$ & -0.8 & 1.8 & 18 & 1181 & 55.3 & 32 & 0.09 \\
\hline $10: 2$ & 431 & $18 \overline{9}$ & 25 & -0.8 & 1.8 & 0.18 & 1184 & 55.3 & 32 & -0.21 \\
\hline $3 \overline{7: 45}$ & 432 & 190 & 25 & -0.8 & 1.8 & 0.19 & 1184 & $55 . \overline{4}$ & 32 & -0.21 \\
\hline $38: 44$ & $433^{4}$ & 191 & 25 & -0.8 & 1.8 & 0.18 & 1181 & 55.4 & 32 & -0.27 \\
\hline 42 & 434 & 191 & 25 & -0.8 & 1.8 & 0.18 & 1183 & 55.4 & 32 & $-0 . \overline{27}$ \\
\hline $0: 41$ & 435 & 190 & 25 & -0.8 & 1.8 & 0.18 & $1180^{\circ}$ & 55.4 & 32 & -0.07 \\
\hline $1: 40$ & 436 & 190 & 25 & -0.8 & 1.8 & $0.16 !$ & 1176 & 55.4 & 32 & -0.07 \\
\hline $39^{\circ}$ & 437 & 191 & 25 & -0.8 & 1.8 & 0.16 & $1173^{\circ}$ & 55.4 & 32 & 0.09 \\
\hline $3: 38$ & $438^{\circ}$ & $193^{\dagger}$ & 25 & -0.8 & 1.8 & 0.18 & $1175^{\dagger}$ & 55.4 & 33 & 0.09 \\
\hline $4: 37$ & $439^{\circ}$ & 194 & 25 & -0.8 & 1.8 & 0.19 & 1178 & 55.5 & 33 & -0.21 \\
\hline 36 & $440^{\circ}$ & 195 & 25 & -0.8 & 1.8 & $0.19^{1}$ & 1182 & 55.4 & 33 & -0.21 \\
\hline$: 35$ & 441 & 197 & 25 & -0.8 & 1.8 & 18 & 183 & 55.4 & 33 & -0.21 \\
\hline 33 & 442 & $19 \overline{8}$ & 25 & -0.8 & 1.8 & 0.18 & 1183 & 55.2 & 33 & -0.21 \\
\hline & 443 & & 25 & -0.6 & 1.8 & 0.16 & $1183^{4}$ & 55.2 & 34 & -0.16 \\
\hline 31 & 444 & 196 & 25 & -0.8 & 1.8 & 0.16 & 1182 & 55.2 & 33 & -0.16 \\
\hline & 445 & 197 & 25 & -0.8 & 1.8 & 0.16 & 1178 & 55.2 & 34 & 0.09 \\
\hline & 446 & 198 & 25 & -0.8 & 1.8 & 0.16 & & $5 \overline{5.2}$ & 34 & 0.09 \\
\hline & 447 & 199 & $25^{\prime}$ & -0.8 & $1.8 !$ & 0.16 & 1173 & 55.2 & 34 & -0.21 \\
\hline & 448 & 201 & 25 & -0.8 & 1.8 & 0.18 & 175 & 55.2 & 34 & -0.21 \\
\hline $44: 25$ & 449 & 202 & 25 & -0.8 & 1.8 & 0.18 & $1179 !$ & 55.2 & 34 & -0.27 \\
\hline 24 & 450 & 203 & 25 & -0.8 & 1.8 & 0.18 & 1182 & 55.2 & 34 & -0.27 \\
\hline & $4 \overline{5} 1$ & 203 & 25 & -0.8 & 1.8 & 0.18 & 11 & 55.2 & 34 & -0.09 \\
\hline & 452 & & 25 & -0.8 & $1.8 !$ & 0.16 & 1182 & 55.1 & 33 & -0.09 \\
\hline $3: 21$ & 453 & 204 & 25 & -0.8 & $1.8 j$ & 0.18 & 1183 & $5 \overline{5.1}$ & 33 & 0.1 \\
\hline 20 & 454 & 204 & 25 & -0.8 & 1.8 & 0.18 & $11 \overline{7} 9$ & 55 & 33 & 0.1 \\
\hline $10: 21: 00: 19$ & 455 & 204 & 25 & -0.8 & 1.8 & 0.18 & 1175 & 54.9 & 33 & -0.27 \\
\hline $10: 21$ & 456 & 205 & 25 & -0.8 & 1.8 & 0.16 & 173 & 54.9 & 34 & -0.27 \\
\hline $10: 2$ & 457 & 205 & 25 & -0.8 & 1.8 & 0.16 & 1174 & 54.9 & 34 & -0.25 \\
\hline & 458 & & 25 & -0.8 & 1.8 & 0.18 & & 55 & 34 & -0.25 \\
\hline & 45 & & 25 & -0.8 & 1.8 & 0. & & 55 & 34 & -0.05 \\
\hline & 46 & & 25 & -0.8 & 1.8 & 0.1 & 1179 & 55 & 34 & -0.05 \\
\hline $6: 12$ & 46 & 209 & 25 & -0.8 & 1.8 & 0.16 & 1182 & 55 & 34 & 0.06 \\
\hline $10: 21: 07: 11$ & 462 & 209 & 25 & -0.8 & 1.8 & 0.16 & 1183 & 55 & 35 & -0.21 \\
\hline $10: 21: 08: 10$ & 463 & 210 & 25 & -0.8 & 1.8 & 0.18 & 1181 & 54.9 & 34 & -0.21 \\
\hline $10: 21: 09: 08$ & 464 & 211 & 25 & -0.8 & 1.8 & 0.16 & 1177 & 54.9 & 34 & -0.21 \\
\hline 10:21:10:07 & 465 & 209 & 25 & -0.8 & 1.8 & 0.18 & 1173 & 54.9 & 34 & -0.21 \\
\hline & 466 & 211 & 25 & -0.8 & 1.8 & 0.1 & 1173 & 54.9 & 35 & -0.06 \\
\hline $10: 21: 12: \overline{05}$ & 467 & 212 & 25 & -0.8 & 1.8 & 0.18 & 1176 & 54.9 & 35 & -0.06 \\
\hline
\end{tabular}




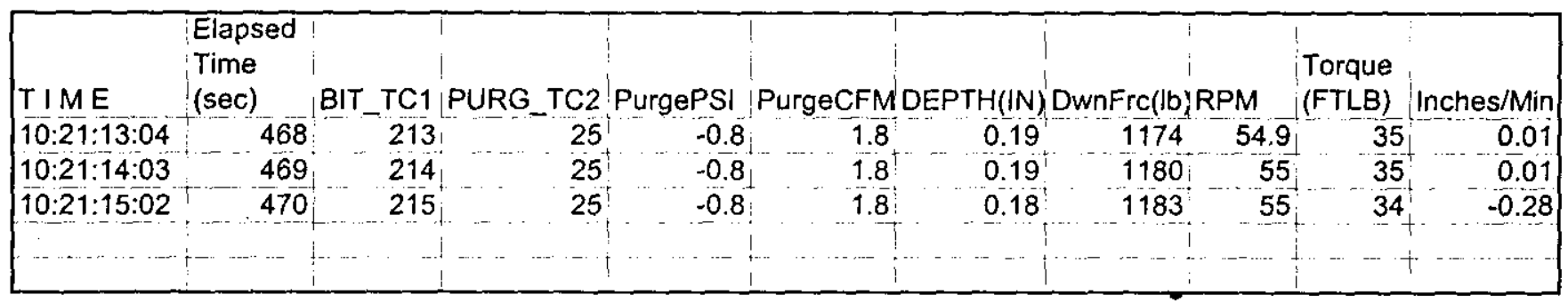




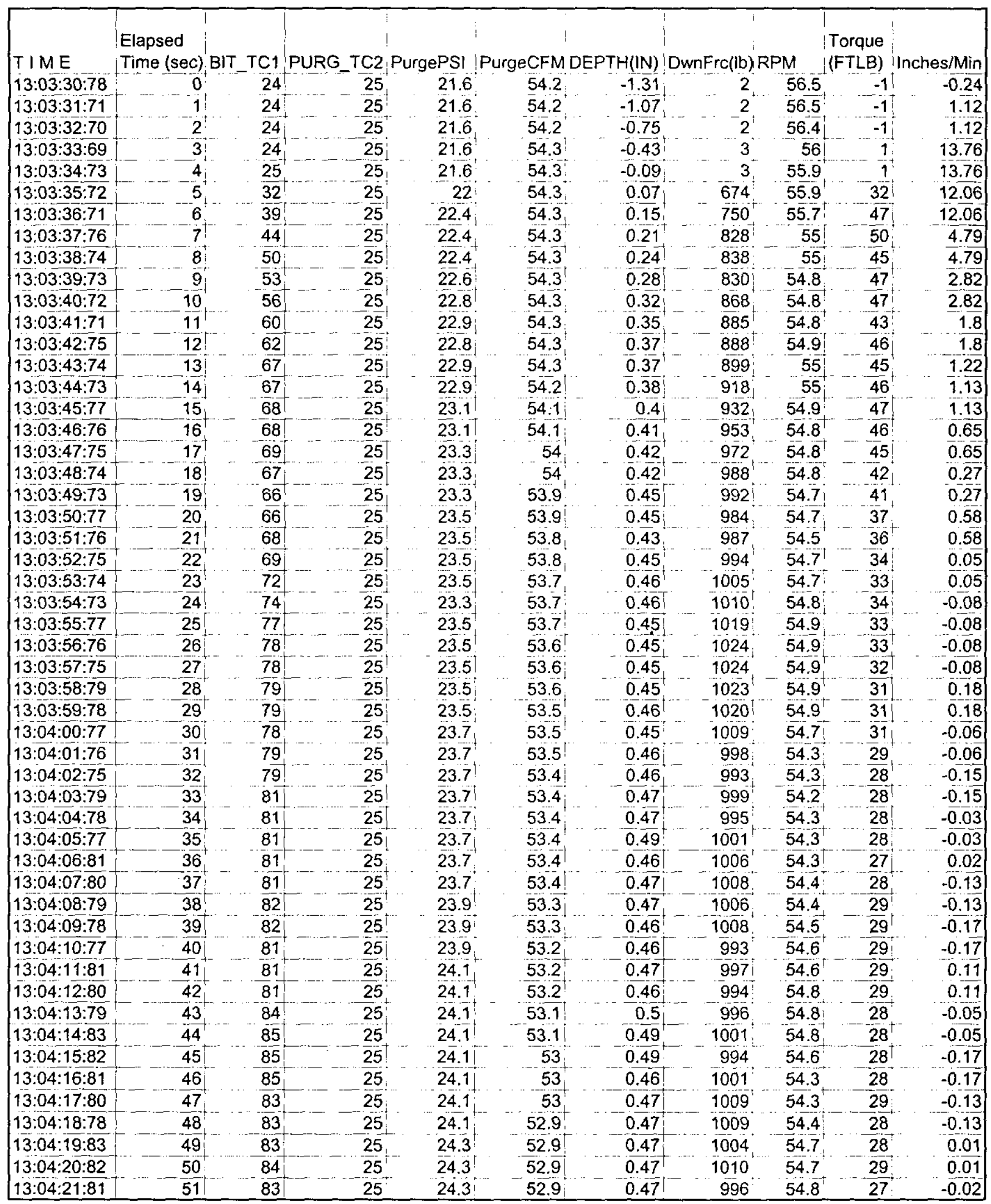




\begin{tabular}{|c|c|c|c|c|c|c|c|c|c|c|}
\hline & Elapsed & & & | & & & ( & & Torque & \\
\hline$T / M E$ & Time (sec) & BIT_TC1 & PURG_TC2 & PurgePSI & PurgeCFM & DEPTH(IN) & DwnFrc(lb) & RPM & (FTLB) & Inches/Min \\
\hline $13: 04: 22: 79$ & 52 & 83 & 25 & 24.3 & $\quad 52.8$ & 0.49 & 997 & 54.9 & 28 & $\quad-0.02$ \\
\hline $13: 04: 23: 78$ & 53 & 85 & 25 & 24.3 & 52.8 & 0.49 & 994 & 54.9 & 28 & -0.27 \\
\hline $13: 04: \overline{24}: \overline{83}$ & $5 \overline{4}$ & 85 & 25 & 24.3 & 52.8 & 0.47 & 999 & 54.8 & 27 & -0.27 \\
\hline $13: 04: 25: 81$ & 55 & 85 & 25 & $24.5^{\circ}$ & $52.8^{\circ}$ & 0.5 & 1005 & 54.9 & 27 & $-0.0 \overline{1}$ \\
\hline $13: 04: 26: 80$ & 56 & 85 & 25 & $24.3^{+}$ & 52.8 & $0 . \overline{49}$ & 1010 & 54.9 & 27 & -0.01 \\
\hline $13: 04: 27: \overline{85}$ & 57 & 85 & 25 & 24.5 & 52.8 & 0.49 & 1008 & 54.9 & 27 & 0.04 \\
\hline $13: 04: 28: 84$ & 58 & 85 & 25 & $24.5^{\dagger}$ & 52.8 & 0.47 & 1014 & 54.8 & 28 & -0.17 \\
\hline $13: 04: 29: 82$ & 59 & 85 & $25:$ & 24.7 & 52.8 & 0.47 & 1013 & $54 . \overline{8}$ & 29 & -0.17 \\
\hline $13: 04: 30: 81$ & 60 & 85 & 25 & 24.7 & 52.7 & 0.49 & 1009 & $54 . \overline{8}$ & 29 ! & $-0.1 \overline{9}$ \\
\hline $13: 04: 31: 80$ & 61 & 84 & 25 & $24.7 !$ & 52.7 & 0.5 & 1003 & 54.9 & 28 & -0.19 \\
\hline $13: 04: 32: 85$ & 62 & 84 & 25 & 24.7 & 52.7 & 0.49 & 1001 & 54.9 & 28 & -0.03 \\
\hline $13: 04: 33: 83$ & 63 & 85 & 25 & 24.7 & 52.7 & 0.49 & 1003 & 55.1 & 28 & -0.03 \\
\hline $13: 04: 34: 82$ & 64 & 86 & 25 & 24.7 & 52.7 & 0.5 & 1003 & 55.2 & 28 & 0.01 \\
\hline $13: 04: 35: 87$ & 65 & 87 & 25 & 24.7 & $52.7 i$ & 0.51 & 1006 & 55.2 & 28 & 0.01 \\
\hline $13: 04: 36: 85$ & 66 & 87 & 25 & 24.7 & 52.7 & 0.51 & $1013^{-1}$ & $5 \overline{5.1}$ & 27 & -0.16 \\
\hline $13: 04: 37: 84$ & 67 & 87 & 25 & 24.7 & 52.7 & 0.49 & 1016 & 55 & 27 & -0.16 \\
\hline $13: 04: 38: 83$ & 68 & $\overline{87}$ & $25 j$ & 24.7 & 52.6 & 0.5 & 1011 & 55 & 27 & -0.16 \\
\hline $13: 04: 39: 82$ & 69 & 87 & 25 & $24.7^{\dagger}$ & 52.6 & 0.5 & $1013^{1}$ & 54.9 & 27 & -0.16 \\
\hline $13: 04: 40: 86$ & 70 & 87 & 25 & 24.7 & 52.6 & 0.5 & 1016 & 55.1 & 27 & -0.02 \\
\hline $13: 04: 4 \overline{1}: 85$ & 71 & 87 & 25 & 24.9 & 52.6 & 0.49 & 1008 & 55.1 & 27 & -0.02 \\
\hline $13: 04: 42: 84$ & 72 & 85 & 25 & 24.9 & 52.6 & 0.49 & 999 & 55.1 & 26 & -0.07 \\
\hline $13: 04: \overline{43: \overline{89}}$ & $\overline{73}$ & 85 & 25 & 24.9 & $52.6^{i}$ & 0.47 & 994 & 55 & 26 & -0.07 \\
\hline $13: 04: 44: 87$ & 74 & 85 & 25 & 24.9 & $52 . \overline{6}$ & 0.49 & 994 & 55 & $26 !$ & -0.22 \\
\hline $13: 04: 45: 86$ & 75 & 87 & $25^{\dagger}$ & 24.9 & 52.6 & 0.51 & 992 & 55 & 25 & -0.22 \\
\hline $13: 04: 46: 85$ & 76 & 87 & 25 & 24.9 & 52.6 & 0.51 & 993 & 55 & 25 & -0.17 \\
\hline $13: 04: 47: 84$ & 77 & 87 & 25 & 25.1 & 52.5 & 0.5 & 997 & 55 & 25 & -0.17 \\
\hline $13: 04: 48: 88$ & 78 & 87 & 25 & 25.1 & 52.5 & 0.51 & 1001 & 55.2 & 24 & 0.05 \\
\hline $13: 04: 49: 87$ & 79 & $8 \overline{7}$ & 25 & $25.1^{\dagger}$ & 52.5 & 0.51 & 996 & 55.2 & 24 & 0,05 \\
\hline $13: 04: 50: 86$ & 80 & 87 & 25 & $25.1^{1}$ & 52.5 & 0.51 & 1001 & 55.2 & $26+$ & -0.08 \\
\hline $13: 04: 51: 85$ & 81 & 87 & 25 & 25.1 & 52.4 & 0.51 & 1001 & 55.1 & $2 \overline{6}^{\top}$ & -0.08 \\
\hline $13: 04: 52: 84$ & 82 & 85 & 25 & 25.1 & 52.3 & 0.51 & 998 & 55 & 26 & -0.18 \\
\hline $13: 04: 53: 88$ & 83 & 86 & 25 & 25.3 & $52.3^{\dagger}$ & 0.47 & 991 & $55^{-}$ & $2 \overline{6}^{\dagger}$ & -0.21 \\
\hline $13: 04: 54: \overline{87}$ & 84 & 86 & $25 !$ & 25.3 & 52.2 & 0.49 & 986 & 55.1 & 26 & -0.21 \\
\hline $13: 04: 55: 86$ & 85 & 86 & 25 & 25.3 & 52.2 & 0.5 & 986 & $5 \overline{5} .2^{\dagger}$ & 25 & 0.07 \\
\hline $13: 04: 56: 90$ & 86 & 87 & 25 & 25.3 & 52.2 & 0.51 & 986 & 55.2 & 24 & 0.07 \\
\hline $13: 04: 57: 89$ & 87 & 86 & $25 !$ & 25.3 & 52.1 & 0.52 & 992 & 55.2 & 23 & -0.11 \\
\hline $13: 04: 58: 88$ & 88 & 87 & $25 !$ & 25.3 & 52.11 & 0.5 & 992 & $55 . \overline{1}$ & 24 & -0.11 \\
\hline $13: 04: 59: 87$ & 89 & $\overline{8} 6$ & $25 !$ & $25.3^{\dagger}$ & $52.1^{1}$ & $0 . \overline{51}$ & 998 & 55.1 & 24 & -0.21 \\
\hline $13: 05: 00: 86$ & 90 & 86 & 25 & $2 \overline{5} .3^{\dagger}$ & 52.1 & 0.51 & 1001 & 55.1 & 24 & -0.21 \\
\hline $13: 05: 01: 90$ & 91 & 86 & 25 & 25.3 & 52.1 & 0.51 & 999 & 55 & 24 & -0.18 \\
\hline $13: 05: 02: \overline{89}$ & 92 & 85 & 25 & $25.3^{i}$ & 52 & 0.51 & 1001 & 55 & 24 & -0.18 \\
\hline $13: 05: 03: 88$ & 93 & 85 & 25 & 25.5 & 52 & 0.5 & $9 \overline{9}$ & 54.9 & 24 & 0.05 \\
\hline $13: 05: 04: 92$ & 94 & 85 & 25 & 25.5 & 51.9 & 0.51 & $993^{1}$ & 55 & 24 & 0.05 \\
\hline $13: 05: 05: \overline{91}$ & 95 & 84 & 25 & $25.5^{i}$ & 51.9 & 0.51 & 988 & 55 & 24 & $-0 . \overline{14}$ \\
\hline 13:05:06:90 & 96 & 84 & 25 & 25.5 & 51.9 & 0.52 & 988 & 55 & 24 & -0.14 \\
\hline $13: 05: 07: 89$ & 97 & 84 & $25^{\dagger}$ & $25.5^{\prime}$ & 51.8 & 0.51 & $989^{\circ}$ & 55 & 24 & -0.24 \\
\hline $13: 05: 08: 88$ & 98 & $\overline{86}$ & $25^{\dagger}$ & $25 . \overline{5}$ & 51.8 & 0.52 & 991 & $55^{\dagger}$ & 24 & -0.24 \\
\hline $13: 05: 09: 92$ & 99 & 86 & 25 & 25.5 & 51.8 & 0.51 & 996 & 55 & 24 & 0.02 \\
\hline $13: 05: 10: 91$ & 100 & 85 & $25^{i}$ & 25.3 & 51.8 & 0.51 & 1001 & 54.7 & 24 & $0.0 \overline{2}$ \\
\hline $13: 05: 11: 90$ & 101 & 85 & 25 & 25.5 & 51.8 & 0.51 & 1005 & 54.7 & 24 & -0.09 \\
\hline $13: 05: 12: 89$ & 102 & 84 & 25 & 25.5 & 51.8 & 0.51 & 1004 & 54.7 & $2 \overrightarrow{4}$ & -0.09 \\
\hline $13: 05: 13: 87$ & 103 & 84 & 25 & 25.5 & 51.8 & 0.5 & 998 & 54.8 & 25 & -0.12 \\
\hline
\end{tabular}




\begin{tabular}{|c|c|c|c|c|c|c|c|c|c|c|}
\hline TIME & $\begin{array}{l}\text { Elapsed } \\
\text { Time (sec) }\end{array}$ & BIT_TC1 & PURG_TC2 & PurgePSI & PurgeCFM & DEPTH(IN) & DwnFrc(ib) & RPM & $\begin{array}{l}\text { |Torque } \\
\text { (FTLB) }\end{array}$ & Inches/Min \\
\hline $13: 05: 14: 92$ & 104 & 84 & 25 & 25.5 & 51.8 & 0.51 & 1004 & 54.8 & 25 & -0.12 \\
\hline $13: 05: 1 \overline{5}: 91$ & $105 !$ & 84 & 25 & $25.5^{i}$ & 51.8 & 0.51 & 997 & 54.9 & 25 & -0.29 \\
\hline 13:05:16:90 & 106 & 83 & 25 & $25.5 \mathrm{i}$ & 51.8 & 0.51 & 992 & 54.9 & 23 & -0.07 \\
\hline $13: 05: 17: 94$ & 107 & 84 & 25 & 25.5 & 51.8 & 0.52 & 993 & 54.9 & 24 & -0.07 \\
\hline $13: 05: \overline{18}: \overline{93}$ & 108 & 84 & 25 & 25.5 & 51.7 & 0.52 & 993 & 54.9 & 24 & -0.02 \\
\hline $13: 05: 19: 92$ & 109 & 85 & 25 & 25.5 & 51.7 & 0.52 & 994 & 54.9 & 24 & -0.02 \\
\hline $13: 05: 20: 91$ & 110 & 84 & 25 & 25.5 & 51.7 & 0.52 & 998 & 54.9 & 23 & -0.11 \\
\hline $13: 05: 21: 89$ & 111 ! & 84 & 25 & 25.7 & 51.7 & 0.52 & 1004 & 54.7 & 23 & -0.11 \\
\hline $13: 05: 22: 94$ & 112 & 84 & 25 & 25.5 & 51.7 & 0.52 & $1007 !$ & 54.6 & 23 & -0.22 \\
\hline $13: 05: 23: 93$ & 113 & 83 & 25 & 25.7 & 51.7 & 0.51 & 1007 & 54.6 & 23 & -0.22 \\
\hline $13: 05: 24: 91$ & 114 & 83 & 25 & $25.9^{+}$ & 516 & 0.51 & 1001 & $54.8^{\dagger}$ & 24 & -0.04 \\
\hline $13: 05: 25: 96$ & $115^{\prime}$ & 83 & 25 & $25.9 !$ & 51.6 & $0.51^{\prime}$ & 1006 & 54.9 & 24 & -0.04 \\
\hline $13: 05: 26: 95$ & $116 !$ & 83 & $25 !$ & 25.9 & 51.5 & 0.52 & 999 & 54.9 & 24 & -0.07 \\
\hline $13: 05: 27: 94$ & 117 & 83 & 25 & 25.9 & 51.5 & 0.51 & $995 !$ & 54.9 & 23 & -0.07 \\
\hline $13: 05: 28: 92$ & 118 & 83 & 25 & 25.9 & 51.5 & 0.52 & $995 !$ & 54.9 & 23 & -0.2 \\
\hline $13: 05: 29: 91$ & 119 & 85 & 25 & 26.1 & 51.5 & 0.52 & 1000 & 54.9 & 23 & -0.2 \\
\hline $13: 05: 30: 96$ & 120 & 85 & $25 !$ & $26.1 !$ & 51.5 & 0.52 & 998 & 54.8 & 23 & -0.22 \\
\hline 13:05:31:95 & 121 & 85 & 25 & $25.9 !$ & $51.5 !$ & 0.52 & 1004 & 54.9 & 23 & -0.22 \\
\hline $13: 05: 32: 93$ & 122 & 85 & 25 & 25.9 & 51.5 & $0.51 i$ & 1009 & 54.9 & 23 & $0.0 \overline{4}$ \\
\hline $13: 05: 33: 98$ & 123 & 84 & 25 & 25.9 & 51.5 & 0.52 & 1013 & $55.1]$ & 23 & 0.04 \\
\hline $13: 05: 34: 97$ & 124 & 84 & 25 & 25.9 & 51.5 & 0.52 & 1011 & 55.2 & 23 & -0.12 \\
\hline $13: 05: 35: 95$ & 125 & 84 & 25 & $26.1^{1}$ & 51.5 & 0.51 & 1005 & 55.2 & 23 & -0.12 \\
\hline $13: 05: 36: 94$ & $126 !$ & 83 & $25 i$ & 26.1 ! & $51.4^{1}$ & $0.51^{?}$ & 1011. & 55.2 & 23 & -0.2 \\
\hline $13: 05: 37: 93$ & 127 & 82 & 25 & 26.1 & 51.4 & 0.51 & 1004 & 55.1 & 22 & -0.2 \\
\hline $13: 05: 38: 98$ & 128 & 82 & 25 & 26.9 & 51.4 & 0.52 & 998 & 55.1 & 22 & -0.22 \\
\hline $13: 05: 39: 96$ & 129 & 82 & 25 & 26.1 & 51.4 & 0.52 & $998^{\dagger}$ & 55.1 & 22 & 0.07 \\
\hline $13: 05: 40: 95$ & $130^{\circ}$ & 86 & $25 !$ & 26.1 & $51.4^{\dagger}$ & 0.51 & $1004 !$ & 55 & 22 & 0.07 \\
\hline $13: 05: 42: 00$ & 131 & 85 & 25 & $26.1^{i}$ & 51.4 & 0.52 & 1002 & 55 & 22 & -0.15 \\
\hline $13: 05: 42: 99$ & 132 & 85 & 25 & 26.1 & 51.4 & 0.52 & 1008 & 55 & 22 & -0.15 \\
\hline 13:05:43:97 & 133 & 83 & 25 & $26.1 !$ & 51.4 & 0.51 & 1014 & 55 & 22 & -0.2 \\
\hline $13: 05: 44: 96$ & 134 & 83 & 25 & 26.1 & 51.4 & 0.52 & 1017 & 55 & 22 & -0.2 \\
\hline $13: 05: 45: 95$ & 135 & 83 & 25 & 26.3 & 51.4 & 0.52 & 1016 & 55.1 & 23 & -0.23 \\
\hline $13: 05: 46: 99$ & 136 & 83 & 25 & 26.3 & $51.3^{\dagger}$ & 0.52 & $1011^{\top}$ & 55.2 & 23 & -0.23 \\
\hline $13: 05: 47: 98$ & -137 & $83 !$ & $25 !$ & 26.3 & 51.3 & 0.52 & 1016 & 55.2 & 23 & 0.07 \\
\hline 13:05:48:97 & 138 & 81 & 25 & 26.3 & $51.3 \mathrm{j}$ & 0.52 & 1010 & 55.2 & 23 & 0.07 \\
\hline $13: 05: 49: 96$ & 139 & 81 & 25 & 26.3 & 51.3 & 0.54 & 1004 & 55.2 & 23 & -0.13 \\
\hline $13: 05: 50: 95$ & $140_{1}^{+}$ & 85 & 25 & $26.3^{3}$ & $51 . \overline{3}$ & 0.54 & 1002 & 55.2 & 22 & -0.13 \\
\hline $13: 05: 51: 99$ & 141 & 85 & 25 & 26.1 & $51, \overline{3}$ & 0.54 & 1006 & 55.1 & 21 & -0.22 \\
\hline $13: 05: 52: 98$ & 142 & 85 & 25 & 26.1 & 51.3 & 0.52 & 1004 & 55 & 21 & $-0 . \overline{22}$ \\
\hline $13: 05: 53: 97$ & 143 & 85 & 25 & $26.1 !$ & 51.3 & 0.52 & $1018:$ & 55 & 22 & -0.18 \\
\hline $13: 05: 55: 01$ & 144 & $8 \overrightarrow{3 i}$ & 25 & 26.3 & 51.3 & 0.52 & 1016 & 55.1 & 22 & -0.18 \\
\hline 13:05:56:00 & 145 & 83 & 25 & 26.3 & 51.3 & 0.52 & $1020^{+}$ & 55.1 & 22 & 0.07 \\
\hline $13: 05: 56: \overline{99}$ & 146 & 83 & 25 & 26.1 & 51.3 & 0.52 & 1021 & 55.1 & 21 & 0.07 \\
\hline $13: 05: 57: 98$ & 947 & 83 & 25 & 26.1 & 51.3 & 0.52 & 1017 & 55.3 & 22 & -0.15 \\
\hline $13: 05: 58: 97$ & 148 & 81 & 25 & 26.3 & $51.3^{\dagger}$ & 0.51 & 1011 & 55.4 & 22 & -0.15 \\
\hline $13: 06: 00: 01$ & 149 & 81 81 & 25 & 26.3 & 51.3 & 0.51 & 1016 & 55.4 & 22 & -0.25 \\
\hline $13: 06: 01: 00$ & 150 & 81 & $25^{i}$ & 26.5 & 51.2 & 0.52 & 1009 & 55.4 & 22 & -0.14 \\
\hline $13: 06: 02: 04$ & 151 & 80 & 25 & 26.3 & 51.2 & 0.52 & 1006 & 55.3 & 22 & -0.14 \\
\hline $13: 06: 03: 03$ & 152 & 83 & 25 & 26.3 & 51.2 & 0.54 & 1008 & 55.3 & 22 & 0.1 \\
\hline $13: 06: 04: 02$ & 153 & 83 & 25 & 26.3 & 51.2 & 0.52 & $1006^{i}$ & 54.9 & 22 & 0.1 \\
\hline $13: 06: 05: 01$ & 154 & 83 & 25 & $26.3^{1}$ & 51.2 & 0.52 & 1012 & 54.8 & 22 & $-0.1 \overline{7}$ \\
\hline $13: 06: 06: \overline{11}$ & 155 & 83 & 25 & 26.3 & 51.2 & 0.54 & 1018 & 54.8 & 22 & -0.17 \\
\hline
\end{tabular}




\begin{tabular}{|c|c|c|c|c|c|c|c|c|c|c|}
\hline & Elapsed I & & | & & ! & & & & ITorque & \\
\hline TIME & Time (sec) & BIT TC1 & PURG TC2 & PurgePSI & PurgeCFM & DEPTH(IN) & DwnFrc(lb) & RPM & (FTLB) & Inches/Min \\
\hline $13: 06: 07: 15$ & $1 \quad \overline{156}$ & -82 & 25 & $\quad 26.3$ & $\quad 51.2$ & $\quad 0.54$ & 1013 & 54.9 & 2. 22 & -0.32 \\
\hline $13: 06: 08: 25$ & 157 & 83 & $25^{i}$ & 26.3 & 51.2 & 0.54 & $1019^{\circ}$ & 55.1 & 21 & -0.32 \\
\hline $13: 06: 09: 35$ & 158 & 84 & $25^{1}$ & 26.3 & $51.2^{\circ}$ & 0.52 & $1015^{\circ}$ & 55.2 & 21 & -0.09 \\
\hline $13: 06: 10: 45$ & 159 & $84 \uparrow$ & 25 & 26.3 & 51.2 & 0.54 & 1021 & 55.2 & 21 & -0.09 \\
\hline $13: 06: 11: 49$ & 160 & $83 !$ & 25 & $26.3^{\dagger}$ & 51.21 & 0.52 & $1018^{\circ}$ & 55.3 & 21 & -0.03 \\
\hline $13: 06: 12: 59$ & 161 & 83 & 25 & 26.3 & 51.2 & 0.54 & 1022 & 55.4 & 21 & -0.12 \\
\hline $13: 06: 13: 69$ & 162 & 83 & 25 & 26.3 & 51.2 & 0.54 & 1021 & 55.4 & 21 & -0.12 \\
\hline $13: 06: 14: 68$ & 163 & $81^{-t}$ & 25 & 26.3 & 51.2 & 0.52 & 1025 & 55.4 & 21 & -0.19 \\
\hline $13: 06: 15: 72$ & 164 & 81 & 25 & 26.3 & 51.2 & 0.52 & 1027 & 55.2 & 22 & -0.19 \\
\hline $13: 06: 16: 71$ & 165 & 81 & 25 & 26.3 & $51.2 !$ & 0.52 & $1024:$ & 55.2 & 22 & -0.17 \\
\hline $13: 06: 17: 81$ & 166 & 81 & 25 & 26.3 & 51.2 & 0.52 & 1028 & 55.2 & 22 & -0.17 \\
\hline $13: 06: 18: 80$ & $167 !$ & 80 & 25 & 26.3 & 51.2 & $0.52^{\circ}$ & 1024 & 55.3 & 22 & 0.02 \\
\hline $13: 06: 20: 06$ & 168 & $80^{\dagger}$ & 25 & 26.3 & 51.2 & 0.54 & 1028 & 55.3 & 21 & 0.02 \\
\hline $13: 06: 21: 05$ & 169 & 80 & 25 & 26.5 & 51.2 & 0.51 & 1024 & 55.3 & 22 & -0.15 \\
\hline $13: 06: 22: 31$ & $170^{\circ}$ & 81 & 25 & 26.3 & 51.2 & 0.52 & 1028 & 55.4 & 21 & -0.29 \\
\hline $13: 06: 23: 63$ & 171 & 81 & 25 & 26.3 & 51.2 & 0.52 & 1025 & $55 . \overline{4}$ & 21 & -0.29 \\
\hline $13: 06: 24: 73$ & 172 & 83 & 25 & $26.3^{1}$ & 51.2 & 0.52 & 1021 & 55.2 & 21 & -0.12 \\
\hline $13: 06: 25: 72$ & 173 & $80^{\dagger}$ & $25^{\circ}$ & 26.3 & 51.2 & $0.54^{\prime}$ & 1026 & 54.9 & 21 & -0.12 \\
\hline $13: 06: 27: 31$ & 174 & 81 & 25 & 26.5 & 51.2 & 0.52 & 1017 & 54.7 & 22 & 0.04 \\
\hline $13: 06: 28: 30$ & 175 & 81 & 25 & 26.5 & 51.2 & 0.52 & 1015 & 54.7 & 22 & -0.14 \\
\hline $13: 06: 29: 34$ & 176 & 81 & 25 & $26 . \overline{5}$ & 51.2 & 0.54 & 1018 & 54.8 & 22 & -0.14 \\
\hline $13: 06: 30: 39$ & 177 & 81 & 25 & 26.5 & 51.2 & $0.52^{i}$ & 1023 & 54.9 & 21 & -0.31 \\
\hline $13: 06: 31: 87$ & 178 & $78^{\dagger}$ & 25 & 26.5 & 51.1 & 0.52 & $1026^{\prime}$ & 55 & $21^{\dagger}$ & -0.04 \\
\hline $13: 06: 32: 91$ & 179 & 78 & 25 & 26.5 & 51.1 & 0.52 & 1019 & 55 & $21^{1}$ & -0.04 \\
\hline $13: 06: 33: 85$ & 180 & 81 & 25 & $26.5^{\dagger}$ & 51.1 & 0.52 & 1016 & 55.1 & 21 & .0 .05 \\
\hline $13: 06: 34: 94$ & 181 & 81 & $25 !$ & 26.5 & $51.1^{\circ}$ & 0.54 & 1018 & $55.1 \mathrm{j}$ & 21 & -0.05 \\
\hline $13: 06: 36: 32$ & 182 & 79 & 25 & 26.5 & 51.1 & 0.52 & 1022 & 55 & 22 & -0.15 \\
\hline $13: 06: 37: 80$ & 183 & $79^{1}$ & 25 & 26.5 & 51.1 & 0.52 & 1032 & 54.8 & 22 & -0.26 \\
\hline $13: 06: 38: \overline{84}$ & 184 & 79 & 25 & 26.5 & 51.1 & 0.54 & $1031^{\circ}$ & 54.8 & 21 & -0.26 \\
\hline $13: 06: 39: 89$ & 185 & 79 & 25 & 26.6 & 51.1 & 0.52 & 1033 & 54.8 & 21 & -0.07 \\
\hline $13: 06: 40: 99$ & 186 & 79 & 25 & 26.6 & 51 & 0.52 & 1033 & 54.8 & 22 & -0.07 \\
\hline $13: 06: 41: 98$ & 187 & 79 & 25 & 26.6 & 51 & $0.52^{-4}$ & $1033^{\circ}$ & 54.8 & 22 & -0.06 \\
\hline $13: 06: 42: 96$ & 188 & 77 & 25 & 26.6 & 51 & 0.52 & 1027 & 54.8 & $21 \mid$ & $-0.0 \overline{6}$ \\
\hline $13: 06: 43: 95$ & 189 & 77 & 25 & 26.6 & 51 & 0.52 & 1020 & 54.8 & 21 & -0.19 \\
\hline $13: 06: \overline{4} 4: \overline{9} 4$ & $190^{\dagger}$ & 80 & 25 & 26.6 & 51 & 0.54 & 1018 & 54.8 & 21 & -0.19 \\
\hline $13: 06: 45: 98$ & 191 & $81\}$ & 25 & 26.5 & 51 & 0.55 & 1021 & 54.6 & 20 & -0.12 \\
\hline $13: 06: 46: 97$ & $19 \overline{2}$ & $81^{\dagger}$ & 25 & 26.6 & 51 & 0.51 & 1018 & 54.3 & 20 & -0.12 \\
\hline $13: 06: 47: 96$ & 193 & 81 & , tan & 26.6 & 51 & 0.52 & 1032 & 54.3 & 20 & -0.07 \\
\hline $13: 06: 49: 01$ & 194 & 79 & 25 & 26.5 & 51 & 0.52 & $1030^{\circ}$ & 54.4 & 21 & -0.07 \\
\hline $13: 06: 49: 99$ & 195 & 78 & $25^{\circ}$ & 26.6 & 51 & 0.54 & 1034 & $5 \overline{4.5}$ & 20 & -0.16 \\
\hline $13: 06: 50: 98$ & 196 & 78 & 25 & 26.6 & $51 !$ & 0.52 & $1033^{\prime}$ & 54.5 & 20 & -0.28 \\
\hline $13: 06: 51: 97$ & 197 & 78 & 25 & 26.6 & 51 & 0.52 & $1026^{\circ}$ & $54.4^{\dagger}$ & 21 & -0.28 \\
\hline $13: 06: 52: 96$ & 198 & 77 & 25 & 26.6 & 50.9 & $0.52^{\circ}$ & 1019 & $54.5^{\dagger}$ & 21 & -0.09 \\
\hline $13: 06: 53: 95$ & 199 & $77^{\dagger}$ & 25 & 26.8 & 50.9 & 0.52 & $1019^{\circ}$ & $54 . \overline{5}$ & 21 & -0.09 \\
\hline $13: 06: 54: 94$ & $200^{\circ}$ & $80^{\dagger}$ & 25 & $26.8^{\dagger}$ & 50.9 & 0.54 & $1019^{\circ}$ & 54.6 & 21 & -0.05 \\
\hline $13: 06: 55: 98$ & 201 & 81 & 25 & 26.8 & 50.9 & 0.54 & $1020^{\circ}$ & 54.8 & 20 & -0.05 \\
\hline $13: 06: 57: 02$ & 202 & 81 & 25 & $26 . \overline{8}$ & 50.9 & 0.54 & 1024 & $54 . \overline{8}$ & 20 & -0.11 \\
\hline $13: \overline{06}: 58: \overline{01}$ & 2031 & 81 & 25 & 26.8 & 50.8 & 0.54 & 1021 & 55.1 & 20 & -0.11 \\
\hline $13: 06: 59: 00$ & 204 & 79 & 25 & 26.8 & $50 . \overline{8}$ & 0.51 & $1033^{\circ}$ & 55 & 20 & -0.22 \\
\hline $13: 06: 59: 99$ & 205 & 79 & 25 & 26.8 & 50.8 & 0.54 & $1031^{\circ}$ & 55 & 20 & -0.22 \\
\hline $13: 07: 00: 98$ & 206 & $79^{\prime}$ & $25^{i}$ & 27 & 50.8 & $0 . \overline{54}$ & 1035 & 55.1 & 21 & -0.12 \\
\hline $13: 07: 02: 02$ & 207 & $79^{\dagger}$ & $\quad 25$ & 27 & 50.8 & 0.54 & 1033 & $5 \overline{5.2}$ & 21 & -0.12 \\
\hline
\end{tabular}




\begin{tabular}{|c|c|c|c|c|c|c|c|c|c|c|}
\hline & Elapsed & & & & & & & & Torque & \\
\hline TIME & Time (sec) & $B I T$ TC1 & PURG_TC2 F & PurgePSI & PurgeCFM & DEPTH $(I N)$ & DwnFrc(lb) & RPM & rLB) & nches/Min \\
\hline $13: 07: 03: 01$ & $\quad 208$ & -77 & 25 & 27 & $\quad 50.8$ & 0.54 & 1029 & $\quad 55.2$ & 21 & -0.04 \\
\hline $13: 07: 04: 00$ & 209 & 77 & 25 & 27 & 50.8 & 0.52 & $1023^{\circ}$ & $55.5^{\circ}$ & $20 !$ & -0.04 \\
\hline $13: 07: 05: 04$ & $210 !$ & 77 & $25:$ & 27 & 50.7 & 0.52 & 1028 & 55.7 & 21 & -0.14 \\
\hline $13: 07: 06: 03$ & 211 & 77 & 25 & 27 & $50.7^{-}$ & 0.52 & 1023 & 55.7 & 21 & -0.14 \\
\hline $13: 07: 07: 02$ & 212 & 80 & 25 & $26.8^{\dagger}$ & 50.7 & 0.55 & $1019^{\prime}$ & 55.7 & 20 & -0.26 \\
\hline $13: 07: 08: \overline{01}$ & 213 & 80 & 25 & 27 & 50.7 & 0.55 & 1021 & 55.7 & 20 & -0.26 \\
\hline $13: 07: 09: 00$ & 214 & 80 & $25 !$ & 27 & 50.7 & 0.54 & 1026 & 55.7 & 20 & 0.04 \\
\hline $13: 07: 10: 04$ & 215 & 80 & 25 & 27 & 50.7 & 0.54 & 1030 & 55.6 & 20 & 0.04 \\
\hline $13: 07: 11: 03$ & $21 \overline{6}$ & 80 & 25 & 27 & 50.7 & 0.54 & 1026 & 55.6 & 20 & -0.09 \\
\hline $13: 07: 12: \overline{02}$ & 217 & 80 & 26 & 27 & 50.6 & 0.52 & 1031 & 55.6 & 20 & -0.09 \\
\hline $13: 07: 13: 06$ & 218 & 79 & $25^{t}$ & 27 & 50.6 & 0.54 & 1035 & 55.9 & 20 & -0.2 \\
\hline $13: 07: 14: 05$ & 219 & 79 & 26 & 27 & 50.6 & 0.54 & 1036 & 55.9 & 20 & -0.22 \\
\hline 13:07 & 220 & 79 & 25 & 27.2 & 50.6 & 0.54 & 1032 & 55.9 & 21 & -0.22 \\
\hline $13: 07: 16: 03$ & 221 & $79^{+}$ & 25 & 27.2 & 50.6 & 0.54 & 1036 & 55.9 & 21 & -0.19 \\
\hline $13: 07: 17: 02$ & 222 & 78 & 25 & 27.2 & 50.6 & 0.54 & 1032 & 56 & 21 & -0.19 \\
\hline $3: 06$ & $223^{+}$ & 78 & 25 & 27.4 & 50.6 & 0.54 & 1026 & 56 & 21 & \\
\hline 05 & 224 & 78 & 25 & 27.4 & 50.6 & 0.54 & 1021 & 56.1 & 21 & \\
\hline $13: 0$ & 225 & 81 & 25 & 27.4 & 50 & 0.54 & 1021 & 56 & 21 & -0.09 \\
\hline $13: 07: 21: 03$ & 226 & 82 & 26 & 27.2 & 50.5 & 0.54 & 1024 & 56 & 20 & -0.09 \\
\hline $13: 07: 22: 02$ & 227 & 82 & 25 & 27.2 & 50.5 & 0.54 & 1028 & 55.8 & 20 & -0.2 \\
\hline$: 06$ & 228 & 81 & 26 & 27.2 & 50.5 & 0.54 & 1025 & 55.9 & 20 & -0.2 \\
\hline $1: 05$ & 229 & $8 \overline{1}$ & 26 & 27.2 & 50.5 & 0.54 & 1030 & 55.9 & 20 & -0.24 \\
\hline $5: 04$ & 230 & 81 & 25 & 27.2 & 50.5 & 0.52 & 1034 & 56 & 20 & -0.24 \\
\hline $13: 07: 26: 08$ & 231 & $8 \overline{1}$ & 26 & $27 . \overline{2}$ & 50.5 & 0.54 & 1036 & 56.1 & 20 & -0.07 \\
\hline $13: 0$ & 232 & 81 & 25 & 27.4 & 50.5 & 0.54 & $1034^{\ddagger}$ & $56.1^{\dagger}$ & $20^{\circ}$ & -0.07 \\
\hline 3:06 & 233 & 80 & 26 & 27.6 & 50.5 & 0.54 & $31 !$ & 56.1 & 21 & -0.05 \\
\hline $9: 05$ & 234 & 80 & 26 & 27.6 & 50.4 & 0.54 & 1036 & 56.1 & $2 \uparrow$ & $-0 . \overline{05}$ \\
\hline $30: 04$ & 235 & 78 & 25 & $27.6 !$ & 50.4 & $0 . \overline{51}$ & 1032 & 56.1 & 21 & -0.11 \\
\hline 1:08 & 236 & 78 & 26 & 27.6 & 50.4 & 0.54 & 1025 & 56 & 21 & -0.11 \\
\hline 207 & 237 & 78 & 26 & 27.6 & 50.4 & 0.52 & $1021 !$ & 55.9 & 21 & -0.24 \\
\hline $33: 06$ & 238 & 78 & 26 & 27.6 & 50.4 & 0.52 & 1022 & 55.9 & 21 & -0.24 \\
\hline 10 & 239 & 82 & 26 & 27.6 & 50. & & 1022 & 55.7 & 21 & -0.16 \\
\hline $5: 09$ & 240 & 82 & 25 & 27.6 & 50.4 & 0.54 & 1023 & 55.7 & 20 & 0.03 \\
\hline 08 & 241 & 82 & 25 & 27.4 & & & & 55.7 & 20 & 0.03 \\
\hline 7:07 & 242 & 82 & 26 & 27.6 & 50.3 & & 1034 & 55.8 & 20 & -0.16 \\
\hline $38: 05$ & 243 & 81 & $26^{\dagger}$ & 27.6 & 50.3 & 0.54 & 1035 & 55.8 & $2 \overline{0}$ & $-0 . \overline{16}$ \\
\hline $13: 07: 39: 10$ & 244 & 81 & 26 & 27.6 & 50.3 & $0.5 \overline{2}$ & 1034 & 55.8 & 20 & -0.21 \\
\hline $13: 07: 40: 09$ & 245 & 81 & $25^{\dagger}$ & 27.6 & 50.3 & 0.54 & $10 \overline{3}$ & 55.6 & $20^{\dagger}$ & -0.21 \\
\hline & 246 & $78^{\dagger}$ & 26 & 27.6 & 50.2 & & 1035 & 55.4 & 20 & -0.2 \\
\hline & 247 & 78 & 26 & 27.8 & 50.2 & 0.5 & 1031 & 55.4 & 21 . & -0.2 \\
\hline $13: 07: 43: 19$ & 248 & 78 & $26 !$ & 27.8 & 50.2 & 0.52 & 1024 & 55.4 & 21 & 0.01 \\
\hline $13: 07: 44: 10$ & 249 & 79 & 26 & 27.8 & 50.1 & 0.52 & 1021 & 55.5 & 21 & 0.01 \\
\hline $13: 07: 45: \overline{08}$ & 250 & 80 & 25 & 27.8 & 50.1 & 0.54 & 1023 & 55.5 & 20 & -0.13 \\
\hline $13: 07: 46: 07$ & 251 & 80 & 26 & 27.8 & 50.1 & 0.54 & 1021 & 55.5 & $20^{\circ}$ & -0.13 \\
\hline $7: 12$ & 252 & 80 & $25 !$ & 27.8 & 50.1 & 0. & 10 & 55.5 & 20 & -0.22 \\
\hline & 253 & 82 & 26 & 27.8 & $50 . \overline{1}$ & & & $55.5^{\dagger}$ & 20 & -0.22 \\
\hline $99: 09$ & 254 & 81 & 26 & 27.8 & 50.1 & 0.5 & 1033 & 55.5 & 20 & -0.24 \\
\hline $13: 07: 50: 08$ & 255 & 81 & 25 & 27.8 & 50.1 & 0.54 & 1036 & 55.5 & 20 & -0.24 \\
\hline $13: 07: 51: 07$ & 256 & 80 & 26 & 27.8 & 50.1 & 0.54 & 1036 & 55.5 & 20 & 0.11 \\
\hline $13: 07: 52: 12$ & 257 & 80 & 26 & 28 & 50.1 & 0.54 & 1036 & 55.5 & $\overline{20}$ & $0 . \overline{11}$ \\
\hline 13:07:53:10 & 258 & 80 & 25 & 28 & 50 & 0.52 & 1036 & 55.4 & 20 . & $-0.1 \overline{9}$ \\
\hline $13: 07: 54: \overline{09}$ & 259 & 77 & 26 & 28 & 50 & 0.54 & 1031 & 55.4 & 20 & -0.19 \\
\hline
\end{tabular}




\begin{tabular}{|c|c|c|c|c|c|c|c|c|c|c|}
\hline & Elapsed & & & & & & ! & & Torque & \\
\hline$T I M E$ & Time (sec) & BIT_TC1 & PURG_TC2 & PurgePSI: & PurgeCFM & DEPTH(IN) & DwnFrc(lb) & RPM & (FTLB) : & Inches/Min \\
\hline $13: 07: 55: 14$ & 260 & $\quad 77$ & 26 & 28 & 50 & 0.54 & $\quad 1026$ & 55.5 & $\quad 20$ & -0.19 \\
\hline $13: 07: 56: 12$ & 261 & 77 & 26 & 28 & $49.9^{\prime}$ & 0.54 & 1031 & 55.6 & 20 & -0.19 \\
\hline 13:07:57:11 & 262 & 77 & 26 & $28 !$ & 49.9 & 0.54 & $1025^{\circ}$ & 55.6 & 20 & -0.22 \\
\hline 13:07:58:10 & $2 \overline{6}$ & 81 & 26 & 28.2 & 49.9 & 0.54 & 1023 & 55.6 & $20^{\circ}$ & -0.22 \\
\hline $13: 07: 59: 09$ & 264 & 81 & 26 & 28.2 & 49.9 & 0.55 & $1025^{\circ}$ & 55.7 & 20 & -0.03 \\
\hline $13: 08: 00: 13$ & 265 & 81 & 26 & 28.2 & 49.9 & 0.54 & $1030^{\circ}$ & 55.7 & 20 & -0.09 \\
\hline $13: 08: 01: 12$ & 266 & 81 & 26 & 28.2 & $49.9^{\dagger}$ & 0.55 & 1035 & 55.8 & 20 & -0.09 \\
\hline $13: 08: 02: 11$ & 267 & 80 & 26 & 28.2 & 49.9 & 0.54 & $1038^{\circ}$ & 55.7 & 20 & -0.13 \\
\hline $13: 08: 03: 16$ & 268 & 80 & 26 & $28.2^{+}$ & 49.9 & 0.55 & 1035 & $55.7^{1}$ & 20 & -0.13 \\
\hline $13: 08: 04: 14$ & 269 & 80 & 26 & 28.4 & 49.9 & 0.54 & 1037 & 55.7 & 20 & -0.29 \\
\hline $13: 08: 05: 13$ & $270^{\circ}$ & 78 & 26 & 28.4 & 49.9 & 0.54 & 1037 & 55.6 & 20 & -0.29 \\
\hline $13: 08: 06: 12$ & 271 & 77 & 26 & 28.4 & $49.9^{\dagger}$ & 0.54 & $1034^{\prime}$ & 55.6 & 20 & -0.09 \\
\hline $13: 08: 07: \overline{11}$ & 272 & 77 & 26 & 28.4 & 49.9 & 0.51 & $1028^{\circ}$ & 55.7 & 20 & -0.09 \\
\hline $13: 08: 08: 15$ & 273 & 77 & 26 & $28.6 !$ & 49.9 & 0.54 & 1034 & 55.7 & 20 & -0.06 \\
\hline $13: 08: 09: 14$ & 274 & 78 & 26 & $28.6 !$ & 49.9 & 0.54 & 1028 & 55.7 & 20 & -0.06 \\
\hline $13: 08: 10: 13$ & 275 & 78 & 26 & $28.6 !$ & 49.9 & 0.54 & 1024 & 55.7 & 19 & -0.14 \\
\hline $13: 08: 11: 17$ & 276 & 78 & 26 & 28.6 & 49.9 & 0.54 & 1025 & 55.8 & 19 & -0.14 \\
\hline $13: 08: 12: 16$ & $27 \overline{7}^{+}$ & $8 \overline{2}$ & 26 & 28.6 & 49.9 & 0.55 & $1030^{\circ}$ & 55.8 & 19 & -0.27 \\
\hline $13: 08: 13: 15$ & $27 \overline{8}$ & 82 & 26 & $28.6^{\prime}$ & $49.9 !$ & 0.54 & 1035 & 55.9 & 19 & -0.27 \\
\hline $13: 08: 14: 14$ & $27 \overline{9}$ & 83 & 26 & 28.6 & 49.9 & 0.54 & 1030 & 55.9 & $19^{\circ}$ & -0.13 \\
\hline $13: 08: 15: 13$ & 280 & 79 & 26 & 28.8 & 49.9 & 0.54 & 1035 & 55.9 & 19 & -0.13 \\
\hline $13: 08: 16: 17$ & 281 & 79 & 25 & 28.8 & 49.9 & 0.54 & $1040^{\circ}$ & $55.9^{+}$ & 20 & 0.03 \\
\hline $13: 08: 17: 16$ & 282 & 79 & 26 & 28.8 & $49.9^{+}$ & 0.54 & 1041 & $55.9^{\dagger}$ & 20 & 0.03 \\
\hline $13: 08: 18: 15$ & $283^{1}$ & 79 & 26 & 29 & 49.9 & 0.54 & 1037 & $55.9^{\dagger}$ & $20^{\prime}$ & -0.16 \\
\hline $13: 08: 19: 14$ & 284 & 77 & 26 & $28.8^{i}$ & 49.9 & 0.54 & $1037^{\circ}$ & 55.8 & 20 & -0.16 \\
\hline $13: 08: 20: 13$ & 285 & 76 & 26 & $29 i$ & 49.9 & 0.54 & 1023 & 55.8 & 20 & -0.22 \\
\hline $13: 08: 21: 17$ & 286 & 76 & 26 & 29 & 49.9 & 0.54 & 1028 & 55.8 & 20 & -0.22 \\
\hline $13: 08: 22: 16$ & 287 & 76 & 26 & $29^{\circ}$ & 49.9 & 0.54 & 1022 & 56 & 19 & -0.24 \\
\hline $13: 08: 2 \overline{3}: \overline{15}$ & 288 & 78 & 26 & 29 & $49.9^{1}$ & 0.55 & 1020 & $56.1^{\dagger}$ & 18 & 0 \\
\hline $13: 08: 24: 19$ & 289 & 79 & 26 & 29 & $49.9^{1}$ & $0.52^{-1}$ & 1021 & 56.1 & 18 & 0 \\
\hline $13: 08: 25: 18$ & 290 & 79 & 26 & 29 & 49.9 & 0.55 & 1019 & 56.1 & 18 & -0.1 \\
\hline $13: 08: 26: 17$ & 291 & 81 & 26 & $29 !$ & 49.9 & 0.55 & $1020:$ & $56.2 !$ & 18 & -0.1 \\
\hline $13: 08: 27: 16$ & 292 & 81 & 26 & 29 & 49.9 & 0.54 & 1024 & 56.2 & 18 & -0.1 \\
\hline $13: 08: 28: 15$ & 293 & 81 & 26 & 29 & 49.9 & 0.54 & 1029 & 56.1 & 18 & -0.1 \\
\hline $13: 08: 29: 19$ & 294 & 78 & 26 & 29 & 49.9 & 0.54 & $1031^{\circ}$ & 56.1 & 18 & -0.31 \\
\hline $13: 08: 30: 18$ & 295 & 78 & 26 & 29 & $49.9^{\prime}$ & 0.54 & $1028^{\circ}$ & 56.2 & 18 & -0.31 \\
\hline $13: 08: 3 \overline{1: 17}$ & 296 & $\overline{78}$ & 26 & 29.2 & 49.9 & 0.54 & $1030^{\circ}$ & 56.2 & 19 & -0.14 \\
\hline $13: 08: 32: 21$ & 297 & 79 & 26 & 29.2 & 49.9 & 0.54 & $1031^{\circ}$ & 56.3 & 19 & -0.14 \\
\hline $13: 08: 33: 20$ & 298 & 75 & 26 & $29.2 !$ & 49.9 & 0.54 & 1029 & 56.4 & 19 & 0.03 \\
\hline $13: 08: 34: 19$ & 299 & 75 & 26 & 29.2 & 49.9 & 0.54 & 1023 & $56.4^{\dagger}$ & 19 & 0.03 \\
\hline $13: 08: 35: 18$ & 300 & 75 & 26 & 29.4 & 49.9 & $0.54^{+}$ & 1018 & 56.5 & 19 & $-0 . \overline{13}$ \\
\hline $13: 08: 36: 17$ & 301 & 77 & 26 & 29.4 & 49.9 & 0.54 & 1014 & 56.5 & 19 & -0.13 \\
\hline $13: 08: 37: 21$ & 302 & 77 & 26 & 29.2 & $49.9^{-}$ & 0.55 & $1018^{\circ}$ & 56.6 & 18 & -0.22 \\
\hline $13: 08: 38: 20$ & 303 & 77 & 26 & 29.4 & 49.9 & 0.54 & 1013 & 56.4 & 18 & -0.22 \\
\hline $13: 08: 39: 19$ & 304 & 81 & 26 & 29.4 & 49.9 & 0.55 & 1014 & 56.4 & 18 & -0.2 \\
\hline $13: 08: 40: 23$ & 305 & 81 & 26 & 29.2 & 49.9 & 0.55 & $1018^{\circ}$ & 56.2 & 18 & -0.2 \\
\hline $13: 08: 41: 22$ & 306 & 81 & 26 & 29.2 & 49.9 & $0.54^{\dagger}$ & 1013 & 56.2 & 18 & -0.06 \\
\hline $13: 08: 42: 21$ & 307 & 82 & 26 & 29.4 & 49.9 & 0.54 & 1025 & 56.2 & 18 & -0.06 \\
\hline $13: 08: 43: 20$ & 308 & 79 & 26 & 29.2 & $49 \overline{9}$ & 0.55 & 1021 & 56.3 & 18 & -0.09 \\
\hline $13: 08: 44: 18$ & 309 & 80 & 26 & 29.4 & 49.9 & 0.54 & 1024 & $56.3^{\dagger}$ & 19 & -0.09 \\
\hline $13: 08: 45: 23$ & 310 & 80 & $2 \overline{6}$ & 29.4 & 49.9 & 0.54 & $1027^{\circ}$ & 56.4 & 19 & -0.1 \\
\hline $13: 08: 46: 2 \overline{2}$ & 311 & 80 & 26 & 29.4 & 49.9 & 0.54 & 1026 & $56 . \overline{4}^{\dagger}$ & $1 \overline{9}$ & -0.29 \\
\hline
\end{tabular}




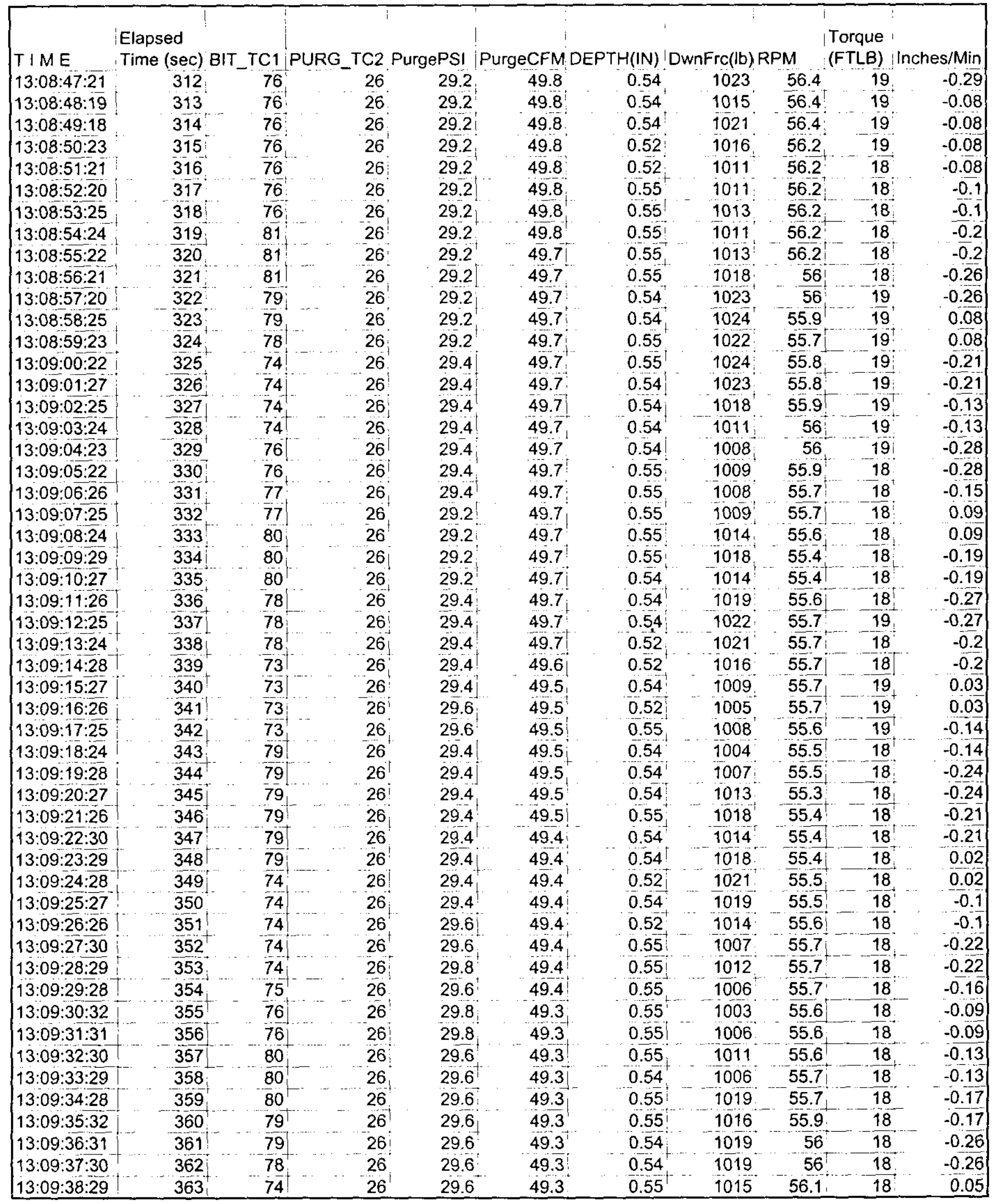




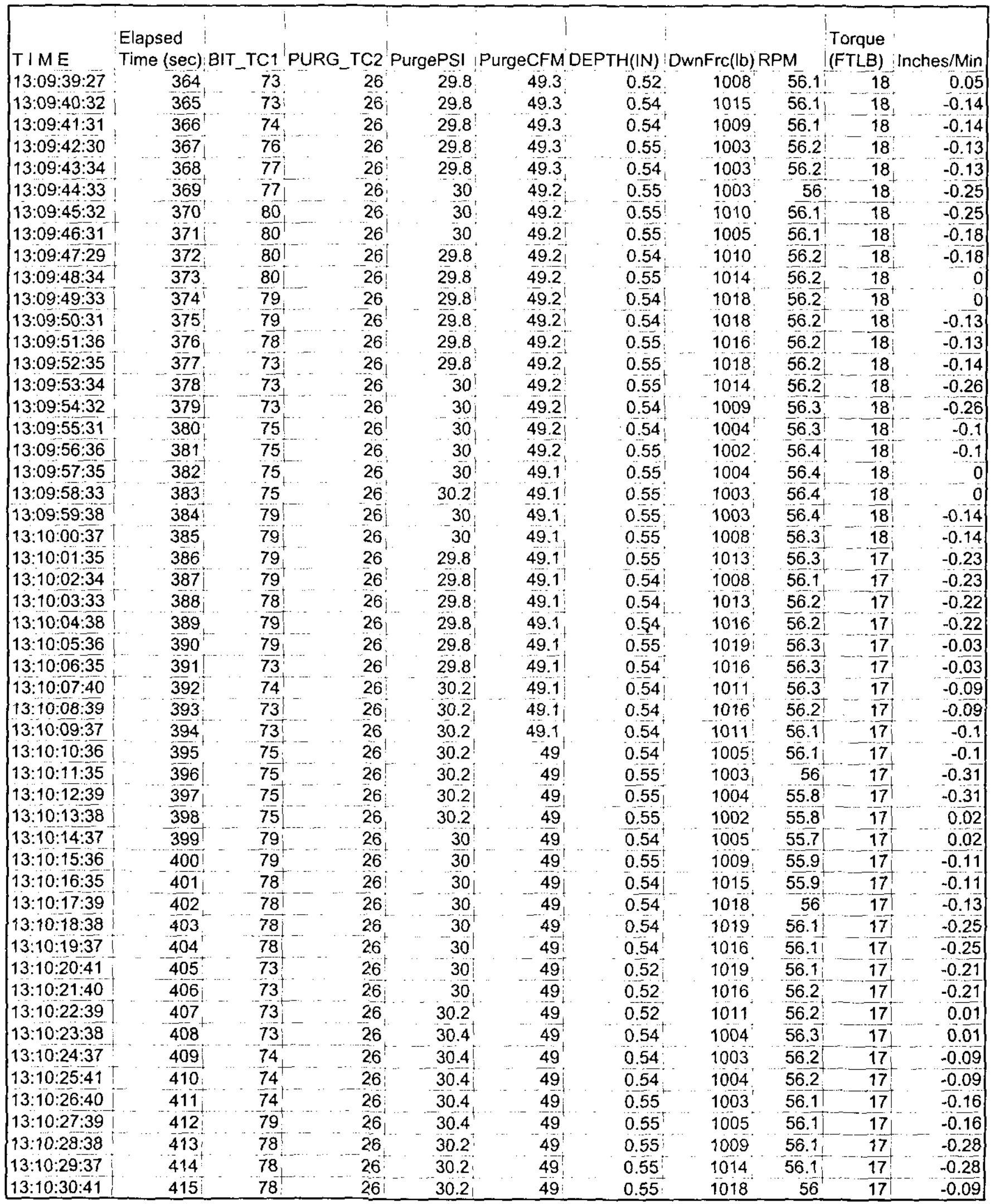




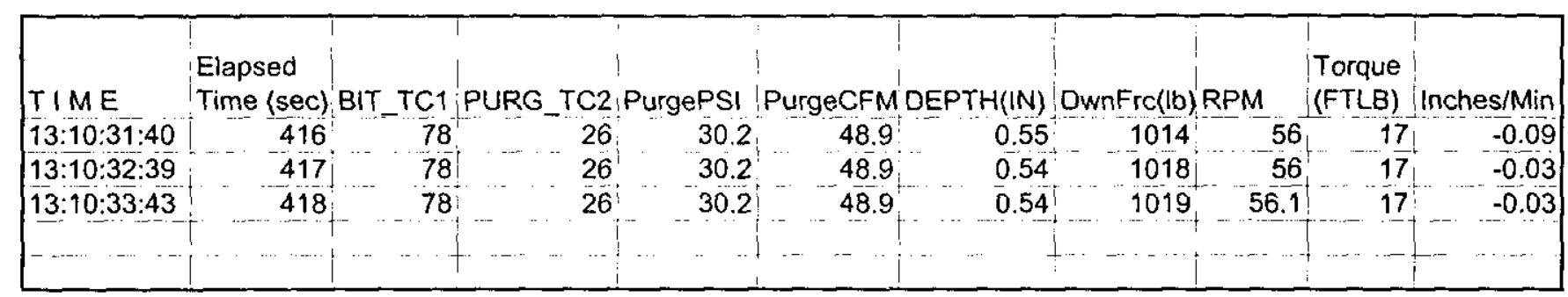




\begin{tabular}{|c|c|c|c|c|c|c|c|c|c|c|}
\hline$T I M E$ & $\begin{array}{l}\text { Elapsed } \\
\text { Time } \\
\text { (sec) }\end{array}$ & BIT_TC1 & PURG_TC2 & PurgePSI & PurgeCFM & DEPTH $(\mathrm{IN})$ & DwnFrc(lb) & RPM & $\begin{array}{l}\text { Torque } \\
\text { (FTLB) }\end{array}$ & Inches/Min \\
\hline $12: 35: 00: 40$ & 0 & -24 & +-25 & $\quad 216$ & 54.2 & $\begin{array}{r}-1.01 \\
\end{array}$ & 1 & 56.6 & 0 & $\quad-0.14$ \\
\hline $12: 35: 01: 39$ & 1. & 24 & 25 & 21.6 & 54.2 & -0.84 & 0 & 56.6 & 0 & -0.14 \\
\hline $12: 35: 02: 38$ & 2 & 24 & 25 & 21.6 & 54.2 & -0.55 & 1 & 56.4 & $\overrightarrow{0}$ & 4.81 \\
\hline $12: 35: 0$ & 3 & 24 & 25 & 21.6 & 54.2 & -0.26 & $1^{t}$ & 56.4 & 0 & 4.81 \\
\hline $04: 35$ & 4 & 25 & 25 & 21.6 & 54.2 & $-0 . \overline{02}$ & $70^{\circ}$ & 56.3 & 4 & 12.11 \\
\hline $55: 34$ & 5 & 26 & 25 & 21.8 & $54 . \overline{2}$ & 0.05 & $709^{\circ}$ & 56.3 & $\overline{27}$ & 12.11 \\
\hline $6: 33$ & 6 & 28 & $25 !$ & 21.8 & 54.2 & 0.06 & 793 & 56.3 & 29 & 5.64 \\
\hline $07: 32$ & 7 & 31 & 25 & 21.8 & 54.2 & 0.09 & 785 & 56 & 31 & 5.64 \\
\hline 31 & $8:$ & 33 & 25 & 21.8 & 54.2 & 0.1 & 789 & 55.5 & 27 & 0.84 \\
\hline 30 & 9 & 34 & 25 & 22 & 54.2 & 0.11 & 798 & 55.5 & 25 & 0.84 \\
\hline 29 & 10 & 36 & 25 & 22 & 54.2 & 0.14 & 823 & 55.5 & 24 & $0 . \overline{8}$ \\
\hline$: 27$ & 11 & $3 \overline{7}$ & 25 & 22 & 54.2 & 0.15 & 842 & 55.5 & 23 & 0.63 \\
\hline$: 26$ & 12 & 40 & 25 & 22 & 54.2 & 0.15 & 860 & 55.5 & 22 & 0.63 \\
\hline 25 & 13 & 41 & 25 & 22.2 & 54.2 & 0.16 & 878 & 55.4 & 19 & 0.11 \\
\hline 24 & 14 & 42 & 25 & 22.2 & 54.2 & 0.15 & 887 & 55.4 & 18 & 0.11 \\
\hline 23 & $15 \mathrm{i}$ & 43 & 25 & 22.4 & 54.2 & 0.14 & 896 & 55.4 & 18 & -0.02 \\
\hline & 16 & 44 & 25 & 22.4 & 54.2 & 0.16 & 903 & 55.3 & 17 & -0.02 \\
\hline 21 & 17 & 44 & 25 & 22.4 & 54.2 & 0.15 & 909 & 55.2 & 17 & 0.13 \\
\hline 20 & 18 & 45 & 25 & 22.4 & 54.2 & 0.16 & 911 & 55.2 & 17 & 0.13 \\
\hline & 19 & 45 & 25 & 22.4 & 54.2 & 0.16 & 915 & 55.2 & 17 & 0.06 \\
\hline $5: 17$ & 20 & 46 & 25 & 22.4 & 54.2 & 0.18 & 914 & 55.2 & 16 & 0.06 \\
\hline & 21 & 46 & 25 & 22.4 & 54.2 & $0.1 \overline{6}$ & 923 & $5 \overline{5} .2$ & 16 & -0.14 \\
\hline 15 & 22 & 47 ! & 25 & 22.4 & 54.2 & 0.16 & 923 & 55.2 & 16 & -0.14 \\
\hline$: 14$ & 23 & 47 & $25^{!}$ & 22.6 & 4.2 & 0.18 & 916 & 55.3 & 16 & -0.25 \\
\hline & 24 & 48 & 25 & 22.6 & $5 \overline{4.2}$ & 0.16 & 918 & 55.3 & 16 & -0.25 \\
\hline & 25 & 48 & 25 & 22.6 & 54.2 & 0.16 & 918 & 55.4 & $\overline{16}$ & 0.09 \\
\hline & 26 & 48 & 25 & 22.6 & 54.2 & 0.16 & 915 & 55.4 & 15 & 0.09 \\
\hline & 27 & 48 & $25^{\prime}$ & 22.6 & 54.2 & 0.16 & 909 & 55.4 & 15 & $-0 . \overline{03}$ \\
\hline & 28 & 49 & 25 & 22.6 & 54.2 & 0.16 & 906 & $55 . \overline{4}$ & 15 & -0.03 \\
\hline & 29 & 49 & 25 & 22.6 & 54.2 & 0.16 & $906^{1}$ & 55.4 & 15 & -0.1 \\
\hline & 30 & 49 & 25 & 22.6 & & 0.16 & 909 & 55.4 & 15 & -0.1 \\
\hline & 31 & 49 & 25 & 22.6 & & 0.16 & 915 & 55.4 & 15 & $-0 . \overline{28}$ \\
\hline & 32 & 50 & 25 & 22.6 & 4.1 & $0 . \overline{16}$ & 911 & 55.4 & 15 & $-0 . \overline{28}$ \\
\hline & 33 & 50 & 25 & 22.6 & 5 & 0.16 & 916 & $55 . \overline{4}$ & 15 & 0.15 \\
\hline & 34 & 50 & $25 !$ & 22.6 & 5 & 0.18 & 919 & 55.3 & 15 & 0.15 \\
\hline & 35 & 50 & 25 & 22.8 & 54.1 & 0.18 & & 55.4 & 15 & -0.07 \\
\hline & 36 & 51 & 25 & 22.8 & 54.7 & 0.18 & $917^{1}$ & 55.4 & 15 & -0.07 \\
\hline & 37 & 51 & 25 & 22.6 & 54.1 & 0.16 & 911 & 55.4 & 15 & $-0 . \overline{1}$ \\
\hline & 38 & 51 & 25 & 22.8 & 54.1 & $0 . \overline{18}$ & 909 & 55.4 & 15 & $-0 . \overline{1}$ \\
\hline & 39 & 51 & 25 & 22.8 & & 0.18 & $910^{\circ}$ & 4 & 15 & -0.33 \\
\hline & 40 & 52 & 25 & 22.8 & & 0.19 & 909 & 55.4 & 15 & -0.33 \\
\hline & 41 & 5 & 25 & 22.8 & & 0.19 & 910 & 55.4 & 15 & 0.07 \\
\hline $1: 92$ & 42 & 52 & 25 & 22.8 & 54.1 & 0.19 & 913 & 55.4 & 15 & 0.07 \\
\hline $2: 91$ & 43 & 52 & 25 & 22.8 & 54.1 & 0.18 & 916 & 55.4 & 15 & 0 \\
\hline $43: 90$ & 44 & 52 & 25 & 22.8 & 54.1 & 0.18 & 918 & 55.4 & 15 & -0.16 \\
\hline $12: 35: 44: 89$ & 45 & 53 & 25 & 22.8 & $5 \overline{4.1}$ & 0.16 & 916 & 55.4 & 14 & -0.16 \\
\hline $12: 35: 45: 88$ & 46 & 53 & 25 & 22.8 & 54 & 0.16 & 911 & 55.4 & 15 & -0.33 \\
\hline $12: 35: 46: 87$ & 47 & 53 & 25 & 22.8 & 54 & 0.18 & 906 & 55.4 & 14 & $-0 . \overline{33}$ \\
\hline $12: 35: 47: 86$ & 48 & 53 & 25 & 22.8 & 54 & 0.18 & 907 & 55.3 & 15 & 0.18 \\
\hline $12: 35: 48: 84$ & 49 & 53 & 25 & 22.8 & 54 & 0.18 & 904 & 55.2 & 15 & 0.18 \\
\hline $12: 35: 49: 83$ & 50 & 53 & 25 & 22.9 & 54 & 0.19 & 902 & 55.2 & 14 & $-0 . \overline{13}$ \\
\hline
\end{tabular}




\begin{tabular}{|c|c|c|c|c|c|c|c|c|c|c|}
\hline TIME & $\begin{array}{l}\text { Elapsed } \\
\text { Time } \\
\text { (sec) }\end{array}$ & BIT_TC1 & PURG_TC2 & PurgePSI & PurgeCFM & DEPTH(IN) & DwnFrc(lb) & RPM & $\begin{array}{l}\text { Torque } \\
\text { (FTLB) }\end{array}$ & Inches/Min \\
\hline $12: 35: 50: 82$ & 51 & -53 & $\quad 25$ & 22.9 & $\quad 54$ & $\quad 0.18$ & 903 & 55.3 & $3 \quad 14$ & $\quad-0.13$ \\
\hline $12: 35: 51: 81$ & 52 & 54 & 25 & 22.9 & 54 & 0.18 & 908 & 55.4 & 14 & -0.14 \\
\hline $12: 35: 52: 80$ & 53 & 54 & 25 & 22.9 & 54 & 0.18 & 913 & 55.4 & 14 & -0.14 \\
\hline 12:35: & 54 & 54 & 25 & 22.9 & 54 & 0.16 & 914 & 55.5 & 14 & $-0 . \overline{32}$ \\
\hline $4: 78$ & 55 & 54 & 25 & 22.9 & 54 & 0.16 & 912 & 55.5 & 14 & -0.32 \\
\hline $12: 35: 55: 76$ & 56 & 54 & 25 & 22.9 & 54 & $0.16^{+}$ & 908 & 55.5 & 14 & 0.04 \\
\hline $56: 75$ & 57 & 54 & 25 & 22.9 & 54 & 0.16 & 911 & 55.4 & 14 & 0.04 \\
\hline $7: \overline{7} 4$ & 58 & 54 & 25 & 22.9 & $53 . \overline{9}^{+}$ & $0.1 \overline{6}$ & 906 & $5 \overline{5.4}$ & 14 & 0.03 \\
\hline$\overline{73}$ & 59 & 54 & 25 & 22.9 & $53.9^{\dagger}$ & $0.1 \overline{9}$ & 904 & 55.4 & 14 & 0.03 \\
\hline $9: 72$ & 60 & 54 & 25 & $22.9 !$ & 53.9 & 0.19 & 903 & 55.5 & 14 & -0.11 \\
\hline $0: 71$ & 61 & 54 & 25 & 22.9 & 53.9 & 0.19 & 904 & 55.4 & 14 & -0.11 \\
\hline$: 70$ & 62 & 54 & 25 & 22.9 & 53.9 & 0.19 & 909 & 55.4 & 14 & $-0 . \overline{3}$ \\
\hline 69 & 63 & 55 & 25 & 23.1 & 53.9 & 0.19 & 914 & 55.6 & 14 & -0.3 \\
\hline 67 & 64 & 55 & 25 & 22.9 & 53.9 & 0.19 & 916 & 55.6 & 14 & -0.01 \\
\hline 66 & 65 & 55 & 25 & 22.9 & $53 . \overline{9}$ & 0.18 & 916 & 55.6 & 14 & -0.01 \\
\hline 65 & 66 & 55 & 25 & 22.9 & 53.8 & 0.18 & 916 & 55.6 & 14 & 0.04 \\
\hline 64 & 67 & 55 & 25 & 22.9 & 53.8 & 0.18 & 914 & 55.6 & 14 & 0.04 \\
\hline $7: 63$ & 68 & 55 & 25 & 23.1 & 53.8 & 0.18 & 909 & 55.6 & 14 & -0.11 \\
\hline $3: 62$ & 69 & 55 & 25 & 23.1 & 53.8 & 0.18 & 906 & 55.6 & 14 & -0.11 \\
\hline 61 & 70 & 55 & 25 & 23.1 & $53.8^{\dagger}$ & 0.19 & 906 & 55.5 & 14 & -0.31 \\
\hline & 71 & 55 & 25 & 23.1 & 53.8 & 0.18 & 908 & 55.5 & 14 & -0.31 \\
\hline 58 & 72 & 55 & 25 & 23.11 & $53.8^{+}$ & 0.18 & 913 & 55.4 & 14 & \\
\hline & 73 & $5 \overline{6}$ & 25 & 23.1 & 53.8 & 0.18 & 909 & 55.3 & 14 & \\
\hline & 74 & 56 & 25 & 23.1 & 53.8 & 0.19 & 914 & 55.3 & 11 & 0.02 \\
\hline & 75 & $5 \overline{6}$ & 25 & $23.1 !$ & 53.7 & 9 & 919 & 55.1 & i) & $0 . \overline{02}$ \\
\hline & 76 & $5 \overline{6}$ & 25 & 23.1 & 3.7 & 0.18 & 919 & 55.1 & 14 & -0.13 \\
\hline 53 & 77 & $5 \overline{6}$ & 25 & 23.1 & 53.7 & $0.1 \overline{9}$ & 916 & 55.1 & 14 & -0.13 \\
\hline$: 52$ & 78 & $5 \overline{5}$ & 25 & 23.1 & .7 & 0.19 & 911 & 55.2 & 14 & -0.28 \\
\hline & 79 & 55 & 25 & 23.1 & $5 \overline{3} .7$ & 0.19 & 909 & 55.2 & 14 & -0.03 \\
\hline & 80 & 55 & 25 & 23.1 & 53.7 & 0.19 & 910 & 55.2 & $1 \overline{4}$ & -0.03 \\
\hline & 81 & $5 \overline{5}$ & 25 & $23.1 !$ & 53.7 & 0.19 & 914 & 55.2 & 14 & 0.06 \\
\hline$: 47$ & 82 & 55 & 25 & 23.1 & 53. & 0.19 & 911 & 55.2 & 14 & 0.06 \\
\hline & 83 & 55 & 25 & 23.1 & 53.6 & 0.19 & 916 & 55.2 & 13 & -0.17 \\
\hline & 84 & 56 & 25 & 23.1 & 53.6 & 8 & 921 & 55.3 & 13 & $-0 . \overline{17}$ \\
\hline $4: 44$ & 85 & 56 & 26 & 23.1 & 53.6 & $0 . \overline{19}$ & $923^{-}$ & 55.3 & 13 & -0.22 \\
\hline & 86 & 56 & 26 & 23.1 & 53.6 & 0.18 & 922 & 55.3 & 13 & -0.22 \\
\hline$: 41$ & 87 & 56 & 26 & 23.1 & 53.6 & 0.18 & 917 & 55.2 & 13 & -0.07 \\
\hline $7: 40$ & 88 & 56 & 26 & 23.1 & 53.6 & 0.19 & 913 & 55.2 & 14 & -0.07 \\
\hline & 89 & 56 & 25 & 23.3 & 53.6 & 0.18 & & 55.2 & 14 & 0.05 \\
\hline & 90 & 56 & 26 & 23.3 & 53 & 0.18 & 9 & 55.2 & 14 & 0.05 \\
\hline & 91 & 56 & 26 & $23.3^{\top}$ & 53.6 & 0.19 & 91 & 55.2 & 14 & -0.13 \\
\hline & 92 & 56 & 26 & 23.3 & 53.6 & 0.19 & & 55.2 & 14 & -0.13 \\
\hline & 93 & 56 & 25 & $23.3^{1}$ & 53.6 & 0.19 & 921 & 55.1 & 14 & -0.32 \\
\hline & 94 & 56 & 25 & 23.3 & 53.6 & 0.19 & 924 & 55.1 & 14 & -0.32 \\
\hline $4: 32$ & 95 & 56 & 25 & 23.1 & 53.6 & 0.18 & 925 & 55.1 & 14 & 0.09 \\
\hline 31 & 96 & 56 & 25 & 23.1 & 53.6 & 0.18 & 921 & 55.1 & 14 & $0.0 \overline{9}$ \\
\hline $36: 30$ & 97 & $5 \overline{6}$ & 25 & 23.1 & $5 \overline{3.6}$ & 0.18 & 916 & 55.1 & 14 & -0.04 \\
\hline $12: 36: 37: 29$ & 98 & 56 & 25 & 23.3 & 53.6 & 0.19 & 913 & 55.1 & 14 & -0.04 \\
\hline $12: 36: 38: 28$ & 99 & 56 & 25 & 23.3 & 53.5 & 0.18 & 914 & 54.9 & 14 & -0.22 \\
\hline $12: 36: 39: 27$ & 100 & 56 & 25 & 23.3 & 53.5 & 0.19 & 912 & 54.9 & 14 & -0.22 \\
\hline $12: 36: 40: 25$ & 101 & 56 & 25 & $23.3^{1}$ & $53 . \overline{5}$ & 0.19 & 914 & 54.8 & 14 & -0.26 \\
\hline
\end{tabular}




\begin{tabular}{|c|c|c|c|c|c|c|c|c|c|c|}
\hline TIME & $\begin{array}{l}\text { Elapsed } \\
\text { Time } \\
(\mathrm{sec})\end{array}$ & BIT_TC1 & PURG_TC2 & PurgePSI & PurgeCFM & DEPTH(IN) & DwnFrc(lb) & RPM & $\begin{array}{l}\text { Torque } \\
\text { (FTLB) }\end{array}$ & Inches/Min \\
\hline $12: 36: 41: 24$ & 102 & 56 & -25 & $\quad 23.3$ & $\quad 53.5$ & 0.19 & $\quad 918$ & 54.8 & 14 & $\quad-0.26$ \\
\hline $12: 36: 42: 23$ & 103 & 56 & 25 & 23.3 & 53.5 & 0.19 & 922 & 54.8 & 14 & $0.0 \overline{9}$ \\
\hline $12: 36: 43: 22$ & 104 & 56 & 25 & 23.3 & 53.5 & 0.19 & 923 & 54.9 & 14 & 0.09 \\
\hline $12: 36: 44: 21$ & 105 & 56 & 25 & 23.3 & 53.5 & 0.19 & 919 & 55.1 & 14 & -0.06 \\
\hline $12: 36: 45: 20$ & 106 & 56 & 26 & 23.3 & 53.4 & 0.19 & 914 & 55.1 & 14 & -0.06 \\
\hline $12: 36: 46: 19$ & 107 & 56 & 26 & 23.3 & 53.4 & 0.19 & 918 & 55.2 & 14 & -0.3 \\
\hline $12: 36: 47: 17$ & 108 & $56^{-1}$ & $26^{\circ}$ & 23.3 & 53.4 & 0.19 & 913 & 55.4 & 14 & -0.3 \\
\hline $12: 36: 48: 16$ & 109 & 56 & 25 & 23.3 & 53.4 & 0.19 & 910 & 55.4 & 14 & -0.17 \\
\hline $12: 36: 49: 15$ & $110^{\dagger}$ & 56 & 26 & 23.3 & 53.4 & 0.2 & 910 & 55.5 & 14 & -0.17 \\
\hline $12: 36: 50: 14$ & 111 & $56^{\dagger}$ & 26 & 23.3 & 53.4 & 0.19 & 913 & 55.4 & 14 & 0.18 \\
\hline $12: 36: 51: 13$ & 112 & 56 & 26 & 23.3 & 53.4 & 0.19 & 917 & 55.4 & 14 & -0.18 \\
\hline $12: 36: 52: 12$ & $113^{\dagger}$ & 56 & 26 & 23.3 & 53.4 & 0.19 & 921 & 55.2 & 14 & -0.18 \\
\hline $111^{\mathrm{i}}$ & 114 & 56 & 25 & 23.3 & $53 . \overline{4}$ & 0.19 & 921 & 55.3 & $13^{\dagger}$ & -0.24 \\
\hline 10 & 115 & $56^{\dagger}$ & 25 & 23.3 & $53.4^{1}$ & 0.19 & 917 & 55.3 & 13 & -0.24 \\
\hline 08 & 116 & 56 & 25 & $23.3^{i}$ & 53.4 & 0.19 & 920 & 55.4 & 13 & -0.21 \\
\hline $5 \overline{6: 07}$ & 117 & 56 & $25^{\dagger}$ & 23.3 & 53.4 & $0.19^{\dagger}$ & 914 & 55.3 & 13 & -0.21 \\
\hline$: 06$ & 118 & $56\rceil$ & $25 !$ & 23.3 & 53.4 & $0.18^{+}$ & 911 & $55.3^{i}$ & 13 & 0.08 \\
\hline $8: 05$ & 119 & $56 !$ & $25^{r}$ & 23.3 & 53.4 & 0.19 & 909 & 55.2 & 14 & 0.08 \\
\hline & 120 & 56 & 26 & 23.3 & 53.4 & 0.19 & 911 & $55.1^{!}$ & 14 & -0.1 \\
\hline $00: 03$ & 121 & 56 & 25 & 23.3 & 53.4 & 0.19 & 915 & $55.1^{\prime}$ & 14 & -0.1 \\
\hline$: 02$ & $122 i$ & $56 !$ & 26 & 23.3 & $53.4 !$ & 0.19 & 919 & 55.1 & 14 & -0.31 \\
\hline$: 00$ & 123 & 561 & $26^{\prime}$ & 23.3 & 53.4 & $0.19^{\prime}$ & 922 & 55 & 14 & -0.31 \\
\hline 99 & 124 & $56]$ & 26 & 23.3 & 53.4 & 0.19 & 921 & 55 & $14^{\dagger}$ & -0.19 \\
\hline & 125 & 56 & 26 & 23.3 & 53.4 & 0.1 & 920 & $55 !$ & 13 & -0.19 \\
\hline & $126 !$ & 56 & 26 & 23.3 & 53. & & 918 & 55 & 13 & 0.05 \\
\hline & $\overline{127}$ & 56 & 26 & 23.3 & 53 & $0.19^{\circ}$ & 913 & 55 & 13 & 0.09 \\
\hline & 128 & 56 & 26 & $23.3_{\mathrm{i}}^{\dagger}$ & 53.4 & 0.18 & 910 & 55.1 & 14 & -0.11 \\
\hline $94^{-1}$ & 129 & 56 & 25 & 23.3 & 53.4 & 0.18 & 911 & 55.1 & 14 & -0.11 \\
\hline $08: 93$ & 130 & 56 & 26 & 23.3 & 53.4 & 0.19 & 913 & 55.2 & 14 & -0.34 \\
\hline 91 & 131 & 56 & 26 & 23.3 & $53.4^{-t}$ & 0.19 & 916 & 55.3 & 13 & -0.34 \\
\hline $10: 90$ & 132 & 56 & $25^{\circ}$ & 23.3 & $53.3^{\dagger}$ & & 91 & 55.3 & 13 & -0.15 \\
\hline $7: 11: 89$ & 133 & 56 & 26 & 23 & $53.3^{-}$ & 0.1 & 916 & $55.1^{\dagger}$ & 13 & -0.15 \\
\hline $12: 88$ & 134 & 56 & 25 & 23.3 & 53.3 & 0.1 & 919 & 55.2 & 13 & 0.12 \\
\hline & 135 & 56 & 26 & 23 & $5 \overline{3}$ & 0.1 & 916 & 55.2 & 13 & 0.12 \\
\hline 86 & 136 & 56 & 26 & 23.3 & 53. & 0.2 & 908 & 55.2 & 13 & -0.18 \\
\hline 85 & 137 & 56 & 26 & 23.5 & $53.3^{1}$ & $0.2^{+}$ & $9 \overline{0} 5$ & 55.1 & 13 & $-0.1 \overline{8}$ \\
\hline $16: 83$ & 138 & 56 & 26 & 23.5 & 53.3 & 0.19 & 903 & 55.1 & 13 & -0.27 \\
\hline $7: 82$ & 139 & 56 & 26 & 23.5 & $53.3^{1}$ & 0.19 & 904 & 55.2 & 13 & -0.27 \\
\hline $18: 81$ & 140 & 56 & $26^{\circ}$ & 23.5 & $53.3^{\dagger}$ & $0.19^{-}$ & 908 & 55.4 & 13 & -0.13 \\
\hline $19: 80$ & $14 \overline{1}$ & $56^{\dagger}$ & 26 & 23.5 & 53.3 & 0.2 & 904 & 55.4 & 13 & -0.13 \\
\hline $20: 79$ & 142 & 56 & 26 & 23.5 & & 0.1 & 908 & 55.5 & 13 & 0.08 \\
\hline & 143 & 56 & 26 & $23.5^{\prime}$ & $53.3^{\top}$ & 0.1 & 912 & 55.4 & 13 & 0.08 \\
\hline & 144 & $56^{\dagger}$ & 26 & 23.5 & 53.2 & 0.19 & 91 & 55.4 & 13 & -0.15 \\
\hline $3: 76$ & 145 & 56 & 26 & 23.5 & 3.2 & 0.1 & 909 & 55.4 & $\overrightarrow{13}$ & -0.32 \\
\hline 74 & $1 \overline{46}$ & $56^{\dagger}$ & 26 & 23.5 & 3.2 & 0.1 & 906 & 55.4 & 13 & -0.32 \\
\hline & $14 \overline{7}$ & 56 & 26 & 23.5 & 53.2 & $0.19^{\prime}$ & 902 & 55.4 & 13 & $-0.0 \overline{9}$ \\
\hline & 148 & 56 & 26 & $23.5 !$ & 53.2 & 0.19 & 901 & 55.4 & $\overrightarrow{13}$ & -0.09 \\
\hline $37: 27: 71$ & 149 & 56 & 26 & 23.5 & 53.1 & $0.2^{\dagger}$ & 901 & 55.3 & 13 & 0.05 \\
\hline $12: 37: 28: 70$ & 150 & 56 & 26 & 23.5 & 53.1 & 0.2 & 901 & $5 \overline{5.3}$ & 13 & 0.05 \\
\hline $12: 37: 29: 69$ & 151 & 56 & 26 & 23.5 & $53.1 \mathrm{j}$ & 0.2 & 903 & 55.4 & 13 & -0.15 \\
\hline $12: 37: 30: 68$ & 152 & 56 & 26 & 23.5 & 53.1 & 0.2 & 908 & 55.6 & 13. & -0.15 \\
\hline
\end{tabular}




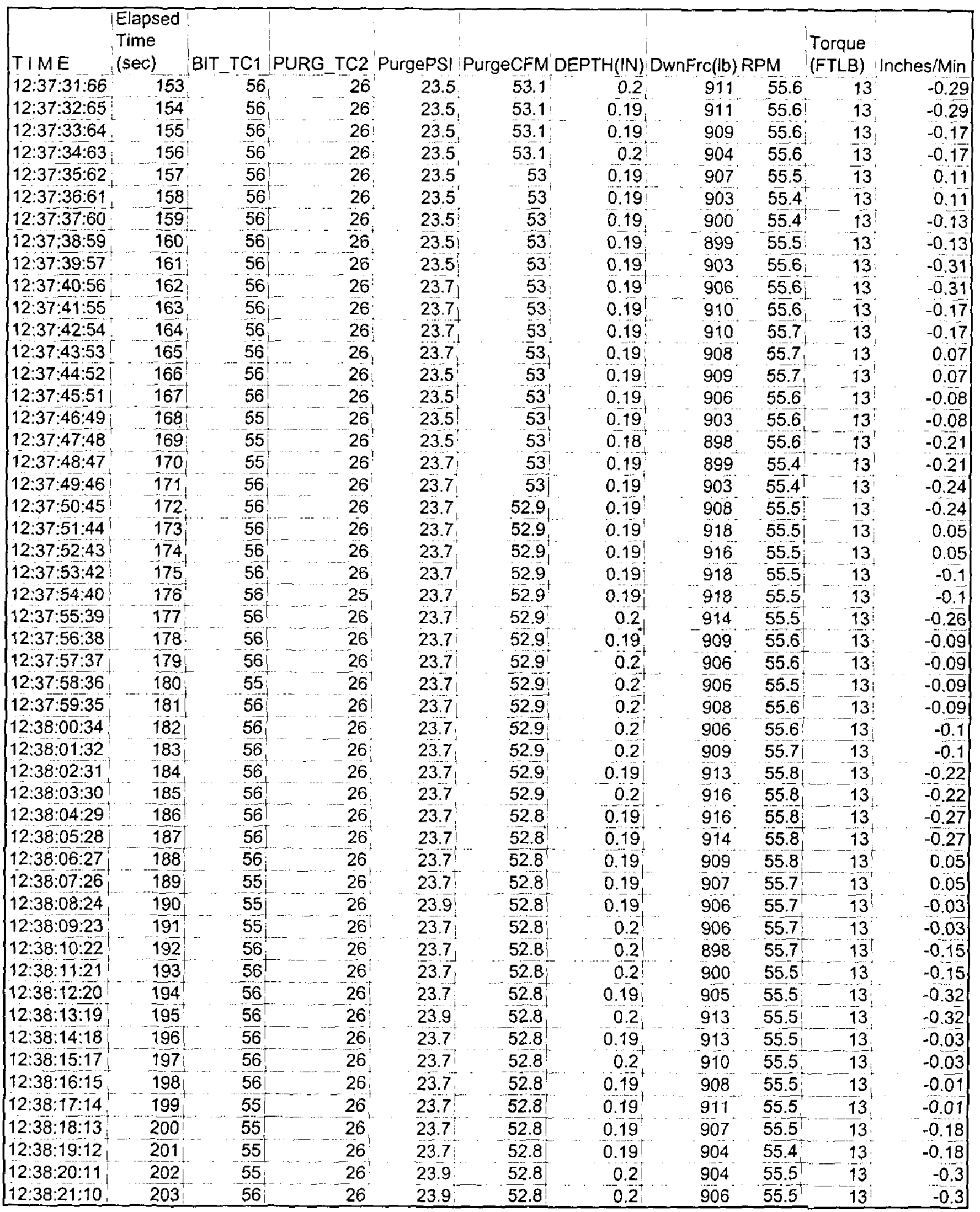




\begin{tabular}{|c|c|c|c|c|c|c|c|c|c|c|}
\hline$T I M E$ & $\begin{array}{l}\text { Elapsed } \\
\text { Time } \\
\text { (sec) }\end{array}$ & BIT_TC1 & PURG_TC2 & PurgePSI & PurgeCFM D & EPTH $(\mathrm{IN})$ & DwnFrc(lb) & RPM & $\begin{array}{l}\text { Torque } \\
\text { (FTLB) }\end{array}$ & Inches/Min \\
\hline $12: 38: 22: 09$ & 204 & 56 & -26 & $\quad 23.9$ & 52.8 & 0.2 & 909 & 55.4 & 13 & 0.12 \\
\hline $12: 38: 23: 07$ & 205 & 56 & 26 & 23.9 & 52.8 & 0.19 & $915^{\circ}$ & 55.3 & 13 & 0.12 \\
\hline $12: 38: 24: 06$ & 206 & 56 & 26 & 23.9 & 52.8 & 0.19 & 915 & 55.3 & $13 !$ & -0.18 \\
\hline $12: 38: 25: 05$ & 207 & 56 & 26 & 23.9 & 52.8 & 0.19 & 916 & 55.3 & 13 & -0.18 \\
\hline $12: 38: 26: 04$ & 208 & 56 & $26^{\dagger}$ & 23.9 & 52.8 & 0.19 & $914^{\circ}$ & 55.3 & 13 & -0.17 \\
\hline $12: 38: 27: 03$ & 209 & 55 & 26 & 23.9 & 52.8 & 0.18 & 910 & 55.3 & 13 & $-0 . \overline{17}$ \\
\hline $12: 38: 28: 02$ & 210 & 55 & 26 & 23.9 & 52.8 & 0.19 & 906 & 55.2 & 13 & -0.29 \\
\hline $12: 38: 29: 01$ & 211 & 55 & 26 & 23.9 & 52.8 & 0.19 & 904 & 55.2 & 13 & -0.29 \\
\hline $12: 38: 30: 00$ & 212 & 55 & 26 & 23.9 & 52.8 & 0.19 & 906 & 55.2 & 13 & 0.01 \\
\hline $12: 38: 30: 98$ & 213 & 55 & 26 & 23.9 & 52.8 & 0.2 & 909 & 55.3 & 13 & -0.05 \\
\hline $31: 97$ & 214 & 55 & $26^{i}$ & 23.9 & $52.8^{4}$ & 0.19 & $914:$ & 55.4 & 13 & -0.05 \\
\hline $12: 38: 32: 96$ & 215 & 55 & 26 & 24.1 & $52.8^{\circ}$ & 0.19 & 916 & 55.4 & 13 & -0.16 \\
\hline $33: 95$ & 216 & 55 & 26 & 24.1 & 52.8 & 0.19 & 915 & 55.2 & 13 & -0.16 \\
\hline $34: 94$ & 217 & $55^{\dagger}$ & 26 & 24.1 & 52.8 & 0.19 & $916^{\circ}$ & 55.2 & 13 & -0.33 \\
\hline $35: 93$ & 218 & 55 & 26 & 24.1 & 52.8 & 0.18 & 913 & 55.1 & 13 & -0.33 \\
\hline $12: 38: 36: 92$ & 219 & 55 & 26 & 24.1 & 52.8 & 0.19 & $908^{4}$ & 55.2 & 13 & 0.01 \\
\hline $37: 90$ & 220 & $55^{+}$ & 26 & 24.1 & 52.8 & 0.19 & $905^{\prime}$ & 55.2 & 13 & $0 . \overline{01}$ \\
\hline $12: 38: 38: 89$ & 221 & 55 & 26 & 24.1 & 52.8 & 0.19 & 906 & 55.2 & 13 & $-0 . \overline{06}$ \\
\hline $39: 88$ & 222 & 55 & 26 & $24.1^{\dagger}$ & 52.8 & 0.2 & 908 & 55.2 & 13 & -0.06 \\
\hline $40: 87$ & 223 & $5 \overline{5}$ & 26 & 24.3 & 52.8 & 0.2 & $912^{-}$ & 55.2 & 13 & -0.19 \\
\hline $1: 86$ & 224 & $55^{t}$ & 26 & 24.3 & 52.8 & 0.21 & 905 & 55.2 & 13 & -0.19 \\
\hline$: 85$ & 225 & 55 & 26 & 24.3 & 52.8 & 0.19 & 910 & 55.1 & 13 & -0.33 \\
\hline$+3: 84$ & 226 & $55^{\dagger}$ & 26 & 24.3 & 52.8 & 0.19 & 910 & 55.1 & 13 & -0.33 \\
\hline $4: 83$ & 227 & 55 & 26 & 24.3 & 52. & 0.19 & 910 & 55.1 & 13 & $\overline{0.04}$ \\
\hline $45: 81$ & 228 & 55 & 26 & 24.3 & 52.8 & 0.19 & 906 & 55.1 & 13 & 0.04 \\
\hline $46: 80$ & 229 & 55 & 26 & 24.3 & 52.8 & 0.19 & 903 & $5 \overline{5.1}$ & 13 & -0.05 \\
\hline $77: 79$ & 230 & 55 & 26 & 24.3 & 52.8 & 0.19 & $900^{\circ}$ & 55.4 & 13 & -0.05 \\
\hline $8: 78$ & 231 & $55^{+}$ & 26 & 24.5 & 52.8 & $0 . \overline{19}$ & 901 & 55.6 & 73 & -0.18 \\
\hline 77 & 232 & 55 & 26 & 24.5 & $52 . \overline{8}$ & 0.2 & $900^{\circ}$ & 55.6 & 13 & -0.18 \\
\hline $5: 76$ & 233 & 55 & 26 & 24.5 & 52.8 & $0 . \overline{2}$ & 901 & 55.7 & 13 & -0.29 \\
\hline :75 & 234 & 55 & 26 & 24.5 & $52.8^{\dagger}$ & 0.19 & 904 & 55.6 & 13 & -0.29 \\
\hline 73 & 235 & 55 & 26 & 24.7 & 52. & 0.2 & 908 & 55.6 & 13 & 0.01 \\
\hline $3: 72$ & 236 & 55 & 26 & 24.7 & 52.8 & 0.19 & 911 & 55.5 & 13 & 0.01 \\
\hline $4: 71$ & 237 & 55 & 26 & 24.7 & 52.8 & 0.19 & 909 & 55.4 & 12 & -0.04 \\
\hline $5: 70$ & 238 & 55 & 26 & 24.7 & 52.8 & 0.19 & 906 & $5 \overline{5.4}$ & 13 & -0.04 \\
\hline $6: 69$ & 239 & 55 & 26 & 24.7 & 52.8 & 0.19 & $903^{\circ}$ & 55.3 & 13 & -0.2 \\
\hline $7: 68$ & $240^{\dagger}$ & $55^{\dagger}$ & 26 & 24.7 & .8 & $0 . \overline{19}$ & 90 & $5 \overline{5.4}$ & 13 & -0.2 \\
\hline 67 & 241 & 55 & 26 & 24.7 & 52.8 & 0.2 & 902 & 55.4 & 13 & -0.34 \\
\hline $9: 66$ & 242 & 55 & 26 & 24.7 & 52.8 & 0.2 & 901 & 55.5 & 13 & -0.34 \\
\hline $00: 64$ & $243^{-1}$ & 55 & 26 & 24.7 & 52.8 & 0.2 & 901 & 55.6 & 13 & 0.06 \\
\hline $12: 39: 01: 63$ & 244 & 55 & 26 & 24.9 & 52.8 & $\overline{0.2}$ & 905 & 55.6 & 13 & 0.06 \\
\hline $12: 39: 02: 62$ & 245 & 55 & 26 & 24.9 & 52.8 & 0.2 & 909 & 55.6 & 13 & -0.08 \\
\hline $12: 39: 03: 61$ & 246 & 55 & 26 & 24.9 & 52.8 & 0.2 & 912 & 55.6 & 13 & -0.16 \\
\hline $12: 39: 04: 60$ & 247 & 55 & 26 & 24.9 & 52.8 & 0.19 & $9+1$ & 55.6 & 13 & -0.16 \\
\hline $12: 39: 05: 59$ & 248 & 55 & 26 & 24.9 & 52.8 & 0.2 & 906 & 55.6 & 13 & -0.24 \\
\hline $12: 39: 06: 58$ & 249 & 55 & 26 & 24.9 & 52.8 & 0.19 & 909 & 55.4 & 13 & -0.24 \\
\hline $12: 39: 07: 56$ & 250 & 55 & 26 & 24.9 & 52.8 & 0.19 & 904 & 55.4 & 13 & -0.1 \\
\hline $12: 39: 08: 55$ & $251 !$ & 55 & 26 & 24.9 & 52.8 & 0.19 & $900^{\circ}$ & 55.4 & 13 & -0.1 \\
\hline $12: 3 \overline{9: 09: 54}$ & 252 & 55 & 26 & 24.9 & 52.7 & 0.2 & 899 & 55.5 & 13 & -0.06 \\
\hline $12: 39: 10: 53$ & 253 & 55 & 26 & 24.9 & 52.7 & 0.2 & $900^{\circ}$ & 55.5 & 13 & -0.06 \\
\hline $12: 39: 11: 52$ & 254 & 55 & 26 & 24.9 & 52.7 & 0.2 & 904 & $5 \overline{5.5}$ & 13 & -0.17 \\
\hline
\end{tabular}




\begin{tabular}{|c|c|c|c|c|c|c|c|c|c|c|}
\hline$T I M E$ & $\begin{array}{l}\text { Elapsed } \\
\text { Time } \\
\text { (sec) }\end{array}$ & BIT_TC1 & PURG_TC2 & PurgePSi & PurgeCFM & DEPTH(IN) & DwnFrc(b) & PM & $\begin{array}{l}\text { Torque } \\
\text { (FTLB) }\end{array}$ & Inches/Min \\
\hline $12: 39: 12: 51$ & $\quad 255$ & 55 & 26 & $\quad 24.9$ & $\quad 52.7$ & $\quad 0.2$ & 908 & 55.4 & 12 & -0.17 \\
\hline $12: 39: 13: 50$ & 256 & 55 & 26 & 24.9 & 52.7 & 0.2 & 909 & 55.4 & 12 & -0.29 \\
\hline $12: 39: 14: 49$ & 257 & 55 & 26 & 25.1 & 52.7 & 0.19 & 909 & 55.3 & 12 & -0.29 \\
\hline $12: 39: 15: 47$ & 258 & 55 & 26 & 25.1 & 52.7 & 0.19 & 910 & 55.3 & 12 & -0.02 \\
\hline $12: 39: 16: 46$ & $259^{\circ}$ & 55 & 26 & 25.1 & 52.7 & $0.2^{+}$ & 908 & 55.3 & 12 & -0.02 \\
\hline $12: 39: 17: 45$ & 260 & 55 & 26 & $2 \overline{5.1}$ & 52.7 & 0.2 & 901 & 55.4 & 12 & -0.06 \\
\hline $12: 39: 18: 44$ & 261 & 55 & 26 & 25.1 & 52.7 & 0.2 & $899^{\circ}$ & $5 \overline{5.4}$ & 13 & -0.06 \\
\hline $12: 39: 19: 43$ & 262 & 55 & 26 & 25.1 & 52.7 & 0.2 & 898 & 55.5 & 13 & -0.17 \\
\hline $12: 39: 20: 42$ & 263 & $5 \overline{5}$ & 26 & 25.1 & 52.7 & 0.2 & 900 & 55.5 & 13 & -0.17 \\
\hline $12: 39: 21: 41$ & 264 & 55 & 26 & 25.1 & 52.7 & 0.2 & 907 & 55.5 & 13 & -0.35 \\
\hline $12: 39: 22: 39$ & 265 & 55 & 26 & 25.1 & 52.7 & 0.21 & 911 & 55.4 & 13 & -0.35 \\
\hline $12: 39: 23: 38$ & 266 & 55 & 26 & 25.1 & 52.7 & 0.2 & $908^{+}$ & 55.3 & 13 & -0.03 \\
\hline $12: 39: 24: 37$ & 267 & 55 & 26 & 24.9 & 52.7 & 0.2 & 911 & 55.3 & 12 & -0.03 \\
\hline $12: 39: 25: 36$ & 268 & 55 & 26 & $24 . \overline{9}$ & 52.7 & 0.2 & 911 & 55.3 & 13 & -0.02 \\
\hline $12: 39: 26: 35$ & 269 & 55 & 26 & 24.9 & 52.7 & 0.2 & 908 & 55.2 & 13 & -0.02 \\
\hline $12: 39: 27: 34$ & 270 & 55 & 26 & 24.9 & 52.7 & 0.2 & 904 & 55.2 & 13 & -0.17 \\
\hline $12: 39: 28: 33$ & 271 & 55 & 26 & 24.9 & 52.7 & 0.2 & 902 & 55.2 & 13 & -0.17 \\
\hline $12: 39: 29: 31$ & $27 \overline{2}$ & 55 & 26 & 24.9 & 52.7 & 0.2 & 903 & 55.2 & 13 & -0.32 \\
\hline $12: 39: 30: 30^{\dagger}$ & 273 & 55 & 26 & 24.9 & 52.7 & 0.21 & 906 & 55.2 & 13 & -0.32 \\
\hline $12: 39: 31: 29$ & 274 & 55 & 26 & 24.9 & 52.7 & 0.21 & 904 & 55.3 & 13 & 0.01 \\
\hline $12: 39: 32: 28$ & 275 & 55 & 26 & 24.9 & 52.7 & 0.2 & $907^{\dagger}$ & 55.3 & 13 & 0.01 \\
\hline $12: 39: 33: 27$ & $2 \overline{76}$ & 55 & 26 & 24.9 & 52.7 & 0.21 & 911 & 55.3 & 13 & -0.06 \\
\hline $12: 39: 34: 26$ & 277 & 55 & 26 & 24.9 & 52.7 & 0.2 & $911^{\prime}$ & 55.3 & 13 & -0.06 \\
\hline $12: 39: 35: 25$ & 278 & 55 & 26 & 24.9 & 52.7 & 0.2 & 909 & 55.2 & 13 & -0.2 \\
\hline $12: 39: 36: 24$ & 279 & 55 & 26 & 24.9 & 52.7 & 0.2 & 905 & 55.2 & 13 & -0.31 \\
\hline $12: 39: 37: 22$ & 280 & 55 & 26 & 24.9 & 52.7 & 0.19 & 901 & 55.1 & $1 \overline{3}$ & -0.31 \\
\hline $12: 39: 38: 21$ & 281 & 55 & 26 & 24.9 & 52.7 & 0.2 & $899 !$ & 55.3 & 13 & 0.03 \\
\hline $12: 39: 39: 20$ & 282 & 55 & 26 & 24.9 & 52.7 & 0.2 & 901 & 55.3 & 13 & 0.03 \\
\hline $12: 39: 40: 19$ & 283 & 55 & 26 & 24.9 & 52.7 & 0.2 & 900 & 55.4 & 13 & -0.07 \\
\hline $12: 39: 41: 18$ & 284 & 55 & 26 & 24.9 & 52.7 & 0.21 & 902 & 55.4 & 13 & -0.07 \\
\hline $12: 39: 42: 17$ & 285 & 55 & 26 & 24.9 & 52.7 & 0.21 & 906 & 55.4 & 13 & -0.17 \\
\hline $12: 39: 43: 16$ & 286 & 55 & 26 & 24.9 & 52.7 & 0.2 & $910^{\circ}$ & 55.3 & 13 & -0.17 \\
\hline $12: 39: 44: 14$ & 287 & 55 & 26 & 24.9 & 52.7 & 0.2 & 911 & 55.2 & 13 & $-0 . \overline{33}$ \\
\hline $12: 39: \overline{45}: 13$ & 288 & 55 & 26 & 24.9 & 52.7 & 0.2 & $908^{\circ}$ & 55.2 & 13 & $-0.3 \overline{3}$ \\
\hline $12: 39: 46: 12$ & 289 & 55 & 26 & 24.9 & 52.7 & 0.2 & 904 & 55.1 & 12 & 0.09 \\
\hline $12: 39: 47: 11$ & 290 & 55 & 26 & 24.9 & 52.7 & 0.19 & 900 & 55 & 13 & 0.09 \\
\hline $12: 39: 48: 10$ & 291 & 55 & 26 & 24.9 & 52.7 & 0.2 & 903 & 55 & 13 & -0.18 \\
\hline $12: 39: 49: 09$ & 292 & 55 & 26 & 24.9 & 52.7 & 0.2 & $899^{\circ}$ & 55.1 & 13 & -0.18 \\
\hline $12: 39: 50: 08$ & 293 & 55 & 26 & 24.9 & 52.7 & 0.2 & 900 & 55.2 & 13 & -0.22 \\
\hline $12: 39: 51: 07$ & 294 & 55 & 26 & 24.9 & $52 . \overline{7}$ & 0.2 & 904 & 55.2 & 13 & -0.22 \\
\hline $12: 39: 52: 05$ & 295 & 55 & 26 & $24 . \overline{9}$ & 52.7 & 0.21 & 908 & 55.2 & 13 & -0.26 \\
\hline $12: 39: 53: 04$ & 296 & 55 & 26 & 24.9 & 52.7 & 0.21 & 911 & $5 \overline{5.2}$ & 12 & -0.26 \\
\hline $12: 39: 54: 03$ & $2 \overline{97}$ & 55 & 26 & 24.9 & 52.7 & 0.2 & $909^{\circ}$ & 55.2 & 12 & 0.15 \\
\hline $12: 39: 55: 02$ & 298 & 55 & 26 & 24.9 & 52.7 & 0.19 & 905 & 55.1 & 12 & 0.15 \\
\hline $12: 39: 56: 01$ & 299 & 55 & 26 & 24.9 & 52.7 & 0.19 & $901^{\circ}$ & 55.2 & 12 & -0.17 \\
\hline $12: 39: 57: 00$ & 300 & 55 & 26 & 24.9 & 52.7 & 0.19 & 904 & 55.2 & 12 & -0.17 \\
\hline $12: 39: 57: 99$ & 301 & 55 & 26 & 24.9 & 52.7 & 0.2 & 901 & 55.5 & 12 & -0.25 \\
\hline 12:39:58:97 & 302 & 55 & 26 & 24.9 & $5 \overline{2.7}$ & 0.2 & 899 & 55.7 & 13 & -0.25 \\
\hline $12: 39: 59: 96$ & 303 & 55 & 26 & 24.9 & 52.7 & 0.2 & 901 & 55.7 & 13 & -0.25 \\
\hline $12: 40: 00: 95$ & 304 & 55 & 26 & 24.9 & 52.7 & 0.2 & 904 & 55.6 & 13 & -0.25 \\
\hline $12: 40: 01: 94$ & 305 & 55 & 26 & 24.9 & 52.7 & 0.2 & 908 & 55.6 & 13 & 0.15 \\
\hline
\end{tabular}




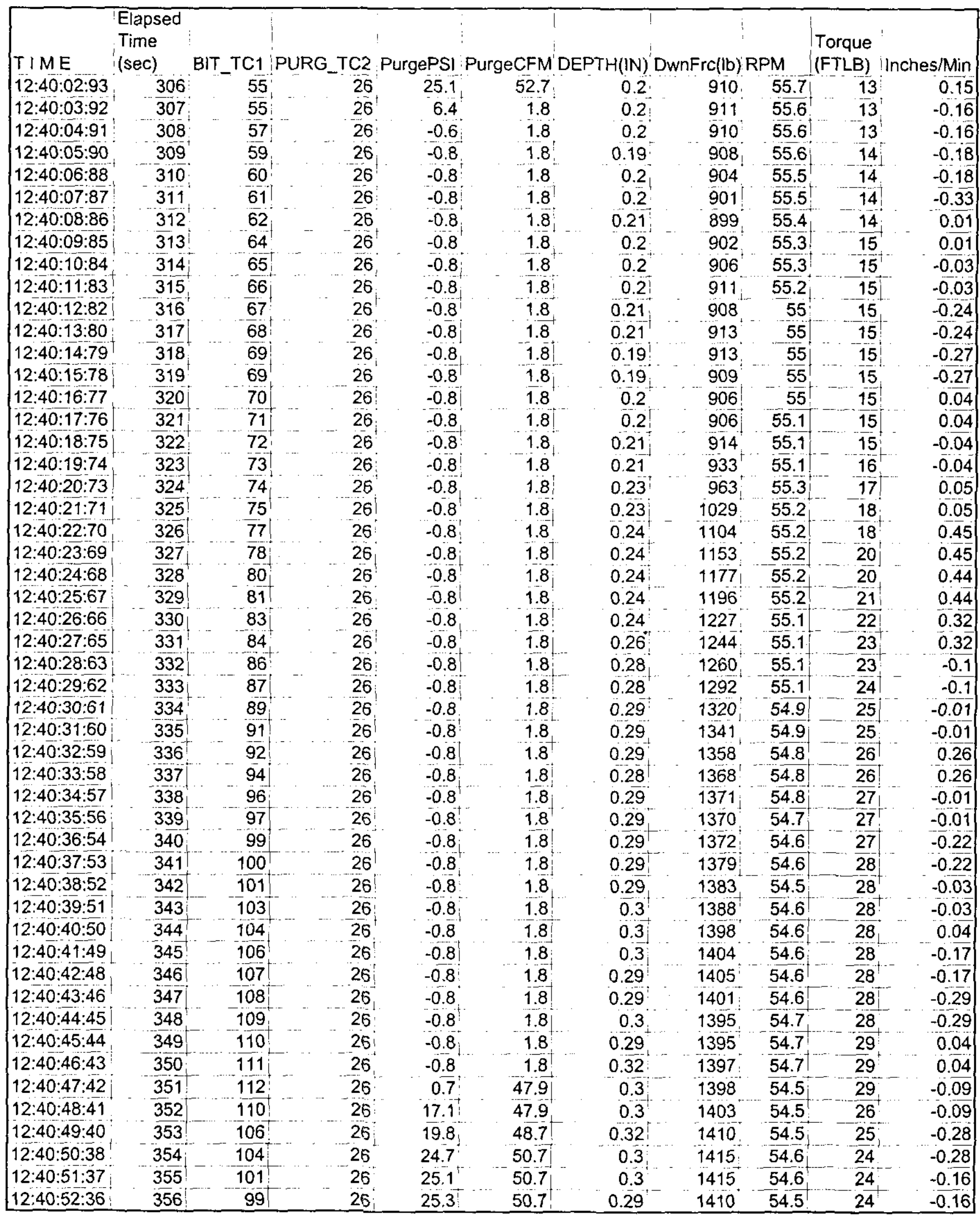




\begin{tabular}{|c|c|c|c|c|c|c|c|c|c|c|}
\hline & $\begin{array}{l}\text { Elapsed } \\
\text { Time }\end{array}$ & & & & 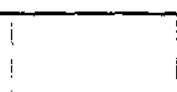 & & & & & \\
\hline TIME & (sec) & BIT_TC1 & PURG_TC2 & |PurgePS| & PurgeCFM & DEPTH(IN) & DwnFrc(lb) & RPM & (FTLB) & Inches/Min \\
\hline $53: 35$ & $\quad 357$ & 97 & 26 & -25.3 & $1 \quad 50.8$ & 0.29 & 1405 & 54.5 & 23 & 0.01 \\
\hline 34 & 358 & 96 & 26 & 25.3 & 50.9 & 0.3 & 1410 & 54.5 & 23 & 0.01 \\
\hline $12: 40: 55: 33$ & 359 & 94 & 26 & 25.3 & 50.9 & 0.3 & 1407 & 54.6 & 23 & -0.26 \\
\hline $12: 40: 56: 32$ & 360 & 93 & 26 & 25.3 & 50.9 & 0.32 & 1407 & 54.6 & 23 & -0.26 \\
\hline 12:4C & 361 & 92 & 26 & 25.5 & 50.9 & 0.3 & 1413 & 54.6 & 22 & -0.39 \\
\hline $12: 40$ & 362 & 91 & 26 & 25.5 & 50.9 & 0.3 & 1425 & 54.6 & 22 & -0.39 \\
\hline $12: 40: 59: 28$ & 363 & 91 & 26 & 25.3 & 50.9 & 0.3 & 1429 & 54.7 & 22 & -0.01 \\
\hline 12:41:00:27 & 364 & 90 & 26 & 25.3 & 50.9 & $0 . \overline{3}$ & 1429 & 54.7 & 22 & -0.01 \\
\hline $12: 41: 0$ & 365 & 89 & 26 & 25.3 & 51 & 0.3 & 1426 & 54.7 & 22 & $-0.0 \overline{5}$ \\
\hline $12: 41: 02: 25$ & 366 & 88 & 26 & 25.3 & 51 & 0.3 & 1432 & 54.5 & 22 & -0.05 \\
\hline
\end{tabular}




\begin{tabular}{|c|c|c|c|c|c|c|c|c|c|c|}
\hline$T \mid M E$ & $\begin{array}{l}\text { Elapsed } \\
\text { Time } \\
\text { (sec) }\end{array}$ & $B I T=T C 1$ & PURG_TC2 & PurgePSI & PurgeCFM & DEPTH(IN) & DwnFrc(lb) & $R P M$ & $\begin{array}{l}\text { Torque } \\
\text { (FTLB) }\end{array}$ & Inches/Min \\
\hline $07: 08: 00: 41$ & 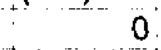 & $=24$ & -25 & $\quad 19.4$ & $\quad 53.4^{\circ}$ & $\quad-1.37$ & 1 & 57.1 & 0 & $\quad-0.22$ \\
\hline $07: 08: 01: 34$ & 1 & 24 & 25 & 19.4 & 53.4 & -1.23 & 0 & 57.1 & 0 & 1.58 \\
\hline $07: 08: 02: 33$ & 2 & 24 & 25 & 19.4 & 53.4 & -0.92 & -2 & 57.1 & 0 & 1.58 \\
\hline $07: 08: 03: 32$ & 3 & 25 & 25 & 19.4 & 53.4 & -0.6 & 1 & 56.8 & 0 & 12.71 \\
\hline 07:08:04:31 & 4 & 24 & 25 & 19.4 & 53.4 & -0.28 & 0 & 56.6 & 0 & 12.71 \\
\hline $07: 08: 05: 30$ & 5 & 25 & 25 & 19.6 & 53.4 & -0.03 & 101 & 56.6 & 5 & 12.48 \\
\hline $5: 29$ & 6 & 26 & 25 & 20.4 & 53.4 & 0.02 & 704 & 56.5 & 41 & 12.48 \\
\hline $07: 08: 07: 28$ & 7 & 30 & 25 & 20.8 & 53.4 & 0.05 & 801 & 56.1 & 35 & 3.62 \\
\hline 26 & 8 & 31 & 25 & 21 & 53.4 & 0.07 & 824 & 56.1 & 33 & 3.62 \\
\hline $9: 25$ & 9 & 33 & 25 & 21 & 53.4 & 0.07 & 852 & 55.7 & 32 & 0.53 \\
\hline $10: 24$ & 10 & 36 & 25 & 21.2 & 53.4 & 0.09 & 876 & 55.7 & 30 & 0.53 \\
\hline $11: \overline{23}$ & 11 & 38 & 25 & 21.2 & 53.4 & 0.09 & 892 & 55.7 & 28 & 0.14 \\
\hline$: 12: 22$ & 12 & 40 & 25 & 21.2 & 53.3 & 0.1 & 905 & 55.8 & $2 \overline{7}$ & 0.14 \\
\hline $3: 21$ & 13 & 42 & 25 & 21.2 & 53.3 & 0.1 & 917 & 55.8 & 26 & 0.05 \\
\hline 20 & 14 & 42 & 25 & 21.2 & 53.3 & 0.1 & 924 & 55.8 & 26 & 0.05 \\
\hline $5: 18$ & 15 & 43 & 25 & 21.2 & 53 & 0.1 & 924 & 55.8 & 26 & 0.14 \\
\hline $6: 17$ & 16 & 44 & 25 & 21.2 & $53.2^{\dagger}$ & 0.09 & 920 & 55.8 & 25 & 0.14 \\
\hline $07: 08: 17: 16$ & 17 & 45 & 25 & 21.2 & 53.2 & 0.09 & 912 & 55.8 & 25 & -0.01 \\
\hline |07:08:18:15 & 18 & 46 & 25 & 21.2 & 53.1 & 0.1 & 915 & 55.8 & 24 & -0.01 \\
\hline 07: & 19 & 46 & 25 & 21.2 & 53.1 & 0.1 & 910 & 55.8 & 24 & -0.21 \\
\hline 13 & 20 & 47 & 25 & 21.2 & 53.1 & 0.1 & 910 & 55.8 & 24 & -0.21 \\
\hline$: 12$ & 21 & 48 & 25 & 21.2 & 3 & 0.1 & 910 & 55.9 & 24 & -0.04 \\
\hline $22: 10$ & 22 & 48 & 25 & 21.2 & 3 & 0.1 & 914 & 55.9 & 24 & -0.04 \\
\hline $3: 09$ & 23 & 49 & 25 & 21.2 & 5 & 0.1 & 918 & 55.8 & 24 & 0.01 \\
\hline & 24 & 49 & 25 & 21.2 & 53 & 0.1 & 922 & 55.8 & 24 & 0.01 \\
\hline 507 & 25 & 50 & 25 & 21.2 & $52 . \overline{9}$ & 0.1 & 921 & 55.8 & 24 & -0.04 \\
\hline $6: 06$ & 26 & 51 & 25 & 21.2 & 52.9 & 0.1 & 917 & 55.8 & 23 & -0.04 \\
\hline$: 05$ & 27 & 51 & 24 & 21.2 & 52.9 & 0.1 & 922 & 55.8 & 23 & -0.18 \\
\hline & 28 & 52 & 25 & 21.4 & 52.9 & 0.1 & 918 & 55.8 & 23 & -0.18 \\
\hline & $29 !$ & 52 & 25 & 21.4 & $52.9 !$ & 0.11 & 914 & 55.8 & 23 & -0.13 \\
\hline & 30 & $5 \overline{3}$ & 25 & 21.4 & 52.9 & 0.11 & 913 & 55.7 & 22 & -0.04 \\
\hline$: 00$ & 31 & 53 & 25 & 21.4 & 52 & 0.11 & 915 & 55.7 & 22 & -0.04 \\
\hline & 32 & 53 & 24 & 21.2 & $52.9^{4}$ & 0.1 & 921 & 55.7 & 22 & -0.03 \\
\hline 07 & 33 & 54 & 25 & 21.2 & 52.9 & 0.11 & 925 & 55.7 & 22 & -0.03 \\
\hline 97 & 34 & 54 & 25 & 21.2 & 52 & 0.11 & 927 & 55.7 & 22 & -0.14 \\
\hline 96 & 35 & 54 & 25 & 21.2 & 52.8 & 0.1 & 925 & 55.7 & 22 & -0.14 \\
\hline $5: 95$ & 36 & 55 & 25 & 21.2 & 52.8 & 0.1 & 927 & 55.8 & 22 & -0.26 \\
\hline & 37 & 55 & 25 & 21.2 & 52.8 & 0.1 & 926 & 55.8 & 22 & -0.26 \\
\hline & 38 & 55 & 25 & 21.4 & & 0.1 & 9 & 55.8 & 22 & -0.02 \\
\hline & 39 & 56 & 25 & & & 0.1 & & 55.9 & 22 & -0.02 \\
\hline & 40 & 56 & 25 & & & 0.11 & 9 & 55.9 & 22 & -0.01 \\
\hline & 41 & 56 & 25 & 21. & 52.8 & $0 . \overline{11}$ & 91 & 55.8 & 22 & -0.01 \\
\hline & 42 & 56 & 25 & 21.4 & 52 & 0.11 & 914 & 55.7 & 21 & -0.1 \\
\hline $42: 87$ & 43 & 57 & 25 & 21.4 & 52.8 & 0.11 & 910 & 55.7 & 21 & -0.1 \\
\hline $3: 86$ & 44 & 57 & 25 & 21.4 & 52.8 & 0.11 & 912 & 55.8 & 22 & -0.19 \\
\hline$: 44: 84$ & 45 & 57 & 25 & 21.4 & 52.8 & 0.11 & 916 & 55.8 & 22 & -0.19 \\
\hline |07:08:45:83 & 46 & 57 & 25 & 21.4 & 52.8 & 0.1 & 920 & 55.8 & 22 & -0.1 \\
\hline $07: 08: 46: 82$ & 47 & 57 & 25 & 21.4 & $52.8 \mathrm{i}$ & 0.1 & 922 & 55.9 & 22 & -0.1 \\
\hline $07: 08: 47: 81$ & 48 & 58 & 25 & 21.4 & 52.8 & $0 . \overline{1}$ & 920 & 55.9 & 22 & -0.03 \\
\hline |07:08:48:80 & $\overline{49}$ & 58 & 25 & 21.4 & $52.8^{\dagger}$ & 0.1 & 916 & 55.9 & 22 & -0.03 \\
\hline 07:08:49:79 & 50 & 58 & 25 & 21.4 & 52.8 & 0.1 & 912 & 55.9 & 22 & -0.05 \\
\hline
\end{tabular}




\begin{tabular}{|c|c|c|c|c|c|c|c|c|c|c|}
\hline$T I M E$ & $\begin{array}{l}\text { Elapsed } \\
\text { Time } \\
\text { (sec) }\end{array}$ & $B \mid T_{-} T C 1_{1}$ & PURG_TC2 & PurgePSI & PurgeCFM & DEPTH(IN) & DwnFrc(lb) & RPM & $\begin{array}{l}\text { Torque } \\
\text { (FTLB) }\end{array}$ & Inches/Min \\
\hline $07: 08: 50: 78$ & $\quad 51$ & $\quad 58^{\dagger}$ & 25 & ; $\quad 21.4$ & 52.8 & $\quad 0.1$ & $\quad 910$. & 56 & 22 & $\quad-0.05$ \\
\hline $07: 08: 51: 76$ & 52 & 58 & 25 & 21.4 & 52.7 & 0.11 & 912 & 56 & $2 \overline{2}$ & -0.26 \\
\hline $07: 08: 52: 75$ & 53 & $59^{\dagger}$ & 25 & 21.4 & 52.7 & 0.12 & 912 & 56 & 22 & -0.26 \\
\hline $07: 08: 53: 74$ & 54 & 59 & 25 & $21.4^{\circ}$ & 52.7 & 0.12 & $913^{\dagger}$ & 55.9 & 22 & -0.17 \\
\hline $07: 08: 54: 73$ & 55 & 59 & 25 & 21.4 & 52.7 & 0.14 & $91 \overline{6}$ & 55.9 & 21 & -0.17 \\
\hline $07: 08: 55: 72$ & 56 & 59 & 25 & 21.4 & 52.7 & 0.12 & 921 & 56 & 22 & 0.03 \\
\hline $07: 08: 56: 71$ & $5 \overline{7}$ & 59 & 25 & 21.4 & 52.7 & 0.12 & 924 & 56 & 22 & 0.03 \\
\hline $07: 08: 57: 70$ & 58 & 59 & 25 & 21.4 & 52.7 & $0.1 \overline{1}$ & 924 & $56 !$ & 22 & -0.04 \\
\hline $58: 69$ & 59 & 59 & 25 & 21.4 & 52.7 & 0.11 & 920 & 55.9 & 22 & -0.04 \\
\hline $07: 08: 59: 67$ & 60 & 60 & 25 & 21.4 & 52.7 & 0.12 & 923 & 55.8 & 22 & -0.2 \\
\hline $07: 09: 00: 66$ & 61 & 60 & 25 & 21.4 & 52.7 & 0.11 & 921 & 55.8 & 22 & -0.2 \\
\hline $07: 09: 01: 65$ & 62 & 60 & 25 & 21.4 & 52.7 & 0.12 & 916 & 55.7 & 21 & -0.23 \\
\hline $07: 09: 02: 64$ & 63 & 60 & 25 & 21.4 & 52.7 & 0.12 & 913 & $55.7^{\mid}$ & 21 & 0.02 \\
\hline $03: 63$ & 64 & 60 & 25 & 21.4 & 52.7 & 0.12 & 914 & 55.7 & 21 & 0.02 \\
\hline 62 & 65 & 60 & 25 & 21.6 & 52.7 & 0.12 & 916 & 55.8 & 21 & -0.07 \\
\hline $05: 61$ & $\overline{66}$ & 60 & 25 & 21.6 & 52.7 & 0.11 & 921 & 55.6 & 21 & $-0.0 \overline{7}$ \\
\hline $07: 09: 06: 59$ & 67 & 60 & 25 & 21.6 & 52.7 & 0.12 & 925 & 55.6 & 22 & -0.15 \\
\hline $07: 58$ & 68 & $60^{\dagger}$ & 25 & 21.6 & 52.7 & 0.12 & 924 & $55.7_{j}^{+}$ & 22 & -0.15 \\
\hline $08: 57$ & 69 & 60 & 25 & 21.6 & 52.7 & 0.11 & 927 & 55.7 & 22 & -0.26 \\
\hline $9: 56$ & 70 & 61 & 25 & 21.4 & 52.6 & 0.11 & 930 & $55.7^{\circ}$ & 22 & -0.26 \\
\hline $10: 55$ & 71 & 61 & 25 & 21.6 & 52.6 & 0.11 & 927 & 55.7 & 22 & $\overline{-0.03}$ \\
\hline $1: 54$ & 72 & 61 & 25 & 21.6 & 52.6 & 0.11 & 923 & 55.7 & 22 & -0.03 \\
\hline 53 & 73 & $61^{\circ}$ & 25 & 21.6 & $52.6^{\dagger}$ & 0.12 & $\overline{9} 17^{\dagger}$ & 55.8 & 22 & -0.01 \\
\hline 13.52 & 74 & 61 & 25 & 21.6 & 52.6 & 0.11 & 916 & 55.9 & 21 & -0.01 \\
\hline $4: 50$ & 75 & 61 & 25 & 21.6 & 52.6 & 0.12 & 919 & 55.9 & 22 & -0.1 \\
\hline $5: 49$ & 76 & 61 & 25 & 21.6 & 52.6 & 0.12 & $922^{1}$ & 55.9 & 21 & -0.1 \\
\hline 48 & 77 & 61 & 25 & 21.6 & 52.6 & 0.12 & 918 & 55.7 & 22 & -0.2 \\
\hline $7: 47$ & 78 & 61 & 25 & 21.6 & $52.6^{+}$ & 0.12 & 922 & 55.7 & 22 & -0.2 \\
\hline$: 46$ & 79 & 61 & 25 & 21.6 & 52.6 & & $929^{\prime}$ & 55.7 & 22 & -0.17 \\
\hline & 80 & 61 & 25 & 21.6 & 52.6 & $0 . \overline{12}$ & 928 & 55.8 & 22 & -0.17 \\
\hline $20: 44$ & 81 & 62 & 25 & $21.6^{\prime}$ & $52.6^{\dagger}$ & 0.11 & 927 & 55.8 & 22 & $0 . \overline{01}$ \\
\hline 21:42 & 82 & 61 & 25 & 21.6 & 52.6 & 0.11 & 923 & 55.8 & 22 & $0 . \overline{01}$ \\
\hline $22: 41$ & 83 & 62 & 25 & 21.6 & 52.6 & 0.11 & 919 & 55.9 & $2 \overline{2}$ & -0.07 \\
\hline $23: 40$ & 84 & 62 & 25 & 21.6 & 52.6 & 0.12 & 916 & 55.9 & 22 & -0.07 \\
\hline $4: 39$ & 85 & 62 & $25^{1}$ & 21.6 & 52.6 & 0.12 & 917 & 55.9 & 22 & -0.26 \\
\hline $5: 38$ & 86 & 62 & 25 & 21.6 & 52.6 & 0.12 & $916^{\circ}$ & 55.9 & 22 & $-0 . \overline{26}$ \\
\hline $6: 37$ & 87 & 62 & 25 & 21.6 & $52 . \overline{6}$ & & 91 & 55.9 & 22 & -0.07 \\
\hline $7: 36$ & 88 & 62 & 25 & 21.6 & 52.6 & & 922 & 55.9 & 22 & -0.07 \\
\hline $28: 35$ & 89 & 62 & 25 & 21.6 & 52.6 & 0.12 & 927 & 55.9 & 22 & -0.1 \\
\hline & 90 & 62 & 25 & 21.6 & 52.6 & 0.12 & 930 & 55.9 & $\overline{22}$ & $-\overline{0.1}$ \\
\hline $00: 32$ & 91 & 63 & 25 & 21.6 & 52.6 & 0.11 & 930 & $55.9^{\circ}$ & 22 & $-0 . \overline{03}$ \\
\hline $07: 09: 31: 31$ & 92 & 63 & 25 & 21.6 & 52.6 & 0.12 & 927 & $55.9^{\dagger}$ & 21 & -0.03 \\
\hline $07: 09: 32: 30$ & 93 & 63 & $25 !$ & 21.6 & 52.6 & 0.12 & $930^{\circ}$ & 55.9 & 21 & $-0 . \overline{22}$ \\
\hline $07: 09: 33: 29$ & 94 & 63 & 25 & 21.6 & 52.6 & 0.12 & 927 & 56 & 21 & -0.22 \\
\hline $07: 09: 34: 28$ & 95 & 63 ! & 25 & 21.6 & 52.6 & 0.12 & 921 & 55.9 & 21 & -0.25 \\
\hline $07: 09: 35: 27$ & 96 & 63 & $25^{\dagger}$ & 21.6 & 52.6 & 0.12 & 916 & $55.9^{\circ}$ & $2 \overline{1}$ & 0.09 \\
\hline $07: 09: 36: 25$ & 97 & $63^{\dagger}$ & 25 & $21.6^{\dagger}$ & $52.6^{+}$ & 0.12 & 916 & 55.9 & 22 & 0.09 \\
\hline $07: 09: 37: 24$ & 98 & 63 & 25 & 21.6 & 52.6 & 0.12 & 919 & 55.9 & $2 \overline{2}$ & $-0.1 \overline{7}$ \\
\hline $07: 09: 38: 23$ & 99 & 63 & 25 & $21.6^{\dagger}$ & 52.6 & 0.12 & 922 & $55.9^{\dagger}$ & 22 & -0.17 \\
\hline & 100 & $63 !$ & 25 & 21.6 & $52 . \overline{6}$ & 0.12 & 926 & 56 & 22 & -0.11 \\
\hline $07: 09: 40: 21$ & 101 & 63 & 25 & $21 . \overline{6}$ & 52.6 & 0.12 & $927^{\circ}$ & 56 & 22 & -0.11 \\
\hline
\end{tabular}




\begin{tabular}{|c|c|c|c|c|c|c|c|c|c|c|}
\hline TIME & $\begin{array}{l}\text { Elapsed } \\
\text { Time } \\
\text { (sec) }\end{array}$ & BIT_TC1 & & PurgePSI & PurgeCFM & DEPTH(IN) & DwnFrc(lb) & RPM & $\begin{array}{l}\text { Torque } \\
\text { (FTLB) }\end{array}$ & Inches/Min \\
\hline $07: 09: 41: 20$ & 102 & $\quad 63$ & 25 & 21.6 & 52.6 & $0 . \overline{12}$ & $\quad 926$ & 56 & $\quad 22$ & -0.27 \\
\hline $07: 09: 42: 19$ & 103 & 63 & 25 & 21.6 & 52.6 & 0.12 & 927 & 56 & 21 & -0.27 \\
\hline $07: 09: 43: 17$ & 104 & $63^{+}$ & 25 & $21.6 !$ & 52.6 & 0.11 & 925 & 55.9 & 21 & 0.02 \\
\hline $07: 09: 44: 16$ & $105^{\prime}$ & 63 & 25 & 21.6 & 52.6 & 0.11 & 921 & 55.9 & 21 & 0.02 \\
\hline 07:0 & 106 & 63 & 25 & 21.6 & 52.6 & 0.11 & 916 & 55.7 & 21 & -0.14 \\
\hline $07: 0$ & 107 & 63 & 25 & 21.6 & 52.6 & 0.11 & 915 & 55.7 & 21 & -0.14 \\
\hline $7: 13$ & 108 & 63 & 25 & 21.6 & 52.6 & 12 & 916 & 55.7 & $21^{+}$ & $-0 . \overline{08}$ \\
\hline$: 12$ & 109 & 63 & 25 & 21.6 & 52.6 & .12 & 919 & 55.9 & 22 & -0.08 \\
\hline $9: 11$ & 110 & 63 & 25 & 21.6 & 52.6 & 0.12 & 916 & 55.9 & 22 & -0.26 \\
\hline $07: 0$ & $1 \overline{11}^{t}$ & 63 & 25 & 21.6 & 52.6 & 0.12 & 919 & 55.9 & 22 & -0.26 \\
\hline $07: 0$ & 112 & 63 & 25 & $21.6 !$ & 52.5 & 0.12 & 923 & 55.9 & 22 & -0.1 \\
\hline $07: 0$ & 113 & 63 & 25 & 21.6 & 52.5 & 0.12 & 925 & 55.9 & 21 & -0.1 \\
\hline 07:0 & 114 & 63 & 25 & 21.6 & 52.5 & 0.12 & 924 & 55.8 & 21 & -0.03 \\
\hline 107:0 & 115 & 63 & 25 & 21.8 & 52.5 & $0 . \overline{12}$ & 922 & 55.8 & 21 & -0.03 \\
\hline & $11 \overline{6}$ & 63 & 25 & 21.8 & 52.5 & 11 & 916 & 55.8 & 21 & -0.11 \\
\hline & 117 & 63 & 25 & 21.8 & 52.5 & & 914 & 55.8 & 21 & -0.11 \\
\hline & $1 \longdiv { 8 }$ & 63 & $25 \mid$ & $2 \overline{11.8}$ & 52.4 & & 916 & 55.7 & 21 & $-0 . \overline{24}$ \\
\hline & 119 & 63 & 25 & 21.8 & 52.4 & 0.12 & 912 & 55.7 & 21 & $-\overline{0.24}$ \\
\hline $07: 0$ & 120 & 63 & $25^{\dagger}$ & 21.8 & 52.4 & 0.12 & 912 & 55.7 & $\overrightarrow{20}$ & -0.13 \\
\hline $07: 0$ & 121 & $6 \overline{3}$ & 25 & 21.6 & 52.4 & 0.14 & 914 & 55.6 & 21 & -0.13 \\
\hline $07: 1$ & 122 & 63 & 25 & 21.6 & 52.3 & 0.14 & 919 & 55.6 & 21 & -0.05 \\
\hline $07: 1$ & 123 & 63 & 25 & 21.6 & 52.3 & & 922 & 55.8 & 21 & $-0 . \overline{05}$ \\
\hline $07: 1$ & 124 & 63 & $25 !$ & 21.6 & 52.3 & & 922 & 55.8 & $21^{1}$ & $-0 . \overline{04}$ \\
\hline & 125 & $6 \overline{3}$ & 25 & 21.8 & 52.3 & & 919 & 55.8 & 21 & -0.04 \\
\hline & 126 & 63 & 25 & 21.6 & $52.3 !$ & & 91 & 55.8 & 21 & -0.18 \\
\hline & $1 \overline{27}$ & 63 & 25 & 21.8 & 52.3 & 12 & 91 & 55.7 & 20 & -0.18 \\
\hline & 128 & 63 & 25 & 1.8 & 52 & & 9 & 55.7 & 20 & -0.21 \\
\hline 89 & 129 & 63 & 25 & 21.8 & 52.3 & 0.12 & 910 & 55.6 & 20 & -0.04 \\
\hline & 130 & 63 & 25 & 21.8 & 52.3 & & 909 & 55.8 & 20 & -0.04 \\
\hline & 131 & 63 & 25 & $21 . \overline{8}$ & 52.3 & 0.12 & 10 & 55.8 & $20^{\dagger}$ & $-0 . \overline{09}$ \\
\hline & 132 & 63 & 25 & 1.8 & $52.3^{\dagger}$ & 0.12 & 914 & 55.7 & 20 & $-0.0 \overline{9}$ \\
\hline & 133 & 63 & 25 & 21.8 & 52.3 & & 918 & 55.7 & 21 & -0.13 \\
\hline & 134 & 63 & 25 & 21.8 & 52.2 & & 920 & .7 & 21 & -0.13 \\
\hline & 13 & 63 & 25 & 21.8 & $5 \overline{2} .2$ & 11 & 91 & 55.9 & 21 & -0.27 \\
\hline 81 & 136 & 63 & 25 & 21.8 & 52.2 & 11 & 920 & 55.8 & 21 & -0.27 \\
\hline 80 & 137 & 63 & 25 & 21.8 & 52.2 & 11 & 917 & 55.8 & 21 & -0.07 \\
\hline 79 & 138 & 63 & 25 & 21.8 & 52.2 & 12 & 913 & 55.9 & 21 & -0.07 \\
\hline 78 & 139 & 63 & 25 & 21.8 & 52.2 & 12 & 908 & 55.9 & 20 & 0.02 \\
\hline & 140 & 63 & 25 & 21.8 & 52.2 & & $905^{1}$ & 55.9 & 20 & 0.02 \\
\hline & 14 & 63 & 25 & 21.8 & 52.2 & & & 5.7 & 20 & -0.12 \\
\hline & 14 & 63 & 25 & 21 & 52.2 & & & & 20 & -0.12 \\
\hline & 143 & 63 & 25 & 21.8 & 52.2 & & 916 & 55.8 & 20 & -0.29 \\
\hline & 14 & 63 & 25 & 21.8 & 52.2 & 0.14 & 912 & 55.8 & 20 & -0.29 \\
\hline $3: 71$ & 145 & 63 & 25 & 21.8 & 52.2 & 0.12 & 916 & 55.9 & 21 & -0.2 \\
\hline 07: & 146 & 63 & 25 & 21.8 & 52.2 & 12 & 919 & 55.9 & 21 & -0.2 \\
\hline 07 & 147 & 63 & 25 & 21.8 & 52.2 & 0.11 & 916 & 55.9 & 20 & 0.12 \\
\hline $26: 68$ & 148 & 63 & 25 & $21 . \overline{8}$ & 52.2 & 0.12 & 913 & 55.8 & 20 & 0.12 \\
\hline $07: 10: 27: 66$ & 149 & 63 & 25 & 21.8 & 52.2 & 0.11 & 909 & 55.8 & $20 !$ & -0.13 \\
\hline $07: 10: 28: 65$ & 150 & 63 & 25 & 21.8 & 52.2 & 0.12 & 906 & 55.8 & 20 & -0.13 \\
\hline $07: 10: 29: 64$ & 151 & 63 & 25 & 21.8 & 52.1 & 0.14 & 906 & 55.8 & $20 !$ & -0.25 \\
\hline $07: 10: 30: 63$ & 152 & 63 & 25 & 21.8 & 52.1 & 0.14 & 905 & 55.8 & 20 & -0.25 \\
\hline
\end{tabular}




\begin{tabular}{|c|c|c|c|c|c|c|c|c|c|c|}
\hline$T / M E$ & $\begin{array}{l}\text { Elapsed } \\
\text { Time } \\
\text { (sec) }\end{array}$ & ¡BIT_TC1 & PURG_TC2 & PurgePSI & $\begin{array}{l}\vdots \\
\text { PurgeCFM }\end{array}$ & DEPTH(IN) & DwnFrc(lb) & $\begin{array}{l}\text { RPM } \\
\text { R }\end{array}$ & $\begin{array}{l}\text { Torque } \\
\text { (FTLB) }\end{array}$ & Inches/Min \\
\hline $07: 10: 31: 62$ & 153 & -63 & -25 & $\quad 21.8$ & $\begin{array}{r}52.1 \\
\end{array}$ & 0.12 & 906 & 55.7 & 20 & $\quad-0.15$ \\
\hline $07: 10: 32: 61$ & 154 & 63 & 25 & 21.8 & 52.1 & 0.14 & 910 & 55.8 & 20 & -0.15 \\
\hline $07: 10$ & 155 & 63 & 25 & 21.8 & 52.1 & 0.12 & $\overline{914}$ & 55.8 & 20 & $-0.0 \overline{6}$ \\
\hline $07: 10: ?$ & $15 \overline{6}$ & 63 & 25 & 21.8 & 52.1 & 0.14 & 917 & 56 & 20 & $-0 . \overline{06}$ \\
\hline $35: 57$ & 157 & 63 & 25 & 21.8 & $52.1^{\dagger}$ & 0.12 & 918 & 56 & 20 & -0.08 \\
\hline 56 & 158 & 63 & 25 & 21.8 & 52.1 & 0.11 & 916 & 55.9 & 20 & -0.08 \\
\hline & 159 & 63 & 25 & 21.8 & 52.1 & 0.12 & 913 & 55.9 & 20 & -0.16 \\
\hline & $\overline{1} \overline{60}$ & $\overline{6} 3$ & 25 & 22 & 52.1 & 0.12 & 909 & 55.9 & 20 & $-0.1 \overline{6}$ \\
\hline & 161 & 63 & 25 & 22.4 & 52.1 & 0.14 & 912 & 56 & 20 & -0.28 \\
\hline 52 & 162 & 63 & 25 & 22.4 & 52.1 & 0.14 & 907 & 56 & 20 & -0.28 \\
\hline 51 & 163 & 63 & 25 & 22.4 & 52.1 & 0.12 & 906 & 56 & 20 & 0.01 \\
\hline $07: 1$ & 164 & $\overline{63}$ & 25 & 22.4 & 52.1 & 0.14 & 907 & 56 & $\overline{2} 0$ & $-\overline{0.07}$ \\
\hline & 165 & 63 & 25 & 22.4 & $5 \overline{2} .1$ & 0.12 & 911 & 55.9 & 20 & $-\overline{0.07}$ \\
\hline & 166 & 63 & 25 & 22.4 & 52.1 & 0.12 & 916 & 55.9 & 20 & -0.1 \\
\hline & 167 & 63 & 25 & 22.4 & $52.1^{\dagger}$ & 0.14 & 918 & 56 & 20 & -0.1 \\
\hline & 168 & 63 & 25 & 22.4 & 52.1 & 0.12 & 918 & 56 & 20 & -0.28 \\
\hline & 169 & 63 & 25 & 22.4 & 52.1 & $0.1 \overline{4}$ & 917 & 56 & 20 & -0.28 \\
\hline & 170 & 63 & 25 & 22.4 & 52.1 & 0.14 & 917 & 55.9 & 20 & -0.09 \\
\hline & 171 & 62 & 25 & 22.4 & 52.1 & 0.12 & $\overline{914}$ & 55.8 & 20 & -0.09 \\
\hline & 172 & 63 & 25 & 22.6 & 52.1 & 0.12 & 911 & 55.8 & 20 & -0.05 \\
\hline & 173 & 62 & 25 & 22.4 & 52.1 & 0.12 & 908 & 55.8 & 20 & -0.05 \\
\hline & 174 & 62 & 25 & 22.4 & 52.1 & 0.14 & 907 & 55.9 & 20 & -0.09 \\
\hline & 175 & $\overline{62}$ & 25 & 22.4 & 52.1 & & 910 & 55.9 & 20 & -0.09 \\
\hline & 176 & 63 & 25 & 22.4 & $2.1 !$ & & 91 & 56 & 20 & -0.24 \\
\hline & 177 & 62 & 25 & 22.4 & 52.1 & 0.15 & 910 & 56 & 20 & -0.24 \\
\hline & 178 & 62 & 25 & 22.4 & 52.1 & 0.12 & 914 & 56 & 20 & -0.16 \\
\hline & 179 & 62 & 25 & 22.4 & 52.1 & 0.12 & 918 & 55.9 & 20 & -0.16 \\
\hline & 180 & 62 & 25 & 22.4 & 52.1 & 0.12 & 919 & 56 & 20 & $0 . \overline{05}$ \\
\hline & $\overline{181}$ & 62 & 25 & 22.8 & $52.1^{1}$ & $0 . \overline{14}$ & 916 & 56 & 20 & 0.05 \\
\hline & 182 & 62 & 25 & 22.9 & 52.1 & $0 . \overline{12}$ & 913 & 55.9 & 20 & $-0.1 \overline{4}$ \\
\hline & 183 & 62 & 25 & 22.9 & 21 & & 910 & 56 & 19 & -0.14 \\
\hline & 184 & 62 & 25 & 22.9 & 52.1 & 0.1 & & 56 & 19 & -0.15 \\
\hline & 185 & 62 & 25 & 22.9 & 52 & 0.1 & 908 & 56.1 & 19 & $-0 . \overline{15}$ \\
\hline & 18 & 6 & 2 & 2.8 & 52 & & & 56.1 & 20 & $-0 . \overline{27}$ \\
\hline & 187 & 62 & 25 & 22.9 & 2.1 & & 908 & 56.1 & 19 & -0.27 \\
\hline & 188 & 62 & 25 & 22.8 & 2.1 & 0.12 & 912 & 56.2 & 19 & -0.07 \\
\hline & 189 & 62 & 25 & 22.9 & & & 916 & 56.1 & 19 & -0.07 \\
\hline & 190 & 62 & 24 & 22.8 & 2.1 & 0.12 & 918 & 56.1 & 19 & 0.01 \\
\hline & 191 & 62 & 25 & 22.8 & & 0.12 & 919 & $5 \overline{6}$ & 19 & 0.01 \\
\hline & $1 \overline{92}$ & 62 & 25 & 22.9 & & 0.12 & 917 & 56 & 19 & -0.1 \\
\hline & 19 & 62 & 25 & & 2.1 & & 914 & 56 & 19 & -0.1 \\
\hline & 19 & 62 & 25 & 22.9 & 52.1 & 0.12 & 917 & 56.1 & 19 & -0.28 \\
\hline & 195 & 62 & 25 & 22.9 & 52.1 & 0.12 & 914 & 56.1 & 19 & -0.28 \\
\hline & 198 & 62 & 25 & $22 . \overline{9}$ & 52.1 & 0.12 & 909 & 56.1 & 19 & -0.11 \\
\hline & 197 & 62 & 25 & 22.9 & 52.1 & 0.1 & 907 & $56^{\dagger}$ & 19 & 0.03 \\
\hline $6: 11$ & 198 & 62 & 25 & 22.9 & 52.1 & 0.15 & 909 & 55.9 & 19 & 0.03 \\
\hline $7: 10$ & 199 & 62 & 25 & 22.9 & 52.1 & 0.15 & 913 & 55.9 & 19 & -0.16 \\
\hline$: 18: 09$ & 200 & 62 & 25 & 22.9 & 52.1 & 0.15 & 917 & 55.9 & 19 & -0.16 \\
\hline $07: 11: 19: 07$ & 201 & 62 & 25 & 22.9 & 52.1 & 0.15 & 919 & 55.9 & 19. & -0.21 \\
\hline $07: 11: 20: 06$ & 202 & 62 & 25 & 22.9 & 52.1 & 0.15 & 917 & 56 & 19 & -0.21 \\
\hline $07: 11: 21: 05$ & 203 & 62 & 25 & 22.9 & $52.1^{1}$ & 0.12 & 919 & 56 & 19 & -0.17 \\
\hline
\end{tabular}




\begin{tabular}{|c|c|c|c|c|c|c|c|c|c|c|}
\hline TIME & $\begin{array}{l}\text { Elapsed } \\
\text { Time } \\
\text { (sec) }\end{array}$ & $B I T$ TC1 & PURG_TC2 & PurgePSi & PurgeCFM & DEPTH(IN) & DwnFrc(lb) & RPM & $\begin{array}{l}\text { Torque } \\
\text { (FTLB) }\end{array}$ & Inches/Min \\
\hline $07: 11: 22: 04$ & 204 & -62 & $\quad 25$ & 22.9 & $\quad 52.1$ & 0.12 & $\quad 919$ & $\quad 56$ & 19 & $\quad-0.17$ \\
\hline $07: 11: 23: 03$ & $205^{\circ}$ & 62 & 25 & $22.9^{\prime}$ & $52.1^{+}$ & 0.12 & $916^{\circ}$ & 55.9 & 19 & -0.04 \\
\hline $07: 11: 24: 02$ & 206 & 62 & 25 & 22.9 & $52.1_{-1}$ & 0.15 & 910 & 55.8 & 19 & -0.04 \\
\hline $07: 11: 25: 01$ & 207 & 62 & 25 & 22.9 & $52.1^{-7}$ & 0.14 & 907 & 55.8 & 19 & -0.08 \\
\hline $07: 11: 26: 00$ & $208^{\circ}$ & 62 & 25 & 22.9 & 52.1 & 0.15 & 908 & 55.7 & 19 & -0.08 \\
\hline $07: 1 \overline{1}: 26: 98$ & 209 & 62 & 25 & 22.9 & 52.1 & 0.12 & 911 & 55.7 & 19 & -0.16 \\
\hline $07: 11: 27: 97$ & $210^{\circ}$ & 62 & 25 & 23.1 & 52.1 & 0.15 & 916 & 55.7 & 19 & -0.16 \\
\hline $07: 11: 28: 96$ & $2 \overline{1} 1$ & 62 & 25 & $23.3 i$ & 52.1 & 0.12 & 911 & 55.6 & $19^{\circ}$ & -0.24 \\
\hline $29: 95$ & 212 & 62 & 25 & 23.1 & $52.1^{1}$ & 0.15 & 916 & 55.6 & 19 & -0.24 \\
\hline $07: 11: 30: 94$ & 213 & 61 & 25 & 23.3 & $52.1^{r}$ & 0.12 & 919 & 55.6 & 19 & -0.09 \\
\hline $07: 11: 31: 93$ & 214 & 62 & 25 & $23.3^{1}$ & $5 \overline{2} .1^{t}$ & 0.12 & $919^{\dagger}$ & 55.7 & 19 & -0.09 \\
\hline $07: 11: 32: 92$ & 215 & 62 & 25 & 23.3 & 52.1 & 0.14 & 916 & 55.8 & 19 & -0.06 \\
\hline $07: 11: 33: 90$ & 216 & 61 & 25 & 23.3 & 52.1 & 0.12 & 912 & $55.8^{\prime}$ & 19 & -0.06 \\
\hline $07: 11: 34: 89$ & 217 & 61 & $25^{\prime}$ & 23.3 & 52.1 & 0.12 & 909 & 55.8 & 19 & -0.07 \\
\hline 88 & 218 & 61 & 25 & 23.5 & 52.1 & 0.14 & $908^{-1}$ & 55.8 & 19 & -0.07 \\
\hline $36: 87$ & 219 & 61 & 25 & $23.3 i$ & 52.1 & 0.14 & $909^{-1}$ & 55.8 & 18 & -0.3 \\
\hline $07: 11: 37: 86$ & 220 & 61 & 25 & 23.3 & 52.1 & 0.14 & 908 & 55.8 & 18 & -0.3 \\
\hline $8: 85$ & 221 & 61 & 25 & $23.5 !$ & 52.1 & 0.15 & 910 & 55.8 & 18 & -0.01 \\
\hline 84 & 222 & 61 & 25 & $23.3 !$ & 52.1 & 0.15 & 914 & 55.8 & 19 & -0.01 \\
\hline $0: 83$ & 223 & 61 & 25 & $23.3 !$ & 52.1 & 0.15 & 919 & 55.8 & 19 & -0.11 \\
\hline $07: 11: 41: 81$ & 224 & $61 \mid$ & 25 & 23.3 & $52.1 !$ & $0 . \overline{12}$ & 919 & 55.8 & 19 & -0.11 \\
\hline $12: 80$ & $225^{1}$ & 61 & 25 & 23.3 & 52.1 & 0.15 & 917 & 55.8 & 19 & -0.09 \\
\hline 79 & 226 & 61 & 25 & 23.3 & $52.1^{\dagger}$ & 0.15 & 914 & 55.9 & 19 & -0.09 \\
\hline $4: 78$ & 227 & 61 & 25 & 23.3 & 52.1 & 0.12 & 918 & 56 & $19^{\top}$ & $-0 . \overline{25}$ \\
\hline $07: 11: 45: 77$ & 228 & 61 & 25 & 23.5 & 52.1 & 0.14 & 914 & 56 & 18 & -0.25 \\
\hline $6: 76$ & $22 \overline{9}^{\circ}$ & 61 & $25^{\prime}$ & 23.3 & 52.1 & 0.14 & 910 & 56 & 18 & $-0 . \overline{19}$ \\
\hline $7: 75$ & 230 & 61 & 25 & 23.5 & 52.1 & 0.14 & 909 & 56 & 18 & 0.06 \\
\hline $3: 73$ & 231 & 61 & 25 & 23.71 & 52.1 & 0.14 & 909 & 56 & 19 & 0.06 \\
\hline $07: 11: 49: 72$ & 232 & $61 !$ & 25 & 24.1 & 52.1 & 0.15 & 913 & 55.8 & 19 & -0.12 \\
\hline 71 & 233 & 61 & 25 & 24.3 & 52.2 & 0.15 & 917 & $55.9^{\dagger}$ & 19 & -0.12 \\
\hline & 234 & 61 & 25 & 24.1 & 52.2 & 0.15 & 919 & 55.9 & 19 & $-0.2 \overline{1}$ \\
\hline 69 & 235 & 61 & 25 & 24.3 & 52.2 & 0.15 & 919 & 55.8 & 19 & -0.21 \\
\hline 68 & 236 & 61 & 25 & 24.1 & $52.3^{2}$ & 0.14 & 919 & 56 & 19 & -0.23 \\
\hline 67 & 237 & 61 & $25^{\prime}$ & 24.1 & 52.3 & 0.14 & 919 & 56 & 19 & -0.23 \\
\hline & 238 & 61 & 25 & 24.3 & 52.3 & 0.12 & 916 & 56 & 19 & 0.06 \\
\hline & 239 & 61 & 25 & 24.3 & 52.3 & 0.14 & 912 & $56 !$ & 18 & 0.06 \\
\hline 63 & 240 & 61 & 25 & 24.3 & 52.3 & 0.14 & 909 & 56 & 18 & -0.15 \\
\hline $8: 62$ & 241 & 61 & 25 & 24.1 & 52.3 & 0.14 & 908 & 56 & $19^{\dagger}$ & -0.15 \\
\hline $59: 61$ & 242 & 61 & 25 & 24.3 & 52.4 & 0.12 & 911 & 55.9 & 18 & -0.13 \\
\hline $0: 60$ & 243 & 61 & 25 & 24.3 & 52.4 & 0.12 & 915 & 55.9 & 19 & -0.13 \\
\hline 1.59 & 244 & $61_{t}^{\dagger}$ & 25 & 24.3 & 52.3 & 0.15 & 912 & 55.9 & 19 & $-0 . \overline{26}$ \\
\hline $07: 12: 02: 58$ & 245 & $61+$ & 25 & 24.3 & 52.4 & 0.12 & 915 & 55.9 & 19 & -0.26 \\
\hline $07: 12$ & 246 & 61 & 25 & 24.3 & 52.4 & 0.12 & 918 & 55.9 & 19 & -0.08 \\
\hline $07: 12:$ & 247 ! & 61 & 25 & 24.3 & 52.4 & 0.12 & 921 & 55.9 & $\overline{19}$ & -0.08 \\
\hline $07: 12: 05: 54$ & $2 \overline{48}$ & 61 & 25 & 24.3 & 52.4 & 0.12 & 919 & 55.9 & 19 & -0.07 \\
\hline $07: 12: 06: 53$ & 249 & 61 & 25 & $24.3^{\top}$ & 52.4 & 0.12 & 916 & $56 !$ & 19 & -0.07 \\
\hline $07: 12: 07: 52$ & 250 & 61 & 25 & $24.3^{1}$ & 52.4 & 0.15 & 912 & 56.1 & 19 & -0.07 \\
\hline $07: 12: 08: 51$ & 251 & 61 & 25 & 24.3 & 52.4 & 0.14 & 908 & 56.1 & 18 & -0.07 \\
\hline $07: 12: 09: 50$ & 252 & 61 & 25 & $24.3^{+}$ & 52.4 & 0.15 & 909 & 56.1 & 18 & -0.25 \\
\hline $07: 12: 10: 49$ & 253 & 61 & 25 & 24.3 & 52.4 & 0.14 & 907 & 55.9 & 19 & -0.25 \\
\hline $07: 12: 11: 47$ & 254 & 61 & 25 & 24.3 & 52.4 & 0.14 & 908 & $55.9^{\circ}$ & 19 & -0.16 \\
\hline
\end{tabular}




\begin{tabular}{|c|c|c|c|c|c|c|c|c|c|c|}
\hline TIME & $\begin{array}{l}\text { Elapsed } \\
\text { Time } \\
\text { (sec) }\end{array}$ & BIT_TC1 & PURG_TC2 & PurgePSI & PurgeCFM & DEPTH(IN) & DwnFrc(lb) & RPM & $\begin{array}{l}\text { Torque } \\
\text { (FTLB) }\end{array}$ & Inches/Min \\
\hline $07: 12: 12: 46$ & 255 & 61 & 25 & $\quad 24.3$ & $\quad 52.4$ & $\quad 0.14$ & $\quad 912$ & 55.9 & $\quad 19$ & $9 \quad-0.16$ \\
\hline $07: 12: 13: 45$ & 256 & 61 & 25 & 24.3 & 52.5 & 0.15 & 916 & 55.9 & 18 & -0.07 \\
\hline $07: 12: 14: 44$ & 257 & 61 & 25 & 24.5 & 52.5 & 0.14 & $919^{\circ}$ & 55.9 & 19 & -0.07 \\
\hline $07: 12: 15: 43$ & 258 & 61 & 25 & 24.3 & 52.5 & 0.14 & 919 & 55.9 & 19 & -0.07 \\
\hline $07: 12: 16: 42$ & 259 & 61 & 25 & 24.3 & 52.5 & 0.14 & 917 & 55.9 & 19 & -0.07 \\
\hline $07: 12: 17: 41$ & 260 & 61 & 25 & 24.5 & 52.5 & 0.12 & 912 & 55.9 & 19 & -0.19 \\
\hline$: 12: 18: 39$ & 261 & 601 & 25 & 24.3 & 52.5 & 0.12 & 915 & 55.6 & 19 & -0.19 \\
\hline $07: 12: 19: 38$ & 262 & 60 & 25 & 24.5 & 52.5 & 0.15 & 910 & 55.5 & 19 & -0.17 \\
\hline $20: \overline{37}$ & 263 & 60 & 25 & $24.3^{1}$ & 52.5 & 0.14 & 907 & 55.5 & 19 & -0.1 \\
\hline $1: 36$ & 264 & 60 & 25 & $24.3^{\prime}$ & 52.5 & 0.14 & 908 & 55.4 & 19 & -0.1 \\
\hline $07: 12: 22: 3 \overline{5}$ & 265 & 60 & 25 & 24.5 & 52.5 & 0.14 & 911 & 55.4 & 19 & -0.05 \\
\hline$: 34$ & 266 & 60 & 25 & 24.5 & 52.5 & 0.15 & $916^{\prime}$ & 55.4 & 19 & -0.05 \\
\hline $4: 33$ & 267 & 60 & 25 & $24.3^{f}$ & $52.5^{1}$ & 0.14 & $919^{\prime}$ & 55.4 & 19 & -0.22 \\
\hline $07: 12: 25: 32$ & 268 & 61 & 25 & 24.5 & 52.5 & 0.14 & $919^{\dagger}$ & 55.4 & 19 & -0.22 \\
\hline$: 12: 26: 30$ & 269 & 61 & $25 !$ & $24.7 !$ & 52.5 & 0.12 & $920^{\dagger}$ & 55.4 & 19 & -0.24 \\
\hline $2: 27: 29$ & 270 & 61 & 25 & 24.5 & 52.5 & 0.14 & 919 & 55.5 & 19 & -0.24 \\
\hline $12: 28: 28$ & 271 & 60 & 25 & 24.7 & 52.5 & 0.14 & 916 & 55.5 & 19 & 0.01 \\
\hline 27 & 272 & 60 & 25 & 24.7 & 52.5 & 0.1 & 910 & 55.5 & 18 & 0.01 \\
\hline & 273 & 60 & 25 & 24.7 & 52.5 & 0.14 & 908 & 55.4 & 19 & -0.1 \\
\hline 25 & 274 & 60 & 25 & $24.7 !$ & 52.5 & 0.14 & $909 ?$ & 55.4 & 18 & -0.1 \\
\hline 24 & 275 & 60 & 25 & 24.7 & 52.5 & 0.14 & 912 & 55.4 & 19 & -0.18 \\
\hline$: 22$ & 276 & 60 & 25 & 24.7 & 52.5 & 0.15 & 917 & 55.5 & 19 & -0.18 \\
\hline 21 & 277 & 60 & 25 & $24.7 !$ & 52.5 & 0.15 & 912 & 55.5 & 19 & -0.2 \\
\hline 20 & 278 & 60 & 25 & 24.9 & 52.5 & 0.12 & 91 & 55.5 & 19 & -0.2 \\
\hline 19 & 279 & 60 & 25 & 24.7 & 52.6 & 0.12 & 919 & 55.5 & 19 & -0.04 \\
\hline & 280 & 60 & 25 & 24.9 & 52.6 & 0.14 & 919 & 55.5 & 19 & -0.04 \\
\hline & 281 & 60 & 25 & 24.9 & 52.6 & & 916 & 55.5 & 18 & -0.11 \\
\hline 16 & 282 & 60 & 25 & 24.9 & 52.6 & 0.12 & 912 & 55.7 & 18 & -0.11 \\
\hline 14 & 283 & 60 & 25 & 24.9 & 52.6 & 0.14 & 909 & 55.8 & 18 & -0.14 \\
\hline$: 13$ & 284 & 60 & 25 & 24.9 & 52.6 & 0.15 & 909 & 55.8 & 18 & -0.14 \\
\hline 12 & 285 & 60 & 25 & 24.9 & 52.6 & 0.15 & 912 & $55 . \overline{6}$ & 18 & -0.25 \\
\hline & 286 & 60 & 25 & 24.9 & 52.6 & & 909 & 55.5 & 18 & -0.25 \\
\hline $4: 10$ & 287 & 601 & 25 & $25.1_{i}$ & 52.6 & 0.14 & 913 & 55.5 & 19 & $-0 . \overline{11}$ \\
\hline & 288 & 60 & 25 & 25.1 & 52.6 & 0.14 & 917 & 55.6 & 19 & $-0 . \overline{1}$ \\
\hline & 289 & 60 & 25 & 25.1 & 52.6 & $0 . \overline{14}$ & 920 & 55.6 & 19 & -0.05 \\
\hline$: 07$ & 290 & 60 & 25 & 24.9 & 52.6 & 0.14 & 919 & 55.6 & 19 & -0.05 \\
\hline 3:05 & 291 & 601 & 25 & 25.1 & 52.7 & 0.12 & 917 & 55.6 & 19 & -0.11 \\
\hline & 292 & 60 & 25 & 25.1 & 52.7 & 0.14 & 912 & 55.6 & 19 & -0.11 \\
\hline & 293 & 60 & 25 & & 2.7 & & 9 & & 18 & -0.3 \\
\hline & 294 & 60 & 25 & 25.1 & 52.7 & & 91 & 55.6 & 18 & -0.3 \\
\hline & 295 & 60 & $25^{\dagger}$ & 25.1 & 52.7 & 0.14 & 908 & $55 . \overline{6}$ & 18 & -0.01 \\
\hline $53: \overline{00}$ & 296 & 60 & 25 & $25.1^{\dagger}$ & 52.7 & 0.15 & $909^{\circ}$ & 55.6 & 18 & -0.01 \\
\hline $3: 99$ & 297 & 60 & 25 & 25.1 & 52.7 & 0.14 & 912 & 55.6 & 18 & -0.1 \\
\hline $4: 97$ & 298 & 60 & 25 & 25.3 & 52.7 & 0.14 & 916 & $55.6^{\dagger}$ & 18 & -0.07 \\
\hline & 299 & 60 & 25 & 25.3 & 52.7 & 0.1 & 919 & 55.6 & 19 & -0.07 \\
\hline & $300^{\dagger}$ & $60 \mathrm{i}$ & 25 & 25.3 & 52.7 & 0.15 & 919 & $55 . \overline{6}$ & 19 & -0.27 \\
\hline $07: 12: 57: 94$ & 301. & 60 & 25 & 25.1 & 52.7 & 0.14 & 917 & 55.6 & 19 & -0.27 \\
\hline $07: 12: 58: 93$ & 302 & 60 & 25 & 25.3 & 52.7 & 0.15 & 919 & 55.8 & 19 & -0.08 \\
\hline $07: 12: 59: 92$ & 303 & 60 & 25 & 25.3 & 52.7 & 0.14 & 916 & $55 . \overline{6}$ & 19 & -0.08 \\
\hline $07: 13: 00: \overline{9} 1$ & 304 & 60 & 25 & 25.3 & 52.7 & 0.15 & 912 & 55.6 & 19 & -0.09 \\
\hline $07: 13: 01: 90$ & 305 & 60 & 25 & 25.3 & 52.7 & 0.12 & 909 & $5 \overline{5} .6$ & 18 & $-0 . \overline{09}$ \\
\hline
\end{tabular}




\begin{tabular}{|c|c|c|c|c|c|c|c|c|c|c|}
\hline$T \mid M E$ & $\begin{array}{l}\text { Elapsed } \\
\text { Time } \\
\text { |(sec) }\end{array}$ & BIT_TC1 & PURG_TC2 & PurgePSI & PurgeCFM & DEPTH(IN) & DwnFrc(lb) & RPM & $\begin{array}{l}\text { Torque } \\
\text { (FTLB) }\end{array}$ & Inches/Min \\
\hline $07: 13: 02: 88$ & 306 & $\begin{array}{r}60 \\
6\end{array}$ & -25 & $\quad 25.1$ & $5 \overline{2} .7$ & 0.15 & 910 & 55.7 & 18 & -0.06 \\
\hline $07: 13: 03: 87$ & 307 & 60 & 25 & 25.3 & 52.7 & 0.94 & 912 & 55.7 & 18 & -0.06 \\
\hline $07: 13: 04: 86$ & 308 & $60 !$ & 25 & 25.3 & 52.7 & 0.15 & 916 & 55.7 & 19 & -0.24 \\
\hline $07: 13: 05: 85$ & 309 & 60 & 25 & 25.1 & 52.7 & 0.16 & 924 & 55.8 & 19 & -0.24 \\
\hline $07: 13: 06: 84$ & 310 & 60 & 25 & 25.1 & 52.7 & 0.14 & 932 & 55.8 & 19 & -0.1 \\
\hline $07: 13: 07: 83$ & 311 & 60 & $25^{1}$ & 25.3 & 52.7 & 0.14 & $950^{\circ}$ & 55.8 & 19 & -0.1 \\
\hline $07: 13: 08: 82$ & 312 & 60 & $25:$ & 25.1 & 52.7 & 0.15 & 984 & 55.8 & 20 & 0.18 \\
\hline $07: 13: 09: 80$ & 313 & 61 & 25 & 25.1 & 52.7 & 0.14 & 1026 & 55.8 & 21 & $0 . \overline{18}$ \\
\hline $07: 13: \overline{10}: \overline{79}$ & 314 & 61 & 25 & 25.1 & $52.7 !$ & 0.16 & 1075 & 55.9 & 22 & 0.44 \\
\hline $07: 13: 11: 78$ & 315 & 61 & 25 & 25.1 & $52.7 !$ & 0.18 & 1110 & 55.9 & 22 & 0.44 \\
\hline $07: 13: 12: 77$ & 316 & 62 & 25 & 25.1 & 52.7 & 0.18 & 1138 & 55.9 & 23 & 0.2 \\
\hline $07: 13: 13: 76$ & 317 & 62 & 25 & 25.1 & 52.7 & 0.19 & 1169 & 55.7 & 23 & 0.2 \\
\hline $07: 13: 14: 75$ & 318 & 63 & 25 & $25.1^{t}$ & 52.7 & 0.2 & 1206 & 55.6 & 24 & 0.1 \\
\hline $07: 13: 15: 74$ & 319 & 63 & 25 & 25.1 & 52.7 & 0.2 & 1242 & 55.6 & 25 & 0.1 \\
\hline $07: 13: \overline{16: 73}$ & 320 & 64 & 25 & 25.1 & 52.7 & 0.21 & 1266 & 55.5 & 25 & 0.41 \\
\hline $3: 17: 7 \overline{1}$ & 321 & 64 & 25 & 25.1 & 52.7 & $0 . \overline{2}$ & 1289 & 55.3 & 26 & 0.41 \\
\hline $07: 13: 18: 70$ & 322 & 65 & 25 & 25.1 & 52.8 & 0.21 & 1309 & 55.3 & 26 & 0.22 \\
\hline $07: 13: 19: 69$ & 323 & 65 & 25 & 25.1 & 52.7 & 0.2 & 1326 & 55.1 & 26 & 0.22 \\
\hline $20: 68$ & 324 & 66 & 25 & $25.1^{\circ}$ & 52.7 & 0.2 & 1338 & $55^{\dagger}$ & 26 & 0.01 \\
\hline $07: 13: 21: 67$ & 325 & 67 & 25 & 25.1 & 52.7 & 0.2 & 1346 & 55 & 27 & 0.01 \\
\hline $07: 13: 22: 66$ & 326 & 67 & 25 & 25.1 & $52.7^{\prime}$ & 0.21 & 1347 & 55 & 27 & -0.1 \\
\hline $07: 13: 23: 65$ & 327 & 67 & 25 & 25.1 & 52.7 & 0.21 & 1351 & 55 & 27 & -0.1 \\
\hline$\overline{24}: \overline{63}$ & 328 & 68 & 25 & 25.1 & $52.8 i$ & & 13 & 55 & 28 & 0.1 \\
\hline & 329 & 68 & 25 & 25.1 & 52.8 & 0.21 & 1357 & 54.8 & 28 & 0.1 \\
\hline $26: 61$ & 330 & 68 & 25 & 25.1 & 52.8 & 0.21 & 1366 & 54.8 & 28 & -0.08 \\
\hline $27: 60$ & 331 & 69 & 25 & $25.1^{+}$ & $52.8^{-1}$ & 0.21 & 1370 & 54.8 & 28 & -0.29 \\
\hline $07: 13: 28: 59$ & 332 & 69 & 25 & 25.1 & 52.8 & 0.21 & 1369 & 54.9 & 28 & -0.29 \\
\hline $07: 13: 29: 58$ & 333 & 69 & 25 & 25.1 & $52.8 !$ & 0.21 & 1366 & 54.9 & 28 & -0.13 \\
\hline $07: 13: 30: 57$ & 334 & 70 & 25 & 25.1 & 52.8 & 0.21 & 1362 & 54.9 & 28 & -0.13 \\
\hline $31: 56$ & 335 & 70 & 25 & 25.1 & 52.8 & 0.2 & 1362 & 55 & 28 & 0 \\
\hline 54 & 336 & 70 & 25 & 25.1 & 52.8 & 0.21 & 1366 & 55 & 28 & 0 \\
\hline 53 & 337 & 70 & 25 & 25.1 & 52.8 & 0.23 & 13 & 55 & 28 & -0.06 \\
\hline 52 & 338 & 70 & 25 & 25.1 & 52.8 & 0.23 & 1371 & 54.9 & 28 & -0.06 \\
\hline $07: 13: 35: 51$ & 339 & 70 & 25 & $25.1^{i}$ & 52.8 & 0.23 & 1377 & 54.9 & 28 & -0.22 \\
\hline $07: 13: 36: 50$ & 340 & 71 & 25 & $25.1^{i}$ & 52.8 & 0.21 & 1383 & 54.9 & 28 & -0.22 \\
\hline $07: 13: 37: 49$ & 341 & 71 & 25 & 25.3 & 52.8 & 0.2 & 1384 & 54.9 & 28 & -0.13 \\
\hline $07: 13: 38: 48$ & 342 & 71 & 25 & 25.1 & $52.8 i$ & 0.21 & 1381 & 54.9 & 28 & -0.13 \\
\hline $3: \overline{39: 46}$ & 343 & 72 & 25 & $25.1^{\circ}$ & 52.8 & 0.21 & 1377 & 55 & 28 & -0.02 \\
\hline $07: 13: 40: 45$ & 344 & 72 & 25 & 25.3 & 52.8 & 0.23 & 1375 & 55 & 28 & -0.02 \\
\hline $07: 13: 41: 44$ & 345 & 72 & 25 & 25.3 & 52.8 & 0.23 & 1377 & 55 & 28 & -0.11 \\
\hline $07: 13: 42: 43$ & 346 & 72 & 25 & 25.1: & 52.8 & 0.23 & 1376 & 54.9 & 28 & -0.11 \\
\hline $3: 43: 42$ & 347 & 72 & 25 & 25.1 & 52.8 & 23 & 1380 & $54.7 !$ & 29 & -0.23 \\
\hline $07: 13: 44: 41$ & 348 & 72 & 25 & 25.1 & 52.8 & 0.23 & 1387 & $54.7 !$ & 29 & -0.23 \\
\hline $07: 13: 45: 40$ & 349 & 72 & 25 & 25.1 & 52.8 & 0.21 & 1392 & 54.7 & 29 & -0.07 \\
\hline $07: 13: 46: 39$ & 350 & 73 & 25 & 25.1 & 52.8 & $0.21^{1}$ & 1393 & 54.8 & 29 & -0.07 \\
\hline $47: 37$ & 351 & 73 & 25 & 25.1 & 52.8 & 0.21 & 1390 & 54.8 & 28 & -0.04 \\
\hline $48: 36$ & 352 & 73 & 25 & 25.3 & 52.8 & $0 . \overline{21}$ & 1385 & 54.9 & 27 & -0.04 \\
\hline $9: 35$ & 353 & 73 & 25 & 25.3 & 52.8 & 0.21 & 1389 & 54.8 & 27 & -0.21 \\
\hline $50: 34$ & 354 & 73 & 25 & 25.1 & 52.8 & 0.23 & 1383 & 54.8 & 28 & -0.21 \\
\hline $1: 33$ & 355 & 73 & 25 & 25.3 & 52.8 & 0.23 & 1383 & 54.6 & 28 & -0.2 \\
\hline $07: 13: 52: 32$ & $356 !$ & 73 & 25 & 25.1 & 52.8 & 0.23 & 1388 & 54.5 & 28 & -0.2 \\
\hline
\end{tabular}




\begin{tabular}{|c|c|c|c|c|c|c|c|c|c|c|}
\hline$T I M E$ & $\begin{array}{l}\text { Elapsed } \\
\text { Time } \\
\text { (sec) }\end{array}$ & BIT_TC1 & PURG_TC2: & PurgePSI & PurgeCFM & DEPTH(IN) & DwnFrc(lb) & RPM & $\begin{array}{l}\text { Torque } \\
\text { (FTLB) }\end{array}$ & Inches/Min \\
\hline $07: 13: 53: 31$ & $\quad 357$ & -73 & 1. -25 & $\quad 25.1$ & $\quad 52.8$ & $\quad 0.23$ & $\quad 1393$ & 54.5 & 28 & -0.03 \\
\hline $07: 13: 54: 29$ & 358 & 73 & 25 & 25.3 & 52.8 & 0.23 & 1399 & 54.6 & 28 & -0.03 \\
\hline $07: 13: 55: 28$ & 359 & 74 & 25 & 25.3 & 52.8 & 0.23 & $1399^{\circ}$ & 54.5 & 28 & -0.08 \\
\hline $07: 13: 56: 27$ & 360 & 74 & 25 & 25.3 & 52.8 & 0.21 & 1396 & 54.5 & 28 & -0.08 \\
\hline $07: 13: 57: 26$ & 361 & $74^{\dagger}$ & 25 & 25.3 & 52.8 & 0.21 & $1399^{\circ}$ & 54.5 & 28 & -0.32 \\
\hline $07: 13: 58: 25$ & 362 & 74 & $25 !$ & $25 . \overline{3}$ & 52.8 & $0.23 !$ & 1393 & 54.5 & 28 & -0.32 \\
\hline $07: 13: 59: 24$ & 363 & 74 & 25 & 25.3 & 52.8 & 0.23 & 1388 & 54.5 & 28 & -0.14 \\
\hline $07: 14: 00: 23$ & 364 & 74 & 25 & 25.3 & 52.8 & 0.23 & 1386 & $54.4 \mathrm{j}$ & 28 & -0.06 \\
\hline $07: 14: 01: 21$ & 365 & 74 & 25 & 25.3 & 52.8 & 0.23 & 1390 & 54.4 & 29 & -0.06 \\
\hline $07: 14: 02: 20$ & 366 & 74 & 25 & 25.3 & 52.8 & 0.23 & 1396 & 54.4 & 29 & -0.17 \\
\hline $07: 14: 03: 19$ & 367 & 74 & 25 & 25.3 & 52.8 & 0.23 & 1398 & 54.5 & 29 & -0.17 \\
\hline $07: 14: 04: 18$ & 368 & 74 & 25 & 25.3 & 52.8 & 0.23 & $1396^{\circ}$ & 54.4 & 29 & -0.22 \\
\hline $07: 14: 05: 17$ & 369 & 75 & 25 & 25.3 & 52.8 & 0.21 & 1389 & 54.4 & 28 & -0.22 \\
\hline $07: 14: 06: 16$ & 370 & 75 & 25 & 25.3 & 52.8 & 0.23 & 1393 & 54.3 & 28 & -0.16 \\
\hline $07: 14: 07: 15$ & 371 & 75 & $25^{\dagger}$ & 25.3 & 52.8 & 0.23 & $1386^{\prime}$ & 54.4 & 28 & -0.16 \\
\hline $07: 14: 08: 14$ & 372 & 75 & $25 !$ & 25.3 & 52.8 & 0.23 & $1381^{\dagger}$ & 54.4 & 28 & -0.04 \\
\hline $07: 14: 09: 12$ & $373 \mid$ & 75 & 25 & 25.3 & 52.8 & 0.23 & 1381. & 54.5 & 28 & -0.04 \\
\hline $07: 14: 10: 11$ & 374 & 75 & 25 & 25.3 & 52.8 & 0.24 & 1385 & 54.6 & 28 & -0.27 \\
\hline $07: 14: 11: 10$ & 375 & 75 & 25 & 253 & 52.8 & 0.24 & $1389^{\circ}$ & 54.6 & $28 !$ & -0.27 \\
\hline $07: 14: 12: 09$ & 376 & 75 & 25 & 25.3 & 52.8 & 0.23 & 1391 & 54.7 & 28 & -0.16 \\
\hline $07: 14: 13: 08$ & 377 & 75 & 25 & 25.5 & 52.8 & $0.23 !$ & 1387. & 54.7 & 28 ! & -0.16 \\
\hline $07: 14: 14: 07$ & 378 & 75 & 25 & $25.5^{\prime}$ & 52.8 & 0.21 & 138 & 54.6 & 28 & -0.07 \\
\hline $07: 1$ & 379 & 75 & 25 & 25.5 & 52.8 & 0.21 & 1383. & 54.6 & 28 & -0.07 \\
\hline $07: 14$ & 380 & 75 & $25 !$ & 25.5 & 52.8 & 0.23 & 1377. & 54.6 & 28 & -0.11 \\
\hline $07: 14$ & 381 & 75 & 25 & 25.3 & 52.8 & 0.24 & 1373 & 54.7 & 28 & -0.11 \\
\hline $07: 14: 18: 02$ & 382 & 76 & 25 & 25.3 & 52.8 & 0.23 & 1373 & 54.8 & 28 & -0.31 \\
\hline $07: 14: 19: 01$ & 383 & 76 & 25 & 25.3 & 52.8 & 0.23 & 1378 & 54.8 & 28 & -0.31 \\
\hline $07: 14: 20: 00$ & 384 & 76 & 25 & 25.3 & 52.8 & 0.23 & 1383 & 54.7 & 28 & -0.15 \\
\hline $07: 14: 20: 99$ & 385 & 76 & 25 & 25.3 & 52.8 & 0.23 & 1383. & 54.7 & 28 & -0.15 \\
\hline $07: 14: 21: 98$ & 386 & 76 & 25 & 25.3 & 52.8 & 0.23 & 1382 & 54.7 & 28 & -0.03 \\
\hline $07: 14: 22: 97$ & 387 & 76 & 25 & 25.5 & 52.8 & 0.21 & 1382. & 54.7 & 27 & -0.03 \\
\hline $07: 14: 23: 95$ & 388 & 76 & 25 & 25.5 & $52 . \overline{8}$ & 0.23 & 1378 & 54.7 & 27 & -0.19 \\
\hline $07: 14: 24: 94$ & 389 & 75 & 25 & 25.5 & 52.8 & 0.23 & 1371 & 54.7 & 27 & -0.19 \\
\hline $07: 14: 25: 93$ & 390 & 75 & 25 & 25.5 & 52.8 & 0.24 & 1366 & 54.7 & 27 & -0.2 \\
\hline 07:14:26:92 & 391 & 76 & 25 & 25.5 & 52.8 & 0.24 & 1368 & 54.5 & 27 & -0.2 \\
\hline $07: 14: 27: 91$ & 392 & 76 & 25 & 25.5 & 52.8 & 0.24 & 1372 & 54.5 & 27 & -0.11 \\
\hline $07: 14: 28: 90$ & 393 & 76 & 25 & 25.5 & 52.8 & 0.23 & 1377 & 54.6 & 28 & -0.11 \\
\hline $07: 14: 29: 89$ & 394 & 76 & 25 & 25.5 & 52.8 & 0.23 & 1379 & $54 . \overline{6}$ & 28 & -0.07 \\
\hline $07: 14: 30: 87$ & 395 & 76 & 25 & 25.5 & 52.8 & 0.21 & $1378 \mathrm{i}$ & 54.6 & 28 & -0.07 \\
\hline $07: 14: 31: 86$ & 396 & 76 & 25 & 25.5 & 52.8 & 0.23 & 1377 & $54.7 !$ & 28 & -0.3 \\
\hline $07: 14: 32: 85$ & 397 & 76 & 25 & 25.5 & 52.8 & 0.23 & 1373. & 54.8 & 28 & -0.22 \\
\hline $07: 14: 33: 84$ & 398 & 76 & 25 & 25.5 & 52.8 & 0.23 & 1366 & 54.8 & 28 & -0.22 \\
\hline $07: 14: 34: 83$ & 399 & $76^{1}$ & 25 & 25.5 & 52.8 & 0.23 & 1361 & 54.8 & 28 & 0.08 \\
\hline $07: 14: 35: 82$ & 400 & 76 & 25 & 25.5 & 52.8 & 0.24 & 1362 & 54.8 & 28 & 0.08 \\
\hline $6: 81$ & 401 & 76 & 25 & 25.5 & 52.8 & 0.23 & 1367 & 54.8 & $28^{\top}$ & $-0.1 \overline{5}$ \\
\hline $7: 80$ & 402 & 76 & 25 & 25.5 & 52.8 & 0.24 & $1371^{\circ}$ & 54.7 & 28 & -0.15 \\
\hline $07: 14: 38: 78$ & 403 & 76 & 25 & 25.5 & 52.8 & 0.23 & 1368 & 54.8 & 28 & -0.29 \\
\hline $39: 77$ & 404 & 76 & 25 & 25.5 & 52.7 & 0.21 & 1371 & 54.8 & 28 & -0.29 \\
\hline $07: 14: 40: 76$ & 405 & 76 & 25 & 25.5 & 52.7 & 0.21 & 1372 & 54.9 & 27 & -0.16 \\
\hline 07:14:41:75 & 406 & 76 & 25 & 25.5 . & 52.7 & 0.23 & 1368. & 54.8 & 27 & -0.16 \\
\hline $07: 14: 42: 74$ & 407 & 76 & 25 & 25.5 & 52.7 & 0.24 & $1361^{\prime}$ & 54.8 & 27 & -0.06 \\
\hline
\end{tabular}




\begin{tabular}{|c|c|c|c|c|c|c|c|c|c|c|}
\hline & $\begin{array}{l}\text { Elapsed } \\
\text { Time }\end{array}$ & & & ! & & & & & & \\
\hline TIME & (sec) & BIT TC1 & PURG TC2 & (PurgePSI I & PurgeCFM & DEPTH $(\mathrm{IN})$ & DwnFrc(lb) & RPM & & hes/Min \\
\hline $43: 73$ & 408 & $\overline{76}$ & -25 & 25.5 & 52.7 & $0 . \overline{23}$ & 1357 & 54.8 & 27 & -0.06 \\
\hline 72 & 409 & 76 & 25 & 25.5 & 2.7 & 0.23 & 1357 & 54.8 & 27 & -0.13 \\
\hline & $4 \overline{10}$ & $7 \overline{6}$ & 25 & 25.5 & 52.7 & 0.24 & 61 & 54.8 & 27 & -0.13 \\
\hline & 411 & 76 & 25 & 25.5 & 52.7 & 0.23 & & 54.7 & 28 & -0.32 \\
\hline & 412 & 76 & 25 & 25.5 & 52.7 & 0.23 & & 54.6 & 28 & -0.32 \\
\hline & 413 & 76 & 25 & 25.7 & 52.7 & 0.21 & 1304 & 54.6 & 27 & -0.47 \\
\hline & 414 & 75 & 25 & 25.7 & 52.7 & 0.21 & 267 & & 26 & -0.47 \\
\hline & 415 & 75 & 25 & 25.7 & 52.7 & 0.21 & $1239^{\dagger}$ & & 26 & 0.33 \\
\hline & 416 & 75 & 25 & 25.7 & 52. & & 12 & & 2 & 0.33 \\
\hline & 417 & 74 & 25 & 25.5 & & 0.2 & 11 & $54 . \overline{9}$ & 24 & -0.48 \\
\hline & 41 & 74 & 25 & 25.5 & 52.6 & 0.21 & & 54.9 & 24 & -0.48 \\
\hline & 419 & 74 & 25 & 25.5 & 52.7 & 0.21 & $1170^{+}$ & $5 \overline{4.9}$ & 24 & -0.31 \\
\hline & 420 & 74 & 25 & 25.5 & 52.6 & 0.2 & 1156 & 54.8 & 24 & -0.31 \\
\hline & 421 & 73 & 25 & 25.5 & 52.6 & 0.2 & 1153 & 54.8 & 24 & -0.2 \\
\hline & 422 & & 25 & 25.5 & 52.6 & 0.2 & & 54.9 & 24 & -0.2 \\
\hline & 423 & 73 & 25 & 25.7 & 52.6 & 0.19 & 1149 & & 23 & -0.15 \\
\hline & 424 & 73 & 25 & 25.7 & 52.6 & 0.1 & & & $2 \overline{3}$ & -0.15 \\
\hline & 425 & 72 & 25 & 25.7 & 52.6 & 0.19 & 1131 & 55.2 & 23 & -0.31 \\
\hline $07: 15: 01: 52$ & 426 & 72 & 25 & 25.7 & 52.6 & 0.2 & 1121 & 55.2 & 23 & -0.31 \\
\hline & & & $\cdots$ & & & & & & & \\
\hline
\end{tabular}




\begin{tabular}{|c|c|c|c|c|c|c|c|c|c|c|}
\hline$T I M E$ & $\begin{array}{l}\text { Elapsed } \\
\text { Time } \\
\text { (sec) }\end{array}$ & $\mathrm{BIT}$ TC1 & PURG_TC2 & PurgePSI & PurgeCFM & DEPTH(IN) & DwnFrc(lb) & RPM & $\begin{array}{l}\text { TORQUE } \\
\left(F T^{\star} L B\right)\end{array}$ & Inches/Min \\
\hline $12: 39: 30: 69$ & 0 & 25 & 26 & $\quad 21.4$ & $\quad 54.5$ & -1.17 & $\quad 0$ & 56.6 & 1 & $\quad-0.11$ \\
\hline $12: 39: 31: 62$ & 1 & 25 & 26 & 21.4 & 54.5 & -0.93 & 0 & 56.7 & 1 & $-0.1 \overline{1}$ \\
\hline $12: 39: 32: 61$ & 2 & 25 & 26 & 21.4 & 54.5 & -0.62 & 0 & 56.7 & 1 & 8.26 \\
\hline $12: 39: 33: 60$ & 3 & 25 & 25 & 21.4 & 54.5 & -0.29 & 2 & 56.5 & 1 & 8.26 \\
\hline $12: 39: 3 \overline{4: 59}$ & 4 & 25 & 26 & 21.4 & 54.5 & -0.02 & 49 & 56.4 & 6 & 12.41 \\
\hline $12: 39: 35: 58$ & 5 & 30 & 26 & 22 & 54.5 & 0.03 & 718 & 56.4 & 35 & 12.41 \\
\hline $12: 39: 36: 57$ & 6 & 32 & 25 & 22.2 & 54.2 & 0.03 & 801 & 56.3 & 37 & $4 . \overline{89}$ \\
\hline $12: 39: 37: 55$ & 7 & 35 & 26 & 22.4 & 54.1 & 0.05 & 824 & $5 \overline{6.1}$ & 36 & 4.89 \\
\hline $12: 39: 38: 54$ & 8 & 36 & 26 & 22.4 & 54 & 0.06 & 853 & 56.1 & 34 & 0.65 \\
\hline $12: 39: 39: 53$ & $\overline{9}$ & 37 & 26 & 22.4 & 54 & 0.09 & $863^{\circ}$ & 55.8 & 33 & 0.65 \\
\hline $12: 39: 40: 52$ & 10 & 39 & 26 & 22.4 & 53.9 & 0.09 & 880 & 55.8 & 31 & 0.11 \\
\hline $12: 39: 41: 5 \overrightarrow{1}$ & 11 & 41 & 26 & 22.6 & 53.9 & 0.09 & 892 & 55.8 & 30 & 0.11 \\
\hline $12: 39: 42: 50$ & 12 & $\overline{41}$ & 26 & 22.6 & $5 \overline{3.8}$ & 0.09 & 900 & $5 \overline{5.8}$ & 29 & 0.18 \\
\hline $12: 39: 43: 49$ & 13 & 42 & 26 & $22 . \overline{6}$ & 53.8 & 0.09 & 904 & 55.8 & 29 & 0.18 \\
\hline $12: 39: 44: 47$ & 14 & 43 & 26 & 22.6 & 53.7 & 0.1 & 905 & 55.8 & 28 & 0.08 \\
\hline $12: 39: 4 \overline{5}: 46$ & 15 & 44 & 26 & 22.6 & 53.7 & 0.1 & 905 & 55.7 & 28 & 0.08 \\
\hline $12: 39: \overline{46}: 45$ & 16 & 45 & 26 & 22.6 & 53.7 & 0.08 & 912 & $5 \overline{5} . \overline{6}$ & 27 & -0.04 \\
\hline $12: 39: 47: 44$ & 17 & 45 & 26 & 22.6 & 53.7 & 0.08 & 912 & 55.6 & 27 & -0.04 \\
\hline $12: 39: 48: 43$ & 18 & 46 & 25 & 22.8 & 53.6 & 0.09 & $910^{\dagger}$ & 55.8 & 27 & -0.21 \\
\hline $12: 39: 49: 42$ & 19 & $4 \overline{7}$ & 26 & 22.8 & 53.6 & 0.09 & 915 & 55.8 & 28 & 0.01 \\
\hline $12: 39: 50: 41$ & 20 & 48 & 26 & 22.8 & 53.6 & 0.1 & 916 & 55.8 & 28 & 0.01 \\
\hline $12: 39: 51: 39$ & 21 & 49 & 25 & 22.8 & 53.6 & 0.1 & 920 & 55.7 & 27 & 0.03 \\
\hline $12: 39: 52: 38$ & 22 & 49 & 26 & 22.8 & 53.6 & 0.09 & 926 & $55 . \overline{6}$ & 27 & 0.03 \\
\hline $12: 39: 53: 37$ & 23 & 50 & 26 & 22.8 & 53.6 & 0.1 & 927 & 55.6 & $2 \overline{7}$ & -0.06 \\
\hline $12: 39: 54: 36$ & $2 \overline{4}$ & 50 & 26 & 22.8 & 53.6 & 0.1 & 928 & 55.7 & 27 & -0.06 \\
\hline $12: 39: 55: 35$ & 25 & 51 & 26 & 22.8 & 53.5 & 0.1 & 922 & 55.6 & 26 & -0.22 \\
\hline $12: 39: 56: 34$ & 26 & 51 & 26 & 22.8 & $5 \overline{3.5}$ & 0.1 & 921 & 55.6 & 26 & -0.22 \\
\hline $12: 39: 57: 33$ & 27 & 52 & 26 & 22.8 & 53.5 & 0.1 & 919 & $5 \overline{5.5}$ & 26 & $-\overline{0.1}$ \\
\hline $12: 39: 58: 32$ & 28 & 52 & 26 & 22.8 & 53.5 & 0.1 & 914 & 55.5 & 26 & -0.1 \\
\hline $12: 39: 59: 30$ & 29 & $5 \overline{3}$ & 26 & 22.8 & 53.5 & 0.1 & 909 & $55 . \overline{6}$ & 26 & 0.01 \\
\hline $12: 40: 00: 29^{\dagger}$ & 30 & 54 & 25 & 22.8 & 53.4 & 0.1 & 905 & 55.7 & 26 & 0.01 \\
\hline $12: 40: 01: 28$ & 31 & 54 & 26 & 22.9 & 53.4 & 0.1 & 907 & 55.7 & 25 & -0.05 \\
\hline $12: 40: 02: 27$ & 32 & 55 & 26 & 22.9 & 53.4 & 0.1 & 910 & 55.6 & 25 & -0.05 \\
\hline $12: 40: 03: 26$ & 33 & 55 & 26 & 22.9 & 53.4 & 0.12 & 904 & 55.7 & 25 & -0.27 \\
\hline $12: 40: 04: 25$ & 34 & 55 & 26 & 22.9 & 53.4 & 0.1 & 909 & 55.7 & 25 & $-0.2 \overline{7}$ \\
\hline $12: 40: 05: 24$ & 35 & 55 & 26 & 22.9 & $53 \overline{4}$ & 0.1 & 912 & 55.6 & 25 & -0.08 \\
\hline $12: 40: 06: 22$ & 36 & 55 & 26 & 22.8 & 53.4 & 0.1 & 911 & 55.6 & 25 & -0.08 \\
\hline $12: 40: 07: 21$ & 37 & 55 & 26 & 22.8 & 53.4 & 0.09 & 910 & 55.6 & 25 & -0.03 \\
\hline $12: 40: 08: 20$ & 38 & 55 & 26 & 22.8 & 53.4 & 0.09 & 906 & 55.6 & 24 & -0.03 \\
\hline $12: 40: 09: 19$ & 39 & 56 & 26 & 22.8 & 53.4 & 0.1 & 902 & 55.6 & 24 & -0.08 \\
\hline $12: 40: 10: 18$ & 40 & 56 & 26 & 22.8 & 53.4 & 0.1 & $900^{\circ}$ & 55.6 & 24 & -0.08 \\
\hline $12: 40: 11: 17$ & 41 & 57 & 26 & 22.8 & 53.4 & 0.09 & 901 & 55.5 & 25 & -0.25 \\
\hline $12: 40: 12: 16$ & 42 & $5 \overline{7}$ & 26 & 22.9 & 53.4 & 0.1 & 900 & $5 \overline{5} .6$ & 25 & -0.25 \\
\hline $12: 40: 13: 15$ & 43 & 57 & 26 & 22.9 & 53.4 & $0 . \overline{09}$ & 902 & 55.6 & 25 & -0.09 \\
\hline $12: 40: 14: 13$ & 44 & 57 & 26 & $22 . \overline{9}$ & 53.4 & 0.1 & 905 & 55.6 & 24 & -0.09 \\
\hline $12: 40: 15: 12$ & 45 & 57 & 26 & 22.9 & 53.4 & 0.1 & 909 & 55.5 & 24 & -0.05 \\
\hline $12: 40: 16: 11$ & 46 & 57 & 26 & 22.9 & 53.4 & 0.1 & 910 & 55.5 & 24 & -0.05 \\
\hline $12: 40: 17: 10^{\dagger}$ & 47 & 57 & 26 & 22.9 & 53.3 & 0.09 & 910 & 55.6 & 24 & -0.02 \\
\hline $12: 40: 18: 09$ & 48 & 58 & 26 & 22.9 & 53.3 & 0.1 & 907 & 55.7 & 24 & -0.02 \\
\hline $12: 40: 19: 08$ & 49 & 58 & 26 & 22.9 & 53.3 & 0.12 & 903 & 55.7 & 24 & -0.24 \\
\hline
\end{tabular}




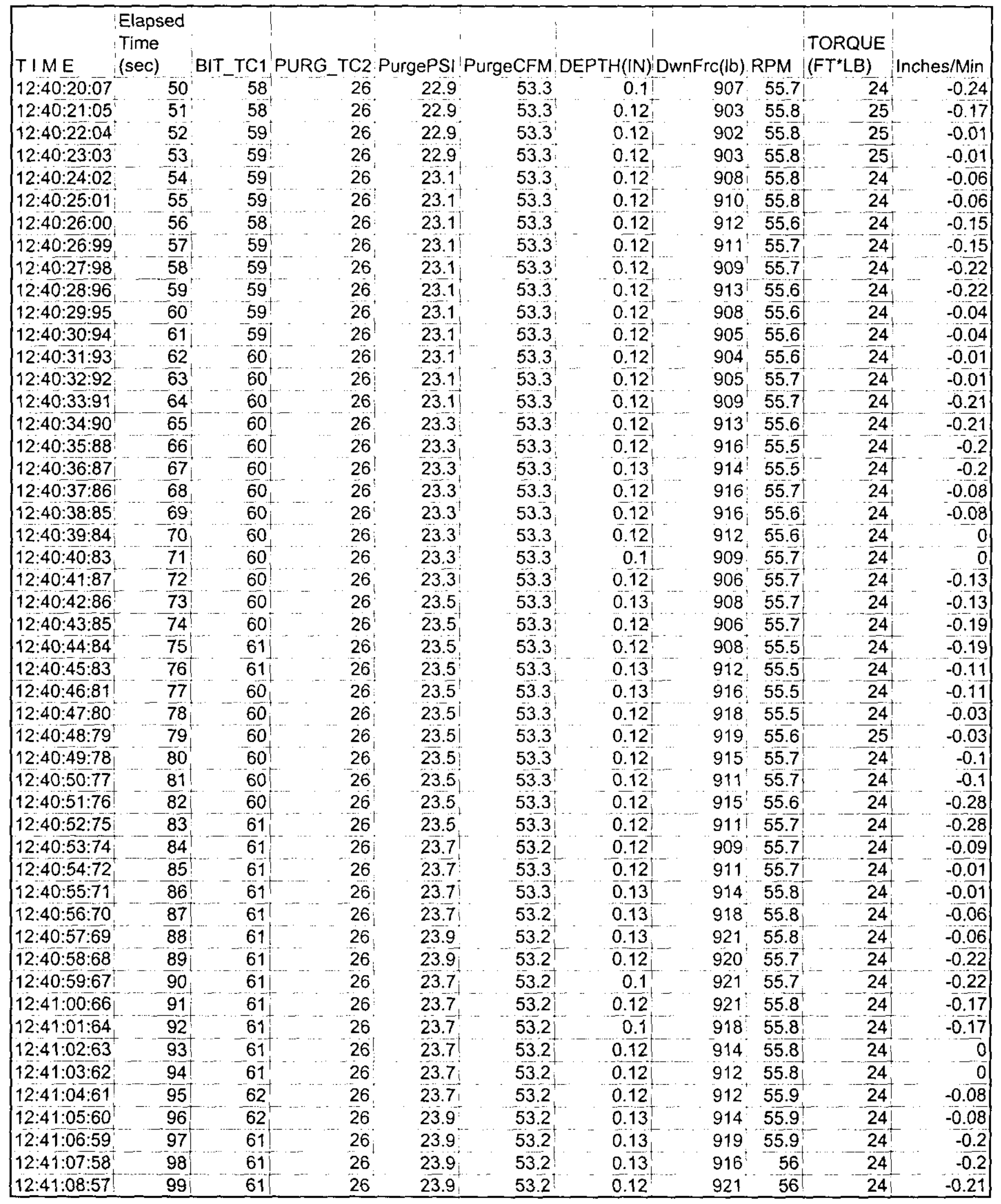




\begin{tabular}{|c|c|c|c|c|c|c|c|c|c|c|}
\hline TIME & $\begin{array}{l}\text { Elapsed } \\
\text { Time } \\
\text { (sec) }\end{array}$ & BIT_TC1 & & & PurgeCFM & & DwnFrc(lb) & RPM & $\begin{array}{l}\text { TORQUE } \\
\left(\mathrm{FT}^{*} \mathrm{LB}\right)\end{array}$ & Inches/Min \\
\hline $12: 41: 09: 55$ & $5 \quad 100$ & $\quad 61$ & 26 & $\quad 23.9^{\circ}$ & $\quad 53.2$ & $\quad 0.12$ & 923 & 56.1 & $1 \quad 24$ & $4 \quad-0.21$ \\
\hline $12: 41: 10: 54$ & 101 & 61 & 26 & 23.9 & 53.2 & 0.12 & 924 & 56.1 & 24 & 0.04 \\
\hline $12: 41: 11: 53$ & 102 & 61 & 26 & 23.7 & 53.2 & 0.12 & 923 & 56.1 & 24 & 0.04 \\
\hline $12: 41: 12: 52$ & 103 & 61 & 26 & 23.7 & 53.2 & 0.12 & 919 & $55.9^{\prime}$ & 24 & -0.08 \\
\hline $12: 41: 13.51$ & 104 & $61 i$ & 26 & 23.7 & 53.2 & 0.12 & 916 & 55.9 & 24 & -0.08 \\
\hline $12: 41: 14: 50$ & 105 & 61 & 26 & 23.7 & 53.2 & 0.12 & 914 & 55.9 & 24 & -0.16 \\
\hline $12: 41: 15: 49$ & 106 & $61 i$ & 26 & 23.5 & 53.2 & 0.12 & $915^{\circ}$ & 56 & 24 & -0.16 \\
\hline $12: 41: 16: 47$ & 107 & 62 & 26 & 23.5 & $53.2^{+}$ & 0.12 & 913 & 56 & 24 & $-0 . \overline{24}$ \\
\hline $12: 41: 17: 46$ & 108 & 62 & 26 & 23.7 & 53.2 & 0.12 & 914 & $56 !$ & 24 & -0.24 \\
\hline $12: 41: 18: 45$ & 109 & 62 & 26 & 23.7 & 53.2 & 0.13 & 917 & 56 & 24 & -0.07 \\
\hline $12: 41: 19: 44$ & 110 & 61 & 26 & 23.7 & 53.2 & 0.13 & 921 & 56 & 24 & -0.07 \\
\hline $12: 41: 20: 43$ & 111 & 61 & 26 & 23.7 & 53.2 & 0.12 & 924 & 56 & 24 & -0.02 \\
\hline $12: 41: 21: 42$ & 112 & 61 & 26 & 23.7 & $53.2^{\dagger}$ & 0.13 & 922 & 55.9 & 24 & $-0 . \overline{02}$ \\
\hline $12: 41: 22: 41$ & 113 & 61 & 26 & 23.7 & 53.2 & 0.12 & 919 & 55.9 & 24 & -0.09 \\
\hline $12: 41: 23: 40$ & 114 & 61 & 26 & $23.5^{i}$ & 53.2 & 0.12 & 914 & 55.9 & 24 & -0.09 \\
\hline $12: 41: 24: 38$ & 115 & 61 & 26 & 23.5 & 53.2 & 0.12 & 917 & 55.9 & 24 & -0.27 \\
\hline $12: 41: 25: 37$ & 116 & 62 & 26 & 23.5 & 53.2 & 0.12 & 913 & 55.7 & 23 & -0.27 \\
\hline $12: 41: 26: 36$ & 117 & $6 \overline{2}$ & 26 & 23.5 & $53 . \overline{2}$ & 0.12 & 909 & 55.7 & 23 & -0.1 \\
\hline $12: 41: 27: 40$ & 118 & 62 & 26 & 23.5 & 53.2 & 0.13 & 909 & 55.8 & 23 & -0.04 \\
\hline $12: 41: 28: 39$ & 119 & 62 & 26 & 23.5 & 53.2 & 0.12 & 912 & 55.9 & 23 & -0.04 \\
\hline $1: 29: 38$ & 120 & 62 & 26 & $23.7 !$ & 53.2 & 0.13 & $915^{4}$ & 55.9 & 23 & -0.05 \\
\hline $30: 37$ & 121 & 62 & 26 & 23.7 & 53.2 & 0.12 & 911 & $55 . \overline{8}$ & 23 & -0.05 \\
\hline $12: 41: 31: 36$ & 122 & 61 & 25 & 23.7 & 53.2 & 0.13 & 915 & 55.7 & 23 & -0.26 \\
\hline $12: 41: 32: 35$ & 123 & 61 & 26 & 23.7 & 53.2 & 0.12 & 916 & 55.7 & 23 & -0.26 \\
\hline $12: 4$ & 124 & $61 !$ & 26 & 23.7 & 53.1 & 0.12 & $916^{\dagger}$ & 55.8 & 23 & -0.17 \\
\hline $12: 41: 3$ & 125 & 61 & $26:$ & 23.7 & 53.1 & 0.13 & 914 & 55.8 & 23 & -0.17 \\
\hline $12: 41: 35: 31$ & 126 & 61 & 26 & 23.5 & 53.1 & 0.12 & $910^{\dagger}$ & 55.9 & 23 & 0.04 \\
\hline $12: 41: 36: 30$ & 127 & 61 & 26 & 23.5 & 53.1 & 0.13 & $905^{\circ}$ & 56 & 23 & 0.04 \\
\hline $12: 41: 37: 29$ & 128 & 62 & 26 & 23.5 & 53.1 & 0.12 & $904^{\prime}$ & 56 & $\overline{23}$ & -0.11 \\
\hline $12: 41: 38: 28$ & 129 & 62 & 26 & 23.5 & 53.1 & 0.12 & 905 & $55 . \overline{9}$ & 23 & -0.11 \\
\hline $12: 41: 39: 27$ & 130 & 62 & 26 & 23.5 & 53.1 & 0.13 & 903 & 55.9 & 23 & -0.22 \\
\hline $12: 41: 40: 26$ & 131 & 62 & 26 & 23.5 & 53.1 & 0.13 & 905 & 55.9 & 23 & -0.22 \\
\hline $1: 4 \overline{1: 25}$ & 132 & 62 & 26 & 23.7 & 53 & 0.13 & 909 & 55.8 & 23 & -0.22 \\
\hline $12: 41: 42: 23$ & 133 & 61 & 26 & $23.7^{+}$ & 53 & 0.13 & $912^{-1}$ & 55.7 & 23 & -0.22 \\
\hline $12: 41: 43: 22$ & 134 & 61 & 26 & 23.5 & $53^{\dagger}$ & 0.12 & 912 & 55.7 & 23 & -0.05 \\
\hline $12: 41: 44: 21$ & 135 & 61 & 26 & 23.7 & 53 & 0.12 & 911 & 55.7 & 23 & -0.05 \\
\hline $12: 41: 45: 20$ & 136 & 61 & 26 & 23.5 & 53 & 0.12 & 907 & 55.7 & 23 & -0.02 \\
\hline $12: 41: 46: 19$ & 137 & 61 & 26 & 23.5 & 53 & 0.1 & 902 & 55.7 & 23 & -0.02 \\
\hline $12: 41: 47: 18$ & $13 \overline{8}$ & 61 & 26 & 23.7 & 53 & 0.13 & 905 & 55.7 & 23 & -0.16 \\
\hline $12: 41: 48: 22$ & 139 & 61 & 26 & 23.5 & $5 \overline{3}$ & 0.12 & 902 & 55.8 & 23 & $-0 . \overline{16}$ \\
\hline $12: 41: 49: 21$ & 140 & 61 & 26 & 23.5 & 53 & 0.13 & $900^{-1}$ & 55.8 & 22 & -0.22 \\
\hline $12: 41: 50: 20$ & 141 & 62 & 26 & 23.5 & 53 & 0.12 & 902 & 55.8 & 23 & -0.22 \\
\hline $12: 41: 51: 19$ & 142 & 62 & 26 & 23.5 & 53 & 0.13 & 905 & 55.9 & 23 & -0.1 \\
\hline $12: 41: 52: 18$ & $\overline{143}$ & 62 & 26 & 23.7 & 53 & 0.12 & 908 & 55.9 & 23 & -0.1 \\
\hline $12: 41: 53: 16$ & 144 & 61 & 25 & $23 . \overline{5}$ & 53 & 0.12 & 910 & $55.9^{\circ}$ & 23 & -0.01 \\
\hline $12: 41: 54: 15$ & 145 & 61 & 26 & 23.5 & 53 & 0.12 & $908 \mathrm{i}$ & 56 & 23 & -0.01 \\
\hline $12: 41: 55: 14$ & 146 & 61 & 26 & 23.5 & 53 & 0.12 & 910 & 56 & 23 & -0.1 \\
\hline $12: 41: 56: 13$ & 147 & 61 & 26 & 23.5 & 52.9 & 0.12 & 909 & 56 & 23 & -0.28 \\
\hline $12: 41: 57: 12$ & 148 & $61^{\dagger}$ & 26 & $23 . \overline{5}$ & $52 . \overline{9}$ & 0.12 & 905 & 56 & 23 & $-0.2 \overline{8}$ \\
\hline $12: 41: 58: 11$ & 149 & 61 & 26 & 23.5 & $52.9^{\circ}$ & 0.13 & $901^{\circ}$ & 56 & 23 & -0.04 \\
\hline
\end{tabular}




\begin{tabular}{|c|c|c|c|c|c|c|c|c|c|c|}
\hline TIME & $\begin{array}{l}\text { Elapsed } \\
\text { Time } \\
\text { (sec) }\end{array}$ & BIT_TC1 & PURG_TC2 & PurgePSI & PurgeCFM & DEPTH(IN) & DwnFrc(lb) & $R P M$ & $\begin{array}{l}\text { TORQUE } \\
\text { (FT*LB) }\end{array}$ & Inches/Min \\
\hline $12: 41: 59: 10$ & 150 & 61 & 26 & 23.7 & $\quad 52.9$ & 0.12 & 897 & 56 & $\quad 22$ & $\quad 0.04$ \\
\hline $12: 42: 00: 09$ & 151 & 61 & 26 & 23.7 & $52.9^{\top}$ & 0.13 & 897 & 56 & 22 & -0.1 \\
\hline $12: 42: 01: 07$ & 152 & 61 & 26 & 23.7 & $52.9^{\prime}$ & $0.1 \overline{3}$ & 900 & 56 & 22 & -0.1 \\
\hline $12: 42: 02: 06$ & 153 & 62 & 26 & 23.7 & 52.9 & 0.13 & $897 !$ & 56.1 & 22 & -0.06 \\
\hline $12: 42: 03: 05$ & 154 & 61 & 26 & 23.7 & 52.9 & 0.13 & $900 !$ & 56 & 22 & -0.06 \\
\hline $12: 42: 04: 04$ & 155 & 62 & 26 & 23.7 & 52.9 & $0.13^{\prime}$ & 904 & 56 & $2 \overline{2}$ & -0.23 \\
\hline $12: 42: 05: 03$ & 156 & 61 & $26 !$ & 23.5 & 52.9 & 0.13 & 905 & 56 & 23 & -0.23 \\
\hline $12: 42: 06: 02$ & 157 & $61 !$ & 26 & 23.5 & 52.9 & 0.13 & 907 & 56.1 & 23 & -0.19 \\
\hline $12: 42: \overline{07: 01}$ & 158 & 61 & 26 & 23.5 & 52.9 & 0.12 & 905 & 56.1 & 23 & -0.19 \\
\hline $12: 42: 07: 99$ & 159 & 61 & 26 & 23.5 & 52.9 & 0.12 & 902 & 56.1 & 23 & -0.06 \\
\hline $12: 42: 08: 98$ & 160 & 61 & 26 & 23.5 & 52.9 & 0.13 & 899 & 56.1 & 23 & -0.06 \\
\hline $12: 42: 09: 97$ & 161 & 61 & 26 & 23.5 & 52.9 & $0 . \overline{13}$ & 901 & 56.1 & 23 & -0.03 \\
\hline $12: 42 ; 10: 96$ & 162 & 61 & 26 & $23 . \overline{7}$ & 52.9 & 0.12 & 897 & 56 & 22 & -0.03 \\
\hline $12: \overline{42}: \overline{11}: 95$ & 163 & 61 & 26 & 23.7 & $52 . \overline{9}$ & 0.13 & 895 & 56 & 22 & -0.17 \\
\hline $12: 42: 12: 94$ & 164 & 62 & $26 !$ & 23.7 & 52.9 & 0.13 & 897 & 56 & 22 & -0.17 \\
\hline $12: 42: 13: 93$ & 165 & 61 & 26 & 23.7 & 52.9 & 0.13 & 900 & 56 & 22 & -0.27 \\
\hline $12: 42: 14: 92$ & 166 & 61 & 26 & 23.7 & $52 . \overline{9}$ & 0.13 & 904 & 56 & 22 & -0.27 \\
\hline $12: 42: 15: 90$ & 167 & 61 & 26 & $2 \overline{3.7}$ & $52 . \overline{9}$ & 0.13 & 905 & 56 & 22 & -0.08 \\
\hline $12: 42: 16: 89$ & 168 & 61 & 26 & 23.5 & 52.9 & 0.13 & 906 & 56 & 22 & -0.08 \\
\hline $12: 42: 17: 88$ & 169 & 61 & 26 & 23.5 & 52.9 & 0.12 & 904 & 56 & 22 & -0.01 \\
\hline $12: 42: 18: 87$ & 170 & 61 & 26 & 23.5 & 52.8 & 0.12 & 905 & 56 & 22 & -0.01 \\
\hline $12: 42: 19: 86$ & 171 & 611 & 26 & 23.5 & 52.8 & 0.13 ? & 904 & 56 & 22 & -0.07 \\
\hline $12: 42: 20: 85$ & 172 & 61 & 26 & 23.5 & 52.8 & 0.12 & 899 & 56 & 22 & -0.07 \\
\hline $12: 42: 21: 84$ & $1 \overline{73}$ & 61 & 26 & 23.5 & 52.8 & 0.12 & 895 & 56 & 22 & -0.24 \\
\hline $12: 42: 22: 82$ & 174 & 61 & 26 & 23.7 & 52.8 & 0.12 & 895 & $55 . \overline{9}$ & 22 & -0.24 \\
\hline $12: 42: 23: 81$ & 175 & 61 & 26 & 23.7 & 52.8 & 0.13 & 897 & 55.9 & 22 & -0.19 \\
\hline $12: 42: 24: 80$ & 176 & 61 & 26 & 23.7 & 52.8 & 0.13 & 902 & 55.9 & 22 & -0.19 \\
\hline $12: 42: 25: 79$ & $177^{+}$ & 61 & 26 & 23.7 & 52.8 & 0.13 & 904 & 55.8 & 22 & 0.01 \\
\hline $12: 42: 26: 78$ & 178 & 61 & 26 & 23.9 & 52.8 & 0.13 & 902 & 55.8 & 22 & 0.01 \\
\hline $12: 42: 27: 77$ & 179 & 60 & 26 & 23.9 & 52.8 & 0.13 & 905 & 55.7 & 22 & -0.07 \\
\hline $12: 42: 28: 76$ & 180 & 60 & $26 !$ & 23.5 & 52.8 & 0.12 & 907 & 55.7 & 22 & -0.07 \\
\hline $12: 42: 29: 75$ & 181 & 60 & 26 & 23.5 & 52.8 & $0.1 !$ & $906^{\prime}$ & 55.7 & 22 & -0.24 \\
\hline $12: 42: 30: 73$ & 182 & 61 & 26 & 23.5 & 52.8 & 0.13 & 904 & 55.7 & 22 & -0.16 \\
\hline $12: 42: 31: 72$ & 183 & 61 & 26 & 23.7 & 52.8 & 13 & 900 & 55.7 & 22 & -0.16 \\
\hline $2: 71$ & 18 & 61 & 26 & 23.5 & 52.8 & 0.13 & 900 & 55.7 & 22 & 0.01 \\
\hline 3.75 & 185 & 61 & 26 & 23.7 & 52.8 & 0.13 & 902 & 55.7 & 22 & 0.01 \\
\hline $4: 74$ & 186 & 61 & 26 & 23.7 & 52.8 & 0.13 & 900 & 55.7 & 22 & -0.03 \\
\hline $12: 42: 35: 73$ & 187 & 61 & 26 & 23.9 & 52.8 & 0.13 & $9 \overline{03}$ & 55.7 & 22 & -0.03 \\
\hline $12: 42: 36: 72$ & 188 & $6 \overline{1}$ & 26 & 23.9 & 52.8 & 0.13 & 907 & 55.6 & 22 & -0.2 \\
\hline $12: 42: 37: 71$ & 189 & 61 & 26 & 23.9 & 52.8 & $0 . \overline{13}$ & 910 & 55.6 & 22 & -0.2 \\
\hline $12: 42: 38: 70$ & 190 & 61 & 26 & 23.9 & 52.8 & $0.1 \overline{3}$ & 912 & 55.6 & 22 & -0.21 \\
\hline $12: 42: 39: 6 \overline{9}$ & 191 & 61 & 26 & 23.9 & 52.8 & 0.12 & 911 & 55.7 & 22 & -0.21 \\
\hline $12: 42: 40: 68$ & 192 & 61 & 26 & 23.7 & 52.8 & 0.12 & 907 & 55.7 & 22 & -0.04 \\
\hline $12: 42: 41: 66$ & 193 & 61 & $26 !$ & 23.7 & 52.8 & 0.12 & 911 & 55.7 & 22 & -0.04 \\
\hline $12: 42: 42: 65$ & 194 & 61 & 26 & 23.7 & 52.8 & 0.12 & 907 & 55.7 & 22 & -0.04 \\
\hline $12: 42: 43: 64$ & 195 & 61 & 26 & 23.7 & 52.8 & 0.13 & 904 & 55.7 & 22 & -0.04 \\
\hline $12: 42: 44: 63$ & 196 & 61 & 26 & 23.7 & 52.8 & 0.13 & 904 & 55.7 & 22 & -0.21 \\
\hline $12: 42: 45: 62$ & 197 & 61 & 26 & 23.7 & 52.8 & 0.13 & 905 & 55.6 & 22 & -0.21 \\
\hline $12: 42: 46: 61$ & 198 & 61 & 26 & 23.9 & 52.8 & 0.13 & 910 & 55.6 & 22 & -0.21 \\
\hline $12: 42: 47: 60$ & 199 & 61 & 26 & 23.9 & 52.8 & 0.12 & 913 & 55.6 & 22 & -0.21 \\
\hline
\end{tabular}




\begin{tabular}{|c|c|c|c|c|c|c|c|c|c|c|}
\hline$T / M E$ & $\begin{array}{l}\text { Elapsed } \\
\text { Time } \\
\text { (sec) }\end{array}$ & BIT_TC1 & PURG_TC2 & PurgePSI & PurgeCFM & DEPTH(IN) & DwnFrc(lb) & RPM & $\begin{array}{l}\text { TORQUE } \\
\text { (FT^LB) }\end{array}$ & Inches/Min \\
\hline $12: 42: 48: 58$ & 200 & -61 & -26 & 23.7 & $\quad 52.8$ & 0.13 & $\quad 914$ & 55.5 & $\quad 22$ & 2: $\quad 0.04$ \\
\hline $12: 42: 49: 57$ & 201 & 60 & 26 & 23.7 & 52.8 & 0.12 & 914 & 55.4 & 22 & 0.04 \\
\hline $12: 42: 50: 56$ & 202 & 60 & 26 & 23.7 & 52.8 & 0.12 & 914 & 55.4 & 22 & $-0 . \overline{12}$ \\
\hline $12: 42: 51: 55$ & 203 & 61 & $26^{\dagger}$ & 23.7 & $52 . \overline{8}$ & 0.13 & 912 & 55.4 & 22 & -0.12 \\
\hline $12: 42: 52: 54$ & 204 & 61 & 26 & 23.7 & 52.8 & 0.12 & 909 & 55.4 & 22 & -0.2 \\
\hline $12: 42: 53: 53$ & 205 & 61 & 26 & 23.7 & $52 . \overline{8}$ & 0.12 & 905 & 55.4 & 22 & -0.2 \\
\hline $12: 42: 54: 57$ & 206 & 61 & 26 & 23.7 & 52.8 & 0.13 & 905 & 55.4 & 22 & -0.2 \\
\hline $12: 42: 55: 56$ & 207 & 61 & 26 & 23.7 & 52.8 & 0.13 & 905 & 55.5 & 22 & -0.2 \\
\hline $12: 42: 56: 55$ & 208 & 61 & 26 & 23.7 & 52.8 & 0.13 & 907 & 55.5 & 22 & $-0.0 \overline{7}$ \\
\hline $12: 42: 57: 54$ & 209 & 61 & 26 & 23.9 & 52.8 & 0.13 & 910 & 55.7 & 22 & -0.07 \\
\hline $12: 42: 58: 53$ & 210 & 61 & 26 & 23.9 & 52.8 & 0.13 & 915 & 55.8 & 22 & 0 \\
\hline $12: 42: 59: 51$ & 211 & 61 & 26 & 23.7 & 52.8 & 0.13 & 917 & 55.8 & 22 & -0.13 \\
\hline $12: 43: 00: 50$ & 212 & 61 & 26 & 23.7 & 52.8 & 0.13 & 918 & 55.8 & 22 & -0.13 \\
\hline $12: 43: 01: 49$ & 213 & 61 & 26 & 23.7 & 52.7 & $0 . \overline{13}$ & 916 & 55.7 & 22 & -0.25 \\
\hline $12: 43: \overline{02}: \overline{4} 8$ & 214 & 61 & 26 & 23.7 & 52.7 & 0.14 & 912 & 55.7 & 22 & -0.25 \\
\hline $12: 43: 03: 47$ & 215 & 61 & 26 & 23.7 & 52.7 & 0.13 & 908 & 55.6 & 22 & $-0 . \overline{07}$ \\
\hline $12: 43: 04: 46$ & 216 & 61 & 26 & 23.7 & 52.7 & $0.1 \overline{3}$ & 912 & 55.6 & 22 & -0.07 \\
\hline $12: 43: 05: 45$ & 217 & 61 & $26 !$ & 23.9 & 52.7 & 0.13 & 909 & 55.6 & 22 & -0.02 \\
\hline $12: 43: 06: 44$ & 218 & 61 & $26 !$ & 23.9 & 52.7 & 0.14 & 909 & 55.8 & 22 & -0.02 \\
\hline $12: 43: 07: 42$ & 219 & 61 & 26 & 23.9 & 52.7 & 0.13 & 912 & 55.7 & 22 & -0.1 \\
\hline $12: 43: 08: 41$ & 220 & 61 & 26 & 23.9 & 52.7 & $0 . \overline{14}$ & 915 & 55.7 & 22 & -0.1 \\
\hline $12: 43: 09: 40$ & $2 \overline{1}$ & 61 & 26 & $23.9^{\dagger}$ & 52.7 & 0.14 & 918 & 55.7 & 22 & -0.28 \\
\hline $12: 43: 10: 39$ & 222 & 61 & 26 & 23.9 & 52.7 & 0.13 & 919 & 55.8 & $2 \overline{2}$ & -0.28 \\
\hline $12: 43: 11: 38$ & 223 & 61 & $26^{\dagger}$ & 23.7 & 52.7 & 0.13 & 918 & 55.8 & 22 & -0.09 \\
\hline $12: 43: 12: 37$ & 224 & 61 & 26 & $23.7^{\dagger}$ & 52.7 & 0.13 & 920 & 55.8 & 22 & -0.09 \\
\hline $12: 43: 13: 36$ & 225 & 61 & 26 & 23.7 & 52.7 & 0.13 & 918 & 55.6 & 22 & -0.02 \\
\hline $12: 43: 14: 34$ & 226 & 61 & $26 !$ & 23.7 & 52.7 & 0.13 & 913 & $55.6^{i}$ & 22 & -0.02 \\
\hline $12: 43: 15: 33$ & 227 & 61 & 26 & 23.7 & 52.7 & 0.14 & 910 & 55.6 & 22 & $-0.0 \overline{9}$ \\
\hline $12: 43: 16: 32$ & 228 & 61 & $26 i$ & 23.7 & $52 . \overline{7}$ & 0.13 & 910 & 55.6 & 22 & -0.09 \\
\hline $12: 43: 17: 31$ & $22 \overline{9}$ & 61 & 26 & 23.9 & 52.7 & 0.14 & 912 & 55.6 & 22 & -0.28 \\
\hline $12: 43: 18: 30$ & 230 & 61 & 26 & 23.9 & 52.7 & 0.13 & 916 & $55 . \overline{6}$ & 22 & -0.28 \\
\hline $12: 43: 19: 29$ & 231 & 61 & 26 & 23.9 & 52.7 & 0.13 & 919 & 55.6 & 22 & -0.13 \\
\hline $12: 43: 20: 28$ & 232 & $\overline{61}$ & 26 & 23.9 & 52.7 & 0.13 & 916 & 55.7 & 22 & -0.13 \\
\hline $12: 43: 21: 27$ & 233 & 61 & 26 & 23.9 & 52.7 & $0.1 \overline{3}$ & 920 & 55.6 & 22 & -0.01 \\
\hline $12: 43: 22: 25$ & 234 & 60 & 26 & 23.9 & 52.7 & 0.13 & 921 & 55.6 & 22 & -0.01 \\
\hline $12: 43: 23: 24$ & 235 & 60 & 26 & 23.7 & 52.7 & 0.13 & 919 & 55.7 & 22 & -0.1 \\
\hline $12: 43: 24: 23$ & 236 & 60 & 26 & 23.7 & 52.7 & 0.13 & 915 & 55.7 & 22 & -0.1 \\
\hline $12: 43: 25: 22$ & 237 & $6 \overline{1}$ & 26 & 23.7 & $52 . \overline{7}$ & 0.14 & 911 & 55.7 & 22 & -0.27 \\
\hline $12: 43: 26: 21$ & 238 & 61 & 26 & 23.7 & 52.6 & 0.13 & 910 & 55.6 & 22 & -0.27 \\
\hline $12: 43: 27: 20$ & 239 & 61 & $26 !$ & $23.7 !$ & 52.7 & 0.14 & 912 & 55.5 & 22 & -0.1 \\
\hline $12: 43: 28: 19$ & 240 & 61 & 26 & 23.9 & 52.6 & 0.13 & 917 & 55.5 & 22 & -0.1 \\
\hline $12: 43: 29: 17$ & 241 & 61 & 26 & 23.9 & 52.6 & $0.1 \overline{3}$ & 914 & 55.5 & 22 & -0.02 \\
\hline $12: 43: 30: 16$ & 242 & 61 & 26 & 23.9 & 52.6 & 0.13 & 917 & 55.6 & 22 & -0.02 \\
\hline $12: 43: 31: 15$ & 243 & 60 & 26 & 23.9 & 52.6 & 0.13 & 921 & 55.6 & 22 & -0.07 \\
\hline $12: 43: 32: 14$ & 244 & 60 & 26 & 23.9 & 52.6 & $0.1 \overline{3}$ & 921 & 55.6 & 22 & -0.26 \\
\hline $12: 43: 33: 13$ & 245 & 60 & 26 & 23.9 & 52.6 & 0.12 & $920^{\circ}$ & 55.6 & 22 & -0.26 \\
\hline $12: 43: 34: 12$ & 246 & 61 & 26 & 23.9 & $52 . \overline{6}$ & 0.12 & $916^{\circ}$ & 55.6 & 22 & -0.18 \\
\hline $12: 43: 35: 11$ & 247 & 61 & 26 & 23.9 & 52.6 & 0.13 & 912 & 55.6 & 22 & -0.18 \\
\hline $12: 43: 36: 10$ & 248 & 60 & 26 & 23.9 & 52.6 & 0.13 & 910 & 55.7 & 22 & 0.01 \\
\hline $12: 43: 37: 08$ & 249 & 61 & 26 & $23.9^{\circ}$ & 52.6 & $0 . \overline{13}$ & $913^{\circ}$ & $55 . \overline{7}$ & 22 & 0.01 \\
\hline
\end{tabular}




\begin{tabular}{|c|c|c|c|c|c|c|c|c|c|c|}
\hline TIME & $\begin{array}{l}\text { Elapsed: } \\
\text { Time } \\
\text { (sec) }\end{array}$ & BIT_TC1 & PURG_TC2 & PurgePSI & PurgeCFM & DEPTH(IN) & DwnFrc(lb) & RPM & $\begin{array}{l}\text { TORQUE } \\
\left(\text { FT }^{*} \mathrm{LB}\right)\end{array}$ & Inches/Min \\
\hline $12: 43: 38: 07$ & $\quad 250$ & 61 & -26 & 23.9 & 52.6 & $\quad 0.13$ & 911 & 55.8 & 22 & $2 \quad-0.04$ \\
\hline $12: 43: 39: 06$ & 251 & 61 & 26 & 23.9 & 52.6 & 0.13 & 914 & 55.9 & 22 & -0.04 \\
\hline $12: 43: 40: 10$ & 252 & 61 & 26 & 23.9 & 52.6 & 0.13 & $917^{\circ}$ & 55.9 & 22 & -0.24 \\
\hline $12: 43: 41: 09$ & 253 & 61 & 26 & 23.9 & 52.6 & 0.13 & 921 & 55.9 & 22 & -0.24 \\
\hline $12: 43: 42: 08$ & 254 & 61 & 26 & $23.9^{i}$ & $52.6 !$ & 0.13 & 921 & 55.7 & 22 & -0.17 \\
\hline $12: 43: 43: 07$ & 255 & 60 & 26 & $23.9^{\circ}$ & 52.6 & 0.13 & 921 & 55.7 & 22 & -0.17 \\
\hline $12: 43: 44: 06$ & 256 & 60 & 26 & $23.9^{\circ}$ & 52.6 & 0.13 & 922 & 55.7 & 22 & -0.09 \\
\hline $12: 43: 45: 05$ & 257 & 60 & 26 & 23.9 & 52.6 & 0.13 & $921^{\circ}$ & 55.7 & 22 & -0.09 \\
\hline $12: 43: 46: 04$ & 258 & 61 & 26 & 23.9 & 52.6 & 0.13 & 917 & 55.7 & 22 & -0.02 \\
\hline $12: 43: 47: 03$ & 259 & 61 & 26 & 23.9 & 52.6 & 0.13 & 912 & 55.7 & 22 & -0.02 \\
\hline $12: 43: 48: 01$ & 260 & $61 !$ & 26 & 23.9 & 52.6 & 0.14 & 911 & $55.7 !$ & 22 & -0.15 \\
\hline $12: 43: 49: 00$ & 261. & 61 & 26 & 23.9 & $52.6^{i}$ & $0.13^{1}$ & 912 & 55.7 & 22 & -0.15 \\
\hline $12: 43: 49: 99$ & 262 & 61 & 26 & $23.9^{\prime}$ & 52.6 & 0.14 & 916 & 55.8 & 22 & -0.21 \\
\hline $12: 43: 50: 98$ & 263 & 61 & $26 i$ & $23.9^{\circ}$ & 52.6 & $0.14 i$ & $921^{\circ}$ & 55.8 & 22 & $-0 . \overline{21}$ \\
\hline $12: 43: 51: 97$ & 264 & 61 & 26 & 23.9 & 52.6 & 0.14 & $917^{\circ}$ & $55.8^{\dagger}$ & 22 & -0.08 \\
\hline $12: 43: 52: 96$ & 265 & $61^{\dagger}$ & $26^{\circ}$ & $23.9^{4}$ & 52.6 & 0.13 & 921 & 55.8 & 22 & -0.08 \\
\hline $12: 43: 53: 95$ & $266^{\dagger}$ & 60 & $26^{\circ}$ & 23.9 & $52.6^{\dagger}$ & 0.13 & $922^{\circ}$ & 55.8 & 22 & -0.02 \\
\hline $12: 43: 54: 93$ & 267 & 60 & 26 & 23.9 & 52.6 & $0.13^{\circ}$ & 922 & 55.8 & 22 & -0.02 \\
\hline $12: 43: 55: 92$ & 268 & 60 & 26 & 23.9 & $52.6^{1}$ & $0.13^{\top}$ & 918 & 55.7 & 22 & -0.1 \\
\hline $12: 43: 56: 91$ & 269 & 60 & $2 \overline{6}^{i}$ & 23.9 & 52.6 & $0.13^{!}$ & 913 & 55.6 & 22 & -0.1 \\
\hline $12: 43: 57: 90$ & 270 & $61 \mathrm{i}$ & 26 & 24.1 & 52.6 & 0.13 & 912 & 55.6 & 22 & -0.22 \\
\hline $12: 43: 58: 89$ & 271 & 61 & 26 & 24.1 & 52.6 & 0.14 & 912 & 55.6 & 22 & -0.22 \\
\hline $12: 43: 59: 88$ & 272 & 61 & 26 & 24,1 & 52.6 & 0.14 & 916 & 55.7 & 22 & -0.12 \\
\hline $12: 44: 00: 92$ & $273^{i}$ & $61 !$ & $26^{\prime}$ & 24.1 & 52.6 & 0.13 & 913 & 55.7 & 22 & -0.12 \\
\hline $12: 44: 0 \overline{1}: 9 \overline{1}$ & 274 & $61^{\circ}$ & 26 & $24.1 !$ & 52.6 & 0.14 & 917 & $55.8^{\dagger}$ & 22 & -0.02 \\
\hline $12: 44: 02: 90$ & 275 & 61 & 26 & $24.1^{\prime}$ & 52.6 & 0.14 & 921 & 55.8 & 22 & -0.08 \\
\hline $12: 44: 03: 89$ & 276 & 60 & 26 & $24.1 !$ & 52.6 & $0.13^{\dagger}$ & 922 & 55.8 & 22 & -0.08 \\
\hline $12: 44: 04: 88$ & 277 & 60 & 26 & $23.9 i$ & 52.6 & 0.13 & 922 & 55.8 & 22 & -0.21 \\
\hline $12: 44: 05: 86$ & 278 & 60 & 26 & 23.9 & 52.6 & 0.13 & 920 & 55.8 & 22 & $-0.2 \overline{1}$ \\
\hline $12: 44: 06: 85$ & 279 & 61 & 26 & 23.9 & 52.6 & 0.13 & 922 & 55.8 & 22 & -0.2 \\
\hline $12: 44: 07: 84$ & $280^{\circ}$ & 61 & 26 & 23.9 & 52.6 & 0.13 & $920^{\circ}$ & 55.8 & 22 & -0.2 \\
\hline $12: 44: 08: 83$ & 281 & 61 & 26 & $23.9^{!}$ & $52.6^{\dagger}$ & 0.13 & 916 & 55.7 & 22 & -0.01 \\
\hline $12: 44: 09: 82$ & 282 & 61 & 26 & 23.9 & 52.6 & 0.13 & 913 & 55.7 & 22 & -0.01 \\
\hline $12: 44: 10: 81$ & 283 & $61 i$ & 26 & $24.1 !$ & 52.6 & 0.13 & 913 & 55.6 & 22 & -0.08 \\
\hline $12: 44: 11: 80$ & 284 & 611 & $26 !$ & $24.1 !$ & 52.6 & $0.13^{!}$ & 917 & 55.6 & 22 & -0.08 \\
\hline $12: 44: 12: 79$ & 285 & $61 j$ & 26 & $24.1 !$ & 52.6 & $0.13^{\top}$ & 921 & 55.7 & 22 & -0.27 \\
\hline $12: 44: 13: 77$ & 286 & $61 j$ & 26 & $24.1^{i}$ & 52.6 & $0.14 !$ & 922 & $55.7 !$ & 22 & -0.27 \\
\hline $12: 44: 14: 76$ & 287 & 61 & 26 & 24.1 & 52.6 & 0.12 & 923 & 55.7 & 22 & -0.17 \\
\hline $12: 44: 15: 75$ & 288 & 60 & 26 & 24.1 & 52.6 & 0.13 & 923 & 55.6 & 22 & -0.17 \\
\hline $12: 44: 16: 74$ & 289 & 60 & 26 & 23.9 & 52.6 & 0.13 & 923 & 55.5 & 22 & -0.01 \\
\hline $12: 44: 17: 73$ & 290 & 60 & 26 & 23.9 & 52.6 & 0.13 & 921 & 55.5 & 22 & -0.01 \\
\hline $12: 44: 18: 72$ & 291 & 61 & 26 & $23.9^{i}$ & 52.6 & 0.14 & 916 & 55.4 & 22 & -0.07 \\
\hline $12: 44: 19: 71$ & 292 & 61 ! & 26 & $23.9^{\circ}$ & 52.6 & 0.13 & 913 & 55.4 & 22 & -0.07 \\
\hline $12: 44: 20: 69$ & 293 & 61 & 26 & 23.9 & $52 . \overline{6}$ & $0.14^{\top}$ & $914^{\circ}$ & 55.4 & 22 & -0.26 \\
\hline $12: 44: 21: 74$ & 294 & 61 & 26 & $23.9^{i}$ & 52.6 & $0 . \overline{14}$ & $918^{\circ}$ & 55.2 & 22 & -0.26 \\
\hline $12: 44: 22: 73$ & 295 & 61 & 26 & 24.1 & $52 . \overline{6}$ & 0.14 & 915 & 55.2 & 22 & -0.19 \\
\hline $12: 44: 23: 72$ & 296 & 61 & 26 & 24.1 & 52.6 & 0.14 & 919 & 55.2 & 22 & -0.19 \\
\hline $12: 44: 24: 70$ & 297 ! & 61 & 26 & 24.1 & 52.6 & 0.13 & 922 & 55.2 & 22 & 0 \\
\hline $12: 44: 25: 69$ & 298 & 60 & 26 & 24.1 & 52.6 & 0.13 & 924 & $55.2^{+}$ & 22 & 0 \\
\hline $12: 44: 26: 68$ & 299 & $60^{\top}$ & 26 & 24.1 & $52 . \overline{6}$ & 0.13 & 922 & 55.2 & 22 & -0.09 \\
\hline
\end{tabular}




\begin{tabular}{|c|c|c|c|c|c|c|c|c|c|c|}
\hline$T I M E$ & $\begin{array}{l}\text { Elapsed } \\
\text { Time } \\
\text { (sec) }\end{array}$ & BIT_TC1 & PURG_TC2 & PurgePSI ! & PurgeCFM & DEPTH(IN)! & DwnFrc(lb) & RPM & $\begin{array}{l}\text { TORQUE } \\
(\text { FT^LB) }\end{array}$ & Inches/Min \\
\hline $12: 44: 27: 67$ & $\quad 300^{1}$ & 60 & 26 & 24.1 & $\quad 52.6$ & 0.12 & $\quad 918$ & 55.2 & $\quad \ldots 22$ & $2 \quad-0.09$ \\
\hline $12: 44: 28: 66$ & 301 & 60 & 26 & 24.1 & 52.6 & 0.12 & $914^{\circ}$ & 55.2 & 22 & -0.25 \\
\hline $12: 44: 29: 65$ & 302 & 60 & 26 & 24.1 & 52.6 & 0.13 & 917 & 55.2 & 22 & -0.25 \\
\hline $12: 44: 30: 64$ & 303 & 60 & 26 & 24.1 & 52.6 & 0.13 & 914 & 55.2 & 22 & -0.17 \\
\hline $12: 44: 31: 62$ & 304 & 61 & $26^{\prime}$ & 24.1 & 52.6 & 0.14 & 914 & 55.3 & 21 & 0 \\
\hline $12: 44: 32: 61$ & 305 & 61 & 26 & 24.1 & 52.6 & 0.14 & 917 & 55.3 & 21 & 0 \\
\hline $12: 44: 33: 60$ & 306 & 61 & 26 & 24.1 & 52.6 & 0.14 & 922 & 55.2 & 21 & -0.06 \\
\hline $12: 44: 34: 59$ & 307 & 60 & 26 & 24.1 & $52.6^{\dagger}$ & 0.13 & 926 & 55.1 & 22 & $-0 . \overline{06}$ \\
\hline $12: 44: 35: 58$ & 308 & 60 & 26 & 23.9 & 52.6 & 0.13 & 925 & 55.1 & 22 & -0.27 \\
\hline $12: 44: 36: 57$ & 309 & 60 & 26 & 23.9 & 52.6 & 0.13 & 922 & 55.1 & 22 & -0.27 \\
\hline $12: 44: 37: 56$ & 310 & $6 \overrightarrow{0}$ & 26 & 23.9 & 52.6 & 0.13 & 918 & 55.2 & 22 & -0.12 \\
\hline $12: 44: 38: 55$ & 311 & 60 & 26 & 23.9 & 52.6 & 0.12 & 919 & 55.2 & 22 & -0.12 \\
\hline $12: 44: 39: 53$ & 312 & 61 & 26 & 23.9 & 52.6 & $\overline{0.13}$ & 915 & 55.3 & 22 & -0.02 \\
\hline $12: 44: 40: 52$ & 313 & 61 & 26 & 24.1 & 52.6 & $0.1 \overline{3}$ & 913 & 55.3 & 21 & -0.02 \\
\hline $12: 44: 41: 51$ & 314 & 61 & 26 & 24.1 & 52.6 & 0.13 & 915 & 55.3 & 21 & -0.09 \\
\hline $12: 44: 42: 50$ & 315 & 60 & 26 & 24.1 & 52.6 & 0.14 & 919 & $55 . \overline{3}$ & 21 & $-0 . \overline{09}$ \\
\hline $12: 44: 43: 49$ & 316 & 60 & 26 & $24.1^{\dagger}$ & 52.6 & 0.14 & 922 & 55.1 & 21 & $-\overline{0.3}$ \\
\hline $12: 44: 44: 48$ & 317 & 60 & 26 & 24.1 & 52.6 & 0.13 & 924 & 55.1 & 22 & -0.3 \\
\hline $12: 44: 45: 47$ & 318 & 60 & 26 & 22.8 & 52.6 & 0.13 & $924^{\circ}$ & 55.2 & $22 !$ & -0.12 \\
\hline $12: 44: 46: 45$ & 319 & 60 & 26 & 3.7 & 1.8 & 0.13 & $919^{\circ}$ & 55.4 & 22 & -0.12 \\
\hline $12: 44: 47: 44$ & 320 & 63 & 26 & -0.8 & 1.8 & 0.14 & 921 & 55.4 & 22 & -0.01 \\
\hline $12: 44: 48: 43$ & 321 & 65 & 26 & -0.8 & 1.8 & 0.14 & 916 & 55.4 & 23 & -0.01 \\
\hline $12: 44: 49: 42$ & 322 & 67 & 26 & -0.8 & 1.8 & $0.14 i$ & 914 & 55.3 & 23 & -0.11 \\
\hline $12: 44: 50: 41$ & 323 & 68 & 26 & -0.8 & 1.8 & 0.14 & 914 & 55.3 & 23 & -0.11 \\
\hline $12: 44: 51: 40$ & 324 & 70 & 26 & -0.8 & 1.8 & 0.14 & 917 & 55.3 & 23 & -0.27 \\
\hline $12: 44: 52: 39$ & 325 & 71 & 26 & $-0.8^{1}$ & 1.8 & 0.14 & 921 & 55.4 & 23 & -0.27 \\
\hline $12: 44: 53: 38$ & 326 & $7 \overline{2}$ & $26^{\prime}$ & -0.8 & 1.8 & 0.14 & 924 & 55.4 & 24 & -0.15 \\
\hline $12: 44: 54: 36$ & 327 & 72 & 26 & -0.8 & 1.8 & 0.14 & 922 & 55.6 & 24 & $-0 . \overline{15}$ \\
\hline $12: 44: 55: 35$ & 328 & 73 & 26 & -0.8 & 1.8 & 0.14 & 925 & 55.6 & 24 & -0.01 \\
\hline $12: 44: 56: 34$ & 329 & 74 & 26 & -0.8 & 1.8 & $0.1 \overline{4}$ & 925 & 55.6 & 24 & -0.01 \\
\hline $12: 44: 57: 33$ & 330 & 75 & 26 & -0.8 & 1.8 & 0.14 & 924 & 55.6 & 24 & -0.07 \\
\hline $12: 44: 58: 32$ & 331 & 76 & 26 & -0.8 & 1.8 & 0.14 & $919^{\circ}$ & 55.5 & 24 & -0.07 \\
\hline $12: 44: 59: 31$ & 332 & 77 & 26 & -0.8 & 1.8 & 0.14 & 916 & 55.5 & 24 & $-0 . \overline{29}$ \\
\hline $12: 45: 00: 30$ & 333 & 78 & $26 !$ & -0.8 & 1.8 & 0.14 & 916 & 55.6 & 24 & -0.29 \\
\hline $12: 45: 01: 28$ & 334 & 79 & 26 & -0.8 & 1.8 & 0.14 & 918 & 55.6 & 24 & -0.14 \\
\hline $12: 45: 02: 27$ & 335 & 80 & 26 & -0.8 & 1.8 & 0.14 & 922. & $5 \overline{5.6}$ & 24 & $-0 . \overline{14}$ \\
\hline $12: 45: 03: 26$ & 336 & 81 & 26 & -0.8 & 1.8 & $0.1 \overline{4}$ & 917 & 55.7 & 24 & -0.01 \\
\hline $12: 45: 04: 25$ & 337 & 82 & 26 & -0.8 & 1.8 & 0.14 & 922 & 55.7 & 25 & -0.01 \\
\hline $12: 45: 05: 24$ & 338 & 82 & 26 & $-0 . \overline{8}$ & 1.8 & 0.15 & 923 & 55.7 & 25 & -0.1 \\
\hline $12: 45: 06: 23$ & 339 & 83 & 26 & -0.8 & 1.8 & 0.14 & 923 & 55.5 & 25 & $-0 . \overline{23}$ \\
\hline $12: 45: 07: 27$ & 340 & 84 & 26 & -0.8 & 1.8 & 0.14 & 922 & 55.5 & 26 & -0.23 \\
\hline $12: 45: 08: 26$ & 341 & 85 & 26 & -0.8 & 1.8 & 0.13 & 917 & 55.5 & 26 & -0.18 \\
\hline $12: 45: 09: 25$ & 342 & $86 !$ & 26 & -0.8 & 1.8 & 0.13 & 921 & 55.6 & 26 & -0.18 \\
\hline $12: 45: 10: 24$ & 343 & 87 & 26 & -0.8 & 1.8 & 0.14 & $917^{\circ}$ & 55.6 & 25 & -0.02 \\
\hline $12: 45: 11: 23$ & 344 & 88 & 26 & -0.8 & 1.8 & 0.14 & 914 & 55.5 & 25 & -0.02 \\
\hline $12: 45: 12: 21$ & 345 & 89 & 26 & -0.8 & 1.8 & 0.14 & 914 & 55.4 & 25 & -0.04 \\
\hline $12: 45: 13: 20$ & 346 & 90 & 26 & -0.8 & 1.8 & 0.14 & 917 & 55.4 & 25 & -0.04 \\
\hline $12: 45: 14: 19$ & 347 & 91 & 26 & -0.8 & 1.8 & 0.15 & 921 & 55.5 & 26 & -0.26 \\
\hline $12: 45: 15: 18$ & 348 & 92 & 28 & -0.8 & 1.8 & 0.14 & 923 & 55.5 & 26 & -0.28 \\
\hline $12: 45: 16: 17$ & 349 & $91 \mathrm{i}$ & 26 & -0.8 & $1.8^{-t}$ & 0.14 & 923 & 55.5 & 26 & -0.16 \\
\hline
\end{tabular}




\begin{tabular}{|c|c|c|c|c|c|c|c|c|c|c|}
\hline$T \mid M E$ & $\begin{array}{l}\text { Elapsed } \\
\text { Time } \\
(\mathrm{sec})\end{array}$ & BIT_TC1 & & PurgePSI & PurgeCFM & DEPTH(IN) & DwnFrc(lb) & $R P M$ & $\begin{array}{l}\text { TORQUE } \\
\text { (FT*LB) }\end{array}$ & !nches/Min \\
\hline $12: 45: 17: 16$ & $\quad 350$ & 92 & 26 & $\quad-0.8$ & $\quad 1.8$ & $\quad 0.13$ & $\quad 923$ & 55.6 & $\quad 26$ & $6 \quad-0.16$ \\
\hline $12: 45: 18: 15$ & 351 & 93 & 26 & -0.8 & 1.8 & 0.13 & 923 & $5 \overline{5.7}$ & 26 & -0.04 \\
\hline $12: 45: 19: 14$ & 352 & 94 & 26 & -0.8 & 1.8 & 0.14 & $921^{\prime}$ & 55.7 & 26 & -0.04 \\
\hline $12: 45: 20: 12$ & 353 & $95 !$ & 26 & -0.8 & 1.8 & 0.14 & 916 & 55.7 & 26 & -0.04 \\
\hline $12: 45: 21: 11$ & 354 & $96 i$ & 26 & -0.8 & 1.8 & 0.14 & 912 & 55.6 & 27 & -0.04 \\
\hline $12: 45: 22: 10$ & 355 & 97 & 26 & -0.8 & 1.8 & 0.14 & 912 & 556 & 26 & -0.23 \\
\hline $12: 45: 23: 09$ & 356 & 98 & 26 & -0.8 & 1.8 & 0.14 & 916 & 55.5 & 26 & -0.23 \\
\hline $12: 45: 24: 08$ & 357 ! & 98 & 26 & -0.8 & 1.8 & 0.14 & 920 & 55.5 & 27 & -0.2 \\
\hline $12: 45: 25: 07$ & 358 & 99 & 26 & -0.8 & 1.8 & 0.14 & 922 & 55.5 & 27 & -0.2 \\
\hline $12: 45: 26: 06^{1}$ & 359 & 100 & 26 & $-0 . \overline{8}$ & 1.8 & 0.13 & 921 & 55.5 & 27 & -0.03 \\
\hline $12: 45: 27: 04$ & 360 & 100 & 26 & -0.8 & 1.8 & 0.13 & 922 & 55.5 & 27 & -0.03 \\
\hline $12: 45: 28: 09$ & 361 & 101 & 26 & -0.8 & 1.8 & 0.13 & 922 & 55.5 & 27 & -0.06 \\
\hline $12: 45: 29: 08$ & 362 & 102 & 26 & $-0 . \overline{8}$ & 1.8 & 0.14 & $919^{+}$ & 55.5 & 27 & -0.06 \\
\hline $12: 45: 30: 07 \mid$ & 363 & 103 & 26 & -0.8 & 1.8 & $0.14 !$ & 914 & $55.5^{\dagger}$ & $\overline{27}$ & -0.21 \\
\hline $12: 45: 31: 05$ & 364 & 104 & 26 & -0.8 & 1.8 & 0.14 & 912 & $55.5^{\dagger}$ & 27 & -0.21 \\
\hline $12: 45: 32: 04$ & 365 & 105 & 26 & -0.8 & 1.8 & 0.14 & 913 & 55.5 & 27 & -0.19 \\
\hline $12: 45: 33: 03$ & 366 & 106 & 26 & -0.8 & 1.8 & $0.14^{\prime}$ & 911 & 55.5 & 27 & -0.19 \\
\hline $12: 45: 34: 02$ & 367 & 107 & 26 & -0.8 & 1.8 & $0.14 !$ & $913^{\prime}$ & 55.5 & 27 & -0.08 \\
\hline $12: 45: 35: 01$ & $368^{\circ}$ & 107 & 26 & -0.8 & 1.8 & 0.14 & 917 & 55.4 & 28 & -0.05 \\
\hline $12: 45: 36: 00$ & 369 & 108 & 26 & -0.8 & 1.8 & 0.14 & $920^{\dagger}$ & 55.2 & 28 & -0.05 \\
\hline $12: 45: 36: 99$ & 370 & 108 & 26 & -0.8 & 1.8 & 0.13 & $921 !$ & 55.2 & 28 & -0.19 \\
\hline $12: 45: 37: 97$ & 371 & 109 & 26 & -0.8 & 1.8 & 0.14 & 921 & 55.2 & 28 & -0.19 \\
\hline $12: 45: 38: 96$ & 372 & 109 & 26 & -0.8 & 1.8 & 0.14 & 917 & 55.2 & 28 & -0.23 \\
\hline $12: 45: 39: 95$ & 373 & 110 & 26 & -0.8 & 1.8 & 0.14 & 920 & 55.2 & 28 & -0.23 \\
\hline $12: 45: 40: 94$ & 374 & 111 & 26 & -0.8 & 1.8 & 0.14 & 916 & 55.2 & 27 & -0.02 \\
\hline $12: 45: 41: 93$ & 375 & 112 & $26 !$ & -0.8 & 1.8 & 0.14 & 912 & 55.2 & 27 & -0.02 \\
\hline $12: 45: 42: 92$ & 376 & 113 & 26 & -0.8 & 1.8 & 0.14 & 911 & 55.2 & 27 & -0.05 \\
\hline $12: 45: 43: 91$ & $37 \overline{7}$ & 114 & 26 & -0.8 & 1.8 & 0.15 & 914 & 55.2 & 27 & -0.05 \\
\hline $12: 45: 44: 90$ & 378 & 115 & 26 & -0.8 & 1.8 & 0.14 & 917 & 55.3 & 27 & -0.24 \\
\hline $12: 45: 45: 88$ & 379 & 115 & 26 & -0.8 & 1.8 & 0.14 & $921^{\circ}$ & 55.3 & 28 & -0.24 \\
\hline $12: 45: 46: 87$ & 380 & 115 & 26 & -0.8 & 1.8 & 0.14 & $921^{\circ}$ & 55.4 & 28 & -0.18 \\
\hline $12: 45: 47: 86$ & 381 & 116 & 26 & -0.8 & 1.8 & 0.13 & $919^{\prime}$ & 55.4 & 28 & -0.18 \\
\hline $12: 45: \overline{48}: 85$ & 382 & 117 & 26 & -0.8 & 18 & 0.14 & 921 & 55.4 & 28 & -0.03 \\
\hline $12: 45: 49: 84$ & $383 !$ & 117 & 26 & -0.8 & 1.8 & 0.14 & 916 & 55.4 & 28 & -0.03 \\
\hline $12: 45: 50: 83$ & 384 & 118 & 26 & -0.8 & 1.8 & 0.14 & 912 & 55.2 & 28 & -0.06 \\
\hline $12: 45: 51: 82$ & 385 & 120 & 26 & -0.8 & 1.8 & 0.14 & 910 & 55.2 & 28 & -0.06 \\
\hline $12: 45: 52: 80$ & 386 & 120 & 26 & -0.8 & 1.8 & 0.13 & 911 & 55.2 & 28 & $-0.2 \overline{3}$ \\
\hline $12: 45: 53: 79$ & 387 & 121 & 26 & -0.8 & 1.8 & 0.14 & 914 & $55 \overline{2}$ & 28 & -0.23 \\
\hline $12: 45: 54: 78$ & 388 & 121 & 26 & -0.8 & 1.8 & 0.14 & 918 & 55.4 & 28 & -0.18 \\
\hline $12: 45: 55: 77$ & $389^{\circ}$ & 122 & 26 & -0.8 & 1.8 & 0.13 & 921 & 55.5 & 28 & -0.18 \\
\hline $12: 45: 56: 76$ & 390 & 123 & 26 & -0.8 & 1.8 & 0.14 & 918 & 55.5 & 28 & 0 \\
\hline $12: 45: 57: 75$ & $391 \mathrm{j}$ & 123 & 26 & -0.8 & 1.8 & 0.13 & 921 & 55.6 & 28 & 8 \\
\hline $12: 45: 58: 74$ & 392 & 123 & 26 & -0.8 & 1.8 & 0.14 & 920 & 55.6 & 28 & -0.07 \\
\hline $12: 45: 59: 73$ & 393 & 124 & 26 & -0.8 & 1.8 & 0.14 & 916 & 55.6 & 28 & -0.07 \\
\hline $12: 46: 00: 71$ & 394 & 125 & 26 & -0.8 & 1.8 & 0.14 & 912 & 55.5 & 28 & -0.24 \\
\hline $12: 46: 01: 70$ & 395 & 126 & 26 & -0.8 & 1.8 & 0.13 & 910 & 55.4 & 28 & -0.24 \\
\hline $12: 46: 02: 69$ & 396 & 127 & 26 & -0.8 & 1.8 & 0.14 & 911. & 55.4 & 28 & -0.2 \\
\hline $12: 46: 03: 68$ & 397 & 128 & 26 & -0.8 & 1.8 & 0.14 & 914 & 55.4 & 29 & -0.2 \\
\hline $12: 46: 04: 67$ & 398 & 129 & 26 & -0.8 & 1.8 & 0.14 & 912. & 55.3 & 29 & 0.01 \\
\hline $12: 46: 05: 66$ & 399 & 128 & 26 & -0.8 & 1.8 & 0.13 & 916 & 55.3 & 29 & 0.01 \\
\hline
\end{tabular}




\begin{tabular}{|c|c|c|c|c|c|c|c|c|c|c|}
\hline TIME & $\begin{array}{l}\text { Elapsed } \\
\text { Time } \\
\text { (sec) }\end{array}$ & BIT TC1 & PURG_TC2 & PurgePSI & & DEPTH(IN) & DwnFrc(lb) & RPM & $\begin{array}{l}\text { TORQUE } \\
\text { (FT*LB) }\end{array}$ & Inches/Min \\
\hline $12: 46: 06: 65$ & 400 & $\quad 129$ & 26 & $\quad-0.8$ & $\quad 1.8$ & $\quad 0.13$ & 918 & 55.1 & $\quad 29$ & $\quad-0.04$ \\
\hline $12: 46: 07: 63$ & 401 & 129 & 26 & -0.8 & 1.8 & 0.14 & 921 & 55 & 29 & -0.23 \\
\hline $12: 46: 08: 62$ & 402 & 130 & 26 & -0.8 & 1.8 & 0.14 & 918 & 55 & 30 & -0.23 \\
\hline $12: 46: 09: 61$ & 403 & $131^{1}$ & 26 & -0.8 & 1.8 & 0.14 & 914 & 55.1 & 28 & -0.2 \\
\hline $12: 46: 10: 60$ & 404 & 131 & 26 & -0.8 & 1.8 & 0.14 & 910 & 55.2 & 28 & -0.2 \\
\hline $12: 46: 11: 59$ & 405 & 133 & 26 & -0.8 & 1.8 & 0.14 & 910 & 55.2 & 28 & -0.01 \\
\hline $12: 46: 12: 58$ & 406 & 134 & 26 & -0.8 & 1.8 & 0.14 & 913 & 55.2 & 28 & -0.01 \\
\hline $12: 46: 13: 62$ & 407 & $134^{\dagger}$ & 26 & -0.8 & 1.8 & 0.15 & 910 & 55.3 & $2 \overline{9}$ & -0.05 \\
\hline $12: 46: 14: 61$ & 408 & $135^{1}$ & 26 & -0.8 & 1.8 & 0.14 & 913 & 55.3 & 29 & -0.05 \\
\hline $12: 46: 15: 60$ & 409 & 135 & 26 & -0.8 & 1.8 & 0.14 & 917 & 55.4 & 29 & -0.26 \\
\hline $12: 46: 16: 59$ & 410 & 135 & 26 & -0.8 & 1.8 & 0.14 & 919 & 55.5 & 29 & -0.26 \\
\hline $12: 46: 17: 58$ & 411 & 136 & 26 & -0.8 & 1.8 & 0.14 & $920^{\prime}$ & 55.5 & 30 & $-0 . \overline{17}$ \\
\hline $12: 46: 18: 56$ & 412 & 136 & 26 & -0.8 & 1.8 & 0.14 & 917 & 55.5 & 30 & -0.17 \\
\hline $12: 46: 19: 55$ & 413 & 137 & 26 & -0.8 & 1.8 & 0.14 ! & 919 & 55.6 & 30 & 0 \\
\hline $12: 46: 20: 54$ & 414 & 138 & 26 & -0.8 & 1.8 & 0.13 & 916 & 55.6 & 30 & 0 \\
\hline $12: 46: 21: 53$ & 415 & 139 & 26 & -0.8 & 1.8 & 0.14 & 910 & 55.5 & 29 & -0.09 \\
\hline $12: 46: 22: 52$ & 416 & 140 & 26 & -0.8 & 1.8 & 0.13 & 909 & 55.4 & 29 & -0.09 \\
\hline $12: 46: 23: 51$ & 417 & 140 & 26 & -0.8 & 1.8 & 0.13 & 910 & 55.4 & 29 & -0.22 \\
\hline $12: 46: 24: 50$ & 418 & $141^{\dagger}$ & 26 & -0.8 & 1.8 & 0.14 & 913 & 55.5 & 29 & -0.22 \\
\hline $12: 46: 25: 49$ & $4 \overline{19}$ & 142 & 26 & -0.8 & 1.8 & 0.14 & 917 & 55.6 & 29 & -0.2 \\
\hline $26: 47$ & 420 & 143 & 26 & -0.8 & 1.8 & 0.14 & 919 & 55.6 & 29 & -0.2 \\
\hline $12: 46: 27: 46$ & 421 & 142 & 26 & -0.8 & 1.8 & 0.13 & 919 & 55.7 & 29 & 0 \\
\hline $12: 46: 28: 45$ & 422 & 142 & 26 & -0.8 & 1.8 & 0.14 & $919^{\prime}$ & 55.6 & 29 & 0 \\
\hline $12: 46: 29: 44$ & 423 & 143 & 26 & -0.8 & 1.8 & 0.13 & 919 & 55.6 & 29 & -0.06 \\
\hline $12: 46: 30: 43$ & 424 & 144 & 26 & -0.8 & 1.8 & 0.13 & 916 & 55.6 & 29 & -0.06 \\
\hline $12: 46: 31: 42$ & 425 & 144 & 26 & -0.8 & 1.8 & 0.14 & 911 & 55.6 & 29 & -0.22 \\
\hline $12: 46: 32: 41$ & 426 & 146 & 26 & -0.8 & 1.8 & 0.14 & $909^{\circ}$ & 55.6 & 29 & -0.22 \\
\hline $12: 4$ & 427 & 146 & 26 & -0.8 & 1.8 & 0.14 & $910^{\circ}$ & 55.3 & 30 & -0.15 \\
\hline 38 & 428 & 147 & 26 & -0.8 & 1.8 & 0.13 & 913 & 55 & 30 & -0.15 \\
\hline $12: 46: 35: 37$ & 429 & 146 & 26 & -0.8 & 1.8 & 0.15 & 917 & 55 & 30 & -0.02 \\
\hline 36 & 430 & 147 & 26 & -0.8 & 1.8 & 0.14 & 919 & 54.9 & 30 & -0.02 \\
\hline 35 & 431 & 147 & 26 & -0.8 & 1.8 & 0.14 & 918 & 55 & 30 & -0.09 \\
\hline $12: 46: 38: 34$ & 432 & 148 & 26 & -0.8 & 1.8 & 0.13 & 919 & 55 & 30 & -0.09 \\
\hline $12: 46: 39: 33$ & 433 & 148 & 26 & -0.8 & 1.8 & 0.14 & 917 & 55.1 & 29 & $-0 . \overline{24}$ \\
\hline $12: 46: 40: 32$ & 434 & $14 \overline{9}$ & 26 & -0.8 & 1.8 & 0.14 & 913 & 55.1 & 29 & -0.19 \\
\hline $12: 46: 41: 30$ & 435 & 150 & 26 & -0.8 & 1.8 & 0.13 & 909 & 55.3 & 29 & -0.19 \\
\hline $42: 29$ & $436 !$ & 151 & 26 & -0.8 & 1.8 & 0.14 & $909^{\circ}$ & 55.4 & 29 & 0 \\
\hline $12: 46: 43: 28$ & $437 !$ & 152 & 26 & -0.8 & 1.8 & 0.14 & 911 & 55.4 & 29 & 0 \\
\hline $12: 46: 44: 27$ & 438 & 152 & 26 & -0.8 & 1.8 & 0.13 & $915^{\circ}$ & 55.5 & 29 & -0.04 \\
\hline $45: 26$ & 439 & 153 & 26 & -0.8 & 1.8 & 0 & 912 & 55.5 & 30 & -0.04 \\
\hline $12: 46: 46: 25$ & 440 & 153 & 26 & -0.8 & 1.8 & 0.13 & 915 & 55.5 & 30 & -0.27 \\
\hline $12: 46: 47: 24$ & 441 & 153 & 26 & -0.8 & 1.8 & 0.14 & 918 & 55.5 & 30 & -0.27 \\
\hline$: 48: 22$ & 442 & 153 & 26 & -0.8 & 1.8 & 0.13 & 918 & 55.6 & 30 & -0.17 \\
\hline $12: 46: 49: 21$ & 443 & 15 & 26 & -0.8 & 1.8 & 0.14 & 916 & 55.6 & 30 & -0.17 \\
\hline $5: 50: 20$ & 444 & 155 & 26 & -0.8 & 1.8 & 0.13 & 911. & 55.4 & 30 & -0.01 \\
\hline $1: 19$ & 445 & 155 & 26 & -0.8 & 1.8 & 0.13 & 908 & 55.4 & 30 & -0.01 \\
\hline & $446^{\prime}$ & 156 & 26 & -0.8 & 1.8 & 0.14 & 909 & 55.4 & 30 & -0.06 \\
\hline$: 17$ & 447 & 157 & 26 & -0.8 & 1.8 & 0.14 & 911. & 55.4 & 30 & -0.06 \\
\hline$: 46: 54: 16$ & 448 & 158 & 26 & -0.8 & 1.8 & 0.14 & 908 & 55.4 & 30 & -0.21 \\
\hline $12: 46: 55: 15$ & $\overline{449}$ & $158^{\dagger}$ & 26 & -0.8 & 1.8 & 0.14 & 912 & 55.4 & 30 & -0.21 \\
\hline
\end{tabular}




\begin{tabular}{|c|c|c|c|c|c|c|c|c|c|c|}
\hline & $\begin{array}{l}\text { Elapsed } \\
\text { Time }\end{array}$ & & & & & 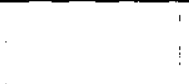 & & & QUE & \\
\hline$T \backslash M E$ & (sec) & BIT_TC1 & PURG_TC2: & PurgePSI! & 'PurgeCFM & DEPTH(IN) & DwnFrc(lb) & RPM i & & Inches/Min \\
\hline $12: 46: 56: 13$ & 450 & 158 & $\quad 26$ & $\quad-0.8$ & 1.8 & 0.14 & 915 & 55.5 & 30 & -0.24 \\
\hline $12: 46: 57: 12$ & 451 & 158 & $26 !$ & -0.8 & 1.8 & 0.13 & 917 & 55.6 & 30 & -0.24 \\
\hline $12: 46: 58: 11$ & 452 & 159 & 26 & -0.8 & 1.8 & $0.14^{\prime}$ & $917^{\circ}$ & 55.6 & 30 & -0.02 \\
\hline $12: 46: 59: 10$ & 453 & 159 & 26 & -0.8 & 1.8 & 0.14 & $916^{i}$ & 55.7 & 30 & -0.02 \\
\hline $12: 47: 00: 09$ & 454 & $1 \overline{60}$ & 26 & -0.8 & 1.8 & 0.14 & 911 & 55.9 & 30 & $-0.0 \overline{6}$ \\
\hline $12: 47: 01: 08$ & 455 & $1 \overline{60}$ & 26 & -0.8 & 1.8 & 0.14 & 907 & 55.9 & 30 & $-0.0 \overline{6}$ \\
\hline $12: 47: 02: 07$ & 456 & $16 \overline{1}$ & 26 & -0.8 & 1.8 & 0.13 & 911 & 55.9 & 30 & -0.19 \\
\hline $12: 47: 03: 05$ & 457 & 162 & 26 & -0.8 & 1.8 & 0.14 & $907^{+}$ & $55.9^{+}$ & 30 & -0.19 \\
\hline $12: \overline{47}: 04: 04$ & 458 & 163 & 26 & -0.8 & 1.8 & 0.14 & 908 & 55.9 & 30 & $-\overline{0.2}$ \\
\hline $12: 47: 05: 03$ & 459 & 163 & $26:$ & -0.8 & 1.8 & $0.14^{\dagger}$ & $910^{\dagger}$ & 55.8 & 30 & -0.2 \\
\hline $12: 47: 06: 02$ & 460 & 164 & 26 & -0.8 & 1.8 & 0.15 & 914 & 55.8 & 30 & -0.08 \\
\hline $12: 47: 07: 01$ & 461 & 164 & 26 & -0.8 & 1.8 & 0.15 & 916 & 55.8 & 30 & -0.08 \\
\hline $12: \overline{4}: \overline{08}: 00$ & 462 & 164 & 26 & $-0.8 \mid$ & 1.8 & 0.14 & 917 & 55.7 & 30 & -0.01 \\
\hline $12: 47: 08: 99$ & 463 & 164 & $26^{+}$ & -0.8 & 1.8 & 0.14 & 916 & 55.7 & 30 & -0.01 \\
\hline $12: 47: \overline{0}: \overline{9} 8$ & 464 & 165 & 26 & -0.8 & 1.8 & 0.14 & 917 & 55.7 & 30 & $-0 . \overline{16}$ \\
\hline $12: 47: 10: 96$ & 465 & 165 & 26 & -0.8 & 1.8 & 0.14 & 916 & $55.8^{\dagger}$ & 30 & -0.16 \\
\hline $12: 47: 11: 95$ & 466 & 166 & 26 & $-0 . \overline{8}$ & $1 . \overline{8}$ & $0.14^{\dagger}$ & 912 & 55.8 & 30 & -0.23 \\
\hline $12: 47: 12: 94$ & 467 & 166 & 26 & -0.8 & 1.8 & 0.14 & 908 & 55.8 & 30 & -0.09 \\
\hline $12: 47: 13: 93$ & 468 & 168 & 26 & -0.8 & 于. & 0.14 & 907 & 55.8 & 30 & -0.09 \\
\hline $12: 47: 14: 92$ & 469 & 168 & 26 & -0.8 & 1.8 & 0.13 & 910 & 55.7 & 30 & -0.04 \\
\hline $12: 47: 15: 91$ & 470 & 169 & 26 & -0.8 & 1.8 & $0.14^{\dagger}$ & $914^{\circ}$ & 55.7 & 31 & -0.04 \\
\hline $12: 47: 16: 90$ & $4 \overline{71}$ & 169 & 26 & -0.8 & 1.8 & $0.14^{\dagger}$ & $917 !$ & $55.6^{\dagger}$ & 31 & -0.13 \\
\hline $12: 47: 17: 88$ & $\overline{472}$ & 169 & 26 & -0.8 & 1.8 & $0.13^{\top}$ & $917^{\circ}$ & 55.5 & 31 & $-0 . \overline{3}$ \\
\hline $12: 47: 18: 87$ & 473 & 169 & 26 & $-0.8^{1}$ & 1.8 & 0.13 & 916 & 55.5 & 31 & -0.24 \\
\hline $12: 47: 19: 86$ & 474 & 169 & 26 & $-0.8 !$ & 1.8 & 0.14 & 917 & 55.4 & 30 & $-0.2 \overline{4}$ \\
\hline $12: 47: 20: 85$ & 475 & 170 & 26 & -0.8 & 1.8 & 0.14 & 916 & 55.5 & 30 & -0.12 \\
\hline $12: 47: 21: 84$ & 476 & 170 & $26 !$ & -0.8 & 1.8 & 0.14 & $912^{+}$ & 55.5 & 30 & $-0 . \overline{12}$ \\
\hline $12: 47: 22: 83$ & $\overline{477}$ & $1 \overline{1} 1$ & 26 & -0.8 & 1.8 & 0.14 & 907 & 55.6 & 30 & 0 \\
\hline $12: 47: 23: 82$ & 478 & $\overline{172}$ & 26 & -0.8 & 1.8 & 0.14 & 907 & 55.6 & 30 & $\overline{0}$ \\
\hline $12: 47: 24: 80$ & 479 & 172 & 26 & -0.8 & 1.8 & 0.14 & $910^{\prime}$ & 55.6 & 30 & -0.1 \\
\hline $12: 47: 25: 79$ & 480 & 173 & 26 & -0.8 & 1.8 & 0.14 & 913 & 55.6 & 31 & -0.1 \\
\hline $12: 47: 26: 78$ & 481 & $1 \overline{74}$ & 26 & -0.8 & 1.8 & 0.14 & 910 & 55.6 & 31 & $-0.2 \overline{7}$ \\
\hline $12: 47: 27: 77$ & $4 \overline{82}$ & 174 & 26 & -0.8 & $1 . \overline{8}$ & 0.14 & 914 & 55.6 & 31 & -0.27 \\
\hline $7: 28: 76$ & $4 \overline{83}$ & 173 & 26 & -0.8 & 1.8 & $0 . \overline{14}$ & 916 & $55 . \overline{5}$ & 31 & $-0 . \overline{01}$ \\
\hline $12: 47: 29: 75$ & 484 & 174 & 26 & -0.8 & 1.8 & 0.14 & 917 & $55.5^{+}$ & 31 & $-0 . \overline{01}$ \\
\hline $12: 47: 30: 74$ & 485 & 174 & 26 & -0.8 & 1.8 & 0.14 & $916^{\prime}$ & 55.3 & 31 & -0.11 \\
\hline $12: 47: 31: 73$ & 486 & 175 & 26 & -0.8 & 1.8 & $\left.0.14\right|^{\dagger}$ & 912 & 55.5 & 30 & -0.11 \\
\hline $12: 47: 32: 71$ & 487 & $1 \overline{7} \overline{6}$ & 26 & $-0.8^{\dagger}$ & 1.8 & 0.13 & 908 & 55.5 & 30 & -0.12 \\
\hline $12: 47: 33: 70$ & 488 & 176 & 26 & -0.8 & 1.8 & 0.14 & $907^{\circ}$ & $55.7 \mathrm{i}$ & 30 & $-0 . \overline{12}$ \\
\hline $12: 47: 34: 69$ & 489 & 177 & 26 & -0.8 & 1.8 & 0.13 & 907 & 55.8 & 30 & $-0.2 \overline{7}$ \\
\hline $12: 47: 35: 68$ & 490 & $17 \overline{8}$ & $26^{-}$ & -0.8 & 1.8 & $0 . \overline{14}$ & 907 & 55.8 & 30 & -0.27 \\
\hline $12: 47: 36: 67$ & 491 & 178 & 27 & -0.8 & $1 \overline{8}$ & 0.14 & $910^{\circ}$ & $55 . \overline{9}^{+}$ & 30 & -0.17 \\
\hline $12: 47: 37: 66$ & 492 & $17 \overline{8}$ & 27 & -0.8 & 1.8 & 0.14 & 914 & 55.7 & 31 & -0.17 \\
\hline $12: 47: 38: 65$ & 493 & 179 & 27 & -0.8 & 1.8 & 0.14 & 916 & 55.7 & 31 & 0 \\
\hline $12: 47: 39: 63$ & 494 & 179 & 26 & -0.8 & 1.8 & 0.14 & 917 & 55.7 & 31 & 0 \\
\hline $12: 47: 40: 62$ & 495 & 179 & 26 & $-0 . \overline{8}$ & 1.8 & 0.14 & 917 & 55.8 & 31 & -0.07 \\
\hline $12: 47: 41: 61$ & 496 & 179 & 26 & -0.8 & $\overline{1.8}$ & $0 . \overline{14}$ & $912^{\circ}$ & 55.8 & 31 & -0.07 \\
\hline $12: 47: 42: 60$ & 497 & 180 & 26 & -0.8 & $1.8 !$ & 0.14 & 908 & 55.6 & 31 & -0.25 \\
\hline $12: 47: 43: 59$ & 498 & 181 & 27 & -0.8 & 1.8 & 0.13 & 912 & 55.5 & 31 & -0.25 \\
\hline $12: 47: 44: 58$ & $4 \overline{9} \overline{9}$ & $18 \overline{1}$ & $26^{1}$ & -0.8 & 1.8 & $0.15^{\dagger}$ & $908^{\circ}$ & 55.5 & 31 & -0.19 \\
\hline
\end{tabular}




\begin{tabular}{|c|c|c|c|c|c|c|c|c|c|c|}
\hline TIME & $\begin{array}{l}\text { Elapsed } \\
\text { Time } \\
\text { (sec) }\end{array}$ & BIT_TC1 & PURG_TC2 $\mathrm{P}$ & PurgePSI & PurgeCFM & DEPTH $(I N)$ & DwnFrc(lb) & RPM & $\begin{array}{l}\text { TORQUE } \\
\text { (FT*LB) }\end{array}$ & Inches/Min \\
\hline $12: 47: 45: 57$ & 500 & 182 & 27 & -0.8 & 1.8 & 0.14 & 907 & 55.6 & 31 & $1 \quad 0$ \\
\hline $12: 47: \overline{46}: 56$ & 501 & 183 & 27 & -0.8 & 1.8 & 0.13 & 909 & 55.6 & 31 & 0 \\
\hline $12: 47: \overline{4} 7: 54$ & 502 & 183 & 27 & -0.8 & 1.8 & 0.14 & $913^{!}$ & 55.6 & 31 & -0.07 \\
\hline $12: 4 \overline{7}: 4 \overline{8}: 53$ & 503 & 182 & 27 & -0.8 & 1.8 & 0.14 & $916^{!}$ & $55.5^{+}$ & 31 & -0.07 \\
\hline $12: 47: 49: 52$ & 504 & $183^{\dagger}$ & 26 & $-0 . \overline{8}$ & 1.8 & 0.13 & 917 & 55.4 & 31 & -0.26 \\
\hline $12: 47: 50: 51^{\prime}$ & 505 & 183 & 26 & -0.8 & 1.8 & 0.13 & 916 & 55.4 & $\overline{31}$ & -0.26 \\
\hline $12: 47: 51: 50$ & 506 & 183 & 27 & -0.8 & 1.8 & $0 . \overline{14}$ & 912 & 55.3 & 31 & -0.2 \\
\hline $12: 47: 52: 49$ & 507 & $18 \overline{4}$ & $26 !$ & -0.8 & 1.8 & 0.13 & 915 & 55.2 & 31 & -0.2 \\
\hline $12: 47: 53: 48$ & 508 & 184 & 26 & -0.8 & $1.8^{-t}$ & 0.14 & $910^{\circ}$ & $55.2 !$ & 31 & $0.0 \overline{7}$ \\
\hline $12: 47: 54: 46$ & 509 & 185 & 26 & -0.8 & 1.8 & 0.13 & 907 & 55 & 31 & 0.07 \\
\hline $7: 55: 45$ & 510 & $186 !$ & 26 & -0.8 & 1.8 & 0.14 & 908 & 55 & 32 & -0.12 \\
\hline $12: 47: 56: 44$ & 511 & 187 & 26 & -0.8 & 1.8 & 0.14 & 912 & 55 & 32 & -0.12 \\
\hline $7: 57: 43$ & 512 & 186 & 26 & $-0.8^{i}$ & 1.8 & 0.14 & 916 & 55.1 & 32 & -0.27 \\
\hline $12: 47: 58: 42$ & 513 & 187 & 26 & $-0.8^{\prime}$ & 1.8 & 0.14 & 917 & 55.1 & 32 & -0.27 \\
\hline $12: 47: 59: 41$ & 514 & 187 & 26 & -0.8 & 1.8 & 0.14 & 917 & 55.1 & 32 & -0.16 \\
\hline$: 00: 40$ & 515 & 187 & 26 & $-0.8 !$ & 1.8 & 0.14 & 918 & 55.2 & 32 & -0.16 \\
\hline $12: 48$ & 516 & 187 & 26 & -0.8 & 1.8 & 0.14 & 916 & 55.3 & 31 & 0.02 \\
\hline $12: 48: 02: 37$ & 517 & 188 & 26 & -0.8 & 1.8 & 0.14 & 913 & 55.3 & 31 & 0.02 \\
\hline $12: 48: 03: 36$ & 518 & 189 & 26 & -0.8 & 1.8 & $0.14^{\dagger}$ & 909 & 55.3 & 31 & -0.09 \\
\hline $12: 48: 04: 35$ & 519 & 189 & 26 & -0.8 & 1.8 & 0.14 & 907 & $55.3^{-1}$ & 31 & -0.09 \\
\hline $12: 4$ & $520^{\circ}$ & 190 & $26 !$ & -0.8 & $1.8 i$ & 0.14 & 909 & 55.3 & 31 & -0.24 \\
\hline 33 & $5 \overline{2} 1$ & 191 & $26^{i}$ & -0.8 & 1.8 & $0.13^{\dagger}$ & $912^{\prime}$ & 55.4 & 31 & -0.24 \\
\hline $7: 32$ & $5 \overline{2} 2$ & $191 \mathrm{f}$ & $266^{\prime}$ & -0.8 & 1.8 & $0.13^{+}$ & 916 & 55.4 & 32 & -0.19 \\
\hline 31 & 523 & 192 & $27 i$ & -0.8 & 1.8 & 0.14 & 913 & $55.7^{+}$ & $3 \overline{3}$ & -0.19 \\
\hline $9: 29$ & 524 & 191 & $26 !$ & -0.8 & 1.8 & 0.14 & 916 & $5 \overline{5} .8$ & 32 & 0 \\
\hline $10: 28$ & 525 & 191 & $26 !$ & -0.8 & 1.8 & 0.14 & 918 & 55.8 & 32 & 0 \\
\hline $1: 27 !$ & 526 & 192 & 27 & -0.8 & $1.8 \mathrm{i}$ & $0.14 !$ & 917 & 55.8 & 32 & -0.04 \\
\hline $12: 26$ & 527 & $192^{\prime}$ & 27 & -0.8 & 1.8 & $0.14 T$ & 914 & 55.8 & 32 & -0.04 \\
\hline $12: 48: 13: 25$ & 528 & 193 & $26:$ & -0.8 & 1.8 & 0.13 & $910^{\circ}$ & $5 \overline{5.8}$ & 32 & $-0 . \overline{24}$ \\
\hline $12: 48: 14: 24$ & 529 & 193 & 27 & -0.8 & 1.8 & 0.14 & $908:$ & 55.7 & 31 & -0.24 \\
\hline $12: 48: 15: 23$ & 530 & 195 & $27 !$ & -0.8 & 1.8 & $0.13 !$ & $909^{\circ}$ & 55.7 & 32 & -0.18 \\
\hline $12: 48: 16: 22$ & 531 & 195 & $27 i$ & -0.8 & 1.8 & 0.14 & 907 & 55.7 & 32 & -0.18 \\
\hline $12: 48: 17: 20$ & $532^{\dagger}$ & $1 \overline{96}$ & 27 & -0.8 & 1.8 & 0.14 & $909^{\circ}$ & $55.7^{\dagger}$ & $\overline{3} 1$ & $-0.0 \overline{1}$ \\
\hline $3: 19$ & 533 & 195 & 26 & -0.8 & 1.8 & 0.15 & 912 & 55.7 & 32 & -0.07 \\
\hline 18 & 534 & $196 !$ & 26 & -0.8 & $1.8^{+}$ & 0.14 & 916 & 55.7 & 32 & -0.07 \\
\hline $12: 48: 20: 17$ & $535^{\prime}$ & $19 \overline{0}$ & 26 & $-0.8^{i}$ & 1.8 & 0.14 & 918 & 55.6 & 32 & -0.21 \\
\hline $12: 48: 21: 16$ & 536 & 195 & 27 & $-0 . \overline{8}$ & 1.8 & 0.13 & 917 & 55.5 & 32 & -0.21 \\
\hline $12: 48: 22: 15$ & 537 & 196 & 27 & -0.8 & 1.8 & 0.13 & 914 & 55.5 & 32 & -0.22 \\
\hline$: 14$ & 538 & 196 & 26 & -0.8 & 1.8 & 0.13 & 910 & 55.6 & 32 & -0.22 \\
\hline $12: 48: 24: 12$ & 539 & 197 | & 26 & -0.8 & 1.8 & 0.14 & 914 & 55.6 & 32 & -0.06 \\
\hline $12: 48: 25: 11$ & 540 & 198 & 27 & -0.8 & 1.8 & 0.14 & 910 & 55.6 & 32 & -0.06 \\
\hline $12: 48: 26: 10$ & $541^{\circ}$ & 198 & 27 & $-\overline{0.8}$ & 1.8 & 0.13 & 908 & 55.6 & 32 & -0.04 \\
\hline $12: 48: 27: 09$ & 542 & 199 & 27 & -0.8 & 1.8 & 0.14 & 910 & 55.4 & 32 & -0.04 \\
\hline $12: 48: 28: 08$ & 543 & 200 & 27 & -0.8 & 1.8 & 0.14 & 914 & 55.4 & 32 & -0.22 \\
\hline $12: 48: 29: 07$ & 544 & 200 & 26 & -0.8 & 1.8 & 0.14 & 917. & 55.3 & 32 & -0.22 \\
\hline $12: 48: 30: 06$ & 545 & 199 & 27 & -0.8 & 1.8 & 0.14 & 918 & 55.1 & 32 & -0.12 \\
\hline $12: 48: 31: 05$ & 546 & 199 & 27 & -0.8 & 1.8 & 0.14 & 916 & 55.1 & 32 & -0.12 \\
\hline $12: 48: 32: 03$ & 547 & 200 & 27 & -0.8 & 1.8 & 0.14 & 912 & 55 & 32 & -0.09 \\
\hline $12: 48: 33: 02$ & 548 & $20 \overline{1}_{t}^{+}$ & $27^{\dagger}$ & -0.8 & 1.8 & 0.14 & $915^{\circ}$ & 55 & 32 & -0.09 \\
\hline $12: 48: 34: 01$ & 549 & 201 & 26 & -0.8 & 1.8 & 0.14 & 911 & 55 & 32 & -0.06 \\
\hline
\end{tabular}




\begin{tabular}{|c|c|c|c|c|c|c|c|c|c|c|}
\hline & $\begin{array}{l}\text { Elapsed } \\
\text { Time }\end{array}$ & & & 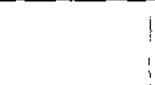 & & ! & & & QUE & \\
\hline$T \backslash M E$ & ! $(\mathrm{sec})$ & BIT_TC1! & PURG_TC2 & PurgePSI: & PurgeCFM & DEPTH $(I N)$ & DwnFrc(lb) & RPM & (FT*LB) & Inches/Min \\
\hline $12: 48: 35: 00$ & 550 & 201 & 27 & -0.8 & 18 & $\quad 0.14$ & $\quad 908$ & 55.1 & 32 & $2 \quad-0.06$ \\
\hline $12: 48: 35: 99$ & 551 & 203 & 27 & -0.8 & 1.8 & 0.14 & 909 & 55.1 & 33 & -0.26 \\
\hline $12: 48: 36: 98$ & 552 & 203 & 26 & -0.8 & $1.8^{\circ}$ & 0.15 & 913 & 55.1 & 33 & -0.26 \\
\hline $12: 48: 37: 97$ & 553 & 204 & 27 & -0.8 & 1.8 & 0.14 & 917 & 55 & 33 & -0.17 \\
\hline $12: 48: 38: 9 \overline{5}$ & 554 & 203 & 26 & -0.8 & 1.8 & 0.14 & 919 & 55.1 & 33 & $-0.1 \overline{7}$ \\
\hline $12: 48: 39: \overline{94}$ & 555 & 201 & 27 & 19.6 & 52.8 & 0.14 & 918 & 55.1 & 32 & -0.02 \\
\hline $12: 48: 40: 93$ & 556 & 191 & $27 !$ & 23.5 & 51.2 & 0.13 & 918 & 55.1 & 28 & -0.02 \\
\hline $12: 48: 41: 92$ & 557 & 185 & 27 ! & 23.7 & 50.9 & $0.14 !$ & 916 & 55.2 & 26 & -0.07 \\
\hline $12: 48: 42: 91$ & 558 & $17 \overline{8}$ & -27 & 23.9 & 50.8 & 0.14 & 910 & 55.2 & 24 & $-0 . \overline{07}$ \\
\hline $12: \overline{48: 43: 90}$ & & 174 ! & 27 & 23.9 & 50.7 & $0 . \overline{14}$ & 906 & 55.4 & 23 & -0.26 \\
\hline $12: 48: 44: 89$ & & 169 & 27 & 23.9 & 50.6 & 0.14 & 905 & 55.5 & 22 & -0.26 \\
\hline $12: 48: 45: 87$ & & 165 & 27 & $23.9 !$ & 50.6 & 0.14 & 905 & 55.5 & 22 & -0.14 \\
\hline $12: 48: 46: 86$ & & 162 & $27^{-1}$ & $23.9^{\circ}$ & 50.6 & 0.15 & 909 & 55.4 & 21 & $-\overline{0} . \overline{4}$ \\
\hline $12: 48: 47: 85$ & & 158 & 27 & $23.9^{i}$ & $50.6^{i}$ & 0.15 & 910 & 55.6 & 21 & -0.04 \\
\hline $12: 48: 48: 84$ & & 155 & $26 !$ & 23.9 & 50.5 & 0.14 & 908 & 55.6 & 21 & -0.04 \\
\hline $12: 48: 49: 83$ & 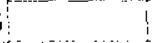 & 151 & 27 & 23.9 & 50.5 & 0.14 & 909 & 55.7 & 20 & -0.05 \\
\hline $12: 48: 50: 82$ & & 148 & 27 & 23.9 & 50.5 & 0.14 & 909 & 55.7 & 20 & -0.24 \\
\hline $12: 48: 51: 81$ & $\cdots$ & 145 & 27 & $23.9^{\dagger}$ & 50.5 & 0.14 & 908 & 55.7 & 20 & -0.24 \\
\hline $12: 48: 52: 80$ & 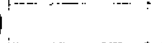 & 143 & 27 & 23.9 & 50.5 & 0.14 & 904 & 55.7 & 20 & -0.22 \\
\hline $12: 48: 53: 78$ & & 141 & 27 & 23.9 & 50.5 & 0.13 & 901 & 55.7 & 19 & -0.22 \\
\hline $12: 48: 54: 77$ & & 138 & 27 & $23.9^{i}$ & $50.5^{\prime}$ & 0.13 & 900 & 55.8 & 19 & -0.04 \\
\hline $12: 48: 55: 76$ & & 137 & 27 & 23.9 & 50.5 & 0.14 & 900 & 55.8 & 19 & -0.04 \\
\hline $12: 48: 56: 75$ & & 134 & 271 & 23.9 & $50.5^{\dagger}$ & 0.15 & 903 & 55.8 & 19 & -0.05 \\
\hline $12: 48: 57: 74$ & & 132 & $27 !$ & 23.9 & 50.5 & 0.14 & 900 & 55.9 & 19 & -0.05 \\
\hline $12: 48: 58: 73$ & & 130 & 27 & 23.9 & 50.5 & 0.14 & 902 & 55.8 & 19 & -0.21 \\
\hline $12: 48: 59: 72$ & & 127 ! & 27 ! & 23.7 & 50.5 & 0.14 & 904 & 55.8 & 19 & -0.21 \\
\hline $12: 49: 00: 70$ & & 125 & 27 & 23.7 & 50.5 & $0.1 \overline{4}$ & 904 & 55.7 & 19 & -0.18 \\
\hline $12: 49: 01: 69$ & 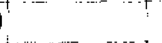 & 123 & 27 & 23.7 & 50.5 & 0.14 & 904 & 55.7 & 19 & -0.18 \\
\hline $12: 49: 02: 68$ & & 121 & 27 & 23.7 & 50.5 & 0.14 & 902 & 55.7 & 19 & -0.11 \\
\hline $12: 49: 03: 67$ & & 119 & 27 & 23.7 & 50.5 & 0.14 & 897 & 55.6 & 19 & -0.11 \\
\hline $12: 49: 04: 66$ & & $118^{+}$ & 27 & 23.7 & 50.5 & 0.14 & 895 & 55.5 & 19 & -0.02 \\
\hline
\end{tabular}




\begin{tabular}{|c|c|c|c|c|c|c|c|c|c|c|}
\hline TIME & $\begin{array}{l}\text { Elapsed } \\
\text { Time } \\
\text { (sec) }\end{array}$ & BIT_TC1 & PURG_TC2 & PurgePSI & PurgeCFM & DEPTH(IN) & DwnFrc(lb) & RPM & $\begin{array}{l}\text { TORQUE } \\
\left(F T^{*} \mathrm{LB}\right)\end{array}$ & !Inches/Min \\
\hline $13: 41: 00: 2$ & 2 & -26 & 27 & $\quad \frac{21}{21}$ & 54.8 & $3 \quad-1.76$ & 1 & 56.9 & -1 & -0.11 \\
\hline $13: 41: 01: 2$ & 1 & 26 & $26^{\circ}$ & 21 & 54.8 & -1.58 & 2 & 56.9 & -1 & 1.7 \\
\hline $13: 41: 02: 2$ & 2 & 26 & 27 & 21 & 54.8 & $-1.27^{\circ}$ & 2 & 56.9 & -1 & 1.7 \\
\hline $41: 03: 2$ & 3 & 26 & 27 & 21 & 54.8 & $-0 . \overline{9}$ & 1 & 56.7 & -1 & 11.54 \\
\hline $13: 41: 04: 2^{+}$ & 4 & 26 & 27 & 21 & $54 . \overline{8}^{\prime}$ & $-0.69^{\prime}$ & 3 & 56.4 & -1 & 11.54 \\
\hline $13: 41: 05: 2$ & 5 & 26 & $27^{\prime}$ & 21.2 & 54.8 & -0.39 & 3 & 56.4 & -1 & 11.9 \\
\hline $41: \overline{06}: 2$ & 6 & 26 & 26 & 21.2 & 54.8 & -0.07 & 5 & 56.4 & -1 & 11.9 \\
\hline $13: 41: 07: 2$ & 7 & 29 & 27 & 21.4 & 54.9 & 0.03 & 521 & 56.3 & 27 & 10.66 \\
\hline $13: 4108: 2$ & 8 & 31 & 27 & 21.6 & 54.9 & 0.05 & $703^{\circ}$ & 56.3 & 30 & 10.66 \\
\hline $13: 41: 09: 2$ & 9 & $33^{\circ}$ & 27 & $21.6^{\dagger}$ & 54.9 & $0.07^{-1}$ & 748 & 56 & 29 & 2.22 \\
\hline $10: 2$ & 10 & 36 & 27 & 21.6 & 54.9 & 0.07 & 772 & 55.6 & 29 & 2.22 \\
\hline $41: 11: 2$ & 11 & 39 & 27 & 21.6 & 54.9 & 0.08 & 782 & 55.6 & 26 & 0.48 \\
\hline $13: 41: 12: 2$ & 12 & 41 & 27 & 21.6 & 54.9 & 0.08 & 788 & 55.5 & 25 & 0.48 \\
\hline $13: 2$ & $\overline{13} !$ & 43 & 27 & 21.6 & 54.9 & $0.1^{i}$ & 794 & 55.5 & $2 \overrightarrow{4}$ & -0.02 \\
\hline $14: 2$ & 14 & 44 & 27 & 21.8 & 54.9 & 0.1 & 801 & $55 . \overline{5}$ & $2 \overline{3}$ & -0.02 \\
\hline $13: 41: 15: 2$ & 15 & 44 & 27 & 21.8 & 54.9 & 0.1 & 803 & 55.3 & 23 & 0.21 \\
\hline $16: 2$ & 16 & 46 & 27 & 21.8 & 54.9 & 0.1 & 814 & 55.3 & 22 & 0.21 \\
\hline $17: 2$ & 17 & $46 !$ & 27 & 21,8 & 54.9 & 0.1 & $831^{\circ}$ & 55.3 & 23 & 0.01 \\
\hline $13: 41: 18: 2$ & 18 & 48 & 27 & $21.8^{\prime}$ & 54.9 & 0.12 & $851^{\circ}$ & 55.4 & 23 & 0.11 \\
\hline $19: 2$ & 19 & 48 & $26:$ & 21.8 & 54.9 & $0.1 \mathrm{i}$ & 866 & 55.2 & $23^{\dagger}$ & 0.11 \\
\hline & 20 & 49 & 27 & 21 & 4.9 & $0 . \overline{12}$ & 874 & $55.2^{\prime}$ & 23 & -0.14 \\
\hline $21: 2^{4}$ & $21 !$ & 50 & $27^{\prime}$ & 21.8 & 54.9 & 0.1 & 879 & $55.3^{\circ}$ & 23 & -0.14 \\
\hline $13: 41: 22: 2$ & 22 & 50 & 27 & 21.8 & 54.9 & 0.1 & $878 !$ & 55 & 23 & -0.02 \\
\hline $23: 2$ & 23 & 51 & 27 & 21.8 & 54.9 & 0.12 & 879 & $55^{\circ}$ & 22 & -0.02 \\
\hline $24: 2$ & 24 & 51 & 27 & 21.8 & 54.9 & 0.12 & 884 & 54.8 & 22 & 0.14 \\
\hline $41: 25: 3$ & 25 & $52^{\prime}$ & 27 & 21.8 & 54.9 & 0.12 & $892^{\circ}$ & 54.8 & 22 & 0.14 \\
\hline $26: 2$ & $26^{\dagger}$ & $52 !$ & 27 & 21.8 & 54.9 & $0.13^{\dagger}$ & 894 & 54.8 & 22 & -0.07 \\
\hline $27: 2$ & $27 i$ & 53 & 27 & 21.8 & $54.9^{\prime}$ & 0.13 & 899 & 54.9 & 23 & -0.07 \\
\hline $41: 28: 2^{\prime}$ & 28 & 53 & 27 & 21.8 & 54.9 & 0.13 & 905 & $54.9^{+}$ & 23 & -0.18 \\
\hline $9: 2$ & 29 & 53 & 27 & & & & 911 & 54.9 & 23 & -0.18 \\
\hline & $30 !$ & 54 & 27 & & 54.9 & $\theta^{+}$ & 915 & 55 & 23 & -0.01 \\
\hline $1: \overline{2}$ & 31 & 54 & 27 & 21.8 & $54.9^{\dagger}$ & 0.13 & 918 & 55 & 23 & -0.01 \\
\hline $32: 2$ & 32 & 54 & 26 & 21.8 & 54.9 & 13 & 919 & 55 & 23 & 0.14 \\
\hline $33: 2$ & 33 & 54 & 27 & & 9 & & 916 & 54.8 & 23 & 0.14 \\
\hline $4: 2$ & 34 & $55 !$ & 27 & 21.8 & 54.9 & 13 & 912 & 54.8 & $23 !$ & -0.2 \\
\hline $35: 3$ & 35 & 55 & 26 & 22 & $54.9^{+}$ & $0.13^{7}$ & 912 & 54.8 & 23 & -0.2 \\
\hline & 36 & 55 & 27 & 22 & & 14 & 9 & 54.9 & 23 & -0.12 \\
\hline & 37 & 55 & 27 & 22 & 54.9 & 0.14 & 915 & 54.8 & 23 & -0.12 \\
\hline $1: 38: 3$ & 38 & 56 & 27 & 22 & 54.9 & $0.1 \overline{14}$ & 912. & 54.8 & 23 & -0.06 \\
\hline $39: 3$ & $39^{t}$ & 56 & 27 & $22^{4}$ & 54.9 & $14^{\dagger}$ & $915^{\circ}$ & 54.7 & 23 & -0.06 \\
\hline & 40 & 56 & 27 & 22 & 54.9 & 0.13 & 917 & 54.6 & 23 & -0.06 \\
\hline & 41 & 56 & 27 & 22 & 54.9 & & 917 & 54.6 & 23 & -0.22 \\
\hline $42: 2$ & 42 & 56 & 27 & 22 & 54.9 & 0.14 & 912 & 54.6 & $23^{\dagger}$ & -0.22 \\
\hline & $43^{\dagger}$ & 56 & 27 & 22 & & & 915 & $54.7 !$ & 23 & $-0 . \overline{16}$ \\
\hline & 44 & 56 & 27 & $22^{-+}$ & 54.9 & 0.13 & & 54.7 & 23 & -0.16 \\
\hline $45: 2$ & 45 & 56 & 27 & 22 & 54.9 & $0 . \overline{13}$ & 906 & 54.8 & 23 & 0.02 \\
\hline $13: 41: 46: 3$ & 46 & 57 & 27 & 22 & 54.9 & 0.14 & 906 & 55 & 23 & 0.02 \\
\hline & 47 & 57 & 27 & 22 & 54.9 & 0.15 & 910 & 55 & 23 & -0.1 \\
\hline & 48 & 57 & 27 & 22 & 54.9 & 0.14 & 914 & $55 . \overline{3}$ & 23 & -0.1 \\
\hline $49: 3$ & 49 & 57 & 27 & 22 & 54.9 & 0.14 & $911^{\circ}$ & $55 . \overline{6}$ & 22 & -0.2 \\
\hline $41: 50: 2$ & 50 & 57 & 27 & 22.2 & 54.9 & 0.14 & $916^{\circ}$ & $55.6^{\prime}$ & 22 & -0.2 \\
\hline $51: 3$ & 51 & 58 & 27 & 22.2 & 54.9 & 0.14 & $918^{\circ}$ & 55.7 & 22 & -0.2 \\
\hline $52: 3$ & 52 & 58 & 27 & 22 & & & $919^{\circ}$ & $55 . \overline{7}$ & 22 & -0.2 \\
\hline & 53 & 58 & 27 & 22.2 & $54 . \overrightarrow{9}$ & 0.14 & 916 & 55.7 & 22 & 0.14 \\
\hline $41: 54: 3$ & $54 !$ & $5 \overline{8}$ & $27^{\circ}$ & 22.2 & $5 \overline{4.9}$ & 0.14 & 913 & 55.7 & 22 & 0.14 \\
\hline $41: 55: 2$ & 55 & 58 & 27 & 22.2 & 54.9 & 0.14 & 917 & 55.6 & 22 & -0.17 \\
\hline $6: 2$ & 56 & & & 22.2 & 549 & 0.14 & $913^{\circ}$ & 55.6 & 22 & -0.1 \\
\hline
\end{tabular}




\begin{tabular}{|c|c|c|c|c|c|c|c|c|c|c|}
\hline TIME & $\mid \begin{array}{l}\text { Elapsed } \\
\text { Time } \\
\text { (sec) }\end{array}$ & & & & & & DwnFrc(b) & RPM & $\begin{array}{l}\text { TORQUE } \\
\left(F^{*} L B\right)\end{array}$ & Inches/Min \\
\hline $13: 41: 57: 2$ & $\quad 57$ & 58 & 27 & 22.2 & 54.9 & $\quad 0.14$ & 911 & 55.7 & 21 & -0.17 \\
\hline $13: 41: 58: 2$ & 58 & 58 & 27 & 22.2 & 54.9 & 0.15 & 911 & 55.7 & 22 & -0.17 \\
\hline $13: 4159: 2$ & 59 & 59 & 27 & 22.2 & $54.9^{\circ}$ & 0.15 & 914 & 55.7 & 21 & -0.28 \\
\hline $13: 42: 00: 2$ & 60 & 59 & 27 & $22.2^{-1}$ & 54.9 & $0.15^{+}$ & 919 & 55.8 & 21 & -0.28 \\
\hline $13: 42: 01: 2$ & 61 & 59 & 27 & 22.2 & 54.9 & 0.14 & 922 & 55.8 & 21 & 0.05 \\
\hline $13: 42: 02: 2$ & 62 & 59 & 27 & $22.2 !$ & 54.9 & 0.15 & 923 & 55.8 & 21 & 0.05 \\
\hline $13: 42: 03: 2$ & 63 & 59 & 27 & 22.2 & $54 \overline{9}$ & 0.14 & 922 & 558 & 21 & .0 .04 \\
\hline $13: 42: 04: 2$ & 64 & 59 & 27 & 22.2 & 54.9 & 0.14 & 924 & 55.9 & 21 & -0.04 \\
\hline $13: 42: 05: 2$ & 65 & 59 & 27 & $22.2^{-1}$ & 54.9 & 0.14 & 922 & 55.9 & 21 & -0.23 \\
\hline $13: 42: 06: 2$ & 66 & 59 & 27 & 22.2 & 54.9 & 0.14 & 918 & 55.9 & $21^{1}$ & -0.23 \\
\hline $13: 42: 07: 2$ & 67 & 59 & $27 !$ & $22.2 !$ & 54.9 & 0.14 & 915 & 55.9 & 21 & -0.23 \\
\hline $13: 42: 08: 2$ & 68 & 59 & 27 & 22.2 & 549 & 0.14 & 912 & $5 \overline{5.9}$ & $29 !$ & -0.14 \\
\hline $13: 42: 09: 2$ & $69^{\dagger}$ & 59 & 27 & 22.2 & 54.9 & 0.13 & $912^{\square}$ & 55.8 & $21 i$ & -0.14 \\
\hline $13: 42: 10: 2$ & 70 & 59 & 27 & 22.2 & 54.9 & 0.14 & 910 & 55.8 & 21 & 0.02 \\
\hline $13: 42: 11: 2$ & 71 & 59 & 27 & 22.2 & 54.9 & 0.15 & 912 & 558 & 21 & 0.02 \\
\hline $13: 42: 12: 2$ & 72 & 59 & 27 & 22.4 & 54.9 & $0 . \overline{14}$ & 915 & 55.8 & $21^{\dagger}$ & -0.23 \\
\hline $13: 42: 13: 2$ & 73 & 59 & 27 & 22.4 & 549 & 0.14 & 917 & 55.6 & 21 & -0.23 \\
\hline $13: 42: 14: 2$ & 74 & 59 & $27 !$ & $22.2 !$ & 549 & 0.14 & 919 & 55.6 & 21 & -0.22 \\
\hline $13: 42: 15: 2$ & 75 & 59 & 27 & 22.4 & $54.9^{1}$ & 0.14 & 916 & 55.5 & 21 & -0.22 \\
\hline $13: 42: 16: 2$ & 76 & 59 & 27 & 22.4 & $54.9]$ & 0.94 & 917 & $55 . \overline{5}$ & 20 & -0.14 \\
\hline $13: 42: 17: 2$ & 77 & 59 & 27 & 22.2 & 54.9 & 0.14 & 915 & 55.5 & 21 & -0.14 \\
\hline $13: 42: 18: 2$ & 78 & 59 & 27 & $22.4^{\dagger}$ & 54.9 & 0.14 & 910 & 55.5 & 21 & 0.04 \\
\hline $13: 42: 19: 2$ & 79 & 59 & 27 & 22.2 & 54.9 & 0.14 & 905 & 55.5 & 20 & 0.04 \\
\hline $13: 42: 20: 2$ & 80 & 59 & 27 & $22.4 !$ & 54.9 & 0.14 & 907 & 55.5 & 21 & -0.28 \\
\hline 212 & 81 & 59 & $27 !$ & 22.4 & 54.9 & $0.14 \mathrm{j}$ & 905 & 55.3 & 21 & -0.28 \\
\hline $13: 42.222$ & 82 & 58 & 27 & 22.4 & 54.9 & 0.14 & 905 & 55.2 & 21 & -0.25 \\
\hline $13: 42: 23: 2$ & $83^{\dagger}$ & 59 & 27 & 22.4 & 54.9 & 0.15 & 910 & 55.2 & 21 & $-0 . \overline{25}$ \\
\hline $13: 42: 24: 2$ & 84 & 59 & 27 & 22.4 & 54.9 & 0.14 & 914 & 55.1 & 21 & -0.12 \\
\hline $13: 42: 25: 2$ & 85 & 59 & $27^{-1}$ & 22.4 & 54.9 & 0.15 & 917 & 55 & 20 & -0.12 \\
\hline $13: 42: 26: 2$ & 86 & 58 & $27 !$ & 22.4 & 54.9 & $0.14 !$ & 915 & 55 & 20 & -0.03 \\
\hline $13: 42: 27: 2$ & 87 & 58 & 27 & 22.4 & 54.9 & 0.14 & 916 & 55.1 & 20 & -0.03 \\
\hline $8: 2$ & 88 & 58 & 27 & 22.4 & 549 & 0.14 & 915 & $55.1 !$ & 20 & -0.29 \\
\hline $13: 42: 29: 2$ & 89 & 58 & $27 i$ & $22.4 !$ & 549 & 0.14 & $911 !$ & 55.1 & $20 !$ & -0.29 \\
\hline $13: 42: 30: 2$ & 90 & 58 & $27 j$ & 22.4 & $54.9 !$ & 0.14 & 907 & 55.2 & 20 & -0.21 \\
\hline $1: 2$ & 91 & 58 & 27 & 22.4 & 54.9 & 0.14 & 907 & 55.5 & 20 & -0.01 \\
\hline & 92 & 59 & 27 & 22.4 & 54.9 & $0.1 \overline{5}$ & 909 & 55.5 & 20 & -0.01 \\
\hline $13: 42: 33: 1$ & 93 & 59 & 27 & 21.8 & 54.9 & 0.14 & 912 & 55.6 & $20_{4}^{+}$ & -0.1 \\
\hline $13: 42: 34: 1$ & 94 & 59 & 27 & 21.8 & 54.9 & 0.15 & 910 & 55.7 & $20^{+}$ & -0.1 \\
\hline $13: 42: 35: 1$ & 95 & 59 & $27 !$ & 21.8 & 54.8 & 0.14 & 913 & 55.7 & 20 & -0.22 \\
\hline $13: 42: 36: 2$ & 96 & 58 & $27 !$ & 21.8 & 54.7 & 0.15 & 916 & 55.8 & 20 & -0.22 \\
\hline $7: 2$ & 97 & 58 & 27 & 21.8 & 54.5 & 0.14 & 917 & 55.7 & 20 & -0.24 \\
\hline $38: 1$ & 98 & 59 & 27 & 21.8 & 54.5 & 0.15 & 917 & 55.7 & 20 & 0.24 \\
\hline $3 \overline{9}: 1$ & 99 & 58 & 27 & $21.8^{+}$ & & 0.15 & 912 & 55.7 & 20 & $-\overline{0.11}$ \\
\hline $40: 1$ & 100 & 58 & 27 & $21.8^{+}$ & 54.4 & 0.14 & 908 & 55.6 & 20 & -0.11 \\
\hline $13: 42: 41: 2$ & 101 & 58 & 27 & 21.8 & 54.3 & 0.14 & $9 \overline{12}$ & 55.6 & 20 & -0.07 \\
\hline $42: 1$ & 102 & 58 & 27 & 21.8 & 54.3 & 0.15 & 907 & 55.6 & $20^{+}$ & -0.07 \\
\hline 43:1 & 103 & 58 & 27 & & & & 907 & 55.7 & 20 & -0.14 \\
\hline $13: 42: 44: 2$ & 104 & 58 & 27 & 21.8 & 54.3 & 0.15 & $909^{7}$ & 55.7 & 20 & -0.14 \\
\hline $13: 42: 45: 2$ & 105 & 58 & $27 i$ & $21 . \overline{8}$ & 54.3 & 0.15 & $907 i$ & 55.7 & $20 !$ & -0.29 \\
\hline $46: 2$ & 106 & 59 & 27 & 21.8 & 54.3 & 0.17 & 910 & $55 . \overline{6}$ & 20 & -0.29 \\
\hline $13: 42: 47: 1$ & $10 \overline{7}$ & 59 & 27 ! & 22 & 54.3 & & 914 & 55.6 & 20 & 0.02 \\
\hline $13: 42: 48: 1$ & 108 & 58 & 27 & 22 & 54.2 & 0.15 & $91 \overline{7}$ & 55.5 & 20 & 0.02 \\
\hline $13: 42: 49: 2$ & 109 & 58 & 27 & 22 & 54.2 & 0.15 & 919 & 55.4 & & -0.2 \\
\hline & 110 & 58 & $27^{\dagger}$ & 22 & 542 & 0.15 & 917 & 55.4 & $20^{\dagger}$ & -0.2 \\
\hline $13: 42: 512$ & 111 & 58 & 27 & 22 & 54.2 & 0.14 & 912 & 55.3 & 20 & -0.16 \\
\hline $13: 42: 52: 1$ & 112 & 58 & $27 !$ & 22 & 54.2 & 0.14 & 916 & 55.4 & 20 & -0.16 \\
\hline $13: 42: 53: 1$ & 113 & 58 & 27 & 22 & 54.2 & $0.15_{1}^{+}$ & 912 & 55.4 & 20 & -0.29 \\
\hline
\end{tabular}




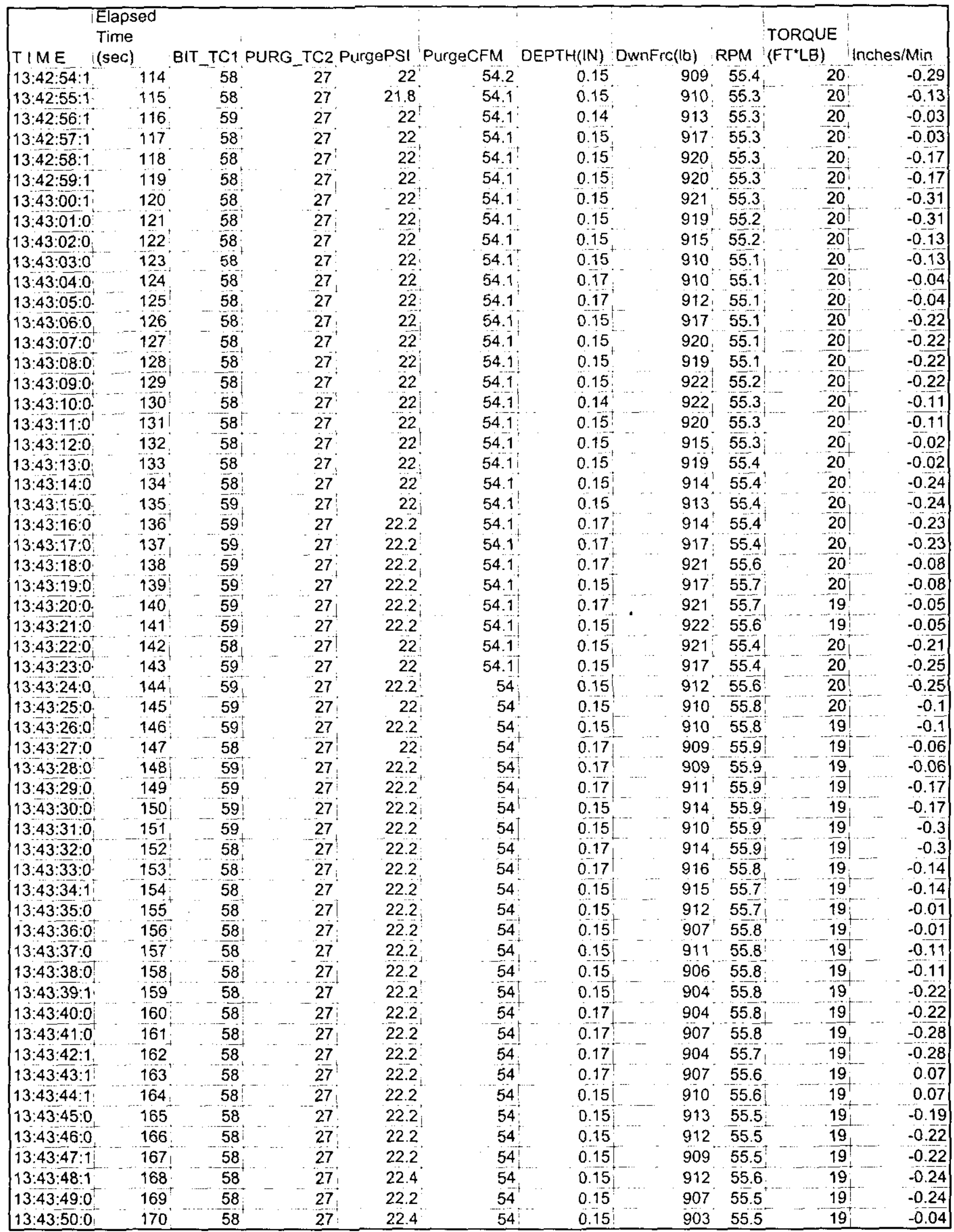




\begin{tabular}{|c|c|c|c|c|c|c|c|c|c|c|}
\hline \multicolumn{2}{|c|}{ 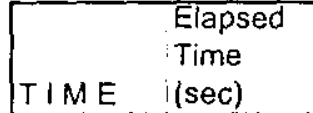 } & BIT_TC1 & \multicolumn{2}{|c|}{ PURG_TC2 PurgePSI } & \multicolumn{2}{|c|}{ PurgeCFM DEPTH(IN) } & DwnFrc(b) & \multicolumn{2}{|c|}{\begin{tabular}{l|l} 
& TORQUE \\
$R P M$ & $\left(\mathrm{FT}^{*} \mathrm{LB}\right)$
\end{tabular}} & 'Inches/Min \\
\hline $13: 43: 51: 0$ & $\quad 171$ & 58 & $\quad 27$ & 22.2 & 54 & $\quad 0.15$ & $5 \quad 902$ & 55.5 & 19 & $\begin{array}{ll}3 & -0.04\end{array}$ \\
\hline $13: 43: 52: 0$ & 172 & 58 & 27 & 22.2 & 53.9 & 0.15 & 903 & 55.5 & 19 & $-0 . \overline{1}$ \\
\hline $13: 43: 53: 0$ & 173 & 58 & 27 & 22.4 & 53.9 & 0.15 & 905 & $55 . \overline{5}^{\prime}$ & 19 & -0.1 \\
\hline $13: 43: 54: 0$ & $174^{\prime}$ & 58 & 27 & 22.4 & $53 . \overline{9}$ & 0.17 & 909 & 55.5 & 19 & -0.22 \\
\hline $13: 43: 55: 0$ & 175 & 58 & 27 & 22.4 & 53.9 & 0.15 & 911 & 55.5 & 19 & -0.22 \\
\hline $13: 43: 56: 0$ & 176 & 58 & 27 & 22.4 & 53.9 & 0.15 & 909 & $55.5^{2}$ & 19 & -0.26 \\
\hline $13: 43: 57: 0$ & 177 & 58 & 27 & 22.4 & $53 . \overline{9}$ & 0.15 & 912 & 55.6 & 19 & -0.26 \\
\hline $13: 43: 5 \overline{7}: 9$ & 178 & 58 & 27 & 22.4 & 53.9 & 0.15 & 910 & 55.5 & 19 & 0.03 \\
\hline $13: 43: 59: 0$ & 179 & 57 & 27 & 22.4 & $53 . \overline{9}$ & 0.15 & 906 & 55.5 & 19 & 0.03 \\
\hline $13: 44: 00: 0$ & 180 & 57 & 27 & $22.4^{\dagger}$ & 53.9 & 0.15 & 902 & $55.5^{\circ}$ & 19 & -0.15 \\
\hline $13: 44: 01: 0$ & 181 & 58 & 27 & 22.2 & 53.9 & 0.17 & 900 & 55.4 & 19 & -0.15 \\
\hline $13: 44: 02: 0$ & 182 & 57 & 27 & 22.2 & 53.9 & 0.17 & 901 & $55.4^{1}$ & 19 & -0.21 \\
\hline $13: 44: 02: 9$ & 183 & $57 !$ & 27 & 22.4 & 53.9 & 0.15 & 900 & 55.4 & 19 & -0.21 \\
\hline $13: 44: 0 \overline{4}: 0$ & 184 & 57 & 27 & 22.4 & 53.9 & 0.17 & 903 & 55.5 & 19 & -0.25 \\
\hline $13: 44: 05: 0$ & 185 & 57 & 27 & $22.4^{i}$ & 53.9 & 0.17 & 906 & $55 . \overline{5}$ & 19 & -0.25 \\
\hline $13: 44: 06: 0$ & 186 & $57 !$ & 27 & $22.4 !$ & 53.9 & 0.17 & 909 & 55.5 & 19 & -0.03 \\
\hline $13: 44: 07: 0$ & 187 & 57 & 27 & $22 . \overline{4}$ & 53.9 & 0.17 & 911 & 55.5 & 19 & -0.03 \\
\hline $13: 44: 07: 9$ & 188 & 57 & 27 & 22.4 & 53.9 & $0 . \overline{17}$ & 910 & $55.5^{t}$ & 18 & -0.03 \\
\hline $13: 44: 08: 9$ & 189 & $5 \overline{7}$ & 27 & 22.2 & 53.9 & 0.17 & 911 & 55.5 & 19 & -0.03 \\
\hline $13: 44: 09: 9$ & 190 & $57^{\prime}$ & 27 & $22.4^{\circ}$ & 53.9 & 0.17 & 909 & $55.5^{+}$ & 19 & -0.25 \\
\hline $13: 44: 10: 9$ & 191 & 57 & 27 & 22.4 & 53.9 & 0.15 & 905 & 55.5 & 19 & -0.25 \\
\hline $13: 44: 119$ & 192 & 57 & 27 & 22.4 & 53.9 & 0.17 & 901 & 55.6 & 19 & -0.21 \\
\hline $13: 44: 12: 9$ & $193^{\dagger}$ & 57 & 27 & 22.4 & 53.9 & 0.17 & 900 & $55 . \overline{5}^{\dagger}$ & 19 & -0.07 \\
\hline $13: 44: 13: 9$ & 194 & 57 & 27 & 22.4 & 53.9 & 0.18 & 904 & 55.5 & 18 & -0.07 \\
\hline $13: 44: 14: 9$ & 195 & 57 & 27 & 22.4 & 53.9 & 0.17 & 906 & 55.4 & 18 & -0.05 \\
\hline $13: 44: 15: 9$ & 196 & 57 & 27 & 22.4 & 53.9 & 0.17 & 904 & 55.3 & 18 & $-0.0 \overline{5}$ \\
\hline $13: 44: 16: 9$ & 197 & 57 & 27 & 22.4 & 53.9 & 0.17 & 907 & 55.3 & 98 & -0.27 \\
\hline $13: 44: 17: 9$ & $198^{\dagger}$ & 57 & 27 & $22.4^{+}$ & 53.9 & 0.15 & 910 & 55.2 & 18 & -0.27 \\
\hline $13: 44: 18: 9$ & 199 & 57 & 27 & 22.4 & 53.9 & 0.17 & 910 & $55 . \overline{2}$ & 18 & $-0.2 \overline{1}$ \\
\hline $13: 44: 19: 9$ & 200 & 57 & 27 & 22.4 & 53.9 & 0.17 & 906 & 55.2 & 19 & $-0.2 \overline{1}$ \\
\hline $13: 44: 20$ & 201 & 57 & 27 & 22.4 & 53.9 & 0.17 & 902 & 55.2 & 18 & -0.02 \\
\hline $13: 44: 21: 9$ & 202 & 57 & 27 & 22.4 & 53.9 & 0.17 & 905 & $55.1^{\dagger}$ & 19 & -0.02 \\
\hline $13: 44: 22: 9$ & 203 & 57 & 27 & 22.4 & 53.9 & 0.17 & 900 & $5 \overline{5} .1$ & 19 & -0.14 \\
\hline $13: 44: 23: 9$ & 204 & 57 & 27 & 22.4 & 53.9 & 0.17 & 900 & 55.3 & 19 & -0.14 \\
\hline $13: 44: 24: 9$ & $205^{\circ}$ & $57^{\dagger}$ & 27 & 22.4 & 53.9 & 0.18 & 902 & 55.6 & 19 & -0.22 \\
\hline $13: 44: 25: 9$ & 206 & 57 & 27 & 22.4 & 53.9 & 0.17 & 904 & 55.6 & 19 & -0.22 \\
\hline $13: 44: 26: 9$ & 207 & 57 & 27 & 22.4 & $5 \overline{3} .8$ & 0.17 & 908 & 55.8 & 19 & -0.19 \\
\hline $13: 44: 27: 9$ & 208 & 57 & 27 & 22.4 & 53.8 & 0.17 & $905^{\circ}$ & $5 \overline{5.9}$ & 19 & -0.19 \\
\hline $13: 44: 28: 9$ & $209^{\circ}$ & 57 & 27 & 22.4 & 53.8 & 0.17 & 908 & 55.9 & 18 & -0.02 \\
\hline $13: 44: 29: 9$ & $210^{\prime}$ & 57 & $27 i$ & 22.4 & 53 & 0.17 & 910 & $55.9^{9}$ & 18 & -0.02 \\
\hline $13: 44: 30: 9$ & 211 & 57 & $27 !$ & 22.4 & 53.8 & 0.17 & 910 & 55.9 & 18 & -0.1 \\
\hline $13: 44: 31: 9$ & 212 & 57 & 27 & 22.4 & $53 \overline{8}$ & 0.17 & 905 & 55.9 & 18 & -0.1 \\
\hline $13: 44: 32: 9$ & 213 & $57 !$ & 27 & 22.4 & 53.8 & 0.15 & 909 & 55.8 & 18 & -0.2 \\
\hline $13: 44: 33: 9$ & 214 & 57 & 27 & 22.4 & 53.8 & 0.15 & 906 & 55.8 & 18 & -0.2 \\
\hline $13: 44: 34: 9$ & 215 & 57 & $27 !$ & 22.4 & 53.8 & 0.15 & 902 & 55.8 & 18 & -0.26 \\
\hline $13: 44: 35: 9$ & 216 & 57 & 27 & 22.4 & 53.8 & 0.17 & 900 & 55.9 & 19 & -0.26 \\
\hline $13: 44: 36: 9$ & 217 & 57 & 27 & 22.6 & 53.8 & 0.17 & 901 & 55.9 & $\overline{19}$ & -0.12 \\
\hline $13: 44: 37: 9$ & 218 & 57 & $27 !$ & 22.6 & 53.8 & 0.17 & 904 & 55.9 & 19 & -0.04 \\
\hline $13: 44: 38: 9$ & 219 & $5 \overline{7}$ & 27 & $10.3^{1}$ & 1.8 & 0.15 & 902 & 55.9 & 19 & -0.04 \\
\hline $13: 44: 39: 9$ & 220 & 58 & 27 & -0.8 & 1.8 & 0.15 & 904 & 56 & 19 & -0.17 \\
\hline $13: 44: 40: 9$ & 221 & 60 & 27 & -0.8 & 1.8 & 0.17 & 907 & 56 & 19 & $-0.1 \overline{7}$ \\
\hline $13: 44: 41: 9$ & 222 & 62 & 27 & -0.8 & 1.8 & 0.17 & 910 & 56.1 & 20 & -0.25 \\
\hline $13: 44: 42: 9$ & 223 & $63^{\dagger}$ & 27 & $-0 . \overline{8}$ & 1.8 & 0.17 & 911 & 56.1 & 19 & -0.25 \\
\hline $13: 44: 43: 9$ & 224 & 64 & 27 & -0.8 & 1.8 & 0.17 & 910 & 56.1 & 20 & -0.18 \\
\hline $13: 44: 45: 0$ & 225 & $65 !$ & 27 & -0.8 & 1.8 & 0.15 & 912 & 55.9 & 20 & -0.18 \\
\hline $13: 44: 46: 0$ & 226 & 67 & 27 & -0.8 & 1.8 & 0.15 & 909 & 55.8 & 20 & 0.04 \\
\hline $13: 44: 46: 9$ & 227 & 68 & 27 & -0.8 & 1.8 & 0.15 & $905^{\circ}$ & 55.8 & 20 & 0.04 \\
\hline
\end{tabular}




\begin{tabular}{|c|c|c|c|c|c|c|c|c|c|c|}
\hline TIME & $\begin{array}{l}\text { Elapsed } \\
\text { Time } \\
\text { (sec) }\end{array}$ & BIT_TC1 & PURG_TC2 & PurgePSI & PurgeCFM & DEPTH(IN) & DwnFrc(lb) & RPM & $\begin{array}{l}\text { TORQUE } \\
\left(\mathrm{FT}^{*} \mathrm{LB}\right)\end{array}$ & Inches/Min \\
\hline $13: 44: 47: 9^{\prime}$ & 228 & 69 & 27 & -0.8 & $3 \quad 1.8$ & $\quad 0.15$ & $\begin{array}{r}9 \\
902\end{array}$ & 557 & 20 & $\begin{array}{ll}-0.14 \\
0\end{array}$ \\
\hline $13: 44: 48: 9$ & 229 & $70:$ & 27 & -0.8 & 1.8 & 0.15 & 903 & 55.6 & 21 & -0.14 \\
\hline $13: 44: 50: 0$ & $230^{\circ}$ & $71 i$ & 27 & -0.8 & 1.8 & 0.17 & 903 & 55.6 & 21 & -0.2 \\
\hline $13: 44: 51: 0$ & 231 & $72 i$ & 27 & -0.8 & $1.8^{\circ}$ & 0.17 & 904 & $5 \overline{5} .6$ & 21 & -0.2 \\
\hline $13: 44: 51: 9$ & 232 & 73 & 27 & -0.8 & 1.8 & 0.17 & 907 & 55.7 & 21 & -0.28 \\
\hline $13: 44: 53: 0$ & 233 & 74 & $27^{\prime}$ & -0.8 & $1.8^{+}$ & 0.17 & $911^{\circ}$ & 55.7 & 22 & -0.28 \\
\hline $13: 44: 54: 0$ & 234 & $7 \overline{5}$ & 27 & -0.8 & 1.8 & 0.17 & 913 & 55.7 & 22 & \\
\hline $13: 44: 5 \overline{5}: 0$ & 235 & 76 & 27 & -0.8 & 1.8 & 0.17 & $914^{\dagger}$ & 55.8 & 22 & 0 \\
\hline $13: 44: 55: 9$ & 236 & 77 & 27 & -0.8 & 1.8 & 0.17 & $914^{\circ}$ & 55.8 & 22 & -0.1 \\
\hline $13: 44: 56: 9$ & $2 \overline{37}$ & 78 & 27 & -0.8 & 1.8 & 0.15 & 915 & 55.8 & 22 & -0.1 \\
\hline $13: 44: 57: 9$ & 238 & 79 & 27 & -0.8 & 1.8 & 0.15 & $913^{\circ}$ & 55.9 & 22 & -0.21 \\
\hline 13:44:58:9' & $239^{\circ}$ & 80 & 27 & -0.8. & 1.8 & 0.17 & $909^{\circ}$ & 55.9 & 22 & -0.21 \\
\hline $13: 44: 59: 9$ & $240^{\dagger}$ & 81 & 27 & -0.8 & $1.8 \mathrm{i}$ & 0.17 & 905 & 55.9 & 23 & -0.08 \\
\hline $13: 45: 00: 9$ & 241 & $82 !$ & 27 & -0.8 & 1.8 & 0.17 & $905^{\circ}$ & 55.7 & 23 & -0.16 \\
\hline $13: 45: 01: 9$ & 242 & 831 & $27^{\prime}$ & -0.8 & 1.8 & 0.17 & $908^{\circ}$ & $55.7^{+}$ & 23 & -0.16 \\
\hline $13: 45: 02: 9$ & 243 & 84 & 27 & $-0.8^{\dagger}$ & 1.8 & 0.17 & 912 & 55.7 & 23 & -0.02 \\
\hline $13: 45: 03: 9$ & 244 & 85 & 27 & $-0.8^{+}$ & 1.8 & 0.17 & $914^{\circ}$ & $5 \overline{5} .7^{+}$ & 23 & -0.02 \\
\hline $13: 45: 04: 8$ & $\overline{2} 45$ & 86 & 27 & $-0.8^{\dagger}$ & $1.8^{\dagger}$ & 0.17 & 912 & $55.9^{\circ}$ & 23 & -0.25 \\
\hline $13: 45: 05: 8$ & 246 & 87 & 27 & -0.8 & $1.8^{\dagger}$ & 0.15 & 915 & $55.9^{\prime}$ & 23 & -0.25 \\
\hline $13: 45: 06: 8$ & 247 & 87 & 27 & -0.8 & 1.8 & 0.15 & $917^{\circ}$ & 55.9 & 23 & -0.22 \\
\hline $13: 45: 07: 8$ & 248 & 88 & $27^{\circ}$ & -0.8 & $1.8^{\prime}$ & 0.15 & 917 & 55.9 & 24 & -0.22 \\
\hline $13: 45: 08: 8^{+}$ & $249^{\circ}$ & 89 & 27 & -0.8 & 1.8 & 0.15 & 912 & 55.8 & 24 & -0.08 \\
\hline $13: 45: 09: 8$ & 250 & 90 & 27 & -0.8 & 1.8 & $0.15^{\prime}$ & 908 & 55.8 & 24 & -0.08 \\
\hline $13: 45: 10: 8$ & 251 & $91 !$ & 27 & -0.8 & 1.8 & 0.17 & 907 & 55.8 & 24 & -0.06 \\
\hline $13: 45: 11: 8$ & $252^{\circ}$ & 92 & 27 & -0.8 & 1.8 & 0.15 & $909^{\circ}$ & 55.8 & 24 & -0.06 \\
\hline $13: 45: 12: 8$ & $253^{\circ}$ & 93 & 27 & -0.8 & 1.8 & 0.171 & $912^{\circ}$ & 55.8 & 24 & -0.12 \\
\hline $13: 45: 13: 7$ & 254 & $\overline{9} 4$ & 27 & $-0.8^{1}$ & $1.8^{+}$ & 0.17 & $910^{\circ}$ & 55.7 & 24 & -0.12 \\
\hline $13: 45: 14: 7$ & $255^{\circ}$ & 95 & 27 & $-0.8^{\prime}$ & 1.8 & $0.17 i$ & 914 & 55.6 & 24 & -0.22 \\
\hline $13: 45: 15: 7$ & 256 & 95 & 27 & $-0.8^{\vdots}$ & $1.8^{4}$ & $0.17 i$ & 916 & 55.6 & 24 & -0.22 \\
\hline $13: 45: 16: 7$ & 257 & 96 & 27 & -0.8 & 1.8 & $0.17 i$ & $919^{3}$ & 55.3 & 24 & -0.15 \\
\hline $13: 45: 17: 7$ & 258 & 97 & 27 & -0.8 & 1.8 & 0.17 & $917^{\circ}$ & 55.4 & 24 & -0.15 \\
\hline $13: 45: 18: 7$ & 259 & 98 & 27 & -0.8 & 1.8 & 0.15 & $913^{\circ}$ & $55 . \overline{4}$ & 24 & -0.07 \\
\hline $13: 45: 19: 7$ & 260 & 98 & 27 & -0.8 & 1.8 & 0.17 & $909^{\prime}$ & 55.5 & 25 & -0.07 \\
\hline $13: 45: 20: 7$ & 261 & $99^{+}$ & 27 & -0.8 & $1.8^{i}$ & 0.15 & $909^{\circ}$ & $55 . \overline{6}^{\prime}$ & 25 & -0.1 \\
\hline $13: 45: 21: 7$ & 262 & $100^{\circ}$ & 27 & -0.8 & 1.8 & 0.17 & $909^{\circ}$ & $55 . \overline{6}^{\dagger}$ & 25 & -0.1 \\
\hline $13: 45: 22: 75$ & 263 & 101 & 27 & -0.8 & 1.8 & 0.17 & $909^{\circ}$ & 55.6 & 25 & -0.31 \\
\hline $13: 45: 23: 7$ & 264 & 102 & 27 & -0.8 & 1.8 & 0.17 & 912 & 55.5 & 25 & -0.31 \\
\hline $13: 45: 24: 7$ & $265^{\circ}$ & 102 & 27 & -0.8 & 1.8 & 0.17 & $916^{\circ}$ & 55.5 & 25 & -0.1 \\
\hline $13: 45: 25: 7$ & 266 & 103 & 27 & -0.8 & 1.8 & 0.17 & $919^{\circ}$ & $5 \overline{5.5}$ & 25 & -0.1 \\
\hline $13: 45: 26: 7$ & $267^{\circ}$ & 104 & 27 & -0.8 & 1.8 & $0.1 \overline{7} \mid$ & 921 & 55.4 & $\overline{2} \overline{5}$ & -0.07 \\
\hline $13: 45: 27: 6$ & $268^{\circ}$ & 105 & 27 & $-0.8^{\dagger}$ & 1.8 & 0.17 & $918^{\circ}$ & 55.4 & 25 & -0.07 \\
\hline $13: 45: 28: 6$ & 269 & 106 & 27 & -0.8 & 1.8 & 0.17 & $920^{\circ}$ & 55.3 & 25 & -0.14 \\
\hline $13: 45: 29: 6$ & 270 & 106 & 27 & -0.8 & 1.8 & 0.15 & 917 & 55.3 & 25 & -0.14 \\
\hline $13: 45: 306$ & $271^{\circ}$ & 107 & 27 & -0.8 & 1.8 & 0.15 & 912 & 55.3 & 25 & -0.3 \\
\hline $13: 45: 31: 6$ & 272 & $1 \overline{07}$ & 27 & -0.8 & 1.8 & 0.17 & 910 & 55.3 & 25 & -0.11 \\
\hline $13: 45: 32: 6$ & 273 & 108 & 27 & -0.8 & 1.8 & 0.17 & $910^{\circ}$ & $55.3^{\dagger}$ & 26 & -0.11 \\
\hline $13: 45: 33: 6$ & 274 & 108 & 27 & -0.8 & 1.8 & $0.15^{\dagger}$ & $913^{\circ}$ & 55.3 & $\overline{26}$ & -0.02 \\
\hline $13: 45: 34: 6^{\prime}$ & 275 & 109 & 27 & -0.8 & 1.8 & 0.17 & 912 & 55.2 & 26 & -0.02 \\
\hline $13: \overline{45}: \overline{35}: 6$ & $276^{\circ}$ & 109 & 27 & -0.8 & 1.8 & 0.17 & 916 & $55.1 !$ & 26 & -0.18 \\
\hline $13: 45: 36: 6$ & 277 & 109 & $2 \overrightarrow{7}$ & -0.8 & 1.8 & $0.17^{\dagger}$ & $919^{\circ}$ & $55.1^{\dagger}$ & 26 & -0.18 \\
\hline $13: 45: 37: 6$ & 278 & 109 & 27 & $-0 . \overline{8}$ & 1.8 & 0.17 & $922^{\circ}$ & $55.2^{\dagger}$ & 25 & -0.22 \\
\hline $13: 45: 38: 6$ & 279 & 110 & 27 & -0.8 & 1.8 & 0.15 & $921^{\circ}$ & 55.3 & 26 & -0.22 \\
\hline $13: 45: 39: 6$ & $280^{\circ}$ & $110^{!}$ & 27 & -0.8 & 1.8 & $0.15^{\dagger}$ & $916^{\circ}$ & $55.3^{\dagger}$ & 26 & -0.12 \\
\hline $13: 45: 40: 6$ & 281 & 113 & 27 & .0 .8 & 1.8 & 0.15 & $921^{\circ}$ & 55.3 & 26 & $-0 . \overline{12}$ \\
\hline $13: 45: 416$ & 282 & 114 & 27 & -0.8 & 1.8 & $0.15^{\dagger}$ & 916 & 55.3 & 26 & -0.03 \\
\hline $13: 4 \overline{5}: 42: 6$ & 283 & 115 & 27 & -0.8 & 1.8 & 0.15 & 912 & 55.3 & 26 & -0.03 \\
\hline $13: 45: 43: 6$ & 284 & 116 & 27 . & -0.8 & 1.8 & 0.17 & 912 & 55.2 & 26 & -0.21 \\
\hline
\end{tabular}




\begin{tabular}{|c|c|c|c|c|c|c|c|c|c|c|}
\hline TIME & $\begin{array}{l}\text { Elapsed } \\
\text { Time } \\
\text { (sec) }\end{array}$ & BIT_TC1 & PURG_TC2 & 2. PurgePSI & PurgeCFM & DEPTH $(\mathrm{IN})$ & DwnFrc(lb) & RPM & $\begin{array}{l}\text { 'TORQUE } \\
\text { (FT*LB) }\end{array}$ & Inches/Min \\
\hline $13: \overline{45}: 44: 6$ & $\quad 285$ & 117 & 27 & $\quad-0.8$ & 1.8 & $\quad 0.18$ & 916 & 55 & $\quad 26$ & -0.21 \\
\hline $13: 45: 45: 6$ & $286^{\prime}$ & 118 & 27 & -0.8 & 1.8 & 0.17 & 921 & 55 & 26 & -0.24 \\
\hline $13: 45: 46: 6$ & 287 & 119 & 27 & -0.8 & 1.8 & 0.18 & 923 & 55 & 26 & -0.24 \\
\hline $13: 45: 47: 6$ & $288^{\circ}$ & 119 & 27 & -0.8 & 1.8 & 0.17 & 921. & 54.9 & 25 & $0.0 \overline{8}$ \\
\hline $13: 45: 48: 6$ & 289 & 120 & 27 & -0.8 & 1.8 & 0.17 & 924 & $54.9^{\circ}$ & 25 & 0.08 \\
\hline $13: 45: 49: 6$ & 290 & 121 & 27 & -0.8 & 1.8 & 0.17 & 923 & 54.7 & 26 & -0.19 \\
\hline $13: 45: 50: 7$ & 291 & 122 & 27 & -0.8 & 1.8 & 0.17 & 918 & 54.8 & $26^{\dagger}$ & -0.19 \\
\hline $13: 45: 51: 6$ & 292 & 122 & 27 & -0.8 & 1.8 & 0.17 & 922 & 54.8 & 25 & $-0.2 \overline{6}$ \\
\hline $13: 45: 52: 6$ & 293 & 123 & 27 & -0.8 & 1.8 & 0.17 & 917 & $54 . \overrightarrow{9}$ & 26 & -0.26 \\
\hline $13: 45: 536$ & 294 & 123 & 27 & -0.8 & 1.8 & 0.17 & 914 & 54.9 & 26 & -0.23 \\
\hline $13: 45: 54: 6$ & 295 & 124 & 27 & -0.8 & 1.8 & 0.18 & 915 & 54.9 & 26 & -0.23 \\
\hline $13: 45: 55: 7$ & 296 & 125 & 27 & -0.8 & 1.8 & 0.18 & 918 & $55.1^{1}$ & 26 & -0.03 \\
\hline $13: 45: 56: 6$ & 297 & 126 & 27 & -0.8 & 1.8 & 0.18 & 922 & 55.1 & 26 & -0.1 \\
\hline $13: 45: 57: 6$ & $298^{\circ}$ & 127 & 27 & -0.8 & 1.8 & 0.18 & 924 & 55.1 & 26 & -0.1 \\
\hline $13: 45: 58: 6$ & 299 & 127 & 27 & $-0.8^{\dagger}$ & 1.8 & 0.18 & 923 & 55.3 & 26 & -0.21 \\
\hline $13: 45: 59: 6$ & 300 & 128 & 27 & -0.8 & 1.8 & 0.17 & 926 & 55.3 & 26 & $-0 . \overline{21}$ \\
\hline $13: 46: 00: 7$ & 301 & 128 & 27 & -0.8 & $1 . \overline{8}$ & 0.17 & 922 & 55.3 & 26 & -0.24 \\
\hline $13: 46: 01: 6$ & 302 & 129 & 27 & -0.8 & 1.8 & 0.15 & 919 & 55.5 & 26 & -0.24 \\
\hline $13: 46: 02: 6$ & $303^{-}$ & 129 & 27 & -0.8 & 1.8 & 0.15 & 914 & 55.6 & 26 & 0 \\
\hline $13: 46: 03: 7$ & 304 & 130 & 27 & -0.8 & 1.8 & $0.15^{\dagger}$ & 918 & 55.6 & 26 & 0 \\
\hline $13: 4$ & 305 & 131 & 27 & -0.8 & 1.8 & 0.17 & 914 & 557 & 26 & -0.1 \\
\hline $13: 46: 05: 6$ & 306 & 132 & $27 !$ & -0.8 & 1.8 & 0.15 & 913 & 55.8 & 26 & -0.1 \\
\hline $13: 46: 06: 6$ & 307 & 132 & 27 & -0.8 & 1.8 & 0.18 & $915^{\prime}$ & 55.8 & 26 & -0.21 \\
\hline $13: 46: 07: 6$ & 308 & 133 & 27 & -0.8 & 1.8 & 0.18 & $919 !$ & 55.8 & 26 & -0.21 \\
\hline $13: 46: 08: 7$ & 309 & $134^{\dagger}$ & 27 & -0.8 & 1.8 & 0.17 & 922 & 55.5 & 26 & -0.21 \\
\hline $13: 46: 09: 7$ & 310 & 134 & 27 & -0.8 & 1.8 & 0.18 & 920 & $55.5^{1}$ & 26 & -0.21 \\
\hline $13: 46: 10: 6$ & 311 & 135 & 27 & $-0.8^{\dagger}$ & 1.8 & 0.17 & 923 & $55.5^{\top}$ & $26{ }_{i}^{\dagger}$ & -0.12 \\
\hline $13: 46: 11: 7$ & $312^{i}$ & 136 & 27 & -0.8 & 1.8 & 0.17 & $926^{\dagger}$ & $55.7^{t}$ & 26 & -0.12 \\
\hline $13: 46: 12: 7$ & 313 & 136 & 27 & -0.8 & 1.8 & 0.17 & $925 !$ & 55.7 & 26 & -0.02 \\
\hline $13: 46: 13: 7$ & 314 & 137 & 27 & $-0 . \overline{8}$ & 1.8 & 0.17 & $921^{\circ}$ & $55.9^{\circ}$ & 27 & -0.02 \\
\hline $14: 7$ & 315 & 137 & 27 & -0.8 & 1.8 & 0.17 & 916 & 55.9 & $27^{\prime}$ & -0.23 \\
\hline $13: 46: 15: 6$ & 316 & 138 & 27 & -0.8 & 1.8 & 0.17 & 921 & 55.9 & 27 & -0.23 \\
\hline $13: 46: 16: 7^{\dagger}$ & 317 & 138 & 27 & -0.8 & 1.8 & 0.17 & 916 & 56 & 27 & $-0 . \overline{23}$ \\
\hline $13: 46: 17: 7_{1}$ & 318 & 139 & 27 & -0.8 & 1.8 & 0.18 & 915 & $55.9 \mathrm{j}$ & 27 & -0.07 \\
\hline 18.7 & 319 & 140 & $27 !$ & -0.8 & 1.8 & 0.17 & 916 & 55.9 & $2 \overrightarrow{1}$ & -0.07 \\
\hline $13: 46: 19: 7$ & $3 \overline{2} 0$ & $1 \overline{41} \mid$ & 27 & -0.8 & 1.8 & 0.17 & 919 & 55.8 & 27 & $-0 . \overline{07}$ \\
\hline $13: 46: 206$ & 321 & 141 & 27 & -0.8 & 1.8 & 0.17 & 922 & 55.7 & 26 & $-0 . \overline{07}$ \\
\hline $13: 46: 21: 6$ & 322 & 141 & $2 \overline{7}$ & -0.8 & 1.8 & 0.18 & 924 & 55.7 & 26 & $-0.1 \overline{6}$ \\
\hline $13: 46: 22: 6$ & 323 & 142 & 27 & -0.8 & 1.8 & 0.17 & 923 & 55.6 & 26 & $-0 . \overline{16}$ \\
\hline $13: 46: 23: 6$ & 324 & 143 & 27 & -0.8 & 1.8 & 0.17 & 926 & $55.7 \mathrm{j}$ & 26 & -0.22 \\
\hline $13: 46: 24: 6$ & 325 & 143 & 27 & -0 & 1.8 & 0.17 & 925 & 55.7 & $2 \overline{6}$ & -0.22 \\
\hline $6: 25: 6$ & 326 & 143 & 27 & -0.8 & 1.8 & 0.18 & 922 & $55 . \overrightarrow{7}$ & 27 & $-0.1 \overline{3}$ \\
\hline $13: 46: 26: 6$ & 327 & 144 & 27 & -0.8 & 1.8 & 0.17 & 918 & 55.7 & 27 & -0.13 \\
\hline & 328 & $14 \overline{4}$ & 27 & $-0.8^{\dagger}$ & 1.8 & $0 . \overline{17}$ & $915 !$ & 55.7 & 27 & -0.07 \\
\hline $46: 28: 6$ & 329 & 145 & 27 & -0.8 & 1.8 & $\underline{0.17}$ & 916 & 55.6 & $2 \overline{7}$ & -0.07 \\
\hline $13: 46: 29: 6$ & 330 & 146 & 27 & -0.8 & 1.8 & 0.18 & 915 & $55.5^{\dagger}$ & 27 & $-0.1 \overline{3}$ \\
\hline $13: 46: 30: 6$ & 331 & 146 & 27 & -0.8 & 1.8 & 0.17 & 917 & 55.5 & 27 & -0.13 \\
\hline & 332 & 147 & $27 !$ & -0.8 & 1.8 & 0.18 & 921 & 55.5 & 27 & $-0 . \overline{3}$ \\
\hline :46:32:6 & 333 & 147 & 27 & -0.8 & 1.8 & 0.18 & 924 & 55.5 & 26 & -0.3 \\
\hline $13: 46: 33: 6$ & 334 & 148 & 27 & -0.8 & 1.8 & 0.17 & 926 & 55.5 & 26 & -0.13 \\
\hline $13: 46: 34: 6$ & 335 & 148 & 27 & -0.8 & 1.8 & 0.18 & 924 & 55.4 & $2 \overline{6}$ & $-\overline{0.13}$ \\
\hline $13: 46: 35: 6$ & 336 & $14 \overline{9}$ & 27 & -0.8 & 1.8 & 0.18 & 926 & 55.3 & 26 & -0.05 \\
\hline $13: 46: 36: 6$ & 337 & 149 & 27 & -0.8 & 1.8 & 0.15 & 925 & 55.3 & 26 & -0.05 \\
\hline $46: 37: 6$ & 338 & 150 & 27 & -0.8 & 1.8 & 0.15 & 919 & 55.3 & 26 & -0.15 \\
\hline $13: 46: 38: 6$ & 339 & 150 & 27 & -0.8 & 1.8 & 0.17 & 915 & 55.1 & 26 & $-0 . \overline{15}$ \\
\hline $13: 46: 39: 6$ & 340 & 151 & 27 & -0.8 & 1.8 & 0.17 & 916 & $55.1^{\dagger}$ & 26 & $-0 . \overline{27}$ \\
\hline $13: 46: 40: 6$ & $341^{+}$ & 151 & 27 & $-0.8^{+}$ & 1.8 & 0.17 & $919^{\circ}$ & $55^{\prime}$ & 26 & -0.27 \\
\hline
\end{tabular}




\begin{tabular}{|c|c|c|c|c|c|c|c|c|c|c|}
\hline TIME & $\begin{array}{l}\text { Elapsed } \\
\text { Time } \\
\text { (sec) }\end{array}$ & BIT_TC1 & PURG_TC2 & PurgePSI & PurgeCFM & DEPTH(IN): & DwnFrc(lb) & RPM & $\begin{array}{l}\text { TORQUE } \\
\left(F T^{*} L B\right)\end{array}$ & Inches/Min \\
\hline $13: 46: 41: 6$ & 342 & 152 & 27 & -0.8 & $\quad 1.8$ & $\quad 0.17$ & 916 & 55.1 & $\quad 26$ & $5 \quad-0.08$ \\
\hline $13: 46: 42: 6$ & 343 & 152 & 27 & -0.8 & 1.8 & 0.17 & 920 & 55.1 & 27 & -0.07 \\
\hline $13: 46: 43: 6$ & 344 & 153 & 27 & -0.8 & 1.8 & 0.17 & 924 & 55.2 & 27 & $-0 . \overline{07}$ \\
\hline $13: 46: 44: 6$ & 345 & 153 & 27 & -0.8 & 1.8 & 0.17 & 926 & 55.3 & 27 & -0.17 \\
\hline $13: 46: 45: 6$ & 346 & 154 & 27 & -0.8 & 1.8 & 0.17 & 924 & 55.3 & 27 & -0.17 \\
\hline $13: 46: 46: 6$ & 347 & 154 & 27 & -0.8 & 1.8 & 0.17 & 926 & 55.5 & 26 & -0.29 \\
\hline $13: 46: 47: 6$ & 348 & 155 & 27 & -0.8 & 1.8 & 0.17 & 927 & 55.6 & 27 & -0.29 \\
\hline $13: 46: 48: 6$ & 349 & 155 & 27 & -0.8 & 1.8 & 0.15 & 923 & 55.6 & 27 & -0.09 \\
\hline $13: 46: 49: 6$ & 350 & 156 & 27 & -0.8 & 1.8 & 0.17 & 918 & 55.7 & 27 & $-0.0 \overline{9}$ \\
\hline $13: 46: 50: 6$ & 351 & 156 & 27 & -0.8 & $1 . \overline{8}$ & 0.15 & 916 & 557 & 27 & -0.07 \\
\hline $13: 46: 51: 6$ & 352 & 157 & 27 & -0.8 & 1.8 & 0.17 & 917 & 55.7 & 27 & -0.07 \\
\hline $13: 46: 52: 6$ & 353 & 158 & 27 & -0.8 & 1.8 & 0.17 & 916 & 55.8 & 27 & -0.15 \\
\hline $13: 46: 53: 6$ & $354^{\prime}$ & 158 & 27 & -0.8 & 1.8 & $0.15^{\circ}$ & 916 & 55.6 & 26 & -0.15 \\
\hline $13: 46: 54: 6$ & 355 & 159 & 27 & -0.8 & 1.8 & 0.17 & 920 & $5 \overline{5} .6$ & 26 & -0.23 \\
\hline $13: 46: 55: 6$ & 356 & 159 & 27 & -0.8 & 1.8 & 0.17 & 924 & $55.7^{\prime}$ & 27 & -0.23 \\
\hline 13:46:56:6 & 357 & 160 & 27 & -0.8 & 1.8 & 0.17 & 927 & 55.7 & 27 & $-0 . \overline{17}$ \\
\hline $13: 46: 57: 7$ & 358 & 160 & 27 & -0.8 & 1.8 & 0.15 & 925 & 55.7 & 27 & -0.17 \\
\hline $13: 46: 58: 7$ & $359 !$ & 161 & 27 & -0.8 & 1.8 & 0.17 & 927 & 55.8 & 27 & -0.01 \\
\hline $13: 46: 59: 6$ & 360 & 161 & 27 & -0.8 & 1.8 & 0.17 & 927 & 55.8 & 27 & -0.01 \\
\hline $13: 47: 00: 6$ & 361 & 161 & 27 & -0.8 & 1.8 & 0.15 & 924 & 55.8 & 27 & -0.11 \\
\hline $13: 47: 01: 6$ & $362 !$ & 162 & 27 & -0.8 & 1.8 & 0.15 & 919 & 55.8 & 27 & -0.11 \\
\hline $13: 47: 02: 7$ & 363 & 162 & 27 & -0.8 & 1.8 & $0.15^{\dagger}$ & 917 & 55.9 & 27 & $-0 . \overline{2}$ \\
\hline $13: 47: 03: 7$ & 364 & 163 & 27 & -0.8 & 1.8 & 0.15 & 919 & 55.9 & 27 & $-0 . \overline{2}$ \\
\hline $13: 47: 04: 6$ & 365 & 163 & 27 & -0.8 & 1.8 & 0.15 & 917 & 55.9 & 27 & -0.28 \\
\hline $13: 47: 05: 7$ & $36 \overline{6}$ & 164 & 27 & -0.8 & 1.8 & 0.17 & 918 & $55.9^{\circ}$ & 27 & 0.01 \\
\hline $13: 47: 06: 7$ & 367 & 165 & 27 & -0.8 & 1.8 & 0.18 & 921 & 55.9 & 27 & 0.01 \\
\hline $13: 47: 07: 7$ & 368 & 165 & 27 & -0.8 & 1.8 & 0.17 & 924 & 55.9 & 27 & -0.08 \\
\hline $13: 47: 08: 6$ & 369 & $16 \overline{6}$ & 27 & -0.8 & 1.8 & 0.17 & 926 & 55.8 & 27 & -0.08 \\
\hline $13: 47: 09: 6$ & 370 & 166 & 27 & -0.8 & 1.8 & 0.17 & 924 & 55.8 & 27 & -0.24 \\
\hline $13: 47: 10: 6$ & $371^{\dagger}$ & 166 & 27 & -0.8 & 1.8 & 0.15 & 927 & 55.8 & 27 & -0.24 \\
\hline $13: 47: 11: 6$ & 372 & 167 & 27 & -0.8 & 1.8 & 0.18 & 928 & 55.9 & 27 & -0.24 \\
\hline $13: 47: 12: 6$ & 373 & 167 & 27 & -0.8 & 1.8 & 0.15 & 925 & 55.9 & 27 & -0.24 \\
\hline $13: 47: 13: 6$ & 374 & 167 & 27 & -0.8 & 1.8 & 0.15 & 920 & 55.9 & 28 & -0.07 \\
\hline $13: 47: 14: 6$ & 375 & 168 & 27 & -0.8 & $1 . \overline{8}$ & 0.17 & 916 & 55.9 & 28 & -0.07 \\
\hline $13: 47: 15: 6$ & 376 & 168 & 27 & -0.8 & 1.8 & 0.15 & 916 & $5 \overline{5}$. & 28 & -0.07 \\
\hline $13: 47: 16: 6$ & 377 & 169 & 27 & -0.8 & 1.8 & 0.17 & 919 & 55.8 & 28 & $-0.0 \overline{7}$ \\
\hline $13: 47: 17: 5$ & $37 \overline{8}$ & 169 & 27 & -0.8 & 1.8 & 0.17 & 916 & 55.8 & 28 & -0.21 \\
\hline $13: 47: 18: 5$ & 379 & 170 & 27 & -0.8 & $1.8^{\dagger}$ & $0 . \overline{7}$ & 919 & 55.8 & 28 & $-0 . \overline{21}$ \\
\hline $13: 47: 19: 5$ & 380 & 170 & 27 & -0.8 & 1.8 & 0.17 & 923 & 55.8 & 27 & -0.25 \\
\hline $13: 47: 20: 5$ & 381 & 171 & 27 & -0.8 & 1.8 & 0.17 & $926^{\circ}$ & 55.9 & 27 & $-0.2 \overline{5}$ \\
\hline $13: 47: 21: 5$ & 382 & 171 & 27 & -0.8 & 1.8 & $0.1 \overline{7}$ & 927 & 55.9 & 27 & -0.1 \\
\hline $13: 47: 22: 5$ & 383 & 171 & 27 & -0.8 & 1.8 & 0.15 & 926 & 55.8 & 27 & -0.1 \\
\hline $13: 47: 23: 5$ & 384 & 172 & 27 & -0.8 & 1.8 & 0.17 & 921. & 55.6 & 27 & -0.02 \\
\hline $13: 47: 24: 5$ & 385 & 172 & 27 & -0.8 & 1.8 & 0.17 & 917 & 55.6 & 27 & -0.02 \\
\hline $13: 47: 25: 5$ & 386 & 172 & 27 & -0.8 & 1.8 & 0.17 & 917 & 55.6 & 28 & -0.12 \\
\hline $13: 47: 26: 4$ & 387 & 173 & 27 & -0.8 & 1.8 & 0.17 & 917 & 55.7 & 28 & -0.12 \\
\hline $13: 47: 27: 4$ & 388 & 173 & 27 & -0.8 & 1.8 & 0.17 & $917^{\circ}$ & 55.7 & 28 & -0.28 \\
\hline $13: 47: 28: 4$ & 389 & 174 & 27 & -0.8 & 1.8 & 0.17 & 919 & 55.8 & 28 & -0.28 \\
\hline $13: 47: 29: 4$ & 390 & 174 & 27 & -0.8 & 1.8 & $0 . \overline{18}$ & 922 & 55.8 & 28 & $-0 . \overline{4}$ \\
\hline $13: 47: 30: 4$ & 391 & 175 & 27 & -0.8 & $1.8^{\dagger}$ & 0.17 & 925 & $55 . \overline{8}$ & 28 & -0.14 \\
\hline $13: 47: 31: 4$ & 392 & 175 & $27^{\dagger}$ & -0.8 & 1.8 & 0.17 & 928 & 55.7 & 27 & -0.02 \\
\hline $13: 47: 32: 4$ & 393 & 175 & 27 & -0.8 & 1.8 & 0.15 & 927 & 55.6 & 27 & -0.02 \\
\hline $13: 47: 33: 4$ & 394 & 176 & 27 & -0.8 & 1.8 & 0.15 & 922 & 55.6 & 27 & -0.14 \\
\hline $13: 47: 34: 4$ & 395 & 176 & 27 & -0.8 & 1.8 & $0 . \overline{17}$ & 927 & 55.7 & 27 & -0.14 \\
\hline $13: 47: 35: 4$ & 396 & 177 & 27 & -0.8 & 1.8 & 0.15 & $922^{\circ}$ & 55.7 & 27 & $-0 . \overline{22}$ \\
\hline $13: 47: 36: 4$ & 397 & 177 & 27 & -0.8 & 1.8 & 0.15 & 919 & 55.7 & 28 & -0.22 \\
\hline $13: 47: 37: 4$ & 398 & $17 \overline{7}$ & 27 & -0.8 & 1.8 & 0.17 & $917^{\circ}$ & 55.7 & 28 & $-0 . \overline{22}$ \\
\hline
\end{tabular}




\begin{tabular}{|c|c|c|c|c|c|c|c|c|c|c|}
\hline TIME & $\begin{array}{l}\text { Elapsed } \\
\text { Time } \\
\text { (sec) }\end{array}$ & BIT_TC1' & PURG_TC2 & PurgePSI & PurgeCFM & DEPTH(IN) & DwnFrc(lb) & RPM & $\begin{array}{l}\text { TORQUE } \\
\text { (FT*LB) }\end{array}$ & Inches/Min \\
\hline $13: 47: 38: 4$ & $\quad 399$ & 178 & -27 & -0.8 & 1.8 & 0.15 & $\begin{array}{r}120^{\circ} \\
\end{array}$ & 55.7 & $\quad 28$ & $\quad 0.03$ \\
\hline $13: 47: \overline{99}: 4$ & 400 & 179 & 27 & -0.8 & 1.8 & 0.17 & 922 & 55.7 & 28 & 0.03 \\
\hline $13: 47: 40: 3$ & 401 & $179^{\prime}$ & $27^{\circ}$ & $-0 . \overline{8}$ & 1.8 & 0.17 & 925 & 55.6 & 28 & -0.18 \\
\hline $13: 47: 41: 3$ & 402 & $180^{\circ}$ & 27 & -0.8 & $1.8^{\circ}$ & 0.17 & 923 & 55.6 & 28 & -0.18 \\
\hline $13: 47: 42: 3$ & 403 & $180^{\circ}$ & $27^{\circ}$ & -0.8 & 1.8 & 0.17 & 926 & 55.6 & 29 & -0.17 \\
\hline $13: 47: 43: 3$ & 404 & 180 & 27 & -0.8 & 18 & 0.17 & 929 & 55.7 & 28 & -0.17 \\
\hline $13: 47: 44: 3$ & 405 & 181 & 27 & -0.8 & 1.8 & 0.17 & 928 & $5 \overline{5.7}$ & $\overline{28}$ & $-0.2 \overline{7}$ \\
\hline $13: 47: 45: 3$ & 406 & 181 & 27 & -0.8 & 1.8 & 0.17 & 924 & 55.7 & 28 & -0.27 \\
\hline $13: 47: 46: 3$ & 407 & 182 & 27 & -0.8 & 1.8 & 0.17 & 920 & 55.7 & 28 & 0.05 \\
\hline $13: 47: 47: 3^{\dagger}$ & 408 & $182^{\prime}$ & 27 & -0.8 & 1.8 & 0.15 & 923 & 55.7 & 28 & 0.05 \\
\hline $13: 47: 48: 3$ & 409 & 183 & 27 & -0.8 & $1.8^{i}$ & 0.17 & 919 & 55.7 & 28 & -0.16 \\
\hline $13: 47: 49: 3$ & 410 : & 183 & 27 & -0.8 & 1.8 & 0.17 & 919 & 55.6 & 29 & -0.16 \\
\hline $13: 47: 50: 3$ & $411 \mathrm{i}$ & 184 & 27 & -0.8 & $1.8^{4}$ & 0.17 & 921 & 55.6 & 28 & -0.2 \\
\hline $13: 47: 51: 3$ & 412 & 184 & 27 & -0.8 & 1.8 & 0.17 & 924 & 55.6 & 28 & -0.2 \\
\hline $13: 47: 52: 3$ & $413^{-1}$ & $185 !$ & $27^{\circ}$ & -0.8 & 1.8 & $0.1 \overline{7}$ & 927 & 55.6 & 28 & -0.24 \\
\hline $13: 47: 53: 3$ & 414 & 185 & 27 & -0.8 & 1.8 & 0.18 & 924 & $55.6^{\circ}$ & 28 & -0.24 \\
\hline $13: 47: 54: 3$ & 415 & 185 & 27 & -0.8 & 1.8 & $0 . \overline{15}$ & 927 & 55.5 & 28 & 0 \\
\hline $13: 47: 55: 3$ & 416 & 185 & $27 !$ & -0.8 & $1 . \overline{8}^{-1}$ & 0.15 & $92 \overline{8}$ & 55.5 & 28 & 0 \\
\hline $13: 47: 56: 3$ & 417 & 186 & 27 & -0.8 & 1.8 & 0.15 & 926 & 55.5 & $2 \overline{8}$ & -0.12 \\
\hline $13: 47: 57: 3$ & $418^{\dagger}$ & 186 & 27 & -0.8 & 1.8 & 0.17 & 922 & 55.2 & 29 & -0.12 \\
\hline $13: 47$ & $419^{\prime}$ & 187 & $27^{\prime}$ & -0.8 & 1.8 & 0.15 & 919 & 55.2 & 29 & -0.21 \\
\hline $13: 47: 59: 3$ & $420^{\circ}$ & $187^{\circ}$ & 27 & -0.8 & 1.8 & 0.15 & 919 & 55.2 & 29 & -0.21 \\
\hline $13: 48: 00: 3$ & 421 & $187 i$ & 27 & -0.8 & $1.8^{\circ}$ & 0.17 & $919^{\circ}$ & $55.3^{4}$ & 29 & -0.24 \\
\hline $13: 48: 01: 3^{\dagger}$ & 422 & 188 & 27 & -0.8 & 1.8 & 0.17 & 919 & $5 \overline{5} .4$ & 30 & -0.24 \\
\hline $13: 48: 02: 3$ & 423 & $189^{\circ}$ & 27 & -0.8 & 1.8 & 0.17 & 922 & 55.4 & 30 & 0.01 \\
\hline $13: 48: 03: 4$ & 424 & $189^{\circ}$ & 27 & $-0.8^{1}$ & $1.8^{i}$ & 0.17 & 926 & 55.4 & 30 & -0.12 \\
\hline $13: 48: 04: 3$ & 425 & 190 & 27 & -0.8 & $1.8 !$ & 0.18 & 929 & 55.4 & 29 & -0.12 \\
\hline $13: 48: 05: 3$ & 426 & $190^{\circ}$ & 27 & -0.8 & 1.8 & 0.17 & 926 & 55.4 & 28 & -0.22 \\
\hline $13: 48: 06: 3$ & 427 & $190 !$ & 27 & $-0 . \overline{8}$ & 1.8 & 0.17 & 931 & 55.3 & 29 & -0.22 \\
\hline $13: 48$ & 428 & 191 & 27 & -1 & 1.8 & 0.15 & $929^{+}$ & 55.2 & 29 & -0.26 \\
\hline $13: 48: 08: 4$ & 429 & 191 & 27 & -0.8 & 1.8 & 0.15 & $925^{\circ}$ & 55.2 & 29 & -0.26 \\
\hline $13: 48: 09: 3$ & 430 & 191 & 27 & -0.8 & 1.8 & 0.15 & 921 & 55.3 & 29 & 0.01 \\
\hline $13: 48: 10: 3$ & 431 & 19 & 27 & -0.8 & 1.8 & 0.17 & 920 & 55.4 & 30 & 0.01 \\
\hline $13: 48: 11: 3$ & 432 & $192^{\dagger}$ & 27 ! & -0.8 & 1.8 & 0.17 & 921 & 55.4 & 30 & -0.12 \\
\hline $13: 48: 12: 3$ & $433^{+}$ & 193 & $27^{\circ}$ & -0.8 & 1.8 & 0.18 & 920 & $55.4^{-1}$ & 30 & -0.12 \\
\hline $13: 48: 13: 4$ & $434 !$ & 193 & 27 & -0.8 & $1.8^{t}$ & 0.17 & 922 & 55.4 & 30 & -0.2 \\
\hline $13: 48: 14: 3$ & 435 & 194 & 27 & -0.8 & 1.8 & 0.17 & 925 & 55.4 & 30 & -0.2 \\
\hline $13: 48: 15: 3$ & 436 & $194 !$ & $27 !$ & -0.8 & 1.8 & 0.17 & 929 & 55.4 & 30 & -0.27 \\
\hline $13: 48: 16: 4$ & 437 & $194\rceil$ & $27 !$ & -0.8 & 1.8 & 0.17 & 926 & 55.4 & 30 & -0.27 \\
\hline $13: 48: 17: 4$ & 438 & 195 & 27 & -0.8 & 1.8 & 0.15 & 929 & 55.4 & 30 & 0.04 \\
\hline $13: 48: 18: 3$ & 439 & 196 & 28 & -0.8 & 1.8 & 0.17 & 931 & 55.4 & 30 & 0.04 \\
\hline $13: 48: 19: 3$ & $4 \overline{40}$ & $196 !$ & 27 & $-0 . \overline{8}$ & $1.8^{-4}$ & 0.17 & 927 & $55.3^{+}$ & 30 & -0.16 \\
\hline $13: 48: 20: 3$ & 441 ? & 196 & 27 & -0.8 & $1.8^{k}$ & 0.17 & 922 & 55.3 & 30 & -0.16 \\
\hline$1 3 \longdiv { 4 8 : 2 1 : 4 }$ & 442 & 197 & 27 & -0.8 & 18 & 0 & 920 & 55.1 & 30 & -0.2 \\
\hline $13: 48: 22: 4$ & $443^{\dagger}$ & 197 & 27 & -0.8 & 1.8 & 0.17 & 922 & $55^{\dagger}$ & 30 & -0.2 \\
\hline $13: 48: 23: 3$ & 444 & 198 & 27 & -0.8 & 1.8 & 0.17 & 921 & 55 & 30 & -0.26 \\
\hline $13: 48: 24: 4$ & 445 & 198 & $2 \overline{7} \dot{j}$ & -0.8 & 1.8 & 0.17 & 922 & $55.2^{+}$ & 30 & 0.03 \\
\hline $13: 48: 25: 4$ & 446 & 199 & 27 & -0.8 & 1.8 & 0.17 & 925 & 55.3 & 30 & 0.03 \\
\hline $13: 48: 26: 4$ & 447 & 199 & 27 & -0.8 & 1.8 & 0.17 & 929 & 55.3 & 30 & -0.16 \\
\hline $13: 48: 2 \overline{7}: 4$ & 448 & $1 \overline{99}$ & 28 & -1 & 1.8 & 0.17 & 931 & 55.3 & 30 & -0.16 \\
\hline $13: 48: 28: 3$ & 449 & 200 & 27 & -0.8 & 1.8 & 0.15 & 931 & 55.2 & 30 & -0.19 \\
\hline $13: 48: 29: 4$ & 450 & 200 & 27 & -0.8 & 1.8 & 0.17 & 931 & 55.3 & 30 & -0.19 \\
\hline $13: 48: 30: 4$ & 451 & 200 & 27 & -0.8 & 1.8 & 0.15 & 930 & 55.3 & 30 & $-0.2 \overline{6}$ \\
\hline $13: 48: 31: 4$ & 452 & 201 & 27 & -0.8 & 1.8 & 0.18 & 925 & 55.3 & 31 & $-0.2 \overline{6}$ \\
\hline $13: 48: 32: 4$ & 453 & 201 & 27 & -0.8 & 1.8 & 0.17 & 921 & 55.4 & 31 & 0.05 \\
\hline $13: 48: 33: 3^{+}$ & 454 & 202 & 28 & $-0.8^{-}$ & $1.8^{\circ}$ & 0.18 & 921 & 55.4 & 31 & 0.05 \\
\hline $3: 48: 34: 3$ & 455 & $200^{\circ}$ & 28 & 18.7 & $51.4^{\prime}$ & 0.18 & 924 & $55 \overline{5}$ & 30 & -0.16 \\
\hline
\end{tabular}




\begin{tabular}{|c|c|c|c|c|c|c|c|c|c|c|}
\hline TIME & $\begin{array}{l}\text { Elapsed } \\
\text { Time } \\
(\mathrm{sec})\end{array}$ & BIT_TC1 & PURG_TC2 & PurgePSi! & PurgeCFM & DEPTH(IN) & DwnFrc(lb) & RPM & $\begin{array}{l}\text { TORQUE } \\
\left(F^{*} L B\right)\end{array}$ & !nches/Min \\
\hline $13: 48: 35: 3$ & 456 & -192 & -28 & 22.2 & $\quad 51.4$ & $\quad 0.18$ & 920 & 55.5 & $\quad 27$ & -0.16 \\
\hline $36: 3$ & 457 & 186 & 28 & 22.4 & 51.4 & 0.18 & 921 & 55.7 & 25 & -0.21 \\
\hline $37: 3$ & 458 & 179 & 28 & 22.6 & 51.4 & 0.18 & 922 & 55.8 & 24 & -0.21 \\
\hline & 459 & 174 & 28 & 22.6 & 51.4 & 0.18 & 926 & 55.8 & 23 & -0.26 \\
\hline $9: 3$ & 460 & 169 & 28 & $22.6 !$ & 51.4 & 0.17 & 926 & 55.8 & 23 & -0.26 \\
\hline $40: 3$ & 461 & 164 & 28 & 22.4 & 51.4 & 0.17 & 924 & 55.8 & 22 & 0.02 \\
\hline & 462 & 160 & 28 & 22.4 & 51.4 & 0.17 & 921 & 55.8 & 21 & 0.02 \\
\hline $2: 3$ & 463 & 157 & 28 & 22.4 & 51.4 & 0.15 & 923 & 55.9 & 21 & -0.13 \\
\hline $3: 3$ & $4 \overline{64}$ & 153 & 28 & 22.4 & 51.4 & 0.17 & 916 & 56 & 21 & -0.13 \\
\hline $44: 3$ & 465 & 149 & 28 & 22.4 & 51.4 & 0.17 & 914 & 56 & 20 & -0.25 \\
\hline $5: 3$ & 466 & 147 & 28 & 22.4 & 51.4 & 0.15 & 912 & 55.9 & 20 & -0.25 \\
\hline 63 & 467 & 144 & 28 & 22.4 & 51.4 & 0.17 & 910 & $55.9^{-t}$ & 20 & -0.19 \\
\hline $13: 48: 47: 3$ & 468 & 140 & 28 & 22.2 & 51.4 & 0.18 & 913 & 55.9 & 20 & -0.19 \\
\hline $8: 3$ & 469 & 137 & 28 & 22.4 & $51 . \overline{4}$ & $0.18_{1}$ & 909 & $5 \overline{6}$ & 19 & -0.14 \\
\hline $9: 3$ & 470 & 135 & 28 & 22.4 & 51.4 & 0.18 & $912^{\dagger}$ & 56 & 19 & $-0 . \overline{14}$ \\
\hline & 471 & 132 & 28 & 22.4 & 51.4 & 0.18 & 916 & 56 & 19 & -0.02 \\
\hline $13: 48: 51: 3$ & 472 & 129 & 28 & $22.4 !$ & 51.4 & 0.17 & 919 & $55.9^{2}$ & 19 & -0.16 \\
\hline $2: 3$ & 473 & 127 & 28 & $22.4 !$ & 51.4 & 0.17 & 918 & 55.9 & 19 & $-0 . \overline{16}$ \\
\hline & 474 & $12 \overline{5}$ & 27 & 22.4 & 51.4 & 0.17 & 916 & 55.9 & 19 & -0.27 \\
\hline $13: 48: 54: 3^{\dagger}$ & 475 & 122 & 28 & 22.4 & 51.4 & 0.17 & $919^{+}$ & $55.9^{\dagger}$ & 18 & -0.27 \\
\hline
\end{tabular}




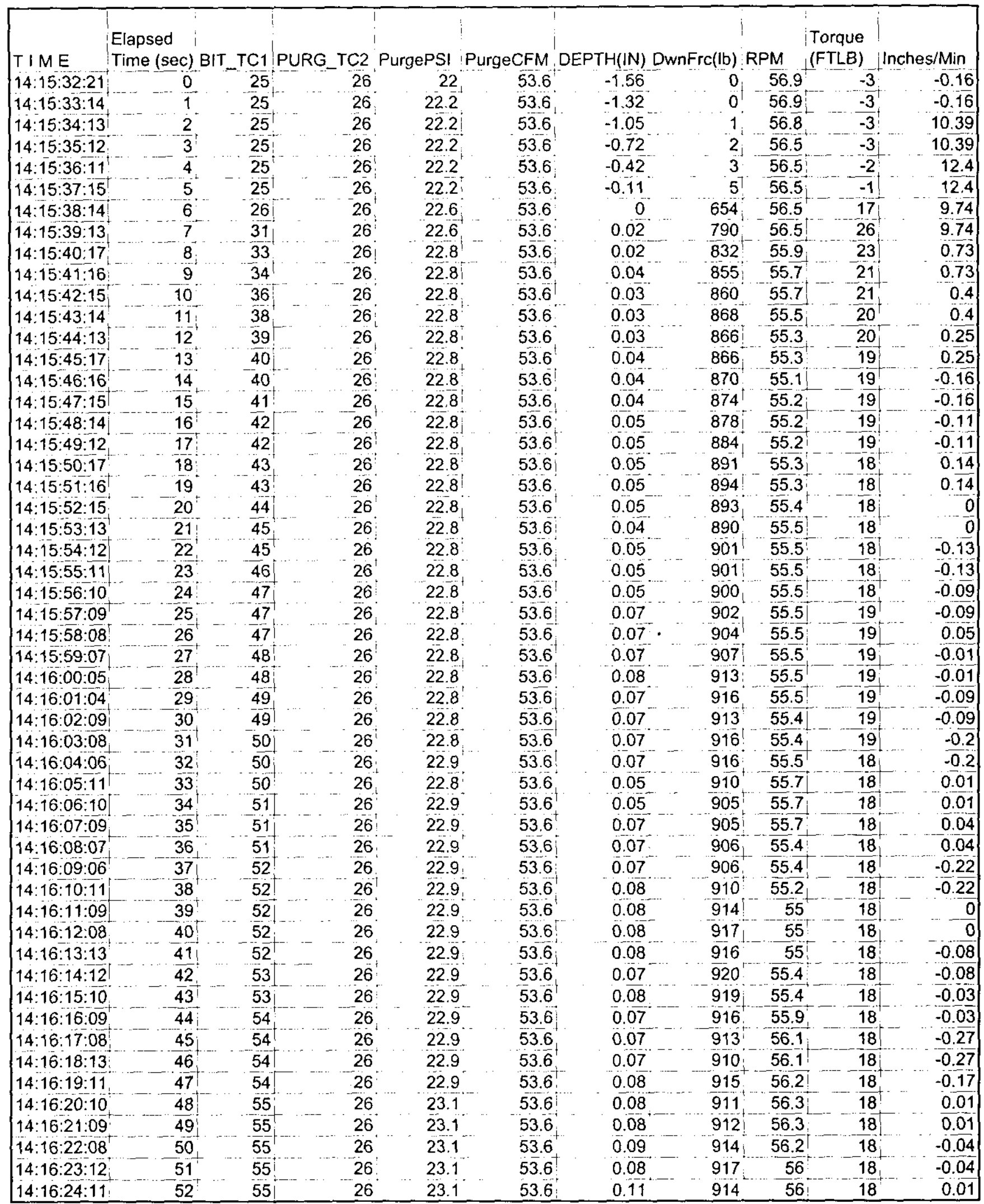




\begin{tabular}{|c|c|c|c|c|c|c|c|c|c|c|}
\hline TIME & \begin{tabular}{|l} 
Elapsed \\
Time (sec)
\end{tabular} & & & PurgePSI & PurgeCFM & DEPTH(IN) & DwnFrc(lb) & RPM & $\begin{array}{l}\text { Torque } \\
\text { ('FTLB) }\end{array}$ & Inches/Min \\
\hline $14: 16: 25: 10$ & $\quad 53$ & 55 & 26 & 3. $\quad 23.1$ & $\quad 53.6$ & 0.08 & $\quad 919$ & $\quad 55.9$ & $9 \quad 18$ & 0.01 \\
\hline $14: 16: 26: 14$ & 54 & 55 & 26 & 23.1 & 53.6 & 0.08 & $92 \overline{3}$ & 56 & 18 & -0.27 \\
\hline $14: 16: 27: 13$ & 55 & 55 & 26 & 23.1 & $53.6^{\circ}$ & 0.08 & $929^{\circ}$ & 56 & 18 & $-0.2 \overline{7}$ \\
\hline $14: 16: 28: 12$ & 56 & $5 \overline{6}$ & 26 & 23.1 & $53.6^{\circ}$ & 0.08 & 928 & 56 & 18 & -0.18 \\
\hline $14: 16: 29: 11$ & 57 & 56 & 26 & 23.1 & 53.6 & 0.08 & 922 & 55.9 & 18 & -0.18 \\
\hline $14: 16: 30: 10$ & 58 & 56 & 26 & 23.1 & 53.6 & 0.08 & 916 & 55.9 & 18 & $0 . \overline{13}$ \\
\hline $14: 16: 31: 14$ & 59 & 56 & 26 & $23.1^{1}$ & 53.6 & 0.09 & 919 & 558 & 18 & 0.13 \\
\hline $14: 16: 32: 13$ & 60 & 56 & 26 & 23.1 & 53.6 & 0.08 & 913 & 55.6 & 18 & -0.09 \\
\hline $14: 16: 33: 12$ & 61 & 56 & 26 & 23.1 & 53.6 & 0.09 & 912. & 55.6 & 19 & -0.09 \\
\hline $14: 16: \overline{34}: 16$ & 62 & 56 & 26 & 23.1 & 53.6 & 0.11 & 915 & 55.4 & 19 & $-0 . \overline{13}$ \\
\hline $14: 16: 35: 15$ & 63 & 56 & 26 & 23.1 & 53.6 & 0.08 & 912 & 55.5 & 19 & -0.13 \\
\hline $14: 16: 36: 14$ & 64 & 56 & 26 & 23.1 & $53.6^{\prime}$ & 0.11 & 921 & 55.5 & 19 & -0.21 \\
\hline $14: 16: 37: 13$ & 65 & 56 & 26 & $23.1 !$ & 53.6 & 0.11 & 919 & 55.4 & $\overline{19}$ & $-0 . \overline{21}$ \\
\hline $14: 16: 38: 12$ & 66 & 57 & 26 & $23.3^{\circ}$ & 53.6 & 0.11 & 922 & 55.2 & 19 & 0.04 \\
\hline $14: 16: 39: 16$ & $67 !$ & 57 & 26 & 23.3 & 53.6 & 0.09 & 921 & 55.2 & 18 & 0.04 \\
\hline $14: 16: 40: 15$ & 68 & 57 & 26 & $23.3 !$ & 53.6 & 0.08 & 918 & 55.1 & 18 & -0.13 \\
\hline $1: 14$ & 69 & 57 & 26 & 23.3 & 53.6 & 0.08 & 913 & 55.3 & 18 & -0.13 \\
\hline $14: 16: 42: 18$ & 70 & 57 & 26 & 23.3 & 53.6 & 0.08 & 914 & 55.3 & 18 & -0.2 \\
\hline $14: 16: 4 \overline{3}: 17$ & 71 & 57 & 26 & 23.3 & 53.6 & 0.08 & 909 & 55.2 & 18 & -0.15 \\
\hline $14: 16: 44: \overline{16}$ & 72 & 57 & 26 & 23.3 & 53.6 & 0.11 & 907 & 55.2 & 18 & -0.15 \\
\hline 15 & 73 & 57 & 26 & $23.3^{4}$ & 53.6 & 0.11 & 909 & 55.2 & 18 & 0 \\
\hline $14: 16: 46: 14$ & 74 & $5 \overline{7}$ & 26 & 23.3 & 53.6 & 0.11 & 912 & 55.2 & 18 & 0 \\
\hline $14: 16: 47: 18$ & 75 & 57 & 26 & 23.3 & 53.6 & 0.11 & 916 & 55.2 & 18 & -0.14 \\
\hline $8: 17$ & 76 & 57 & 26 & 23.3 & 53.6 & 0.09 & 914 & 55.2 & 18 & -0.14 \\
\hline $9: 16$ & 77 & 57 & 26 & 23.5 & 53.6 & 0.11 & $917 !$ & 55.1 & 18 & -0.22 \\
\hline $14: 16: 50: 15$ & 78 & 57 & 26 & 23.5 & 53.6 & 0.09 & 920 & 55.1 & 18 & -0.22 \\
\hline $14: 16: 51: 14$ & 79 & 57 & 26 & 23.5 & $53.6^{\prime}$ & 0.09 & 917 & 55.1 & 18 & $-0.1 \overline{3}$ \\
\hline $14: 16: 52: 18$ & 80 & 57 & 26 & 23.5 & 53.6 & 0.08 & 911 & $54.9^{\prime}$ & 18 & -0.13 \\
\hline $3: 17$ & 81 & 57 & 26 & 23.5 & 53.6 & 0.08 & 912 & 54.8 & 17 & -0.08 \\
\hline $14: 16: 54: 16$ & 82 & 57 & 26 & 23.5 & 53.6 & 0.09 & 907 & 54.8 & 17 & -0.08 \\
\hline $14: 16: 55: 20$ & 83 & 57 & 26 & 23.5 & 53.6 & & 905 & 54.7 & 17 & -0.13 \\
\hline $14: 16: 56: 19$ & 84 & 57 & 26 & 23.5 & $53.6^{\dagger}$ & 0.11 & 907. & 54.7 & 17 & $-0 . \overline{13}$ \\
\hline $7: \overline{18}$ & 85 & 57 & 26 & 23.5 & 53.6 & 0.11 & 912 & 54.7 & 17 & -0.25 \\
\hline $14: 16: 58: 17$ & 86 & 57 & 26 & 23.5 & 53.6 & 0.11 & 914 & 54.7 & 17 & -0.25 \\
\hline $14: 16: 59: 15$ & 87 & 58 & 26 & 23.5 & 53.6 & 0.1 & 914 & 54.8 & 17 & -0.03 \\
\hline $14: 17: 00: 20$ & 88 & 57 & 26 & 23.5 & 53.6 & 0.11 & 917 & 54.8 & 17 & -0.03 \\
\hline $14: 17: 01: 19$ & 89 & 57 & 26 & 23.5 & 53.6 & $0 . \overline{11}$ & 917 & 54.8 & 17 & -0.04 \\
\hline $14: 17: 02: 18$ & 90 & 57 & 26 & 23.7 & 53.6 & & 912 & 54.7 & 17 & -0.04 \\
\hline $14: 17: 03: 22$ & 91 & 57 & 26 & 23.5 & & & 908 & 54.7 & 17 & -0.15 \\
\hline $14: 17: 04: 21$ & 92 & 57 & 26 & 23.7 & 53.6 & 0.08 & 912 & 54.6 & 17 & -0.15 \\
\hline $14: 17: 05: 20$ & 93 & 57 & 26 & 23.7 & 53.6 & 0.11 & 908 & 54.9 & 17 & -0.27 \\
\hline $14: 17: 06: 19$ & 94 & 57 & 26 & 23.7 & 53.6 & 0.11 & 908 & 54.9 & 17 & 0.12 \\
\hline $14: 17: 07: 17$ & 95 & 57 & 26 & 23.7 & 53.6 & & 911 & 55.2 & 17 & 0.12 \\
\hline $14: 17: 08: 22$ & 96 & 58 & 26 & 23.7 & 53.6 & $0.11^{\prime}$ & 914 & 55.5 & 17 & -0.15 \\
\hline $14: 17: 09: 21$ & 97 & 58 & 26 & 23.7 & 53.6 & 0.09 & $918^{-}$ & 55.5 & 17 & -0.15 \\
\hline $14: 17: 10: 19$ & 98 & 58 & 26 & $23.7^{\dagger}$ & 53.6 & 0.11 & 916 & 55.7 & 17 & -0.17 \\
\hline $14: 17: 11: 24$ & 99 & 58 & 26 & $23 . \overline{7}$ & 53.6 & 0.09 & 919 & 55.6 & 17 & -0.17 \\
\hline $7: 12: 23$ & $100^{\dagger}$ & 58 & 26 & $2 \overline{7} .7$ & 53.6 & 0.11 & 921 & 55.6 & 17 & -0.05 \\
\hline $14: 17: 13: 22$ & 101 & 58 & 26 & 23.7 & 53.6 & 0.09 & 918 & 55.5 & 17 & -0.05 \\
\hline $14: 17: 14: 20$ & 102 & 58 & 26 & 23.7 & 53.6 & 0.09 & 914 & 55.4 & 17 & -0.11 \\
\hline $14: 17: 15: 19$ & 103 & 58 & 26 & 23.7 & 53.6 & 0.11 & 910 & 55.4 & 17 & -0.11 \\
\hline $14: 17: 16: 24$ & 104 & 58 & 26 & 23.7 & 53.6 & 0.11 & 914 & 55.7 & 17 & 0.01 \\
\hline $14: 17: 1 \overline{7}: 23$ & 105 & 58 & 26 & 23.7 & 53.6 & 0.09 & 910 & 55.8 & $8 \quad 17$ & 0.01 \\
\hline
\end{tabular}




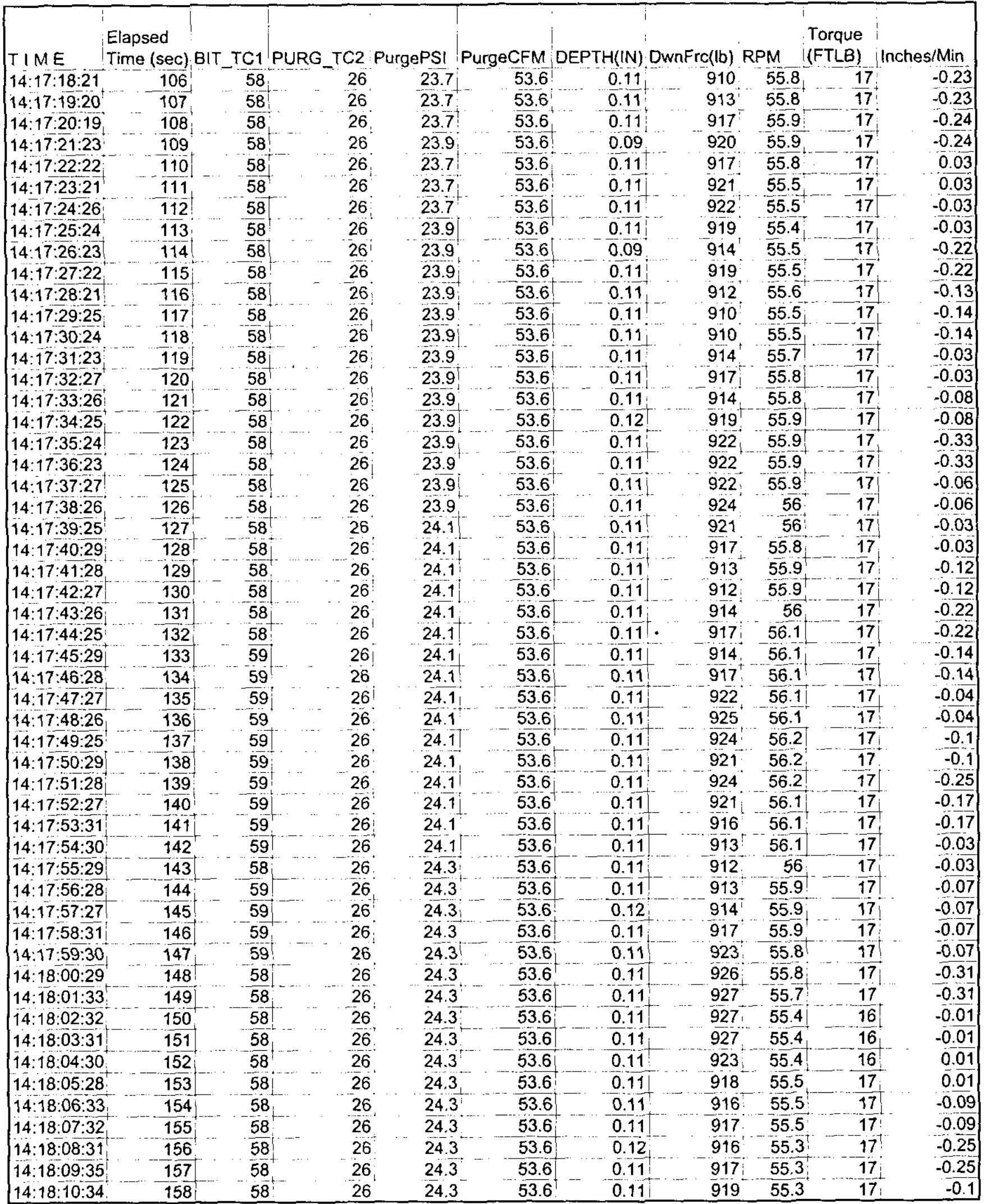




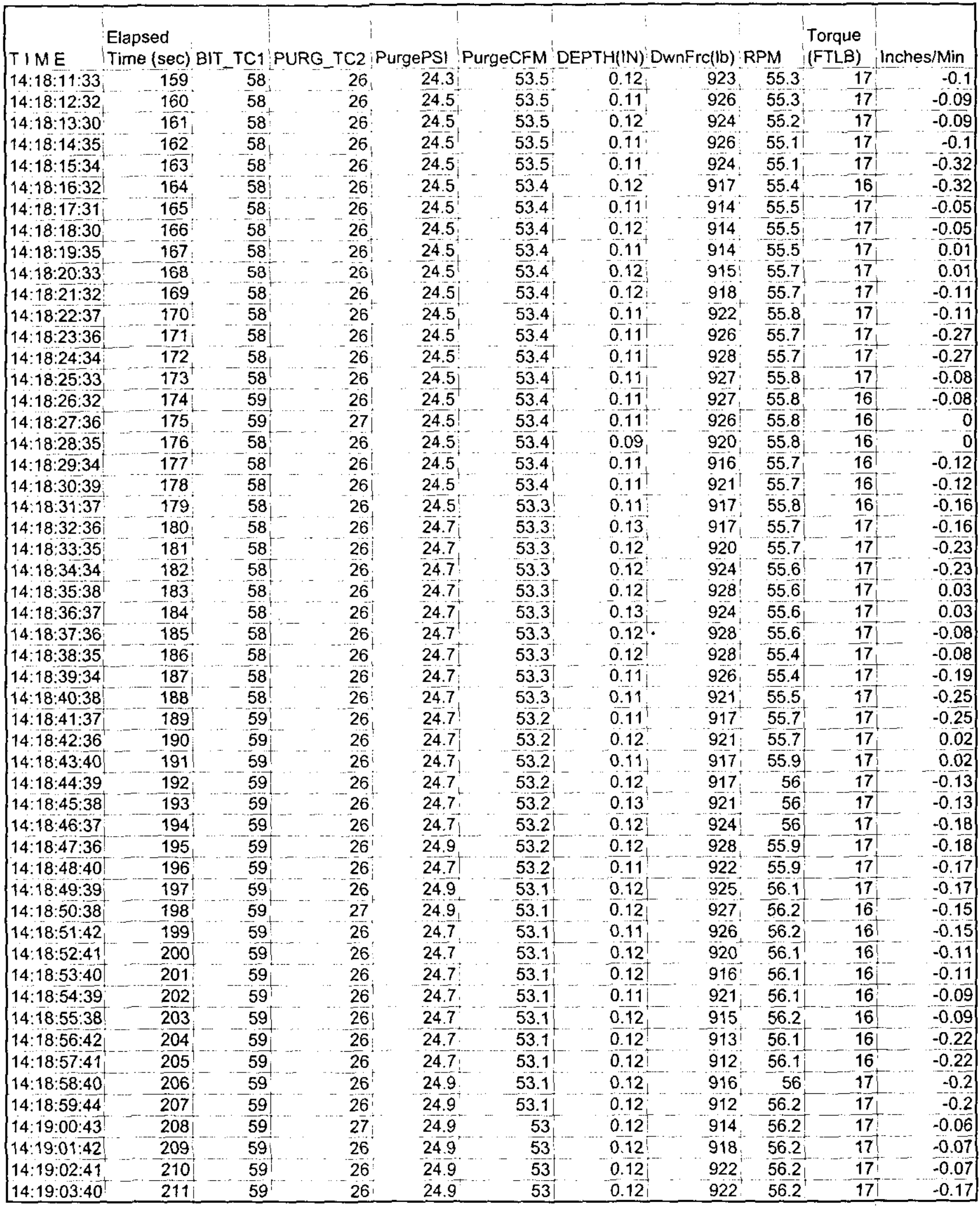




\begin{tabular}{|c|c|c|c|c|c|c|c|c|c|c|}
\hline & $\begin{array}{l}\text { Elapsed } \\
\text { Time (sec) }\end{array}$ & BIT_TC1 & & Purgepsi & PurgeCFM & DEPTH(IN) & DwnFrc(lib) & & $\begin{array}{l}\text { Torque } \\
\text { (FTLB) }\end{array}$ & ches/Min \\
\hline $04: 44$ & 212 & 59 & 26 & $\quad 24.9$ & 53 & 0.12 & 921 & 56 & 16 & -0.17 \\
\hline & 213 & 59 & 26 & & 53 & 0.11 & $922^{\circ}$ & 56 & 16 & -0.27 \\
\hline & 214 & $59^{+}$ & 26 & 24.9 & 53 & 0.11 & $920^{\circ}$ & 56 & 16 & -0.27 \\
\hline & 215 & 59 & 26 & $24.9^{4}$ & 53 & 0.12 & 915 & 56.2 & 16 & -0.09 \\
\hline & 216 & 59 & 26 & 24.9 & 53 & 0.11 & 910 . & $56 . \overline{2}$ & 16 & -0.09 \\
\hline & 217 & 59 & 27 & & 53 & 0.09 & $909^{\circ}$ & 56.2 & 16 & -0.03 \\
\hline & 218 & 59 & $27 !$ & & $53^{1}$ & & 910 & 56.1 & 16 & 0.03 \\
\hline & 219 & 59 & 27 & 24 & $53 !$ & 0.12 & 909 & 56.2 & 17 & 0.18 \\
\hline & 220 & 59 & 27 & 9 & $5 \overrightarrow{3}$ & 0.12 & 909 & 56.2 & 17 & 0.18 \\
\hline & 221 & 59 & 27 & $24.9^{\prime}$ & 53 & & 912 & 56.4 & $17 !$ & 0.37 \\
\hline & 222 & 59 & 27 & 24.9 & 53 & 0.12 & 916 & 56.5 & 17 & -0.37 \\
\hline & 223 & 59 & 27 & & 53 & & 918 & 56.5 & 17 & 0.01 \\
\hline & 224 & 59 & 27 & $24.9^{+}$ & 53 & 0.12 & 920 & 56.3 & 17 & 0.01 \\
\hline & $225^{\dagger}$ & 59 & 26 & 25.1 & 52.9 & 0.12 & 918 & 56.3 & 17 & 0.11 \\
\hline & 226 & 59 & 26 & 24.9 & 52.9 & & 920 & 56.3 & 16 & 0.11 \\
\hline & 227 & 59 & 26 & 24.9 & 52.9 & & 918 & 56.3 & 16 & 0.07 \\
\hline & 228 & 59 & 26 & 24.9 & 52.9 & & 913 & 56.3 & 16 & -0.07 \\
\hline & 229 & 59 & 26 & $24.9^{\circ}$ & 52.9 & & & 56.3 & 16 & -0.2 \\
\hline & 230 & 59 & 26 & 24.9 & 52.9 & & 07 & 0.3 & 16 & -0.2 \\
\hline & 231 & 59 & 26 & 24.9 & 52.9 & & 908 & 56.1 & 16 & 2.26 \\
\hline & 232 & 59 & 26 & & 52.9 & & 906 & 56.1 & 16 & 0.05 \\
\hline & 233 & 59 & 27 & 25.1 & 52.9 & & 08 & 8 & 17 & 0.05 \\
\hline & 234 & 59 & 27 & 1 & 52.9 & & 911 & 5.7 & 17 & 0.01 \\
\hline & 235 & 59 & 27 & 25.1 & $52.9^{\dagger}$ & & 916 & 5.7 & 17 & -0.01 \\
\hline & 236 & 59 & 27 & 25.1 & 52.9 & & 919 & 55.8 & 17 & $0 . \overline{24}$ \\
\hline & 237 & 59 & 27 & 25.1 & 52.9 & & 915 & 55.7 & 17 & -0.24 \\
\hline & 238 & 59 & 27 & $25.1^{\dagger}$ & 52.9 & & & 0.7 & 17 & $=0.21$ \\
\hline & 239 & 59 & 27 & 25.1 & 52.9 & 0.12 & 916 & 55.5 & 17 & 0.21 \\
\hline & 240 & 59 & 27 & & 52.9 & & & 5.2 & 16 & -0.09 \\
\hline & 241 & 59 & 26 & 25.1 & $52.9^{\dagger}$ & & 907 & 55.2 & 16 & -0.09 \\
\hline & 242 & 59 & 27 & 25.1 & 52.8 & & 910 & 55.3 & 16 & 0.03 \\
\hline & 243 & 59 & 27 & & 52.8 & & 906 & 5.3 & 16 & 0.03 \\
\hline & 244 & 59 & 27 & 25.1 & 52.8 & & 905 & 5.3 & 16 & 27 \\
\hline & 245 & 58 & 27 & 251 & 52.8 & & 908 & 55.3 & 16 & -0.27 \\
\hline & 246 & 58 & 26 & 25.1 & 52.8 & & & 55.2 & 16 & -0.18 \\
\hline & 247 & 58 & 27 & 25.1 & 52.8 & & 91 & 55.2 & 16 & $=0.18$ \\
\hline & 248 & 59 & 26 & & 52.8 & & 914 & 55.3 & 16 & 0 \\
\hline & 249 & 59 & 27 & 25.1 & 52.8 & & & 55.4 & 16 & $\frac{0}{7}$ \\
\hline & 250 & 59 & 27 & 25.1 & 52.8 & & 917 & 55.4 & 16 & -0.07 \\
\hline & 251 & 59 & 26 & 25.1 & 52.8 & & & 55.5 & 16 & -0.07 \\
\hline & 252 & 59 & 27 & 25.1 & 52.8 & & 8 & 55.5 & 16 & -0.28 \\
\hline & 25 & 59 & 27 & & 52.8 & & & 55.5 & 16 & $-0 . \overline{28}$ \\
\hline & 254 & 59 & 27 & 25 & 52.8 & & & 55.4 & 16 & -0.23 \\
\hline & 255 & 59 & 27 & 25.3 & 52.8 & & 905 & 55.4 & 16 & -0.02 \\
\hline & 256 & 59 & 27 & 25.3 & 52.8 & & 37 & 55.4 & 16 & -0.02 \\
\hline & 257 & 59 & 27 & 25.3 & 52.8 & 0.12 & 910 & 55.2 & 16 & -0.05 \\
\hline 52 & 258 & 59 & 27 & 25.3 & 52.8 & & 908 & 55.3 & 16 & -0.05 \\
\hline & 259 & $5 \overline{9}$ & 27 & 25.3 & 52.8 & 0.12 & 911 & 55.3 & 16 & $-0 . \overline{3}$ \\
\hline & 260 & 59 & 27 & 25.3 & 52.8 & & 915 & 55.4 & 16 & -0.3 \\
\hline 49 & 261 & 59 & 27 & 25.3 & 52.8 & 0.11 & 917 & 55.5 & 16 & -0.21 \\
\hline & 262 & 59 & 27 & 25.1 & 52.8 & 0.11 & 916 & 55.5 & 16 & -0.21 \\
\hline & 263 & 59 & 27 & 25.3 & 52.8 & 0.12 & 910 & 55.5 & $16 \vec{j}$ & 0.11 \\
\hline $14: 19: 56: 51[$ & $\quad 264$ & 59 & 27 & 25.3 & 52.8 & 0.11 & 914 & 55.3 & 16 & 0.11 \\
\hline
\end{tabular}




\begin{tabular}{|c|c|c|c|c|c|c|c|c|c|c|}
\hline IIME & $\begin{array}{l}\text { Elapsed } \\
\text { Time (sec) }\end{array}$ & BIT_TC1 & PURG_TC2 & PurgePSI & PurgeCFM & DEPTH $(I N)$ & & & $\begin{array}{l}\text { Torque } \\
\text { (FTLB) }\end{array}$ & Inches/Min \\
\hline $14: 19: 57: 55$ & 265 & 59 & 27 & 25.3 & $\quad 52.8$ & $0 . \overline{11}$ & $\quad 910$ & 55.3 & $-\frac{1}{16}$ & $\quad-0.16$ \\
\hline $14: 19: 58: 54$ & 266 & 59 & 27 & 25.3 & 52.8 & 0.11 & 905 & $55.3^{\dagger}$ & 16 & -0.16 \\
\hline $59: 53$ & 267 & 59 & 27 & 25.3 & 52.7 & 0.11 & 905 & 55.6 & 16 & -0.19 \\
\hline 52 & $268^{\circ}$ & 59 & 27 & 25.5 & 52.7 & 0.12 & $908^{\dagger}$ & 55.7 & $\overline{16}$ & -0.19 \\
\hline $01: 51$ & 269 & 59 & 27 & $25.5^{\dagger}$ & $52 . \overline{7}$ & 0.12 & 912 & 55.7 & 16 & $-0 . \overline{16}$ \\
\hline 02.55 & $270^{\circ}$ & 59 & 27 & 25.5 & 52.7 & 0.13 & 907 & 55.7 & $\frac{7}{16}$ & -0.16 \\
\hline 54 & 271 & 59 & 27 & 25.5 & 52.7 & 0.13 & 912 & $55 . \overline{7}$ & 16 & -0.04 \\
\hline 4.53 & 272 & 59 & 27 & 25.5 & 52.7 & 0.13 & 914 & 55.7 & 16 & -0.04 \\
\hline 57 & 273 & 59 & 27 & 25.5 & 52.7 & 0.12 & 916 & 55.7 & $\overline{16}$ & -0.13 \\
\hline $06: 56$ & 274 & 59 & 27 & 25.7 & 52.7 & 0.12 & $915 !$ & 55.7 & 16 & -0.13 \\
\hline 55 & 275 & 58 & 27 & 25.5 & 52.7 & 0.12 & 908 & 55.7 & 16 & $-0 . \overline{18}$ \\
\hline $54^{\dagger}$ & 276 & 58 & & 25.5 & 52.7 & 0.12 & 913 & 55.6 & 16 & -0.18 \\
\hline 53 & $27 \overline{7}$ & 58 & $\overline{2} 7^{\prime}$ & $2 \overline{5.7}$ & 52.7 & $0.12 i$ & 908 & 55.6 & 16 & $-0 . \overline{27}$ \\
\hline 57 & 278 & 58 & 27 & 25.7 & 52.7 & 0.13 & 904 & 55.6 & 16 & 0.09 \\
\hline & 279 & 58 & 27 & 25.7 & 52.7 & 0.12 & 904 & 55.7 & 16 & 0.09 \\
\hline 55 & 280 & 58 & 26 & 25.7 & 52.7 & $0.12 !$ & 907 & 55.7 & 16 & -0.18 \\
\hline 54 & 281 & 58 & 27 & 25.7 & 52.7 & 0.12 & 904 & 55.7 & 16 & $-0 . \overline{18}$ \\
\hline & 282 & 58 & 27 & 25.9 & 52.7 & $5.13^{+}$ & 907 & 55.8 & 16 & -0.07 \\
\hline & 283 & $5 \overline{8}$ & 27 & 25.9 & 52.7 & & 911 & 55.9 & 16 & -0.07 \\
\hline & 284 & 58 & 27 & 25.9 & 52.7 & $0.13^{!}$ & $914^{\dagger}$ & 55.9 & 16 & -0.24 \\
\hline 55 & 285 & 58 & 26 & 25.9 & 52.7 & 0.13 & 916 & 55.8 & 16 & -0.24 \\
\hline & 286 & 58 & 27 & 25.9 & 52.7 & 0.12 & 915 & 55.8 & 16 & -0.21 \\
\hline & 287 & 58 & 27 & 25.9 & 52.7 & & 909 & 55.8 & 16 & -0.21 \\
\hline $0: 51$ & 288 & 58 & 27 & 25.9 & 52.7 & 0.1 & $914^{\dagger}$ & 55.8 & $\overline{16}$ & $-0 . \overline{04}$ \\
\hline & 289 & 58 & 27 & 25.9 & 52.7 & & 909 & 55.8 & 16 & -0.04 \\
\hline 49 & 290 & 58 & & 25.9 & 52.7 & & & 55.8 & 16 & -0.11 \\
\hline 48 & 291 & 58 & 27 & 25.9 & 52.7 & 0.13 & 904 & 55.7 & 16 & -0.11 \\
\hline & 292 & 58 & 27 & 26.1 & 52.7 & & 906 & 55.7 & 16 & $-0 . \overline{21}$ \\
\hline & 293 & 58 & 27 & $26.1^{\dagger}$ & 52.7 & & 909 & 55.8 & 16 & -0.21 \\
\hline $44^{\dagger}$ & 294 & 58 & 27 & 26.1 & 52.7 & & 913 & 55.9 & 16 & -0.1 \\
\hline 49 & $295^{\prime}$ & 58 & 27 & 26.1 & 52.7 & 0.1 & 915 & 55.9 & 16 & $-0 . \overline{1}$ \\
\hline & 296 & 58 & 27 & 26.1 & 52.7 & & 913 & 55.8 & 16 & -0.09 \\
\hline & 297 & 58 & 27 & 26.1 & 52.7 & & & 55.8 & 16 & -0.09 \\
\hline & 298 & 58 & $2 \overline{7}$ & 26.1 & 52.7 & & 914 & 55.8 & 16 & -0.1 \\
\hline $5 \overline{0}$ & 299 & 58 & 26 & 26.1 & 52.7 & 3 & 908 & 55.9 & 16 & -0.1 \\
\hline & 300 & 58 & 26 & 26.1 & 52.7 & & 905 & 55.8 & 16 & -0.2 \\
\hline & 301 & 58 & 27 & 26.3 & 52.7 & $0.11^{\top}$ & & 55.8 & 16 & -0.2 \\
\hline & 302 & 58 & 27 & 26.3 & 52.7 & 0.1 & 905 & 55.8 & 16 & -0.21 \\
\hline 51 & 303 & 58 & 27 & 6.8 & 1.9 & 0.12 & $903^{\dagger}$ & 56 & 16 & -0.04 \\
\hline & 304 & 59 & 27 & -0.8 & 1.9 & 0.1 & 904 & 56 & 17 & -0.04 \\
\hline$: 48$ & 305 & 61 & $27^{\dagger}$ & -0.8 & 1.9 & & & $\overline{56}$ & 17 & -0.07 \\
\hline 53 & 306 & 62 & 27 & -0.8 & 1.9 & 0.1 & 911 & 55.9 & 17 & -0.07 \\
\hline 52 & 307 & 64 & 27 & -0.8 & 1.9 & 0.13 & $908^{\circ}$ & 56.1 & 17 & -0.16 \\
\hline & 308 & 65 & 27 & -0.8 & 1.9 & 0.12 & 912 & 56.1 & 17 & -0.16 \\
\hline & 309 & $66^{7}$ & 27 & -0.8 & 1.9 & 0.1 & 91 & 56.2 & 18 & $-0 . \overline{24}$ \\
\hline & 310 & 67 & $26^{\prime}$ & -0.8 & 1.9 & & 91 & 56.2 & 18 & -0.24 \\
\hline $43: 53$ & 311 & 68 & -27 & -0.8 & 1.9 & 0.12 & 914 & 56.2 & $\overline{18}$ & -0.01 \\
\hline $14: 20: 44: 51$ & 312 & 69 & 27 & -0.8 & 1.9 & 0.12 & 917 & 56 & 18 & -0.01 \\
\hline & 313 & 70 & 27 & -0.8 & 1.9 & 0.12 & 914 & 56 & 18 & -0.1 \\
\hline & 314 & $71\}$ & 27 & -0.8 & 1.9 & & 909 & 56.1 & 18 & -0.1 \\
\hline $20: 47: \overline{48}$ & 315 & 72 & 27 & -0.8 & 1.9 & 0.12 & $905^{\dagger}$ & 56.2 & 19 & -0.07 \\
\hline $14: 20: 48: 52$ & 316 & 73 & 27 & -0.8 & 1.9 & 0.12 & 905 & 56.2 & 19 & -0.07 \\
\hline $14: 20: 4 \overline{9: 51}$ & 317 & 74 & 27 & -0.8 & 1.9 & 0.13 & 907 & 56.2 & 19 & -0.17 \\
\hline
\end{tabular}




\begin{tabular}{|c|c|c|c|c|c|c|c|c|c|c|}
\hline TIME & $\begin{array}{l}\text { Elapsed } \\
\text { Time (sec) }\end{array}$ & & PURG TC2 & PurgePSI & PurgeCFM & DEPTH(IN) & DwnFrc(tb) & !RPM & $\begin{array}{l}\text { Torque } \\
\text { (FTLB) }\end{array}$ & Inches/Min \\
\hline $14: 20: 50: 50$ & 318 & 74 & 27 & -0.8 & $\quad 1.9$ & 0.12 & 905 & 56.1 & 19 & $-\overline{0.17}$ \\
\hline $14: 20: 51: 54$ & $\overline{319}$ & 75 & 27 & -0.8 & 1.9 & 0.13 & 909 & 56.1 & 19 & -0.27 \\
\hline $14: 20: 52: 53$ & 320 & 76 & 27 & -0.8 & $1.9^{\prime}$ & $0.13^{\circ}$ & 912 & 56.1 & 20 & -0.27 \\
\hline $14: 20: 53: 52$ & 321 & 77 & 27 & -0.8 & 1.9 & 0.12 & 916 & 56.1 & 19 & 0.01 \\
\hline $14: 20: 54: 51$ & 322 & 78 & 27 & -0.8 & 1.9 & 0.12 & 921 & 56.1 & 20 & 0.01 \\
\hline $14: 20: 55: 50$ & 323 & 78 & 27 & -0.8 & 1.9 & 0.12 & 919 & 56.1 & 20 & -0.02 \\
\hline $14: 20: 56: 54$ & $\overline{324}$ & $7 \overline{9}$ & 27 & -0.8 & 1.9 & 0.12 & $\overline{921}$ & 55.9 & 20 & -0.02 \\
\hline $14: 20: 57.53$ & 325 & 80 & 27 & -0.8 & 1.9 & 0.12 & 921 & 55.8 & 20 & -0.23 \\
\hline $1: 58: 52$ & 326 & 81 & 27 & -0.8 & 1.9 & 0.11 & 915 & 55.8 & 20 & $-0 . \overline{21}$ \\
\hline $14: 20: 59: 56$ & 327 & 82 & 27 & -0.8 & 1.9 & 0.12 & $9 \overline{11}$ & 55.9 & 20 & -0.21 \\
\hline $14: 21: 00: 55$ & 328 & 83 & 27 & -0.8 & $1.3 !$ & 0.12 & 909 & 55.9 & 20 & -0.05 \\
\hline $1: 54$ & 329 & 83 & 27 & -0.8 & 1.9 & 0.12 & 910 & 55.9 & 21 & -0.05 \\
\hline $02: 53$ & 330 & 84 & 27 & -0.8 & $1.9^{\prime}$ & 0.13 & 908 & 55.8 & 21 & -0.03 \\
\hline $14: 21: 03: 52$ & 331 & $85^{\dagger}$ & 27 & -0.8 & 1.9 & 0.12 & 909 & 55.7 & 21 & -0.03 \\
\hline $14: 21: 04: 56$ & 332 & 86 & 27 & -0.8 & 1.9 & 0.12 & 912 & 55.7 & 21 & $-0 . \overline{18}$ \\
\hline $5: 55^{\prime}$ & 333 & 87 & 27 & $-0 . \overline{8}$ & 1.9 & 0. & 917 & 55.7 & 21 & -0.18 \\
\hline $14: 21: 06: 54$ & 334 & 88 & 27 & -0.8 & 1.9 & 0.12 & 921 & 55.8 & 21 & -0.26 \\
\hline $14: 21: 07: 58$ & 335 & 88 & 27 & -0.8 & 1.9 & 0.12 & 918 & 55.8 & 21 & -0.26 \\
\hline 8.57 & 336 & 89 & 27 & -0.8 & 1.9 & 0.12 & 921 & 55.8 & 21 & -0.01 \\
\hline $09: 56$ & 337 & 90 & 27 & -0.8 & $1.9^{\circ}$ & 0.12 & 921 & 55.8 & 21 & -0.01 \\
\hline $14: 21: 10: 55$ & 338 & 91 & 27 & -0.8 & 1.9 & 0.11 & 915 & 55.8 & 21 & -0.12 \\
\hline $14: 21:$ & 339 & 92 & 27 & -0.8 & $1.9^{\circ}$ & 0.12 & 911 & 55.7 & 21 & -0.12 \\
\hline 258 & 340 & 93 & 27 & -0.8 & 1.9 & 0.12 & 909 & 55.7 & 21 & -0.04 \\
\hline $5 \overline{5}$ & 341 & 94 & 27 & -0.8 & $1.9^{\circ}$ & 0.12 & 911 & 55.7 & 21 & $-0.0 \overline{4}$ \\
\hline 56 & 342 & 94 & 27 & -0.8 & 1.9 & 0.13 & 909 & 55.8 & 21 & -0.3 \\
\hline 55 & 343 & 95 & 27 & -0.8 & 1.9 & 0.13 & 910 & 55.9 & 22 & -0.3 \\
\hline 54 & $\overline{344}$ & 96 & 27 & -0.8 & 1.9 & 0.12 & 912 & 55.9 & 22 & $0 . \overline{05}$ \\
\hline $7: 58$ & 345 & 96 & 27 & -0.8 & 1.9 & 0.12 & 917 & 55.7 & 22 & 0.05 \\
\hline $18: 57$ & 346 & $97 \overrightarrow{0}$ & 27 & -0.8 & 1.9 & 0.12 & 914 & 55.7 & 22 & $-0 . \overline{15}$ \\
\hline $9: 56$ & 347 & 98 & 27 & -0.8 & 1.9 & 0.12 & 919 & 55.7 & 23 & -0.15 \\
\hline$: 60$ & 348 & 99 & 27 & -0.8 & 1.9 & 0.12 & 922 & 55.7 & 23 & -0.09 \\
\hline $21 \overline{59}$ & 349 & 100 & 27 & -0.8 & $1.9 !$ & 0.12 & 921 & 55.8 & 23 & -0.27 \\
\hline $22: 58$ & 350 & 101 & 27 & -0.8 & $1.9^{\circ}$ & & 918 & 55.8 & 22 & -0.27 \\
\hline & 351 & 101 & 27 & -0.8 & 1.9 & 0.12 & 912 & 55.9 & 22 & -0.1 \\
\hline 55 & 352 & 102 & 27 & -0.8 & $1.9^{\circ}$ & 0.12 & 910 & 55.7 & 22 & $-\overline{0.1}$ \\
\hline 60 & 353 & 103 & 27 & -0.8 & 1.9 & 0.12 & 913 & 55.7 & 22 & -0.08 \\
\hline & 354 & 103 & 27 & -0.8 & 1.9 & 0. & 910 & 55.8 & 22 & $-0 . \overline{08}$ \\
\hline $7: 58$ & 355 & 104 & 27 & $-0 . \overline{8}$ & 1.9 & 0.12 & 911 & 55.8 & 22 & -0.13 \\
\hline $88: 62$ & 356 & 104 & 27 & -0.8 & $1.9^{\dagger}$ & 0.13 & 914 & 55.8 & 23 & $-0 . \overline{13}$ \\
\hline $14: 21: 29: 61$ & 357 & 104 & 27 & -0.8 & 1.9 & 0.13 & 912 & 55.8 & 23 & -0.19 \\
\hline & 35 & 105 & 27 & -0.8 & 1.9 & & 922 & 55.8 & 23 & -0.19 \\
\hline & 35 & $\overline{105}$ & 27 & -0.8 & 1.9 & 0.1 & 919 & 55.8 & 23 & $-0 . \overline{07}$ \\
\hline $2: 57$ & 360 & 105 & 27 & -0.8 & $1.9^{\dagger}$ & 0.13 & 922 & 55.7 & 23 & -0.07 \\
\hline $14: 21: 33: 62$ & 361 & 105 & 27 & -0.8 & 1.9 & 0.12 & 923 & 55.7 & 23 & -0.1 \\
\hline $4: 61$ & 362 & 106 & 27 & -0.8 & 1.9 & 0.1 & 920 & 55.7 & 23 & -0.1 \\
\hline & 363 & 106 & 27 & -0.8 & 1.9 & 0. & 915 & 55.6 & 22 & -0.06 \\
\hline & 364 & $\overrightarrow{106}$ & 27 & -0.8 & $1.9^{\prime}$ & 0.12 & 919 & 55.6 & 23 & -0.06 \\
\hline $14: 21: 37: 63$ & 365 & 107 & 27 & -0.8 & 1.9 & 0.12 & 914 & 55.6 & 23 & -0.24 \\
\hline $14: 21: 38: 62$ & 366 & 107 & 27 & -0.8 & $1 . \overline{9}$ & 0.12 & 912 & 55.7 & 23 & -0.24 \\
\hline $14: 21: 39: 6 \overline{0}$ & 367 & 107 & 27 & -0.8 & 1.9 & 0.12 & 912 & 55.5 & 23 & $-0 . \overline{12}$ \\
\hline $14: 21: 40: 59$ & 368 & 108 & 27 & -0.8 & 1.9 & 0.12 & 916 & 55.5 & 23 & -0.12 \\
\hline $11: 64$ & 369 & 108 & 27 & -0.8 & 1.9 & 0.12 & 921 & 55.5 & 23 & -0.09 \\
\hline $14: 21: 42: 63$ & 370 & 108 & 27 & -0.8 & 1.9 & 0.13 & 917 & 55.7 & 23 & $-0 . \overline{09}$ \\
\hline
\end{tabular}




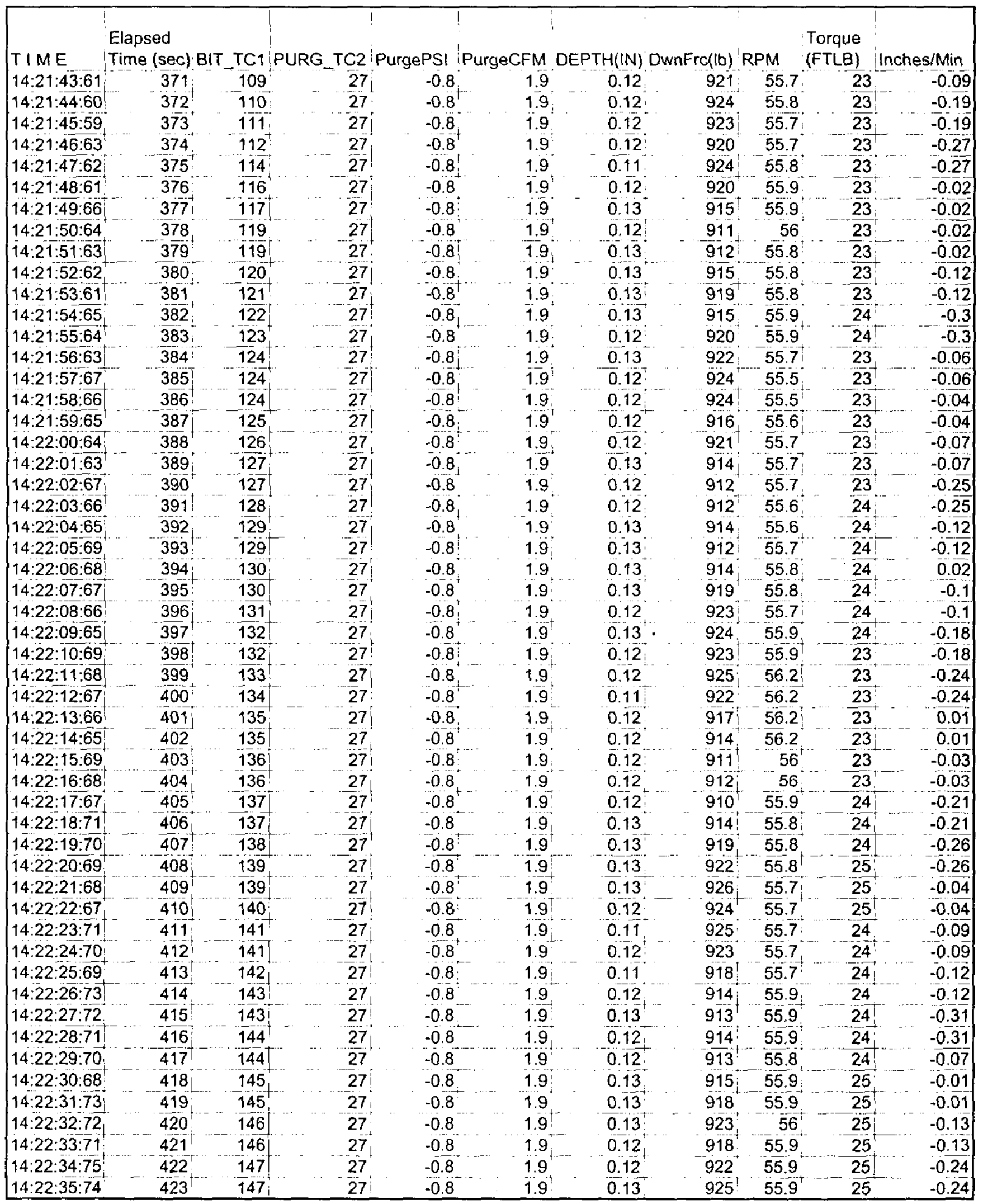




\begin{tabular}{|c|c|c|c|c|c|c|c|c|c|c|}
\hline $\mathrm{T} I \mathrm{ME}$ & $\begin{array}{l}\text { Elapsed } \\
\text { Time (sec) }\end{array}$ & & PURG_TC2 & PurgePSI & & DEPTH(IN) & DwnFrc(lb) & RPM & $\begin{array}{l}\text { Torque } \\
\text { (FTLB) }\end{array}$ & Inches/Min \\
\hline $14: 22: 36: 73$ & $\quad 424$ & 148 & 27 & -0.8 & $\begin{array}{r}1.9 \\
\quad 19\end{array}$ & $\quad 0.12$ & $\quad 924$ & $\begin{array}{ll}55.9 \\
\end{array}$ & 25 & $5-\quad-0.24$ \\
\hline $14: 22: 37: 72$ & 425 & 148 & 27 & -0.8 & 1.9 & 0.12 & 920 & 55.9 & 25 & -0.24 \\
\hline $14: 22: 38: 70$ & $426 !$ & 149 & 27 & -0.8 & 1.9 & 0.12 & 915 & $55 . \overline{9}$ & 24 & 0.05 \\
\hline $14: 22: 39: 75$ & 427 & 149 & 27 & -0.8 & 1.9 & 0.12 & 920 & 55.8 & 24 & 0.05 \\
\hline $14: 22: 40: 74$ & 428 & 150 & 27 & -0.8 & 1.9 & 0.12 & 915 & 55.8 & 24 & -0.07 \\
\hline $14: 22: 41: 72$ & $429^{i}$ & 150 & 27 & -0.8 & 1.9 & 0.12 & 914 & 55.8 & 24 & -0.07 \\
\hline $14: 22: 42: 71$ & 430 & $1 \overline{51}$ & 27 & -0.8 & 1.9 & 0.13 & 914 & 55.7 & 24 & -0.24 \\
\hline $14: 22: 43: 70$ & 431 & 151 & 27 & -0.8 & 1.9 & 0.13 & 918 & 55.7 & 24 & -0.24 \\
\hline $14: 22: 44: 75$ & 432 & $15 \overline{2}$ & 27 & -0.8 & 1.9 & 0.13 & 923 & 55.6 & 25 & -0.21 \\
\hline $14: 22: 45: 73$ & 433 & 152 & 27 & -0.8 & 1.9 & 0.13 & 919 & 55.6 & 25 & -0.21 \\
\hline $14: 22: 46: 72$ & 434 & 153 & 27 & -0.8 & 1.9 & 0.13 & 924 & 55.6 & 25 & 0.05 \\
\hline $14: 22: 47: 77$ & 435 & 153 & 27 & -0.8 & 1.8 & 0.13 & 926 & 55.4 & 25 & 0.05 \\
\hline $14: 22: 4 \overline{8}: 76$ & 436 & 154 & 27 & -0.8 & $1 . \overline{9}$ & 0.12 & 926 & 55.5 & 25 & -0.09 \\
\hline $14: 22: 49: 74$ & 437 & 155 & 27 & -0.8 & 19 & 0.12 & 921 & 55.5 & 25 & -0.09 \\
\hline $14: 22: 50: \overline{73}$ & 438 & 155 & 27 & -0.8 & $1 . \overline{9}$ & 0.12 & 925 & 55.6 & 25 & -0.2 \\
\hline $14: 22: 51: 72$ & $439 \dagger$ & 156 & 27 & -0.8 & 1.9 & 0.12 & 919 & $5 \overline{5} 6$ & 25 & -0.2 \\
\hline $14: 22: 52: 76$ & 440 & 156 & 27 & -0.8 & 1.9 & $0 . \overline{12}$ & 915 & 55.6 & 25 & -0.21 \\
\hline $14: 22: 53: 75$ & 441 & 157 & 27 & $-0 . \overline{8}$ & 1.9 & $\overline{0.12}$ & 914 & 55.6 & 25 & 0.07 \\
\hline $14: 22: 54: 74$ & 442 & 157 & 27 & -0.8 & 1.9 & 0.12 & 916 & 55.5 & 25 & 0.07 \\
\hline $14: 22: 55: 79$ & 443 & 157 & 27 & -0.8 & 1.9 & 0.13 & 920 & 55.5 & 25 & -0.1 \\
\hline $14: 22: 56: \overline{77}$ & 444 & 158 & 27 & -0.8 & 1.9 & 0.13 & 917 & 55.4 & 25 & -0.1 \\
\hline $14: 22: 57: 76$ & 445 & 158 & 27 & -0.8 & 1.9 & 0.13 & 922 & 55.4 & 25 & -0.21 \\
\hline $14: 22: 58: 75$ & 446 & 159 & 27 & -0.8 & 1.9 & 0.13 & 926 & 55.4 & 25 & -0.21 \\
\hline $14: 22: 59: 74$ & $44 \overline{7}$ & 159 & 27 & -0.8 & 1.9 & 0.13 & 927 & 55.3 & 25 & -0.22 \\
\hline $14: 23: 00: 78$ & 448 & 160 & 27 & -0.8 & 1.9 & 0.13 & 924 & 55.4 & 25 & -0.22 \\
\hline $14: 23: 01: 77$ & 449 & 160 & 27 & -0.8 & 1.9 & 0.13 & 926 & $5 \overline{5.4}$ & 25 & 0.09 \\
\hline $14: 23: 02: 76$ & 450 & 161 & 27 & -0.8 & 1.9 & 0.12 & 922 & 55.4 & 25 & 0.09 \\
\hline $14: 23: 03: 75$ & 451 & 161 & 27 & -0.8 & 1.9 & 0.12 & 917 & 55.4 & 25 & -0.16 \\
\hline $14: 23: 04: 74$ & 452 & 162 & 27 & -0.8 & 1.9 & 0.13 & 914 & 55.4 & 25 & -0.16 \\
\hline $14: 23: 05: 78$ & 453 & 162 & 27 & -0.8 & 1.9 & $0 . \overline{13}$ & 914 & 55.5 & 26 & -0.2 \\
\hline $14: 23: 06: 77$ & 454 & 163 & 27 & -0.8 & 1.9 & 0.13 & $91 \bar{t}$ & 55.6 & 26 & -0.2 \\
\hline $14: 23: 07: 76$ & 455 & 163 & 27 & -0.8 & 1.9 & 0.13 & 915 & 55.6 & 26 & -0.21 \\
\hline $14: 23: 08: 80$ & $\overline{456}$ & 164 & 27 & -0.8 & 1.9 & 0.13 & 918 & 55.6 & 26 & -0.21 \\
\hline $14: 23: 09: 79$ & 457 & 164 & 27 & -0.8 & $1 . \bar{s}$ & 0.13 & 923 & 556 & 26 & -0.03 \\
\hline $14: 23: 10: 78$ & 458 & 164 & 27 & -0.8 & 1.9 & 0.13 & 925 & 55.6 & 26 & -0.03 \\
\hline $14: 23: 11: 77$ & 459 & 165 & 27 & -0.8 & 1.9 & 0.12 & 926 & $5 \overline{5} .4$ & 26 & -0.03 \\
\hline $14: 23: 12: 76$ & 460 & 165 & 27 & -0.8 & 1.5 & 0.12 & 922 & 55.5 & 25 & -0.03 \\
\hline $14: 23: 13: 80$ & 461 & 166 & 27 & -0.8 & 1.9 & 0.12 & 917 & 55.5 & 25 & -0.25 \\
\hline $14: 23: 14: 79$ & 462 & 166 & 27 & -0.8 & 1.9 & 0.12 & 922 & 55.6 & 25 & -0.22 \\
\hline $14: 23: 15: 78$ & 463 & 167 & 27 & -0.8 & 9.9 & 0.12 & 917 & 55.6 & 25 & -0.22 \\
\hline $14: 23: 16: 82$ & 464 & 167 & 27 & -0.8 & 1.9 & $0 . \overline{12}$ & 915 & 55.6 & 25 & 0 \\
\hline $14: 23: 17: 81$ & 465 & 168 & 27 & -0.8 & 1.9 & 0.13 & 916 & 55.5 & 25 & 0 \\
\hline $14: 23: 18: 80$ & 466 & 168 & 27 & -0.8 & 1.9 & 0.12 & 919 & 55.5 & 25 & -0.05 \\
\hline $14: 23: 19: 79$ & 467 & 169 & 27 & -0.8 & 1.9 & 0.13 & 917 & 55.5 & 26 & -0.05 \\
\hline $14: 23: 20: 78$ & 468 & 169 & 27 & -0.8 & 1.9 & 0.13 & 921 & 55.4 & 26 & -0.24 \\
\hline $14: 23: 21: 82$ & 469 & 170 & 27 & -0.8 & 1.8 & 0.13 & 924 & 55.5 & 26 & -0.24 \\
\hline $14: 23: 22: 81$ & 470 & 170 & 27 & $-0 . \overline{8}$ & 1.8 & 0.13 & 927 & 55.5 & 26 & -0.25 \\
\hline $14: 23: 23: 80$ & $4 \overline{71}$ & 171 & 27 & -0.8 & 1.9 & 0.12 & 926 & 55.5 & 26 & -0.25 \\
\hline $14: 23: 24: 84$ & 472 & 171 & 27 & -0.8 & 1.9 & 0.12 & 921 & 55.6 & 26 & 0.04 \\
\hline $14: 23: 25: 83$ & 473 & 172 & 27 & -0.8 & 1.8 & 0.12 & 926 & 55.6 & 26 & 0.04 \\
\hline $14: 23: 26: 82$ & 474 & 172 & 27 & -0.8 & 1.9 & 0.12 & 921 & 55.7 & 26 & -0.1 \\
\hline $14: 23: 27: 81$ & 475 & 173 & 27 & $-0 . \overline{8}$ & 1.9 & 0.12 & 916 & 55.7 & 26 & -0.1 \\
\hline $14: 23: 28: 80$ & 476 & 173 & 27 & -0.8 & 1.8 & 0.13 & 914 & 55.7 & 26 & -0.15 \\
\hline
\end{tabular}




\begin{tabular}{|c|c|c|c|c|c|c|c|c|c|c|}
\hline TIME T & $\begin{array}{l}\text { Elapsed } \\
\text { Time (sec) }\end{array}$ & BIT_TC1 & PURG_TC2 $\mid F$ & PurgePSI & PurgeCFM & DEPTH $(I N)$ & DwnFrc(lb) & RPM & $\begin{array}{l}\text { Torque } \\
\text { (FTLB) }\end{array}$ & Inches/Min \\
\hline $14: 23: 29: 84$ & $\quad 477$ & 174 & 27 & $\quad-0.8$ & 1.9 & 0.13 & 916 & 55.6 & $2 \quad 26$ & -0.15 \\
\hline $14: 23: 30: 83$ & 478 & 174 & 27 & -0.8 & 1.9 & 0.12 & 914 & 55.6 & 26 & -0.29 \\
\hline $14: 23: 31: 82$ & $479^{\circ}$ & 175 & $27 !$ & -0.8 & $1 . \overline{9}$ & 0.12 & 917 & 55.6 & 26 & -0.29 \\
\hline $14: 23: 32: 86$ & 480 & 175 & 27 & -0.8 & 1.9 & 0.13 & 921 & 55.7 & 26 & 0.02 \\
\hline $14: 23: 33: 85$ & 481 & 175 & 27 & -0.8 & $1 . \overline{9}^{\dagger}$ & 0.13 & 924 & 55.7 & 26 & 0.02 \\
\hline $14: 23: 34: 84$ & 482 & 176 & 27 & -0.8 & 1.9 & $0 . \overline{13}$ & 928 & 55.7 & 26 & -0.1 \\
\hline $14: 23: 35: 83$ & 483 & 176 & $27 !$ & -0.8 & 1.9 & 0.12 & 926 & 55.7 & 26 & $=0.1$ \\
\hline $14: 23: 36: 82$ & 484 & 177 & 27 & -0.8 & 1.9 & 0.12 & 928 & 55.8 & 26 & -0.1 \\
\hline $14: 23: 37: 86$ & 485 & 177 & 27 & -0.8 & 1.9 & 0.12 & 926 & 55.8 & 26 & -0.1 \\
\hline $14: 23: 38: 85$ & 486 & 178 & 27. & -0.8 & 1.9 & 0.12 & 921 & 55.9 & 26 & -0.29 \\
\hline $14: 23: 39: 84$ & 487 & 179 & 27 & -0.8 & 1.9 & 0.13 & 916 & 55.9 & 26 & 0.07 \\
\hline $14: 23: 40: 82$ & 488 & 179 & 27 & $-0 . \overline{8}$ & 1.9 & 0.12 & 915 & 55.9 & 27 & 0.07 \\
\hline $14: 23: 41: 81$ & 489 & 180 & 27 & -0.8 & 1.9 & 0.13 & 917 & 55.7 & 27 & $-0 . \overline{15}$ \\
\hline $14: 23: 42: 86$ & $490^{\circ}$ & 180 & 27 & -0.8 & 1.9 & 0.12 & 916 & 55.8 & 27 & -0.15 \\
\hline $14: 23: 4385$ & 491 & 181 & 27 ! & -0.8 & 1.9 & 0.13 & 917 & 55.8 & 27 & -0.09 \\
\hline $14: 23: 44: 83$ & 492 & 181 & 27 & -0.8 & 1.9 & 0.13 & 921 & 55.8 & 27 & -0.09 \\
\hline $14: 23: 45: 88$ & $493^{+}$ & 182 & 27 & -0.8 & 1.9 & 0.13 & 926 & 55.8 & 27 & -0.28 \\
\hline $14: 23: 46: 87$ & 494 & 182 & 27 & -0.8 & 1.9 & 0.12 & 929 & 55.8 & 27 & -0.28 \\
\hline $14: 23: 47: 86$ & 495 & 182 & 27 & -0.8 & 1.9 & 0.13 & 927 & 55.8 & 26 & -0.05 \\
\hline $14: 23: 48: 84$ & 496 & 183 & 27 & -0.8 & 1.9 & 0.13 & 927 & 55.7 & 26 & -0.05 \\
\hline $14: 23: 49: 83$ & 497 & 183 & 27 & -0.8 & 1.9 & 0.12 & 927 & 55.7 & 26 & -0.1 \\
\hline $14: 23: 50: 88$ & 498 & 183 & 27 & -0.8 & 1.9 & 0.12 & 922 & 55.6 & 26 & -0.1 \\
\hline $14: 23: 51: 86$ & 499 & 184 & 27 & -0.8 & 1.9 & 0.12 & 918 & 55.7 & 26 & -0.08 \\
\hline $14: 23: 52: 85$ & 500 & 184 & 27 & -0.8 & 1.9 & 0.13 & 916 & 55.7 & 26 & -0.08 \\
\hline $14: 23: 53: 84$ & 501 & 185 & 27 & $-0.8^{\dagger}$ & 1.9 & 0.13 & 917 & 55.9 & 27 & -0.23 \\
\hline $14: 23: 54: 83$ & 502 & 185 & 27 & -0.8 & 1.9 & 0.13 & 916 & 55.7 & 27 & -0.23 \\
\hline $14: 23: 55: 8 \overline{7}$ & 503 & 186 & 27 & -0.8 & 1.9 & 0.13 & 918 & 55.7 & 27 & -0.22 \\
\hline $14: 23: 56: 86$ & 504 & 186 & $27 !$ & -0.8 & 1.9 & 0.13 & 921 & 55.7 & 27 & -0.22 \\
\hline $14: 23: 57: 85$ & 505 & 186 & 27 & -0.8 & 1.9 & 0.13 & 926. & 55.8 & 27 & $0 . \overline{02}$ \\
\hline $14: 23: 5 \overline{8}: 90$ & 506 & 187 & 27 & $-\overline{0.8}$ & 1.9 & 0.13 & $929^{\circ}$ & 55.8 & 27 & 0.02 \\
\hline $14: 23: 59: 88$ & 507 & 187 & 27 & -0.8 & 1.9 & $0 . \overline{12}$ & 927 & 55.9 & 27 & -0.09 \\
\hline $14: 24: 00: 87$ & 508 & 188 & 27 & -0.8 & 1.9 & 0.12 & 929 & 55.9 & 26 & -0.09 \\
\hline $14: 24: 01: 92$ & 509 & 188 & $27^{\dagger}$ & -0.8 & 1.9 & 0.12 & 927 & 55.9 & 26 & -0.22 \\
\hline $14: 24: 02: 90$ & $510^{\dagger}$ & 189 & 27 & -0.8 & $1 \overline{9}$ & 0.12 & 923 & 55.8 & 26 & -0.24 \\
\hline $14: 24: 03: 89$ & 511 & 189 & 27 & -0.8 & 1.9 & 0.12 & $9 \overrightarrow{919}$ & 55.8 & 26 & $-\overline{0.24}$ \\
\hline $14: 24: 04: 88$ & 512 & 190 & 27 & -0.8 & 1.9 & 0.12 & 923 & 55.8 & 26 & 0.01 \\
\hline $14: 24: 05: 87$ & 513 & 191 & 27 & $-\overline{0} .8$ & 1.9 & 0.12 & 919 & 55.9 & 26 & 0.01 \\
\hline $14: 24: 06: 91$ & 514 & 191 & 27 & -0.8 & $1 . \overline{9}$ & 0.13 & 917 & 56 & 26 & -0.08 \\
\hline $14: 24: 07: 90$ & 515 & 191 & 27 & -0.8 & 1.9 & 0.13 & 917 & 56 & 28 & $-0.0 \overline{8}$ \\
\hline $14: 24: 08: 89$ & 516 & 192 & 27 & -0.8 & 1.9 & 0.13 & 921 & 55.9 & 27 & -0.14 \\
\hline $14: 24: 09: 88$ & 517 & 192 & 27 & -0.8 & 1.9 & 0.13 & 926 & 55.7 & 28 & -0.14 \\
\hline $14: 24: 10: 87$ & 518 & 192 & 27 & -0.8 & 1.9 & 0.13 & 929 & 55.7 & 28 & -0.29 \\
\hline $14: 24: 11: 91$ & 519 & 193 & 27 & -0.8 & 1.9 & 0.13 & 926 & 55.8 & 28 & $-0 . \overline{29}$ \\
\hline $14: 24: 12: 90$ & 520 & 193 & 27 & $-0 . \overline{8}$ & 1.9 & $0 . \overline{13}$ & 929 & 55.9 & 28 & 0.03 \\
\hline $14: 24: 13: 89$ & 521 & 194 & 27 & -0.8 & 1.9 & 0.13 & 928 & 55.9 & 28 & 0.03 \\
\hline $14: 24: 14: 93$ & 522 & 194 & 27 & -0.8 & 1.9 & 0.13 & 924 & 55.7 & 27 & -0.13 \\
\hline $14: 24: 15: 92$ & 523 & 195 & 27 & -0.8 & 1.9 & 0.12 & 928 & 55.7 & 27 & -0.13 \\
\hline $14: 24: 16: 91$ & 524 & 195 & $27^{\circ}$ & $-0 . \overline{8}$ & 1.9 & 0.12 & 917 & 55.7 & 27 & $-0 . \overline{1}$ \\
\hline $14: 24: 17: 90$ & 525 & 196 & 27 & -0.8 & $1 \overline{9}$ & 0.12 & 920 & 55.9 & 27 & -0.1 \\
\hline $14: 24: 18: 89$ & 526 & 196 & 27 & -0.8 & 1.9 & 0.12 & 917 & 55.9 & 27 & $-0.2 \overline{8}$ \\
\hline $14: 24: 19: 93$ & 527 & 197 & 27 & -0.8 & 1.9 & 0.13 & 917 & 55.9 & 27 & $-0 . \overline{28}$ \\
\hline $14: 24: 20: 92$ & 528 & 197 & 27 & $-0 . \overline{8}$ & 1.9 & 0.12 & 921 & 55.9 & 27 & -0.14 \\
\hline $14: 24: 21: 91^{\dagger}$ & 529 & 197 & 27 & -0.8 & 1.9 & 0.13 & 924 & 55.8 & 28 & -0.14 \\
\hline
\end{tabular}




\begin{tabular}{|c|c|c|c|c|c|c|c|c|c|c|}
\hline$T I M E$ & $\begin{array}{l}\text { Elapsed } \\
\text { Time (sec) }\end{array}$ & BIT_TC1 & PURG_TC2 & PurgePS| & PurgeCFM & DEPTH(IN) & DwnFrc(lb) & RPM & $\begin{array}{l}\text { Torque } \\
\text { (FTLB) }\end{array}$ & Inches/Min \\
\hline $14: 24: 22: 95$ & 530 & -198 & 27 & $\quad-0.8$ & 1.9 & $\quad 0.13$ & $\quad 921$ & 558 & $\quad 28$ & $\begin{array}{ll}8 & 0.01\end{array}$ \\
\hline $14: 24: 23: 94$ & 531 & 198 & 27 & -0.8 & 1.9 & 0.12 & 925 & 55.6 & 28 & -0.09 \\
\hline $14: 24: 24: 93$ & 532 & $19 \overline{8}$ & 27 & -0.8 & 1.9 & $0 . \overline{13}$ & 929 & 55.5 & 28 & -0.09 \\
\hline $14: 24: 25: 92$ & 533 & $199^{\prime}$ & 27 & -0.8 & 1.9 & 0.12 & 929 & 55.5 & 28 & -0.26 \\
\hline $14: 24: 26: 91$ & 534 & 199 & 27 & -0.8 & 1.9 & 0.12 & 926 & 55.6 & 28 & -0.26 \\
\hline $14: 24: 27: 95$ & 535 & 200 & 27 & -0.8 & 1.9 & 0.13 & 921 & 55.7 & 28 & -0.16 \\
\hline $14: 24: 28: 94$ & 536 & 200 & 27 & -0.8 & 1.9 & 0.12 & 924 & 55.7 & 28 & -0.16 \\
\hline $14: 24: 29: 93$ & 537 & 201 & 27 & -0.8 & 1.9 & 0.12 & 920 & 55.8 & 28 & -0.02 \\
\hline $14: 24: 30: 97$ & 538 & 201 & 27 & -0.8 & 1.9 & 0.12 & $\overline{917}$ & 55.9 & 28 & -0.02 \\
\hline $14: 24: 31: 96$ & 539 & 202 & 27 & -0.8 & 1.9 & 0.12 & 916 & 55.9 & 29 & -0.08 \\
\hline $14: 24: 32: 95$ & 540 & 202 & 27 & -0.8 & 1.9 & 0.13 & 919 & 56 & 29 & -0.08 \\
\hline $14: 24: 33: 94$ & 541 & 202 & 27 & -0.8 & 1.9 & 0.13 & $9 \overline{16}$ & 56 & 29 & -0.22 \\
\hline $14: 24: 34: 93$ & 542 & 202 & 27 & -0.8 & 1.9 & 0.13 & 919 & 56 & 29 & -0.22 \\
\hline $14: 24: 35: 97$ & 543 & 203 & 27 & -0.8 & 1.9 & $0.1 \overline{3}$ & 924 & 56 & 28 & -0.21 \\
\hline $14: 24: 36: 96$ & 544 & 203 & 27 & -0.8 & 1.9 & 0.13 & 927 & 56 & 28 & -0.21 \\
\hline $14: 24: 37: 95$ & 545 & 204 & 27 & -0.8 & 1.9 & 0.13 & 929 & 56 & 28 & 0 \\
\hline $14: 24: 38: 94$ & 546 & 204 & 27 & -0.8 & 1.9 & 0.13 & 926 & 55.9 & 28 & 0 \\
\hline $14: 24: 39: 92$ & 547 & 204 & 27 & 13.4 & 48.8 & 0.12 & 929 & 55.9 & 28 & -0.06 \\
\hline $14: 24: 4 \overline{0}: 9 \overline{7}$ & 548 & 200 & 27 & 25.7 & 50.2 & 0.12 & 925 & 55.9 & 24 & $-0.0 \overline{6}$ \\
\hline $14: 24: 41: 96$ & 549 & 193 & 27 & 27.8 & 50.2 & 0.12 & 920 & 55.9 & 22 & -0.13 \\
\hline $14: 24: 42: 95$ & 550 & 189 & 27 & 28.2 & 50.2 & 0.12 & 914 & 56 & 23 & -0.13 \\
\hline $14: 24: 43: 93$ & 551 & 183 & 27 & 28.4 & 50.2 & 0.12 & 912 & 56 & 21 & -0.28 \\
\hline $14: 24: 44: 92$ & 552 & 178 & 27 & 28.2 & 50.2 & 0.13 & 912 & 56.2 & 21 & -0.28 \\
\hline $14: 24: 45: 97$ & 553 & 173 & 27 & 28.2 & 50.2 & 0.13 & 910 & 56.2 & 20 & -0.01 \\
\hline $14: 24: 46: 96$ & 554 & 169 & 27 & 28.2 & 50.2 & 0.13 & 911 & 56.2 & 19 & -0.1 \\
\hline $14: 24: 47: 94$ & 555 & 164 & 27 & 28.2 & 50.2 & 0.13 & 913 & 56.2 & 19 & -0.1 \\
\hline $14: 24: 48: 99$ & 556 & 161 & 27 & 28.2 & 50.2 & 0.12 & 916 & 56.4 & 19 & -0.07 \\
\hline $14: 24: 49: 98$ & 557 & 158 & $27 !$ & 28 & 50.2 & 0.12 & 917 & 56.4 & 18 & -0.07 \\
\hline $14: 24: 50: 96$ & 558 & 154 & 27 & 28 & 50.2 & 0.13 & 917 & 56.4 & 18 & -0.21 \\
\hline
\end{tabular}

